\title{
'Non senza scandalo delli convicini': pratiche musicali nelle istituzioni religiose femminili a Napoli 1650-1750
}

Angela Fiore

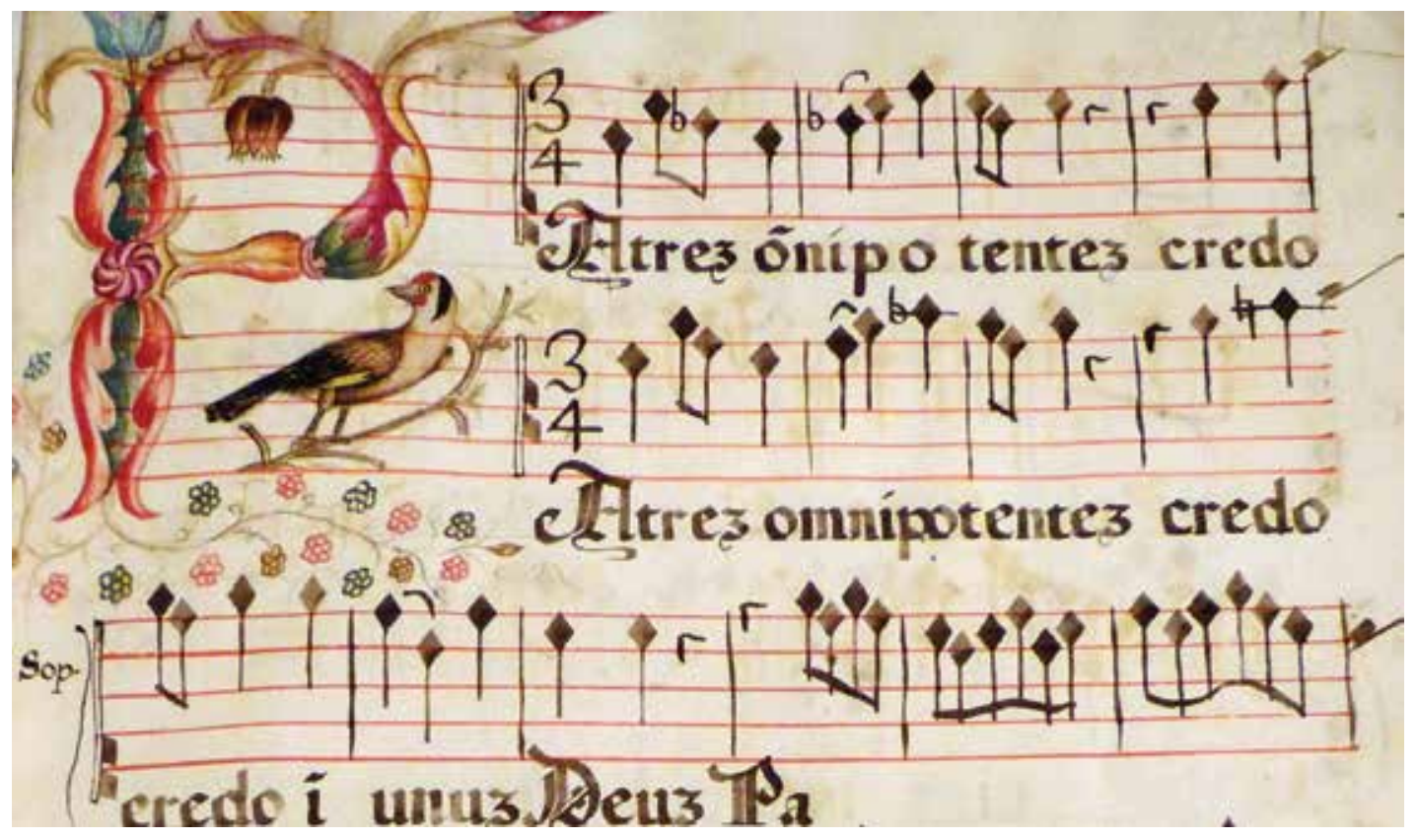

Peter Lang 


\section{SMG Schweizerische Musikforschende Gesellschaft \\ SSM Société Suisse de Musicologie \\ SSM Società Svizzera di Musicologia}

\section{Serie II - Vol. 58}

Le istituzioni femminili partenopee furono centri indiscutibili di cultura, fucine di mecenatismo, laboratori privilegiati di attività artistiche e di produzioni musicali e diedero un contributo incisivo alla vita culturale e artistica della Napoli vicereale. Monasteri e conservatori impiegarono nel corso dei secoli i musicisti più rappresentativi della città di Napoli a sostegno delle cerimonie liturgiche o per curare la formazione musicale delle religiose e delle figlie ospiti.

Il presente studio si propone di indagare e ricostruire le attività musicali di questi enti attraverso lo studio della documentazione proveniente dai principali archivi presenti nella città di Napoli. Le fonti d'archivio permettono infatti di comprendere quale funzione avesse l'arte musicale nella vita dei chiostri. La ricerca intende inoltre considerare la realtà monastica femminile nel suo insieme, esaminando la presenza delle comunità religiose nella città e il loro ruolo all'interno delle dinamiche della vita urbana. L'indagine condotta restituisce oggi una visione attendibile del fenomeno sonoro nel quotidiano delle realtà claustrali, portandoci a comprendere quanto le comunità monastiche femminili contribuirono allo sviluppo di tradizioni musicali, spettacolari, liturgiche.

Angela Fiore si è laureata in musicologia presso l'Università degli Studi di Pavia nel 2004 ed ha conseguito nel 2015 il dottorato di ricerca presso l'Università di Fribourg (Svizzera). Le sue ricerche si occupano prevalentemente di musica sacra del Sei e Settecento italiana ed europea. E' inoltre diplomata in violino e specializzata nel repertorio barocco. 
'Non senza scandalo delli convicini': pratiche musicali nelle istituzioni religiose femminili a Napoli 1650-1750 
Publikationen der Schweizerischen

Musikforschenden Gesellschaft

Publications de la Société Suisse

de Musicologie

Serie II - Vol. 58

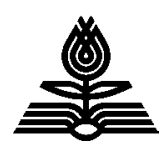

PETER LANG

Bern $\cdot$ Bruxelles $\cdot$ Frankfurt am Main $\cdot$ New York $\cdot$ Oxford $\cdot$ Warszawa $\cdot$ Wien 


\section{Angela Fiore}

\section{'Non senza scandalo delli convicini': pratiche musicali nelle istituzioni religiose femminili a Napoli 1650-1750}


Information bibliographique publiée par «Die Deutsche Nationalbibliothek» «Die Deutsche Nationalbibliothek» répertorie cette publication dans la «Deutsche Nationalbibliografie»; les données bibliographiques détaillées sont disponibles sur Internet sous ‘http://dnb.d-nb.des.

La prestampa e l'edizione digitale di questo volume sono state realizzate grazie al sostegno del Fondo Nazionale Svizzero per la ricerca scientifica (FNS). La pubblicazione del volume ha ricevuto il finanziamento della AMS 75 PAYS ENDOWMENT dell'American Musicological Society-NATIONAL ENDOWMENT for the HUMANITIES/ ANDREW W. MELLON FOUNDATION.

Tesi di dottorato presentata presso la Facoltà di Lettere dell'Università di FriburgoSvizzera, approvata dalla Facoltà di Lettere su proposta dei professori Luca Zoppelli (premier rapporteur) e Claudio Toscani (second rapporteur). Friburgo, 20 aprile 2015, il decano Prof. Marc-Henry Soulet.

In copertina: Messa a due voci. Pater Noster.

Biblioteca del Monastero di Santa Chiara (Napoli), 1723.

ISSN $1012-8441 \mathrm{br}$.

ISBN 978-3-0343-2340-6 br.

ISBN 978-3-0343-2348-2 eBook ISBN 978-3-0343-2349-9 EPUB

\section{PETER LANG}

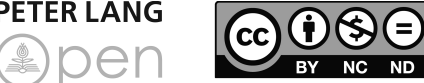

Open Access: Cette œuvre est mise à disposition selon les termes de la Licence Creative Commons Attribution - Pas d'Utilisation Commerciale - Pas de Modification 4.0. Pour consulter une copie de cette licence, visitez le site internet https://creativecommons.org/licenses/by-nc-nd/4.0/

(C) Angela Fiore, 2017

Peter Lang AG

International Academic Publisher

Bern 


\section{Indice}

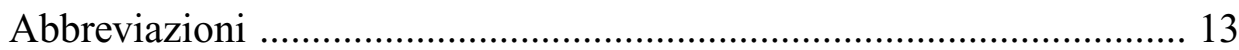

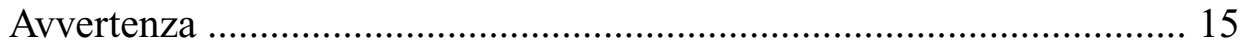

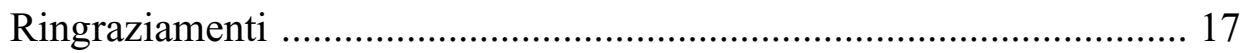

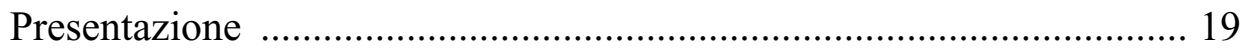

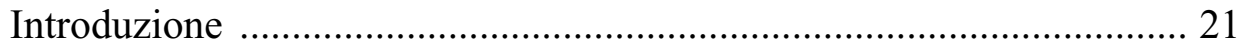

Parte I: La vita dei chiostri fra consuetudini e proibizioni .......................31

I. Il buon governo degli istituti ..................................................... 33

1. La riforma dei monasteri femminili ...................................... 33

2. Gli strumenti di controllo ................................................... 36

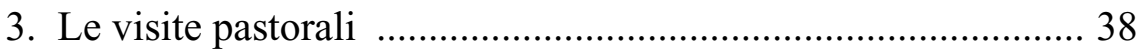

4. I sinodi e gli arcivescovi della Diocesi .................................. 40

5. Editti e lettere pastorali ......................................................... 44

II I monasteri e i legami con la città ................................................ 49

1. Norme, tempi e luoghi ......................................................... 49

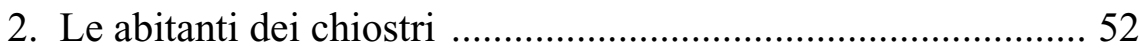

3. Città 3 aristocrazia e istituzioni regie .................................... 54

III. Liturgie ordinarie e straordinarie ............................................... 59

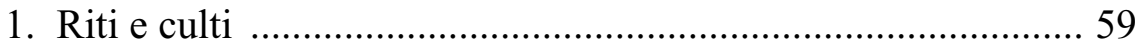

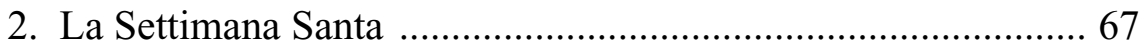

3. Liturgie 'straordinarie' ............................................................ 72

4. Le cerimonie di 'monacazione' .............................................. 75

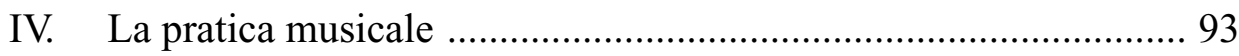

1. Maestri di musica e di strumento .......................................... 93

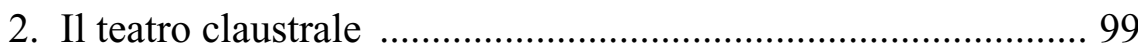

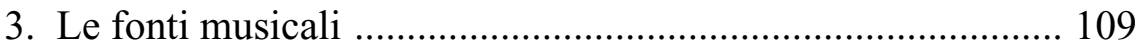




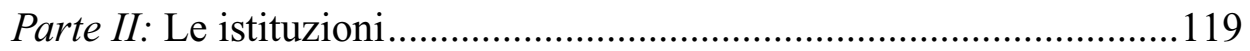

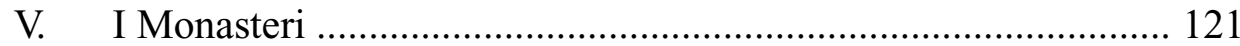

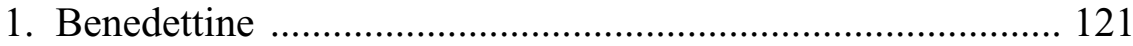

1.1 Ss. Marcellino e Festo ..................................................... 121

1.2 San Potito ........................................................................ 123

1.3 Santa Maria Donnalbina ............................................... 126

1.4 Santa Maria Donna Romita ............................................ 128

1.5 San Gregorio Armeno ....................................................... 131

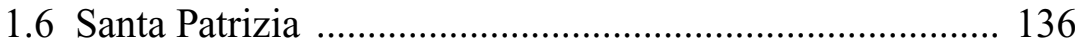

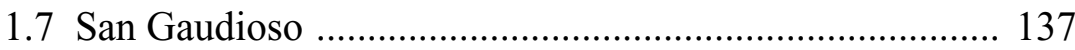

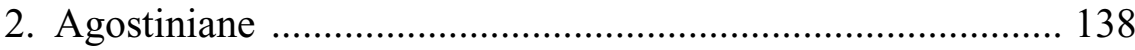

2.1 Santa Maria Egiziaca Maggiore a Forcella e Santa Maria Egiziaca a Pizzofalcone ............................. 138

2.2 San Giuseppe dei Ruffi .............................................. 141

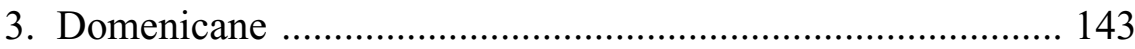

3.1 Santa Maria del Divino Amore ......................................... 143

3.2 Santa Maria Maddalena delle Spagnole .......................... 145

3.3 Santa Caterina da Siena ................................................ 146

3.4 Ss. Pietro e Sebastiano ...................................................... 146

3.5 Santa Maria della Sapienza .......................................... 148

4. Francescane ...................................................................... 149

4.1 Santa Maria Donnaregina …........................................... 149

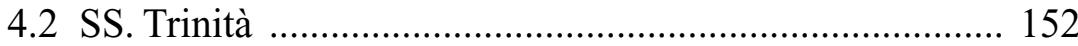

4.3 San Francesco delle Cappuccinelle ................................ 155

4.4 San Francesco dell'Osservanza ....................................... 156

4.5 Sant'Antonio a Port'Alba ............................................... 157

4.6 Santa Maria Maddalena Maggiore ................................. 157

4.7 Santa Maria della Provvidenza ai Miracoli .................... 159

5. Canonichesse Lateranensi .................................................... 161

5.1 Santa Maria Regina Coeli .............................................. 161

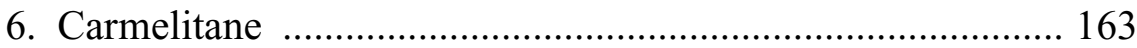

6.1 La Croce di Lucca .......................................................... 163

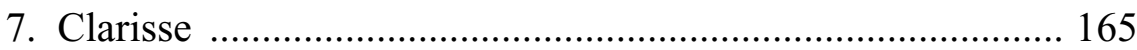

7.1 Santa Maria del Gesù ........................................................ 165

7.2 San Francesco degli Scarioni .......................................... 166 
VI. Il monastero delle clarisse di Santa Chiara ............................... 169

1. Il monastero e il suo archivio ............................................... 169

2. La vita musicale dell' istituto .............................................. 171

3. Quotidianità liturgica e prassi musicale ............................... 173

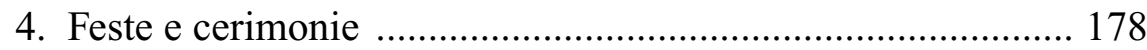

5. La venuta del nuovo $\operatorname{Re}$.................................................... 182

VII. I conservatori e gli istituti assistenziali .................................. 185

1. Uno sguardo d'insieme ........................................................ 185

2. L'educazione delle fanciulle .................................................. 186

3. Le diverse tipologie di istituti ............................................ 189

4. Conservatori napoletani e ospedali veneziani: un possibile

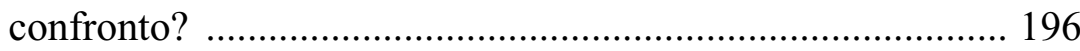

VIII. Il conservatorio di Nostra Signora della Solitaria ...................... 203

1. La fondazione ................................................................... 203

2. La Soledad e la Cappella Reale .......................................... 205

3. Le festività ....................................................................... 211

4. La formazione musicale ...................................................... 213

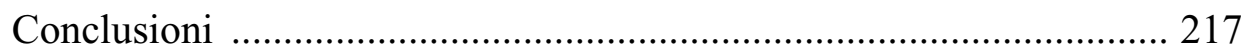

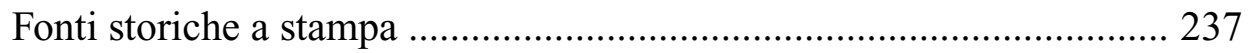

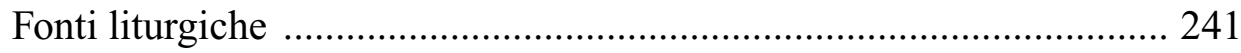

Bibliografia ............................................................................ 243

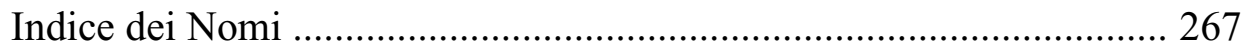





\section{Indice delle Tabelle}

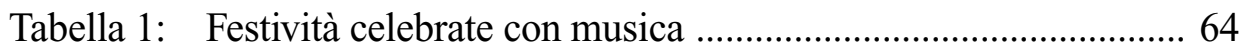

Tabella 2: Rito di consacrazione delle vergini ......................................... 79

Tabella 3: Ricostruzione dell'organico per la monacazione di donna Petronilla Chirico presso il conservatorio di Santa Maria

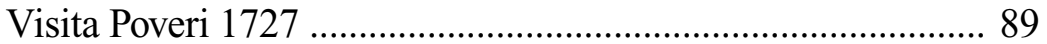

Tabella 4: Maestri di canto e di strumento ................................................. 97

Tabella 5: Fonti drammatiche dei chiostri napoletani fra XVII

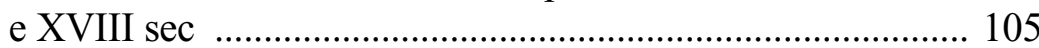

Tabella 6: Partiture dedicate a religiose ............................................... 115

Tabella 7: Partiture per monasteri .......................................................... 117

Tabella 11: Attività e maestranze musicali dei monasteri femminili napoletani XVII-XVIII ....................................................... 221

Tabella 12: Attività e maestranze musicali degli istituti di carità napoletani XVII-XVIII ....................................................... 232 



\section{Indice delle Illustrazioni}

1. Costituzioni dei monasteri del Divino Amore e di San Giuseppe dei Ruffi. Frontespizi .......................................................................... 50

2. Alessandro Speranza. Cinque antifone. Frontespizio .......................... 81

3. Alessandro Speranza, Posuit signum in faciem meam ......................... 82

4. Il trionfo della fede nel martirio di Santa Lucia, frontespizio del libretto a stampa ................................................................................ 104

5. Gaetano Veneziano, Zain. Repulit Dominus altare suum .................. 110

6. Gaetano Veneziano, Daleth. Tetendit arcum suum ............................ 111

7. Alessandro Speranza, La Gara tra la Fede e la Fortezza.

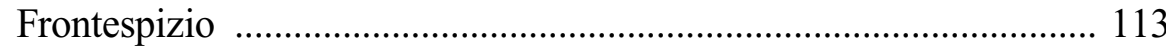

8. Alessandro Speranza, Sinfonia introduttiva ....................................... 114

9. Gaetano Manna, Inno della Gloriosa S. Maria Egiziaca ................... 141

10. Messa a due voci composta da suor Delia Bonito. Frontespizio ....... 175

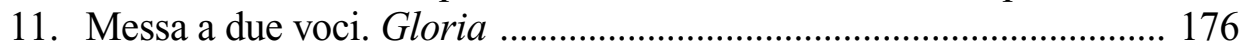

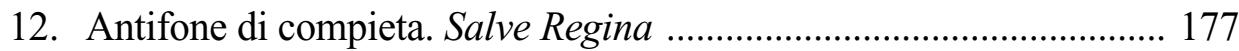

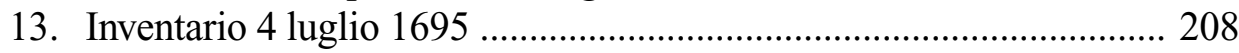

14. Libro Maggiore 1695-1703, 1/I/1697, c. 144v ................................ 215

15. La città di Napoli e le istituzioni religiose femminili ........................ 220 



\section{Abbreviazioni}

AsN - Archivio di Stato di Napoli

MS - Fondo Corporazioni Religiose Soppresse [Monasteri soppressi]

AsDn - Archivio Diocesano di Napoli

AsBN - Archivio Storico del Banco di Napoli

BPI - Banco della Pietà

BPO - Banco del Popolo

BPV - Banco dei Poveri

BSA - Banco del Salvatore

BSE - Banco di Sant'Eligio

BSG - Banco di San Giacomo

BSS - Banco dello Spirito Santo

S. Chitara - Biblioteca del monastero di Santa Chiara

Solitaria - Archivio Storico della Solitaria

Asv - Archivio Segreto Vaticano

GDN - Gazzetta di Napoli

BNN - Biblioteca Nazionale di Napoli 'Vittorio Emanuele'

I-Nc - Biblioteca del Conservatorio di Napoli San Pietro a Majella

Loreto - Fondo antichi conservatori: Santa Maria di Loreto

I-Nf - Archivio della congregazione dell'Oratorio dei Padri filippini [Girolamini] 



\section{Avvertenza}

$\mathrm{Al}$ presente volume è allegato un $\mathrm{CD}-\mathrm{ROM}$ nel quale è stata inserita l'appendice documentaria contenente lo spoglio di tutta la documentazione archivistica relativa alle istituzioni religiose femminili. Per ogni citazione presente nel testo si riporta in nota l'indicazione della fonte archivistica e il rimando all'appendice documentaria.

Nella trascrizione dei documenti ci si è attenuti agli aspetti grafici e fonetici dei manoscritti, limitando l'intervento allo scioglimento delle abbreviazioni e delle forme contratte più rare. Nel complesso, è stato seguito un criterio conservativo, che ha mantenuto gli errori di scrittura, punteggiatura, l'uso di maiuscole, accenti e virgole o le diverse versioni della stessa parola contenuta nel testo.

L'unità monetaria in uso nel viceregno napoletano era il ducato. Il ducato si divideva in 5 tarì, ognuno dei quali composto da 100 grana. Considerando i numerosi fattori e parametri di calcolo, è arduo ipotizzare il valore del ducato utilizzato fra XVII e XVIII sec. Tuttavia a titolo orientativo si propone il riferimento del valore del ducato a circa 30 euro odierni. 



\section{Ringraziamenti}

Il presente studio rappresenta il frutto della ricerca dottorale svolta dal 2011 al 2015 presso l'Istituto di Musicologia dell'Università di Friburgo condotta grazie al sostegno del Fondo Nazionale Svizzero per la ricerca scientifica.

Desidero esprimere la mia gratitudine a tutte le persone che hanno reso possibile a vario titolo la realizzazione della mia ricerca e la pubblicazione di questo volume. Rivolgo un sincero grazie al mio tutor dottorale Luca Zoppelli, nonché al correlatore Claudio Toscani, i cui consigli e suggerimenti hanno di certo reso migliore questo lavoro. Ringrazio inoltre la Società Svizzera di Musicologia per aver accolto questo studio nella collana Publications de la Société Suisse de Musicologie e per aver conferito alla tesi il premio Handschin 2016. È stato per me un grande onore ricevere tale prestigiosa onorificenza e un riconoscimento per tutto il lavoro svolto in questi anni.

Ringrazio ancora tutti i bibliotecari e gli archivisti incontrati, in particolar modo il personale dell'Archivio di Stato di Napoli e dell'Archivio Diocesano di Napoli, la Soprintendenza Archivistica della Campania, il conservatorio di Nostra Signora della Solitaria, il personale della Biblioteca di Santa Chiara di Napoli e della biblioteca del conservatorio San Pietro a Majella di Napoli.

Dedico questo volume alla mia famiglia, che in questo percorso ha saputo sempre sostenermi con amore, condividendo con me ogni momento di difficoltà e di gioia.

Angela Fiore

Padova, ottobre 2017 



\section{Presentazione}

Now that the study of musical practice in early modern female monasteries has extended over a generation of scholarship, it is a particular pleasure to welcome Angela Fiore's detailed work on some twenty-seven houses, plus the Conservatorio della Solitaria. Based on extensive archival research, and following on the work of historians such as Elisa Novi-Chavarria, Fiore is able to offer both a diachronic and citywide account of the conditions - teaching, liturgy, and not least role in civic ritual life — of music in these institutions, as well as detailed studies of each individual foundation.

In addition to these accounts of how female communities managed to maintain their traditions against a background of changing archiepiscopal decrees and the Spanish Habsburg-Austrian Habsburg-Bourbon transition in the city, Fiore also gives a comprehensive list of the sacred music written for monastic singers, by composers ranging from Caresana to Hasse to Leo to Porpora. By detailing these pieces for Mass, the Office, and other occasions, she is able to concretize at least some parts of what must have been a large repertory heard in urban houses. Furthermore, we gain a sense of individual voices by looking at the styles and pieces written for women in any given institution. Finally, her study contributes valuable new information on music education and the practice of teaching the figlie di coro inside houses, as well as the presence of the ducal Real Cappella in the liturgical life of the Solitaria as one of the Neapolitan conservatories. This monograph marks an important contribution to both religious history in the city as well as to the evergrowing panorama of nuns musicians in early modern Italy.

Robert L. Kendrick University of Chicago 



\section{Introduzione}

\section{Il contesto storiografico}

Negli ultimi decenni si è fatta strada l'esigenza di un dibattito attorno al ruolo culturale dei monasteri e del monachesimo femminile di età moderna. L'impulso della letteratura gender ha incrementato varie ed articolate ricerche in questo settore, portando alla luce un grande dinamismo delle realtà claustrali femminili in diversi contesti geografici. Attraverso il lavoro di storici come Gabriella Zarri, la storiografia monastica ha restituito centralità all'educazione conventuale femminile, allontanandosi da un'immagine negativa del monastero visto essenzialmente come luogo di reclusione. ${ }^{1}$

La storia dei monasteri è stata indagata spesso sotto diversi profili: da quello urbanistico a quello sociale, alla funzione culturale ed educativa svolta da questi istituti. Gli studi di Miller Lawrence, Kate Lowe hanno affermato la vivacità culturale delle comunità monastiche femminili, in riferimento al loro contributo nella committenza artistica, nella letteratura, nell'arte e nell'architettura. ${ }^{2}$

Per quanto concerne la ricerca musicologica, di fondamentale importanza sono stati gli studi di Craig Monson, legati all'attività del monastero bolognese di Santa Cristina e alla figura della monaca compositrice Lucrezia Orsini Vizzani. ${ }^{3}$ Essi sono stati lo spunto per analoghe ricerche su specifici ambienti monastici femminili legati a città del nord e del centro Italia. Di grande importanza il celebre Celestial sirens di Robert Kendrick, che ripercorre le attività musicali dei conventi milanesi e ne analizza le tradizioni musicali in relazione alle differenti occasioni liturgiche. Kendrick ha avuto il merito non solo di riscoprire la vitalità dei chiostri milanesi, ma anche di portare alla luce figure di monache compositrici come Chiara Margherita Cozzolani. Sono da citare ancora i contributi Colleen Reardon per Siena, Enrico Peverada per Ferrara che hanno ricostruito la storia delle istituzioni e la loro offerta musicale. ${ }^{4}$ L'ambiente veneziano ha beneficiato invece degli studi di Helen Geyer che si è lungamente occupata dell'attività delle 'figlie di coro' e della produzione musicale degli ospedali veneziani. Anche Pier Giuseppe Gillio ha dedicato una ricerca ventennale ai quattro celebri ospedali. Attraverso materiale

1 ZARri/Pomata 2000; Zarri 1986; Zarri 1990; ZarRi 1997.

2 Grieco/Zarri 2000; Miller LaWrence 1996; Lowe 2004.

3 Monson 1995; si veda anche Monson 1993, pp. 143-160.

4 Dedicati all'ambiente milanese sono Kendrick 1996 e Kendrick 2002; per Siena Reardon 2002 e Reardon 2005; per Ferrara Peverada 1991 e Peverada 1997; per Venezia oltre a Gillio 2006 si rimanda anche agli studi di Geyer/Osthoff 2004; Over 1998. 
d'archivio inedito, Gillio ha ricostruito l'organizzazione interna degli istituti e ha mostrato i molteplici aspetti dell'attività musicale dei cori.

Minori, ma non per questo secondarie, sono le indagini sulle realtà monastiche del Sud Italia. Sono da citare gli studi ad opera di Maria Grazia Melucci, Angela Morgese e Annamaria Bonsante sulle benedettine del monastero pugliese di San Lorenzo, grazie ai quali è stata riscoperta e valorizzata una delle principali collezioni di musica monastica femminile esistente. ${ }^{5}$

Il dibattito storiografico sulle comunità monastiche del Meridione ha prodotto contributi anche riguardo Napoli. Un discreto numero di studiosi ha indagato singoli aspetti della storia delle istituzioni napoletane. Ricordiamo principalmente la monografia di Carla Russo basata sulla ricostruzione della vita interna dei monasteri femminili di Napoli nel Seicento, attraverso le visite pastorali dell'arcivescovo Filomarino. ${ }^{6}$ Di grande importanza sono poi gli studi di Giuliana Boccadamo relativi alla storia delle istituzioni caritative dell'Italia meridionale e alle forme e contenuti dell' istruzione religiosa. ${ }^{7}$ Da citare ancora Adriana Valerio che si impegna da tempo nella ricostruzione della memoria delle donne nella storia del cristianesimo e dell'esperienza religiosa femminile nel Mezzogiorno di età moderna. Si sottolinea soprattutto il contributo della Valerio sulla vita religiosa e culturale del monastero di San Gregorio Armeno e sulla storia del sito archeologico sul quale la celebre istituzione si è sviluppata. ${ }^{8}$ Dedicato invece ai cambiamenti dell'architettura conventuale napoletana a seguito del Concilio di Trento il volume Invisible City di Helen Hills. L'analisi meticolosa della Hills fornisce interessanti riflessioni sulle strutture familiari e sulla rete di relazioni sociali del monachesimo femminile partenopeo. ${ }^{9}$ I chiostri partenopei intesi come «spazi sociali ed economici aperti» sono oggetto di alcune pubblicazioni di Elisa Novi-Chavarria volte a mostrare gli stretti legami esistenti fra le comunità cittadine, le élites dirigenti, e gli istituti monastici femminili. ${ }^{10}$ Citiamo infine Vittoria Fiorelli che, da diverse angolature, ha indagato i tratti della religiosità al femminile e il ruolo delle donne e delle istituzioni nel contesto cittadino e in periferia. ${ }^{11}$

Dunque, anche in ambito napoletano il monachesimo risulta essere significativo per l'affermazione sociale e culturale della donna.

5 Cfr. Melucci/Morgese 1993; Bonsante/Pasquandrea 2010.

6 Russo 1970.

7 BocCadamo 1996; BocCadamo 1999; BocCadamo 2001.

8 Galasso/Valerio 2001; Valerio 2006; Valerio 2007; Spinosa/Pinto/Valerio 2013.

9 HiLls 2004.

10 Novi-Chavarria 1993, pp. 84-111; Novi-Chavarria 1997, pp. 339-367; Novi-Chavarria 2001.

11 Fiorelli 2001; Fiorelli 2003. Si veda anche Illibato 1985; Facchiano 1992; Hills 2004. Si rimanda alla bibliografia per l'elenco completo degli studi sulle istituzioni monastiche napoletane. 
La letteratura musicologica relativa alla storia della musica napoletana ha invece trascurato l'apporto di questa tipologia di istituzioni. Di certo, il difficile reperimento delle fonti e l'inaccessibilità di alcuni archivi, hanno frenato nel corso degli anni studi approfonditi. Le stesse fonti scritte di musica sacra - abbondatissime nella Napoli dei sec. XVII-XVIII - sono spesso prive di qualsiasi riferimento su usi e appartenenza istituzionale, e non possono dunque, da sole, far presagire quale tipo di attività musicale si celasse all'interno dei chiostri.

Alcune indagini sono state condotte da Carla Conti che, nel volume Nobilissime allieve, racconta della pratica musicale al femminile nella Napoli tra Sette e Ottocento, dedicando un contributo anche agli spazi claustrali. La Conti si sofferma principalmente su alcune figure femminili di particolare rilevanza come le sorelle Capece Minutolo, che nel tempo crearono e conservarono un vasto patrimonio della cultura musicale, poi donato al Conservatorio San Pietro a Majella di Napoli. ${ }^{12}$

Un breve ma significativo tentativo di indagine sui documenti del fondo $\mathrm{Mo}$ nasteri Soppressi dell' Archivio di Stato di Napoli, venne intrapreso intrapreso nel 2005 dal "Gruppo di lavoro Napoli” dell'Università Ca' Foscari di Venezia, team coordinato da Dinko Fabris e David Bryant che portò alla luce una serie di informazioni inedite sull'utilizzo della musica nei monasteri partenopei sia femminili che maschili. ${ }^{13}$ Questa tipologia di lavoro a campionatura, pur non consentendo di ricostruire pienamente la vita reale che doveva animare i monasteri femminili, ha proposto sufficienti elementi per intuire quanto essa fosse segnata da complesse dinamiche di relazioni sociali, culturali e religiose.

Tuttavia poco è stato fatto per illuminare, nelle forme di una descrizione densa, il paesaggio musicale diffuso della città, il suo quotidiano, le implicazioni funzionali dell'attività sonora nelle decine di istituzioni che le davano forma, secondo una prospettiva metodologicamente più aggiornata. Mancano lavori di ampio respiro che analizzino le istituzioni ecclesiastiche e la loro organizzazione, sia dal punto di vista culturale sia da quello delle inquietudini religiose che influenzarono la vita delle stesse istituzioni.

Il presente studio, condotto grazie al sostegno del Fondo Nazionale Svizzero, ha quindi lo scopo di approfondire, indagare e ricostruire le vicende musicali delle diverse tipologie di istituzioni religiose femminili napoletane, attraverso una ricerca più mirata e consapevole. La delimitazione temporale scelta - 1650/1750 - ha inteso coprire un periodo nevralgico della storia di Napoli, nel quale il viceregno passò dal dominio spagnolo a quello austriaco, per poi divenire regno autonomo: il rapporto fra gli elementi di discontinuità e la longue durée in ambito politico

12 Conti 2003.

13 Si ringrazia David Bryant per avermi concesso di consultare tutta la documentazione relativa al progetto "Gruppo di lavoro Napoli”, in buona parte pubblicata in BRYANT/QUARANTA 2005. Nell'appendice documentaria verrà fatto esplicito riferimento alle unità archivistiche consultate dal gruppo dell'Università Ca' Foscari di Venezia. 
e culturale sono infatti particolarmente interessanti. Inoltre, da metà Seicento a metà Settecento furono celebrati a Napoli numerosi sinodi diocesani alla luce dei quali leggere i mutamenti di vita e concezione religiosa. Inoltre, un approccio pluridisciplinare ha permesso un ampliamento delle prospettive: l'incrocio fra storia, antropologia, storia dell'arte, storia della liturgia e delle istituzioni religiose con la storia della musica ha consentito di contestualizzare i diversi aspetti del vissuto monastico, chiarire l'organizzazione del tempo e dello spazio e comprendere quale peso avesse la musica nella quotidianità di queste istituzioni.

\section{Fonti e metodologie}

La ricerca si è concentrata non tanto sulle fonti scritte della musica, quanto piuttosto sulla documentazione d'archivio, capace di registrare puntualmente il sistema di consumo in funzione del quale hanno origine gli stessi repertori musicali. Le fonti d'archivio, testimoni dei meccanismi di produzione e committenza, permettono di osservare l'incidenza delle istituzioni sacre sul circuito spettacolare cittadino e comprendere quale funzione avesse l'arte musicale nella vita di questi istituti.

Il lavoro ha avuto inizio attraverso un'individuazione degli istituti religiosi femminili presenti nella città di Napoli, mediante la verifica delle fonti archivistiche e bibliografiche, delle piante urbane storiche e dell'osservazione diretta del territorio.

Alcune informazioni sulla storia dei chiostri partenopei si trovano nelle guide e nelle cronache dell'epoca come Carlo Celano, Carlo De Lellis e nei giornali di Napoli di Innocenzo Fuidoro e Domenico Confuorto. ${ }^{14}$ Queste fonti fanno però riferimento, quasi esclusivamente, alla descrizione storico-artistica delle istituzioni, lasciando solo in qualche caso trapelare un'attività musicale pubblica, legata soprattutto alle funzioni liturgiche.

Fonti di particolare importanza che hanno permesso l'identificazione degli istituti "musicalmente" attivi, sono state le cedole di pagamento dei giornali copiapolizze dei sette antichi istituti di credito napoletani custoditi oggi presso l'Archivio Storico dell'Istituto Banco di Napoli. ${ }^{15}$ Esse documentano le fedi di credito e le

14 Celano 2001; Confuorto 1930; D’Engenio 1624; Fuidoro 1943. Si rimanda alla bibliografia dove sono segnalate tutte le fonti storiche prese in considerazione.

15 Lo spoglio di alcuni giornali copiapolizze dell'Archivio Storico del Banco di Napoli è stato oggetto di un progetto di ricerca promosso dalla Fondazione Pergolesi Spontini di Jesi nel 2007. Il gruppo di ricerca, cui io stessa ho preso parte, è stato coordinato da Francesco Cotticelli e Paologiovanni Maione ed ha portato avanti e concluso un progetto che aveva avuto inizio già nel 1999 per iniziativa di Francesco Degrada volto allo studio dei materiali di interesse teatrale e musicale del decennio 1726-1736 desunti dai giornali di cassa dei sette antichi istituti di credito napoletani. I risultati del progetto sono stati pubblicati in CotTiCelli/Maione 2015. Si rimanda inoltre ai due studi precedentemente apparsi e dedicati allo spoglio dei giornali copia- 
polizze utilizzate per i pagamenti da istituzioni, privati, enti laici ed ecclesiastici. Nelle polizze i clienti usavano specificare la causale del pagamento, consentendoci dunque di ottenere dati significativi circa l'attività di molte istituzioni della città, fra le quali anche i monasteri e i conservatori. Dallo spoglio delle carte si riscontrano infatti molti riferimenti ad un'importante 'tradizione' musicale dei monasteri sia pubblica che privata.

Necessaria è stata poi una classificazione delle diverse categorie di istituzioni: monasteri, conservatori, ospedali, collegi, ritiri. La terminologia utilizzata dalle fonti archivistiche, e in alcuni casi dalla stessa storiografia dell'epoca, non aiuta a designare e distinguere le diverse realtà, ma genera, al contrario, un'ambiguità di fondo: il termine 'monastero' è infatti utilizzato per ogni tipologia di istituto. Probabilmente questo accadeva perché anche gli istituti assistenziali, senza obbligo di clausura, adottavano regole e schemi monastici per la tutela e l'educazione delle figlie che ospitavano.

Le istituzioni sono state quindi suddivise in due categorie: con e senza obbligo di clausura. Alle istituzioni con obbligo di clausura appartengono i monasteri. Sono stati individuati 27 monasteri dei seguenti ordini monastici: sette istituti di benedettine, sette di francescane, cinque di domenicane, tre di agostiniane, tre di clarisse, uno di canonichesse laternanensi, uno di carmelitane.

Le istituzioni senza obbligo formale di clausura sono invece i conservatori, $i$ ritiri, gli educandati, i collegi, gli ospedali. Si tratta di istituti che vengono definiti 'assistenziali' o 'caritativi'. Essi affiancarono i monasteri con lo scopo di accogliere e istruire fanciulle bisognose. Ciascun conservatorio aveva una finalità ben precisa: vi erano enti destinati a ragazze disagiate, alle orfane con natali onorati, a ragazze povere o semplici educande di particolari corporazioni di arti e mestieri. Sedici sono gli istituti di assistenza di cui abbiamo notizie relativamente alla loro attività formativa e musicale.

Se da un lato è necessario studiare il monachesimo femminile senza porre recinti terminologici troppo rigidi, d'altra parte, bisogna fare luce e chiarire quali erano gli ambiti di azione e le differenze fra le diverse tipologie di istituzioni. La difficoltà nel differenziare $\mathrm{i}$ vari istituti ha ostacolato, anche dal punto di vista musicologico, la comprensione della loro finalità. Pensiamo ad esempio ai conservatori femminili napoletani, realtà completamente dimenticata dagli storici della musica. Chiarire che le istituzioni assistenziali, differentemente dai monasteri, avevano come scopo primario l'accoglienza e la formazione di giovani donne, cambia notevolmente lo scenario dell'insegnamento della musica a Napoli e ne

polizze dell'Asbn: Maione 2000, pp. 1-129; Cotticelli/Maione 2006, pp. 21-54. Si ringrazia Paologiovanni Maione e Francesco Cotticelli per aver permesso la pubblicazione parziale dei documenti connessi alle istituzioni religiose femminili desunti dal progetto promosso dalla Fondazione Pergolesi Spontini. 
deriva, di contro, un accostamento e una comparazione alle omonime realtà maschili maggiormente conosciute.

Lo studio delle fonti archivistiche ha poi previsto lo spoglio sistematico dei documenti delle singole istituzioni custoditi presso il fondo Corporazioni Religiose Soppresse dell'Archivio di Stato di Napoli. La soppressione degli ordini e delle corporazioni religiose nel regno di Napoli avviata nel decennio francese e sancita definitivamente da regio decreto nel 1866, comportò, durante i primi anni dell'Ottocento, la dispersione e la distruzione, di gran parte degli archivi appartenenti agli ordini monastici in tutto il Sud. Tuttavia a Napoli, il trasferimento negli archivi dello Stato della documentazione delle corporazioni religiose soppresse, ne ha consentito in molti casi la sopravvivenza.

Si tratta di documentazione in massima parte contabile: rendiconti dell'amministrazione, libri maggiori, registri di introito ed esito, conti di chiesa e di sacrestia, a cui si aggiungono fasci miscellanei composti da documenti di vario tipo. Lo studio della descrizione di tutte le entrate percepite dalle diverse istituzioni e il confronto fra relative somme e le corrispondenti voci di spesa, ha permesso di portare alla luce numerose informazioni su usi e consuetudini della vita claustrale. La documentazione del fondo Corporazioni Religiose Soppresse per alcune delle istituzioni indagate è molto scarsa (ad esempio il gruppo degli istituti caritativi); in altri istituti invece è abbastanza lacunosa per il periodo preso in esame, e dunque si è ritenuto opportuno ampliare in questi casi l'arco temporale, in modo da comprendere se in anni precedenti o posteriori vi fosse comunque traccia di una qualche attività musicale. Ugualmente per alcune istituzioni in cui si riscontra una vivace attività musicale su più fronti, si è creduto opportuno studiarne l'evoluzione su di un periodo più esteso.

Parallelamente è stato condotto lo spoglio di alcuni fondi dell'Archivio Diocesano di Napoli. In primo luogo i resoconti delle Sante Visite contenuti nei Libri Visitationum Monialium Monasteriorium Civitatis Neapolitanae. Si tratta delle relazioni delle visite pastorali effettuate dai cardinali di Napoli fra XVII e XVIII sec. Esse danno un quadro abbastanza chiaro degli aspetti meno conosciuti della vita claustrale e forniscono indicazioni riguardo le disposizioni disciplinari che potevano seguire la visita nelle istituzioni più ribelli. ${ }^{16}$ Le informazioni desunte dalle visite pastorali sono state poi confrontate con la documentazione del fondo Pastorali e Notificazioni, ovvero decreti, editti, disposizioni, proibizioni e lettere pastorali che i diversi arcivescovi inviarono nel corso dei secoli a tutte le istituzioni femminili. Inoltre sono stati studiati gli atti dei Sinodi Diocesani, un imponente complesso di norme che stabiliva la disciplina della vita monastica.

16 Alcuni verbali delle Sante Visite si trovano anche presso la Biblioteca Nazionale di Napoli, ms. XI.E.29: Acta visitationis monasteriorum sanctimonialium neapolitanarum, anno D.ni 1642; altri atti si trovano nelle carte del fondo Vicario delle Monache, infine alcuni verbali sono presenti nella documentazione dei singoli monasteri presso il fondo Monasteri soppressi dell'Archivio di Stato di Napoli. 
La documentazione più cospicua indagata presso l'Archivio Diocesano e che ha maggiore rilevanza per lo studio delle istituzioni religiose femminili corrisponde al fondo Vicario delle Monache, relativo all'ecclesiastico che su delega dell'arcivescovo trattava ordinariamente relazioni e problematiche con i diversi monasteri della città. Il fondo, suddiviso in tre sezioni denominate Esplorazioni (esame della volontà delle monacande), Badesse (relativo alla nomina delle badesse) e Miscellanea, contiene notizie di grande interesse soprattutto in quest'ultima sezione, riguardante documenti vari sulla fondazione dei monasteri, sull'ingresso delle future monache in clausura, atti amministrativi, corrispondenza, permessi. Alle sopracitate fonti si è ritenuto poi opportuno aggiungere la consultazione del fondo dedicato agli Arcivescovi, contenente le scritture private intercorse fra arcivescovi e autorità religiose e civili, in cui si trovano notizie fondamentali per le attività religiose e anche di grande rilevanza per la storia delle istituzioni; e infine i Diari dei Cerimonieri, concernenti le cerimonie svolte nelle principali chiese e monasteri della città alla presenza dell'arcivescovo, con riferimenti a particolari liturgie solenni come i riti di professione monastica.

Alla documentazione archivistica del fondo Monasteri soppressi e dell'Archivio Diocesano sono stati aggiunti alcuni fondi di archivi privati quali quelli del conservatorio di Nostra Signora della Solitaria e del monastero di Santa Chiara. La possibilità di consultare i fondi archivistici privati ha consentito un'analisi più attenta e particolareggiata di queste due istituzioni che risultano essere le realtà monastiche più interessanti della città. La documentazione indagata per entrambe le istituzioni, restituisce numerose informazioni sulla pratica musicale legata alle celebrazioni ufficiali, alla "ricreazione" personale e alla formazione musicale.

Inoltre per la ricostruzione di questa complessa realtà, mi sono avvalsa delle cronache della «Gazzetta di Napoli», strumento fondamentale per la conoscenza della vita e delle attività musicali della città fra XVII e XVIII secolo. La settimanale «Gazzetta di Napoli» iniziò le sue pubblicazioni nel 1675, e svolse la funzione di organo ufficiale del governo dal 1675 fino al 1768, quando il periodico assunse una diversa fisionomia e un'intestazione propria (quella di «Foglio ordinario»). Tale periodico è prodigo di notizie riguardanti la musica e lo spettacolo, essendo riportata in esso la cronaca degli eventi "spettacolari" della città molti dei quali legati alle istituzioni religiose. Lo spoglio delle cronache della «Gazzetta», relativamente alle notizie di interesse musicale, è stato pubblicato da Ausilia Magaudda e Danilo Costantini nel 2009. ${ }^{17}$

17 Ausilia Magaudda e Danilo Costantini hanno localizzato le gazzette superstiti e individuato tutte le cronache con contenuti musicali nel periodo 1675-1768: si tratta di 3.188 notizie relative alla città e provincia di Napoli, e 1. 526 relative agli altri centri del Regno di Napoli. Nei prossimi capitoli, così come nell'appendice documentaria, saranno dunque citati i riferimenti relativi ai monasteri femminili estrapolati dalla «Gazzetta di Napoli» e pubblicati in MAGAUDDa/Costantini 2009. 
L'approfondita conoscenza del sistema di consumo ha consentito, nell'ultima fase di studio, di gettare nuova luce sulla musica appositamente prodotta per l'utilizzo nell'ambito del sistema. Purtroppo i fondi documentari dei monasteri dei diversi archivi cittadini non custodiscono fonti musicali. La maggior parte della produzione musicale sacra napoletana è ancora oggi conservata presso l'Archivio dei Girolamini, chiuso al pubblico da oltre trent'anni. L'archivio possiede buona parte delle composizioni di autori come Cristofaro Caresana, Gaetano Veneziano, Gennaro Ursino, etc. maestri di musica e di cappella in diverse istituzioni cittadine. È stata dunque condotta un'indagine sul fondo dei Girolamini attraverso l'unico catalogo esistente, elaborato nel 1918 da Salvatore Di Giacomo. Parallelamente sono stati consultati alcuni manoscritti digitalizzati, appartenenti allo stesso fondo musicale dei Girolamini, e consultabili oggi online. Questo ha permesso di individuare una serie di composizioni destinate all'ambiente claustrale femminile.

\section{Elementi di novità}

Il presente studio ha voluto considerare la realtà monastica femminile nel suo insieme, esaminando la presenza delle comunità religiose nella città e il loro ruolo all'interno delle dinamiche della vita urbana, sia dal punto di vista socioistituzionale, sia dal punto di vista culturale. In questo senso, l'analisi della situazione e del sistema dei monasteri femminili contribuisce a ricostruire tasselli meno noti della storia della città e a riscoprire una parte significativa della storia della musica napoletana in cui le donne hanno avuto un ruolo di primo piano. Fondamentale ai fini della ricostruzione del contesto nel quale queste istituzioni si mossero è l'analisi del rapporto fra autorità religiose e istituzioni femminili in relazione alla pratica musicale. I conflitti tra religiose e gerarchie ecclesiastiche caratterizzano tutto il periodo in questione anche perché l'attuazione della riforma era soggetta a svariate interpretazioni individuali, a pressioni politiche, al gusto delle famiglie. Le autorità ecclesiastiche ritenevano che la musica potesse entrare in conflitto con la morale e ne contrastavano dunque una pratica troppo intensa. Le restrizioni proibivano la polifonia, il canto figurato, il possedere strumenti musicali nelle celle, accogliere insegnanti di musica esterni. Restrizioni che non si ritrovano nelle stesse tipologie di istituzioni monastiche maschili e che venivano puntualmente violate, grazie anche al sostegno delle famiglie aristocratiche di provenienza. Le grate dei parlatori non servirono ad evitare contatti con il mondo esterno, anzi l'alto livello qualitativo della musica prodotta, commissionata ed eseguita presso le istituzioni femminili fu tale da essere spesso oggetto di cronache coeve.

Se da un lato la clausura ebbe ricadute nell'organizzazione della vita monastica, dall'altro i legami che le istituzioni femminili mantennero con la vita civica e gli usi rappresentativi del patriziato napoletano sono testimonianza di quanto la rifor- 
ma non fosse riuscita a porre fine al controllo delle famiglie. Gli istituti di maggior prestigio erano retti da badesse provenienti da famiglie legate all'aristocrazia. La famiglia destinava parte dei propri beni al sostegno o alla creazione di una comunità monastica e controllava, in tal modo, attraverso le proprie figlie, non soltanto i chiostri, ma anche grandi patrimoni e strategici nodi politici e istituzionali. Le religiose tendevano a fare dei loro cenobi dei centri culturali oltre che spirituali, mentre $\mathrm{i}$ vincoli familiari permettevano loro di svolgere un ruolo importante nel contesto pubblico e cittadino. Sotto questo profilo le donne furono abilitate a giocare un ruolo di primo piano nella storia sociale, religiosa e culturale della città. In questo scenario la musica rappresenta uno dei punti di contatto fra comunità e vita cittadina, ed è attraverso la committenza artistica, l'organizzazione di liturgie e cerimonie, che le monache napoletane mostrano una volontà di interagire attivamente con la città.

Una delle caratteristiche più sorprendenti è la commissione e il consumo di musica ad opera dei monasteri. L'anno liturgico di ciascun istituto era costellato da numerose devozioni: tridui per Santi patroni, novene e ottavari, liturgie di professione religiosa, cerimonie 'straordinarie' per vittorie, nascite, matrimoni degli esponenti dell'aristocrazia, andavano ad aggiungersi alle festività canoniche. Si pensi quindi alla quantità smisurata di musica prodotta e commissionata da queste istituzioni. L'organizzazione musicale delle celebrazioni cambiava a seconda del prestigio economico dei culti, dell'importanza degli istituti e delle famiglie nobiliari che spesso li patrocinavano. Pertanto vi erano realtà che beneficiavano del sostegno dei grandi complessi musicali cittadini come la Cappella musicale della Cattedrale o la Cappella Reale, altre invece che riunivano pochi cantori e strumentisti a sostegno delle liturgie. Solo il confronto fra fonti liturgiche, documentazione d'archivio, cronache e testimoni musicali permette oggi la comprensione dell'impiego che veniva fatto di musica e musicisti e la ricostruzione di tempi e azioni di alcune liturgie.

Le numerose istituzioni religiose femminili accolsero nei secoli uno stuolo di musicisti tra i più rappresentativi della scena musicale partenopea, ed ebbero pertanto un ruolo centrale nella circolazione di tante maestranze musicali. La documentazione archivistica rivela nomi, ruoli e paghe assunte per lo svolgimento degli incarichi, restituendoci un quadro estremamente vario dell'attività musicale napoletana. I musicisti attivi presso i monasteri avevano principalmente il compito di sostenere con la musica le numerose devozioni e le funzioni più importanti dell'anno liturgico. Negli istituti assistenziali, come i conservatori, i musicisti erano chiamati anche a provvedere alla formazione musicale di religiose ed educande. Accanto a nomi prestigiosi quali Marchitelli, Caresana, Veneziano, Feo, Durante, si trovano anche nomi di artisti meno noti in qualità di maestri di musica, canto o di un qualche strumento.

Infine, la disciplina musicale poteva avere funzione anche di semplice divertimento grazie ai 'trattenimenti', 'ricreazioni musicali' e alle svariate forme di 
rappresentazioni sceniche organizzate in occasioni festive. Anche a Napoli, così come in altri contesti europei, troviamo diversi esempi di 'teatro claustrale', che prevedevano in alcuni casi la partecipazione attiva delle stesse religiose.

L'idea alla base di questa indagine non è stata tanto quella di ricostruire in senso prettamente documentario una serie di piccoli quadri a sé stanti su ognuna delle istituzioni cittadine indagate, ma piuttosto di determinare quale sia stato il contributo fornito dal complesso delle istituzioni alla vita musicale cittadina attraverso il significato della tradizione e della consuetudine. Contemporaneamente si è cercato di approfondire il ruolo degli ordini femminili nella produzione e nella committenza musicale, e di converso, sul piano dell'antropologia e della storia culturale, i modi attraverso cui l'attività musicale organizza la presenza e la funzione femminile nella società. L'analisi delle consuetudini di produzione e consumo ci dona una visione attendibile del fenomeno sonoro nel quotidiano delle realtà femminili e nel quadro cittadino, portandoci a comprendere quanto le comunità monastiche femminili contribuirono allo sviluppo di tradizioni musicali, spettacolari, liturgiche. I monasteri rappresentarono una parte soltanto apparentemente secondaria nella storia musicale della città e per tale motivazione è necessario riscoprirne il ruolo culturale centrale. 
Parte I

La vita dei chiostri fra consuetudini e proibizioni 



\section{Il buon governo degli istituti}

\section{La riforma dei monasteri femminili}

I monasteri femminili napoletani furono luoghi privilegiati per assicurare un onorato collocamento alle figlie della nobiltà destinate, per vocazione o per scelta familiare, alla vita del chiostro piuttosto che all' istituto matrimoniale. La vita religiosa era una condizione spesso imposta, e pertanto la monacazione era in molti casi una parte essenziale delle strategie familiari di conservazione e arricchimento del patrimonio. ${ }^{1}$ Le fanciulle erano fatte entrare nel monastero ancora bambine, molto prima che potessero prendere i voti solenni e, in cambio del versamento della dote monastica, ricevevano un certo grado di istruzione. I monasteri erano assimilabili a degli educandati, in cui l'applicazione delle regole era abbastanza elastica.

La vita che si conduceva in monastero agli inizi del XVI secolo era infatti abbastanza libera e l'osservanza della clausura moderata: si ricevevano visite, era possibile uscire dai chiostri, si amministravano beni personali. Alcuni monasteri, pur avendo adottato la clausura ai tempi della fondazione, l'avevano poi col tempo abbandonata, allontanandosi dagli ideali che ne avevano ispirato la costituzione. ${ }^{2}$ Col tempo inoltre, le attività di lavoro femminile, le spese superflue, il lusso nelle cerimonie o gli svaghi, avevano indotto le religiose a violare i principi fondanti della clausura e aggirare la limitazione dei rapporti con il mondo esterno, facendo sì che il posto riservato alla preghiera fosse tutt'altro che prioritario.

Come in altre parti d'Italia, anche nel viceregno napoletano, si era poco per volta diffusa la contrapposizione tra monasteri 'chiusi', che seguivano strettamente le norme claustrali, e i monasteri 'aperti', dove invece le religiose risiedevano nei propri lussuosi appartamenti, protette da reti di relazioni con l'esterno. ${ }^{3}$ Pochi i monasteri napoletani che godevano di una buona reputazione. Le carte degli arcivescovi napoletani ne indicano all'incirca tre: la Sapienza, San Giovanni Battista, Santa Maria di Gerusalemme. Uno dei monasteri più prestigiosi e indisciplinati, maggiormente messo sotto tiro dalle autorità ecclesiastiche fu quello delle clarisse di Santa Chiara. Le clarisse si distinsero nel corso dei secoli per la magnificenza e il benessere della vita che conducevano dietro le grate e il loro comportamento fu spesso oggetto di interventi riformatori. Già nel 1536, padre Giacomo d'Ancona,

1 Sulle monacazioni forzate cfr. CANosa 1991, pp. 133-136; ZARri 1997; Novi-ChavarRia 2001, pp. 67-70; PAOLIN 1996; Monson 1992, pp. 15-47.

2 Prima del concilio la clausura non era prevista né applicata da tutti gli ordini monastici, si veda Miele 2001, pp. 91-138.

3 Sulla contrapposizione fra monasteri aperti e chiusi si veda CREYTENs 1965, pp. 45-84. 
generale dei convenutali incaricati della guida spirituale dell'istituzione, lamentava il carattere mondano delle cerimonie organizzate dalle clarisse, specificando, ad esempio, che durante la festa del Corpus Domini troppi secolari entrassero nel monastero di Santa Chiara dove si faceva "troppa musica". ${ }^{4}$

La reintroduzione della clausura rappresentò quindi l'elemento essenziale del programma di riforma dei monasteri proposto dal Concilio di Trento. I padri conciliari si occuparono infatti specificamente della questione monastica attraverso il Decretum de regularibus et monialibus, ${ }^{5}$ un documento che ribadiva l'osservanza dei tre voti di povertà, castità e obbedienza e mirava all'applicazione dell'istituto claustrale a tutte le comunità femminili. La clausura venne così imposta obbligatoriamente alle monache di qualunque ordine, anche lì dove non era mai stato previsto nella regola originaria e nelle costituzioni dei singoli istituti. L'obiettivo era quello di agire sulla disciplina interna dei chiostri infrangendo così circuiti e legami tra professe e gruppi parentali. Le nuove norme tridentine furono diffuse con la costituzione Circa pastoralis emanata da Papa Pio V nel $1566 .{ }^{6}$ Il primo risultato pratico che si ottenne a Napoli, fu la chiusura di alcuni monasteri ritenuti inadatti alla vita claustrale. ${ }^{7}$ Il cardinale Alfonso $\mathrm{Carafa}^{8}$ infatti, procedette a sopprimere e unire diverse strutture, trasferendo le monache da un istituto all'altro. ${ }^{9}$ $\mathrm{Fu}$ inoltre resa obbligatoria la professione solenne e Pio V stabilì espressamente che anche le converse e le novizie fossero tenute ad osservare la clausura. Nessuna religiosa avrebbe più potuto lasciare il monastero, neanche per breve tempo, se non

$4 \quad$ Miele 2001, p. 97.

5 Conciliorum Decreta 1973, pp. 777-778.

6 Sulla riforma dei monasteri femminili si rimanda a: Galasso/VAlerio 2001; Creytens 1965; Novi-Chavarria 2001, pp. 67-70; PASchini 1960; Zarri 1999; Donadi 2003, pp. 37-38; MieLE 1990, pp. 123-204; BocCADAMO 1990.

7 Sant'Aniello, Sant'Agata, Santa Maria della Misericordia, San Benedetto e San Festo furono i primi monasteri a subire questo provvedimento. Le monache dei primi due istituti vennero unite a quelle di Donnalbina, mentre le religiose di Santa Maria della Misericordia e San Benedetto furono trasferite al monastero Sant'Arcangelo a Baiano. Le monache di San Festo furono unite a San Marcellino. Cfr. Galasso/VAlerio 2001, p. 102. Sull'accorpamento dei monasteri di San Festo e San Marcellino si veda il cap. V, pp. 121-123.

8 Alfonso Carafa fu arcivescovo di Napoli fra 1540 e 1565, Cfr. De Maio 1997 pp. 28-34.

9 Sul clima riformistico napoletano e sul malcontento delle monache vi è la preziosa testimonianza di Fulvia Caracciolo. Nata nel 1539 fu portata, a soli 2 anni, al monastero di San Gregorio Armeno per diventare monaca nel 1547. Fu autrice del celebre Brieve compendio della fundatione del Monistero di San Gregorio Armeno detto San Ligoro di Napoli (1580) e inoltre del Borro, o sia esemplare delle nobile memorie della R.a D. Fulvia Caracciola 1577, sulle cose succedute nel nostro monastero nella restrittione delle regole e clausura (oggi conservato presso l'Asn, MS, f. 3435). Nelle sue memorie la Caracciolo espresse tutto il suo disappunto in merito all'applicazione delle normative tridentine. Si tratta di un documento eccezionale per le informazioni che la Caracciolo dà circa la vita quotidiana di un monastero femminile italiano della metà del Cinquecento e, soprattutto, per le reazioni che registra rispetto alle imposizioni dettate dal Concilio. Si veda anche Spinosa/Pinto/Valerio 2013; Carrino 2013. Si rimanda inoltre alle pagine dedicate al monastero di San Gregorio Armeno, cap. V, pp. 131-136. 
per legittimo motivo, approvato dal vescovo ordinario. Inoltre, senza il permesso dell'ordinario, nessuno sarebbe più potuto entrare a fare visita alle monache sotto pena di scomunica. ${ }^{10}$

L'adeguamento alle nuove normative impose inoltre una ridistribuzione degli spazi claustrali. Prima della riforma tridentina, l'organizzazione dei chiostri era basata sul cosiddetto "sistema delle celle". La cella rappresentava una sorta di microcosmo in cui venivano svolte buona parte delle attività giornaliere e dove era possibile condurre una vita quasi privata. Infrangere tale sistema, significava anzitutto donare nuovamente importanza alla struttura organizzativa della comunità. Pertanto i monasteri divennero cantieri permanenti e interi edifici vennero trasformati, ampliati o costruiti ex novo. Per rimarcare la condizione di separazione del monastero dal mondo circostante, vennero posti portoni, mura, grate, ruote, chiavistelli, finestre murate od oscurate. Furono però predisposti anche giardini, logge e portici ampi, per consentire alle recluse di uscire all'esterno senza essere viste da sguardi indiscreti. La clausura impose anche la creazione del parlatorio. Si trattava di una stanza intermedia tra l'interno del chiostro e l'esterno. Uno spazio simbolico di confine che permetteva alle religiose di parlare con i visitatori attraverso delle grate senza essere viste. Il parlatorio era costantemente sorvegliato da una monaca 'ascoltatrice' che presenziava ai colloqui fra religiose e parenti o fornitori. Vi erano tuttavia norme che regolavano l'accesso ai monasteri e i colloqui al parlatorio: erano ammesse soltanto quelle persone a cui era concessa esplicita licenza per motivi di parentela o d'ufficio. Tanto rigore e tanta severità erano giustificati dall'intenzione di proteggere in tutti i modi le professe, garantire la loro diligenza agli uffici divini e assicurare la loro totale concentrazione durante la preghiera, che doveva costituire lo scopo principale della loro vita.

La clausura contribuì anche alla rivalutazione del ruolo assunto dagli ecclesiastici all'interno delle comunità monastiche femminili. Confessori, cappellani e predicatori, divennero una presenza costante nella vita delle religiose. Il loro sostegno era giudicato fondamentale ai fini di una pratica sacramentale e devozionale più cosciente.

Badesse e superiori avevano poi il compito di preoccuparsi affinché tutto fosse attentamente rispettato. In caso di disordini rischiavano di incorrere loro stesse in gravi pene.

Inutile dire che la resistenza da parte dei monasteri fu enorme. A mettere maggiormente in allarme le monache erano le norme che riducevano al minino i loro contatti col mondo esterno, le restrizioni riguardo visite e doni, le limitazioni riguardo l'organizzazione di feste e liturgie. Il malcontento delle religiose, costrette a misurarsi con cambiamenti che sembravano per loro sconcertanti, fu sostenuto dai parenti e dalle comunità locali. La clausura veniva interpretata come un tentativo della gerarchia ecclesiastica di sciogliere quei legami che fino ad allora avevano 
collegato in vario modo le professe ai loro nuclei parentali di origine. I genitori temevano che l'eccessivo rigore della normativa tridentina avrebbe spinto molte fanciulle ad abbandonare il chiostro, con tutte le conseguenze patrimoniali che ne sarebbero derivate per le famiglie. Nella società napoletana infatti il controllo esercitato dalle famiglie nobili sulle istituzioni ecclesiastiche cittadine era forse più radicato che in altri contesti geografici e l'atteggiamento verso la disciplina imposta dalla riforma era fortemente condizionato dal senso dell'onore della casata. ${ }^{11}$

La riforma, come vedremo nelle pagine seguenti, non attecchì mai seriamente nel viceregno napoletano. I brevi interventi degli arcivescovi Acquaviva, Carafa e Boncompagni non sortiranno grandi esiti. ${ }^{12}$ Solo anni più tardi, a metà Seicento, l'arcivescovo Ascanio Filomarino si dedicò con decisione alla questione monastica. $^{13}$

\section{Gli strumenti di controllo}

Le disposizioni conciliari post-tridentine posero il controllo dei monasteri femminili sotto l'autorità dei vescovi ordinari che stavano a capo delle varie diocesi locali. ${ }^{14}$ A loro volta i vescovi nominavano dei vicari, ovvero ecclesiastici che su delega, trattavano ordinariamente relazioni e problematiche con i diversi monasteri della città. I poteri del vicario sui chiostri femminili consistevano nella supervisione amministrativa dello stato economico e della gestione dei beni temporali degli istituti. Il vicario era responsabile anche della disciplina, dell'osservanza dei tre voti monastici, del rispetto della clausura e dell'applicazione della normativa tridentina. ${ }^{15} \mathrm{Si}$ occupava inoltre dell'esplorazione della volontà di monacande e novizie; ne approvava i confessori e i maestri ed assisteva all'elezione della superiora. Una funzione più o meno simile era svolta dal cardinale protettore, in genere esponente di grandi famiglie, deputato alla supervisione delle istituzioni di regio patronato. Non è sempre facile stabilire quali comunità ricadessero sotto la giurisdizione del vicario o dei protettori, né ricostruire come tali autorità si alternarono nel corso dei secoli.

11 Sul ruolo esercitato dalle famiglie napoletane a seguito della riforma si veda GaLAsso/VALErio 2001; Novi-Chavarria 1993, pp. 84-111; Novi-Chavarria 2001; Boccadamo 2001, pp. 77-106.

12 Ottavio Acquaviva d'Aragona fu arcivescovo di Napoli dal 1605 al 1612; Decio Carafa dal 1613 al 1626; Francesco Boncompagni dal 1626 al 1641.

13 Sui diversi interventi riformatori da parte degli arcivescovi della Diocesi si veda De MaIo 1997, pp. 28-34; Strazzullo 1968; StrazZullo 1978.

14 Si vedano i cap. V e IX del Decretum de Regularibus et Monialibus, cfr. Conciliorum Decreta 1973, p. 179.

15 Cfr. ZARRI 1999, pp. 347-368. 
La rete di controllo che tutelava le comunità monastiche partenopee non si limitava all'attività del vescovo locale e dei vicari da lui delegati, ma si avvaleva anche dell'intervento delle congregazioni curiali presso la Santa Sede come la Congregazione del Concilio e in modo particolare la Congregazione dei Vescovi e Regolari. ${ }^{16}$ Deputata a vigilare sulla condotta di prelati, religiosi e religiose e a dirimerne le controversie, la Congregazione dei Vescovi e Regolari era ben informata su ogni aspetto della vita delle comunità religiose in modo da poter esercitare un controllo abbastanza capillare sulla loro condotta. Essa si attivava soprattutto in risposta ai memoriali che laici, ecclesiastici e nunzi apostolici inviavano a Roma per denunciare le situazioni più disparate o semplicemente per domandare un intervento risolutore nelle questioni più spinose. La Congregazione dei Vescovi e Regolari esercitava anche sui monasteri napoletani una giurisdizione piuttosto ampia, che abbracciava svariati aspetti della vita claustrale: disciplinare, patrimoniale, amministrativo e giudiziario. Si trovano, ad esempio, testimonianze dell'intervento di questa Congregazione anche riguardo l'utilizzo della musica nelle comunità monastiche. Alcuni dei provvedimenti da essa disposti circa l'utilizzo della musica nella liturgia, sono presenti nella lacunosa corrispondenza intercorsa fra le congregazioni curiali e il vicario delle monache custodita presso 1'Archivio Diocesano di Napoli. ${ }^{17}$

La Congregazione dei Vescovi e Regolari era coadiuvata nella sua opera di controllo dalla Congregazione del Concilio, sorta nel 1563 come commissione temporanea incaricata di sovrintendere all'applicazione delle decisioni tridentine. Le istituzioni ecclesiastiche e la stessa Congregazione dei Vescovi e Regolari, pur controllando la condotta delle religiose, tendevano a trovare dei compromessi e ad evitare imposizioni che avrebbero potuto sortire esiti negativi nelle alleanze politiche.

La coesistenza di organismi differenti, tutti deputati al controllo delle istituzioni napoletane, produsse spesso conflitti di competenza. Tuttavia le monache napoletane non furono mai destinatarie passive delle decisioni altrui ma, beneficiando anche del sostegno dei parenti, tentarono di far sentire la propria voce nelle questioni che le riguardavano, non da ultimo quelle musicali.

16 La Congregazione dei Vescovi e Regolari sorse alla fine del XVI secolo dalla fusione di due distinte congregazioni: la Sacra Congregatio super consultationibus episcoporum et aliorum prelatorum e la Sacra Congregatio super consultationibus regularium.

17 Purtroppo si hanno solo pochi riferimenti nelle carte d'archivio napoletane riguardo gli interventi delle congregazioni curiali. La documentazione completa si conserva presso l'Archivio Segreto Vaticano. Ad oggi, per Napoli, sono stati compiuti solo pochi sondaggi circa l'impegno sinodale dei vescovi riformatori e delle congregazioni della Santa Sede in applicazione delle risoluzioni tridentine. Si tratta di studi che si occupano però specificamente di aspetti legati all'antropologia e alla storia delle istituzioni, cfr. Russo 1970; VALERIo 2006; BocCADAMO 1990, alla storia dell'arte e all'architettura, STRAZzULlo 1968, e non si occupano della pratica musicale. 


\section{Le visite pastorali}

Il Concilio di Trento aveva inoltre ribadito il dovere della visita pastorale dei vescovi. ${ }^{18}$ Le visite consentivano una maggiore conoscenza della realtà sociale e spirituale della diocesi, erano un'occasione di controllo dei fedeli e del clero, e un mezzo tramite il quale trasmettere modelli morali, devozionali, etici. ${ }^{19}$ L'arcivescovo aveva la libertà di eseguire la visita ogni volta che lo avesse ritenuto opportuno. Effettuarla non era però cosa semplice e scontata da parte dei vescovi, anche perché non tutti i monasteri ricadevano sotto il controllo arcivescovile. I problemi si ponevano soprattutto nelle istituzioni 'regie' che non erano controllate direttamente dalla curia. ${ }^{20}$ La visita era vista come una vera e propria inchiesta, in cui ogni aspetto dell'organizzazione religiosa delle singole istituzioni veniva investigato dal vescovo in persona o dai suoi delegati: lo stato di conservazione di chiese, cappelle e altari, il numero e la condotta delle religiose, il numero delle messe e delle processioni, la presenza di associazioni devozionali laiche, le eventuali condotte sospette.

Le visite fornivano alle gerarchie ecclesiastiche informazioni preziose sulla situazione religiosa, sulla composizione sociale degli istituti, offrendo un interessante quadro d'insieme della clausura napoletana. I documenti che ne scaturivano rappresentano dunque una fonte ricca delle notizie più varie. Essi fanno luce sull'effettivo grado di conformità dei monasteri ai dettami tridentini e forniscono

18 La questione era stata specificata nel canone VIII della XXII sessione conciliare «I vescovi, anche come delegati della Sede Apostolica, nei casi concessi dal diritto, saranno gli esecutori di tutte le disposizioni pie, sia di quelle che sono espressione delle ultime volontà, che di quelle tra vivi. Abbiano la facoltà di visitare gli ospedali, i collegi di qualsiasi specie, le confraternite laicali, anche quelle che chiamano 'scuole' o con qualsiasi altro nome; non però quelle che sono sotto la immediata protezione dei re, senza loro espressa licenza»; e nel III canone della XXIV sessione «I patriarchi, i primati, i metropoliti e i vescovi non manchino di visitare personalmente la propria diocesi; se ne fossero legittimamente impediti, lo facciano per mezzo del loro vicario generale o di un visitatore. Se ogni anno non potessero visitarla completamente per la sua estensione, ne visitino almeno la maggior parte, in modo tale, però, che nel giro di due anni, o personalmente o per mezzo dei loro visitatori, terminino di visitarla», cfr. CONCILIORUM DeCRETA 1973.

19 Le ricerche sulle visite pastorali effettuate dagli arcivescovi napoletani hanno prodotto diversi contributi. Si veda: Russo 1970; Boccadamo 1999, pp. 277-315. Numerosi riferimenti alle Sante Visite si trovano anche in De Maio 1997, pp. 35-40; Strazzullo 1978.

20 Si ricorda, ad esempio, la tenace resistenza delle monache di Santa Chiara, istituzione per l'appunto di regio patronato, ai tentativi di visita pastorale del cardinale Alfonso Gesualdo nel 1596, e in seguito del cardinale Ascanio Filomarino. Nel marzo 1652 le clarisse fecero barriera con i loro corpi per non permettere l'accesso nel monastero al cardinale presentatosi a sorpresa alle porte del monastero pretendendo di visitarlo. All'episodio fece seguito un interdetto con la privazione della celebrazione di liturgie. Qualche anno più tardi nel 1654 le clarisse acconsentirono alla visita pastorale. Cfr. VALERIO 2006, pp. 127-139. 
anche informazioni sulle condizioni materiali e patrimoniali dei monasteri, sull'urbanistica e la storia della città.

I diversi arcivescovi furono più o meno severi verso le inadempienze delle monache riscontrate attraverso le visite. Di certo, nessuno ebbe lo stesso persistente interesse per la questione come Ascanio Filomarino. Ossessionato dal rispetto della disciplina claustrale, egli lamentava, nelle sue relazioni inviate a Roma, che il più grave danno arrecato ai monasteri era stato l'alleggerimento della clausura. Filomarino si pose pertanto l'obiettivo di restituire dignità religiosa alle monache, non giudicando abbastanza efficaci gli interventi dei suoi predecessori. ${ }^{21} \mathrm{Fu}$ pertanto particolarmente assiduo alla visita pastorale, effettuando tre cicli di visite, nel 1642, dal 1648 al 1654 e infine, dal 1657 al 1662, affermando in tal modo la propria giurisdizione. Egli emanò una serie di particolareggiati decreti per la salvaguardia della clausura, tendenti ad evitare occasioni di contatto con il mondo esterno. Inoltre, a seguito della visita del 1642, istituì una congregazione apposita per vigilare sulle spese sostenute dai monasteri e verificare che fossero realmente conformi alle esigenze degli istituti. ${ }^{22}$

Le relazioni delle visite contenevano in genere una breve introduzione storica sulla fondazione della comunità monastica e sulla regola osservata, a cui faceva seguito l'esame della chiesa adiacente al singolo chiostro con il controllo di altari, cappelle, stato delle suppellettili sacre, etc.; a volte si allegava anche un accurato elenco delle reliquie conservate e delle celebrazioni che si effettuavano. Terminata l'analisi della chiesa, veniva fatta una descrizione delle condizioni del monastero e dello stato della vita religiosa che vi si conduceva. I verbali erano spesso corredati da alcune disposizioni generali relative a varie questioni: licenze di entrata e uscita dal chiostro, modalità di utilizzo di parlatori, norme concernenti la gestione temporale del chiostro, amministrazione del denaro, e ovviamente impiego e utilizzo della musica. Ne troviamo testimonianza nelle visite del cardinale Giacomo Cantelmo del 1691, in cui l'arcivescovo si espresse sul divieto di prendere lezioni di musica da parte delle abitanti dei chiostri:

\footnotetext{
L'Abbadesse, ò superiore di qualsisia Monastero non permettano in conto veruno, che tanto dalla parte delle Grati [sic], quanto della Chiesa qualsivoglia monaca professa, novitia, educanda, ò conversa pigli lettione di cantare, ò sonare qualsivoglia instrumento, ne permettano che in coro, ò nelle Grate si canti figurato, ma solo in semplice canto gregoriano, sotto pena della privazione di officio ipso facto all'Abbadessa, ò superiore, che lo permetteranno, e di scomunica ipso facto à noi riserbata rispetto alle Monache et à chi l'insegnerà essendo secolare, et essendo persona à noi soggetta di carcere formale per sei mesi [...]. 19 dicembre 1691 Giacomo Cardinal Cantelmo Arcivescovo. ${ }^{23}$
}

21 I predecessori di Filomarino, Carafa e Buoncompagni non svolsero un'azione efficace sui monasteri. Basti pensare che il cardinale Boncompagni in quindici anni di governo visitò solo sei monasteri napoletani. Cfr. De Maio 1997, pp. 28-34.

22 Ne fa menzione un editto del 1643, si veda Asdn, Vicario, 259 D, app. p. 171.

23 AsDn, Sante Visite, IV, app. p. 342. 
Quando il giudizio della visita era negativo, venivano aggiunte istruzioni disciplinari. Un esempio riguardo la pratica della musica è contenuto nella visita al monastero di Santa Maria della Provvidenza effettuata nel 1744 dal cardinal Spinelli. Nei decreti emessi al termine della visita si legge:

$3^{\circ} \mathrm{Nel}$ Coro poi proibiamo affatto altro Canto, che il Canto fermo, à qual fine ordiniamo, che non si debbano le monache servire di altre carte, né di altri libri, se non quelli, che sono stati contrassegnati ed approvati dal nostro mastro di Cappella, ed ordiniamo, che le Religiose che presiedono al coro, diano anco à Noi raguaglio di qualunque trasgressione, che si commetta in maniera di coro. ${ }^{24}$

Ad ogni modo, nelle relazioni delle visite non si hanno grandi informazioni circa l'utilizzo di musica nei monasteri. Si ha piuttosto l'impressione che l'interesse della visita si concentrasse sull'applicazione architettonica delle norme imposte dalla clausura e sul ristabilimento della vita comunitaria. ${ }^{25}$

\section{I sinodi e gli arcivescovi della Diocesi}

I sinodi diocesani furono lo strumento maggiormente utilizzato dagli arcivescovi per riformare la Curia e gli istituti religiosi. Gli atti dei sinodi costituiscono infatti una rilevante fonte per la storia ecclesiastica napoletana: essi permettono di conoscere gli ideali di società cristiana che i diversi arcivescovi perseguirono e i provvedimenti necessari per attuarli. ${ }^{26}$ I sinodi rispecchiavano di solito le esperienze delle Sante Visite e si ponevano l'obiettivo di promuovere nuovi programmi e prospettive pastorali. Nei diversi sinodi napoletani, particolare attenzione venne appunto riservata alle comunità monastiche, e si tentò di stabilire, attraverso decreti e norme, una comune disciplina della vita claustrale. Dal 1644 al 1726, furono celebrati a Napoli dodici sinodi diocesani.

Ascanio Filomarino fu arcivescovo di Napoli dal 1641 al 1667, ${ }^{27}$ periodo turbolento caratterizzato principalmente dalla rivolta di Masaniello, ${ }^{28}$ dalla conseguente

24 AsDn, Vicario, 471, app. p. 216.

25 Dai verbali delle visite si apprende, ad esempio, dell'esistenza di muri di cinta non sufficientemente alti, dove spesso esistevano fori comunicanti con l'esterno, oppure di finestre che davano sulla strada o su altri edifici etc.

26 Sui sinodi diocesani napoletani si veda Da Nadro 1960; Miele 1979; Caserta 1983; De Maio 1997 pp. 35-40 e pp. 203-211; Strazzullo 1968.

27 Ascanio Filomarino (1583-1666) fu cameriere segreto di papa Urbano VIII. Nel 1641 divenne cardinale e venne nominato arcivescovo di Napoli. Filomarino è anche noto per essere stato uno dei principali protagonisti della rivolta napoletana del 1647-1648 contro il malgoverno dei funzionari spagnoli. Cfr. De Maio 1997, pp. 28-29.

28 Le difficili condizioni economiche e sociali del Viceregno, il carico fiscale imposto dalla Spagna, la caotica situazione abitativa avevano provocato nel Seicento numerose sommosse popolari, fra cui la più importante fu quella capeggiata da Masaniello. La rivolta fu soffocata nell'aprile 
proclamazione della Real Repubblica Napoletana nel 1647 e dalla devastante peste del 1656. Nei vent' anni del suo servizio napoletano furono celebrati cinque sinodi diocesani. ${ }^{29}$ Negli atti si trovano numerosi emendamenti relativi al buon governo delle istituzioni femminili. Una serie di prescrizioni riguardavano l'utilizzo della musica ed erano volte a condannare l'esecuzione di canti e musiche sconvenienti durante le sacre funzioni; proibire la partecipazione delle religiose a rappresentazioni sceniche anche di soggetto sacro e il mascherarsi per divertimento; indossando abiti secolari maschili o femminili. Per evitare confusioni fra sacro e profano, le processioni dovevano essere effettuate con sobrietà, vi era infatti la proibizione di danze e giochi di fanciulli vestiti da angeli e santi. Fu però con il sinodo del 1646 che Filomarino emanò una specifica istruzione dedicata ai monasteri femminili. La normativa riguardante le monache, presentata come una sorta di compendio denominato Institutio de vita et honestate monialium, ricordava i motivi ascetici della consacrazione verginale e ribadiva nuovamente l'impossibilità per le vergini consacrate di utilizzare in maniera impropria la musica, di mascherarsi nel Carnevale e di partecipare a rappresentazioni con danze, musica e canti. ${ }^{30}$

Ad Ascanio Filomarino successe Innico Caracciolo, ${ }^{31}$ che si impegnò subito nella riforma dell'organizzazione della sua diocesi e nell'indirizzare verso forme nuove l'educazione religiosa e la vita di fede della città partenopea. Nei quattro sinodi svolti, ${ }^{32}$ egli prospettò un'individuazione precisa delle problematiche, ${ }^{33}$ proponendo una nuova serie di canoni punitivi. I nuovi decreti sulla musica e sul canto tendevano a tutelare la sacralità delle esecuzioni con l'assoluto divieto che cantanti definiti "profani" potessero esibirsi durante le liturgie. Veniva inoltre ribadito il divieto di allestire drammi anche di soggetto sacro, in quanto si riteneva che le rappresentazioni della Passione o della vita della Madonna e dei Santi, anziché suscitare devozione, provocassero riso e ilarità non adeguate. Le rappresentazioni infatti potevano essere permesse solo in casi particolari, con licenza scritta dell'arcivescovo e garanzie di un'esecuzione edificante. Vi era inoltre il divieto ai laici e chierici di insegnare canto e musica senza licenza apposita.

del 1648. Tuttavia l'effimera rivolta di Masaniello e i successivi avvenimenti della Repubblica segnarono un periodo assai critico della storia del Regno di Napoli. Cfr. D’Alessio 2007; Galasso 1982.

29 I sinodi indetti da Filomarino si tennero nel 1644, 1646, 1649, 1658, 1662.

30 Cfr. CiCalese, 1972, pp. 208-240; Miele 1979, p. 112.

31 Innico Caracciolo (1607-1685) fu alla guida della diocesi partenopea dal 1668 al 1685. Gli anni del suo ministero a Napoli coincisero con l'avvio del processo di rinnovamento filosofico e scientifico, favorito dai più frequenti contatti con uomini e istituzioni culturali straniere. Cfr. De MaIo 1997, pp. 29-30.

32 I sinodi si svolsero nel 1669, 1672, 1676, 1680.

33 Oltre alle irregolarità riscontrate nei monasteri femminili, i numerosi decreti emanati si riferivano all'amministrazione dei sacramenti, alle cerimonie liturgiche, all'immunità ecclesiastica, alla condotta dei chierici, al concubinaggio, all'ordinazione e all'istruzione dei novelli sacerdoti. 
I molti decreti emanati da Caracciolo erano del tutto simili a quelli dei suoi predecessori, a testimonianza del fatto che abusi e inosservanze riscontrati in precedenza, non erano mai stati definitivamente debellati. La riforma dunque non aveva avuto piena attuazione, non tanto nelle forme esteriori della vita monastica, ma soprattutto nello spirito.

Fece seguito il governo del cardinale Giacomo Cantelmo Stuart. ${ }^{34}$ Ancora una volta i due sinodi seguenti, il diocesano del 1694 e quello provinciale del 1699 , non fecero altro che ribadire con vigore, la disciplina e i decreti auspicati dai predecessori. Anche Cantelmo non mancò di emanare un nuovo ordine per il buon governo dei monasteri femminili, chiedendo a tutte le istituzioni religiose il rispetto dei voti e della disciplina.

Con il governo pastorale dell'arcivescovo Cantelmo si chiudeva un secolo denso di attività sinodali da cui era scaturito un imponente complesso di norme. Il sinodo diocesano successivo si terrà infatti solo trentadue anni dopo, per iniziativa dell'arcivescovo Francesco Pignatelli. ${ }^{35}$

Francesco Pignatelli fu nominato arcivescovo di Napoli in un momento storico critico e pieno di cambiamenti. La lunga dominazione spagnola si avviava al tramonto, ad essa era subentrata la dominazione austriaca conclusasi poi con la ricostruzione del regno autonomo per opera di Carlo di Borbone nel 1734. Il sinodo, unico del secolo, si svolse dal 9 al 12 giugno 1726 con una serie rinnovata di decreti punitivi. Oltre a ribadire l'importanza di un rigido tenore di vita per le vergini consacrate, il sinodo affermava, in merito alla disciplina musicale, che né monache né educande o novizie dovessero prendere lezioni di musica e che non facessero utilizzo del canto figurato:

V. [...] Nulli vero neque Moniali, neque Educandae, neque Novitiae, aut cuicunque, quocunque nomine in Monasteriis degenti bus, liceat Musicam, quem Canto Figuratum vocant, addiscere sub poena excommunicationis; neque permittendam sancimus ulli unquam facultatem hunc Cantum in Monasteriis degentes edocendi sub eadem poena. ${ }^{36}$

Era inoltre ribadita l'impossibilità di introdurre insegnanti di musica o musicisti entro la clausura, anche in celebrazioni particolari come la notte di Natale, sotto pena di scomunica:

XX. Monasteriorum Superioribus quomodocunque vocatis, Sacristis, aut Monialibus quibuscunque non liceat in nocte Nativitatis Domini nostri Jesu Christi Musicos in Ecclesias suorum

34 A Caracciolo in realtà subentrò Antonio Pignatelli eletto nel 1686. Egli rimase in carica però solo fino al 1691 in quanto eletto Papa sotto il nome di Innocenzo XII. La documentazione sul suo governo napoletano è dunque scarsa. Giacomo Cantelmo Stuart fu arcivescovo di Napoli dal 1691 al 1702. Cfr. De Maio 1997, pp. 32-35.

35 Francesco Pignatelli (1652-1734), fu elevato al rango di cardinale da Papa Clemente XI nel dicembre 1703 e immediatamente fu nominato arcivescovo di Napoli. Morì il 5 dicembre 1734 a Napoli all'età di 82 anni. 
Monasteriorum introducete, sub poenis arbitrio nostro; Musici vero,qui fortasse accesserint, excommunicatione plectentur, aliisque etiam poenis nostro arbitrio, prout sevandum mandavimus speciali Edicto die 15. Decembris $1716 .^{37}$

Parimenti era disposto che non venissero fatte rappresentazioni teatrali:

XXVII. In observantiam Decretorum Synodalium Praedecessorum nostro rum, et S. Congregationis interdictrum sit Monialibus omnibus, et quibuscunque quovis tempore, praesertim vero recreationum et Bacchanalium, habitus seculares viriles, vel etiam muliebres, aut cujuslibet generis personas, vulgo Maschere, ad animi relaxationem iduere, nec commedia, fabulas repraesentationes agere; quae dissolutionem Ecclesiasticae disciplinae inducunt, et Sacras Virgines a studio Orationis, et colestis Sponsi deliciis avertunt. ${ }^{38}$

Pignatelli si espresse anche sul numero e tipologia di musicisti da poter interpellare durante le celebrazioni ufficiali:

Si ricorda alli Signori della Congregazione del Sinodo, chè la S. M. del Cardinal Caracciolo per evitare l'estorsioni, è spese superflue alli Monasteri, è Conservatori soggetti alla sua Giurisdizione, ordinò, chè nelle feste delle loro chiese, anche in occasione di monacazione, ò di altra qualunque funtione, si dovessero servire della musica della Chiesa Arcivescovale, ò pure di quella del Conservatorio di Santa Maria della Colonna detto de' Poveri di Gesù Cristo, come Conservatorio immediatamente soggetto all'Arcivescovo, tassando lo stipendio dell'uno, è dell'Altro Luogo: è chè in caso fusse chiamata la Musica della Cattedrale, è non quella del detto Conservatorio, fusse tenuto il Maestro di Cappella chamar egli dal detto Conservatorio due violini, et una viola, ò sia violoncello in ogni occasione, è congiontura, come di sopra, è pagarli à raggione di Carlini trè per ciasched'uno di detti Istrumenti, chè tutti fanno Carlini nove per ogni servizio; quali Carlini nove dovesse il Maestro di Cappella cavarli dall'intiera Summa, chè esso riceve dalle sudette funzioni; è si ordinò ancora, chè li Monasteri in occasione di dette feste havendo di bisogno di clerici estraordinari per servire le Messe, guardar'Altari, et altro, havessero è chiamare li figlioli di detto Conservatorio, è non di Altri Conservatori, però con lo stesso Stipendio, chè suole pratticarsi con tutti gl'Altri $[\ldots] .^{39}$

I decreti prescrivevano per le istituzioni religiose il ricorso ai musicisti della Cappella dell'Arcivescovo detta anche della Cattedrale e agli allievi del conservatorio dei Poveri di Gesù Cristo, istituto direttamente legato alla curia, bandivano pertanto il ricorso ai musicisti della Cappella Reale di Palazzo, istituzione alle dipendenze dei viceré.

Il sinodo del 1726 chiuse un'epoca della storia ecclesiastica di Napoli. Tuttavia le monache si presero numerose libertà in materia di musica come testimonia anche la cronaca fatta dall'abate senese Giovan Nicola Bandiera di passaggio a Napoli nel 1726 circa il canto delle monache durante gli uffici:

37 Asdn, Sinodo 1726, app. p. 338.

38 AsDn, Sinodo 1726, app. p. 338.

39 AsDn, Sinodo 1726, app. pp. 338-339. Il documento è citato anche in BaCCIAGALuppi 2010, p. 72. 
A sentir gli offici per lo più si va a' monasteri di monache, che per la verità generalmente cantano egregiamente; è però incredibile la pratica e l'invito che fanno i cicisbei delle monache canterine, e la cosa si riduce a tale che vi si sentono i viva come alle cantatrici de' teatri. ${ }^{40}$

Il problema di fondo è che vi era una percezione diversa di cosa fosse moralmente improprio: le autorità ecclesiastiche vedevano come devianza ciò che invece le monache consideravano un'antica consuetudine. Le religiose pertanto non riuscivano a comprendere né ad accettare il motivo per il quale dovessero essere mutate pratiche consuete fino ad allora svolte in maniera del tutto indisturbata e si ostinavano pertanto a non volerle abbandonare. ${ }^{41}$

\section{Editti e lettere pastorali}

Molti riferimenti alla pratica musicale si trovano nei decreti, editti, disposizioni, proibizioni e lettere pastorali che i diversi arcivescovi inviarono nel corso dei secoli a tutte le istituzioni femminili. In quanto documenti di natura pubblica essi contenevano precetti di tipo dogmatico, disposizioni su culto e devozione, ordinanze relative alla denuncia della mancanza di disciplina, prescrizioni per cerimonie, e potevano rivolgersi alle singole istituzioni oppure alla collettività dei monasteri.

A partire dalla fine del Cinquecento è possibile trovare ordini e proibizioni che richiamavano a costumi di vita più morigerati, oltre a numerose proibizioni legate all'uso e consumo di musica. Nel 1586, l'arcidiocesi di Napoli inviava, a tutti gli istituti religiosi femminili della città, un ordine tramite il quale veniva vietato alle monache l'utilizzo del canto figurato e si prescriveva l'abolizione dell'impiego di qualsiasi strumento ad eccezione degli organi, permessi comunque solo nei luoghi non vincolati dalla clausura:

Non si permetta à monache nelle lor Chiese canto figurato, mà solamente il canto fermo, et di più si toglino e cavino da ciascun monastero tutti i musici instromenti, salvo gl'organi i quali si permettono solamente nelle Chiese fuori della clausura. ${ }^{42}$

Alle monache infatti non era consentito avere nelle proprie celle strumenti musicali:

Tutti gli Instrumenti musicali eccettuato il Rigalo et l'Alpicordo [sic] o Monocordio siano cavati dal Monasterio in termine di un Mese, sotto la pena contenuta nelle Constitutioni generali di

40 Strazzullo 1978, p. 18.

41 Alla morte di Pignatelli subentrò Giuseppe Spinelli (1694-1763), arcivescovo dal 1734 al 1754. Durante il suo ventennio napoletano tentò di reintrodurre l'istituzione del Santo Uffizio. I napoletani chiesero l'intercessione del nuovo sovrano Carlo di Borbone, il quale impose la destituzione dell'istituto. Cfr. De Maio 1997, pp. 183-186. 
Napoli di privatione di voce attiva et passiva alle Monache: di officio alla Madre Abbadessa; e di espulsione alle Novitie et alle secolari $[\ldots]{ }^{43}$

La pena per chi avesse trasgredito si traduceva in un interdetto della chiesa con il ritiro del Sacramento e la sospensione di tutte le celebrazioni pubbliche di culto. ${ }^{44}$ In alcuni casi era addirittura prevista la scomunica, come si legge in un documento inviato dall'arcivescovo Filomarino al monastero di San Giovanni Battista, le cui abitanti avevano avuto l'ardire di porre un organo in chiesa e di farlo suonare:

\begin{abstract}
Havendomi presentito da più giorni, che nella Chiesa del Monastero di S. Giovanni Battista di questa città da diverse persone vi si canti a suono d'organo portatile posto nel pavimento di detta Chiesa non senza scandalo delli Convicini, et affatto contrario alla Regolar Disciplina, per lo che subito havutomi tal notitia ordinassimo all'Infrascritto nostro Mastro delle monache, che facesse precetto alla Madre Priora di detto Monastero che subito havesse fatto levar l'organo sudetto dalla medesima Chiesa per toglier via ogni Inconvenienti, del qual precetto fattogli tuttavia continua nella disobedienza. Per tanto col presente ordiniamo alla sudetta Madre Priora, e sacristana di detto Monasterio sotto pena di scomunica late sententiae ipso facto incurrenda a noi riserbata, che per tutta la presente giornata faccino levar via da detta lor Chiesa l'organo sudetto [...]. Napoli 14 gennaro $1664 . .^{45}$
\end{abstract}

Gli editti prescrivevano inoltre l'utilizzo del solo canto gregoriano, proibivano qualsiasi lezione di canto o di strumento e si esprimevano anche sulla moderazione nelle spese per l'organizzazione di festività e cerimonie:

[...] essendosi introdotto per occasione delle Feste, e sollennità, che si fanno ne i Monasteri di Monache l'uso della Musica vocale, \& di instrumenti con Trombe, Piffari, Naccare, Ciaramelle, \& altri, \& della Musica di figlioli vestiti da Angeli, \& anco di fare luminarij, fuochi artificiali \& sparatorij de maschi, \& parendoci, che queste siano spese superflue, \& che le feste in honore de Santi si debbiano solennizare con la divotione intrinseca, \& non con le pompe, \& apparati esterni: perciò prohibiamo per hora, $\&$ espressamente comandiamo in virtù di questo nostro ordine, che in qualunque occasione di festa, o solennità de Monasteri, ò in altro tempo, non possano le Monache pigliar Musica ne di voce, ne d'istrumenti, ne di figlioli vestiti da Angeli, ne far luminarij, fuochi artificiali, sparatorij, \& altro come sopra, senza nostra espressa licenza. Incarichiamo però alle Superiore, \& Sagrestane de Monasteri l'osservanza del presente Ordine sotto pena à quelle, che trasgrediranno di privatione di voce attiva, $\&$ passiva, $\&$ di altre à nostro arbitrio. Datato in Napoli nel nostro Palazzo Arcivescovale a di 16 Novembre 1643. Ascanio Cardinal Filomarino Arcivescovo di Napoli [...]. ${ }^{46}$

Nonostante a seguito delle prescrizioni fioccassero interdetti e sanzioni di vario genere, le religiose sembra non riuscissero a contenere la loro estrosità. Le monache di Regina Coeli, ad esempio, in occasione di una novena, avevano violato

43 Asn, MS, f. 2702, app. p. 262.

44 Si veda ad esempio l'interdetto posto proprio dall'arcivescovo Cantelmo al monastero di Donna Romita avendo le monache fatto ricorso ai musici della Cappella Reale per la visita della Viceregina in occasione delle celebrazioni per la festa di San Giovanni Battista. Cfr. cap. V, p. 130.

45 Pur riferendosi al monastero di San Giovanni Battista l'ordine è contenuto nella documentazione del monastero della SS. Trinità, cfr. AsDn, Vicario, 348 D, app. p. 190. 
anche l'interdetto che era stato loro posto, facendo ugualmente celebrazioni sontuose:

[...] per parte delle Monache fatto lacerare l'interdetto, et aperta la loro Chiesa con sono festivo di Campane, organo e con canti, e si è similmente continuato, celebrando in essa Messe, e Divini Officij $[\ldots]$ non senza disprezzo dell'autorità ordinaria $[\ldots] .^{47}$

Anche per i riti della Settimana Santa veniva richiesta una maggiore moderazione nei costumi, come possiamo vedere in un editto del cardinale Pignatelli del 1705:48

S'ordina alle Reverende Signore Madri Abbadesse, Priore, Sagrestane, et altre Monache delli infrascritti Monasteri a Noi soggetti sotto pena d'Interdetto di loro Chiese, ed in virtù di Santa Ubidienza, che debbano osservare, e farle osservare respettivamente le cose seguenti [...]. Per cantare il Passio, nella prossima Settimana Santa, non si possano servire d'altri Preti, ò Clerici, che di quelli delle loro Chiese, e non essendovi il numero sufficiente, possano pigliare altri con nostra licenza in scriptis, con darci prima nota de' loro Preti, e Clerici. Avvertendo, che non si ammettano à cantare il Passio altri, se non che Sacerdoti, ed ogn'uno di essi canti di canto fermo, e la sua parte solamente. Proibendo espressamente a' detti Sacerdoti di cantare la parte della Turba in terzetto, ma che non recedono, né pure una nota dal canto fermo. L'Offici, Lamentazioni, Lezzioni, e Responsorii, si cantino di canto fermo, senza trilli, e passaggi di sorte alcuna, e senza Organo, dalle Monache solamente, e non dalle figliuole secolari [...]. L'Evangelio non si possa cantare con canto figurato, ma solamente in canto fermo sotto pena di scomunica alle Abbadesse, Superiore, Sacrestane, ò altre, che ciò faranno, e rispetto alli Preti sotto pena di carcere, ed altre a nostro arbitrio [...]. Non si chiamino a cantare il Passio, o all'assistenza di Messe, Processioni, o altra funzione nella prossima Settimana Santa, Eddomadarij, o altri Preti, o Chierici della Chiesa Arcivescovale, né meno si chiamino Musici, o Periti di canto figurato [...]. Dato dal Palazzo Arcivescovale questo dì 7 Marzo $1705 .{ }^{49}$

L'intento delle gerarchie ecclesiastiche era appunto di evitare che riti e liturgie finissero per somigliare a feste profane. In questo senso, grande attenzione era riservata alle liturgie di professione religiosa, veri e propri matrimoni di stampo laico che si svolgevano usualmente in un contesto di grande sfarzo. ${ }^{50}$

Numerosi decreti e ordini disciplinari erano dedicati alla consuetudine di organizzare commedie o rappresentazioni sceniche nei monasteri o in luoghi esterni ad essi adiacenti:

Non si facciano comedie, né presentationi sotto qual si voglia colore [sic] i pretesto, né la Madre Abbadassa lo permetta in modo alcuno sottopena della privatione dell'officio suo. ${ }^{51}$

Nel 1697, un commissario dello stesso Papa Innocenzo XII, comandava alle religiose del monastero dei Ss. Pietro e Sebastiano la sospensione di commedie, in-

47 AsDn, Vicario, 334 D, app. p. 230. Il documento manca di data.

48 Sulle celebrazioni in uso nei monasteri durante la Settimana Santa si rimanda al cap. III, pp. 67-71.

49 Asdn, Vicario, 259 D, app. p. 172.

50 Si veda cap. III, pp. 75-91.

51 Asn, MS, f. 2702, app. p. 262. Si tratta degli Ordini del Reverendissimo Padre Frà Bonaventura Caltagirone Ministro Generale dell'ordine di San Francesco fatti per le monache del Monastero di Santa Chiara in Napoli l'anno del Signore 1594. 
termezzi o rappresentazioni teatrali ad opera delle religiose, educande o converse. Si specificava inoltre che non era concesso alle monache il cambiarsi d'abito o mascherarsi:

Similmente sotto precetto formale, e scomunica late sententie ipso facto incurrenda, commandiamo alle Madri Priora, e sottopriora pro tempore, che non permettano nel sudetto Monastero si rappresentino dalle Religiose, anche converse e non meno dalle Signore educande, comedie, Intermezzi, opere ò rappresentazioni di qualsisia maniera, ne mutino habito, ò mascherarsi sotto qualsisia titolo, ò pretesto. ${ }^{52}$

Nel tardo Settecento, anche il cardinale Giuseppe Capece Zurlo ${ }^{53}$ richiamò le religiose riguardo l'organizzazione di spettacoli profani e l'utilizzo di musicisti che provenissero da altre realtà musicali diverse dalla cappella dell'Arcivescovado:

Avendo preso S. M. in seria considerazione l'abuso delle Musiche, che si sono introdotte ne' Santuarj delle Monache, Debitore a Dio ed alla Religione, essendo Egli ancora il primo Vindice, e Custode de' Canoni, e della Disciplina Ecclesiastica: E volendo che le Chiese consecrate al Culto Divino, alla Orazione, ed alla santificazione de' Fedeli, non si convertano in ispettacoli profani, e Teatrali, ed a Scuole d'indecenza; Comanda, che Vostra Eminenza col suo noto, e commendabile Zelo, e con quella prudenza, e carità, di cui è adorna, v'introduca il buon ordine, e lo spirito della Chiesa: E che quando si voglia una Musica ne' Casi di Solennità, permetta solo quella dell'Arcivescovado, che suppone adattabile alla gravità, ed al decoro dell'Ecclesiastiche Funzioni [...]. Arcivescovado 13 Giugno $1792 .{ }^{54}$

Il contrasto evidente tra il lusso combattuto e persistente e la sobrietà richiesta dalla Chiesa che si palesa dallo spoglio e dal confronto con la documentazione interna degli istituti, ci porta a comprendere quanto il 'modus vivendi' dei chiostri risultasse essere tutt'altro che improntato su una ferrea osservanza dei voti e dei regolamenti. Dunque, se da un lato le autorità cercavano di eliminare ogni fasto superfluo, dall'altro le monache continuarono ad agire secondo abitudini radicate, beneficiando soprattutto del sostegno dei parenti. Lo spirito dei voti e di preghiera a cui erano chiamate le religiose attraverso sinodi e prescrizioni, non aveva di certo fatto molta strada fra i chiostri napoletani.

52 Asn, MS, f. 1407, app. pp. 158-159.

53 Giuseppe Maria Capece Zurlo (1711-1801) divenne arcivescovo di Napoli nel 1782, successore del cardinal Serafino Filangieri, carica che ricoprì fino alla morte avvenuta nel 1801.

54 Asdn, Pastorali e Notificazioni, 13 giugno 1792, app. pp. 340-341. 



\section{I monasteri e i legami con la città}

\section{Norme, tempi e luoghi}

La vita claustrale era scandita da una serie di norme stabilite dalle regole monastiche. Le regole, soprattutto le più antiche come quella benedettina, agostiniana o francescana, rimasero praticamente invariate nel corso dei secoli dal punto di vista dei contenuti. La regola infatti serviva a creare l'unità e l'identità di una famiglia monastica, aveva una validità permanente, era immodificabile e le stesse comunità femminili utilizzavano sostanzialmente quelle scritte per gli ordini monastici maschili. Costituzioni e statuti invece risultavano essere un adeguamento delle regole ai bisogni di ogni singola comunità. Le costituzioni scandivano la vita interna dell'istituto e la pratica religiosa. Si trattava in sostanza di 'leggi' organizzative della comunità e contenevano le prescrizioni da osservarsi per la convivenza quotidiana, orientate anche dagli ordini e decreti vescovili che interagivano con la vita claustrale. ${ }^{1}$ Regole e costituzioni erano dipendenti le une dalle altre e pertanto spesso venivano edite nello stesso volume. ${ }^{2}$ Le costituzioni contenevano capitoli relativi ai requisiti delle fanciulle da ammettere nel chiostro, ai tre voti monastici, alla clausura, all'organizzazione dell'ufficio divino, alle varie cariche monastiche, all'elezione delle badesse, etc. A volte, erano aggiunte anche le formule di vestizione e professione e il cerimoniale di votazione della superiora. Infine, regole e costituzioni, redatte o ristampate a seguito del Concilio di Trento, potevano includere anche una sintesi dei decreti post-tridentini riguardanti la disciplina monastica.

Nelle costituzioni veniva fissato anche il numero delle religiose ammesse in un determinato istituto. Il Concilio aveva infatti stabilito che ciascun monastero potesse accogliere, in base al proprio stato patrimoniale, un numero massimo di professe.

Oltre al numero di professe, le norme delle varie comunità, potevano anche richiedere una serie di caratteristiche specifiche per restringere l'accesso al loro monastero e di conseguenza selezionare gli ingressi. Questo comportò una differenziazione sociale tra chiostro e chiostro in base alla tipologia di donne che venivano ammesse all'abito.

1 Le regole a volte comprendevano anche un compendio con gli usi osservati dalla comunità negli anni e secoli precedenti alla stampa.

2 Purtroppo per la città di Napoli non si conservano le costituzioni di ciascun istituto, anzi ad oggi ben pochi esemplari sono consultabili. 

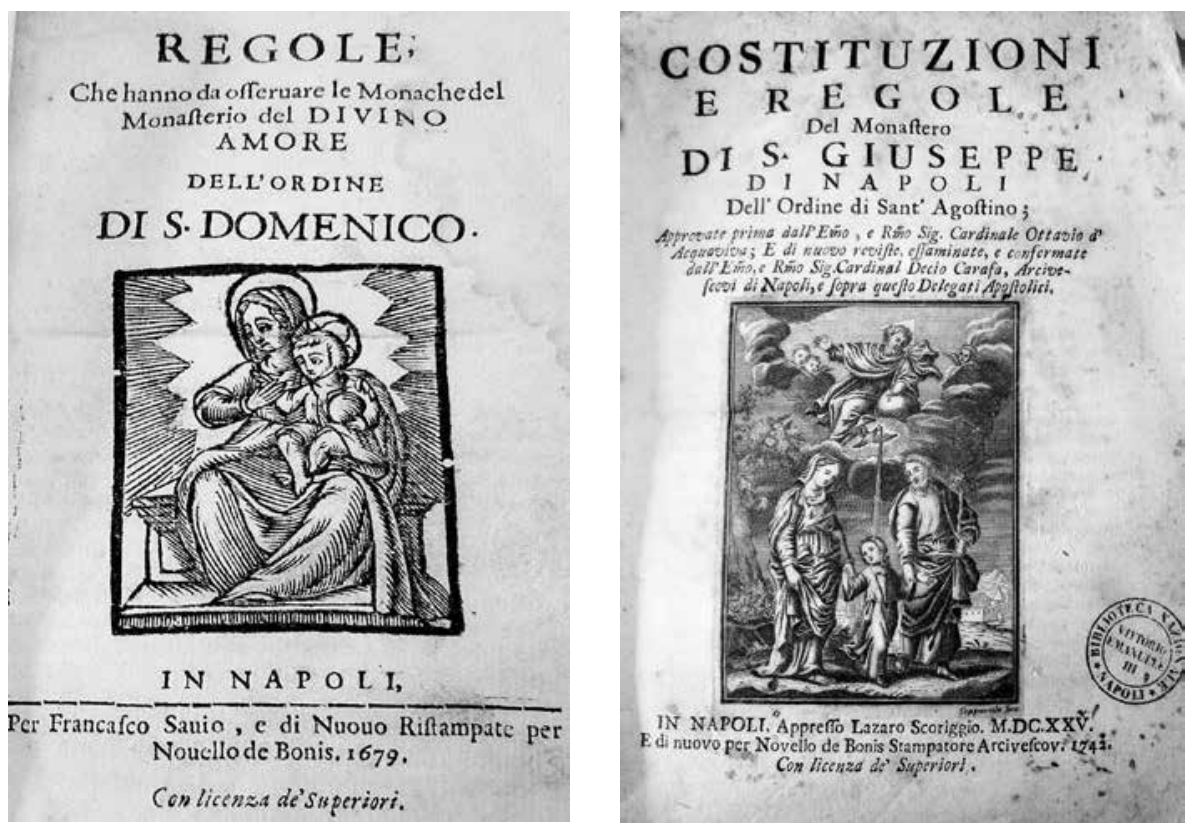

1. Costituzioni dei monasteri del Divino Amore e di San Giuseppe dei Ruffi. Frontespizi. Napoli, Biblioteca Nazionale.

Regole e costituzioni forniscono indicazioni anche riguardo ai tempi della vita claustrale. Ad una perfetta religiosa era richiesta la frequenza al coro, partecipazione agli uffici divini, orazione mentale, letture spirituali, modestia, silenzio. La giornata delle monache, secondo uno schema alquanto generico desunto dall'incrocio fra i documenti delle visite pastorali e dalle costituzioni a noi pervenute, doveva presentarsi come segue: dopo essersi recate nel coro, le monache recitavano l'Ufficio, partecipavano alla messa e facevano l'orazione mentale, poi si pranzava nel refettorio in silenzio, ascoltando la lettura di libri spirituali fatta da una monaca corista. Alla fine del pranzo vi era la visita al SS. Sacramento seguita da un momento di ricreazione. Al termine ognuna rientrava nella sua cella e si osservava il silenzio, tramite anche la lettura di libri spirituali. Al vespro si andava in coro nuovamente per le preghiere serali. Seguiva la cena in refettorio. Quindi ancora un periodo di ricreazione e il ritiro notturno.

La messa quotidiana, la lettura della Scrittura in refettorio, eventuali esercizi spirituali, così come la severità nei gesti e nelle coercizioni potevano differenziarsi a seconda dell'istituto e dell'ordine di appartenenza o delle specifiche devozioni seguite. Ad esempio, a riguardo del Divino Ufficio al monastero di San Giuseppe dei Ruffi era vietato sia il canto fermo che il canto figurato, ${ }^{3}$ mentre a San Gregorio 
Armeno per i «Divini Ufizi nel coro» era concesso «il canto Monastico grave e semplice $[\ldots]$ con licenza de superiori». ${ }^{4}$

Le regole prevedevano sezioni riguardanti aspetti del comportamento e della morale. Nelle regole del conservatorio dei Ss. Gennaro e Clemente si trovano ad esempio indicazioni su come preservare castità e modestia delle fanciulle:

Capo III. Della Castità.

Questa è quella virtù, che presa nel suo grado più nobile rende gli uomini simili agli Angeli: E se da tutti deve essere on ogni diligenza, e gelosia custodita, ciò deve intendersi più particolarmente delle Donne, le quali per la loro leggerezza, inconsiderazione, e facilità sono molto fragili; per riparo della quale fragilità Dio ha posto in loro maggiore la verecondia [...]. Proibiamo ancora espressamente ogni sorta di Ballo, ed anche ogni canto e suono profano, massime fuori del Conservatorio; e similmente l'intervenire a' balli, spettacoli, e comedie, ancorche spirituali o dentro, o fuori del Conservatorio [...].

Capo VII. Della Modestia.

La modestia è la virtù, che tra gli altri suoi ufizi custodisce la castità [...]. Colla modestia regolino la loro lingua, acciocchè non si allarghi in discorsi anche leciti, ma non necessarj, e geniali con uomini di qualsivoglia condizione, anche Ecclesiastici [...]. Regolino ancora le loro orecchie per non ascoltare ciò che non sarà lecito, e convenevole a proferire: ed a tal fine proibiamo l'accesso in que' luoghi del Conservatorio, donde si possa sentire cosa non conforme allo stato, come di canzone profane, e di suoni lascivi, volendo che sieno punite coloro, che si troveranno colle scale esser salite nelle finestre per vedere, o ascoltare simili inezie $[\ldots] .5$

Alcuni ordini prescrivevano almeno una volta alla settimana il "capitolo delle colpe" che consisteva nella pubblica denuncia delle proprie mancanze. ${ }^{6}$ Generalmente, le colpe erano divise in gravi e leggere, ma in alcuni casi erano previste anche quelle denominate 'mezzane', e infine le colpe 'più gravi' e 'gravissime'. Colpa leggera era considerata, ad esempio presso le agostiniane del Divino Amore, il mancato adempimento di un ufficio:

Colpa leggiera è s'alcuna [...] non adempisse attentamente l'offitio ad essa imposto per leggere, cantare, overo s'havendo lei à cominciare Antifona, ò Responsorio, conturbasse il coro, s'alcuna ancora leggendo ò cantando male non subito in presenza di tutte se humiliasse [...]. ${ }^{7}$

4 Maniere, e costumi cò quali vivono le Sig.re Moniche, Sig.re Educande, e converse, cfr. SpInosa/ Pinto/Valerio 2013, appendice B, Pinto, Ricerca 2011.

5 Le regole di Ss. Gennaro e Clemente si trovano in AsDN, Vicario 427, si veda app. pp. 306-307. Una sintesi del documento viene citata anche in Illibato 1985 e in Conti 2003 pp. 25-28.

6 Le monache si riunivano nella sala capitolare e ciascuna confessava davanti alle altre le mancanze che riteneva di avere commesso e riceveva dalla badessa eventuali penitenze. Alla fine della riunione, alcune comunità facevano in gruppo la "disciplina" - ossia si flagellavano. La motivazione di tale pratica consisteva nell'avvicinarsi alla sofferenza vissuta dal Cristo e garantiva l'umiliazione del corpo.

7 BNn, Regole Divino Amore, app. p. 146. 
Nonostante la disciplina imposta da Trento e lo stile di vita comunitario, le monache continuarono nei secoli a condurre una sorta di vita privata. Secondo le costituzioni, le celle avrebbero dovuto essere semplici e nude, in realtà erano considerate come dei veri e propri appartamenti, sistemate con cura e arredate a piacimento con ricche suppellettili e, talvolta, con strumenti musicali. ${ }^{8}$ Nelle camere venivano svolte inoltre attività lavorative che le monache eseguivano sulla base di commissioni esterne al monastero. ${ }^{9}$

Presenza costante nei monasteri era il coro, da cui le religiose partecipavano non viste a tutte le liturgie. Arredato generalmente con stalli lignei e con la caratteristica presenza delle grate, esso rappresentava il filtro tra la dimensione claustrale e lo spazio collettivo. ${ }^{10}$

Un altro luogo simbolico e fulcro di un monastero era il chiostro: attorno ad esso si concentrava la maggior parte degli ambienti e dei settori della comunità. Era in genere provvisto di porticati su uno o due livelli, ed era affiancato da un cortile in cui vi era lo spazio per l'orto, il pollaio oltre a giardini e fontane. ${ }^{11}$

\section{Le abitanti dei chiostri}

L'autorevolezza dei monasteri si palesava soprattutto attraverso la figura della badessa, per lo più discendente del fondatore o appartenente alla famiglia più potente. Alla badessa era affidato il governo dell'istituzione e da lei dipendeva la distribuzione degli uffici, la sorveglianza sulla vita morale e religiosa delle monache, l'osservanza dei voti e della clausura.

La badessa era coadiuvata nel suo ministero da una vicaria che, come si legge nella corrispondenza fra il vicario delle monache e il monastero di Regina Coeli, poteva essere eletta ed espletare questa funzione qualora non avesse superato

8 A conferma di questo i numerosi decreti riguardanti l'impossibilità di custodire strumenti nelle celle. Cfr. cap. I, pp. 45-47.

9 Si veda quanto detto nel cap. I, pp. 35-36 riguardo al sistema delle celle e ai tentativi per debellarlo.

10 Celebre è il coro delle monache del monastero di San Gregorio Armeno, con stalli intagliati del sec. XVI. Da esso si aveva acceso agli ambienti del monastero, tra cui il cosiddetto "corridoio delle monache", con altarini arricchiti nel tempo da opere d'arte d'ogni epoca portate in dote dalle novizie.

11 Uno degli esempi più significativi è l'imponente chiostro maiolicato delle monache di Santa Chiara. La struttura trecentesca del chiostro fu completamente trasformata nel 1739 da Domenico Antonio Vaccaro. Il chiostro comprende due viali che, incrociandosi, dividono il giardino in quattro settori. Fiancheggiano i viali 64 pilastri rivestiti da maioliche con scene vegetali. I pilastri maiolicati sono collegati tra loro da sedili sui quali, con la stessa tecnica, sono rappresentate scene tratte dalla vita quotidiana dell'epoca. Le pareti dei quattro lati del chiostro sono interamente coperte da affreschi secenteschi, raffiguranti santi, allegorie e scene dell'Antico Testamento. Cfr. Gallino 1951; PANe 1954. 
gli «anni trenta d'età, et anni diece di professione». Inoltre per essere «eliggibile» all'aspirante vicaria era chiesto di saper «ben leggere» e «cantare il canto fermo», in modo da poter «lodevolmente esercitare il suo officio di reggere il Choro $[. ..] \gg .{ }^{12}$ La vicaria, assieme ad una procuratrice, si occupava dei beni materiali del monastero, mentre le econome erano addette all'amministrazione degli istituti. ${ }^{13}$

La scansione delle attività comunitarie della vita monastica auspicata dopo Trento, comportava una suddivisione della giornata in cui si riducevano i tempi individuali. Il lavoro si svolgeva nella comunità o al servizio di essa, in quegli uffici necessari per la riorganizzazione interna del monastero.

A seconda dell'istituto, ogni monaca poteva ricoprire uno o più uffici e ciascuna, nel disbrigo del proprio compito, godeva di una larga autonomia amministrativa. Vi era dunque la 'sagrestana' addetta alla sacrestia e quindi all'organizzazione degli uffici liturgici e della chiesa; la 'portinara' o 'ruotara', responsabili delle porte e della ruota e garanti della clausura, ${ }^{14}$ con il compito di controllare gli accessi al monastero; la 'cellarara' addetta alla cantina, la 'pratera' o 'giardiniera' al giardino, la 'panettiera' al forno, la 'cuciniera' alla cucina, l' 'ascoltatrice', a guardia del parlatorio, e l' 'accompagnatrice' che trattava con le diverse maestranze del monastero, e ancora l' 'infermiera' e le maestre delle educande, novizie o converse. ${ }^{15}$ Le 'coriste' erano le monache preposte al coro, il cui compito principale era attendere all'Ufficio divino, ossia alla preghiera, secondo la liturgia delle ore canoniche. ${ }^{16}$

All'interno dei chiostri si riproduceva la rigida gerarchia di valori sociali che esisteva nella realtà secolare. Accanto alle coriste, vi erano infatti le converse, in genere ragazze di bassa estrazione sociale. Giudicate inferiori e trattate come tali, esse erano chiamate a svolgere i compiti più umili, come ad esempio occuparsi delle faccende domestiche del monastero; in questo modo sollevavano le monache altolocate dalle occupazioni più gravose e le lasciavano libere di dedicarsi completamente alla preghiera e alle cariche più rilevanti per la comunità. In alcune realtà vi era anche la presenza delle cosiddette serve, donne che non prendevano i voti, ma che comunque erano tenute all'osservanza della vita claustrale finché rimanevano a lavorare nel monastero.

12 Asdn, Vicario, 335 D, app. p. 230.

13 In queste pagine si fornisce un quadro di riferimento generico circa ruoli, nomi e competenze espletati dalle monache. Ogni monastero aveva poi le sue differenze, ad esempio, a Santa Chiara l'ufficio dell'economa era gestito dalla 'camerlenga'.

14 Una ruota, posizionata in genere sul portone d'ingresso di un istituto, permetteva di controllare minuziosamente ciò che entrava e usciva dal monastero.

15 ILlibato 1985.

16 Hills 2004, pp. 115-119; Zarri 2000, pp. 87-89; PAolin 1996, p. 35 e sg. 
In alcuni monasteri erano presenti delle educande. ${ }^{17}$ Queste appartenevano in genere a famiglie benestanti o patrizie, pagavano una retta mensile ed erano in numero inferiore rispetto alle monache che le ospitavano. Le educande erano tenute a seguire le leggi della clausura e una volta entrate non potevano uscire dal chiostro fino al momento del matrimonio oppure di prendere i voti. Dovevano avere più di 7 anni e meno di 25, ed era obbligo che il numero di educande ammesse non superasse il numero prefisso indicato dalle costituzioni di ciascun monastero. ${ }^{18}$ Anche le educande avevano una loro maestra che doveva istruirle nella dottrina cristiana, nel modo di confessarsi e comunicarsi, di recitare le preghiere e le altre pratiche religiose. La maestra doveva inoltre sorvegliare che le fanciulle non cantassero e non leggessero libri profani. Così infatti ci informano le costituzioni di San Gregorio Armeno:

\footnotetext{
Per quanto tocca poi alle Sig.re che si ricevono in detto Mon.ro con licenza questo si prattica compiti li sette anni, e si chiamano Educande, sono sottoposte alla direzzione d'una Monica, la quale si chiama Maestra dell'Educande, nella tenera loro età apprendono il santo timor di Dio, ed j regolari precetti, legendo libri spirituali, ed altre recite di preci vocali, e nel tempo che rimane, acciò non regni l'ozio, si applicano à qualche esercizio manuale nel luogo particolare che detto Mon.ro tiene, chiamato l'educazione, contribuendo $\mathrm{j}$ Parenti di ciascheduna educanda annuj docati $72 \mathrm{col}$ pagamento del semestre anticipato, accio il Mon.ro non venghi gravato, e per l'anno del noviziato contribuiscono doc.ti $60 .{ }^{19}$
}

\section{Città, aristocrazia e istituzioni regie}

L'immagine che regalava Napoli fra Sei e Settecento, era quella di una metropoli in cui sovrabbondavano edifici religiosi a scapito delle abitazioni civili. L'eccessivo numero di chiese e monasteri costituiva infatti un motivo di sorpresa per i numerosi forestieri che visitavano la città. Fra XVII e XVIII sec., si registrò un moltiplicarsi delle istituzioni religiose femminili e della popolazione monastica in rapporto sproporzionato rispetto al numero della popolazione civile. ${ }^{20}$ Il Celano annotava infatti che «per il numero e la vastità dei suoi edifici sacri e dei suoi chiostri, Napoli poteva dirsi una città conventuale». ${ }^{21} \mathrm{Nel}$ Seicento Napoli era divisa in 29 ottine ${ }^{22}$ raggruppate in nove quartieri e intorno al 1620, si contavano circa 400 istituzioni religiose, di cui 31 monasteri femminili.

17 La presenza delle educande era una prerogativa degli istituti assistenziali che avevano il compito di ospitare "in educazione" giovani fanciulle. Si veda il cap. VII.

18 Illibato 1985, p. 15 e seg.

19 Spinosa/Pinto/Valerio 2013, appendice A.

20 Sui rapporti fra edilizia sacra e civile si veda Strazzullo 1968.

21 Celano 2001, Giornata I, p. 120.

22 L'ottina indicava l'area territoriale appartenente ad una parrocchia della città. Il termine probabilmente derivava dal nome dell'originario organismo di rappresentanza territoriale costituito da otto notabili eletti per ogni zona. 
I monasteri rappresentarono per la capitale del viceregno un valore aggiunto: al pari delle corti aristocratiche, furono importanti centri di produzione, di consumo e di ridistribuzione delle risorse finanziarie e materiali. Nel corso dei secoli, molti istituti femminili si trovarono ad accumulare e a gestire enormi risorse economiche, grazie ad una fitta rete di scambi e di relazioni con il territorio. Scorrendo i registri contabili si legge che le comunità monastiche amministravano case e terre, e, coadiuvate da procuratori e governatori, gestivano investimenti finanziari. Alcuni monasteri erano delle vere e proprie aziende addette alla fabbricazione di stoffe, dolci, pane, pasta, conserve, il cui ricavato costituiva una rendita per la comunità. Tutto ciò era in paradossale controtendenza con quanto la riforma post-tridentina aveva tentato di fare, separando vita claustrale e mondo esterno. L'attività commerciale invece contribuiva a intrecciare sempre più i chiostri con le dinamiche della vita economica e sociale del territorio.

I patrimoni, testimoniati dai libri dei conti, mettono a nudo interessi e relazioni economiche. Anche il numero e l'estrazione sociale delle monache che vi risiedevano concorreva alla costituzione del patrimonio grazie al deposito della dote e al ruolo e all'influenza delle famiglie. Le monache tendevano a ritagliarsi spazi propri all'interno della gestione economica dei chiostri e al momento di fare la professione, pur rinunziando ai loro beni, si riservavano somme di denaro che gestivano autonomamente. Queste risorse, di cui però non si trova traccia nei registri contabili, costituivano un aspetto non secondario dell'economia monastica. Accumulandosi negli anni esse furono delle forme di risparmio per attività finanziarie o di committenza artistica, per l'acquisto di opere d'arte, arredi per le celle, biancheria pregiata etc. ${ }^{23}$ Spendere somme di denaro ragguardevoli attingendo dai vitalizi personali o dal reddito comune era un abuso incompatibile con il voto di povertà. Tuttavia la consultazione dei registri d'esito conferma le ingenti spese per festività e regali. I destinatari erano di solito i benefattori, i confessori e i predicatori, ma anche i maestri, i viceré e l'arcivescovo con i suoi collaboratori.

La rilevanza dei monasteri era soprattutto determinata dai rapporti che essi intrattenevano con gli esponenti della gerarchia ecclesiastica, l'aristocrazia locale e le famiglie del patriziato. L'ingresso in uno dei chiostri cittadini di qualche esponente femminile di un gruppo parentale altolocato, garantiva alla famiglia ascesa e rilancio sociale nel panorama politico e aristocratico della città. ${ }^{24}$

Nei registri di istituzioni come Santa Chiara, la Croce di Lucca, Regina Coeli, San Gregorio Armeno, Donnaregina, San Potito, si incontrano e si ripetono cogno-

23 Le monache esercitarono un'importante azione di patrocinio nel campo dell'arte e dell'architettura e permisero ad artisti di grande fama di cimentarsi nell'abbellimento di chiese e istituti. Operarono nei chiostri napoletani artisti quali Cosimo Fanzago, Luca Giordano, Domenico Antonio Vaccaro, Francesco Solimena, Francesco De Mura, Luigi Vanvitelli etc. Cfr. VALERIo 2007, p. 14.

24 Sui rapporti fra monasteri, città e poteri locali si veda: Novi-Chavarria 1993, pp. 84-111; NOVI-CHAVARRIA 2001. 
mi prestigiosi (Pignatelli, Caracciolo, Capece Minutolo), anche perché le famiglie aristocratiche erano spesso imparentate tra di loro. Esisteva inoltre un rapporto preciso tra la dislocazione del monastero, la presenza al suo interno di gruppi familiari e l'appartenenza ad uno dei seggi della capitale. Le famiglie nobiliari napoletane erano infatti raggruppate in cinque 'seggi'. Roccaforti del patriziato e organi amministrativi del governo cittadino ${ }^{25}$ i seggi contribuivano ad esercitare numerose forme di controllo sulle comunità monastiche femminili, orientandone i criteri di gestione patrimoniale e le modalità di reclutamento delle religiose. Ad esempio monasteri come San Gregorio e Santa Patrizia erano riservati ai nobili di Nido e Capuana, cui facevano capo le famiglie di maggior peso politico, rappresentando essi la nobiltà antica, quella originaria della città.

I legami fra patriziato e istituzioni religiose si esplicitavano in maniera ancora più decisiva nei monasteri di regio patronato, una tipologia di istituzione che si sottraeva all'autorità episcopale per obbedire direttamente al Pontefice tramite i nunzi apostolici. L'essere denominati istituzioni regie permetteva ai monasteri di essere maggiormente legati al potere sovrano, di godere di benefici e privilegi, di essere liberi di agire e di disporre dei propri beni in quanto sottratti all'autorità episcopale. Questo però comportava il verificarsi di numerosi conflitti tra poteri laici, gerarchie ecclesiastiche e nobiltà, soprattutto perché gli equilibri fra le diverse parti, erano resi ancora più precari dall'intervento di autorità come quelle dei nunzi apostolici e dei viceré. ${ }^{26}$

Le famiglie aristocratiche crearono reti di alleanze per accaparrarsi il controllo dei monasteri più in vista e dei loro patrimoni. Le istituzioni regie rappresentavano una vera e propria forma di investimento economico e davano la possibilità di offrire una onorevole protezione alle nobildonne non destinate al matrimonio. Pertanto era interesse dell' aristocrazia garantire un sostegno a queste istituzioni.

Essendo slegate dalle autorità ecclesiastiche locali, le istituzioni di regio patronato avevano maggiori concessioni anche riguardo l'impiego di musica nelle cerimonie ordinarie e straordinarie. Differentemente dagli altri monasteri, le fondazioni regie usufruivano dei musicisti provenienti dalla prestigiosa Real Cappella di Palazzo per il corredo musicale di feste e liturgie e per la formazione musicale delle educande e delle figlie ospiti. ${ }^{27}$ Questo fece si che tali istituzioni divenissero

25 I seggi, detti anche 'sedili' o 'piazze', erano organismi con funzioni amministrative e politiche che ed avevano il compito di conciliare le esigenze dei cittadini ed il potere del sovrano. I sedili nobili erano cinque: Capuana, Montagna, Nido, Porto e Portanova - tutti suddivisi in ottine, ad essi si aggiungeva il sedile del Popolo che comprendeva la popolazione non nobile di tutta la città. I seggi più rinomati erano quelli di Capuana e Nido. Sui rapporti fra nobiltà di seggio e monasteri femminili si veda: Novi-CHAVARrIA 1993.

26 Sulle istituzioni sotto patronato regio si veda: Russo 1970; Novi-ChavarRIa 1993, pp. 84-111; Novi-Chavarria 2001; Fiore 2015², pp. 33-60.

27 Si rimanda in proposito a quanto detto nel cap. I, p. 43 a riguardo delle disposizioni sull'utilizzo di musicisti provenienti dalla Cappella Reale. 
veri e propri cenacoli di musicisti tra i più rappresentativi della città. Le vicende storiche e musicali di istituzioni regie quali il monastero di Santa Chiara e il conservatorio di Nostra Signora della Solitaria, come vedremo nelle pagine a loro dedicate, testimoniano perfettamente questa sintesi fra aristocrazia e poteri locali. ${ }^{28}$ 



\section{Liturgie ordinarie e straordinarie}

\section{Riti e culti}

L'organizzazione delle celebrazioni liturgiche ordinarie o straordinarie ad opera di monasteri e conservatori è un chiaro esempio di quanto la musica fosse un punto di contatto fra comunità claustrale e città, e di quanto fosse viva la volontà delle monache di prendere parte pienamente alla vita sociale, senza per questo negare la dimensione della fede, della ritualità e della devozione. Le cerimonie costituiscono inoltre un ottimo punto di osservazione per comprendere le relazioni di potere che intercorrevano fra comunità monastiche e autorità civili o religiose. Attraverso feste e liturgie le istituzioni acquisivano una visibilità nello spazio pubblico che permetteva loro di affermare il proprio peso nell'ambito cittadino e manifestare la propria identità.

Ogni monastero aveva una propria attività liturgica ed ingaggiava compositori e musicisti per le differenti celebrazioni. In questo senso le diverse istituzioni giocarono un ruolo non secondario nei meccanismi di committenza e produzione di molta musica sacra. ${ }^{1}$ Il compito della musica era infatti quello di rendere solenne e fastoso ogni rituale. Al contempo architetti e artisti, attraverso sofisticati apparati, trasformavano uno spazio consueto come quello di una chiesa in una sorta di 'teatro' allestito per la liturgia. Ogni dettaglio contribuiva alla magnificenza di tali eventi e al coinvolgimento dei fedeli.

Il corredo musicale festivo era affidato, nella maggior parte dei monasteri, alla Cappella della Cattedrale detta anche dell'Arcivescovado. Si trattava infatti di un organico formato da cantanti e strumentisti alle dipendenze dell'arcivescovo di Napoli. ${ }^{2}$ Come indicato dagli editti diocesani, la Cappella della Cattedrale era la sola a poter soddisfare l'offerta liturgica delle istituzioni sacre partenopee. ${ }^{3}$ Tuttavia i musicisti dell'arcivescovo si contendevano la scena sacra con la prestigiosa Cappella Reale di Palazzo da sempre considerata la principale istituzione musicale napoletana, il cui compito era quello di accompagnare le cerimonie religiose e mondane della corte. Dal momento della sua costituzione in poi, la Cappella Reale raccolse attorno a sé compositori e soprattutto celebri organisti come Jean

1 Sui meccanismi di produzione e committenza delle istituzioni sacre a Napoli e in altri contesti italiani si rimanda a BRYANT/QUARANTA 2005.

2 È interessante notare che i documenti d'archivio fanno utilizzo della dicitura "coro" per indicare l'insieme dei cantanti di questa compagine.

3 Si veda quanto detto al cap. I, p. 43. Purtroppo ad oggi non vi sono studi specifici legati a questa istituzione che doveva avere un peso non secondario nel panorama spettacolare napoletano. 
de Macque, Giovanni Maria Trabaci, Ascanio Majone, Andrea Falconieri, Filippo Coppola, Pietro Andrea Ziani, Alessandro Scarlatti. ${ }^{4}$ La compagine vicereale ebbe stretti legami anche con diverse istituzioni femminili: ${ }^{5}$ furono soprattutto i monasteri di regio patronato a beneficiare dei musicisti e cantanti della Cappella Palatina, supportati dalla protezione dei viceré. Questa situazione non mancò di suscitare spesso severi rimproveri da parte della Curia.

A fianco delle cappelle musicali stabili, si riscontra anche la partecipazione di compagini ad organico variabile che potremmo definire occasionali: composte da musicisti provenienti dai gruppi strumentali cittadini, erano organizzate dal maestro di cappella o dall'organista in servizio presso l'istituzione e remunerate in base ad una determinata occorrenza festiva. La composizione e la grandezza degli organici rispecchiava in genere l'importanza dell' istituzione promotrice di un evento e la rilevanza dell'evento stesso, assieme a ragioni di ordine economico e allo spazio a disposizione. ${ }^{6}$ Il numero dei musicisti stabili era inoltre destinato ad aumentare in occasione delle festività più solenni con l'arruolamento di artisti aggiunti, detti anche "soprannumerari". ${ }^{7}$ Nella celebrazione delle liturgie

4 La Cappella Reale venne fondata nel 1555 dal viceré Pedro de Toledo, Diego Ortiz fu il primo maestro di cappella. Sulla Cappella Reale si vedano: Рrota-Giurleo 1952, pp. 19-77; Dietz 1972², pp. 379-406; MaIONe 2005, pp. 309-341; Fabris 1983, pp. 63-110; FABRIs 2001, pp. 235-250; Krause 1993, pp. 235-257 e Krause 1998, pp. 271-295; Fiore 2012, pp. 25-44.

5 L'esempio che maggiormente testimonia e chiarisce i rapporti fra Cappella Reale e istituzioni religiose è il conservatorio di Nostra Signora della Solitaria, cfr. cap. VIII, pp. 203-216. Il modello di organizzazione delle attività musicali delle istituzioni religiose napoletane trova un rispecchiamento quasi perfetto nel variegato ambito palermitano, caratterizzato da profili istituzionali pressoché identici, nonché dalla circolazione di medesimi generi e repertori. Fra XVI e XVIII sec. la situazione politica e culturale di Napoli e Palermo presenta caratteristiche fra loro assimilabili e al contempo assai diverse da quelle delle altre corti europee, essendo entrambe province spagnole. La fitta rete di interscambi fra monasteri, conventi e chiese si strutturava attraverso la presenza delle cappelle musicali, e in particolar modo delle rispettive Cappelle Reali. Proprio i legami della Cappella palermitana con quella partenopea, forniscono importanti informazioni sui repertori, sugli organici, e soprattutto sulle interazioni con il tessuto sociale, tali da far comprendere che si trattasse di realtà pressoché gemelle. I primi risultati di questo lavoro di comparazione sono confluiti in un paper intitolato Musica nelle istituzioni religiose del Meridione d'Italia: ipotesi di confronto fra le Cappelle Reali di Napoli e Palermo, presentato assieme ad Ilaria Grippaudo dell'Università di Palermo al «III Encontro Nacional de Investigação em Música (ENIM 2013)» della Sociedade Portuguesa de Investigação em Música (Cascais, Palácio da Cidadela, 1-3 novembre 2013).

6 Oltre alla Cappella Reale e alla Cappella della Cattedrale a Napoli esistevano anche altri organici stabili legati a specifiche istituzioni religiose. La più prestigiosa di esse era la cappella musicale del Tesoro di San Gennaro, ensemble destinato ad avere un ruolo di grande importanza nel tessuto artistico cittadino nel corso dei secoli. La minuta ricostruzione delle vicende artistiche e musicali della Cappella di San Gennaro è oggetto del volume: Columbro/Maione 2008. Anche la Casa Santa dell'Annunziata si avvaleva di un complesso musicale stabile di cantori e strumentisti. Sull'attività musicale dell'Annunziata si veda Columbro 2001, pp. 42-78; cfr. cap. VII, pp. 192-193.

7 Sul meccanismo dell'impiego e della soprannumerarietà si rimanda a CotTiCELLi/MAione 1996, pp. 28-35; TuFAno 2009, pp. 773-804. 
in genere la cappella musicale era posizionata in appositi spazi allestiti per l'occasione e denominati "orchestri", mentre i cantanti erano ospitati nei "coretti".

Fondamentale per Napoli furono poi i sodalizi che le istituzioni religiose femminili stipularono con i quattro conservatori maschili: Santa Maria della Pietà de' Turchini, Santa Maria di Loreto, Sant'Onofrio a Capuana e i Poveri di Gesù Cristo. In questi istituti caritativi si formarono figure musicali di alto profilo, poi assurti alla notorietà nella capitale del viceregno, come Francesco Provenzale, Alessandro Scarlatti, Giovanni Paisiello o Niccolò Piccinni. ${ }^{8}$ Gli allievi, citati spesso come "figlioli", venivano impiegati per soddisfare servizi liturgici e musicali di chiese, cappelle, monasteri, congregazioni, confraternite e ordini ecclesiastici. Molto spesso gli allievi dei conservatori intervenivano alle liturgie con le famose «flottole», ovvero con stuoli di ragazzini a volte travestiti da angeli, con un ruolo non sempre legato alla musica, ma spesso semplicemente come ornamento.

Ecco dunque che il conservatorio dei Poveri di Gesù Cristo assicurava la presenza dei propri «figlioli» a San Gregorio Armeno «avanti la statua e sangue del loro Glorioso San Giovanni Battista», ${ }^{9}$ oppure per le festività di Sant'Agata presso il monastero benedettino di Donnalbina, dove si riscontra la presenza sia di allievi ingaggiati per il corteo processionale, sia dei «musici» che intervenivano invece nella liturgia:

Alli Musici de Poveri di Gesù Cristo che hanno cantato Vespero e Messa in detta Festa, come anche alli Figlioli di detto Conservatorio, che hanno accompagnato la processione colla statua di Sant'Agata due volte cioè il giorno della vigilia della Santa quando escì dal Monastero, e quando ritornò, se li sono pagati d. nove di limosina fatta dentro del Monisterio. ${ }^{10}$

Le date del calendario liturgico solennizzate con musica erano numerose e differenti per ciascun ente, per lo più legate ai tridui per i santi patroni e alle ricorrenze mariane. ${ }^{11}$ L'anno liturgico dei monasteri risulta essere infatti ricco di devozioni legate al culto di Maria: cinque istituti solennizzano la ricorrenza dell'Immacolata Concezione, sette quella dell'Assunta, cinque la Natività della Vergine, tre il titolo di Santa Maria delle Grazie, ma si trovano ancora la festa della Madonna del Rosario, della Visitazione e della Purificazione della Vergine, della Madonna del Carmelo e della Madonna dei Dolori.

Tra i momenti del normale calendario liturgico che maggiormente richiedevano l'intervento di musicisti ingaggiati, spiccano naturalmente le celebrazioni relative all'Avvento, al Natale e all'Epifania, quattordici istituti annoverano spese per cantori e strumentisti per messe solenni e per la novena di Natale. Ben sedici istituzioni

8 Sull'attività dei quattro conservatori maschili si vedano: Florimo 1882; Di Giacomo 1928; Dietz 1972, pp. 419-429; Fabris 1983; Cafiero/Marino 1987; Fabris 1994; Cotticelli/ Maione 1996; Del Prete 1999, vol. II, pp. 671-715; Del Prete 1999², pp. 413-464; Olivieri 1999; Maione 2000; Cotticelli/Maione 2006.

9 Asbn, BPO 983 9/X/1728, app. p. 105.

10 Asn, MS f. 3249, app. p. 61.

11 Sull'avvicendarsi delle differenti festività nella Napoli vicereale si veda: FABRIs 2007; CAMPANELLI 2009. 
registrano spese per la musica del periodo quaresimale e per la Pasqua. ${ }^{12}$ Anche le celebrazioni per il Carnevale, che avevano inizio il 17 gennaio con la festa di Sant'Antonio Abate, coinvolgevano alcune istituzioni femminili. In questa occasione, fra i vicoli cittadini, si assisteva a maschere, giochi, scene improvvisate della commedia dell'arte cui prendevano parte tutti, dalla nobiltà alla borghesia, ai ceti popolari, agli stessi monasteri: ${ }^{13}$ sette istituti infatti organizzano commedie e rappresentazioni sceniche per questa occorrenza festiva, con l'allestimento di un vero e proprio teatro all'interno delle mura claustrali.

Le strade della città erano spesso invase da colorate processioni caratterizzate da macchine effimere costruite da qualificati artisti e accompagnate da cori di voci e strumenti, arricchite da effetti coreografici e da stendardi delle confraternite, attorno ai quali si stringevano parrocchie, monasteri e aggregazioni di fedeli. Fra le più sfavillanti processioni vi erano quelle legate al culto dell'Eucarestia, con l'ostensione del Santissimo Sacramento in occasione del Corpus Domini. Le celebrazioni dedicate alla solennità del Corpus Domini duravano una settimana ed il monastero delle clarisse di Santa Chiara era indiscusso protagonista dell'organizzazione cittadina del culto. ${ }^{14}$ A seguito della fastosa processione a Santa Chiara era organizzato un solenne ottavario, di cui fa menzione ripetutamente la «Gazzetta di Napoli».

Le processioni non erano organizzate soltanto in onore dell'Eucaristia o di Maria, ma anche dei santi protettori della città come Santa Chiara, San Lorenzo, San Giovanni e San Benedetto.

Pervasa da elementi folcloristici e da grande partecipazione di popolo, aristocrazia e istituzioni era la festività di San Giovanni Battista. Le celebrazioni per la festività del Battista erano una sorta di spettacolo itinerante per le vie cittadine con una serie di apparati scenografici di grande rilievo: cortei, tableaux vivants, rappresentazioni sacre, pantomime cui prendevano parte gli stessi monasteri femminili. ${ }^{15}$ Ben tre istituti erano stati custodi del prezioso sangue che era annualmente soggetto a liquefazione: Santa Maria di Donna Romita Sant'Arcangelo a Baiano e San Gregorio Armeno. ${ }^{16}$

La musica era di certo il mezzo privilegiato per donare dignità e solennità alle feste e per aiutare il fedele alla contemplazione del soprannaturale. Purtroppo per le istituzioni napoletane, i documenti d'archivio non forniscono molte indicazioni sulla tipologia di repertorio utilizzato nelle differenti festività: spesso si riscontra una generica indicazione di messe e vespri cantati, solenni esposizioni, ottavari, novene e tridui con musica. Inoltre, non sempre si hanno chiare informazioni sul

12 Si vedano le pp. 64-67 di questo capitolo.

13 FABRIS 2007 pp. $2-5$.

14 Si veda cap. VI, pp. 178-179.

15 Sulla festività cittadina di San Giovanni e sugli apparati 'giovannei' si veda: Megale 2013, pp. 387-413.

16 Sulle celebrazioni per San Giovanni si veda anche il cap. V, pp. 129-130. 
numero di musicisti impiegati o sulla compagine. In alcuni casi nei registri contabili vengono riportati i pagamenti per il numero o l'insieme dei musicisti coinvolti e viene nominato soltanto il maestro di cappella o l'organista, come ad esempio, nel caso della messa cantata per la solennità dell'Epifania celebrata nel monastero benedettino di San Potito nel 1719:

In gennaro $[\ldots]$ per la messa cantata dell'Epifania del Signore $[\ldots]$

Per sedici Istrumenti d. 25.3.10

A sette voci d. 23

Al mastro di Cappella Don Angelo Durante d. 4

A Don Andrea Amendola organista d. 2

Per affitto d'organo d. 1.2.10

Alli trombettieri numero cinque d. $2.1^{17}$

I dati indicati dal documento sopracitato sono pochi ma ugualmente utili per dedurre che in questa celebrazione vi era stata la partecipazione della cappella dell'Arcivescovado, di cui facevano parte Andrea Amendola e Angelo Durante. In casi più fortunati si trovano invece informazioni dettagliate che permettono la ricostruzione pressoché integrale degli organici vocali e strumentali intervenuti nelle cerimonie. ${ }^{18}$

Aveva infatti poca importanza citare i nominativi dei singoli musicisti, bastava più che altro evidenziare i nomi illustri, perché appartenenti alla Cappella Reale piuttosto che a quella della Cattedrale. L'esplicito riferimento, nella documentazione d'archivio o nelle cronache, a musicisti di chiara fama, contribuiva infatti a sottolineare l'importanza della manifestazione.

Risulta quindi complicato comprendere quale fosse il ruolo delle istituzioni femminili nella committenza e discernere se e quando i pagamenti facciano riferimento alla composizione o alla sola esecuzione di musiche già composte $\mathrm{e}$ riutilizzate per l'occasione. Abbiamo infatti solo pochi riferimenti espliciti che attestano somme stanziate per la composizione di musiche originali. Ne è un esempio il documento seguente, tratto dalla documentazione del monastero di Regina Coeli, che attesta la commissione di una composizione in occasione del Natale, senza tuttavia specificare il nome del compositore ingaggiato:

Spese estraordinarie 1739-40

Donna Marianna Scalera Abbadessa tanti consegnati contanti à Donna Chiara Maria d'Andrea per spenderli in una composizione di Musica per la notte di Natale d. $22 .{ }^{19}$

17 Asn, MS, f. 2940, app. p. 21.

18 Si veda ad esempio il documento relativo alla festività di Santa Maria Egiziaca del 26 e 27 aprile 1749 avvenuta nell'omonimo monastero che riporta l'indicazione dei musicisti e dei cantanti ingaggiati, con la specifica del canto dei mottetti a loro affidato. Cfr. cap. V, pp. 139-140.

19 MS, f. 1990, app. p. 228. 
Nello schema seguente si propone un calendario delle festività solennizzate con musica dalle istituzioni femminili napoletane fra XVII e XVIII secolo: ${ }^{20}$

Tabella 1: Festività celebrate con musica.

\begin{tabular}{|c|c|c|}
\hline Festività & Data & Istituzione \\
\hline Circoncisione & 1 gennaio & $\begin{array}{l}\text { Donnalbina; } \\
\text { S. Potito }\end{array}$ \\
\hline Epifania & 6 gennaio & $\begin{array}{l}\text { Donnalbina; } \\
\text { S. Potito; Regina Coeli }\end{array}$ \\
\hline S. Potito & 14 gennaio & S. Potito \\
\hline S. Antonio Abate & 17 gennaio & $\begin{array}{l}\text { S. Antonio a Port.; } \\
\text { Donna Romita }\end{array}$ \\
\hline Ss. Fabiano e Sebastiano & 20 gennaio & Ss. Pietro e Seb.; Donnaregina \\
\hline $\begin{array}{l}\text { Candelora/Purificazione di } \\
\text { Maria }\end{array}$ & 2 febbraio & $\begin{array}{l}\text { Donnalbina; Croce di Lucca; } \\
\text { SS. Trinità; S. M. del Gesù }\end{array}$ \\
\hline S. Biagio & 3 febbraio & $\begin{array}{l}\text { Donna Romita; S. Gregorio; } \\
\text { SS. Pietro e Sebastiano; Donnaregina }\end{array}$ \\
\hline Sant'Agata & 5 febbraio & Donnalbina \\
\hline S. Scolastica & 10 febbraio & Donnalbina \\
\hline Carnevale & - & $\begin{array}{l}\text { Donnalbina; } \\
\text { Egiziaca Pizz.; Donnaregina; Provvidenza; } \\
\text { S. M. del Gesù; S. Chiara; } \\
\text { S. M. Maddalena delle Sp. }\end{array}$ \\
\hline S. Giuseppe & 19 marzo & $\begin{array}{l}\text { S. Potito; } \\
\text { S. Giuseppe Ruffi; } \\
\text { S. M. Maddalena delle Sp.; } \\
\text { S. M. del Gesù; } \\
\text { Provvidenza }\end{array}$ \\
\hline S. Benedetto & 21 marzo & $\begin{array}{l}\text { Donnalbina; } \\
\text { S. Gregorio; } \\
\text { S. Patrizia; } \\
\text { S. Potito }\end{array}$ \\
\hline Annunciazione & 25 marzo & S. M. del Gesù \\
\hline Quaresima & - & $\begin{array}{l}\text { Solitaria; } \\
\text { S. Caterina da Siena }\end{array}$ \\
\hline Domenica di Passione & $\begin{array}{l}\text { Domenica di } \\
\text { Passione }\end{array}$ & Solitaria \\
\hline
\end{tabular}

20 Nel seguente schema sono state confrontate le festività citate nei documenti delle singole istituzioni con due differenti edizioni del Messale Romano post-tridentino: Missale 1998; Missale 1994. 


\begin{tabular}{|c|c|c|}
\hline Festività & Data & Istituzione \\
\hline Conversione M. Maddalena & $\begin{array}{l}\text { Giovedì dopo la } \\
\text { domenica di Passione }\end{array}$ & S. M. Maddalena delle Sp. \\
\hline Madonna dei Dolori & $\begin{array}{l}\text { Venerdì dopo la } \\
\text { domenica di Passione }\end{array}$ & Solitaria; Donnalbina \\
\hline Domenica delle Palme & - & Donnalbina; Egiziaca Mag. \\
\hline Settimana Santa & - & $\begin{array}{l}\text { Donnalbina; } \\
\text { S. Potito; } \\
\text { Egiziaca Mag.; S. Giuseppe Ruffi; } \\
\text { Croce di Lucca; } \\
\text { S. M. Maddalena delle Sp.; } \\
\text { S. M. Maddalena Mag.; Donnaregina; } \\
\text { SS. Trinità; } \\
\text { S. Caterina da Siena; } \\
\text { S. Francesco Capp; } \\
\text { S. Antonio a Port.; } \\
\text { Regina Coeli; } \\
\text { Provvidenza; } \\
\text { Solitaria }\end{array}$ \\
\hline Pasqua & - & $\begin{array}{l}\text { Divino Amore; } \\
\text { S. Caterina da Siena; } \\
\text { Solitaria; Donnalbina }\end{array}$ \\
\hline Pentecoste & - & $\begin{array}{l}\text { Donnalbina; Croce di Lucca; } \\
\text { S. Caterina da Siena; } \\
\text { Spirito Santo }\end{array}$ \\
\hline SS. Trinità & - & $\begin{array}{l}\text { Donnalbina; Divino Amore; } \\
\text { Provvidenza; } \\
\text { SS. Trinità }\end{array}$ \\
\hline Corpus Domini & - & $\begin{array}{l}\text { Donnalbina; } \\
\text { S. Chiara; } \\
\text { Egiziaca Mag.; } \\
\text { Croce di Lucca; } \\
\text { S. Francesco Oss.; } \\
\text { Ss. Marcellino e Festo; } \\
\text { S. Antonio a Port. }\end{array}$ \\
\hline S. M. Egiziaca & 26 aprile & Egiziaca Mag.; Egiziaca Pizz. \\
\hline S. Caterina da Siena & 30 aprile & S. Caterina da Siena \\
\hline S. Croce & 3 maggio & Croce Lucca \\
\hline S. Michele & 8 maggio & Solitaria \\
\hline S. Antonio da Padova & 13 giugno & Provvidenza; S. Antonio a Port. \\
\hline S. Giovanni Battista & 26 giugno & Donnalbina; Donna Romita; S. Gregorio \\
\hline Ss. Pietro e Paolo & 29 giugno & $\begin{array}{l}\text { Donnalbina; Ss. Pietro e Sebastiano; } \\
\text { Regina Coeli }\end{array}$ \\
\hline Visitazione di Maria & 2 luglio & Donnalbina \\
\hline
\end{tabular}




\begin{tabular}{|c|c|c|}
\hline Festività & Data & Istituzione \\
\hline S. M. delle Grazie & 5 luglio & $\begin{array}{l}\text { S. Chiara; } \\
\text { S. M. del Gesù; } \\
\text { S. M. delle Grazie }\end{array}$ \\
\hline S. Silvano & 13 luglio & S. Francesco Oss. \\
\hline S. M. del Carmelo & 16 luglio & Croce di Lucca \\
\hline S. M. Maddalena & 22 luglio & $\begin{array}{l}\text { S.M. Maddalena delle Sp.; } \\
\text { S. M. Maddalena Mag.; Donnalbina }\end{array}$ \\
\hline S. Giacomo & 25 luglio & Donnalbina \\
\hline S. Anna & 26 luglio & S. M. del Gesù; Donnalbina \\
\hline S. Domenico & 4 agosto & $\begin{array}{l}\text { Divino Amore; S. M. Maddalena delle } \\
\text { Sp.; Ss.Pietro e Seb.; } \\
\text { S. Caterina da Siena; Donnaregina }\end{array}$ \\
\hline S. Donato & 7 agosto & Ss. Marcellino e Festo; Donnaregina \\
\hline S. Lorenzo & 10 agosto & Donnalbina \\
\hline S. Chiara & 12 agosto & $\begin{array}{l}\text { S. Chiara; } \\
\text { S. Francesco Scarioni }\end{array}$ \\
\hline Assunzione & 15 agosto & $\begin{array}{l}\text { Donnalbina, Donna Romita; Regina } \\
\text { Coeli; Divino Amore, Donnaregina; } \\
\text { Provvidenza; } \\
\text { S. Francesco Scarioni. }\end{array}$ \\
\hline S. Rocco & 16 agosto & Donnaregina \\
\hline S. Bartolomeo & 24 agosto & Donnaregina \\
\hline S. Patrizia & 25 agosto & S. Patrizia \\
\hline S. Agostino & 28 agosto & $\begin{array}{l}\text { Egiziaca Mag.; Egiziaca Pizz.; } \\
\text { S. Giuseppe Ruff; } \\
\text { Regina Coeli; Divino Amore }\end{array}$ \\
\hline Decollazione del Battista & 29 agosto & Donna Romita, S. Gregorio \\
\hline Natività di Maria & 8 settembre & $\begin{array}{l}\text { Donna Romita; Donnalbina; } \\
\text { S. Potito; Regina Coeli; } \\
\text { S. M. del Rifugio } \\
\end{array}$ \\
\hline Esaltazione della Croce & 14 settembre & $\begin{array}{l}\text { Egiziaca Mag; Egiziaca Pizz.; Croce } \\
\text { Lucca }\end{array}$ \\
\hline $\begin{array}{l}\text { Conversione di S. Maria } \\
\text { Egiziaca }\end{array}$ & 14 settembre & S. M. Egiziaca Mag.; S. M. Egiziaca Pizz. \\
\hline S. Gennaro & 19 settembre & $\begin{array}{l}\text { Donnalbina, Provvidenza, SS. Filippo e } \\
\text { Giacomo }\end{array}$ \\
\hline S. Gregorio Armeno & 20 settembre & S. Gregorio \\
\hline S. Matteo & 21 settembre & S. Gregorio \\
\hline S. Michele & 29 settembre & Solitaria \\
\hline
\end{tabular}




\begin{tabular}{|c|c|c|}
\hline Festività & Data & Istituzione \\
\hline S. Francesco & 4 ottobre & $\begin{array}{l}\text { Donnaregina; } \\
\text { S. M. del Gesà; } \\
\text { S. M. Maddalena Mag.; } \\
\text { S. Francesco Oss.; } \\
\text { S. Francesco Scarioni; } \\
\text { SS. Trinità; Provvidenza; } \\
\text { S. Chiara. }\end{array}$ \\
\hline Madonna del Rosario & 7 ottobre & $\begin{array}{l}\text { S. Caterina da Siena; } \\
\text { S. M. del Rifugio }\end{array}$ \\
\hline S. Orsola & 21 ottobre & SS. Trinità \\
\hline S. Martino & 11 novembre & Donnalbina \\
\hline $\begin{array}{l}\text { Presentazione di Maria al } \\
\text { Tempio }\end{array}$ & 21 novembre & Scorziata; Paparelle \\
\hline S. Andrea & 30 novembre & $\begin{array}{l}\text { Donnaregina; } \\
\text { S. Chiara }\end{array}$ \\
\hline S. Nicola & 6 dicembre & S. Francesco Capp. \\
\hline Immacolata Concezione & 8 dicembre & $\begin{array}{l}\text { Donnalbina; Provvidenza; S. M. del Gesù; } \\
\text { Ss. Marcellino e F.; } \\
\text { Concezione a Montecalv. }\end{array}$ \\
\hline S. Aniello & 14 dicembre & Donnalbina \\
\hline Natale & 24-25 dicembre & $\begin{array}{l}\text { Donnalbina, Donna Romita; Egiziaca } \\
\text { Mag.; Egiziaca Pizz.; Regina Coeli; Croce } \\
\text { Lucca; } \\
\text { S. Francesco Capp.; } \\
\text { S. Francesco Oss.; S. Francesco Scarioni; } \\
\text { SS. Trinità; Sapienza; } \\
\text { S. Caterina da Siena; } \\
\text { Solitaria; } \\
\text { S. M. Costantinopoli }\end{array}$ \\
\hline
\end{tabular}

S. Giovanni Evangelista 27 dicembre $\quad$ Provvidenza

\section{La Settimana Santa}

Le principali istituzioni ecclesiastiche partenopee intensificavano apparati scenici e spettacolari in occasione delle celebrazioni della Settimana Santa. Nonostante lo spirito penitenziale che questo momento liturgico avrebbe dovuto comportare, si assiste invece ad una lunga successione di sfarzose liturgie durante tutto il periodo quaresimale fino alla domenica di Pasqua. La Settimana Santa doveva probabil- 
mente rappresentare il momento di maggiore impiego per i musicisti e le cappelle della capitale del viceregno. ${ }^{21}$

Testimonianza del contrasto fra la sobrietà imposta dalla Chiesa e gli usi delle istituzioni si trovano nelle guide e nei diari di viaggiatori stranieri in visita a Napoli. Jean-Jacques Bouchard durante il suo soggiorno napoletano nel 1632, descrisse nel suo celebre Journa $l^{2}$ le tradizioni partenopee e le liturgie a cui aveva avuto modo di assistere. Bouchard osserva le processioni della domenica delle Palme e le funzioni del Giovedì Santo, il triduo delle Tenebre, sottolineando quanto la preparazione della Pasqua a Napoli fosse caratterizzata da poco riguardo nei confronti delle restrizioni imposte dalla Chiesa e quanto il lato spettacolare delle cerimonie religiose prendesse il sopravvento sul clima quaresimale. Lo spettacolo era di certo un elemento essenziale del culto, il fedele era visto come uno spettatore, e si contravveniva costantemente al silenzio prescritto per la settimana auspicato dal Concilio di Trento.

Anche nei registri contabili dei monasteri femminili numerose risultano essere le voci di spesa destinate all'organizzazione del culto e degli apparati della Settimana Santa. Diversi riferimenti si trovano per il canto liturgico del "Passio", una pratica musicale inserita nella liturgia di rito romano fin dal Medioevo volta all'intonazione del racconto evangelico della Passione di Cristo. Le monache di San Gregorio Armeno, ad esempio, chiedevano espressa licenza alle autorità ecclesiastiche, affinché fosse loro concesso di far cantare il "Passio" da specifici cantori:

Si concede licenza alle signore Moniche del Venerabile Monistero di San Ligorio, che in questa Settimana Santa possano far cantare il Passio dalli Reverendi Don Domenico Basso per la parte di Cristo, Don Francesco d'Orsi da Testo, e Don Diego Fiore per la Turba, ed anco di far componere il sepolcro sopra la porta grande [...]. Oggi Marzo 1735. ${ }^{23}$

Il monastero di Donnalbina si serviva anche dell'intervento degli allievi dei conservatori maschili:

Alli clerici straordinari per detta causa et cantori del Passio et Exultatur [sic] col canto delli figlioli del Conservatorio d.18 [...]. ${ }^{24}$

Anche nelle comunità monastiche femminili gli usi e i costumi della Settimana Santa erano però decisamente differenti dalle proibizioni. Il cardinale Pignatelli, nel 1705, ordinava che «Offici, Lamentazioni, Lezzioni e Responsorii» durante la Settimana Santa venissero cantati soltanto in «canto fermo, senza trilli, e passaggi di sorte alcuna», senza alcun accompagnamento strumentale, anche «senza Organo». Era inoltre proibito l'intervento di musicisti esterni o «periti di canto 
figurato». ${ }^{25}$ L'uso del canto fermo non solo non veniva rispettato, ma le celebrazioni erano invece arricchite con musica e con l'intervento di molti cantanti e strumentisti.

Il rito delle Tenebre rappresentava il momento di maggiore suggestione del ciclo pasquale. Si svolgeva durante il Sacrum Triduum della Settimana Santa, dal Giovedì al Sabato Santo e ciascun giorno del triduo prevedeva una struttura simmetrica di tre notturni. ${ }^{26}$ Ciascun notturno era composto da una serie di tre antifone, tre salmi e tre lezioni seguite a loro volta da 3 responsori a cui seguivano le lodi. La liturgia impiegava nelle lezioni i testi delle lamentazioni di Geremia. Ogni lamentazione aveva inizio con l'intonazione di una lettera dell'alfabeto ebraico (Aleph, Beth, Gymel...). Ogni giornata terminava con l'invocazione Hierusalem, Hierusalem, convertere ad Dominum Deum tuum.

La denominazione di "Tenebre" si riferiva al passaggio dalla luce all'ombra all'interno della stessa liturgia che terminava infatti a lumi spenti. All'inizio del rito su di un candeliere di forma triangolare venivano accese quindici candele che venivano spente progressivamente, una dopo ciascun salmo, fino alla completa oscurità seguita dallo Strepitus ${ }^{27}$ momento in cui fedeli e celebranti battevano con vigore arnesi di legno per riprodurre il suono della terra scossa dalla morte di Cristo. Lo stesso Bouchard aveva preso parte al rito delle Tenebre presso l'oratorio di San Giovanni dei Fiorentini:

Le mercredi saint septieme Avril. La plus belle musique que l'on fasse est au vieus palais, à la chapelle du viceroi [...]. Le soir, à une heure de nuit, se dirent tenebres à l'oratoire de St Jan des Florentins, où la plus part des musiciens du palais se treuverent. La musique fut assez bone, mais courte, car ils ne chanterent que le repons et antienes et une seule leçon. ${ }^{28}$

Questo genere costituì un terreno fertile di creazione musicale in cui si cimentarono numerosi compositori napoletani e non. ${ }^{29}$ Proprio alcuni compositori e maestri di cappella attivi presso i monasteri femminili furono autori di lezioni e lamentazioni per la Settimana Santa, pensiamo infatti a Cristoforo Caresana e Gaetano Veneziano. ${ }^{30}$

Molte delle lezioni composte da Veneziano sono dedicate ad una voce solista con accompagnamento di archi e alcune di esse sono esplicitamente dedicate a

28

29

30

AsDn, Vicario, 259 D, app. p. 172.

L'indebolimento della consuetudine del clero secolare della preghiera notturna, lentamente impostosi, anticipò la celebrazione del mattutino alla sera del giorno prima, il che giustifica il ritrovare nel Seicento partiture destinate ai notturni dal Mercoledì Santo al Venerdì.

27 Nella tradizione meridionale chiamato anche Terremoto.

BOUCHARD 1977, p. 186.

Cfr. Fabris 2007, pp. 4-5; Bernardi 1991, pp. 316-319.

Si veda anche il cap. IV, pp. 109-112. 
religiose. ${ }^{31}$ E probabile che queste tipologie di composizioni venissero cantate direttamente dalle suore per le celebrazioni liturgiche della Settimana Santa, contravvenendo ancora una volta alla pratica del solo canto gregoriano prescritta dagli ordini disciplinari. ${ }^{32}$

L'esempio più rappresentativo di quanto accadeva nei chiostri durante questo periodo, ci viene fornito dal conservatorio della Solitaria, particolarmente rinomato per i riti della Settimana Santa. Presso questa istituzione era prevista musica «a 4 voci, e 4 instrumenti dalla Domenica delle olive per tutto il giorno di Pasqua di Resurrettione», ${ }^{33} \mathrm{e}$ ad essere protagonisti di questi eventi erano Gennaro Ursino, Gaetano Veneziano, Pietro Bartilotti, ovvero tutti esponenti della Cappella Reale. ${ }^{34}$ Presso l'istituzione spagnola l'evento di maggior impatto emotivo era la rinomata e suggestiva processione detta appunto "della Solitaria", che si snodava per le vie di Napoli nella notte del Venerdì Santo, portando in strada il mistero della morte di Cristo. Il canonico Carlo Celano nelle sue cronache fa infatti riferimento a «una divotissima processione con i misteri della Sacra Passione» cui prendevano parte «tutti i capi dei Tribunali e Ministri, in modo che vi si vedono torchi accesi al numero di duemila e forse più». ${ }^{35}$

Fede, pathos, folclore si mescolavano nella ricostruzione visiva del dramma della Passione. Ma il potente fascino di questo rituale era dato dalla teatralizzazione del dolore, che prendeva vita non solo attraverso canto e parola, ma anche con la gestualità dei corpi, i giochi di luce, i rumori. Purtroppo non si posseggono testimonianze iconografiche relative alla processione, ma la descrizione del Bouchard può essere utile a comprendere in che modo la processione avanzasse per le vie cittadine:

Après ces battans, suivent / quantité de faquins tous vestus de longues aulbes de toile et la face couverte, qui portent les uns au bout de longues perches de grans chaudrons pleins de poix, graisse et huile alumez, les autres des cierges de cire blanche au bout de certaines cannes fendues en quattre, et entourent tout le mystere, au devant duquel marche un choeur de musique, et au derriere le cavalier il quale conduce il misterio, avec tous ceus qu'il a invitez, ayants tous un cierge allumé en main. En ce mesme ordre suivent tous les autres mysteres [... ${ }^{36}$

31 Probabilmente non tutte le lezioni e responsori di ciascun notturno erano messi in musica. Durante la controriforma, dagli ultimi decenni del ' 500 , molti salmi, i responsori e una parte delle lezioni, erano cantati in polifonia, mentre le altre parti restavano in canto gregoriano.

32 Esempi di lamentazioni messe in musica per religiose si trovano anche in altri contesti italiani. Colleen Reardon nel saggio «Ha innalzato un muro attorno a me»: Lamentazioni per monache senesi, si interroga sul significato di questa tipologia di composizioni in relazione anche alla vasta diffusione in diverse regioni d'Italia. La Reardon propone un esame del simbolismo presente nelle Lamentazioni di Geremia rispetto al contesto monastico e ipotizza che queste composizioni possano essere considerate alla stregua dei pianti rituali che le donne usavano compiere durante i riti funebri in occasione della morte dei parenti. Si veda ReArdon 2000, pp. 27-44.

33 Solitaria, Giornale di introito ed esito 1697-1704, app. p. 329.

34 La "Domenica delle olive" indicava la Domenica delle Palme. Solitaria, Libro Maggiore 1704-1710, app. p. 330.

35 Celano 2001, Giornata V, p. 588.

36 BOUCHARD 1977, pp. 189-190. 
La processione era arricchita da passi processionali, i cosiddetti "misteri", raffigurazioni artistiche della Passione e Morte di Cristo, che si ergevano su una base sagomata di legno detta "vara", realizzati sia in legno che in cartapesta dagli artigiani napoletani. I gruppi si ispiravano all'iconografia classica, oppure ad episodi citati nei Sacri Testi o nei Vangeli Apocrifi. Le statue, illuminate in modo da far risaltare i tratti del volto, le movenze di dolore e sofferenza, venivano spesso arricchite con preziosi ornamenti argentei ed elaborate composizioni floreali che ne esaltavano la bellezza. Alla processione prendevano parte, oltre al popolo ai clerici e sacerdoti, il viceré, tutte le alte autorità cittadine compreso i corpi militari, assieme ad uno stuolo di battenti, ovvero fedeli che accompagnavano il corteo con lunghe tuniche e cappucci e armati di flagelli, aggiungendo in tal modo alla preghiera la mortificazione fisica, in segno di penitenza e di partecipazione alla Passione di Cristo ${ }^{37}$ Ad essi si aggiungevano i musici della Cappella Reale e le 'flotte' dei Conservatori.

Fino ad oggi le svariate carte d'archivio esaminate non ci restituiscono chiarimenti sul concreto impiego della musica nella processione. Molto spesso nei documenti contabili l'utilizzo di musica e musicisti viene semplicemente accennato senza precisi dettagli relativi alla prassi e alle modalità di esecuzione. Si trovano indicazioni in tal senso a partire dal 1602, in cui nelle spese relative alla processione si annotano i compensi ad Ascanio Meo «mastro de capilla de Santiago», cui venivano corrisposti «d. 7 por la musica del Viernes Santo y dia de Resurection», ${ }^{38}$ così come nel 1606 don Agustin Agresta «mastre de Capilla», viene ricompensato con «dies ducados por lo que ha trabajado con la musica la noche del Viernes Santo con la procession, y el domingo siguiente de la Resurection de Nostro Señor». ${ }^{39}$

La lunga maratona dei riti della Settimana Santa annoverava un'altra importante processione che si svolgeva il Sabato Santo, in qualche modo connessa a quella della Solitaria per l'utilizzo dei "misteri". Era la cosiddetta processione dei Battaglini, realizzata dalla Reale Arciconfraternita di Montecalvario che portava in processione la statua della Madonna con la raffigurazione dei Misteri che rappresentavano alcuni episodi della vita di Gesù e Maria. Questa processione coinvolgeva i viceré, la nobiltà, i funzionari più distinti dello stato. Era la più grandiosa $\mathrm{e}$ sontuosa manifestazione cittadina, e anche in essa si riscontrava la partecipazione della Real Cappella di Palazzo, sei bande di dieci strumenti ciascuna, cinque "flottole" di voci e strumenti del conservatorio de Poveri di Gesù Cristo e tre del conservatorio della Pietà de' Turchini. ${ }^{40}$

37 Si tratta di una tradizione dalle origini spagnole dove le rappresentazioni erano frutto del 'teatro de los misterios' che, con la dominazione spagnola, cominciavano lentamente a diffondersi identificandosi come processioni penitenziali sui misteri dolorosi.

38 Solitaria, Registro d'Introito 1602-1609, app. p. 311.

39 Solitaria, Registro d'Introito 1602-1609, app. p. 311.

40 Sulla Processione dei Battaglini si rimanda a: Nappi 2001; MANCini 1968; Cotticelli/ MAIONE 2006. 


\section{Liturgie 'straordinarie'}

Vi sono poi cerimonie che potremmo definire 'straordinarie' perché organizzate in relazione a degli avvenimenti pubblici o a delle specifiche necessità della città o di un' istituzione..$^{41}$ Appartengono a questa categoria le celebrazioni per la dedicazione del titolo di un monastero; il culto di un santo patrono o protettore cui era riservata una particolare solennità e concorso di popolo; le cerimonie legate alla storia della città o all'emissione di un voto, istituite per ringraziamento dell'intervento salvifico della Beata Vergine piuttosto che di un santo.

La straordinarietà di un culto era data dallo spettacolo offerto al fedele attraverso la ricchezza dei paramenti liturgici e l'impiego di sontuosi apparati. Le chiese si riempivano di luci e di effetti sbalorditivi, per una mise en scène provvisoria che mirava a suscitare la meraviglia del fedele. La musica costituiva ovviamente uno dei mezzi privilegiati per l'esaltazione di un cerimoniale che in genere aveva, nel canto del Te Deum, il suo fulcro. ${ }^{42}$

Le cronache della «Gazzetta di Napoli» sono testimoni indiscussi dei molteplici eventi spettacolari che affollavano le vie e le istituzioni napoletane. Dalla loro lettura è possibile ripercorrere gli eventi storici della capitale del Viceregno.

Napoli fu, per un arco assai esteso di tempo, capitale di un vicereame senza una corte o un governatore stabili, in quanto gli avvicendamenti del rappresentante di Spagna si susseguivano a pochi anni di distanza, o, in alcuni casi, a pochi mesi l'uno dall'altro. Il forte legame con Madrid si esplicitava anche attraverso le celebrazioni di ringraziamento per battaglie, incoronazioni, vittorie militari, date che riguardavano i regnanti e le famiglie aristocratiche. I genetliaci dei viceré beneficiavano di messe solenni e musicisti della Cappella Reale, ${ }^{43}$ mentre per la recuperata salute di nobili e aristocratici, si rendeva grazie con solenni esposizioni del Santissimo Sacramento. Stesso dicasi per i riti funebri in onore di sovrani, esponenti di famiglie aristocratiche e clero. Ad esempio, in occasione della morte di Carlo II re di Spagna, avvenuta il 1 novembre 1700, il conservatorio dello Spirito Santo celebrò una messa commemo-

41 I testi liturgici ufficiali relativi a riti e cerimonie (rubriche dei messali e breviari, pontificale, cerimoniale) non utilizzano una distinzione fra liturgie ordinarie e straordinarie. Si comprende però che le liturgie ordinarie erano semplicemente quelle previste dal calendario liturgico universale o proprio di una diocesi. Sulla distinzione fra cerimonie ordinarie e straordinarie si rimanda a HAMELINE 2009, pp. 19-31, e in generale sulle cerimonie straordinarie in differenti contesti europei si rimanda a DOMPNIER 2009.

42 I documenti riferiscono genericamente di liturgie, non sempre si specifica la tipologia di liturgie organizzate. Non è scontato che esse prevedessero la celebrazione di una messa o l'ufficio dei vespri. Costante, invece, è la presenza del canto del Te Deum.

43 GDN, 1 aprile 1681 «Giovedì 27 del passato [marzo] questo eccellentissimo sig. viceré marchese de los Velez, solennizzò il giorno natalizio della maestà della regina sposa [...] e dopo si portò a tener cappella reale nella chiesa del monasterio della Concezione delle monache spagnole, ove si cantò solenne Te Deum e messa [...]». cfr. Magaudda/Costantini 2009, appendice, p. 15. 
rativa del defunto monarca con l'accompagnamento musicale «delle virtuosissime monache di quel medesimo luogo»». ${ }^{44}$ Le monache di Santa Chiara organizzarono invece solenni funerali con «scelta musica $\rangle^{45}$ per la prematura morte del figlio del Conte Daun, viceré di Napoli nel 1715; «messa cantata con musica» invece per i funerali del «fu mons. Viglino, vescovo di Tricarico $»^{46}$ e benefattore del conservatorio dell'Arte della Lana.

Nel 1707 Napoli passò sotto governo austriaco ed ecco apparire nelle cronache una numerosa serie di celebrazioni legate alla casa imperiale d'Austria. L'incoronazione a re d'Ungheria di Carlo VI d'Asburgo, imperatore del Sacro Romano Impero, avvenuta il 22 maggio 1712, venne festeggiata nella chiesa di Santa Chiara «da quelle dame religiose fatta magnificamente adornare», cantando un «solenne Te Deum e messa da' musici della R. Cappella, animata dagli armonici concenti del rinomato Matteucci Sassano, la cui funzione seguì sotto la triplice scarica dell'archibugio del battaglione alemanno $[\ldots] \gg{ }^{47}$ Te Deum e messe di ringraziamento furono celebrati per le diverse gravidanze della consorte di Carlo VI e per «l'augustissima prole al nostro imperadore». In occasione della nascita del primogenito di Carlo VI, Leopoldo Giovanni d'Asburgo, le clarisse di Santa Chiara predisposero una solenne esposizione del Santissimo Sacramento, con un «vaghissimo ornamento» disegnato da Francesco Solimena e Nicolò Amenta. I festeggiamenti, ci indica la «Gazzetta», proseguirono poi la mattina successiva, giorno di Pentecoste, al conservatorio dello Spirito Santo, dove «da dieci figliuole di esso cantossi un bellissimo componimento del medesimo Amenta per l'accennata nascita». ${ }^{48}$

Il viceregno austriaco si concluse nel 1734, quando con Carlo III di Borbone, il regno tornò indipendente e iniziò una sua vita autonoma pur rimanendo strettamente legato alla Spagna. Il 10 maggio 1734 Carlo fece il suo solenne ingresso nella città di Napoli, salutato da una folla festante sulla quale, come riportato da cronache del tempo, il novello re faceva ricadere monete d'oro. Numerose istituzioni cittadine si prepararono a festeggiare tale evento storico: le clarisse di Santa Chiara predisposero una serie di artificiosi apparati ${ }^{49}$, mentre le benedettine di Santa Patrizia, «in attestato del godimento che han sentito per il felicissimo ingresso di sua maestà in questo Regno», illuminarono per tre sere il loro monastero, «e nell'ultimo giorno [...] fecero comparire la chiesa riccamente abbellita e cantarono il Te Deum con sceltissima musica $[\ldots] \gg .^{50}$

Nel 1737 Maria Amalia di Sassonia divenne promessa sposa di Carlo di Borbone. Ottenuta infatti la dispensa papale resa necessaria per sanare un impedimento

44 GDN, 16 febbraio 1701 (1), app. p. 293.

45 GDN, 20 agosto 1715 (1), app. pp. 273-274.

46 Gdn, 13 agosto 1720 (1), cfr. Magaudda/Costantini 2009, appendice, p. 340.

47 GDN, 21 giugno 1712 (1), app. p. 273.

48 GDN, 2 giugno 1716 (1), app. p. 294. Nicolò Amenta era uno stimato letterato e commediografo.

49 Si veda cap. VI, pp. 182-184.

50 GDN, 25 maggio 1734 (5), app. p. 112. 
causato da un rapporto di parentela, la cerimonia nuziale venne pubblicamente annunciata alla fine dell'ottobre 1737. Le cronache della «Gazzetta» palesano l'euforia con la quale fu accolta la notizia a Napoli. Dal gennaio 1738 si susseguirono infatti numerose cerimonie di ringraziamento per l'annuncio del matrimonio: le monache di Santa Chiara «fecero illuminare tutto il monastero e cantare un solenne Te Deum nella loro chiesa con scelta musica, magnifico apparato e continue orazioni» $; ;^{51}$ «pomposo apparato» e «scelta musica $\rangle^{52}$ a San Potito, mentre «l'inesplicabile divozione» all' «invittissimo monarca» delle monache di Santa Maria Maddalena, veniva suggellata da un «ricco altare d'argenti a lume di più torce [...] Te Deum e solenne messa a più cori di scelta musica [...] in ringraziamento all'Altissimo delle stabilite nozze della maestà sua colla serenissima R. principessa di Polonia $[\ldots] \gg .{ }^{53}$ Anche presso il conservatorio della Solitaria si «rese le grazie a Iddio» facendo «ornare la chiesa con magnifico apparato» e con la celebrazione di una messa «con tutta la sacra pompa e con musica sceltissima, dopo la quale furon rese per la seconda volta le grazie a Dio col Te Deum, cantato maravigliosamente da' migliori virtuosi della città $[\ldots] » ;^{54}$ stesso dicasi delle monache del monastero di Santa Maria Egiziaca Maggiore ${ }^{55}$ e delle Carmelitane Scalze. ${ }^{56}$

La celebrazione del matrimonio avvenne poi a Dresda 1'8 maggio 1738. La quattordicenne Maria Amalia sposò per procura Carlo di Borbone. La cerimonia precedette il lungo viaggio della sposa verso Napoli. ${ }^{57}$ Per l'occasione al conservatorio della Solitaria si fecero «pubbliche preghiere con esposizione del Santissimo e musica per impetrare da Dio felice viaggio e prospero arrivo della M. della nostra regina in questa capitale $[\ldots] »{ }^{58}$ assieme ad un «solenne Te Deum con musica, messa grande» per «la lieta notizia gionta del matrimonio già contratto». ${ }^{59} \mathrm{La}$ coppia si incontrò per la prima volta il 19 giugno in un villaggio sulla frontiera del Regno e fece il suo ingresso a Napoli il 4 luglio, in un clima di festa generale. I novelli sposi furono salutati dalle monache della Solitaria con «tre sere di vaga e ricca illuminazione [...], nel giorno de' 23 del caduto si cantò solenne Te Deum con sceltissima musica $[\ldots] »{ }^{60}$ Le religiose di Ss. Pietro e Sebastiano infine «dimostrarono anche il loro singolare giubilo, con averne fatto dire il Te Deum, in ringraziamento al Signore [...] nella loro regal chiesa, con musica solenne fatta dal maestro della R. Cappella Domenico Sarro $[\ldots] \gg .{ }^{61}$

51 GDN, 7 gennaio 1738 (2), app. p. 274.

52 GDN, 21 gennaio 1738 (5), app. p. 40.

53 GDN, 4 febbraio 1738 (1), app. p. 211.

54 GDN, 11 febbraio 1738 (1), app. p. 334.

55 GDN, 25 febbraio 1738 (4), app. p. 119.

56 Gdn, 25 marzo 1738 (1), cfr. Magaudda/Costantini 2009, appendice, p. 563.

57 Il fratello di Maria Amalia, Federico Cristiano di Sassonia rappresentava lo sposo.

58 GDN, 20 maggio 1738 (1), app. pp. 334-335.

59 GDN, 3 giugno 1738 (1), app. p. 335.

60 GDN, 1 luglio 1738 (2), app. p. 335.

61 GDN, 8 luglio 1738 (5), app. p. 160. 
Anche le diverse gravidanze della regina Maria Amalia videro una grande partecipazione da parte delle istituzioni cittadine. La Solitaria cantò solenne Te Deum per la prima nascita, nel 1740, di Maria Isabella Antonia, ${ }^{62}$ mentre le monache di Santa Chiara cantarono un solenne Te Deum per quella di Maria Josefa Antoinette, ${ }^{63}$ deceduta poco dopo. Per la nascita del principe Filippo, il conservatorio dei Ss. Filippo e Giacomo celebrò una «gran messa [...] più cori di scelta musica» e un immancabile Te Deum in ringraziamento «all' Altissimo di una grazia sì segnalata $[\ldots] \gg,{ }^{64} \mathrm{e}$ ancora le monache del conservatorio dell'Arte della Lana, «con apparato, esposizione del Santissimo, messa cantata, musica e gran sparo di mortaletti» ${ }^{65}$ festeggiarono la nascita del piccolo Carlo, destinato a diventare Carlo IV, continuatore del ramo spagnolo dei Borbone.

A seguito invece della morte della figlia di Carlo di Borbone, Maria Teresa Anna, deceduta due mesi dopo la nascita, furono organizzati a Santa Chiara solenni funerali e un imponente corteo funebre:

[...] seguita in esso giorno [1 maggio] e sullo spuntare del medesimo la morte dell'ultima serenissima infantina [...], fu trasportata nella chiesa del R. monistero di S. Chiara [...] e chiudeva la marcia una compagnia delle reali guardie d'infanteria italiana con bandiera spiegata e tamburo battente $[\ldots] \mathrm{e}$, situata sopra alto, maestoso e nobilmente ideato feretro tutto copiosamente adorno di lumi [...], così restò esposta fino alla seguente mattina di domenica [3 maggio]. E, dopo esserli recitate le solite preci, secondo il rituale della santa romana Chiesa, da cento religiosi di ciascuna delle quattro Religioni Mendicanti, siccome pure da tutti i musici della R. Cappella, successivamente $[\ldots]$ fu collocata provvisionalmente in una nicchia d'una cappella d'essa R. chiesa $[\ldots]{ }^{66}$

\section{Le cerimonie di 'monacazione'}

Il potere delle famiglie aristocratiche si esplicitava soprattutto nell'organizzazione delle sontuosissime cerimonie di professione religiosa, che segnavano l'ingresso di giovani donne nell'universo claustrale. L'ostentazione e la pompa di molte cerimonie di monacazione, in linea con le forme di rappresentazione della religiosità dell'epoca, segnavano pubblicamente il cambiamento nello status di vita della donna e l'inizio di un'esistenza nuova.

La liturgia della 'monacazione' appariva una trasposizione sul piano mistico del rito matrimoniale, del quale conservava l'uso dei simboli come velo e corona, quali segni di unione e fedeltà di una vergine al suo Sposo celeste. ${ }^{67} \mathrm{Vi}$

62 GDN, 15 marzo 1740 (3). app. p. 335.

63 GDN, 23 gennaio 1742 (4), app. p. 274.

64 GDN, 18 luglio 1747 (3), app. p. 281.

65 Gdn, 26 novembre 1748 (2), cfr. Magaudda/Costantini 2009, appendice, p. 689.

66 GDN, 5 maggio 1750 (1), app. p. 274.

67 Sul parallelismo tra nozze mistiche e nozze profane si vedano: ZARRI 2000, pp. 385-388; EvanGELISTI 2000, pp. 233-247; LowE 2002, pp. 41-65. 
era infatti l'uso di espressioni quale sponsae Christi, puellae Christi, Christo maritatae, Deo nuptae etc. quasi a rendere palese il significato sponsale della scelta e dello stato di vita di donne che per amore di Cristo rinunciavano al matrimonio secolare.

La documentazione archivistica esplorata per la città di Napoli, riporta alla luce una svariata serie di cerimonie di monacazione senza mai arrivare a configurare una forma comune e condivisa di azioni liturgiche e rituali. L'uniformità dei culti post-tridentina e l'idea del centralismo romano, non sembrano in realtà essere state assorbite dai diversi ordini monastici napoletani. Molto spesso la professione monastica viene identificata dalla fonti con i termini di "monacazione" e "monacaggio", o ancora più genericamente col "prender l'abito". Questa terminologia poteva riferirsi però ai rituali di vestizione, professione o consacrazione di una vergine. ${ }^{68}$

Per i rituali di vestizione e professione ci vengono in aiuto le descrizioni presenti nelle costituzioni delle singole istituzioni monastiche o nei rituali e cerimoniali previsti dalle regole dell'ordine a cui apparteneva il chiostro, fonti purtroppo scarse per il contesto napoletano. ${ }^{69}$ Risulta quindi difficile una puntuale ricostruzione delle diverse azioni liturgiche che potevano variare a seconda dell'ordine e delle regole. ${ }^{70} \mathrm{Vi}$ è inoltre da dire che fra le comunità religiose femminili, non solo di

68 Sui diversi significati di professione e consacrazione si rimanda a: ZARRI 2000, p. 375; METZ 2001.

69 Per XVII e XVIII sec. si conservano pochissimi testimoni di rituali di professione religiosa per la città di Napoli. Qualche informazione è possibile desumerla da alcune regole e costituzioni di singole istituzioni: La regola quale devono osservare le donne et le figliole che nel Monastero della Santissima Trinità di Napoli vivono, Napoli, Giovan Giacomo Carlino, 1615; Constitutioni delle monache del Monastero di S. Andrea di Napoli, Napoli, Orazio Salviani, 1589; Regole che hanno da osservare le monache del monasterio del Divino amore dell'Ordine di S. Domenico, Napoli, Francesco Savio stampatore della Corte Arcivescovile, 1644. Risalente al 1847 invece un cerimoniale contenente i riti di vestizione e professione religiosa del monastero di San Francesco delle Cappuccinelle, Asdn, San Francesco delle Cappuccinelle, Cerimoniale per novizie, professe e abbadesse sec. XVIII-XIX.

70 La difficoltà di reperire un unico codice di informazioni riguardanti i riti di professione viene segnalata in un manuale di vestizione e professione del monastero di Santa Chiara di Manfredonia del 1743. Nella prefazione si legge: «Questo breve Libretto, non si è scritto ad altro fine, che per dar comodo al Sacerdote che fà la funzione di vestire, o di professare la Religiosa: perchè si osserva, non senza qualche ammirazione, e disordine: che il Sacerdote, (che al più delle volte sono gli stessi Prelati) sogliono andar, come suol dirsi, scartabellando, nell'atto stesso, che si sta facendo la funzione, per cercar questa, e quell'altra cosa; per i Messali; per i Cerimoniali; per le Regole; e per alcune carte disperse di quà, e di là, onde non riesce con decoro una funzione così tenera, e così grave; a Dio cotanta grata, com'è la Vestizione, e la Professione, d'una Vergine Religiosa [...]». Manuale ove si contengono le due formole; di vestir l'abito, e di far la professione nell'ordine della madre S. Chiara. Giusta il cerimoniale de' Frati Minori, e le regole chiarine; e secondo si pratica dalle religiose di esso ordine nel monastero di S. Chiara della città di Manfredonia. Cosagrato alla Madre Santa Chiara da una religiosa sua figlia, professa nel monastero predetto di essa città di Manfredonia 1743, Biblioteca del Convento di San Matteo, S. Marco in Lamis, Foggia. 
ambito napoletano, vi fu una grandissima varietà in relazione al cerimoniale di vestizione e professione. Alcuni monasteri trascuravano il rito liturgico per dare spazio ad elementi devozionali e spettacolari, altri invece seguivano uno schema rituale comune, a tratti assimilabile a quello della consacrazione. ${ }^{71}$

Dal confronto fra le fonti superstiti esaminate possiamo affermare che, nella maggior parte dei casi, il rito si svolgeva alla presenza del vescovo o del cardinale protettore del monastero, oppure di un suo delegato. ${ }^{72}$ La fanciulla da 'vestire' veniva condotta in chiesa da due matrone, abbigliata pomposamente come una sposa, tanto da indossare un ricco abito bianco, con gioielli e fiori nelle chiome, simboleggiando che in tal modo la donna proveniente dalla mondana vita secolare si spogliava, accettando la povertà della nuova condizione sponsale. ${ }^{73} \mathrm{La}$ liturgia si apriva con la celebrazione della messa votiva dello Spirito Santo, con una colletta pro Virgine ${ }^{74}$ Terminata la messa aveva inizio l'esplorazione da parte del vescovo della volontà della monacanda, a seguito della quale alla postulante veniva imposto un nuovo nome. Seguivano orazioni e litanie che terminavano con il canto del Veni Creator Spiritus. Il rito prevedeva poi la benedizione della veste e del velo bianco. La badessa, o in alcuni casi lo stesso vescovo, recideva i capelli alla fanciulla, le faceva indossare la nuova veste, il velo, le corde e lo scapolare ove previsto dall'ordine, imponeva sul suo capo una corona e infine le venivano consegnati il salterio, una croce e una candela. ${ }^{75}$ Dopo nuove orazioni, il rito giungeva al fine con il canto del Te Deum, e nel rientrare in clausura, le consorelle intonavano il salmo Ecce quam bonum o più comunemente l'antifona Veni Sponsa Christi.

Terminato l'anno di noviziato, si procedeva alla cerimonia di professione. Il rito era pressoché il medesimo: di nuovo la fanciulla si prostrava a terra, venivano benedetti e poi consegnati una nuova tonaca, un nuovo velo, la corona, il salterio, quindi la postulante dichiarava la sua professione solenne. La neoprofessa moriva al mondo e alle sue inquietudini e rinasceva a una nuova vita, a uno stato superiore e più alto, quello di Sponsa Dei.

71 Sulle differenze fra i vari rituali di professione in uso a Napoli si veda anche Novi-ChavarRIA 2009, pp. 31-43.

72 Prima del Concilio di Trento, la funzione veniva in genere presieduta da un chierico o da un membro di un ordine regolare, in seguito per conferire maggiore solennità alla cerimonia i riti si svolsero nella maggior parte dei casi alla presenza del vescovo.

73 La cerimonia poteva avvenire anche in forma 'privata', in tal caso si svolgeva dietro le grate della clausura. Nel monastero di Santa Patrizia ad esempio non era prevista alcuna formulazione solenne dei voti, ma solo una professione tacita, cfr. Facchiano 1992, p. 24 e seg.; NoviChavarria 2009, pp. 33-34; Novi-Chavarria 2009², pp. 349-364.

74 Se la monacazione avesse avuto luogo di domenica o in un giorno festivo, la messa avrebbe seguito il rito della festa corrente conservando la colletta Pro Virgine.

75 La corona indicava fortezza, gloria e onore, il velo era indice di obbedienza e purezza, mentre l'abito doveva costituire il segno evidente della scelta di vita appena compiuta, la candela infine simboleggiava la luce divina che avrebbe illuminato il cammino della professa da allora in poi. 
Il significato sponsale della professione monastica era rimarcato in maniera ancora più forte dalla cerimonia di consacrazione ${ }^{76}$ che accompagnava o seguiva la professione religiosa, rappresentando un atto pubblico in cui formalmente si prendeva possesso, davanti alla Chiesa, della decisione di un'esclusiva dedicazione e donazione a Dio. Non tutte le professe però ricevevano la consacrazione, alcune facevano voto privato conosciuto dall'autorità ecclesiastica ma non confermato da una cerimonia ufficiale.

Differentemente dai riti di vestizione e professione, celebrati a seguito della messa, la consacrazione si collocava nella liturgia, fra l'epistola e il Vangelo, così come previsto nel Pontificale. ${ }^{77}$ Dopo il canto del graduale le vergini uscivano processionalmente dalla clausura al canto dell'antifona Prudentes Virgines, accompagnate da due matrone e precedute dalla croce portata da due accoliti, con la partecipazione di chierici, sacerdoti, canonici. Le consacrande si presentavano e si prostravano a terra davanti al vescovo e una volta alzatesi si disponevano a semicerchio intorno al celebrante e ascoltavano esortazioni sul significato dei voti che avrebbero di lì a poco pronunciato. Seguiva l'antifona Venite, venite, venite filiae, una triplice esortazione tramite la quale il vescovo chiamava le vergini chiedendo ripetutamente la volontà nel seguire Cristo in obbedienza, castità e povertà. Le fanciulle accettavano la chiamata rispondendo Et nunc sequimur in toto corde. $\mathrm{Al}$ termine di questo lungo e suggestivo dialogo fra il celebrante e le future consacrate, si passava alla benedizione degli oggetti rituali. La liturgia a questo punto prevedeva tre momenti processionali differenti, relativi all'accettazione dei simboli nuziali: velo, anello e corona. La velazione era forse il momento maggiormente evocativo della cerimonia nunziale, aveva inizio con il canto dell'antifona Posuit signum in faciem meam e proseguiva con la consegna dell'anello, durante la quale le vergini alzavano simultaneamente la mano inanellata cantando Anulo suo subarravit. ${ }^{78}$ L'imposizione di una corona donava loro il diritto di essere chiamate e riconosciute dal mondo come spose di Cristo. Infine il vescovo si congedava dai presenti con la pronuncia di un anatema contro coloro che avrebbero potuto insidiare la verginità delle monache o che avrebbero tentato di usurpare i loro beni. Terminata la parte rituale proseguiva la messa con orazioni espressamente rivolte alle giovani.

76 Sull'evoluzione del rito di consacrazione si veda: Metz 2001; ZARRI 2000, pp. 251-388; BOLCHI 2002.

77 Nei libri pontificali vengono specificati inoltre i giorni in cui poteva essere celebrato il rito: il giorno dell'Epifania, durante la settimana in albis, nelle feste dei Santi Apostoli o nelle domeniche. Si veda anche: Buommattei 1773; Metz 2001, pp. 152-178.

78 Il termine velatio si riferisce all'antica tradizione di velare le giovani spose nel rito matrimoniale. Questo elemento simbolico venne ripreso nelle cerimonie di consacrazione religiosa, spesso identificate con il solo termine di velazione. Nel corso dei secoli, con l'adozione di nuovi elementi simbolici del rito liturgico quali anello e corona, volti a rimarcare il significato sponsale della cerimonia, si preferì utilizzare i termini più ampi di consacrazione o benedizione. 
Si riassumono nello schema successivo le principali azioni rituali della cerimonia, così come compaiono nel Pontificale Romano, ${ }^{79}$ evidenziando le principali sezioni cantate affidate di volta in volta alle monache ${ }^{80}$ al celebrante e al coro:

Tabella 2: Rito di consacrazione delle vergini.

\begin{tabular}{|c|c|c|c|}
\hline Processione & Presbitero & Antifona & Prudentes virgines \\
\hline \multirow[t]{2}{*}{ Interrogazione } & Celebrante & Antifona & $\begin{array}{l}\text { Venite venite venite } \\
\text { filiae }\end{array}$ \\
\hline & Monache & Antifona & $\begin{array}{l}\text { Et nunc sequimur in } \\
\text { toto corde }\end{array}$ \\
\hline \multirow{2}{*}{$\begin{array}{l}\text { Benedizione } \\
\text { delle vesti }\end{array}$} & Monache & Responsorio & Regnum Mundi \\
\hline & Celebrante e coro & Responsorio & Veni electa mea \\
\hline \multicolumn{4}{|l|}{$\begin{array}{l}\text { Imposizione dei } \\
\text { simboli: }\end{array}$} \\
\hline \multirow[t]{2}{*}{ Velo } & $\begin{array}{l}\text { Monache } \\
\text { Monache }\end{array}$ & $\begin{array}{l}\text { Antifona } \\
\text { Antifona }\end{array}$ & $\begin{array}{l}\text { Ancilla Christi sum } \\
\text { Posuit signum in } \\
\text { faciem meam }\end{array}$ \\
\hline & Celebrante e coro & Antifona & $\begin{array}{l}\text { Desponsari dilecta } \\
\text { mea }\end{array}$ \\
\hline \multirow[t]{3}{*}{ Anello } & Monache & Antifona & Ipsi sum desponsata \\
\hline & Monache & Antifona & Anulo suo subarravit \\
\hline & Celebrante & Antifona & Veni Sponsa Christi \\
\hline Corona & Monache & Antifona & Induit me Dominus \\
\hline Professione dei voti & Monache & $\begin{array}{l}\text { Antifona } \\
\text { Antifona }\end{array}$ & $\begin{array}{l}\text { Ecce quod concupivi } \\
\text { Mel et lac ex eius ore } \\
\text { suscepi }\end{array}$ \\
\hline Anatema & Celebrante & - & - \\
\hline \multicolumn{4}{|l|}{ Alleluia } \\
\hline Communio & Monache & Antifona & $\begin{array}{l}\text { Mel et lac ex eius ore } \\
\text { suscepi }\end{array}$ \\
\hline
\end{tabular}

La celebrazione del rito di consacrazione divenne qualcosa di abbastanza raro fra Sei e Settecento, perché la sola professione ribadiva già da se stessa l'impegno che la persona si assumeva entrando nella vita religiosa, pertanto questo rito si era in qualche modo fuso alla professione stessa ${ }^{81}$ D'altra parte professione e

79 Lo schema seguente deriva dalla consultazione dell'edizione del 1663 del Pontificale Romano post-tridentino di Clemente VIII pubblicato per la prima volta nel 1596, cfr. PonTIFICALE 1663.

80 Le antifone riservate al canto delle monache sono tratte dalla Passio Sancte Agnetis, racconto ad opera di Sant'Ambrogio dedicato alla vita della martire Agnese, considerata l'archetipo delle vergini consacrate a Dio.

81 L'edizione del Pontificale di Clemente VIII segnò un punto di arresto nella storia dell'evoluzione del rito, tanto che tutte le edizioni seguenti del Pontificale Romano (Urbano VIII nel 1645, 
consacrazione rappresentavano forme celebrative di amore sponsale per Cristo, e pur prevedendo azioni e tempi differenti, avevano di certo una notevole capacità di integrarsi vicendevolmente.

Come componente essenziale della liturgia, la musica costituiva un elemento determinante per il fasto della cerimonia. Probabilmente le stesse professe partecipavano al rito con il canto delle antifone così come prescritto dal Pontificale, con le formule di intonazione o attraverso l'uso del canto figurato. Una testimonianza interessante a proposito della pratica di cantare le promesse ci viene offerta da cinque antifone per contralto composte nel 1781, per la monacazione della «Reverendissima Signora Mariantonia Pignatelli», da Alessandro Speranza, maestro di cappella del monastero di Regina Coeli. ${ }^{82}$ Si tratta delle antifone corrispondenti ai momenti simbolici e alla proclamazione dei voti da parte delle postulanti presenti nel rito di consacrazione. Le antifone si presentano disposte, all'interno del fascicolo che le contiene, in ordine diverso rispetto alla liturgia prevista dal Pontificale: ${ }^{83}$

1. Posuit signum in faciem meam ut nullum praeter eum amatorem ad mittam

2. Induit me Dominus Cyclade auro textaet immensis monilibus ornavit me.

3. Anulo suo subhonoravit me Dominus et tam quam sponsam decoravite me corona

4. Ipsi sunt desponsata cujus pulchritudinem sol et luna mirantur

5. Mel et lac ex eius ore suscepi et sanguinis eius ornavit genas meas.

Benedetto XIV nel 1754 e Leone XIII nel 1888) si limitarono a riportare il testo del 1596 senza apportare modifiche rilevanti. Un intervento di revisione fu realizzato dall'abate benedettino dom Guéranger che preparò nel 1868 un rituale in cui in un'unica celebrazione erano fuse la consecratio virginum e la professione monastica. Tale rito venne approvata dalla Santa Sede e denominato Ritus sacrae professionis virginis. Cfr. BolCHI 2002, pp. 27-54.

82 Le antifone sono custodite presso la Biblioteca privata Giuseppe Pastore di Lecce. Ringrazio Sarah $\mathrm{M}$. Iacono per avermi segnalato queste fonti e per averle fotografate in mia vece e ringrazio ancora la famiglia Pastore per avermi concesso di pubblicare le riproduzioni dei manoscritti. Si rimanda inoltre ad un piccolo contributo scritto da Giuseppe Pastore relativo a questo manoscritto da lui custodito: PAstore 2007, pp. 55-64. Si rimanda inoltre a Fiore/IAcono 2016.

83 Si veda la disposizione delle antifone presente nella tabella 2, p. 79. 


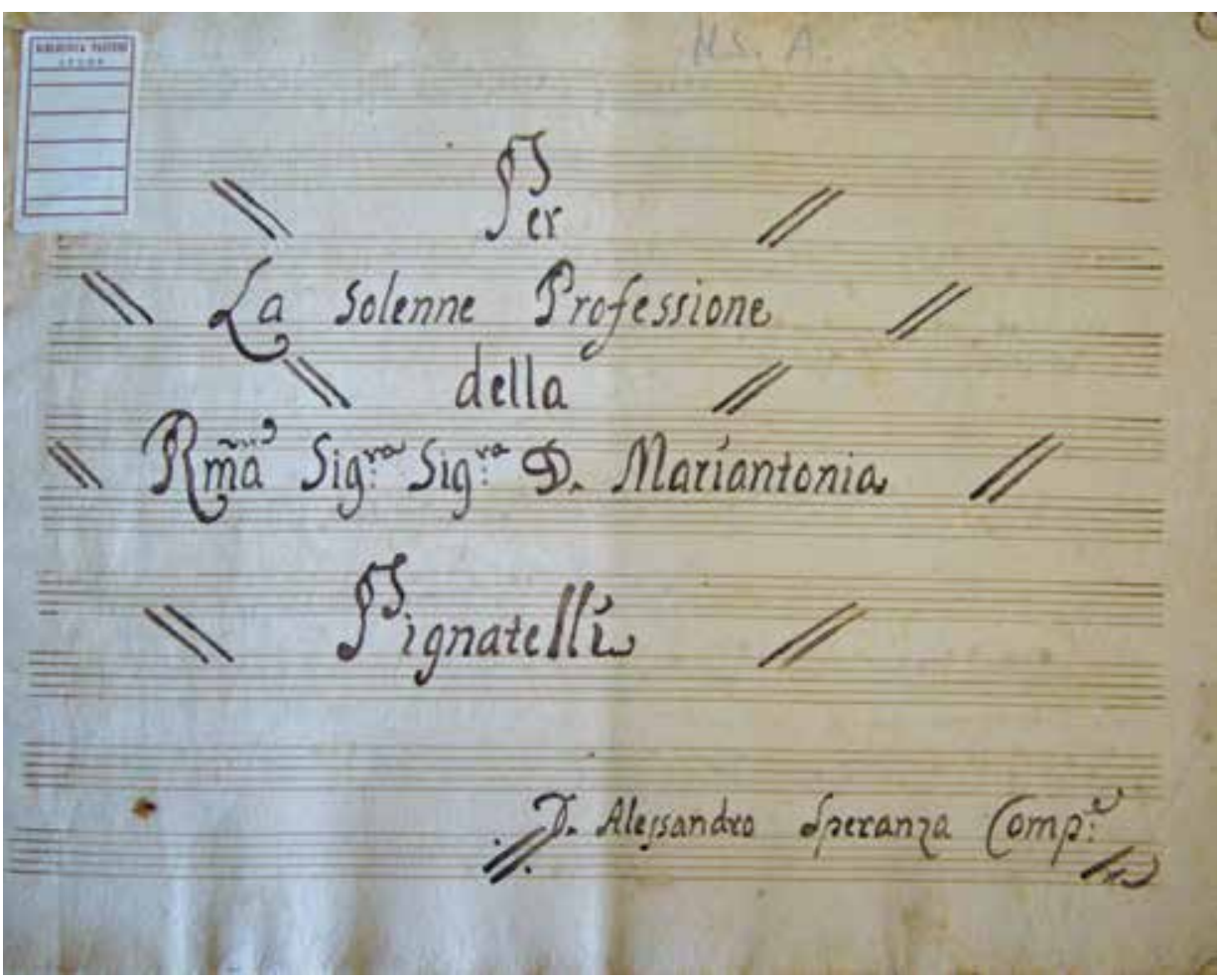

2. Alessandro Speranza, Cinque antifone. Frontespizio. ${ }^{84}$ Biblioteca G. Pastore.

Si tratta di antifone scritte in notazione moderna, con una melodia ornamentata con reminiscenze di stile operistico. ${ }^{85}$ Purtroppo per Napoli non abbiamo sufficienti informazioni per poter supporre o affermare che il canto delle promesse attraverso l'uso del canto figurato, ad opera delle stesse religiose, fosse una pratica generalizzata o relativa ad un ordine monastico, così come avveniva in altri contesti

84 A riprova della mistione dei riti di professione e consacrazione, è da notare in questo testimone la dicitura di «solenne professione», tuttavia le antifone presenti nel manoscritto appartengono alla liturgia di consacrazione descritta nel Pontificale.

85 Non è ben chiaro l'organico strumentale previsto per queste antifone. Il manoscritto non reca alcuna indicazione in merito. La scrittura del rigo superiore farebbe pensare ad un violino, tuttavia vi sono accordi difficilmente eseguibili per uno strumento ad arco. L'ipotesi avanzata dallo stesso Giuseppe Pastore nel suo articolo sarebbe del semplice accompagnamento tastieristico ma con una diversa disposizione delle parti: il primo rigo sarebbe relativo alla mano destra e l'ultimo alla sinistra, la voce di contralto al centro. Anche questa ipotesi sembra alquanto dubbia, non soltanto per l'inusuale disposizione delle parti ma anche per la presenza di accordi di undecima affidati alla sola mano destra. Si potrebbe forse avanzare l'ipotesi di una viola d'amore. 
europei ${ }^{86}$ Le antifone di Speranza risultano essere ad oggi l'unica testimonianza a noi pervenuta per l'ambito napoletano. Di certo le monache, così come prescritto dalle costituzioni e dalle regole interne ai monasteri, erano istruite per poter partecipare attraverso il canto alle liturgie, e non stupisce quindi che esse potessero poi cimentarsi anche in melodie più complesse.

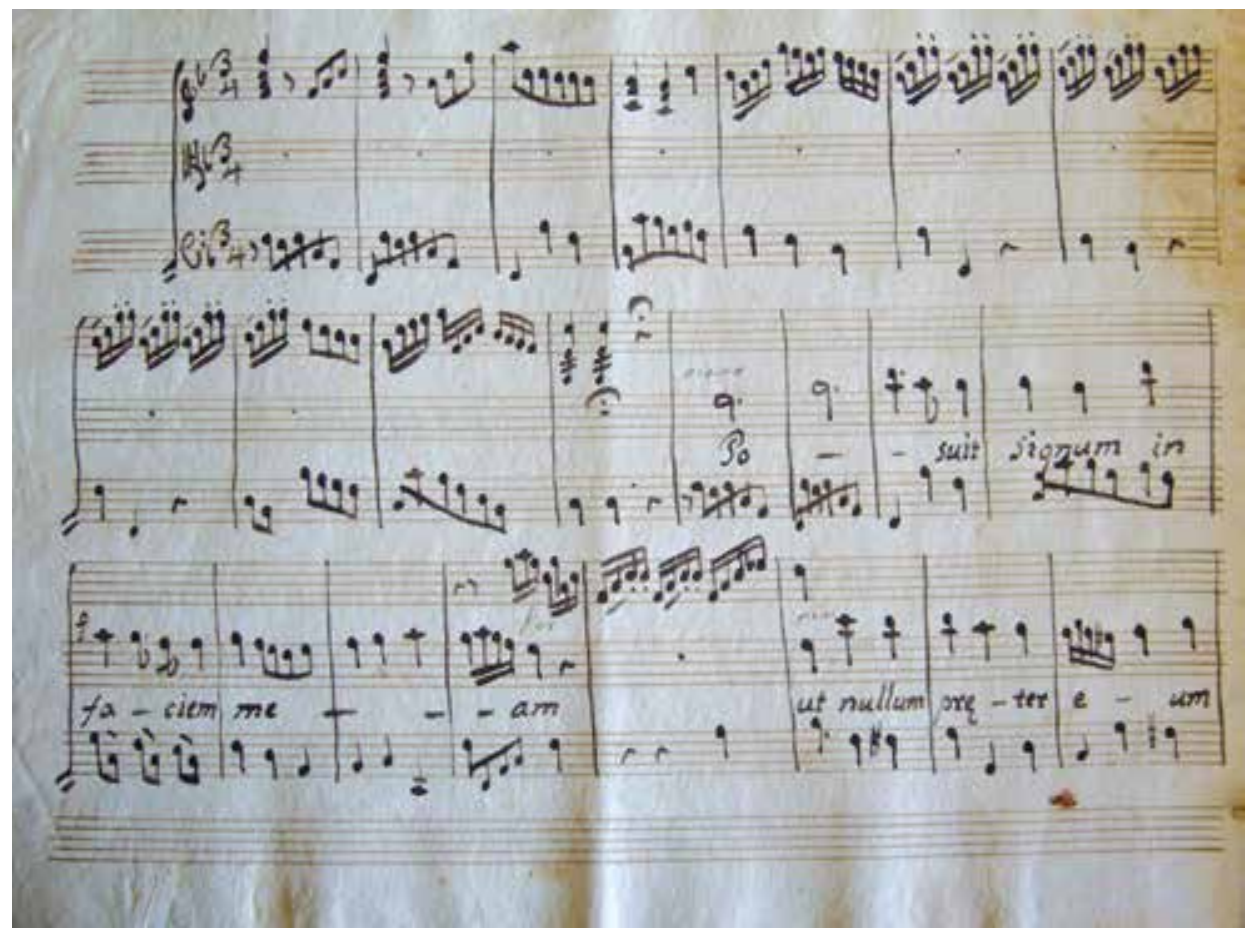

3. Alessandro Speranza, Posuit signum in faciem meam. Biblioteca G. Pastore.

Le gerarchie ecclesiastiche tendevano a proibire qualsiasi eccesso in queste cerimonie, negando sia la possibilità di decorare la chiesa con paramenti vari, sia di eseguire canto figurato e musica con qualsiasi strumento eccettuato l'organo, ammettendo soltanto che le monache si esibissero nel canto fermo durante la liturgia. Si tentava inoltre di evitare che la pubblicità di tali eventi favorisse l'andirivieni di persone che potevano mettere a rischio la clausura delle monache già professe. Un documento del 1690, proveniente dal monastero delle clarisse di Santa Chiara,

86 Un esempio è rappresentato da alcune comunità monastiche portoghesi che prevedevano la pratica di cantare i voti o le promesse sia con l'utilizzo del cantus planus che del canto figurato, in alcuni casi ciò avveniva in portoghese, discostandosi quindi dal latino. Cfr. FerNANDES 1998, pp. 59-94; Fernandes 2015. 
indica quella che sarebbe dovuta essere la regola circa l'utilizzo della musica proprio durante le cerimonie di vestizione e professione:

Ordini dell'Eminentissimo Signor Cardinale Protettore per li Monasteri di Santa Chiara e della Madalena, circa la musica, ristretti in alcuni punti.

1. Si prohibisce [...] il cantare in Musica in ogn'altra occasione, fuorchè di Vestimento, di Professione, d'elettione, ò conferma dell'Abbadessa [...]

2. Se bene da Sua Eminenza si concede la musica ne sopradetti casi; si prohibisce però sempre il cantar cose vane, e recitare, havendo ciò più del comico, che del religioso; e solo si permette il canto di cose serie e spirituali, che muovino a devozione.

3. Si sfugga in occasione di Musica, ogn'invito di Cavalieri, essendo ciò vietato non solo espressamente da Sua Eminenza ma anche da Sua Santità medesima.

4. Ritrovandosi accidentalmente cavalieri in Chiesa, non invitati a simili funzioni $[\ldots]$ non si permetta l'ingresso dentro le cancellate $[\ldots]$.

5. Nel far dette musiche per messe, quelle che canteranno doveranno essere secolari, ò educande modestamente vestite; e volendo cantar le Professe o à mente, ò con la carta, doveranno comparir con gl'habiti religiosi, ne sopra quelli porteranno, ò si lascieranno mai vedere con addobbi non convenienti al loro stato, si nella pretiosità portando perle $[\ldots]$ volendo $[\ldots]$ che le religiose in qualsivoglia funtione [...] comparendo avanti il secolo, siano conosciute, e nel canto, e nella modestia degl'habiti degne spose di Giesù Christo $[\ldots] .{ }^{87}$

Di conseguenza, numerosissime furono le richieste delle famiglie affinché, in occasione della vestizione delle loro parenti, si potesse far musica e soprattutto si potesse aumentare il numero dei musicisti proprio per conferire maggiore importanza alla cerimonia, come si legge dalla seguente licenza risalente al 1732 del monastero di San Gregorio Armeno:

Si concede licenza alla signora Donna Anna Revertera che Domenica ventidue del Corrente Mese di Giugno possa prendere l'Abito di Monica nella Chiesa del Venerabile Monastero di San Ligorio con la Musica del Arcivescovato con altre voci, è Istrumenti agionti [...] ${ }^{88}$

Alcune fonti documentarie napoletane riportano la descrizione sommaria o puntuale di queste liturgie e ci forniscono in tal modo una ricostruzione visiva del rito restituendoci un quadro delle modalità e delle pratiche gestuali, delle vesti e degli apparati liturgici e spettacolari. Già agli inizi del XVII secolo il conservatorio della Solitaria di Napoli annota alcune indicazioni circa le monacazioni:

En 8 de septembre del año 1602 [...] se dio el abito de monjas a las tres hijas desta Santa Casa [...]. Primeramente el Reverendissimo Señor don Jeronimo Bernardo de quiros obispo de Castel Amar vestido con su capa de coro de brocado bendixo los abitos con mucha authoridad [...], se siguio la misa $[. .$.$] cantandose el credo, y muchos motetes a dos coros con la musica del señor$ Virrey $[\ldots] \cdot{ }^{89}$

87 Asn, MS, f. 2702, app. pp. 263-264.

88 Asdn, Vicario, 170 D, app. p. 100.

89 Solitaria, Elenchi e notamenti, app. p. 310. 
Nelle sporadiche indicazioni da seguire durante l'organizzazione delle cerimonie di monacazione, le monache della Solitaria si assicuravano che la messa dovesse essere cantata con «mucha solenidad $\rangle^{90}$ e che nel momento di prendere l'abito venisse intonato un «moteto a proposito como Veni Esponsa Christi o otro», ${ }^{91}$ con il concorso della musica «de palacio», ${ }^{92}$ ovvero della prestigiosa Real Cappella di Palazzo. ${ }^{93}$ Qualche particolare in più relativo alle cerimonie viene menzionato nella descrizione della monacazione di due blasonate fanciulle:

In primis essendo venute dette due figliole con la carrozza della signora Principessa del Colle assieme con la Signora principessa di Forino, furono introdotte in Chiesa, anco dalla signora Duchessa di Madaloni, e Principessa di Loporano; et si inginocchiarono sopra due coscini ricamati preparati vicino l'antiporta, et ivi dalle dette due Padrine se li sono poste in testa le corone di fiori portateli dal Maestro di Cerimonie dentro due bacini d'Argento; mentre che da musici si cantava il Veni Sponsa Christi [...]. Doppo dal signor Governatore furono introdotte nella sacristia delle Monache, dove stava il Padre Priore di Santo Spirito e diedero la volontà di prendere l'habito in sue mani; separatamente cioè una doppo l'altra [...]. Cominciata la messa Cantata che si celebrò dal detto Padre Priore di Santo Spirito, e dal medesimo fù fatto il sermone doppo la Comunione. Al tempo della Comunione furono dalle signore Padrine portate all'altare dove dalla medesima furono levate le corone, et li furono posti in testa due veli bianchi per riverenza del sacramento; et doppo comunicate li furono levati detti veli, e riposte le corone in testa. Finita la messa, sono entrate con le Padrine nel Comunichino, dove dalla Madre Rettora, se li sono posti l'habiti, e tagliati li capelli dalle reverende monache fù cantato il Te Deum. ${ }^{94}$

Anche i diari dei Cerimonieri risultano essere importanti testimoni di questi eventi e ci portano a comprendere quanto numerose e diverse fossero tra di loro le cerimonie. La solennità era scandita secondo schemi differenti a seconda del rango sociale della professa e dell'investimento economico a lei riservato dalla famiglia.

Le liturgie inoltre potevano vedere protagonista una sola fanciulla o riguardare più ragazze contemporaneamente. Il monastero benedettino di Donna Romita fu infatti la prestigiosa cornice della consacrazione di ben venti vergini, una liturgia particolarmente suggestiva che aveva commosso, come si intuisce dalla lettura del documento, lo stesso cronista:

Domenica 14 febbraio 1649 si fa quella sollenne consecratione de' Venti Vergini monache di quel Venerabile, et antico Monisterio di Donna Romita [...] dal Reverendissimo Monsignor Vescovo di Sira $[\ldots]$, furono osservate $[. .$.$] tutte le cerimonie che comanda il Pontificale [...]. Vedendo$ tante Vergini rinunciare il Mondi, e spose di Christo (cosa da intenerire i sassi): la divozione poi con che si sposarono con Dio [...] lo lascio pensare perché questo fecero con tanta divotione,

90 Solitaria, Elenchi e notamenti, app. p. 310.

91 Solitaria, Elenchi e notamenti, app. p. 310.

92 Solitaria, Elenchi e notamenti, app. p. 310. Il documento si riferisce alla monacazione di «Sor Maria Felice del Spiritus Santo» avvenuta nel maggio del 1639.

93 Cfr. Fiore 2012.

94 Solitaria, Elenchi e notamenti, app. p. 309. 
quanta era la loro nobiltà. Le corone con le quali furono coronate, non posso io descriverne qui le gioie, e li diamanti, i zaffiri, et i topazij e tutte sorte di gemme [...] e gli occhi delle dame, e tante signore ch'erano ivi venuti spettatori di tante belle e sante vite che parevano (senza affezione dico) tante imperatrici [...]. Il signor Eminentissimo Cardinale Filomarino Arcivescovo di Napoli gli concesse ancora musica che cantarono sempre in tutta detta attione molti mottetti, oltre dell'antifona che deve il Coro, col Veni Creator Spiritus, et il Te deum laudamus. ${ }^{95}$

Occorre inoltre aggiungere che il grado di fastosità e pubblicità della cerimonia, contribuivano a rimarcare e rendere visibile il prestigio sia della comunità monastica in cui la novizia entrava che della novizia stessa e della sua famiglia di origine. Ad esempio, la professione religiosa della figlia dell'illustre principe di Cellamare, fu un vero e proprio connubio tra poteri civili ed ecclesiastici. Avvenuta nel febbraio del 1645 presso il monastero della Croce di Lucca, ${ }^{96}$ la liturgia vide la partecipazione del cardinale arcivescovo Filomarino:

S'apparò tutta la Chiesa, il trono di Sua Eminenza di broccato si preparò a man dritta dell'Altare maggiore $[\ldots]$ in sotto all'organo con tre gradini $[\ldots]$. Si pose uno scanno di damasco rosso per i prelati con spalliere [...]. Si posero 12 candelieri, e si tutti accesero nella funzione [...]. Finita la Messa il sig. Cardinale con mitra e bacolo andò al trono ove levatosi il manipolo e la pianeta prese il piviale rosso [...] intonò il Veni Creatori Spiritus mentre i musici seguitarono detto, il Signor Cardinale benedisse nel medesimo trono le vesti e altre cose per la monaca [...]. Fatta questa benedizione il Signor Cardinale con mitra e bacolo andò alla sedia vicino al Communicatorio ove stando in silentio i musici vestì la monaca [...]. Vestita la monaca nel medesimo luogo intonò il Te Deum Laudamus poi mentre questo hinno si seguitava [...] presa la mitra e il bacolo andò al trono ove stette con piede senza la mitra fino al fine dell'hinno [...]. Mentre il signor Cardinale vestì la monaca, i Vescovi stettero seduti nello scanno loro. Mentre Sua Eminenza disse la messa et anco prima i musici cantarono mottetti nell'organo essendo mastro di Cappella Falconiero. ${ }^{97}$

Un evento per l'intera città dovette essere, nel 1649, anche la monacazione di Isabella Carafa, figlia del Duca di Noja, uomo di punta della politica partenopea. La professione di una nobile, che oltretutto apparteneva in questo caso a una famiglia celebre come i Carafa, rappresentava difatti un avvenimento urbano e somigliava decisamente ad una festa di stampo laico:

[...] festa della Monacazione della Signora Isabella Carrafa figliola del signor Duca di Noia di anni 15 giovinetta bianca e bella e di biondissimi capelli [...]. La Chiesa [...] del Giesù delle Monache della porta di San Gennaro fù superbissimamente parata tutta di drappi d'oro, l'altare di argenti abondevoli $[\ldots]$, due cori di musici $[\ldots]{ }^{98}$

95 Asdn, Cerimonieri, Vol. III, app. p. 84. Si veda anche Novi-Chavarria 2009, pp. 37-43.

96 Sui rapporti fra il principe di Cellamare e il monastero della Croce di Lucca si veda cap. V, pp. 163-165.

97 Asdn, Cerimonieri, Vol. II, app. p. 241. Da notare la presenza di Andrea Falconieri indicato in questo documento del 1645, come maestro di cappella. Falconieri ritorna a Napoli nel 1639 risulta essere fino al 1647 liutista della Cappella Reale di Palazzo per poi prenderne la direzione nel 1648 alla morte di Giovanni Maria Trabaci. Cfr. FABRIs 1987. 
La chiesa risultava essere gremita di dame e cavalieri esponenti della nobiltà napoletana, tanto che il cronista annota come nella strada adiacente al monastero, fossero presenti duecento carrozze che impedivano il passaggio degli stessi invitati. A rimarcare questa rappresentazione simbolica del potere politico e sociale della nobile famiglia di origine, la fanciulla venne accompagnata in chiesa dal viceré in persona:

Cominciò la messa e seguitò alla spagnola, con musica [...]. Finita la messa [...] si spogliò detto Monsignor Vicario della pianeta e manipolo, e dispose il piviale in cornu epistole ove in vase d'argento vennante [sic] li vestimenti monacali, quelli benedisse [...] asperse e incensò; $\mathrm{e}$ subito si fa segno à musici che cantarono il Te Deum Laudamus; et in quel tempo la fanciulla con le matrone, appoggiata al braccio destro del Viceré si condusse sino alla porta del Monasterio, e le stava vestita di intensissimi drappi tempestati di gioie e di gemme; et in testa nei suoi capelli apparivano tante stelle di diamanti e altre preziosissime gioie; così come nelle mani: se ne andò così con un seguito de signore e signori e fù ricevuta dalle Monache velate col canto Veni Sponsa Christi [...].99

I viceré, in quanto invitati d'onore alla vestizione di monache di clausura, agivano come padrini o madrine, accompagnando personalmente le novizie all'ingresso della clausura. Nel 1739 la figlia del Principe di Belvedere beneficiò dell'assistenza della regina Maria Amalia di Sassonia, consorte di Carlo di Borbone. La cerimonia fu «solennizzata con molte pompe, e con musica scelta» presso il monastero di Donnaregina ove «la Maestà sua vi andò appresso per vederla spogliare e vestire $[\ldots]$ e si portò nel communicatorio à far la funzione $[\ldots]$... Il vicario delle monache «principiò à tagliarli i capelli, e poi andò dalla Signora Abbadessa per far il resto [...] se li pose la corona, si dissero le solite orazioni, e poi d'intonò il Te Deum, che dalli Musici si cantò, e mentre si cantava la detta signora monaca andò a baciar la mano alla Maestà sua $[\ldots] \gg{ }^{100}$

La ricchezza degli apparati, l'ostentazione e la pompa, la grandeur propria del barocco permettevano al rito di divenire metafora del potere. Per questo liturgie come le monacazioni cariche di una grande teatralità, mettevano l'osservatore del tempo di fronte ad una percezione del 'sacro' prevalentemente offerta all'artificio ove la musica era mezzo privilegiato per la costruzione di una liturgia esibita più che di mistero partecipato.

Un'altra consuetudine fu quella di chiamare illustri cantanti per far cantare mottetti o 'mottettoni' ${ }^{101}$ con la partecipazione di più voci contemporaneamente. In queste occasioni venivano scritturati i migliori cantanti partenopei o di passaggio nella città, perché magari coinvolti nelle stagioni operistiche dei teatri cittadini. Il celebre tenore Angelo Maria Amorevoli venne infatti ingaggiato su concessione dell'arcivescovo Giuseppe Spinelli nel 1739 presso il monastero di Donna Romita

99 AsDn, Cerimonieri, Vol. II, app. p. 246.

100 AsDn, Cerimonieri, Vol. XVI, app. p. 173.

101 Sul mottettone si veda: BACCIAGALuPPI 2009, pp. 329-360. 
in occasione della professione religiosa della figlia del Conte di Potenza, purché, teneva a sottolineare l'arcivescovo, questa concessione non avesse poi dato adito all'introduzione di nuove spese "straordinarie" destinate alla musica:

\section{Illustrissimo Signore.}

Mi viene fatta istanza per parte del Signor Conte di Potenza, che in occasione di dover professare in Donna Aromita [sic] la signora sua Figlia, si permetta ad Amorevoli di potervi cantare, [...]: io le rispondo che quando la detta supposta musica sia solita, otterranno da lei la licenza, perché vi canti il nominato musico, e che ne do a lei l'ordine, dichiarandonsi però, ch'io non intendo con questo di permettere la detta nuova musica, quando non sia cosa solita, non volendo introdurre nel Monisterio questa nuova spesa non necessaria [...]. ${ }^{102}$

Amorevoli era uno dei maggiori virtuosi dei palcoscenici settecenteschi, presente in prestigiose produzioni operistiche a Torino, Milano e in altre città dell'Italia settentrionale e d'Europa. Protagonista fra l'altro dell'A Achille in Sciro di Domenico Sarro, dramma che inaugurò il teatro San Carlo nel 1737. ${ }^{103}$ Anche il «rosignolo» ${ }^{104}$ della città di Napoli, Matteo Sassano, illustre cantore della Cappella Reale e protagonista indiscusso della scena sacra partenopea, fu ambitissimo per le monacazioni delle figlie appartenenti alle più nobili famiglie della città. Il suo nome infatti compare nei registri contabili di numerosi monasteri che riferiscono dei compensi dati al «musico Matteuccio», ma è presenza costante anche in cronache e gazzettieri che riferiscono spesso della «sceltissima musica» che accompagnava questi riti. Ad esempio nel 1724 per la monacazione di Emilia Carafa, sorella del potente duca di Maddaloni, vi furono ingaggiati Matteo Sassano e Farinelli, come si legge dal seguente documento:

102 Dovrebbe trattarsi della corrispondenza fra l'arcivescovo Spinelli e il canonico Majella, vicario delle monache. Pur riferendosi al monastero di Donna Romita, il documento si trova nelle carte del monastero di San Gregorio Armeno. Si veda l'appendice documentaria. Asdn, Vicario, 172 D, app. p. 102.

103 Amorevoli (Venezia 1716 - Dresda 1798) aveva iniziato la sua carriera prestissimo debuttando in Amore e Gratitudine e nell'Ottone in Villa di Vivaldi nel 1729, e nel Mitridate e Siface di Porpora. Arrivò a Napoli nel 1736 e vi restò fino al 1740 dove la sua presenza è attestata in ben 11 opere. Nel 1739 fu inoltre a Vienna, per il Feraspe di Vivaldi. Il 26 dicembre del 1740 partecipò anche all'inaugurazione del Nuovo Teatro Regio di Torino nell'Arsace di Francesco Feo. Nel 1741 si esibì prima a Firenze, città nella quale fu alquanto apprezzato, e quindi a Londra, dove dall'ottobre del 1741 al maggio del 1743 cantò diversi produzioni al King's Theatre. Tornato in Italia, nel 1744-45 si esibì a Milano e nel 1745 decise di trasferire la propria dimora a Dresda al fine di poter cantare nelle opere di Hasse. Nel 1748 fu ancora a Vienna, dove prese parte all'inaugurazione del Burgtheater esibendosi nel ruolo di Ircano ne La Semiramide riconosciuta di Gluck. Ritiratosi dalle scene, terminò la sua carriera a Dresda rientrò nella corte sassone, come cantante da camera e da chiesa, posizioni che tenne sino al 1771 circa. Cfr. Selfridge-Field 1985; MAMY 1994.

104 Matteo Sassano (San Severo, 1667 - Napoli, 15 ottobre 1737), conosciuto come "Matteuccio", fu uno dei più celebri castrati attivi a Napoli, soprannominato per l'appunto «il rosignuolo di Napoli». Si formò al conservatorio dei Poveri di Gesù Cristo, e successivamente entrò a far parte della Cappella Reale di Palazzo. Nel 1695 e 1700 fu attivo anche a Vienna e a servizio della corte di Spagna a Madrid. Oltre alla sua attività nei teatri napoletani e italiani, il suo nome compare di frequente nelle cronache di eventi legati alle famiglie aristocratiche e agli ambienti religiosi. Cfr. Confuorto, 1930, II, p. 245; Prota-Giurleo 1966. 
Mercordì della scorsa settimana [15 novembre] nel monistero della Sapienza di monache della primaria nobiltà di questa capitale, con vaghissimo apparato e sceltissima musica a più cori de' principali virtuosi che qui si ritrovano, frà quali il marchese Matteo Sassani e Carlo Broschi detto Farinello, sotto la direzione del rinomato maestro di cappella Tommaso Carapella, si fece la funzione della monacazione di D. Emilia Carafa, degnissima sorella di D. Domenico Marzio Carafa duca di Madaloni e principe del S.R.I [...]. ${ }^{105}$

Abbiamo però anche testimonianze di cerimonie in cui erano le stesse professe a cantare i mottetti:

[...] Il vicario del cardinale celebra la messa, al termine della quale si avvicina alla grata per comunicare la monaca. I paggi di Sua Eccellenza devono stare inginocchiati fino alla fine della comunione, quando si rialzano. Alla fine della messa, il vicario si reca vicino alla grata dove si trovano anche Sua Eccellenza con la sua sedia e il vicario con il suo banchetto. La monaca canta qualche mottetto e il vicario le benedice l'abito che poi le pasa attraverso la grata. Intona il Te Deum e i musici rispondono. Sua Eccellenza, intanto, rimane in piedi. Dopo, il vicario recita un'altra orazione e, al termine, saluta Sua Eccellenza e se ne va. [...]. ${ }^{106}$

I registri contabili delle istituzioni religiose femminili napoletane, così come i giornali copiapolizze dei banchi napoletani, aggiungono dettagli relativamente alle spese che venivano sostenute in occasione delle monacazioni, e ci informano al contempo sulle maestranze coinvolte. Il monastero della Sapienza pagava Leonardo Leo per la musica «d'ogni sorte d'instrumenti di fiato, e di voci, affitto d'organi, e maestro di Cappella, ed ogni altro che per detta causa habi sonato nella festa della professione di Lucia Maria Arcangela Mirelli». ${ }^{107}$ Al Collegio di Santa Maria ante Secola de' Vergini, Andrea Amendola, maestro della Cappella della Cattedrale di Napoli provvedeva alla musica per la monacazione di «donna Candida Anastasio [...]», con «sette voci, otto violini, un Arceliuto, un violoncello un controbasso, e un buè inclusovi in detti duc. 33.2.10 anche l'affitto dell'organo [...]». ${ }^{108}$ Nicola Fago interveniva al «Venerabile Monastero di monache di San Gaudioso [...] nella monacatione di Donna Violante del Tufo», con «voci [...] et instrumenti, et anco pagare il fitto delle due organi $»^{109}$ mentre Domenico Sarro solennizzava con la sua musica «l'ammonacazione di donna Teresa Caravita ${ }^{110}$ nel monastero dei Ss. Pietro e Sebastiano. Duemilaottocento ducati vennero impiegati per le spese della professione di Aurelia Caracciolo «figlia di Don Aniello Ettore Caracciolo, marchese di Barisciano»e «per la musica di 60 istrumenti e 30 voci», eseguita nel monastero di San Gaudioso. ${ }^{11}$ Un esempio particolarmente significativo è costituito da un documento del 9 ottobre

105 GDN, 21 novembre 1724, app. p. 164.

106 La descrizione è riportata nei libri dei cerimoniali (1650-1717) in uso presso la corte del viceré, cfr. ANTONELLI 2012, pp. 240-243.

107 Asbn, BSS 1194 28/I/1729, app. p. 163.

108 Asbn, BSE 974 22/V/1731. Cfr. Соtticelli/Maione 2015.

109 AsBn, BSS 1231 9/IV/1731 app. pp. 113-114.

110 AsBn, BPV 1126 12/VII/1731, app. p. 159.

111 AsBn, BSS 1152 16/III/1726, app. p. 113. 
del 1727, presente in un giornale copiapolizze del Banco dello Spirito Santo, in cui si fa riferimento alla monacazione di donna Petronilla Chirico avvenuta presso il conservatorio di Santa Maria Visita Poveri nel 1727. Il documento cita i nomi dei musicisti e cantanti intervenuti nella cerimonia, il ruolo ricoperto e i relativi compensi, permettendo una ricostruzione attendibile della compagine musicale che aveva preso parte alla liturgia:

Tabella 3: Ricostruzione dell'organico per la monacazione di donna Petronilla Chirico presso il conservatorio di Santa Maria Visita Poveri 1727..12

$\begin{array}{lll}\text { Maestro di Cappella } & \text { Pisano [Gaetano] } & \text { d. } 1 \\ \text { I soprano } & \text { Egittio [Domenico Gizzi] } & \text { d. } 9 \\ \text { II soprano } & \ldots & \text { d. } 1.2 .10 \\ \text { Contralto } & \text { Tadeo [Taddeo Mallozzi] } & \text { d. } 1.20 \\ \text { Tenore } & \text { Pertici } & \text { d. } 1.20 \\ \text { Basso } & \text { Fiorillo } & \text { d. } 1 \\ \text { I violino } & \ldots & \text { d. } 1 \\ \text { II violino } & \text { Apice } & \text { g. } 60 \\ \text { violino } & \text { Cantalon } & \text { g. } 50 \\ \text { violino } & \text { Canonico } & \text { g. } 50 \\ \text { violino } & \text { Mero } & \text { g. } 50 \\ \text { violino } & \text { Carcai [Saverio Carcais] } & \text { g. } 50 \\ \text { violino } & \text { Marra [Andrea Manna] } & \text { g. } 50 \\ \text { violino } & \text { Gurriero } & \text { g. } 50 \\ \text { violino } & \text { Cataneo } & \text { g. } 50 \\ \text { contrabbasso } & \text { Bruno [Gioacchino Bruno] } & \text { g. } 50 \\ \text { II contrabbasso } & \text { De Vita [Francesco De Vita] } & \text { g. } 50 \\ \text { violoncello } & \text { Pallino [Ferdinando Pallino] } & \text { g. } 50 \\ \text { arciliuto } & \text { Sarraca } & \text { g. } 50 \\ \text { I corno da caccia } & \ldots & \text { g. } 50 \\ \text { II corno da caccia } & \ldots & \text { g. } 50 \\ \text { Organista } & \text { Pisano [Nicola] } & \text { d. } 2 \\ & & \end{array}$

Al pari di una festa nuziale alle liturgie di monacazione facevano seguito ricchissimi ricevimenti, con di lussuosi banchetti, 'ricreazioni', 'trattenimenti musicali' e spettacoli teatrali. Le compagini che vi prendevano parte erano in genere le stesse

112 AsBn, BSS 1177 9/X/1727, app. p. 291. In appendice si trova la trascrizione completa del documento. Altri documenti simili, che riferiscono dell'intero organico presente alle cerimonie di monacazione si trovano nelle pagine dedicate al monastero della SS. Trinità, cap. V, pp. 152-154. 
cui era stato affidato il compito di solennizzare le liturgie mattutine. La «Gazzetta di Napoli» riporta molto spesso le cronache di questi ricevimenti, informandoci ad esempio della monacazione della figlia del Duca d'Atri, al secolo donna Foresta Acquaviva, avvenuta nell'aprile del 1720 presso il monastero di Donnaregina. La fastosa liturgia con «musica a quattro cori», aveva previsto la partecipazione degli «istessi cori di musici» per l'intrattenimento «nel dopo pranzo» alla presenza dell'arcivescovo allora cardinale di Napoli Francesco Pignatelli e «gran concorso di nobiltà». ${ }^{113}$

Anche il 'mistico sposalizio' aveva una dimensione assolutamente sociale, e grazie a quella che potrebbe essere definita a tutti gli effetti un'attenta strategia matrimoniale, vi era la possibilità di consolidare alleanze politiche tra famiglie e gerarchie ecclesiastiche, più che ottemperare a una scelta individuale delle future spose di Cristo. A riprova di questo vanno segnalati anche i numerosi regali e donazioni che in qualche modo veicolavano e favorivano l'ingresso delle nuove professe nella comunità, permettendo di guadagnare più facilmente consenso e una 'carriera' futura nei vari uffici del monastero. Difatti per la solenne professione di «Donna Marianna Lucina» nel monastero di Santa Maria la Nova di Nola, duecentocinquanta ducati rappresentavano la somma «per abito [...] Musica di Nola, Messa Cantata», per «una serie di musici di Napoli; et apparato per la Chiesa» ma anche per i «regali soliti da farsi cosi à Monsignor Vescovo, come, à Monsignor Vicario, et agl'invitati, et altri à quali è solito farsi [...]». ${ }^{114}$ Alle spose celesti venivano inoltre dedicati sonetti, rime, corone poetiche e composizioni musicali. Il costume di festeggiare le fanciulle che prendevano il velo attraverso componimenti celebrativi a tema religioso come cantate, oratori, sacri drammi è diffusa ed è documentata in diverse fonti non soltanto napoletane. ${ }^{115}$

La citazione costante delle cerimonie di monacazione da parte delle cronache coeve testimonia quanto questi riti fossero intesi a pieno titolo come eventi spettacolari e mondani.

Si comprende bene dunque quanto i monasteri, con le loro liturgie, devozioni, musiche e apparati, furono, per tutto il XVII e XVIII sec., un grande polo di attrazione, pur essendo proprio feste e cerimonie potenziali motivi di condanna e ammonimento da parte delle autorità ecclesiastiche.

Organizzare sontuose e spettacolari celebrazioni significava ostentare il potere che sottendeva ognuna di queste ricorrenze, intese come eventi non solo religiosi ma sociali che disegnavano anche un'evoluzione del costume e della cultura. Le spese per la gloria di Dio erano assolutamente necessarie nell'economia di ogni istituzione per garantire, accrescere e confermare il proprio posto nella gerarchia sociale della città. D'altra parte vi è da dire che le nobili famiglie di origine ten-

113 GDN, 9 aprile 1720 (3), app. p. 175.

114 Asbn, BPI 1633 13/III/1731, cfr. Cotticelli/Maione 2015.

115 Si veda anche il cap. IV, pp. 99-108; pp. 113-114. 
tavano di rendere meno amara la segregazione nei monasteri, riproducendo nei chiostri il modello di vita mondana che era stato precluso a fanciulle molto giovani e spesso senza alcuna vocazione.

I frutti dell'azione moralizzatrice della Chiesa furono dunque piuttosto effimeri: più che moderare o sopprimere abusi, la riforma sarebbe dovuta intervenire nel cambiare certe abitudini mentali. Le disposizioni tridentine infatti avevano cercato di salvaguardare la libertà delle fanciulle nello scegliere o meno la vita monastica, ma in effetti non avevano risolto quel principale problema che consisteva nell'interpretare la monacazione come la migliore e strategica soluzione sociale di molte vite femminili. Questo era uno egli ostacoli di base ad una vera riforma della vita monastica. 



\section{La pratica musicale}

\section{Maestri di musica e di strumento}

Monasteri e conservatori impiegarono nel corso dei secoli i musicisti più rappresentativi della città per le diverse occasioni liturgiche o paraliturgiche e per provvedere all'istruzione musicale delle religiose.

In genere ogni istituto aveva un proprio maestro di cappella che, come una sorta di impresario, aveva l'onere di scritturare cantanti e strumentisti per soddisfare le differenti celebrazioni. Spesso il maestro di cappella riceveva una remunerazione complessiva da parte dei governatori di un monastero o direttamente dalle sacrestane, preposte alla cura delle liturgie, e provvedeva poi a ripartire la somma tra tutti gli artisti 'scritturati'.

I nominativi dei musicisti chiamati a ricoprire la carica di maestro di cappella sono spesso altisonanti e di frequente ingaggiati da differenti istituzioni: ad esempio troviamo Nicola Fago presso le domenicane del Divino Amore e al monastero di San Gaudioso; a Regina Coeli erano presenti Giuseppe e Nicola Conti mentre Francesco Durante era maestro di cappella all'ospedale di Sant'Eligio. Giuseppe De Bottis era invece maestro di cappella al conservatorio della Solitaria e al contempo al collegio di Santa Maria delle Grazie. Andrea Amendola, alla guida della cappella dell'Arcivescovado, era impiegato anche presso il monastero della SS. Trinità e similmente provvedeva alla musica nella festività delle benedettine dei Ss. Marcellino e Festo e di Santa Patrizia, San Gregorio Armeno e del monastero di Santa Maria della Provvidenza. ${ }^{1}$

L'appartenenza alla Real Cappella piuttosto che all'ensemble della Cattedrale non impediva ai musicisti di essere attivi anche nelle confraternite, oppure di interagire con i conservatori e i monasteri in veste di semplici maestri di musica e ancora di essere coinvolti nelle stagioni operistiche dei teatri cittadini. Raramente infatti un musicista era legato ad un'unica istituzione, in genere negli stessi anni ricoprivano ruoli molteplici per conto di istituzioni differenti. ${ }^{2} \mathrm{Vi}$ sono poi casi eccezionali di musicisti estremamente dinamici, i cui nomi si impongono sulle scene napoletane. Ad esempio Francesco Feo, formatosi presso il conservatorio della Pietà de' Turchini, attivo come operista al Teatro San Bartolomeo, maestro al

1 Maione 2000; Cotticelli /Maione 2006; Cotticelli /Maione 2015.

2 Le numerose istituzioni ecclesiastiche così come i teatri cittadini alimentarono un'intensa circolazione di musica e musicisti tale da far nascere e accrescere la fama di Napoli quale capitale musicale. Sul mestiere del musicista a Napoli si veda: Tufano 2009, pp. 773-804; LARson 1983, pp. 63-77; Fabris 1994, pp. 779-800; Columbro/Intini 2000; Cotticelli /Maione 1996. 
conservatorio di Sant'Onofrio e successivamente ai Poveri di Gesù Cristo, collaborò contemporaneamente con la Cappella musicale dell'Annunziata, il monastero di Santa Maria Egiziaca a Pizzofalcone, la congregazione dei nobili eretta dentro la Casa professa del Gesù, la chiesa di Santa Maria delle Grazie alla Pietra del Pesce, il monastero della SS. Trinità, la chiesa delle Anime del Purgatorio ad Arco e ancora la chiesa del Monte della Madonna dei Poveri. Stesso dicasi per Gaetano Veneziano, Cristoforo Caresana, Domenico Sarro, Leonardo Leo, nomi di primo piano della scuola napoletana. Ma non stupisce di riscontrare la stessa poliedricità anche in personalità poco conosciute. ${ }^{3}$

Numerose anche le maestranze chiamate a provvedere alla formazione musicale di religiose ed educande. Le educande beneficiavano di un'offerta culturale piuttosto ampia. La disciplina musicale era un elemento indispensabile per animare le funzioni liturgiche quotidiane e l'alfabetizzazione musicale faceva semplicemente parte del programma formativo offerto da un istituto. ${ }^{4}$

I "permessi di ingresso" nei chiostri testimoniano che le monache ricevevano musicisti esterni per lezioni di musica e i registri contabili ne attestano gli stipendi. Alcuni di essi avevano un impiego stabile, altri sembrano essere ingaggiati occasionalmente.

Documentata è l'attività di maestri di canto e di musica. ${ }^{5}$ Le prime informazioni relative all'insegnamento del canto, ci giungono dal conservatorio della Solitaria. Già nel 1601, ovvero pochi anni dopo la fondazione del conservatorio, si trovano compensi per maestri di canto:

3 I numerosi riferimenti relativi ai musicisti attivi nelle istituzioni religiose femminili sono stati lo spunto per l'avvio di un nuovo progetto intitolato Musico Napolitano: indice delle maestranze musicali attive a Napoli. Il progetto si propone, attraverso la creazione di un database di mettere in relazione i molteplici dati riguardanti i professionisti dello spettacolo attivi a Napoli, emersi dallo spoglio di fonti di diversa natura quali cronache, documenti contabili, atti notarili, documentazione interna alle istituzioni, corrispondenza etc., utili a ricostruire i percorsi biografici e le carriere di artisti noti e meno noti. Le 'maestranze' oggetto d'indagine inserite nel database sono non soltanto i musicisti in senso stretto (cantanti, suonatori, musicisti, maestri di cappella), ma anche tutti quei nomi apparentemente meno rilevanti e in parte obliati (librettisti, impresari etc.) che si rivelano importanti anelli per la ricostruzione del tessuto spettacolare e musicale partenopeo. La creazione di un indice di semplice consultazione e in continua fase di aggiornamento, rappresenta la possibilità di beneficiare di una banca dati con indicazioni biografiche più circostanziate su ciascun nominativo, permettendo di osservare anche la presenza e l'integrazione dei musicisti nel tessuto urbano e la mobilità di essi sulla scena musicale partenopea. Il database è stato creato grazie al sostegno del pool de recherche dell'Università di Friburgo. Il comitato editoriale è costituito da Angela Fiore e Claudio Bacciagaluppi, la realizzazione informatica è di Rodolfo Zitellini. Cfr. musiconapolitano.unifr.ch. Si rimanda inoltre a BaCCiagaluppi/Fiore/Zitellini 2015.

4 Si veda il cap. VII.

5 Probabilmente la generica dicitura maestro di canto e di musica si riferiva anche all'insegnamento del solfeggio. Negli ospedali veneziani si riscontrano esempi simili. Cfr. GILlio 2006 pp. 165-184. Si veda quanto detto nel cap. VII, p. 199. 
Banco de Santyago y Vitoria Pagaran por nos Administradores del Colegio de nostra Senora de la Soledad a Don Dominico Manso mastro de canto de las viergines deste dicho Santo Colegio, cinco ducados que se le dan por su salario de cinco meses de primero Agosto 1601 por todo dicembre siguiente $[\ldots] .^{6}$

L'insegnamento del canto avrebbe dovuto corrispondere all'insegnamento del canto fermo, essendo il canto figurato espressamente proibito:

Illustrissimo e Reverendissimo Signor mio Osservantissimo

Vostra Signoria Illustrissima si dignirà di prohibire affatto con l'autorità dilla sacra Congregazione che al Monasterio delle monache di Regina Coeli di Napoli non vadano laici ad imparare alle monache il canto figurato, ne meno, che le medesime monache possano cantarlo in chiesa sotto le pene, che pareranno a lei, poiché ciò disturba molto il vivere regolare, et io le baccio con questo humilissimamente le mani. Di Roma 20 febraro 1609 [... $]^{7}$

Essendo la giornata scandita dalla preghiera liturgica, il canto aveva un ruolo fondamentale nei gradi festivi semplici e quotidiani. Esso non era solo musica vocale ma mezzo di preghiera. Il canto fermo inoltre sembra andare di pari passo con l'insegnamento della grammatica e della letteratura. In alcuni contesti gli stessi maestri di grammatica provvedevano alla formazione musicale:

Al Canonico Don Domenico de Fusco Rettore d. dieci e per esso à Don Gennaro Grasso et esserno per l'onorario, che paga il Conservatorio di Santi Gennaro, e Clemente per l'incomodo ci prende di dare lettione di Canto fermo, leggere, e scrivere tanto alle monache quanto alle secolare $[\ldots]^{8}$

Era inoltre fondamentale che l'insegnamento della musica, così come di altre discipline, non impedisse la preghiera e i compiti a cui ciascuna religiosa era chiamata:

Si concede licenza alli sottoscritti Maestri di lingua latina, di lingua francese e di Musica che possano dare respettivamente lezioni alle sottoscritte educande del Venerabile Monastero di San Gregorio Armeno per mesi sei dalle solite grate e nell'ore che non s'impedisce la recitazione del Divino Officio, e coll'assistenza della Reverenda Signora Badessa o d'altra Religiosa dalla medesima destinanda e con osservanza delle solite condizioni; e rispetto alli Maestri di Musica che le lezioni non siano di arie profane, ma di cose appartenentino a Divini Officij e nelle ore che non si impediscono gli esercizij della Religione. Napoli 1 luglio 1790 [...]. ${ }^{9}$

Quasi inesistenti le informazioni circa le modalità di insegnamento, la frequenza e la durata delle lezioni, così come i contenuti. Difficile dunque ad oggi ricostruire l'istruzione delle religiose che praticavano musica, le fonti d'ispirazione, le norme tecniche e le teorie estetiche eseguite.

Ciascun maestro poteva essere chiamato a istruire una sola fanciulla, o più ragazze contemporaneamente. A San Gregorio Armeno, dove è attestata una grande

6 Solitaria, Registro Copiapolizze 1601-1603, app. p. 310.

7 AsDn, Vicario, 472 D, app. p. 341. Si tratta della corrispondenza fra la congregazione dei vescovi e regolari e i vescovi della diocesi. Questa lettera, scritta dal cardinale Gallo era indirizzata al cardinale Acquaviva, all'epoca arcivescovo di Napoli.

8 AsBn, BPV 1201 20/IX/1736, app. p. 308.

9 AsDn, Vicario, 174 D, app. p. 103. 
presenza di maestri di musica 'personali', ${ }^{10}$ troviamo anche testimonianza di un anziano maestro che si prendeva cura di ben venti educande. Le benedettine di San Gregorio Armeno si preoccupavano del suo carico di lavoro e del poco tempo a disposizione dell'insegnante e ne chiedevano pertanto la sostituzione:

Havendo Sua Eminenza concesso il Maestro di canto fermo nel Monastero di San Ligorio,
Monsignor Vicario conobbe espediente darci Don Vito Carrero che dava lettione à Donna Regi-
na, e molti altri Monasteri, con che stando apprezzato in tanti luoghi non à potuto dar nessuna
sodisfatione nel Monastero di San Ligorio, non avendogli dato più di trè quarti d'hora di tempo,
e per essere in detto luogo venti educande, non à potuto sodisfar d'ottener l'intento della virtù si
desidera, e lui da se stesso per essere vecchio con età avanzato, non à voluto proseguire à venire in
detto nostro Monastero di San Ligorio, [... . si supplica Sua Eminenza à concederci il Reverendo
Don Gaetano Crocco ò puro Don Antonio Liola et ogn'altro che fusse idoneo per tal virtù $[\ldots] .{ }^{11}$

Documentata è anche la presenza di maestri di strumenti ad arco e a tastiera.

Il violino fa la sua comparsa assai tardi a Napoli. Nel più antico dei quattro conservatori maschili, Santa Maria di Loreto, la prima notizia relativa all'insegnamento del violino si ha nel $1634 .{ }^{12}$ Nei conservatori maschili e anche femminili sopravviveva l'uso della ribeca dotata di sole tre corde. Ancora una volta, esempi significativi sono presenti presso il conservatorio della Solitaria, dove alcuni documenti testimoniano l'uso della ribeca e la successiva introduzione degli strumenti a corda. ${ }^{13}$ Nel 1652 Onofrio Mirabello, «Maestro de tocar rebichèn», viene stipendiato «por el travajo de enseñar las monjas de esta Santa casa a tocar rebichon $[\ldots] \gg,{ }^{14}$ a cui susseguono diversi insegnanti fino al 1695, quando è Pietro Marchitelli ad essere investito della carica di maestro di violino delle monache. Marchitelli godeva della stima e della protezione delle più importanti famiglie nobili della città, e riuscì gradualmente a consolidare il suo prestigio, al punto da ottenere, nel 1707, la nomina a governatore e tesoriere della congregazione dei Musici di Palazzo. Occupò inoltre la carica di primo violino della Real Cappella per oltre 50 anni. Era denominato "Petrillo" e fu l'unico violinista che aveva osato umiliare il divino Arcangelo Corelli giunto a Napoli nel 1702 in occasione delle solenni celebrazioni per l'arrivo di Filippo $\mathrm{V}^{15}$

La Solitaria non fu l'unica istituzione ad avere un maestro di violino: Bonaventura Veneziano e Francesco Sabatini risultano essere maestri di violino presso Santa Maria di Costantinopoli; Nicola Consolo era attivo invece al conservatorio

10 Cfr. cap. V, pp. 133-135.

11 AsDN, Vicario, 170 D, app. p. 101.

12 Cfr. Fabris 1994, pp. 779-800; Di Giacomo 1928.

13 La ribeca è uno strumento a tre corde di provenienza araba. Il termine ribeca veniva utilizzato a Napoli per identificare il violino prima della completa affermazione di questo strumento. Il nome deriva dall'arabo rebab: nei documenti della Solitaria si riscontrano diverse denominazioni come rebichèn, rabel, ravel. A fine Cinquecento la ribeca si identificò con la pochette impiegata dai maestri di ballo per accompagnare la danza. Cfr. FABRIs 1994, pp. 779-800.

14 Solitaria, Manual 2 1644-1654, app. p. 319.

15 Lo storico resoconto si può leggere in BURNEY 1957, vol. II, pp. 439-440. 
dell'Arte della Seta; Nicola Di Napoli viene citato come maestro di viola delle Figliole di San Gennaro; un anonimo maestro di violino dava «lettione alle monache» presso il collegio di Santa Maria della Carità. ${ }^{16}$

In tali contesti le lezioni non sembrano essere indirizzate alla formazione professionale, ma tendono piuttosto ad offrire un complemento al percorso educativo e, forse, a garantire l'autosufficienza musicale degli istituti nella celebrazione delle funzioni liturgiche.

Nella tabella seguente si riportano tutti i maestri di canto e strumento desunti dalla documentazione interna delle istituzioni, la carica, il loro periodo di attività ove possibile e l'istituzione di riferimento.

Tabella 4: Maestri di canto e di strumento.

\begin{tabular}{|c|c|c|c|}
\hline Nominativo & Ruolo & $\begin{array}{l}\text { Periodo di } \\
\text { attività }\end{array}$ & Istituzione \\
\hline Domenico Manso & maestro di canto / canto & $1601-1604$ & Solitaria \\
\hline Aurelio Sassano & maestro di canto & $1603-1616$ & Solitaria \\
\hline Andrea Ansalone & maestro di cappella / canto & $1652-1655$ & Solitaria \\
\hline Jacinto de Ortega & maestro di ribeca & 1652 & Solitaria \\
\hline Onofrio Mirabella & maestro di ribeca / violino & 1652 & Solitaria \\
\hline Gian Angelo D’Urso & maestro di violino & $1653-1655$ & Solitaria \\
\hline Cristofaro Caresana & maestro di musica & $1659-1670$ & Solitaria \\
\hline $\begin{array}{l}\text { Francesco Antonio } \\
\text { D'Angelo }\end{array}$ & maestro di violino & $1659-1666$ & Solitaria \\
\hline Pietro Bartilotti & maestro di musica & $1671-1698$ & Solitaria \\
\hline Pietro Marchitelli & maestro di violino & $1695-1698$ & Solitaria \\
\hline Domenico De Majo & maestro di cappella & $1695-1703$ & Solitaria \\
\hline Gaetano Veneziano & maestro di cappella & $1704-1707$ & Solitaria \\
\hline Gennaro Ursino & maestro di musica & $1708-1724$ & Solitaria \\
\hline Nicola Marinotti & $\begin{array}{l}\text { «lezione di canto fermo e } \\
\text { suono» }\end{array}$ & 1723 & S. Francesco Scarioni \\
\hline Paolo Giugno & «lezione» & $1725-1735$ & S. Francesco Scarioni \\
\hline Bonaventura Veneziano & maestro di violino & $1727-1735$ & S. M. Costantinopoli \\
\hline Nicola Di Napoli & maestro di viola & $1728-1731$ & Figliole di S. Gennaro \\
\hline Filippo Prota & maestro di musica & $1728-1731$ & Figliole di S. Gennaro \\
\hline Francesco Tringhera & «lettione di musica» & $1728-1733$ & Sant'Eligio \\
\hline Nicola Labruzzo & maestro di violino & $1729-1731$ & Arte della Seta \\
\hline
\end{tabular}

16 Si veda anche il cap. VII. 


\begin{tabular}{|c|c|c|c|}
\hline Nominativo & Ruolo & $\begin{array}{l}\text { Periodo di } \\
\text { attività }\end{array}$ & Istituzione \\
\hline Andrea Barile & maestro di canto & $1730-1734$ & S. M. del Rifugio \\
\hline Francesco Sabatini & maestro di violino & $1731-1734$ & S. M. Costantinopoli \\
\hline Giacomo Sarcuni & maestro di cappella & 1732 & S. M. Costantinopoli \\
\hline Giuseppe De Bottis & maestro di musica & 1732 & Solitaria \\
\hline Nicola Consolo & Maestri di violino & $1733-1734$ & Ss. Filippo e Giacomo \\
\hline Michele de Falco & maestro di musica & 1735 & S.M. Costantinopoli \\
\hline Gennaro Grasso & «lettione di canto fermo» & 1736 & $\begin{array}{l}\text { SS. Gennaro e } \\
\text { Clemente }\end{array}$ \\
\hline Carlo Terillo & maestro di canto & 1759 & S. Caterina da Siena \\
\hline Giuseppe de Magistris & «lezioni canto e cembalo» & 1778 & S. Gregorio \\
\hline Giuseppe Arena & «ammaestrare di canto» & XVIII sec. & S. Gregorio \\
\hline Michele Perla & «maestro di musica» & XVIII sec. & S. Gregorio \\
\hline Giuseppe Elia & «lezione di musica» & XVIII sec. & S. Gregorio \\
\hline $\begin{array}{l}\text { Giovanni Battista } \\
\text { Barbatiello }\end{array}$ & «lezioni di musica» & XVIII sec. & S. Gregorio \\
\hline Vito Carrero & maestro di canto & XVIII sec. & S. Gregorio \\
\hline Gaetano Crocco & maestro di canto & XVIII sec. & S. Gregorio \\
\hline Giacomo Monopoli & «lezioni di musica» & XVIII sec. & S. Gregorio \\
\hline Angelo Vinditti & «lezioni di musica» & XVIII sec. & S. Gregorio \\
\hline Giacomo Tritto & «insegna una messa cantata» & XVIII sec. & S. Gregorio \\
\hline Filippo Cinque & «lezioni di musica» & XVIII sec. & S. Gregorio \\
\hline Domenico Scitta & «lezione di musica» & 1815 & S. Gregorio \\
\hline
\end{tabular}

Frequenti inoltre sono anche i pagamenti per i maestri organisti, organari e cembalari, stipendiati per garantire utilizzo e ordinaria accordatura degli strumenti in uso nei diversi istituti. Spesso l'organista assolveva anche alla funzione di maestro di cappella, destreggiandosi fra la direzione e l'accompagnamento all'organo. La documentazione d'archivio non fa esplicitamente riferimento alla tipologia di impiego musicale prescritto ad un organista, ma di certo la funzione espletata doveva ricoprire numerose attività, che potevano andare dall'esecuzione di brani strumentali per la liturgia, all'accompagnamento delle voci delle cappelle musicali o delle religiose, alla composizione stessa. ${ }^{17}$

17 Sull'arte organaria e cembalaria napoletana: Sisto/CARdi 2005; Columbro 2005; Nocerino 1998, pp. 85-109; Nocerino 2005, pp. 105-115; Nocerino 2009; Romano 1980; РrotaGiURLEO 1961, pp. 109-126. 
Organari e cembalari provvedevano invece alla manutenzione degli strumenti in uso presso i monasteri. Le maestranze stabilivano in genere dei sodalizi con le strutture e venivano stipendiati mensilmente o trimestralmente. Questa tipologia di incarico veniva affidato solitamente sempre agli stessi organari o a esponenti della stessa famiglia, essendo questo uno dei tipici lavori tramandato a livello generazionale. ${ }^{18} \dot{E}$ il caso ad esempio di Francesco e Fabrizio Cimino, provenienti da una delle più note famiglie di organari napoletani. Essi furono attivi in numerosissimi istituti come il Divino Amore, Ss. Marcellino e Festo, la Solitaria, Donna Romita, Santa Maria del Gesù, San Francesco degli Scarioni. Ma troviamo anche Francesco e Carmine Scoppa, Nicola Muscato, Tommaso De Martino a San Gregorio Armeno, Geronimo Ferraro a San Giuseppe dei Ruffi. E potremmo citare ancora Andrea Basso, Cesare Catarinozzi, Geronimo Baffi e il «cimbalaro» Nicola Cennamo stipendiato nel 1728 «per accomodare il cembalo per la musica del conservatorio dell'Arte della Seta $[\ldots] \gg .^{19}$

Affiancavano organisti e organari, una serie di professionalità legate all'attività organaria: indoratori, intagliatori, falegnami, artisti che contribuivano a creare vere e proprie opere d'arte. Ma anche personaggi senza alcuna qualifica come facchini, tiratori di mantici che permettevano l'utilizzo stesso degli organi.

\section{Il teatro claustrale}

La musica poteva costituire anche un piacevole passatempo. Nei monasteri vi era infatti l'uso di allestire rappresentazioni sacre come 'ricreazione' in occasione di feste solenni. ${ }^{20} \mathrm{Il}$ teatro claustrale fu una pratica comune a molteplici istituzioni religiose di differenti regioni della penisola, così come si evince da una vasta documentazione sull'argomento fra Cinque e Seicento. Il teatro aveva il duplice scopo di offrire occasioni di svago a tutte le ospiti di un monastero e ne ampliava contemporaneamente la preparazione culturale. Di certo si tentava di rendere gli svaghi delle fanciulle il più possibile "spirituali", ma i momenti ricreativi costituivano soprattutto una salutare occasione di convivialità e di evasione dalla vita contemplativa.

Le botteghe dei maestri organari erano situate nello stesso luogo, addirittura nella stessa via che veniva pertanto indicata come «strada degli organari». Cfr. Celano 2001, Giornata III, p. 825.

19 Asbn, BSA 914 20/7/1733, app. p. 280.

20 Purtroppo si dispone di una scarsa bibliografia riguardo ai drammi sacri allestiti nei monasteri, soprattutto per il sud Italia. Le ricerche condotte da Antonio Dell'Olio sui drammi sacri pugliesi hanno evidenziato anche nella provincia pugliese del viceregno napoletano, una grande partecipazione delle istituzioni monastiche alle sacre rappresentazioni. Cfr. Dell'Olio 2013. Sulle diverse forme di rappresentazioni sceniche in uso a Napoli si veda: CAFIEro/Marino 1987, pp. 465-510; Magaudda/Costantini 2015, pp. 243-278. 
Le forme drammatiche utilizzate nei monasteri vengono indicate con differenti denominazioni: operette spirituali, commedie, drammi sacri o spirituali, dialoghi drammatici. Si rappresentavano testi su soggetti sacri, scene della Passione di Cristo, biografie di santi, con lo scopo di infondere maggiore cultura religiosa e umanizzare lo stesso concetto di 'Santità' e 'Divinità'. Gli autori dei testi potevano essere predicatori e sacerdoti, ${ }^{21}$ ma anche poeti e letterati e, in molte parti d'Italia, si trovano testimonianze di testi scritti dalle stesse suore. ${ }^{22}$

Quella teatrale era una pratica assolutamente consueta nei chiostri napoletani. Ne sono conferma i diversi decreti e ordini disciplinari, citati in precedenza, riguardo la sospensione delle diverse forme di 'teatro' ad opera delle religiose. ${ }^{23}$ Essendo viste anche come una forma d'istruzione religiosa, le rappresentazioni sortivano, in molti casi, il beneplacito dei diversi arcivescovi della città, che concedevano licenze affinché le monache potessero qualche volta assistere alla recita di operette spirituali:

\footnotetext{
Illustrissimo e Reverendissimo Signore

Avendomi fatta istanza coteste buone Religiose di Santa Chiara per la permissione di sentir recitare al solito nel prossimo Carnevale avanti la Porta del loro Monastero qualche operetta spirituale, io mi sono mosso più che di buon animo a condescendere a questo loro innocente, $\mathrm{e}$ convenevole divertimento. Prego però l'Illustrissima di volerci mandare ad'assistere qualche suo Ministro, affinché passino le cose con tutta la migliore ordinanza e quiete. E con la solita stima bacio intanto a Vostra Signoria Illustrissima le mani. Roma 25 gennaio 1727. ${ }^{24}$
}

Avevano luogo soprattutto nel Carnevale, in occasione di festività di santi patroni e ancora durante i banchetti che facevano seguito alle professioni religiose. Si trattava in genere di azioni brevi con 2 o 3 atti, che potevano prevedere un allestimento scenico. In genere, le rappresentazioni avevano luogo negli spazi esterni adiacenti agli edifici religiosi, tuttavia i registri contabili indicano la costruzione di scene e teatri allestiti appositamente all'interno del monastero. Ad esempio presso il monastero della SS. Concezione, nel 1733, si fa riferimento a diverse spese per il «tavolato della comedia, che ha da fare con Suoni e Scene», ${ }^{25}$ mentre per il Carnevale, presso l'ospedale di San Giacomo, venivano corrisposti quindici ducati a: «Suor Marianna Coviedo [...] per el gasto dell'Intavolato della Comedia che hanno fatto fare con sue tramoije y otro nelli giorni di Carnevale», ${ }^{26}$ e quello della Maddalena affittava «quattro abiti nuovi, Gioie, Barrettoni, penne

21 L'uso di allestire rappresentazioni sceniche si ricollega alle predicazioni gesuitiche e alla tradizione oratoriale. Cfr. Croce 1966; De Maio 1997.

22 Al teatro monastico è dedicato il volume di WEAVER 2002.

23 Cfr. cap. I, pp. 42-46.

24 Asn, MS, f. 2702, app. p. 265. Lettera inviata al nunzio apostolico di Napoli, purtroppo non vi è l'indicazione del mittente, presumibilmente poteva trattarsi del cardinale protettore del monastero o di un esponente della congregazione dei Vescovi e Regolari.

25 Asbn, BSG 806 14/1/1733, cfr. Cotticelli/Maione 2006, appendice.

26 Asbn, BSG 854 7/II/1736, CotticelLi/Maione 2015. 
Sciabole, Manti, et un altro abito semplice, con altri fornimenti [...] per uso della Comedia fatta per Donna Celeste, Donna Luisa, e Donna Zeza d'Aponte [...]». ${ }^{27}$

Raramente si trovano informazioni circa i testi teatrali o i titoli delle commedie rappresentate, stesso dicasi riguardo gli autori della musica. Un esempio significativo è rappresentato da alcuni documenti del 1716 appartenenti al monastero benedettino di Donnalbina, in cui si fa cenno ad un'opera rappresentata all'interno del monastero. Un primo documento attesta che nel novembre 1716 veniva corrisposto un ducato alle educande per comprare dei «lumi» in vista della rappresentazione effettuata dalle stesse figliole:

Per tanti dati alle signore educande per li lumi dell'opera da esse rappresentanda dentro del Monisterio d. $1 .{ }^{28}$

Un secondo documento, che riporta la stessa data, aggiunge un dettaglio significativo, precisando il nome dell'opera: Santa Rosalia.

A 28 detto dar alle signore educande per ordine della Signora Badessa per servirsene per li lumi per l'opera rappresentata di Santa Rosalia dentro del Monastero d.2. ${ }^{29}$

L'opera di Santa Rosalia è meglio conosciuta come La colomba ferita, ed è uno dei capolavori teatrali di Francesco Provenzale su libretto di Giuseppe Castaldo. Si sa che quest'opera venne rappresentata probabilmente per la prima volta nel 1670 presso il conservatorio di Santa Maria di Loreto di Napoli, e più tardi nel 1672 presso il monastero di Donnaregina di Napoli. Il libretto della seconda rappresentazione era infatti dedicato ad Isabella Caracciolo dei Duchi di Martina, badessa nel monastero di Donnaregina.$^{30}$ Definito come "dramma armonioso" nel libretto del 1670, se ne conoscono i libretti relativi alle riprese del 1672 presso il monastero di Donnaregina e del 1696, corrispondente ad una ripresa a Calvizzano da parte degli allievi del conservatorio della Pietà de' Turchini. Due riprese finora sconosciute si ebbero ad Aversa e a Capri nel 1674, così come citato da alcuni registri contabili presenti presso l'Archivio Storico del conservatorio San Pietro a Majella di Napoli. Oggi possiamo ipotizzare, con buona probabilità, che la Santa Rosalia rappresentata a Donnalbina corrispondesse proprio ad un'ulteriore rappresentazione dell'opera di Provenzale in un monastero femminile, probabilmente ad opera delle educande stesse. Le rappresentazioni infatti erano spesso affidate agli allievi di uno dei quattro conservatori cittadini, in alcuni casi invece, le attrici erano le stesse monache ed educande. Probabilmente La colomba ferita, che rappresentava una sorta di poema didattico con finalità educative

30 I-Nc, Loreto, ${ }^{\text {n. }}$ 14, Libro Maggiore 1668-1678, c. 365. La partitura manoscritta, custodita nella biblioteca del conservatorio di Napoli senza indicazioni di data per la perdita del frontespizio, fu rintracciata da Pietro Andrisani e Domenico Antonio D’Alessandro. 
per gli allievi del conservatorio di Santa Maria di Loreto, ebbe lo stesso scopo per le educande del monastero di Donnalbina.

Molto attivo sotto il profilo teatrale fu il monastero di Santa Chiara: si trovano numerosi riferimenti a vere e proprie rappresentazioni teatrali, operette spirituali, balletti mascherati e teatri di burattini. I registri contabili delle clarisse riferiscono di spese «per l'operetta» ${ }^{31}$, e per «l'operette spirituali», ${ }^{32}$ a cui assistevano dalle grate donne secolari e spesso anche sacerdoti e frati. La corrispondenza fra i monasteri e la Sacra Congregazione dei Vescovi e dei Regolari, contiene numerosi riferimenti a questa pratica, soprattutto in rapporto alle frequenti infrazioni ai regolamenti. In un documento del 1650 si legge che nella chiesa «di dentro» del monastero di Santa Chiara si era fatta «una rappresentazione della Natività con nove personaggi» in cui le monache avevano recitato «in abito secolare decente, conforme al solito, in presenza del padre guardiano Cirillo da Varese e tutti frati nella grata ordinaria [...]». L'eco di questo episodio giunse alla Sacra Congregazione di Vescovi e Regolari che puntualmente rimproverò il padre guardiano in quanto aveva avuto l'ardire «non solo di permettere a dette monache di recitar a 15 di maggio nella chiesa di dentro una rappresentazione con nove personaggi travestiti d'abito secolare, ma anche non si sia vergognato di assistere egli medesimo spettatore, insieme con molti suoi frati alle grate del coro»». ${ }^{33}$ Le autorità ecclesiastiche cercavano per quanto possibile, di sopprimere questo genere di attività, soprattutto perché le rappresentazioni comportavano spesso l'utilizzo di abiti secolari ed erano a volte accompagnate da musica, cori e intermezzi. I libretti superstiti riportano infatti nei titoli indicazioni come «componimento sacro da cantarsi», «dramma sacro per musica», «azione sacra da cantarsi», «cantata a due voci da cantarsi» etc.

Sempre a Santa Chiara, nelle voci di spesa relative all'organizzazione di festività, si trovano riferimenti come il seguente:

Nel divertimento dato col permesso del Signor Cardinal Protettore di ben sette oprette trà spirituali, e morali alla Porta per divertimento innocente in tutto il triennio; anzi la prima di esse ritrovata composta propriamente per noi in Musica con Prologo, e stampa de' libretti, e l'altre tutte con intermezzi ed introduzzione in musica: con esservi dato ancora ogn'anno libre due dolci con acqua annevata, [...] ed in ogni volta cioccolata calda con biscottini dentro, e fuori, spesovi con musica, Teatri, lumi,affitto de' Vesti, e recognizioni à Recitanti con ogni altra minuzia d. $867 \cdot .^{34}$

32 Asn, MS, f. 2669, app. p. 259.

33 L'intero episodio è in ASV, Congregazione vescovi e regolari, registra monialium, 15 luglio 1650, cc. 351v-352r, Napoli, monastero di Santa Chiara, ed è riportato anche in Weaver 2002, pp. $25 ; 80$.

34 S. Chiara, Conto Carmignano, app. p. 271. 
Il catalogo di Claudio Sartori ${ }^{35}$ riporta i libretti a stampa napoletani composti in occasione del Carnevale per il monastero di Santa Chiara e presso la biblioteca del conservatorio San Pietro a Majella di Napoli si conservano alcuni libretti superstiti relativi a queste occasioni, come Il trionfo della fede nel Martirio di Santa Lucia, opera sacra composta per il Carnevale del 1735. Nella dedica alla badessa del monastero Antonia Capece si intuisce la consuetudine delle clarisse nell'allestire ogni anno una rappresentazione teatrale:

\section{Reverendissima Signora}

Siccome è gloria del suo ben degno governo, il mantenere in questi Reali Chiostri, non meno il pregio di decorosa stima, che la strettezza di Regolare Osservanza, così riescono con degne parti della vostra innata gentilezza, il confermare, e permettere alle sue suddite Dame Religiose, quelli innocenti divertimenti, da' quali niente và disgiunta la ritiratezza Monastica, non che l'esemplarità di vita innocente; Quindi è, che essendo toccata a noi la sorte in quest'anno, per l'officio di Refettoriere, il preparare la recita della presente sacra Operetta, stimiamo qual parto della vostra benignità. Che comparisca alla luce col pregio del vostro veneratissimo nome, che può renderla plausibile [...].

Nelle avvertenze al lettore si legge inoltre l'intenzione dell'autore di rendere degna del luogo tale rappresentazione e gli espedienti utilizzati affinché ciò fosse stato possibile:

Dovendo andare in Iscena questa Sagra rappresentazione nel presente Carnevale, egli è stato necessario per lo maggior commodo, diletto, e giovamento del luogo e delle persone, alle quali rappresentar si dee, riformarla in molto, così nella ripartizione degli atti, da cinque che egli erano ridotta in trè, come nella di lei tessitura, sentenza, dizzione, costume, ed episodj: ed in alcuna parte gastigare, ed in alcun'altra adornare il suo lepido, ingrandire il serio colla più propria locuzione per l'espression degli affetti, ordinare ne' loro siti la proposizione, l'azione, il contrasto, e lo scioglimento [...]; e finalmente si è fatigato a purgarla di quanto render lo potea forsi nojosa, ed increscevole, e d'arricchirla di nuovi avvenimenti serj, e piacevoli [...]. ${ }^{36}$

La vicenda ambientata a Siracusa racconta, in maniera del tutto originale, la storia del martirio di Santa Lucia. Lucia promessa sposa del giovane Filargiro accompagnata da sua madre Eutizia, gravemente malata, si reca in pellegrinaggio al sepolcro di Sant'Agata. Qui la madre riceve per intercessione di Sant'Agata il miracolo della guarigione e in cambio della grazia ricevuta, Lucia decide di consacrarsi a Dio. 


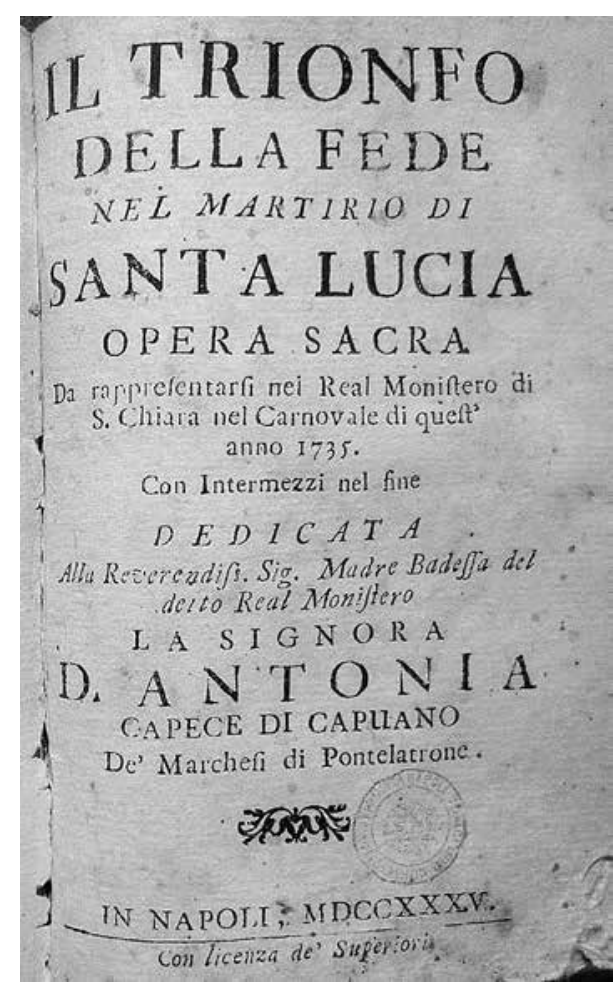

4. Il trionfo della fede nel martirio di Santa Lucia, frontespizio del libretto a stampa.

Napoli, Biblioteca del Conservatorio San Pietro a Majella.

Da questo punto in poi, il racconto del martirologio si mescola ad una serie di elementi popolari e semicomici che stravolgono la storia. Se nella realtà la giovane promessa, dichiaratasi cristiana, viene condannata ad una serie di tribolazioni e alla morte da parte del governatore Pascasio, nel libretto napoletano Pascasio si invaghisce della ragazza e assolda la maga Teurgica per riuscire nella sua impresa amorosa. Nonostante i numerosi tentativi della maga e l'intervento del Demonio in persona, Lucia non si piega al volere di Pascasio. La maga, per non perdere di credibilità agli occhi di Pascasio, prenderà le sembianze della stessa Lucia concedendosi alle lusinghe di Pascasio. L'inganno sarà sciolto dalla stessa Lucia che, brandendo un crocifisso, 'esorcizzerà' la maga costringendola a ritornare nelle proprie sembianze. Lucia verrà dunque imprigionata in una torre e martirizzata. Affiancano i personaggi 'storici' del dramma, figure come l'Angelo e il Demonio che ne supportano le gesta benefiche e malefiche. Il commento della vicenda è invece affidato ad un povero mendicante napoletano denominato "Cocomero napoletano", che si trova in Sicilia a tentare fortuna e che si esprime in dialetto donando al dramma un risvolto buffo. A lui viene affidata la conclusione dell'atto finale, con il proposito di tornare a Napoli e di dedicare a "Santa Lucia" il luogo più bello della città. 
Se da un lato il martirio della Santa viene presentato come ideale di vita cristiana e dunque, come motivo di emulazione da parte delle giovani ospiti del monastero, dall'altro la vicenda è intessuta di elementi buffi. Nonostante nella premessa del dramma l'autore del libretto esprima l'intenzione di "epurare" la vicenda in virtù del luogo e delle sue abitanti, la sensazione nella lettura è che si cercassero motivazioni o dei 'travestimenti' morali per giustificare e promuovere il teatro al di là del semplice divertimento.

Come anticipato nel capitolo precedente, le rappresentazioni sceniche potevano anche far seguito alle cerimonie di professione religiosa. Il costume di festeggiare le fanciulle che prendevano il velo attraverso componimenti celebrativi a tema religioso come cantate, oratori, sacri drammi è diffusa ed è documentata in diverse fonti non soltanto napoletane. Sono in genere composizioni d'occasione, strutturate secondo uno schema abbastanza fisso: due atti, una sinfonia strumentale introduttiva, cui seguiva un prologo e l'alternanza di recitativi e arie. Gli organici erano abbastanza minuti con la presenza di due, tre voci soliste. Tratti da soggetti biblici o allegorici, i testi poetici di questi componimenti, facevano emergere l'elemento celebrativo-sponsale. In alcuni casi era prevista anche la stampa del libretto, un ulteriore elemento per sottolineare l'eccezionalità dell'occasione celebrativa: non era scontato che questi eventi così effimeri ne prevedessero la pubblicazione. Presso il monastero di Regina Coeli, ad esempio, vi era l'uso di stampare libretti delle rappresentazioni teatrali, svoltesi in occasioni delle professioni religiose, così come a Santa Chiara in occasione del Carnevale. Probabilmente si trattava di una consuetudine propria di queste due istituzioni. ${ }^{37}$

Si propone di seguito un elenco in ordine cronologico di tutte le fonti drammatiche indicate dal catalogo Sartori, citate dai documenti d'archivio e desunte dalle partiture superstiti. Per ogni fonte viene fornita la trascrizione del titolo, il genere, l'autore del testo e della musica (se indicati), elenco dei personaggi, istituto dove era avvenuta la rappresentazione, eventuali dediche e note.

Tabella 5: Fonti drammatiche dei chiostri napoletani fra XVII e XVIII sec.

\section{6}

tit. Opera di S. Rosalia

ist. monastero di S.M. di Donnalbina

note: Probabilmente si tratta de La Colomba Ferita-opera sacra di Santa Rosalia composta da Francesco Provenzale su libretto di Giuseppe Castaldo, cfr. Sartori 5903; 5904

fonti: ASN, MS f. $3241 . .^{38}$

\section{5}

tit. La Genevieffa ovvero L'Innocenza difesa dall'inganno. Dramma sacro per musica da rappresentarsi nel real monastero di S. Chiara di Napoli dagli alunni del real conservatorio della Pietà de' Torchini. In questo presente carnevale 1735. Dedicato all'incomparabile merito della ill. e reverendissima signora D. Antonia Capace [...].

37 Come si vedrà nella tabella seguente, i pochi libretti superstiti connessi ai monasteri femminili provengono essenzialmente da questi due monasteri. 
gen. Dramma sacro

ded. Antonietta Monfort a Caterina Sersale.

pers./interp. Luigi Giuliano (Genevieffa), Giuliano Nicoletti (Sifrido), Battista Zingone (Benoni); Domenico Geronimo Malizia (Romildo); Pompeo Melospirito (Golo); Nicola Pellegrino (D. Ciarletta); Gennaro Sarnicchiaro (Marcone).

mus. Giuseppe Bonno

istit. monastero di Santa Chiara

fonti: Sartori 11513

\section{5}

tit. Il Trionfo della Fede nel martirio di Santa Lucia, opera sacra da rappresentarsi nel real Monistero di S. Chiara nel carnevale di quest'anno 1735. Con Intermezzi nel fine. Dedicata alla reverendiss. sig. madre badessa del detto real monistero [...] D. Antonia Capece di Capuano de' marchesi di Pontelatrone. ded.: Dedica di C. S. e A. M.

ind. Con Prologo e 2 Intermezzi.

istit. monastero di Santa Chiara

note: «Si avvisa il Lettore che l'Autore dell'Opere e dell'Intermezzi non ha avuta la sorte di comporre anco il Prologo, essendo questo di altra dotta mano».

fonti: Sartori 23829

\section{7}

tit. La Teodora, dramma sacro per musica di Gennarantonio Federico napoletano da rappresentarsi nel Real Monistero di S. Chiara nella fine del carnovale di questo anno 1737.

gen. Dramma sacro

ded. Gennarantonio Federico all' [...] D. Carlo Francesco Molew Trivulzio

pers./interpr. Gennaro de Magistris (Valente); Francesco Bilanzoni (Placido); Giovanni Manzuoli (Marcella); Francesco Pasquale (Teodora); Francesco Giacchi (Didimo); Ferdinando Trojano (Paolina); Nicolo’ de Simone (Cianne).

mus.: Papebrochio Fungoni.

scene: Giuseppe Baldi.

istit. monastero di Santa Chiara

note: Pag. 90: Gennaroantonio Federico a lettori: «[...] nostro presente Dramma è lo stesso, che quello della Tragedia Francese del [...] M. Pietro Cornelio [...]».

fonti: Sartori 23018

\section{6}

tit. La Betulia Liberata, sacro dramma per la monacazione dell'Ecc.ma Sig.ra D. M.a Teresa Francone.

gen. Sacro dramma

libr. Pietro Metastasio

mus. Pasquale Cafaro

pers. Ozia, Principe di Betulia (tenore), Giuditta, vedova di Manasse (canto primo), Amital, nobile israelita (canato secondo), Achior principe degli Ammoniti (alto) (Chabri, capo del popolo (canto terzo), Charmi, capo del popolo (basso).

organico: vl I,II, vla, ob 2, corni da caccia, tr 2, bc.

fonti: partitura, Bibliothèque nationale de France, département Musique, MS-1661 (1)

\section{7}

tit. Cantata a due voci, da cantarsi nel regal Monistero di Reginaceli in occasione della monacazione dell'eccellentiss. signora D. Giovanna Pignatelli de' duchi di Monteleone, etc.

gen. Cantata a due voci

istit. monastero di Regina Coeli

fonti: Sartori 4794 


\section{9}

tit. Rut nel campo di Booz, Azione sacra da cantarsi nel regal Monistero di Reginaceli in occasione della monacazione dell'eccellentissima signora D. Giulia Riario Ruspigliosi de' duchi Riario, marchesi di Corleto ec.

gen. Azione sacra

pers. Rut, Booz, Siba

libr. Tegrillo Alfirense P. A. (=Nicolò Recco).

mus. Nicolò Conti

istit. monastero di Regina Coeli

fonti: Sartori 20237

\section{0}

tit. Lo sposalizio di Rebecca, componimento sacro da cantarsi in occasione che veste l'abito religioso nel real Monastero di Reginaceli di Napoli l'eccellentissima [...] D. Francesca Maria Caracciolo de' principi d'Avellino. Dedicato a sua eccellenza [...] D. Maria Antonia Caraffa principessa d'Avellino ec. ec. amantissima genitrice della medesima.

gen. Componimento sacro

ded. Madrigale di dedica di Fisanio Teutronio P.A.

pers. Rebecca, Eliezer

mus. un nobile dilettante.

istit. monastero di Regina Coeli

fonti: Sartori 22508

\section{5}

tit. Li Furbi, intermezzo fatto per L'Ecc.me Sig.re Dame Monache In S. Chiara di me Giacomo Tritto 1765.

gen. Intermezzo

mus. Giacomo Tritto.

istit. Monastero di Santa Chiara

organico: S, S, T, B, fl I,II, ob I,II, trI,II corI,II vlI,II, bc.

fonti: partitura, I-Nc Rari: 2.4 .3

\section{7}

tit. La Genevieffa ovvero L'Innocenza difesa dall'inganno, dramma sacro per musica da rappresentarsi nel real monastero di S. Chiara di Napoli dagli alunni del real conservatorio di S. Onofrio in questo presente carnevale 1767. Dedicato all'eccell. [...] signora D. Cattarina Maria Sersale de' duchi di Cerisano attuale abadessa in detto real monistero.

gen. Dramma sacro

ded. Agnese di Gennaro camerlenga: «Ecco la saggia Geneviefa $[\ldots]$ che dopo il giro di ben sei lustri a comparir qui torno $[\ldots]$ ».

pers./interpr. Allievi conservatorio S. Onofrio: Angiolo Bucci (Geneviefa); Nicola Delmonti (Sigfrido); Antonio Pio (Benoni); Giuseppe Mancinelli (Romildo); Pantalone Nelvi (Golo); Giuseppe Caricato (D.Ciraletta); D. Pasquale de Marco (Marcone).

mus. Simone Leclerc

istit. monastero di Santa Chiara

note: Con Ballo.

fonti: Sartori 11514

\section{7}

tit. Lo sposalizio di Abigaille con Davide, componimento sacro per musica da cantarsi in occasione che veste l'abito religioso nel real Monastero di Reginaceli di Napoli la eccellentissima signora D. Argentina Mollo de' duchi di Lusciano. gen. Componimento sacro 
pers. Abigaille, Davide, coro di fanciulli

mus. Antonio Sacchini

istit. monastero di Regina Coeli

fonti: Sartori 22504

\section{9}

tit. Il fortunato incontro degli innocenti sposi Isacco e Rebecca, sacro componimento a due voci per musica da cantarsi nell'occasione che veste l'abito religioso fra le dame canonichesse lateranensi nel regal monistero di Reginaceli di Napoli l'eccellentissima signora D. Maria Carmela d'Anna de' duchi di Laviano.

gen. Sacro componimento a due voci per musica

libr. Poesia di Prineo Tissoate, P. A. Accad. Ercolanese ed Etrusco (Basso Bassi).

mus. Giuseppe de Magistris

istit. monastero di Regina Coeli

fonti: Sartori 10806

\section{9}

tit. Il Trionfo della Croce, componimento sacro per musica da cantarsi in occasione che veste l'abito religioso nel real Monistero di Reginaceli di Napoli l'eccellentissima signora D. Marianna Pignatelli de' principi di Marsico Nuovo presentato a sua eccellenza il signor D. Girolamo Pignatelli principe di Marsico Nuovo, Moliterno ecc. ecc. gentiluomo di camera di esercizio di S. M. il re delle Sicilie padre amantissimo della medesima.

gen. Componimento sacro per musica

ded. Dedica in versi di Tisanio Teutronio P.A.

libr. Tisanio Teutronio P.A.

mus. Giuseppe de Magistris

istit. monastero di Regina Coeli

fonti: Sartori 23789

\section{6}

tit. La Gara tra la Fede e la Fortezza, cantata a tre voci, in occasione «che veste l'abito religioso l'Eccellentissima Signora D. Maria Luisa de' Principi Pignatelli nel Real Monistero di Regina Coeli di Napoli

gen. cantata sacra a tre voci

organico: vl I/II, corni 2, tr 2, ob2, coro ST

musica: Alessandro Speranza

istit. monastero di Regina Coeli

fonti: partitura, Biblioteca Privata Giuseppe Pastore di Lecce

\section{SENZa DATA}

tit. Il Trionfo dell'Amor Divino, ${ }^{39}$ componimento drammatico di Tersalgo Lidiaco da cantarsi vestendo l'abito religioso nel real Monastero di Regina-Coeli S. E. la signora D. M.a Antonia Pignatelli.

gen. Componimento drammatico

libr. Clemente Filomarino Della Torre

pers. Amor divino, Amor profano, Sposa, Sposo

istit. monastero di Regina Coeli

fonti: Sartori 23713

39 Questo libretto, sprovvisto di data, potrebbe forse ricondursi alla professione religiosa di donna Maria Antonia Pignatelli avvenuta presso il monastero di Regina Coeli nel 1781. Della stessa professione si conservano le 5 antifone utilizzate durante la liturgia e composte da Alessandro Speranza, cfr. cap. III, pp. 80-82. 


\section{Le fonti musicali}

Alla ricchezza dell'attività musicale dei monasteri napoletani, purtroppo non fa riscontro un'adeguata presenza di fonti musicali. Se da un lato i documenti d'archivio ci vengono in aiuto dandoci informazioni sulla commissione e sull'utilizzo di musica, essi non possono essere da soli reali e completi testimoni del fenomeno musicale. Purtroppo i fondi archivistici delle singole istituzioni custodite presso l'Archivio di Stato e l'Archivio Diocesano di Napoli, non conservano partiture, probabilmente andate disperse nel corso dei secoli. Bisogna inoltre ricordare che la quasi totalità della produzione musicale sacra napoletana è ancora oggi custodita presso l'Archivio dei Girolamini, chiuso agli studiosi da più di trenta anni. I manoscritti in essa presenti, appartengono ad autori legati alla scuola napoletana fra Sei e Settecento fra cui Cristofaro Caresana, Gaetano Veneziano, Gennaro Manna, ovvero tutti compositori attivi presso i monasteri femminili. ${ }^{40} \mathrm{Da}$ questo scaturisce dunque l'impossibilità di un confronto fra fonti musicali e documenti d'archivio. Tuttavia, il catalogo elaborato nel 1918 da Salvatore Di Giacomo sul fondo dei Girolamini, ${ }^{41}$ lo spoglio archivistico condotto per il presente studio e le recenti ricerche di studiosi della Napoli vicereale, permettono d'individuare una serie di composizioni destinate all'ambiente claustrale femminile che riassumiamo di seguito.

Ad un primo gruppo appartengono una serie di composizioni esplicitamente dedicate a religiose. In esse non sempre si deduce il monastero di riferimento, spesso sono indicate solo le destinatarie dei brani. Nella maggior parte dei casi il nome della religiosa viene riportato senza cognome, questo rende pressoché impossibile determinare la famiglia di appartenenza e quindi un collegamento ad un'istituzione.

Si tratta di composizioni destinate alle celebrazioni liturgiche: salmi per l'ufficio delle ore, cantate per l'esposizione del Santissimo Sacramento, lezioni per la Settimana Santa inserite nel triduo delle Tenebre. Le lezioni presenti nel fondo dei Girolamini sono composte da autori quali Johann Adolph Hasse, Leonardo Leo, Nicola Porpora e soprattutto Gaetano Veneziano. ${ }^{42}$ Negli anni in cui Veneziano era attivo alla Cappella Reale, la sua presenza si ricontra anche presso il conservatorio di Nostra Signora della Solitaria, per il quale le fonti attestano la composizione e l'esecuzione di musica per il triduo pasquale e per

40 Il patrimonio musicale della Biblioteca copre l'arco dei secoli che vanno dal XVI al XX e registra ben oltre 6.500 composizioni. Ad oggi il prezioso patrimonio dell'archivio musicale dei Girolamini, chiuso dal 1980, è consultabile in parte online grazie alla digitalizzazione di diversi manoscritti. Sul problema delle fonti napoletane di musica sacra si veda MARINo 2009, pp. 823-923.

41 Di Giacomo 1918.

42 Gaetano Veneziano (1665-1716) pugliese di nascita, era giunto a Napoli all'età di 10 anni. Entrò nel conservatorio di Santa Maria di Loreto e divenne allievo di Provenzale. Veneziano fu maestro nello stesso conservatorio di Loreto nel 1684 e poi stabilmente dal 1695 alla morte divenendo per alcuni anni, fra 1704 e 1707, maestro della Real Cappella. Si veda Turano 1988, pp. 37-48. 
la Domenica di Pasqua ${ }^{43}$ Veneziano compose anche per il monastero di Santa Chiara, Santa Maria Maddalena delle Spagnole e il conservatorio dello Spirito Santo, così come leggiamo nelle dediche delle sue composizioni. ${ }^{44}$ Molte delle lezioni di Veneziano sono dedicate ad una voce solista con accompagnamento di archi. È il caso della Lettione terza da cantare durante la sera del Giovedì Santo composta da Gaetano Veneziano nel 1700, in cui si legge chiaramente la dedica ad una certa «suor Chiara», e della Lettione del Giovedi Santo del Primo Notturno dedicata a «Suor Maria Gioseppa»:45

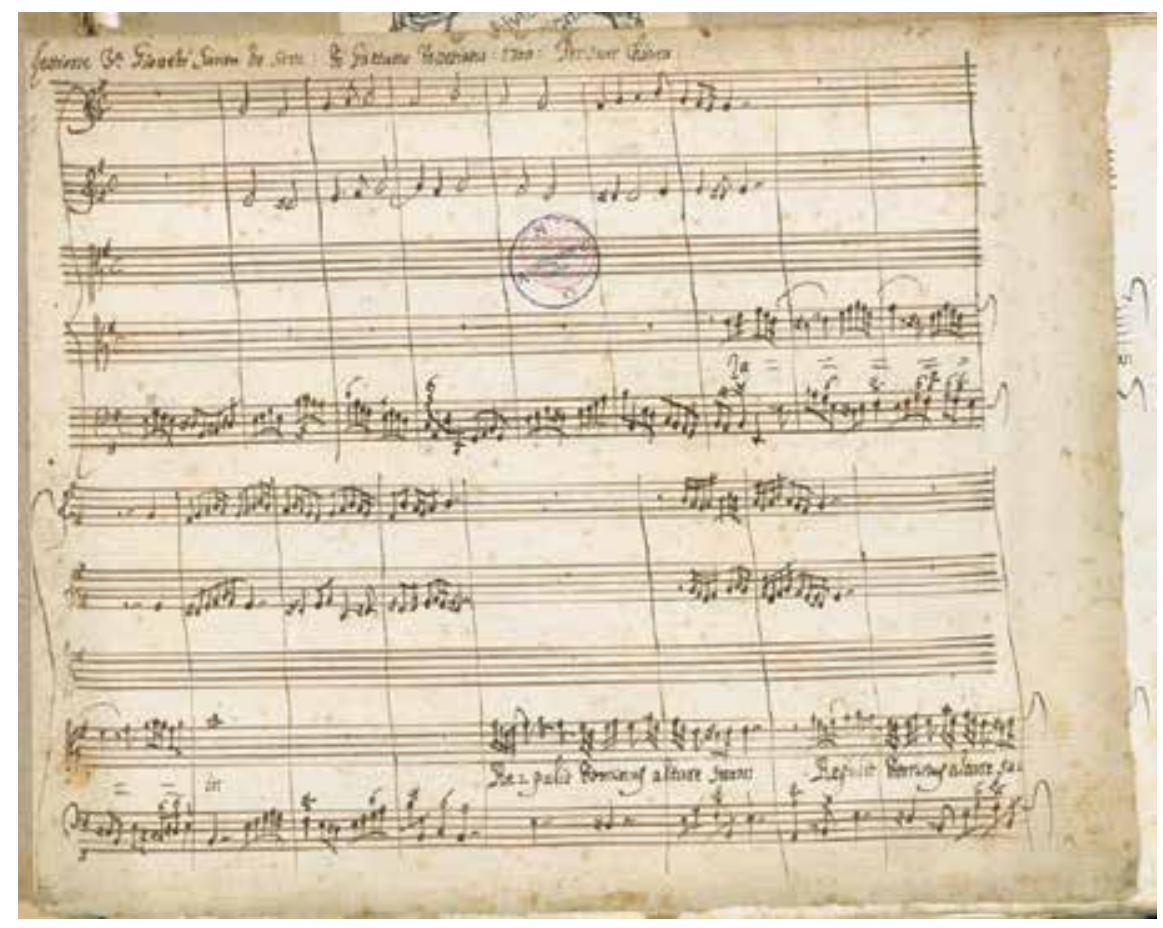

5. Gaetano Veneziano, Zain. Repulit Dominus altare suum,

Napoli, Archivio musicale della Congregazione dell’Oratorio.

L'interpretazione che Veneziano fa del testo di Geremia lascia grande spazio al virtuosismo canoro attraverso melodie che richiedono una notevole destrezza nella parte vocale: non si ha infatti l'impressione di essere di fronte a partiture per così dire scolastiche.

43 Sulle Lamentazioni si veda anche cap. III, pp. 69-70.

44 Si veda la tabella 6 p. 115 relativa alle partiture dedicate a religiose.

45 Si veda quanto detto a proposito delle proibizioni sui riti della Settimana Santa nel cap. I, p. 46. 


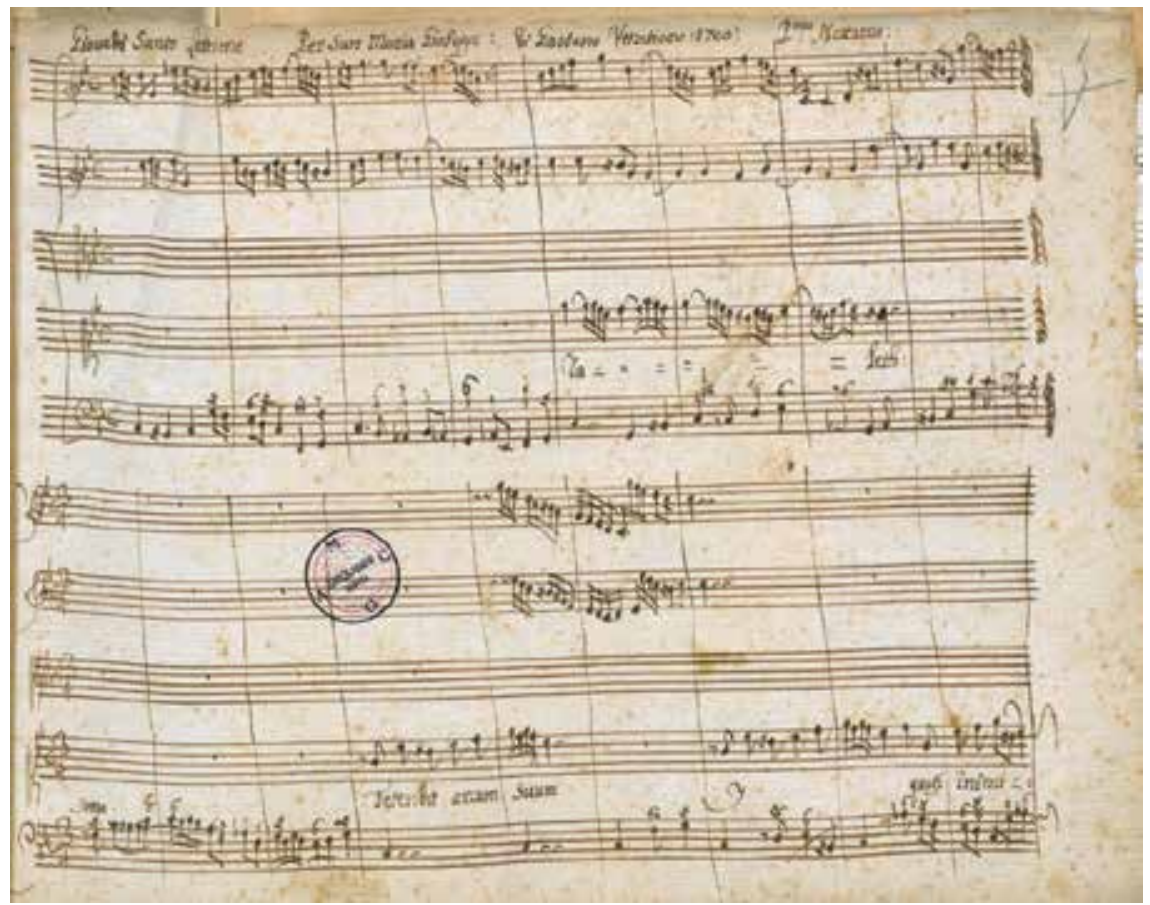

6. Gaetano Veneziano, Daleth. Tetendit arcum suum. ${ }^{46}$

Napoli, Archivio musicale della Congregazione dell'Oratorio.

Vi è inoltre l'alternanza di situazioni musicali diverse, motivi della tradizione popolare si mescolano in giusto equilibrio al repertorio colto. Inoltre le sezioni strumentali solistiche, l'uso del basso, rendono l'idea del livello dei musicisti o delle musiciste a cui erano destinate queste composizioni. ${ }^{47}$

Vi sono poi una serie di fonti musicali che si riferiscono a specifiche occorrenze liturgiche proprie di un'istituzione. Sono per la maggior parte inni composti per le celebrazioni dei santi venerati nei singoli monasteri. Ne è un esempio l'inno di Gaetano Manna in occasione della festività Santa Maria Egiziaca, commissionato dalla badessa dell'omonima istituzione, ${ }^{48}$ oppure l'inno del XVIII sec., O Clara, da eseguire «per quando entra la statua di s. Chiara in detta Chiesa», composto probabilmente per la processione organizzata dal monastero di Santa Chiara in occasione della festività della Santa. Il sontuoso corteo partiva dalla Cattedrale cittadina, ove era custodita la statua d'argento raffigurante la Santa e giungeva a Santa Chiara dove aveva inizio un

46 Le due partiture sono custodite presso l'Archivio dei Girolamini di Napoli, la riproduzione fotografica è tratta da <http://www.internetculturale.it $>$.

47 Entrambe queste composizioni si possono considerare per molti versi analoghe alle più conosciute lamentazioni di Alessandro Scarlatti, in comune hanno l'esecuzione a voce sola con archi e la costante alternanza di situazioni musicali molto diverse fra loro.

48 Cfr. cap. V, pp. 140-141. 
solenne triduo. ${ }^{49}$ È probabile che questi esempi musicali fossero prodotti e commissionati per le cerimonie ufficiali e dunque eseguiti dalle cappelle musicali (Arcivescovado o Cappella Reale) chiamate a realizzare la parte musicale del rito liturgico.

Provengono dal monastero di Santa Chiara alcune fonti musicali destinate alla quotidianità liturgica: una messa e tre antifone di compieta a due voci, appartenenti alla tradizione della polifonia semplice, composte nel 1733 dalla maestra di coro del monastero di Santa Chiara, Delia Bonito. ${ }^{50}$ Ad oggi sono gli unici esempi provenienti dai chiostri napoletani ad essere stati composti da mano femminile. Sono fonti che si collegano alla pratica del canto fratto. Si tratta di un repertorio assai diffuso in tutta Europa e testimoniato da numerosi libri liturgici sia manoscritti sia a stampa e da una notevole diffusione sul territorio europeo tra Cinque e Settecento. In altre parti d'Italia come Trento, Bologna etc., soprattutto sotto l'impulso delle comunità monastiche appartenenti alla famiglia francescana, in pieno Settecento venivano composti interi Kyriali, Graduali etc. nello stile del canto fratto, talvolta anche a due o tre voci. La scoperta, anche per il Meridione d'Italia, di nuove fonti musicali manoscritte in canto fratto, conferma che si trattava di una tradizione assai più diffusa di quanto non ci facciano credere le rare testimonianze scritte rimaste. Essendo fonti composte dalla maestra di coro del monastero, possiamo supporre che fossero destinate alle monache coriste di Santa Chiara. Questo confermerebbe la partecipazione attiva delle stesse religiose al servizio musicale delle celebrazioni liturgiche giornaliere. ${ }^{51}$

Sono presenti poi alcune composizioni per celebrazioni liturgiche specifiche dei monasteri femminili, come le liturgie di professione religiosa. Si tratta quasi sempre di mottetti composti sul testo dell'antifona Veni Sponsa Christi o dell'inno Veni Creator Spiritus presenti nei riti di professione religiosa composti da Francesco Feo, Gennaro Manna, Girolamo Abos, Giacomo Sarcuni, cui si aggiungono cinque antifone relative al canto dei voti pronunciati dalle professe e composte da

50 Su queste composizioni si veda cap. VI, pp. 173-178.

51 La maggior parte degli studi sulle istituzioni monastiche e sulla musica composta per la liturgia si è concentrata sulle occasioni del far musica più solenni, eccezionali e fastose, con largo impiego di professionisti e di tutte le possibilità che lo sviluppo delle cappelle musicali presso le istituzioni religiose poneva a disposizione dei compositori. In passato inoltre le ricerche riguardanti la vita liturgica e musicale degli ordini monastici sono state fortemente condizionate da lacune causate dalla mancata conoscenza e dal difficile reperimento delle fonti, cui si aggiungeva anche - per l'ambito liturgico quotidiano - la consuetudine di valorizzare soltanto le testimonianze più antiche, emarginando tutto ciò che era considerato tardo rispetto all'aurea età medievale. Questo ha significato l'esclusione di un patrimonio musicale che non appartiene direttamente al canto detto 'gregoriano' ma che di fatto da esso deriva, e che ha avuto piena cittadinanza nelle azioni liturgiche: il canto fratto. Le indagini sulle consuetudini musicali degli ordini monastici femminili e il ritrovamento di questa tipologia di fonti musicali aprono nuovi filoni di ricerca e nuovi interrogativi. Napoli e il Meridione d'Italia potrebbero non essere stati esclusi dal fenomeno del canto fratto anche in pieno Settecento. 
Alessandro Speranza..$^{52}$ Nei riti di monacazione era particolarmente viva la consuetudine di chiamare illustri cantanti per far cantare mottetti o di farli eseguire direttamente alle religiose ${ }^{53} \mathrm{Il}$ mottetto solistico era infatti il genere musicale maggiormente utilizzato nelle professioni religiose: esso permetteva agli artisti coinvolti di esprimere al meglio tutte le loro abilità vocali.

Infine citiamo le partiture superstiti relative alle diverse forme drammatiche che spesso facevano seguito ad alcune ricorrenze festive. A questa categoria appartiene ad esempio la cantata sacra a tre voci La Gara tra la Fede e la Fortezza, composta, nel 1776, in occasione «che veste l'abito religioso l'Eccellentissima Signora D. Maria Luisa de' Principi Pignatelli», per il monastero di Regina Coeli e attribuita al compositore Alessandro Speranza. ${ }^{54}$

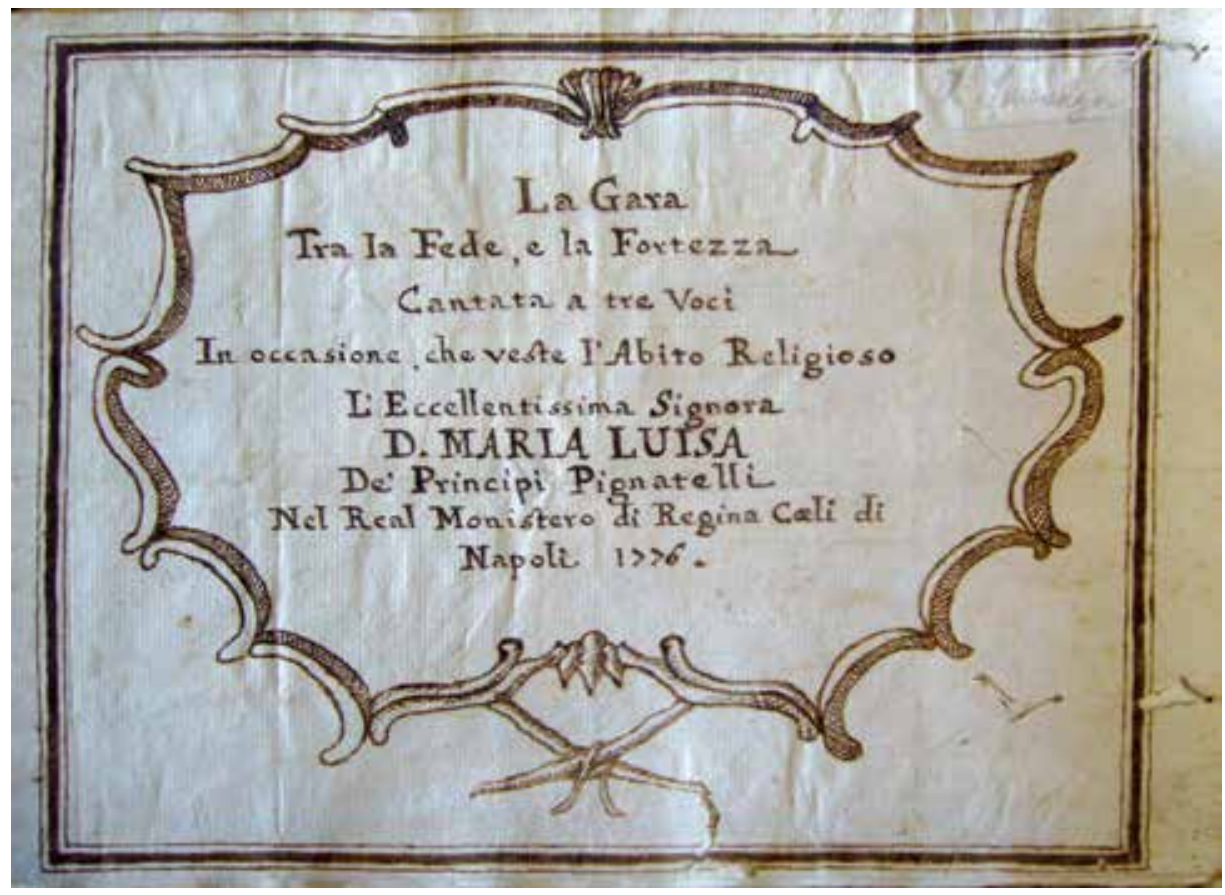

7. Alessandro Speranza, La Gara tra la Fede e la Fortezza. Frontespizio. ${ }^{55}$ Lecce, Biblioteca G. Pastore.

52 Cfr. cap. III, pp. 80-82.

53 Cfr. cap. III, pp. 86-88.

54 Il manoscritto non presenta né il nome dell'autore dei versi né del compositore. Tuttavia sul frontespizio, in alto a destra, è presente il nome di Alessandro Speranza a matita. Speranza fu attivo a Regina Coeli come maestro di cappella e sacerdote dal 1764. Cfr. PAStore 2007; Fiore/ IACONO 2016.

55 Anche il presente manoscritto fa parte della collezione privata della Biblioteca Giuseppe Pastore di Lecce. 
L'organico è formato da tre soprani, un ensemble strumentale costituito da archi, due corni, due trombe e due oboi, un coro di soprani e tenori. La cantata si apre con una sinfonia introduttiva tripartita, cui fanno seguito recitativi ed arie che vedono protagonisti Amor Divino Fede e Fortezza che si vantano reciprocamente di aver concesso ad una semplice fanciulla le doti necessarie per diventare perfetta sposa di Cristo.

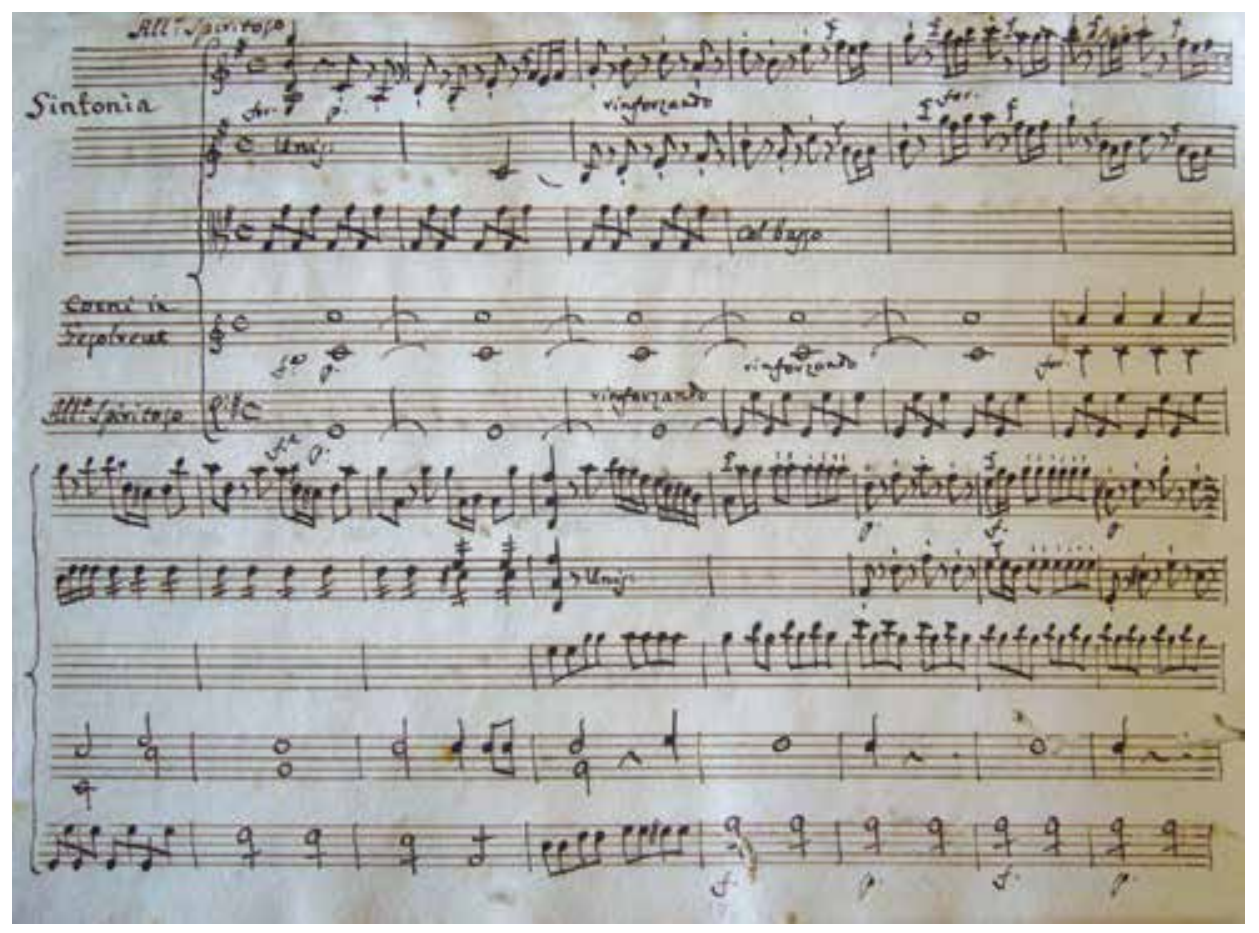

8. Alessandro Speranza, Sinfonia introduttiva.

Lecce, Biblioteca G. Pastore.

Alle partiture sopracitate si potrebbe ancora aggiungere La Betulia liberata, «sacro dramma», la cui musica risulta essere composta da Pasquale Cafaro per la monacazione «dell'Eccellentissima D. M. Teresa Francone» nel 1749. La partitura, oggi conservata presso la Biblioteca Nazionale di Francia, non riporta il nome dell'istituzione in cui aveva avuto luogo il rito di professione. Cafaro, compositore di origini pugliesi, risiedeva a Napoli già dal 1735, essendo allievo di Nicola Fago e Leonardo Leo al conservatorio della Pietà de' Turchini, per poi prenderne lui stesso la direzione nel 1759. È verosimile dunque ipotizzare che anche questa testimonianza sia di ambito napoletano. 
Queste fonti musicali, diverse fra loro, sono molto significative, essendo espressione di usi e costumi delle comunità claustrali. Osservate nel loro insieme costituiscono una testimonianza preziosa dell'ambiente del quale sono emanazione.

Si propone nelle seguenti tabelle, l'elenco di tutte le composizioni riconducibili agli ambienti monastici femminili napoletani emerse dalla documentazione d'archivio, dalle fonti musicali a disposizione e segnalate nel catalogo Di Giacomo:

Tabella 6: Partiture dedicate a religiose.

\begin{tabular}{|c|c|c|c|c|}
\hline Sec. XVII & $\begin{array}{l}\text { Bartolomeo } \\
\text { Ferrari }\end{array}$ & $\begin{array}{l}\text { Risorto il Signore, aria a } \\
\text { solo }\end{array}$ & $\begin{array}{l}\text { Per una signora } \\
\text { monaca }\end{array}$ & I-Nf \\
\hline $\begin{array}{l}\text { Inizio } \\
\text { sec. XVIII }\end{array}$ & $\begin{array}{l}\text { Gaetano } \\
\text { Veneziano }\end{array}$ & $\begin{array}{l}\text { Laudamus a voce sola con } \\
\text { violini unisoni }\end{array}$ & $\begin{array}{l}\text { Per suor Eugenia } \\
\text { alla Maddalena delle } \\
\text { Spagnole }\end{array}$ & $\mathrm{I}-\mathrm{Nf}$ \\
\hline $\begin{array}{l}\text { Inizio } \\
\text { sec. XVIII }\end{array}$ & $\begin{array}{l}\text { Gaetano } \\
\text { Veneziano }\end{array}$ & Cantata per il SS.mo & $\begin{array}{l}\text { Per la signora d. Luisa } \\
\text { de Franchis in Santa } \\
\text { Chiara }\end{array}$ & $\mathrm{I}-\mathrm{Nf}$ \\
\hline $\begin{array}{l}\text { Inizio } \\
\text { sec. XVIII }\end{array}$ & $\begin{array}{l}\text { Gaetano } \\
\text { Veneziano }\end{array}$ & $\begin{array}{l}\text { Lezione II noct. I per la } \\
\text { notte del SS. Natale }\end{array}$ & $\begin{array}{l}\text { Per suor Rosa Mancini } \\
\text { al Spirito Santo }\end{array}$ & $\mathrm{I}-\mathrm{Nf}$ \\
\hline $\begin{array}{l}\text { Inizio } \\
\text { sec. XVIII }\end{array}$ & $\begin{array}{l}\text { Gaetano } \\
\text { Veneziano }\end{array}$ & $\begin{array}{l}\text { Lezione II noct. I } \\
\text { Mercoledì Santo }\end{array}$ & $\begin{array}{l}\text { Per suor Giuseppa } \\
\text { Conti dentro lo Spirito } \\
\text { Santo. }\end{array}$ & $\mathrm{I}-\mathrm{Nf}$ \\
\hline $\begin{array}{l}\text { Inizio } \\
\text { sec. XVIII }\end{array}$ & $\begin{array}{l}\text { Gaetano } \\
\text { Veneziano }\end{array}$ & $\begin{array}{l}\text { Versetto del Benedictus e } \\
\text { versetto del Miserere }\end{array}$ & $\begin{array}{l}\text { Per suor Eugenia } \\
\text { dentro lo Spirito Santo }\end{array}$ & I-Nf \\
\hline $\begin{array}{l}\text { Inizio } \\
\text { sec. XVIII }\end{array}$ & $\begin{array}{l}\text { Gaetano } \\
\text { Veneziano }\end{array}$ & $\begin{array}{l}\text { Lezione III noct.I per la } \\
\text { notte del S. Natale }\end{array}$ & $\begin{array}{l}\text { Per suora Giuseppa } \\
\text { Conte dentro lo Spirito } \\
\text { Santo }\end{array}$ & I-Nf \\
\hline $\begin{array}{l}\text { Inizio XVIII } \\
\text { sec. }\end{array}$ & $\begin{array}{l}\text { Gaetano } \\
\text { Veneziano }\end{array}$ & $\begin{array}{l}\text { Salmo I di compieta a } 4 \\
\text { con violini }[\ldots] \text { Venerdì } \\
\text { Santo di sera. Lezione I } \\
\text { noct. III }\end{array}$ & Per suor Colomba & $\mathrm{I}-\mathrm{Nf}$ \\
\hline 1693 & $\begin{array}{l}\text { Gaetano } \\
\text { Veneziano }\end{array}$ & $\begin{array}{l}\text { De Lamentatione Giovedì } \\
\text { Santo la sera }\end{array}$ & $\begin{array}{l}\text { Per suor Maria } \\
\text { Gioseppa }\end{array}$ & $\mathrm{I}-\mathrm{Nf}$ \\
\hline 1700 & $\begin{array}{l}\text { Gaetano } \\
\text { Veneziano }\end{array}$ & $\begin{array}{l}\text { Lettione del Giovedì Santo } \\
\text { noct. I }\end{array}$ & $\begin{array}{l}\text { Per suor Maria } \\
\text { Gioseppa }\end{array}$ & $\mathrm{I}-\mathrm{Nf}$ \\
\hline $\begin{array}{l}18 \text { febbraio } \\
1700\end{array}$ & $\begin{array}{l}\text { Gaetano } \\
\text { Veneziano }\end{array}$ & $\begin{array}{l}\text { Lettione III Giovedì Santo } \\
\text { de sera }\end{array}$ & Per suor Chiara & I-Nf \\
\hline $\begin{array}{l}\text { Inizio } \\
\text { sec. XVIII }\end{array}$ & $\begin{array}{l}\text { Gaetano } \\
\text { Veneziano }\end{array}$ & $\begin{array}{l}\text { Magnificat «volgare» a } 2 \\
\text { con violini }\end{array}$ & $\begin{array}{l}\text { Per le ss.re monache } \\
\text { del Spirito Santo }\end{array}$ & $\mathrm{I}-\mathrm{Nf}$ \\
\hline Sec. XVIII & Nicola Grillo & Lectio III Giovedì Santo & $\begin{array}{l}\text { Per la Signora d. Maria } \\
\text { Anna Scalea }\end{array}$ & $\mathrm{I}-\mathrm{Nf}$ \\
\hline Sec. XVIII & $\begin{array}{l}\text { Johann Adolph } \\
\text { Hasse }\end{array}$ & $\begin{array}{l}\text { Lectio II noct.I Mercoledì } \\
\text { Santo }\end{array}$ & $\begin{array}{l}\text { Per l'Ill.ma sig.ra d. } \\
\text { Anna d'Andrea }\end{array}$ & $\mathrm{I}-\mathrm{Nf}$ \\
\hline
\end{tabular}




\begin{tabular}{|c|c|c|c|c|}
\hline Sec. XVIII & $\begin{array}{l}\text { Johann Adolph } \\
\text { Hasse }\end{array}$ & Lectio III Venerdì Snto & $\begin{array}{l}\text { Per la sig.ra d. Chiara } \\
\text { d'Andrea }\end{array}$ & $\mathrm{I}-\mathrm{Nf}$ \\
\hline $\begin{array}{l}\text { Dicembre } \\
1720\end{array}$ & Leonardo Leo & Lectio III Giovedì Santo & $\begin{array}{l}\text { Per servizio dell'ill. } \\
\text { ma sig.ra d. Chiara } \\
\text { d'Andrea }\end{array}$ & $\mathrm{I}-\mathrm{Nf}$ \\
\hline $\begin{array}{l}\text { Dicembre } \\
1720\end{array}$ & Leonardo Leo & Lectio III Giovedì Santo & $\begin{array}{l}\text { Per servizio dell'ill.ma } \\
\text { sig.ra [illegibile] }\end{array}$ & $\mathrm{I}-\mathrm{Nf}$ \\
\hline $\begin{array}{l}\text { Inizi } \\
\text { sec. XVIII }\end{array}$ & Leonardo Leo & $\begin{array}{l}\text { Veni sponsa Christi a } 5 \text { con } \\
\text { violini }\end{array}$ & $\begin{array}{l}\text { Per le signore monache } \\
\text { del Spirito Santo }\end{array}$ & I-Nf \\
\hline Sec. XVIII & $\begin{array}{l}\text { Francesco de } \\
\text { Majo }\end{array}$ & $\begin{array}{l}\text { Lamentatio I Mercoledì } \\
\text { Santo }\end{array}$ & $\begin{array}{l}\text { Per la sig.ra donn' } \\
\text { Agnese di Gennaro }\end{array}$ & $\mathrm{I}-\mathrm{Nf}$ \\
\hline XVIII sec. & Nicola Conti & Messa & $\begin{array}{l}\text { Sig.re Isabella, Rosa, } \\
\text { Ottavia }\end{array}$ & $\mathrm{I}-\mathrm{Bc}$ \\
\hline Ottobre 1732 & Nicola Porpora & $\begin{array}{l}\text { Lezione III Mercoledì } \\
\text { Santo }\end{array}$ & $\begin{array}{l}\text { Per l'ill.ma sigr.a d. } \\
\text { Isabella d'Andrea }\end{array}$ & I-Nf \\
\hline 1746 & $\begin{array}{l}\text { Pasquale } \\
\text { Cafaro }\end{array}$ & La Betulia Liberata & $\begin{array}{l}\text { per la monacazione } \\
\text { «dell'Eccellentissima } \\
\text { D. M. Teresa Francone }\end{array}$ & F-Pn \\
\hline 17 luglio 1747 & $\begin{array}{l}\text { Gennaro } \\
\text { Manna }\end{array}$ & $\begin{array}{l}\text { Gioas re di Giuda. Oratorio } \\
\text { a } 6\end{array}$ & $\begin{array}{l}\text { A richiesta della } \\
\text { signora } d . \text { Vittoria } \\
\text { Colonna }\end{array}$ & I-Nf \\
\hline $\begin{array}{l}\text { Fine XVIII } \\
\text { sec. }\end{array}$ & $\begin{array}{l}\text { Donato } \\
\text { Ricchezza }\end{array}$ & $\begin{array}{l}\text { Lamentatio I Mercoledì } \\
\text { Santo a sera coll'arciliuto }\end{array}$ & $\begin{array}{l}\text { Per la sig.ra suora } \\
\text { Antonia }\end{array}$ & I-Nf \\
\hline 1776 & $\begin{array}{l}\text { Alessandro } \\
\text { Speranza }\end{array}$ & La Gara tra Fede Speranza & $\begin{array}{l}\text { Per Ecc. Sig.ra } \\
\text { D. Maria Luisa de, } \\
\text { Principi Pignatelli, } \\
\text { Regina Coeli }\end{array}$ & $\begin{array}{l}\text { I-LE } \\
\text { pastore }\end{array}$ \\
\hline 1789 & $\begin{array}{l}\text { Alessandro } \\
\text { Speranza }\end{array}$ & 5 antifone & $\begin{array}{l}\text { Per la monacazione } \\
\text { della «R. Sig. } \\
\text { ra Mariantonia } \\
\text { Pignatelli», Regina } \\
\text { Coeli }\end{array}$ & $\begin{array}{l}\text { I-LE } \\
\text { pastore }\end{array}$ \\
\hline $1791-1810$ & Anonimo & $\begin{array}{l}\text { Festinantes ad Praesepe } \\
\text { Mottetto | Ripieno in } \\
\text { Pastorale } \mid \text { A quattro voci, } \\
\text { cioé di Basso, tenore, } \\
\text { Contralto, e | di Soprano }\end{array}$ & $\begin{array}{l}\text { Per le Sig.re Monache } \\
\text { di S.a M.a Della Purità }\end{array}$ & $\mathrm{I}-\mathrm{Nc}$ \\
\hline
\end{tabular}


Tabella 7: Partiture per monasteri. ${ }^{56}$

\begin{tabular}{|c|c|c|c|c|}
\hline Sec. XVII & Antonio Nola & Veni de Libano & per le Monache & $\mathrm{I}-\mathrm{Nf}$ \\
\hline Sec. XVII & Anonimo & $\begin{array}{l}\text { Inno a } 5 \text { Concinant } \\
\text { plebs fidelium }\end{array}$ & $\begin{array}{l}\text { Per Santa } \\
\text { Chiara }\end{array}$ & $\mathrm{I}-\mathrm{Nf}$ \\
\hline 1723 & Delia Bonito & Messa & S. Chiara & S. Chiara \\
\hline 1723 & Delia Bonito & Salve Regina & & S. Chiara \\
\hline 1723 & Delia Bonito & Regina Coeli & & S. Chiara \\
\hline 1723 & Delia Bonito & $\begin{array}{l}\text { Ave Regina } \\
\text { coelorum }\end{array}$ & & S. Chiara \\
\hline Sec. XVIII & Anonimo & $\begin{array}{l}\text { Hymnus Lux o } \\
\text { decora Gregori, } \\
4 \mathrm{vv}\end{array}$ & $\begin{array}{l}\text { Per San } \\
\text { Gregorio } \\
\text { Armeno }\end{array}$ & I-Nf \\
\hline- & Francesco Feo & $\begin{array}{l}\text { Pater superni } \\
\text { luminis }\end{array}$ & $\begin{array}{l}\text { Per S. Maria } \\
\text { Maddalena de } \\
\text { Pazzi }\end{array}$ & I-Nf \\
\hline Sec. XVIII & Francesco Feo & $\begin{array}{l}\text { Veni sponsa Christi } \\
\text { a S solo }^{57}\end{array}$ & $\begin{array}{l}\text { Per } \\
\text { monacazione }\end{array}$ & $\mathrm{I}-\mathrm{Nf}$ \\
\hline 1765 & Giacomo Tritto & Li Furbi & $\begin{array}{l}\text { Intermezzo } \\
\text { fatto per L'Ecc. } \\
\text { me Sig.re Dame } \\
\text { Monache In } \\
\text { S. Chiara }\end{array}$ & I-Nc \\
\hline Aprile 1789 & Gaetano Manna & $\begin{array}{l}\text { Inno della gloriosa } \\
\text { S. Maria Egiziaca }\end{array}$ & $\begin{array}{l}\text { Per comando } \\
\text { della signora } \\
\text { dama m.a } \\
\text { Giuseppe } \\
\text { d'Aragona } \\
\text { ad uso della } \\
\text { real chiesa di } \\
\text { S.M. Egiziaca } \\
\text { di Pizzofalcone }\end{array}$ & I-Nf \\
\hline Sec. XVIII & Gennaro Manna & Veni sponsa Christi & $\begin{array}{l}\text { Per } \\
\text { monacazione }\end{array}$ & I-Nf \\
\hline
\end{tabular}

56 Si segnalano anche le seguenti partiture riconducibili a dei monasteri femminili pur non avendo un esplicito riferimento: due inni dedicati a Santa Maria Maddalena di Gaetano Veneziano sec. XVIII e Cristofaro Caresana del 1678, probabilmente composti per una delle istituzioni femminili cittadine dedicate alla Maddalena; e ancora Giuseppe Alfieri, Hynno per Santa Maria Egyptiaca a 4, sec. XVI; Stamigna Hino per S. Maria Magdalena de Pactis a 9 voci, sec. XVII; Gaetano Veneziano, In festo S. Mariae Magdalena a 5 voci co' violini, 16 luglio 1700; Gaetano Veneziano, due inni carmelitani per S. Maddalena dei Pazzi, 1689.

57 Salvatore Di Giacomo indica anche un altro mottetto Veni sponsa Christi composto da Feo ove però non vi è l'esplicita indicazione della monacazione. 


\begin{tabular}{lllll}
\hline- & Girolamo Abos & $\begin{array}{l}\text { Veni Creator } \\
\text { Spiritus }\end{array}$ & $\begin{array}{l}\text { Per } \\
\text { monacazione }\end{array}$ & I-Nf \\
\hline Sec. XVIII & $\begin{array}{l}\text { Giacomo } \\
\text { Sarcuni }^{58}\end{array}$ & $\begin{array}{l}\text { Veni Creator } \\
\text { Spiritus }\end{array}$ & $\begin{array}{l}\text { Pel velo di } \\
\text { monaca }\end{array}$ & I-Nf \\
\hline Sec. XVIII & Nicolò Veneziano & $\begin{array}{l}\text { O Clara, «hinno } \\
\text { per quando entra la } \\
\text { statua di s. Chiara } \\
\text { in detta Chiesa». }\end{array}$ & Shiara & I-Nf \\
& & & \\
& & & \\
\hline
\end{tabular}

58 Di Sarcuni è presente presso i Girolamini anche un Veni Sponsa Christi a due Chori senza specifica destinazione alla funzione della monacazione. 
Parte II

Le istituzioni 



\section{I Monasteri}

\section{Benedettine}

\subsection{Ss. Marcellino e Festo}

Il monastero dei Santi Marcellino e Festo ebbe origine dalla fusione di due distinti monasteri preesistenti: il monastero basiliano dei Ss. Marcellino e Pietro e quello benedettino dei Ss. Festo e Desiderio. A seguito del sinodo diocesano del 1565, si decise di accorpare il monastero dei Ss. Festo e Desiderio, noto per decadenza morale, ${ }^{1}$ a quello di San Marcellino, ormai riformato. Pertanto, alla fine del Cinquecento, le monache di San Festo si unirono a quelle di San Marcellino e il monastero prese il nome dei Ss. Marcellino e Festo. ${ }^{2}$

L'accorpamento dei due monasteri non dovette però cambiare di molto la situazione. Una lettera inviata al Papa Clemente XI dall'arcivescovo Pignatelli, ci informa sulla mancata osservanza di voti e disposizioni:

\footnotetext{
Santissimo Padre

Già che Vostra Santità sin dal principio dell'Assunzione al suo Pontificato hà cominciato con apostolico zelo à sbarbicare gl'abusi introdotti in varij monasteri di monache in questa Città, $[\ldots]$ e frà queste, e molte altre Religiose, che sono in questa Città, vi sono quelle del Monastero de SS. Marcellino, e Festo [...] persona, che desidera la salute dell'anime di dette Religiose, hà stimato bene rappresentare à Vostra Santità molti abusi, et inconvenienti, che sono nati, $\mathrm{e}$ nascono alla giornata in detto Monastero $[\ldots]$

Primo, per quello riguarda il Voto della Povertà, non s'osserva, mentre ogni Religiosa spende à su modo il livelo, in quello, che li piace con far donativi a' parenti, ed altre persone, le stanze adornate in miglior modo, di quelle de secolari, e facendosi cucinare ogn'una separatamente, e senza mangiare al Commune Refettorio [...]

$3^{\circ}$ La maggior parte delle monache dormono in una istessa stanza, anzi in un medesimo letto; di che nascono molti inconvenienti, e Vostra Santità m'intende ne io l'esprimo per non tediar le sue caste Orecchie [...]
}

1 Per il ripristino della disciplina, gli stessi parenti delle religiose sollecitarono l'intervento di papa Paolo IV e nel 1577 dell'arcivescovo Alfonso Carafa. Si veda Valerio 2006, p. 357.

2 Durante il decennio francese le monache furono trasferite a San Gregorio Armeno, l'edificio fu donato ad alcune suore salesiane che vi rimasero fino al 1829. In seguito il complesso venne trasformato in educandato femminile. La chiesa è attualmente sconsacrata. Sulla storia del complesso si vedano le fonti storiche: De Stefano 1560, pp. 173-174; D’Engenio 1624, pp. 314 315; Celano 2001, Giornata III, pp. 707-711; Galante 1985, Giornata VI, pp. 216-217, 219. Si veda anche: Valerio 2006, pp. 358-365; STRAZZUllo 1955, pp. 437-438. 
XVI. Procuri Vostra Santità che nel Cantare li Passij, Evangelij, et altre funzioni ecclesiastiche si canti Canto fermo Gregoriano, perche ne nascono inconvenienti, e risate, con sentir quelli trilli di musici $[\ldots]$

Santissimo Padre questi abusi sono stati riferiti da monaca zelante del Monastero onde Vostra Santità Procuri per ora riparar presto a' questi inconvenienti [...] Napoli dal Monastero de' SS. Marcellino e Festo. 4 Gennaro. $1710 .^{3}$

La visita pastorale del cardinale Spinelli del 1742, di cui si conserva la relazione completa, ci informa sulla «Maniera e costume di vivere di dette Signore Monache»:

L'osservanza della Regola di tutte le predette Signore Monache stà addetta sotto quella del Padre San Benedetto [...] la prattica della quale principalmente consiste nelli quattro voti di Povertà, obedienza, celibato, e perpetua clausura [...]. Perciò che riguarda al voto d'Ubidienza, questo distintamente incaricato dal P. S. Benedetto nel Capitolo della sua Regola [...] viene da tutte esattamente osservato colla totale dipendenza dalla volontà della Superiora, la quale non trascurando il proprio dovere, non manca da tempo in tempo ricordare publicamente in Capitolo, e privatamente alle sue Religiose la prattica di regolare li Istituti [...] e precisamente nella frequenza de Divini Ufficij à tenore della Regola [...] né quali convengono tutte paritamente cinque volte il giorno, cioè à dire avanti l'alba la mattina al mattutino colle Laudi, alle quali s'aggiunge anche l'ora di prima, e dato lo spazio di circa un'ora di più per la seconda volta, si conviene in coro alle ore di Terza, e Sesta, al qual tempo si canta ancor la Messa tutti i giorni festivi di Precetto, e di divozione, che vale à dire la maggior parte dell'anno, per la terza volta si conviene à Divini Ufficij per l'ora di nona, la quale né giorni di digiuno si dice avanti la Mensa; ad ora debita si ritorna per la quarta volta nel coro a cantare i vespri. E per ultimo circa l'ore 24 si dice la compieta, alla quale in tempo di quaresima si permette la lezzione spirituale ordinata dal Capitolo 42 della Regola, ed in tutta la salmodia si frequenta il canto monastico grave e semplice, per insegnare il quale colla licenza de' superiori, talvolta qualche prete morigerato, e probo dà lezzioni dalla Grata della Chiesa, per non esservi altro luogo à proposito [...]. ${ }^{4}$

I maggiori riferimenti all'attività musicale dell'istituzione sono infatti relativi alla pratica musicale vocale legata al canto liturgico e a quella organistica. Erano in servizio presso le monache di Ss. Marcellino e Festo gli organari Nicola Muscato e Francesco Scoppa, i rinomati Francesco e Fabrizio Cimino assieme al maestro di cappella Nicola Buscé. Inoltre è attestata, nel 1731, la presenza di un maestro di canto per la formazione musicale di un'educanda:

Al Dottor Giovanni Grimaldi a disposizione del Regio Consigliero Signor Don Matteo de Ferrante duc. uno E per esso alla Signora Donna Maria Moles educanda nel Monastero di San Marcellino di questa Città, e Sono per la mesata del Mastro di Canto maturata ad ultimo Febraro 1731 e Stà Sodisfatta della mesata di Gennaro $1731[\ldots] .^{5}$

Il resoconto della visita pastorale del 1742 ci informa anche delle festività celebrate nel monastero:

3 Asdn, Arcivescovi-Pignatelli II, app. p. 17. Ringrazio Claudio Bacciagaluppi per avermi segnalato il documento.

4 Asn, MS, f. 2878, app. p. 16.

5 AsBn, BPO 1020 17/III/1731, app. p. 19. 
Si celebrano per le Feste della Santissima Concezzione, della Trasfigurazione di Nostro Signore, di San Benedetto, di Santa Felicita, di San Marcellino, di San Donato, nell'esposizione di tutte le reliquie, sempre però con pompa decenza allo stato monastico. Altresì Si espone il venerabile senza però invito in apparati et ordinariamente senza musica nel tempo della novena della nascita di nostro Signore, nelli tre ultimi giorni di Carnevale, in tutti li venerdì di marzo, nell'ottavario di Corpus Domini. ${ }^{6}$

Spese per la musica vengono registrate quasi esclusivamente in occasione delle festività del Corpus Domini e di San Donato, in cui il sostegno musicale era affidato agli allievi dei conservatori maschili, cui potevano aggregarsi voci e strumenti aggiuntivi, come ad esempio nel 1749: «musica cioè d. 8 al Conservatorio, d. 12 per trè voci aggiunte e d. 3 per due violini aggiunti d. 23». ${ }^{7}$ Nei giornali di esito sono registrati numerosi pagamenti a musicisti, senza alcuna specifica di festività, ma legati magari a celebrazioni che richiedevano semplicemente un corredo sonoro più importante. Nel 1748, ad esempio, in occasione della «Messa Cantata del Vicario», si predisponevano «musica in due servizij» con «due violini aggiunti $[\ldots]$, un soprano $[\ldots]$ un contraldo». ${ }^{8}$

Le autorità ecclesiastiche raccomandavano alle benedettine di Ss. Marcellino e Festo che la monacazione non dovesse prevedere una festa «molto apparente e sontuosa». Al contrario, sia la professione che la 'velazione', celebrata presso le benedettine «doppo molti anni di professione», avrebbero dovuto svolgersi «senza superfluità», rispettando così la «maggior decenza». ${ }^{9}$ Sono attestate però monacazioni sontuose di giovani rampolle dell'aristocrazia, alle quali interveniva la Cappella della Cattedrale guidata dal maestro di cappella Andrea Amendola, stipendiato nel 1726 per «tutta la musica fatta nella Chiesa di S. Marcellino a lo febbraio corrente per la Professione di donna Angela de Liguoro [..]», ${ }^{10}$ e nel 1728 per « la musica così di voce come d'Istromenti» in occasione della monacazione di «donna Andreina e Donna Chiara Brancaccia». ${ }^{11}$

\subsection{San Potito}

Il monastero risale al IV secolo ed era situato nella "regione di Pozzo Bianco" nella zona detta dell'Anticaglia. Inizialmente ospitava monaci benedettini e in seguito, nel XII secolo, passò alle monache dello stesso ordine. Successivamente le monache decisero di conformarsi maggiormente ai dettami del Concilio di Trento, cercando una sistemazione più consona alla clausura richiesta dalla

6 Asn, MS, f. 2878, app. p. 16.

7 Ass, MS, f. 2755, app. p. 11.

8 Asn, MS, f. 2755, app. p. 12.

9 Asn, MS, f. 2878, app. p. 16.

10 AsBn, BSS 1156 13/II/1726, app. p. 18.

11 Asbn, BPI 1579 12/IV/1728, app. p. 18. 
riforma. Così, nel 1615, acquistarono un nuovo palazzo e ne affidarono la trasformazione in monastero all'architetto Pietro de Marino. ${ }^{12}$ La soppressione del 1808 costrinse le monache di San Potito a trasferirsi presso il monastero di San Gregorio Armeno. ${ }^{13}$

San Potito fu una delle roccaforti del patriziato cittadino. Molteplici e ricche dunque le festività in esso celebrate. Il mese di gennaio era un susseguirsi di appuntamenti: l'arrivo del nuovo anno era festeggiato con una «messa Cantata di Capo d'anno» con la «Cappella del'Arcevescovado [...]», «Voce Aggionte [...]», «Istrumenti [...] Orghanisto [...] Affitto d'Orghano», assieme a «trommettieri», «processione», «clerici e cinque figlioli de poveri»; ${ }^{14}$ per la messa della festività della Circoncisione, tredici strumentisti e cinque voci si aggiungevano alla Cappella dell'Arcivescovado, ${ }^{15}$ che interveniva anche nella messa cantata dell'Epifania con «sedici Istrumenti [...], sette voci» e la partecipazione di Angelo Durante come maestro di cappella e Andrea Amendola come organista. ${ }^{16}$ Il 14 gennaio cadeva poi la celebrazione della festa del santo titolare del monastero: San Potito. Anche in questa occasione si trovano diversi riferimenti alla «musica extraordinaria», con l'utilizzo di «violini particolari»». ${ }^{17}$ La «Gazzetta di Napoli» così si esprimeva a riguardo della celebrazione della festa nel 1720:

Dovendosi celebrare nella venerabile chiesa di S. Petito di dame monache la festa di detto glorioso santo la mattina del dì 13 del corrente [...], la musica, così nel primo e secondo vespro, come nella mattina di detta festività, fu delle migliori voci ed istrumenti di questa capitale, e particolarmente vi cantò due mottetti il tanto celebre e virtuoso musico, il marchese sig. Matteo Sassano, per sua divozione $[\ldots] .{ }^{18}$

Anche in occasione della festività di San Benedetto è documentata la presenza di celebri musicisti, di cui religiose annotano i soprannomi con cui erano maggiormente conosciuti:

In marzo $[\ldots]$ san Benedetto $[\ldots]$

Dato al sig. Aquilano per le due vesperi e Messa d.4

Dato al sig. Traschilio e sig. Battestino .8

12 Pietro de Marino fu un architetto, ingegnere e cartografo italiano, attivo a Napoli tra il 1629 e il 1666.

13 Nel 1780 il monastero fu oggetto di un restauro ad opera dell'architetto Giovan Battista Broggia. L'edificio fu poi adibito a fanteria nel 1808. Per la storia del monastero si rimanda: De STEFano 1560, p. 175, D’Engenio 1624, p. 599; D’Aloe 1883, p. 719; Celano 2001, Giornata VII, pp. 236-238; Galante 1985, Giornata XII, p. 406; Valerio 2006, pp. 321-325; Boccadamo 2001, pp. 77-106.

14 Ass, MS, f. 2941, app. p. 23.

15 Asn, MS, f. 2941, app. p. 23.

16 Ass, MS, f. 2940, app. p. 21.

17 Asn, MS, f. 2946, app. p. 27.

18 GDN, 23 gennaio 1720 (2), app. pp. 39-40. 
Dato per diece Istromenti d.20

Dato alli Poveri di Giesu Xristo d.9 [...]

Dato a chi a tirato li Mantici d.0.1.10

Dato per affitto di un organo d.1.2.10

L'«Aquilano» altri non era che Domenico Melchiorre, musico della Cappella Reale, mentre il «Battestino» potrebbe probabilmente corrispondere al violoncellista di origine tedesca Jean-Baptiste Stuck, che, nel Settecento, aveva prestato servizio a Napoli presso la contessa di Lemos..$^{20} \mathrm{Nel} 1728$, figura invece la partecipazione di Giuseppe Avitrano, ${ }^{21}$ virtuoso del violino e membro della Cappella Reale alla fine del 1690:

1728 In Marzo, Aprile, Maggio e Giugno

[...] Festa di San Benedetto

Per la musica d. 58

Per li violini particolari d. 6 [...]

Per affitto d'organo d. 1.2.10

Per la sedia al Mastro di Cappella d. 0.2.10

Per assistenza del signor Giuseppe Avitrano d.6 $6^{22}$

La presenza di musici come Domenico Melchiorre e Matteo Sassano, del violinista Giuseppe Avitrano e di violini definiti come «particolari», potrebbe far pensare all'intervento di alcuni musicisti della Cappella Reale - probabilmente i migliori virtuosi - che andavano ad accrescere l'organico della Cappella della Cattedrale nelle occasioni più importanti. Gli stessi virtuosi compaiono anche nel 1725, in occasione della messa cantata del giorno di San Giuseppe:

In febraro e Marzo [...] per la messa cantata il giorno di San Giuseppe [...]

Dato al Conservatorio delli Poveri di Giesu Cristo d. 9

Dato al sig. Aquilano d. 4

Dato al Sig. Battestino d. 4

Dato al sig. Agostiniello per la Messa cantata e trattenimento d. 2

Dato per sei istromenti carlini venti per uno d. 12

Dato a chi a tirato li Mantici d. 0.1.10

Dato al Sig. Francesco Cimino per aver accomodato l'organo fuori e dentro il Monastero d. $2^{23}$

Infine per la festività della Natività di Maria, l'8 settembre, si trova la presenza «delli Figlioli rossi $»^{24}$ e «Figlioli del Conservatorio de Poveri di Sant'Onofrio

19 Asn, MS, f. 2943, app. p. 25.

20 Dominguez 2015, pp. 63-98.

21 Giuseppe Avitrano (Napoli 1670-1756) fu attivo come violinista presso la Cappella Reale dal 1690 in poi. Il suo nome è legato soprattutto alla produzione di sonate a quattro, per tre violini e basso op. 3, pubblicate nel 1713, che si basano sul modello, sviluppato proprio a Napoli alla fine del Seicento da Pietro Marchitelli e Giancarlo Cailò. Cfr. Olivieri 2000.

22 Ass, MS, f. 2946, app. p. 26.

23 Ass, MS, f. 2943, app. p. 25.

24 Asn, MS, f. 2940, app. p. 22. 
vestiti di campagna con voci, ed Istromenti aggionti». ${ }^{25}$ Le carte sottolineano anche la partecipazione dei musici «Taddeo» e «Nicolino» che affiancavano per l'occasione «tre sonatori» durante la messa cantata. ${ }^{26}$

\subsection{Santa Maria Donnalbina}

Le origini del monastero di Donnalbina risalgono al medioevo e svariate sono le ipotesi sul significato del suo nome. Alcuni storici riferiscono che in età ducale la zona era denominata "albinense", altri si collegano invece ad una torre eretta in età romana da un certo Albino. ${ }^{27}$ A queste attribuzioni, nel tempo, è andata ad aggiungersi un'antica leggenda napoletana che vedeva la fondazione del monastero legata alla storia delle tre figlie del Barone di Toraldo chiamate Albina, Regina e Romita. Innamoratesi tutte dello stesso uomo, per non farsi torto a vicenda, decisero di consacrarsi a Dio e di fondare tre monasteri, corrispondenti oggi alle omonime istituzioni. ${ }^{28}$

L'opposizione delle benedettine di Donnalbina alla riforma monastica tridentina fu molto dura. ${ }^{29}$ L'arcivescovo Carafa inviò pertanto, nel 1564, Eugenia Villani, canonichessa di Regina Coeli, per tentare di attuare la riforma con maggiore efficacia. La disciplina prevista dal Concilio però fu accettata ufficialmente solo alla fine del Cinquecento. L'istituzione fu soppressa nel 1808 e le religiose si trasferirono nel monastero di San Giovanni Battista. Il monastero di Donnalbina ospitò inoltre le monache provenienti dai monasteri soppressi di Sant'Agata a Mezzocannone e di Sant'Agnello al Cerriglio, che trasferendosi, nel 1563, portarono con loro reliquie di ogni genere. ${ }^{30}$

Non si riscontrano fino al 1690 informazioni dettagliate circa l'utilizzo della musica nelle liturgie e festività. L'attività musicale sembra essere organizzata dall'organista titolare e maestro di cappella Giovanni Maria Sabino ${ }^{31}$ cui subentra

26 Asn, MS, f. 2943, app. p. 25.

27 Sui cenni relativi alla fondazione dell'istituzione e alla sua storia si vedano: De STEFANo 1560, p. 173; D’Engenio 1624, pp. 499-501; D’Aloe 1883, pp. 512-513; Celano 2001, Giornata IV, pp. 42-45, Galante 1985, Giornata IV, pp. 140-14; Boccadamo 2001, pp. 77-106; VAlerio 2006, pp. 279-285.

28 L'episodio viene raccontato da Matilde Serao nelle sue Leggende napoletane. Cfr. SERAo 1970.

29 Le monache infatti avevano malmenato il delegato dell'arcivescovo Carafa che chiedeva di effettuare la visita al monastero. Cfr. VAlerio 2006, pp. 279-285.

30 Si conservavano: una spina della corona di Cristo, una gruccia di Sant'Agnello, un pezzo di grasso di San Lorenzo, che si liquefaceva nella ricorrenza del martire ed una mammella di Sant'Agata. Le principali feste celebrate presso il monastero sono appunto quelle dei santi di cui il monastero possedeva le reliquie.

31 Giovanni Maria Sabino citato dalle fonti del monastero di Donnalbina è attivo come organista titolare dal 1690 al 1716. Il ‘Giovanni Maria Sabino’ di cui si conoscono cenni biografici e attivi- 
Domenico Altamura. Dal 1708 in poi i registri di esito sono più circostanziati e i riferimenti musicali sono presenti sia nei Conti di Sacristia e nei Conti dei Provisionati, in cui compare la voce del «mastro di cappella» con annesso dettagliato conto.

Pagamenti ai musicisti e agli allievi dei conservatori si evincono anche dalle spese per «complimenti» e «inferte», ovvero regalie in dolciumi o piccole somme di denaro, elargite in occasione di alcune festività o per il 'badessato' di una monaca. ${ }^{32}$

La principale festività celebrata con grande pompa e apparati musicali, era quella del santo patrono dell'ordine: San Benedetto. Nel 1670 infatti, a seguito di un interdetto, la badessa del monastero tentò di trovare un espediente per celebrare la festa di San Benedetto con musica, senza che però essa potesse rappresentare motivo di scandalo. Si proponeva dunque l'utilizzo del solo canto fermo, come si legge nel documento seguente:

Reverendissimo Signore

Intorno al negotio de la musica per gratia de Dio l'ho accomodato in questo modo, [per evitare che possa] succedere qualche scandalo in giorno di San Benedetto dove se dovea celebrare con più devotione $[. .$.$] voleano ubedire con cantare la vespera ferma con tutte l'altre monache; \mathrm{e}$ questo l'ho palesato per il Monasterio è sono rimaste tutte edficate di questa partita $[\ldots] .{ }^{33}$

Negli anni venti del Settecento, la medesima festività veniva celebrata con «la musica dell'Arcivescovado con quattro voci estraordinarie di più per le prime e seconde vespere e messa cantata», ${ }^{34}$ con la partecipazione di Angelo Durante in qualità di maestro di cappella. "Musica nelle prime vesperi messa cantata e trattenimento nel giorno per le seconde vesperi con due voci di più estraordinarie ${ }^{35}$ erano previste per la festa di Sant'Aniello, con cantanti di prim'ordine come 1'Aquilano. ${ }^{36}$ Per la festa della Purificazione e per quella di Sant'Agata, vespri e messa cantata affidata ai «Musici de Poveri di Gesù Cristo», ${ }^{37}$ mentre i «figlioli» dello stesso conservatorio provvedevano al corteo processionale che accompagna-

tà dovrebbe essere nato nel 1588 e morto dopo il 1645, data in cui è attestato il suo ultimo compenso. Probabilmente si tratta di un caso di omonimia: il Sabino attivo a Donnalbina doveva essere semplicemente uno degli esponenti di questa grande famiglia di musicisti napoletani di cui fino ad oggi non si aveva notizia. Altrimenti andrebbero riviste le date di nascita e di morte del celebre Giovanni Maria Sabino attivo come compositore, musicista e insegnante in diverse istituzioni napoletane. Si veda la voce Giovanni Maria Sabino curata da Dinko Fabris presente nel Grove 2001.

32 Inteso quale giorno festivo, il badessato corrispondeva all'inizio del servizio di badessa di una monaca.

33 Asdn, Vicario, 240 D, app. pp. 72-73. Lettera inviata dalla badessa del monastero Agata Caprile al vicario delle monache Don Antonio Tango.

34 Ass, MS, f. 3246, app. p. 57.

35 Asn, MS, f. 3246, app. p. 58.

36 Ass, MS, f. 3249, app. p. 61.

37 Asn, MS, f. 3249, app. p. 61. 
va la statua di Sant'Agata. Ancora si trovano note di spese per la musica eseguita quasi sempre dalla Cappella della Cattedrale, in occasione del Natale, Settimana Santa, Madonna dei Sette Dolori, Immacolata, Capodanno, Natività di San Giovanni Battista, Visitazione della Beata Vergine Maria, Santa Maria Maddalena, San Giacomo e Sant'Anna.

Particolarmente nutrita risulta essere la festa di San Lorenzo di cui si riporta un'esatta cronaca di tutte le voci di spesa occorse per l'anno 1762:

Per la festa di s. Lorenzo

Per la processione d. 4

A 40 padri di S. Maria La Nuova d.4

Al Conservatorio d. 3

A 8 sacerdoti per l'aste del Pallio d. 0.80

Alli sonatori della banda d. 2.40

A 4 facchini che portano la varcha della statua d. 0.80

Alli clerici ordinarij della chiesa, e conversa per la detta festa e per quelle dell'Assunta d. 4.50

All'organista d. 1

Alli due preti aver assistito alle due vesperi e messa ponteficale d. 140

Al maestro di cerimonie della cattedrale d. 0.40

A due clerici della medesima d. 0.50

Al clerico straordinario d. 0.50

A 18 clerici straordinarij ch'anno assistito alla messa piana ed in sacrestia d.1.85

Alli musici da voci ed strumento per tre servizij, vespero, messa cantata, trattenimento e secondo vespero si sono pagato videlicet d. 56.50

Alla cappella della cattedrale [ $\mathrm{manca}$ ]

Alle voci aggiunte ed istrumenti ed organiste [...] Per tre voci soprani d. 9

Al tenore d. 3

A 8 violini d. 12

Al Boe con due corni d. 4.50

Alla violoncella e contro basso d. 3

All'organista d. $2^{38}$

Da citare ancora la presenza di spese per «far accomodare il salterio $»^{39}$ e la costruzione di due organi. Il primo ad opera di Carmine Scoppa nel 1700 e un altro costruito da Fabrizio Cimino nel 1731.40

\subsection{Santa Maria Donna Romita}

Il monastero venne fondato nell'VIII secolo da alcune monache fuggite da Costantinopoli a seguito delle persecuzioni iconoclaste. La primitiva intitolazione era Santa Maria a Percejo di Costantinopoli o delle Donne Romite di Costantino- 
poli, ${ }^{41}$ per contrazione, divenne «Donna Romita». Secondo lo storico Bartolomeo Capasso, invece, la denominazione deriverebbe dal nome della famiglia Aromata che fondò il monastero prima dell'anno $1025 .{ }^{42}$

Il monastero ospitava fanciulle provenienti dalle famiglie Pappacoda, Caracciolo, Minutolo, Mormile, grazie alle quali intratteneva numerosi rapporti con altre istituzioni cittadine. Che il monastero fosse sede di cerimonie e che fosse attivo musicalmente, se ha testimonianza già dal 1582 , data in cui i sacerdoti della vicina chiesa di Sant'Angelo a Nido lamentavano che le monache facessero musica fino a tarda ora con le porte aperte in occasione dell'Assunta e della festività di Sant'Antonio Abate:

Noi sacristano et preti di Sant'Angelo a Nido facemo fede come nel di di nostra donna di agusto passato nel monastero delle donne monache di Santa Maria donna Aromata [sic] si fa musica di voci con la porta aperta et in quella entravano molte persone a prendere l'indulgentia in detta ecclesia, et anco nel di di Santo Antonio di gennaro molte volte hanno fatto festa con la porta aperta et questo, e la verità et ne fa plena et indubitata fede. Datum Neapolis die 4 januarij $1578 .{ }^{43}$

Probabilmente le monache dovevano cimentarsi anche in pièces teatrali, se qualche anno più tardi, nel 1599, un ordine dell'arcivescovo proibiva l'esecuzione di opere profane rappresentate delle stesse religiose, per evitare alle sedicenti attrici una mancata assoluzione:

Si ordina da parte dell'Eccellente [...] N. Sig. Curtio Palumbo- vicario delle monache, et della Corte Arcivescovile di Napoli In civilibus luogotenente generale, in virtù di Santa ubedientia della Madre Abbadessa Priora, è Decana del Monastero di Santa Maria Donna Romita, che non vogliano far recitare un'opera profana, è meno honesta dalle monache dentro del loro Monastero, avertendole che si ciò permetteranno non potranno essere assolute dal loro confessore e poi che celo reserviamo à Noi espressamente questi peccato di trasgressione, tanto si permetteranno, quanto si espressamente non le prohiberanno, e così anco ci reserviamo l'assolutione per quelle che recitavanno, di modo tale che non possano essere assolute, ordinandoli di più che vogliano di ciò far avisare le monache presente acciò non possano allegar causa d'ignorantia, è questo oltre l'altre pene à Noi arbitrarie, anco per la privazione dell'officio per l'Abbadessa. Dato in Napoli li 14 mensis Aprile 1599.44

La festività più rilevante è quella di San Giovanni Battista. Durante le due date legate al Santo - il 24 giugno in cui si celebrava la nascita e il 29 agosto la de-

41 Il monastero venne soppresso nel 1808, le monache tuttavia rimasero in esso fino al 1824 per poi aggregarsi al monastero di San Gregorio Armeno. Durante la seconda guerra mondiale il complesso venne danneggiato, ma nel dopoguerra fu restaurato e riportato all'originale stile rinascimentale. Oggi ospita alcuni dipartimenti dell'Università di Napoli Federico II, cfr.: DE Stefano 1560, pp. 175; Celano 2001, Giornata III, pp. 648-651; Galante 1985, Giornata VI, pp. 224, 227; CAPASSO 1882, pp. 874-875; Si veda anche: VALERIO 2006, pp. 271-277; BoCCADAMO 2001, pp. 77-106.

42 Sulla leggenda legata al nome di Donna Romita si veda anche quanto scritto nel paragrafo precedente riguardo la fondazione del monastero di Donnalbina, p. 126.

43 Asdn, Vicario, 275 D, app. p. 83.

44 AsDn, Vicario, 275 D, app. p. 83. 
collazione - aveva infatti aveva luogo lo scioglimento del sangue. ${ }^{45}$ Queste due occasioni solenni prevedevano spesso l'intervento dei viceré, così come testimonia un interdetto del 1695 dell'allora arcivescovo di Napoli, il cardinale Giacomo Cantelmo Stuart, avendo le monache chiamato i musicisti della Cappella Reale per celebrare la visita della Viceregina: ${ }^{46}$

La sera antecedente a detto giorno fù la Signora Viceregina nella sudetta Chiesa di Donna Romita a visitare il glorioso sangue di San Giovanni Battista e per la festa che si celebrava in detta con l'ottava per causa del possesso come padrone di Napoli conforme si è detto di sopra coll'occasione [...] vi stava in detta Chiesa la musica di palazzo, cosa che dispiacque molto a Sua Eminenza non per la visita di detta Signora, ma per la musica, onde nella stessa sera che fù il sabbato antecedente li 25 giugno pose l'interdetto à detta Chiesa. ${ }^{47}$

Nonostante la possibilità di ricevere ulteriori sanzioni, la tradizionale visita dei viceré è attestata anche nel Settecento, con la partecipazione di rinomati cantanti come Domenico Gizzi, musico della Real Cappella: ${ }^{48}$

Al 3 settembre detto 1729 pagato per la Musica in occasione che il signor Vice Re e Vice Regina e suoi signori figli vennero a vedere la Reliquia di San Giovanni Battista alle sottoscritte persone sono cioè:

Pagato al Mastro di Cappella dell'Arcivescovado d.1

Pagato per numero 4 Istrumenti d.2

Pagato alla prima voce Gizij d.1.2.10

Pagato alla seconda voce Don Diego del Vasto d. $1[\ldots]^{49}$

La «Gazzetta di Napoli» menziona invece la presenza del celebre 'Matteuccio' in occasione della festa di San Giovanni del 1717: «ritrovandosi la chiesa riccamente apparata con quattro cori di scelti musici, con essersi celebrate prime e seconde vespere [sic], e la mattina vi fu a cantare il renomato marchese Matteo Sassano [...]». ${ }^{50}$

Si registrano inoltre spese che ci informano del compenso dovuto agli organisti attivi presso l'istituzione. Preposti a tale servizio erano l'organista Tommaso Tango e Filippo Prota, mentre anonimi organari erano stipendiati «per aver accordato 3 volte l'organo in occasione di cantare le Figlie d.1.1»..$^{51}$ Purtroppo i documenti tacciono informazioni aggiuntive sulle 'cantanti' di cui fanno menzione.

45 Sulla festa di San Giovanni Battista si veda anche cap. III, p. 62.

46 Sull'impiego della Cappella Reale al posto di quella dell'arcivescovo si rimanda al cap. I, p. 43.

47 Asdn, Cerimonieri,Vol. VIII, app. p. 84.

48 Domenico Gizzi (1687-1758) fu un celebre castrato attivo a Napoli in diverse istituzioni come la Cappella Reale e la Cappella del Tesoro di San Gennaro. Partecipò a numerose opere di autori quali A. Scarlatti, Vinci, Leo, Porpora. Cfr. Di Giacomo 1920; Prota-Giurleo 1952; СотtiCelli/ Maione 1993.

49 Ass, MS, f. 4009, app. p. 78. Il documento è citato anche in BaCCiAgaluppi 2010, p. 71.

50 GDN, 31 agosto 1717, app. p. 85.

51 Asn, MS, f. 4012, app. pp. 81-82. 


\subsection{San Gregorio Armeno}

Il monastero benedettino di San Gregorio Armeno fu un vero e proprio centro della vita musicale della città di Napoli. Secondo la tradizione, il monastero era sorto nell'VIII secolo sui resti del tempio di Cerere Attica, fondato da un gruppo di monache dell'ordine di San Basilio fuggite da Costantinopoli con le reliquie di San Gregorio, vescovo d'Armenia. La chiesa e il monastero, tuttora attivi, sono monumenti di straordinaria bellezza, decorati da opere di Luca Giordano, arricchiti da stucchi e marmi da imponenti organi e da due cantorie in legno intagliato.

Il monastero ospitava le figlie delle migliori famiglie napoletane, basti pensare che per entrare in San Gregorio Armeno era necessaria una dote di 1500 ducati. ${ }^{52}$ Durante il periodo di noviziato veniva corrisposta una retta per il mantenimento dell'educanda e a volte, assieme alla dote, veniva versato dalle famiglie anche un vitalizio affinché la professa potesse avere una somma da spendere per le proprie necessità. I decreti seguiti al termine del Concilio di Trento, cambiarono irrimediabilmente lo stile di vita delle monache anche a San Gregorio Armeno. Innanzitutto furono necessari alcuni interventi architettonici per adeguare l'edificio alle norme prescritte, ${ }^{53}$ e progressivamente, la libertà delle religiose provenienti dal più alto patriziato napoletano, subì una incredibile restrizione, di cui è testimonianza diretta il famoso compendio scritto da Fulvia Caracciolo.$^{54} \mathrm{I}$ primi accenni di un cambiamento, si erano già avuti nel 1554 attraverso la restrizione della libera uscita dal monastero e nel 1561 con la costruzione del muro di clausura, ma è solo con il cardinale Alfonso Carafa che si ebbero gli interventi più incisivi che suscitarono le reazioni delle monache. Prima del Concilio, le religiose vivevano ciascuna in un appartamento che affacciava su di un cortile interno, provvisto di tutte le comodità necessarie alla comunità: mulino, pozzo, cisterne, cantine, magazzini. Carafa procedette a numerose riforme architettoniche e ad unire diversi monasteri. San Gregorio Armeno vide infatti l'arrivo delle monache dal soppresso monastero di Sant'Arcangelo a Baiano, Donna Romita e più tardi, nel 1864 le monache di Santa Patrizia. Questo accorpamento di più istituzioni comportò l'arrivo di una numerosa serie di reliquie. Le benedettine,

52 Sul valore del ducato si veda quanto detto a p. 15.

53 L'antico complesso fu stravolto e modificato, vennero innalzate mura e inferriate. La chiesa fu spostata al centro del monastero. Cfr. VALERIO 2006, pp. 193-201. Attualmente il monastero è occupato dalle Suore Crocifisse Adoratrici dell'Eucaristia. Cfr. Caracciolo 1864; Celano 2001, Giornata III, pp. 756-760; D’Engenio 1624, pp. 341-363, Galante 1872, Giornata V, pp. 200-204; VAlerio 2006, pp. 193-201; Spinosa/Pinto/Valerio 2013.

54 Si veda quanto detto al cap. I, pp. 33-35 e nella nota 9. Sulla vita che si conduceva a San Gregorio Armeno bisogna citare il libro di Enrichetta Caracciolo del 1864 Misteri del chiostro napoletano, nel quale la suora narrava la propria vita da reclusa, denunciando la vita oppressiva del monastero. Cfr. CARACCIOLO 1864. 
a fine Cinquecento, si trovavano dunque ad essere custodi de: «la testa di Santo Stefano, la testa di San Biaso coverte d'argento, parte del legno della croce di Christo posta parimente in argento, il braccio di San Lorenzo, il braccio di San Panataleone coverti d'argento, il sangue di Santo Stefano, la catena del nostro San Gregorio Armeno e li scorriati, ${ }^{55}$ con li quali l'istesso santo fu battuto, dalli quali e dalla catena ogni giorno si veggono per la Dio grazia stupendi miracoli». ${ }^{56}$ Si celebravano quindi con «sollenne religiosa pompa»:

[...] cinque giorni festivi con musica e panegirici a 3. Febraro la festività di S. Biaggio Vescovo e Martire a 21. marzo la festività di S. Benedetto a 29 ag.o la festività della decollazione di S. Gio: Batta a 21 sett.e la festa di S. Matteo Apostolo a p.mo ott.re la festa di S. Gregorio Armeno titolare del Mon.rio. S'espone il venerabile augustissimo Sacramento, senza musica, una colla debita proprietà della novena della nascita del Sig.re, tutti li venerdi di Marzo, l'ultimi tre giorni di Carnevale, e tutto l'ottavario del Corpus D.ni. ${ }^{57}$

Inoltre oggetto di grande culto era la reliquia del sangue di San Giovanni Battista soggetto miracolosamente a liquefazione ogni anno il 29 agosto.$^{58}$ In occasione della festa di San Giovanni Battista, sono presenti numerosi pagamenti ai musicisti della Cattedrale e ai maestri di cappella che la governarono fra Sei e Settecento come Pietro Bartilotti e Andrea Amendola. Ai musicisti della Cattedrale si aggiungevano «musici estraordinari», «voci aggionte»e «instromenti per lo trattenimento havuto in chiesa». ${ }^{59}$ Per la festa di San Biagio invece:

Venivano i maestri de la chiesa di detto glorioso santo, come hoggi dì anco vengono, e con solenne processione con trenta o quaranta torchi accesi, accompagnati da diverse sorte di suoni e da infinito numero di genti, entravan nella nostra chiesa et andavano insino all'altare maggiore e dalla nostra abbadessa et anco dalle sacristane li era consegnata la testa di detto glorioso San Biaso per spazio di due hore e si mandavano dieci o dodici preti di casa acciò l'accompagnassero et guardassero, come cosa propria del monistero et la riponevan sopra l'altare di San Gennarello, finché fosse fornita la messa solenne; fra questo tempo dimoravano le nostre genti, acciò chè, finita la messa, la riportassero con la medesima processione $[\ldots]^{60}$

Della festività di San Gregorio, ci informa la «Gazzetta di Napoli»:

Essendo stato acclamato, come si scrisse, per nuovo padrone di questa città l'invitto martire di Cristo S. Gregorio vescovo d'Armenia, mercordì [30 settembre] dalle signore monache del suo monastero, ne fu celebrata la festa di detta padronanza, con occasione ch'era il giorno festivo di detto santo. Martedì mattina fu portata la statua con la reliquia dal Tesoro con numerosa proces-

55 Il termine dialettale indica una sorta di frusta, scudiscio.

56 CARRINo 2013, pp. 113-114.

57 Spinosa/Pinto/Valerio 2013, Appendice A, p. 803.

58 A fine Cinquecento le monache di San Gregorio Armeno disputarono a lungo con le benedettine di San Potito per il possesso della reliquia di San Giovanni Battista. Erano in gioco non solo questioni di prestigio, ma anche introiti economici che potevano derivare dalla venerazione delle reliquie da parte dei fedeli.

59 Asn, MS, f. 3356, app. p. 91.

60 Il passo è tratto dalle memorie di Fulvia Caracciolo. Cfr. CARrino 2013, p. 63. 
sione [...] nella sua chiesa, che si vide superbamente apparata di contratagli e ricca d'argenti, che così è stata per tutta l'ottava e con più cori di musica e l'affacciata [sic] della medesima parimente apparata con l'effigie di detto santo padrone, sì come tutta la piazza, in capo della quale vi erano l'archi trionfali, che con esquisiti colori esprimevano diverse imprese del santo e le di lui virtù $\left[\ldots . .{ }^{61}\right.$

Per la festività di San Matteo troviamo informazioni nel 1748, anno in cui viene retribuito il «magnifico Costantino de Ruberto per la musica fatta nella nostra Chiesa», «per il coro dell'Arcivescovado»e «per il trattenimento». ${ }^{62} \mathrm{Il}$ fasto delle differenti celebrazioni, a San Gregorio Armeno così come in altre comunità, era garantito anche dalla presenza dei «figliuoli» dei conservatori. Il conservatorio dei Poveri di Gesù Cristo assicurava la presenza dei propri figlioli per la processione del Battista, oppure per le festività dell'Ascensione, di San Biagio, San Benedetto. In alcune occasioni festive si registra anche la presenza di pagamenti alle sacrestane donna Beatrice di Sangro, donna Eleonora Pignatelli, donna Chiara della Marra, donna Cornelia Milano per «servizi di musica». ${ }^{63}$ Non è chiaro se il loro compito fosse poi quello di distribuire semplicemente le paghe ai musicisti, oppure se anche loro ricoprissero un ruolo nel servizio musicale.

Particolare solennità era riservata anche alle celebrazioni pasquali, così come, ancora una volta, ci informa Fulvia Caracciolo:

Similmente nel giorno delle Palme veniva come hoggi anco viene, il parrocchiano di San Gennarello con pluviale e con dui ministri et altre persone confrati di decta chiesa. Uno dei ministri portava una croce et l'altro una palma di dattoli et, nell'entrare alla porta del nostro monastero, lo parrocchiano pigliava la croce nelle sue mani et inchinandosi fino a terra faceva riverenza ad una nostra croce antichissima [...]. Giunto, poi, alla porta de la chiesa, toccando la croce sua con la nostra, diceva cantando: Benedictus qui venit in nomine Domini, osanna fili David, et donava la palma di dattoli all'abbadessa, la quale li donava alcuni dinari per elemosina con alcune cortesie di zuccaro $[\ldots] .^{64}$

Numerose anche le informazioni relative agli organisti e organari attivi presso l'istituto. ${ }^{65}$ Ricordiamo infatti che San Gregorio Armeno possedeva a fine Settecento ben quattro organi: due costruiti da Tommaso De Martino nel 1737 e nel 1742, uno di Domenico Antonio Rossi del 1769 e infine uno strumento di Francesco Cimino del 1790.

La vocazione musicale del monastero è testimoniata anche da una numerosa serie di riferimenti relativi ai maestri di musica delle educande e monache benedettine. Giuseppe de Magistris, ad esempio, era stipendiato nel dicembre del 1778

61 GDN, 6 ottobre 1676 (1), app. p. 108.

62 Asn, MS, f. 3366, app. p. 96.

63 Cfr. Asbn, BPO 982 8/X/1728, app. p. 105; BPO 1013 11/X/1730; BPO 1023 12/X/1731, app. p. 106.

64 CARrino 2013, p. 64.

65 Per i nominativi dei musicisti si rimanda all'appendice documentaria e alla tabella 11 a p. 221. 
«per la lezzione di canto alla signora educanda» ${ }^{66} \mathrm{e}$ «per la lezione di cembalo». ${ }^{67}$ Non mancano le licenze concesse dalle autorità religiose concernenti l'ingresso di maestri di musica, purché gli insegnanti avessero un'età adeguata:

Si concede licenza al Signor Don Giuseppe Arena, Mastro di Cappella, di està [sic] sopra à sissanta, di ammaestrare di canto le Signore Educande di San Gregorio Armeno. ${ }^{68}$

Inoltre bisognava assicurarsi che l'insegnamento fosse in linea con gli orari e le prescrizioni della vita claustrale:

Per i Maestri di Musica che possono dare rispettivamente lezioni alle educande e monache del Venerabile Monisterio di San Gregorio Armeno per mesi sei dalle solite grate, e nel ore che non s'impedisce la recitazione del Divino Officio, e coll'assistenza della Reverenda Signora Badessa o d'altra Religiosa dalla Medesima destinanda, e coll'osservanza delle solite condizioni, e rispetto alli Maestri di Musica che le lezioni non siano di arie profane, ma di cose appartenevoli a Divini Officij e nelle ore che non s'impediscono gli Esercizij della Religione. Napoli 17 Agosto 1799. Maria Giuseppe de Medici Abbadessa. ${ }^{69}$

Le lezioni di musica dovevano inoltre svolgersi alle grate e in presenza di testimoni o di altre persone deputate a garantire l'innocenza di ogni incontro:

Si concede licenza al Maestro di Cappella, Don Domenico Scitta, di venire nella grata del Monastero di San Gregorio, per dar lezione di Musica, alle figlie del Principe di Pettoranello. E saranno nella lezione assistite dalle di loro zie. ${ }^{70}$

Era anche necessario che le licenze concesse venissero rinnovate di anno in anno. Si evince inoltre che i maestri deputati all'insegnamento della musica potevano variare e probabilmente essere scelti dalle stesse monache o dalle loro famiglie. Affiorano così nomi del tutto obliati chiamati a servire l'educazione delle figlie della nobiltà: il «Signor Don Michele Perla» era «Maestro di Cappella per le Figlie di Buccino e per la Figlia di Fringuiano [...]», mentre «Il Signor Giuseppe Elia» era incaricato di «dar lezione di Musica alla Figlia del duca di Monteleone», il «Signor don Giovanni Battista Barbatiello per dar lezzione di Musica a donna Mariangela Caracciolo di Melissano a donna Chiarina di Tocco e donna Maria Antonia Caracciolo di Marano», ${ }^{71}$ "Giacomo Monopoli per la lezione di Musica alle Figlie del Principe di Scilla». «Don Gaetano Barbatiello» era incaricato «per la lezione di Musica alla Signora Donna Giustiniana Caracciolo di Melizzano a Donna Chiarina di Tocco, Donna Maria Antonia Caracciolo, Donna Maria Camilla Galiota», «il signor Angelo Vinditti» provvedeva invece alla «lezione di musica alle Figlie del Principe di Scilla», «Don Giuseppe Elia»

66 Asn, MS, f. 3395, app. p. 99.

67 Asn, MS, f. 3395, app. p. 100.

68 Asdn, Vicario, 170 D, app. p. 101.

69 AsDn, Vicario, 172 D, app. p. 102.

70 Aspn, Vicario, 174 D, app. p. 103.

71 Asdn, Vicario, 172 D, app. p. 103. 
era chiamato «per dar lezione di Musica alla Figlia del Signor Duca di Monteleone Donna Costanza Pignatelli»e infine «Don Filippo Cinque per dar lezione di musica alle figlie di Montemiletto»..$^{72}$

Un documento del 1808 testimonia che lo scopo didattico era finalizzato anche alla pubblica esecuzione ad opera delle stesse religiose. Don Giacomo Tritto ${ }^{73}$ veniva ricompensato con 4 ducati «per la messa cantata insegnata alle Signore Moniche» e nello stesso documento si fa menzione del compenso dovuto alle religiose ovvero «22 Stracchini di Sorbetta per le Signore che hanno cantato la messa figorata per San Giovanni $[\ldots] \gg .^{74}$

Il ricchissimo monastero è l'unico ad oggi a custodire un fondo musicale organizzato e preservato nel corso dei secoli proprio dalle monache benedettine. ${ }^{75}$ Sono presenti in esso numerosi manoscritti databili fra la metà del Settecento e fine Ottocento. Si tratta soprattutto di copie di composizioni sacre e profane, testi didattici, liturgici, letteratura per tastiera, che lasciano presupporre il loro utilizzo in una dimensione ricreativa spesso testimoniata dalle spese sostenute dal monastero per i trattenimenti, ricreazioni etc. Gli autori di cui si ritrovano le composizioni sono Girolamo Abos, Giuseppe Avitrano, Gennaro Manna, Nicola Sabatino, al fianco di stranieri come Schuster, Johann Christian Bach, Mysliveček, Handel.

I manoscritti recano spesso i nominativi delle religiose a cui erano destinati. Caterina Pignatelli, Maddalena Sersale, Maria Anna Filangieri, risultano essere dedicatarie di numerose partiture. A Caterina Pignatelli vengono dedicate alcune parti del Confitebor di Pergolesi, così come la copia del Laudate pueri, e alcune parti parti dello Stabat. Marianna Filangieri, ${ }^{76}$ fu invece destinataria di alcune composizioni di Giovanni Paisiello. Vi sono infine conservati anche solfeggi per soprano di Giuseppe Aprile, Vincenzo Righini, Giacomo Insanguine, Cesare Giannoni, Gerolamo Crescentini etc.

Se da un lato il fondo di San Gregorio rappresenta un caso unico e di fondamentale importanza per comprendere quanto l'utilizzo della musica all'inter-

AsDN, Vicario, 174 D, app. p. 103.

73 Giacomo Tritto fu allievo di Nicola Fago, Girolamo Abos e Pasquale Cafaro, nel 1799 fu nominato primo maestro del Conservatorio della Pietà de' Turchini. Nel dicembre del 1806, dopo l'unificazione di tutti i conservatori napoletani, entrò nella direzione del Real Collegio di Musica assieme a Giovanni Paisiello e Fedele Fenaroli. Conservò la prestigiosa carica fino al 1813. Pur vantando anche un'attività come operista, Tritto viene ricordato soprattutto come insegnante. Fu autore infatti di due opere didattiche Partimenti e regole generali per conoscere qual numerica dar si deve ai vari movimenti del basso, Milano, 1816 e Scuola di contrappunto, ossia Teorica musicale, Milano, 1816.

74 Cfr. Bacciagaluppi 2010, p. 72.

75 Del fondo musicale di San Gregorio Armeno non esiste ad oggi un catalogo, esso tuttavia ha una sua sigla RISM I-Ng. Sul fondo musicale del monastero si rimanda a BonsANTE 2013, pp. 267-281. 
no dei chiostri fosse assolutamente comune, bisogna anche tener presente che alcuni di questi manoscritti potrebbero semplicemente essere stati destinati alle nobildonne ancora prima di entrare nel monastero. Potrebbe quindi trattarsi di partiture portate in dote, assieme agli effetti personali. I beni appartenuti alle monache divenivano poi, alla loro morte, di proprietà dell'istituzione. Non è dunque certo che tutta questa musica venisse eseguita realmente dalle benedettine. Alcune partiture, come le arie profane e le pagine operistiche in voga nei teatri cittadini, potrebbero essere state semplicemente custodite, testimoniando dunque la moda del collezionismo che dilagava fra le giovani di alta società a fine XVIII secolo.

\subsection{Santa Patrizia}

Il monastero dapprima consacrato ai Ss. Nicandro, Marciano e Patrizia, si intitolò alla sola Santa Patrizia a partire dal XIII sec., periodo nel quale si affermò il culto della Santa a Napoli. Il monastero consolidò nel tempo la sua posizione economica grazie a lasciti e donazioni che lo fecero divenire, in età angioina, uno dei cenobi di maggiore prestigio sociale e politico. ${ }^{77}$ Le monache infatti appartenevano quasi tutte ai seggi nobiliari di Nido e Capuana. La difesa della propria libertà, autonomia e potere politico indusse infatti le monache ad opporsi tenacemente alle riforme avviate nel Cinquecento. Nel 1625, le benedettine ottennero la concessione che Santa Patrizia fosse eletta patrona di Napoli e pertanto ogni anno una statua d'argento,$^{78}$ contenente una reliquia della Santa, veniva condotta in processione dalla Cattedrale al monastero, dove rimaneva fino al termine delle cerimonie religiose.

La musica per la festa di Santa Patrizia, prevedeva l'assistenza dei «Figli de Poveri di Giesù Christo» ${ }^{79} \mathrm{e}$ con molta probabilità, l'intervento della Cappella della Cattedrale, così come testimoniano alcune polizze del Banco di Napoli. Esse riferiscono di compensi al maestro di cappella Andrea Amendola, a capo della compagine dell'arcivescovo:

Al Monasterio di Santa Patrizia d. ventidue, e con firma di Donna Maria Emanuele Orsini Abbadessa a Don Andrea Amendola, dette sono per la Musica, da esso fatta nella Loro Chiesa, nelli giorni de 25 e 26 agosto 1726, per la Festività della loro Gloriosa Santa Patrizia e resta sodisfatto e per esso ad Ignatio Amendola. ${ }^{80}$

77 Sulle vicende storiche del monastero si veda: De Stefano 1560, pp. 172; D’Engenio 1624, pp. 178-180; Celano 2001, Giornata II, pp. 81-83; Galante 1985, Giornata III, pp. 84-85. Si veda anche: VAlerio 2006, pp. 313-319; FACCHIANo 1992.

78 La statua d'argento era stata donata con una solenne cerimonia la badessa Agnese Maria Carafa consegnò il 6 aprile 1642 alla cappella del Tesoro di San Gennaro.

79 AsBn, BPI 1577 29/V/1728, app. p. 111.

80 Asbn, BPO 967 17/IX/1727, app. p. 111. 
Durante la festività avveniva lo scioglimento del sangue della Santa, in virtù del quale le monache erano solite ricevere la visita dei viceré:

[...] il giorno appresso [26 agosto] furono [il viceré e la viceregina] a venerare le reliquie di

S. Patrizia nella sua chiesa delle monache benedettine, ove con $[\ldots]$ scelta musica se ne celebrava la festività $[\ldots] .^{81}$

Le spese ingenti per le fastose cerimonie contribuirono, accanto a un alto tenore di vita, a indebolire le finanze del monastero. Le monache, non si risparmiavano neanche nel festeggiare eventi civili o ricorrenze dell' aristocrazia. Nel 1734 celebrarono infatti solennemente l'ingresso di Carlo di Borbone a Napoli. ${ }^{82}$

Il monastero venne soppresso definitivamente nel 1861, le monache rimanenti si trasferirono a San Gregorio Armeno, portando con sé il corpo di Santa Patrizia e i reliquiari d'argento. Tutt'oggi è vivo il culto della Santa con lo scioglimento del sangue.

\subsection{San Gaudioso}

Il complesso di San Gaudioso fu fondato nel V secolo da Settimio Celio Gaudioso, vescovo africano naufragato a Napoli e qui rimasto a vivere. Tre secoli dopo cadde in rovina e fu acquistato dalle monache benedettine che lo riadattarono. L'espansione vera e propria del complesso avvenne però tra il XVI e il XVII secolo. ${ }^{83}$

Il monastero, dalle cronache coeve, risultava essere uno dei più indisciplinati $\mathrm{e}$ dei meno propensi ad accettare gli ordini della riforma. Così come per San Gregorio Armeno e Santa Patrizia, anche San Gaudioso ospitava figlie provenienti dalle famiglie dei Seggi di Nido e Capuana.

Purtroppo la documentazione relativa a San Gaudioso è molto lacunosa. Pochissimi riferimenti ad una pratica musicale che probabilmente aveva comunque fatto parte della vita delle monache.

L'unico musicista citato dalle fonti è l'organista Francesco Galletti, stipendiato dalle monache fra 1734 e 1735. Qualche indicazione è relativa alla festività di San

81 GDN, 30 agosto 1712 (1), app. p. 112.

82 Si veda cap. III, p. 73 e GDN, 25 maggio 1734 (5), app. p. 112.

83 Il restauro seicentesco fu opera di Cosimo Fanzago. Danneggiato nel 1799, dal 1807 al 1819 l'edificio fu sede dell'Osservatorio Astronomico e successivamente dell'Osservatorio di Marina. Il monastero fu demolito completamente tra il 1920 e il 1950 per realizzarvi le cliniche universitarie. Nelle demolizioni andarono perdute testimonianze pittoriche di artisti come Battistello Caracciolo, Francesco Solimena e Luca Giordano. Cfr. De Stefano 1560, p. 176; D’Engenio 1624, pp. 195, 197-200, 202; Celano 2001, Giornata II, pp. 64-66; Galante 1985, Giornata III, p. 91; Filangieri 1888. Si veda anche: Valerio 2006, pp. 175-181. 
Gaudioso, per la quale dovevano celebrarsi «li primi vesperi e messa cantata [...] giusta l'antico solito». ${ }^{84}$

Alle scarse testimonianze sulle festività religiose, si contrappone invece la copiosità di indicazioni circa le sontuose monacazioni avvenute nel monastero: «Musica delle voci e istrumenti più scelti $[\ldots]$... erano previsti in occasione che «prese l'abito di monica nel monistero di S. Gaudioso, per mano dell'eminentissimo nostro arcivescovo Pignatelli, D. Virginea [sic] Colonna, figlia del principe di Sonnino» ${ }^{85}$ Nicola Fago interveniva con la Cappella dell'Arcivescovado assieme con «tutte l'altre voci, et instrumenti, et anco pagare il fitto delle due organi» per la monacazione della figlia del marchese del Tufo. ${ }^{86} \mathrm{Il}$ «nuovo soprano» Angelo Monticelli, ${ }^{87}$ «qui ancora non inteso» come precisava la «Gazzetta di Napoli», presenziò invece nell'aprile del 1735 alla professione religiosa della figlia del principe di Marano. Nell'occasione «si vide la chiesa assai nobilmente apparata, coll'altar maggiore meravigliosamente concertato dal celebre ingegnere D. Domenico-Antonio Vaccaro» ${ }^{88}$

\section{Agostiniane}

\subsection{Santa Maria Egiziaca Maggiore a Forcella e Santa Maria Egiziaca a Pizzofalcone}

La chiesa di Santa Maria Egiziaca venne fondata nel 1342 per volere della regina Sancia assieme ad un monastero destinato ad accogliere le cosiddette donne traviate, essendosi rivelato insufficiente il monastero della Maddalena, ${ }^{89}$ anch'esso fondato dalla pia moglie di Roberto d'Angiò. Sancia volle dedicare chiesa e monastero a Santa Maria Egiziaca che dopo essere stata 'peccatrice' come la Maddalena, aveva poi trascorso quarantasette anni di vita eremitica nel deserto d'Egitto. ${ }^{90}$ Per volere della regina, al monastero dell'Egiziaca, venne assegnata una consistente rendita annuale e venne stabilito che le monache vivessero sotto la regola di Sant'Agostino. Col tempo il monastero perse gradualmente il suo

86 AsBN, BSS 1231 9/IV/1731, app. pp. 113-114.

87 Angelo Maria Monticelli, sopranista milanese, era giunto a Napoli da Roma per esibirsi nelle opere della stagione 1735-1736. cfr. Magaudda/Costantini 2009, p. 62.

88 GDN, 26 aprile 1735 (1), app. p. 114.

89 Cfr. pp. 157-159.

90 Santa Maria Egiziaca vissuta nel IV sec. d.C., era stata infatti una prostituta prima di divenire monaca ed eremita. Il culto della santa, venerata come la protettrice delle prostitute pentite, si diffuse in occidente e nell'oriente cristiano. 
carattere assistenziale e ospitò le figlie della nobiltà cittadina, ingrandendo considerevolmente il suo patrimonio. ${ }^{91}$

La documentazione archivistica risulta essere lacunosa, tuttavia i pochi registri contabili riportano discrete indicazioni circa l'attività musicale dell'istituto.

La festa di Sant'Agostino prevedeva «musica delle 2 vespri e messa cantata del Conservatorio ${ }^{92}$ con l'ausilio delle flotte dei conservatori maschili, il Santo Natale prevedeva l'utilizzo delle caratteristiche «sampogne», ${ }^{93}$ mentre le sontuose monacazioni e l'ottavario del Corpus Domini venivano celebrati con «la musica a più cori de' più rinomati virtuosi di questa capitale». ${ }^{94}$ I maggiori fasti erano però riservati alla solennità della santa titolare, che vantava ben due celebrazioni l'anno. Il 14 settembre, in occasione della sua conversione, e il 26 aprile vera e propria festa. Le cronache ci informano che per l'occasione si predisponeva un «capriccioso e ricco apparato», e che alla festa vi era un «infinito concorso di dame e cavalieri $\rangle^{95} \mathrm{e}$ «più cori di scelta musica». ${ }^{96}$ I suddetti fasti trovano riscontro anche in un documento del 1749 che riporta l'organico strumentale intervenuto alla festa della patrona e che si propone di seguito:

Musica di Santa Maria Egiziaca 26, 27 Aprile 1749

Bilancione con mottetto d.3.2.10

Pascalino con mottetto d.3.2.10

Don Giovanni Battista Sericci con mottetto d.3.2.10

Don Francesco Orsi con mottetto d.3.2.10

Palumbo con mottetto d.3.2.10

Amadoro per un mottetto d.3

Violini

Meola d.1.1.

Moro d.1.1

Capone d.1.1.

Pirozi d.1.1

Almonte d.1.1

Muschese d.1.1

Camardella d.1.1

Clerici d.1.1

Orgetano d.1.1

Beneduce per il Signor Vicario d.1.1

91 De Stefano 1560, p. 195; D’Engenio 1624, pp. 425-426. 429-430; Celano 2001, Giornata III, pp. 825-829; Galante 1985, Giornata VII, pp. 259-260. Si veda anche: Valerio 2006, pp. 297-301.

92 Asn, MS, f. 5146, app. p. 115.

93 Asn, MS, f. 5146, app. p. 116.

94 GDN, 11 giugno 1720, app. p. 119.

95 GDN, 3 maggio 1718 (3), app. p. 119.

96 GDN,12 maggio 1722 (3), app. p. 119. 


\author{
Trombe \\ Caglione d.1.1 \\ Marotta d.1.1 \\ Violoncello \\ Prota d.1.1 \\ Controbassi \\ Teodoro d.1.1 \\ Blando d.1.1 \\ Organista \\ Ambrisi d.1.2.10 \\ Maestro di Cappella \\ Rossi d.3.2.1097
}

Nel 1639, alcune monache dell'Egiziaca, vollero allontanarsi dal monastero per vivere secondo una regola più rigorosa. Fondarono dunque un nuovo istituto, anch'esso dedicato all'Egiziaca, nella zona collinare denominata Pizzofalcone. L'antico e originario istituto assunse allora la denominazione di Santa Maria Egiziaca Maggiore, detta anche popolarmente Santa Maria Egiziaca all'Olmo ${ }^{98}$ per distinguersi dal nuovo complesso denominato 'Egiziaca a Pizzofalcone'. ${ }^{99}$

I maestri di cappella dell'Egiziaca a Pizzofalcone furono Gaetano Pisano, Nicola Pisano e soprattutto Francesco Feo, uno dei più illustri rappresentanti della scuola napoletana settecentesca, in servizio all'Egiziaca tra 1735 e 1753. Egli provvedeva infatti alla musica per la notte di Natale, alla festa dell'Esaltazione della Croce e a vari «servizij di musica con voci ed istrumenti fatti e fatti fare nella Festa del Santo Agostino a 28 Agosto». Feo era affiancato da Lorenzo Planelli, organista titolare dell'istituto, i cui compensi sono regolarmente registrati dal 1739 al 1748 . Non mancano solenni professioni religiose come quella di Donna Livia Grimaldi e sua sorella Placida per la cui celebrazione nel 1720 vi fu «sceltissima musica» con la partecipazione dei virtuosi Matteo Sassano e Francesco Vitale. ${ }^{100}$

La solennità di Santa Maria Egiziaca veniva festeggiata anche nel complesso di Pizzofalcone. La biblioteca oratoriana dei Girolamini custodisce oggi il manoscritto dell'Inno a 5 voci a Santa Maria Egiziaca composto da Gaetano Manna su commissione di Donna Giuseppa d'Aragona nel 1789 per l'occasione festiva da celebrarsi nel monastero di Pizzofalcone.

97 Asn, MS, f. 5156, app. pp. 117-118.

98 Denominata così per il grande albero sotto il quale si raccoglievano i negozianti del luogo.

99 Sulla storia del monastero si vedano: Celano 2001, Giornata V, pp. 586-588; Galante 1985, Giornata XI p. 377; VALERIO 2007, pp. 355-361.

100 GDN, 14 maggio 1720 (3), app. p. 130. 


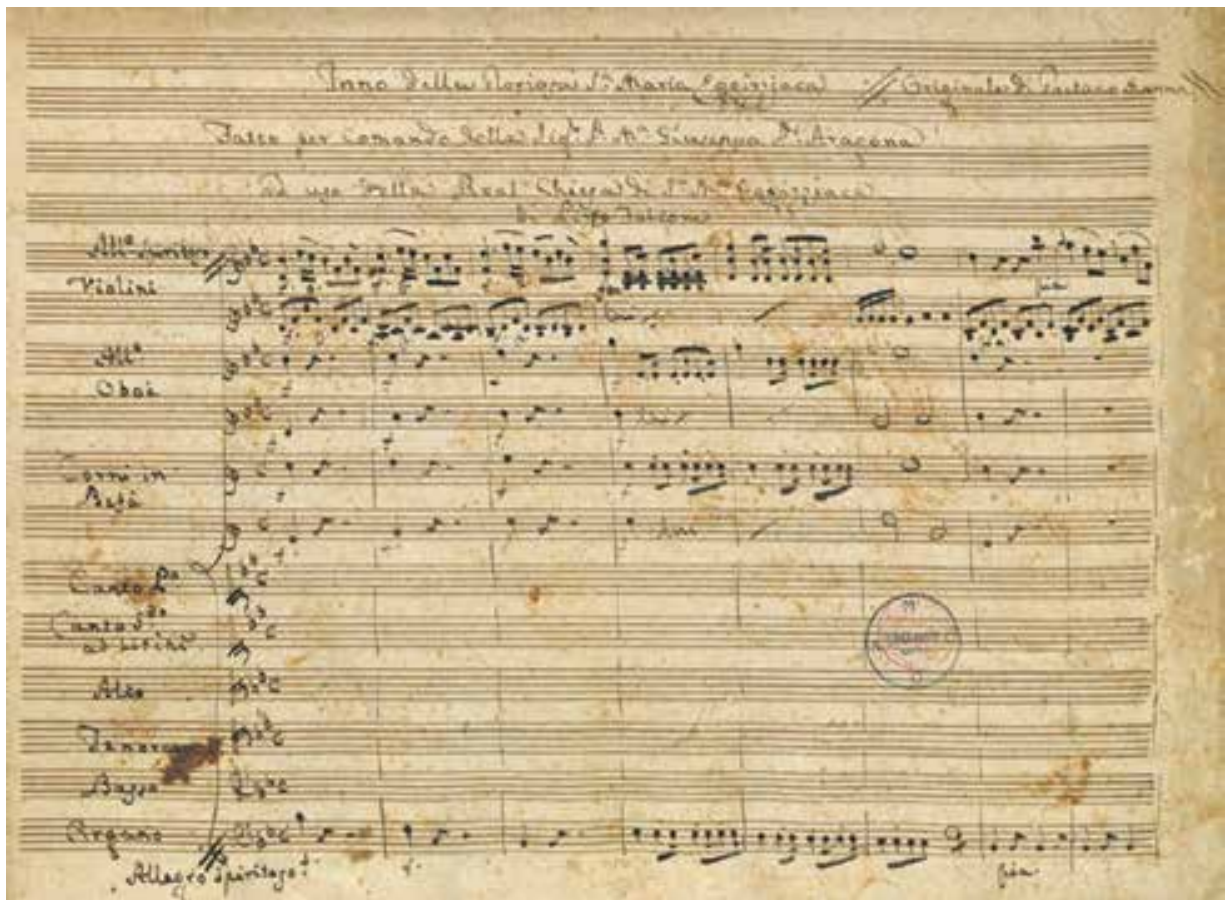

9. Gaetano Manna, Inno della Gloriosa S. Maria Egiziaca,

Napoli, Archivio musicale della Congregazione dell'Oratorio. ${ }^{101}$

\subsection{San Giuseppe dei Ruffi}

Ippolita e Caterina della dinastia Ruffo assieme a Caterina Tomacelli, nobildonne napoletane, ritirate a vita monastica in un palazzo sito presso il Seggio di Capuana, furono le prime assidue frequentatrici di una cappella già dedicata a San Giuseppe. Il monastero venne ufficialmente fondato nel 1604. Inizialmente vi dimorarono monache benedettine e la struttura rispose per molto tempo al nome di monastero di Santa Maria degli Angeli, soppresso come tale nel 1611 per mancanza di vocazioni. La struttura fu allora affidata ad alcune monache agostiniane fino al 1826, anno in cui le religiose vennero trasferite alla Croce di Lucca. ${ }^{102}$

101 La partitura di Manna è custodita presso l'Archivio dei Girolamini di Napoli, la riproduzione fotografica è tratta da $<$ http://www.internetculturale.it $>$.

102 Due anni più tardi le Suore Perpetue Adoratrici, dette "Sacramentine", vi si insediarono e tutt'oggi vi risiedono. Sulla storia dell'istituto si vedano: D'Engenio 1624, pp. 173-175; CELANo 2001, Giornata I, pp. 239-240; Galante 1985, Giornata III, pp. 658-663; Sigismondo 1789, vol. I, pp. 136-138; VALERIo 2007, pp. 207-215. 
Del monastero di San Giuseppe si conservano le costituzioni a stampa, dalla cui lettura si comprende una certa rigidità dei costumi adottati. Ad esempio, per quanto concerne gli uffici divini si annotava:

Tutte le Monache professe diranno l'officio del Signore, e della Madonna ogni giorno, con tutte l'ore Canoniche nel Coro di Chiesa; dove anco diranno l'officio de' morti, li Salmi Penitenziali, e Graduali, secondo la forma delle Rubriche del Breviario Romano riformato [...]. L'officio si dirà leggendo, in tuono ordinario di Religiosi, senza canto fermo, e figurato; e l'uno e l'altro canto talmente si proibisce, che né la Priora, né tutto il Capitolo congregato insieme possa dispensarci. Per questo si proibisce, che Monaca alcuna possa imparare, né canto, né suono e se alcuna l'avesse imparato nel secolo, le sia proibito di essercitarlo. E per questo non si ammetterà alcuna sorte di istromento, né di libri o composizioni musicali nel Monastero; si permettono solo le laudi spirituali, cantate ad aria per loro divozione, e ricreazione; ma non in Coro. ${ }^{103}$

Inoltre lo spazio adibito alla recita degli uffici, doveva essere protetto da qualsiasi sguardo esterno, il coro dunque doveva essere «fatto \& accomodato in modo, che le Monache non possino vedere, né esser viste; e però sarà dalla banda della Chiesa serrato con gelosie, e con una tela, per la quale le Monache non si possino scorgere di fuora». ${ }^{104}$

Particolare cura si aveva per i riti della Settimana Santa, in cui le monache agostiniane si sforzavano di trovare la giusta misura fra diletto e devozione. Per tal motivo, nel XVII sec., avevano compilato un rubricario con precise indicazioni sul modo di cantare la messa e celebrare il triduo pasquale. La mattina del Giovedì Santo aveva luogo la celebrazione eucaristica seguita da una piccola processione:

[...] Subito che il celebrante incomincia la Messa con li Ministri, le Monache dal choro cantaranno nel suono loro ordinario ma solenne l'introito Nos aure gloriari oportet sino al fine, dopo il quale intonato che havrà il gloria in excelsis seguitaranno a cantarlo tutto conforme allo stile ordinario [...]. Mentre si intona Gloria in excelsis si sonaranno le campane della chiesa e del Monastero [...]. Finito che haverà il suddiacono di cantare l'epistola le Monache non diranno De gratias, ma cantaranno subito il Graduale Christus factus est pro nobiscum sino al fine come sta nel Messale [...]. Doppo l'evangelio intonarà il celebrante il Credo in unum Deum, e le Monache nel suono loro seguiteranno à cantarlo sino al fine [...]. Finita la messa seguirà la processione [...] e le Monache tra tanto diranno cantando Tantum ergo sacramenturm sino al fine. [...]. Mentre s'avvia e camina la processione le Monache cantaranno Pange lingua gloriosi Corporis, e seguitaranno sino che il sacerdote habbia riposto il sagramento sopra l'altare dove hà da stare [...]. Mentre si comincia à spogliare l'altare le Monache dal choro intonano l'Antifona divise e la cantano tutta, poi il diacono alternatamente il Salmo Deus Deus meus sino al fine come sta notato nel Messale [...]..$^{105}$

Il Venerdì Santo era il giorno dedicato al silenzio, non aveva luogo la celebrazione della messa ma esclusivamente le lezioni. Pertanto le monache «deputate per cantare» dovevano disporsi «nel mezzo del choro aciò si possano accordare nel canto e non si dilongaranno dall'altre mentre si canta per vedere le cirimonie $[\ldots]$... Du-

103 BNn, Costituzioni S. Giuseppe, app. p. 136.

104 BnN, Costituzioni S. Giuseppe, app. p. 136.

105 Ass, MS, f. 4925, app. p. 133. 
rante l'adorazione della Croce le monache avrebbero dovuto «cantare l'improperij, tutti o parte, quanto riserva il tempo che dura l'adorazione [...]», e fare «la loro adorazione, sino all'ultima conversa». Il canto non avrebbe dovuto abbandonare il momento meditativo, si prescriveva pertanto che «quelle che cantano l'improperij, non lasciaranno di cantare tutte insieme, per andare all'adoratione $[\ldots] \gg{ }^{106}$ Infine il Sabato Santo «all'ufficio in cui si Cantaranno le profezie per ordine conforme allo stile loro $[\ldots]$ », le monache avrebbero dovuto aggiungere «le litanie che stanno stese nel Messale con suono solenne» e al termine della messa «subito si suonaranno tutte le campane, e le monache seguitaranno a cantare tutto il resto sino al fine $[\ldots] \gg . .^{107}$

Il monastero si avvaleva della collaborazione dell'organaro Giuseppe Gualberto Ferreri, e soprattutto dei musicisti della Cappella dell'Arcivescovado sotto la guida dei maestri di cappella Pietro Cortillatto, Francesco Marinelli e Domenico Arcucci che intervenivano nelle due principali festività di Sant'Agostino e di San Giuseppe:

Al Reverendo Don Domenico Arcucci edomadario [sic] e Mastro di Cappella della Chiesa Arcivescovile di Napoli d. 33 per la musica a due cori per le prime e seconde vesperi, e Musica nel Giorno del Glorioso Patriarca San Gioseppe, e due voci forastiere. ${ }^{108}$

$\mathrm{La}$ «Gazzetta di Napoli» ci informa inoltre di un oratorio realizzato a San Giuseppe in onore di Sant'Anna, composto da Scipione Cigala nel 1746 e «rappresentato dalli celebri virtuosi Eggizziello, Barbi e Barialdo, con la musica composta dal maestro di cappella Niccolò Locroscino» ${ }^{109}$ che aveva sortito un grande consenso da parte della nobiltà e della popolazione tutta.

\section{Domenicane}

\subsection{Santa Maria del Divino Amore}

Il complesso fu istituito dalla nobildonna Beatrice Villani che decise di far diventare luogo di culto un palazzo di proprietà della sua famiglia. ${ }^{110}$ L'opera fu affidata all'architetto Francesco Antonio Picchiatti che costruì la chiesa nell'atrio del palazzo, con una splendida vista sul mare e sulla collina di San Martino. Nel 1866 il

106 Asn, MS, f. 4925, app. p. 133.

107 Asn, MS, f. 4925, app. p. 134.

108 Asn, MS, f. 4875, app. p. 132.

109 Gdn, 5 luglio 1746, app. p. 135. Sull'episodio si veda Magaudda/Costantini 2009, appendice, nota 1541 .

110 Proprio per questo, la chiesa non ha una vera e propria facciata, essendo stata costruita all'interno di una struttura originariamente destinata a svolgere altre funzioni. 
monastero venne soppresso e destinato ad abitazioni private, mentre le monache furono trasferite nelle strutture di Santa Chiara. ${ }^{111}$

Nel 1727 venne commissionato a Francesco e Fabrizio Cimino, per 150 ducati, un organo per la chiesa, approvato da Nicola Fago, in quel periodo maestro di cappella del monastero. Lo strumento fu eseguito talmente bene che il lavoro venne pagato immediatamente, pur essendo stato pattuito un pagamento tardivo rispetto alla consegna:

A Don Carlo Capecelatro d. cinquanta; e per esso alli Mastri Fabrizio, e Francesco Cimmino organari disse pagarceli in nome, e parte di Donna Orsola Comite monica professa nel venerabile nostro monastero del Divino Amore in nome della quale da esso se li diede a detti Mastri fare l'organo per detta sua Chiesa e assegnandolo già di tutta sua sodisfattione e di don Nicolò favo [Fago] Mastro di Cappella al quale era rimesso l'approvazione di detto organo di sua bontà e perfettione e detti $\mathrm{d}$. cinquanta sono a compimento di $\mathrm{d}$. centrocinquanta intiero prezzo di detto organo pattuito, $[. .$.$] con il presente pagamento e consegna fatta di detto organo restano per$ intiero sodisfatti così da detta Signora, come da esso che ne hà avuto il pensiero e si tanto da detta Signora le sodette summe non haver che pretendere altra cosa e detto pagamento benche né fusse il patto di pagarli questa summa dopo sei mesi dalla consegna di detto organo tutta volta detta Signora si è compiaciuta farla adesso la finale per sua benevolenza per la sodisfattione avuta di detto organo $[\ldots] .{ }^{112}$

Diversi «Servitij di Musica» ${ }^{113}$ erano assicurati dalla collaborazione con il conservatorio dei Poveri di Gesù Cristo, mentre le feste del Divino Amore, San Domenico, Sant'Agostino, Assunta, Settimana Santa, così come le numerose e fastose monacazioni, prevedevano il coinvolgimento dei maestri della Cappella della Cattedrale, cui spesso si aggiungevano strumentisti aggiuntivi o virtuosi come Matteo Sassano. Il "Matteuccio" risulta infatti essere protagonista di diverse monacazioni e festività, come attesta anche il seguente pagamento che figura nelle «spese di sacrestia» del monastero del 1723:

Per musica nelle due feste della nostra Chiesa a Poveri di Jesù Xristo d. 20

A 4 Voci estraordinarie d. 36

All'istromenti estraordinari d. 39.3.10

All'Istromenti portati dal Musico Matteucci d.4.2.10 [...]

Per affitto d'organo d. 2.4

Per tiratura di detto d. $0.3^{114}$

$111 \mathrm{Nel} 1870$ la chiesa venne affidata ai frati crociferi. Le monache trasferirono nella loro nuova dimora le opere contenute nella loro vecchia chiesa, tra cui la Concezione di Maria, opera di Francesco De Mura, e la Deposizione, attribuita alla scuola di Mattia Preti. Sulla storia dell'istituzione si rimanda a Celano 2001, Giornata III, pp. 777-780; Galante 1985, Giornata VI, pp. 196-197. Si veda anche: VALERIo 2007, pp. 249-255.

112 AsBn, BPV 1075 11/IX/1727, app. p. 144.

113 AsBn, BSE 975 4/V/1731, app. p. 144.

114 Il pagamento si riferisce ai mesi di luglio e agosto, in cui si celebravano San Domenico e Sant'Agostino, è probabile dunque che la musica sopracitata fosse relativa a queste due festività. Ass, MS, f. 3733 , app. p. 140. 


\subsection{Santa Maria Maddalena delle Spagnole}

Il monastero di Santa Maria Maddalena delle Convertite Spagnole, conosciuto anche come Maddalenella degli Spagnoli, venne eretto alla fine del XVII secolo dalla nobildonna Isabella Alarcon y Mendoza. ${ }^{15}$

Purtroppo su questo istituto non vi sono grandi testimonianze documentarie concernenti l'attività musicale. La prima notizia che si ha è datata 1698 ed è relativa ad un anonimo mastro «ch'accomoda il cimbalo de Signore Monache». ${ }^{116}$ L'archivio delle Corporazioni Religiose Soppresse, riporta poche indicazioni anche sulle festività in esso celebrate. Qualche accenno alle feste di Santa Maria Maddalena, che avevano luogo il 22 luglio e il giovedì dopo la domenica di Passione in memoria della conversione della Santa. In occasione di quest'ultima si trovano spese per «la musica per le prime e seconde vespere» ai «Figlioli della Pietà»». ${ }^{117}$ Maggiori indicazioni si ricavano dalle polizze del Banco di Napoli riguardo le celebrazioni organizzate in occasione della Settimana Santa, San Giuseppe e San Domenico e sui maestri di cappella. Nel 1731 si ha notizia di Dioniso Zamparelli cui viene affidato il compito di intervenire alla festa di San Domenico con «4. voci, 5. violini, violongello, leuto, controbasso, due trombe, ed un boè, con affitto dell'organo»». ${ }^{118}$ Fra 1732 e 1733 è Saverio Ranucci ad essere maestro di cappella: si trovano pagamenti per la festività di Santa Maria Maddalena alla quale Ranucci prendeva parte con «sei voci, sette violini, violoncello, liuto, Controbasso, quattro Istromenti di fiato, ed organista [...]. ${ }^{119}$ Qualche anno più tardi, nel 1762, troviamo invece la presenza, in qualità di maestro di cappella, di Pietro Auletta, una delle figure più poliedriche della Napoli vicereale: ${ }^{120}$

Banco dello Spirito Santo pagate a Don Pietro Auletta Mastro di Cappella d. 47.60: dite sono così per sua ricognizione dell'organista, come per salario de Musici di voce, ed Istromento: per la musica dal medesimo fatta nella Chiesa del nostro Real Monastero nel giorno che si è celebrata nel passato mese di luglio del corrente anno la festività della Gloriosa S.M. Madalena, così la mattina per il trattenimento, messa cantata, e dunzione d'una religiosa corista, che hà professato, come per una conversa, che hà preso l'abito,come per le seconde vesperi il giorno inclusovi tra detti d.47.60 d. quattro per li cartocci d'esso Mastro di Cappella Organista, e Musici sudetti, giusta la nosta, che si conserva nelle cautele $[\ldots] .{ }^{121}$

115 Sulla storia del monastero: Galante 1985, Giornata X p. 371; Sigismondo 1789, vol. II, pp. 294-295; VALERIO 2007, pp. 375-379.

116 Asn, MS, f. 6600, app. p. 149.

117 Asn, MS, f. 6600, app. p. 149.

118 Asbn, BSG 789 22/VIII/1731, app. p. 150.

119 AsBn, BSG 817 14/8/1733, app. p. 150.

120 Pietro Auletta era infatti attivo come maestro di cappella presso le chiese di Santa Maria la Nova, San Luigi di Palazzo e Sant'Agostino, compositore per il Teatro Nuovo e maestro di musica presso i palazzi nobiliari del Duca di Parete e del Principe di Belvedere. È interessante quindi notare come informazioni desunte da questa tipologia di documento aggiungano tasselli nelle biografie delle maestranze napoletane, consentendoci di ampliare maggiormente le nostre conoscenze sui musicisti e sulla scena spettacolare partenopea.

121 Asn, MS, f. 4625, app. p. 148. 


\subsection{Santa Caterina da Siena}

Si presume che la chiesa sia stata eretta nel XVI secolo da Don Giovanni d'Austria. Il luogo era stato scelto per le sue qualità ambientali, come sito dove costruire un ospedale intitolato a "Santa Maria della Vittoria", in ricordo della sconfitta subita dall'armata turca a Lepanto nel 1571. Nel XVII secolo il trasferimento delle strutture ospedaliere e della chiesa presso l'attuale piazza denominata 'della Vittoria', aveva reso liberi gli ambienti originari, acquistati poi dal frate domenicano Feliciano Zuppardi ed affidati ad alcune religiose domenicane. Il monastero venne soppresso nell'Ottocento e successivamente ospitò le monache del conservatorio della Solitaria. ${ }^{122}$

Pochissime le testimonianze relative all'attività musicale dell'istituzione, complice anche la mancanza di documentazione archivistica. Le uniche informazioni riguardano gli organisti attivi presso il monastero come Giuseppe Filomena nel 1748. Più interessante è la documentata presenza del maestro di canto Carlo Terillo cui, nel 1759, vengono corrisposti compensi per insegnare «di canto fermo le ss.re monache coriste del medesimo monastero». ${ }^{123}$ Nello stesso periodo un pagamento testimonia l'acquisto di «un cembalo fatto comprare per servizio delle nostre monache coriste che s'imparano di canto fermo». ${ }^{24} \mathrm{Dal} 1782$ si riscontra la presenza di Gaetano Manna ${ }^{125}$ maestro di cappella cui era affidata la musica de «primi e secondi vespri e messa» ${ }^{126}$ in occasione delle festività di San Domenico e di Santa Caterina.

\subsection{Ss. Pietro e Sebastiano}

Nel 1424 alcune monache domenicane, provenienti dal monastero di San Pietro a Castello, raso al suolo a causa della guerra tra Alfonso d'Aragona e Luigi III d'Angiò, furono collocate, per disposizione di papa Martino V, nell'antica sede del monastero benedettino di San Sebastiano. Si trattava di un monastero di regio patronato che vantava del patrimonio del monastero di San Pietro a Castello, le

122 La chiesa di Santa Caterina conserva ancora oggi affreschi di Mario Gioffredo e Fedele Fischetti oltre a tele di Francesco De Mura, Luca Giordano, Giacinto Diano, Paolo De Matteis. Sulla storia del complesso si veda: D’Engenio 1624, p. 570; D’Aloe 1883, p. 142; De Lellis 1654, vol. IV, ff. 155-157; Celano 2001, Giornata V, pp. 574-575; Galante 1985, Giornata X, p. 372; Sigismondo 1789, vol. I, pp. 291-292; Bulifon 1932 p. 93; VAlerio 2007 pp. 157-161.

123 Asn, MS, f. 4357, app. p. 155.

124 Asn, MS, f. 4358, app. p. 156.

125 Gaetano Manna (1751-1804) fu allievo di Pietro Antonio Gallo e Fedele Fenaroli al conservatorio di Santa Maria di Loreto, fu anche maestro di cappella dell'Annunziata e collaborò con diverse istituzioni ecclesiastiche napoletane, fra cui la Cattedrale. Cfr. Dietz 1972.

126 Asn, MS, f. 4360, app. p. 156. 
cui abitanti erano strette consanguinee di Elisabetta d'Ungheria. Il monastero, sottoposto all'osservanza della regola agostiniana, intrecciò nel corso dei secoli, relazioni con diverse comunità domenicane femminili del Mezzogiorno, per le quali divenne un importante punto di riferimento. L'istituto venne soppresso nel 1808 e l'edificio monastico venne destinato alla sede di un conservatorio di musica e infine si trasformò nel Convitto Nazionale Vittorio Emanuele II. ${ }^{127}$

Le fonti pervenute danno un quadro abbastanza prospero e dinamico della vita che conducevano le monache. Un documento del 1692 testimonia la presenza di cento monache coriste, dieci educande e diciannove monache converse, il cui numero si ridurrà drasticamente nel Settecento. Nel 1753 si contavano infatti solo una quarantina di religiose fra monache ed educande.

Negli ordinamenti dei «Maestri generali» si esortavano le monache a seguire i dettami delle costituzioni, si ribadiva l'obbligo dei sacramenti, del silenzio, si vietavano le visite di dame e cavalieri, si raccomandava la preghiera. In una lettera inviata dal maestro generale, fra Antonino Cloche, si raccomandava che la musica fosse prevista solo in tre festività l'anno: San Pietro, San Sebastiano e San Domenico, e che tutte le altre feste fossero celebrate solo con la messa solenne:

Ordiniamo [...] che nella Chiesa di codesto nostro Monasterio si facciano solamente trè feste l'anno, l'una di San Pietro, l'altra di San Sebastiano titolati in detta Chiesa, e la terza del nostro Padre San Domenico, volendo che le due prime feste si facciano con le solite solennità, cioè con Musica, predica, processione delle reliquie, e compieta de Padri al ritorno delle dette reliquie, e nella 3a debba esservi solamente la musica per sodisfattione di un legato fatto al Monastero per questi effetto comandando espressamente che in ogni altra festa introdotta fuori delle tre accennate non si possa fare cosa alcuna delle nominate, mà solo si possa cantare la messa del Padre Priore $[\ldots] . .^{128}$

Le cronache relative al monastero riguardano la festa dei Ss. Fabiano e Sebastiano, che veniva celebrata con musica e cappella reale fino al 1734. La festa dei Ss. Pietro e Paolo, citata già nel 1687, era invece contraddistinta dalla «ricchezza dell'apparato e squisitezza della musica a più cori»». ${ }^{129}$ Stesso dicasi per la festa di San Domenico, solennizzata generalmente con "sceltissima musica». ${ }^{130}$ Le celebrazioni del 1732 avevano visto la partecipazione di «Domenico Sarro Vice Maestro della Regal Cappella [...] per li tre esercitij e trattenimenti di musica dal medesimo fatti nella Regal Chiesa di San Pietro e Sebastiano di Monache nel giorno di San Domenico, così per voci, come per istrumenti $[\ldots] \gg .^{131}$

127 Sulla storia dell'istituto: De Stefano 1560, pp. 177-179; D'Engenio 1624, pp. 222, 226-227; D’Aloe 1883, p. 336, 724-723; Celano 2001, Giornata II, pp. 303-304; Galante 1985, Giornata IV, pp. 109-111; VALERIO 2006, pp. 367-371.

128 Asn, MS, f. 1483, app. p. 159.

129 GDN, 1 luglio 1687, app. p. 160.

130 GDN, 6 agosto 1720, app. p. 160.

131 Asbn, BPV 1144 19/8/1732, app. p. 159. 
Le monache tuttavia non disdegnavano di organizzare sontuose celebrazioni per compiacere l'aristocrazia e gli stessi regnanti. Nel 1738 l'arrivo a Napoli della regina Maria Amalia fu festeggiato con un Te Deum e «musica solenne fatta dal maestro della R. Cappella Domenico Sarro». ${ }^{132}$ È probabile inoltre che fosse in uso, presso il monastero, organizzare rappresentazioni teatrali. A tal proposito si riscontra una proibizione del visitatore generale di Papa Innocenzo XII, fra Salvatore Ascanio, riguardo il costume di allestire «rappresentazioni di qualsisia maniera» da parte delle monache domenicane. ${ }^{133}$

\subsection{Santa Maria della Sapienza}

Nel 1507 il cardinale Carafa cominciò a restaurare un edificio denominato "la Sapienza" perché potesse essere utilizzato come ricovero per studenti poveri. La morte del cardinale, però, fermò i lavori di costruzione di questa casa. Nel 1511, gli eredi cedettero l'intera struttura ai fratelli Pietro e Marino Stendardo e a Giovanni Latro che, dal 1519, decisero di istituirvi un monastero di clarisse, la cui direzione venne affidata a Lucrezia Dentice, proveniente dal monastero di Santa Maria del Gesù. ${ }^{134}$ Il luogo divenne subito frequentato da religiose provenienti da famiglie nobili, le cui cospicue donazioni vennero riutilizzate per acquistare gli edifici circostanti ed espandere la struttura. Alla morte della Dentice, ne assunse la direzione Sancia Carafa, del monastero di Donna Romita, con l'intento di creare un monastero di rigida clausura sotto la regola domenicana. Il monastero acquisì nel tempo sempre maggior fama e crebbe il numero delle postulanti, esponenti delle famiglie Piccolomini, d'Aragona, Orsini, Carafa.

Probabilmente in virtù delle severe norme di vita di cui godeva l'istituto, non sembra esserci stata una cospicua attività musicale. La documentazione inoltre risulta essere molto lacunosa. Nella «Gazzetta di Napoli», sono citate numerose cerimonie di professione religiosa, in cui si attesta la presenza di «trombe e musicali istrumenti» ${ }^{135}$ per la monacazione della figlia del duca di Gravina, «quattro cori di sceltissima musica» ${ }^{136}$ per la figlia della principessa della Riccia, «scelta

132 GDN, 8 luglio 1738 (5), app. p. 160.

133 Il documento è citato al cap. I, p. 47 si veda anche AsN, MS, f. 1407, app. pp. 158-159.

134 I lavori per la sua costruzione della chiesa cominciarono nel 1625, vi presero parte molti prestigiosi architetti tra cui Cosimo Fanzago e Dioniso Lazzari. Nel 1799 si trasferirono alla Sapienza alcune suore di San Gaudioso e nel 1825 arrivarono anche le domenicane del Divino Amore, nel 1864 le monache di San Giovanni. Nel 1886 si decise di abbattere il monastero per costruire un Policlinico Universitario. Di tutto il complesso oggi rimane solo la chiesa. Sulla storia del monastero si veda: De Stefano 1560, p. 179; D’Engenio 1624, pp. 70-71; D’Aloe 1883, pp. 526-527; Celano 2001, Giornata II, pp. 55-57; Galante 1985, Giornata III, pp. 105-106; VALERIO 2006, pp. 235-239.

135 GDN, 10 giugno 1693 (1), app. p. 163.

136 GDN, 11 marzo 1710 (1), app. p. 164. 
musica a più cori mattina e giorno $[\ldots] \gg{ }^{137}$ per la figlia del principe di Marzano, e ancora musica «mattina e sera» con «voci istromenti e affitto d'organo» ${ }^{138}$ per la figlia del Duca di Bagnara. Al monastero risiedeva anche la sorella di Papa Benedetto XIII, suor Maria Scolastica Ursini. Nei diari dei Cerimonieri è riportata la cerimonia funebre che vide la partecipazione dell'arcivescovo Pignatelli e della Cappella della Cattedrale:

Mercoledì 2 giugno 1728 Funerale ed una castellana con 100 e più lumi fatta à spese di Sua Eminenza nella Chiesa della Sapienza per la sudetta signora sorella del Papa. Il Signor Cardinale Pignatelli Arcivescovo verso le 14 hore con tutto il suo capitolo, cioè canonici, eddomadarij, 40; e seminario andò nella Chiesa della Sapienza assistè alla messa in cappa, la quale fu cantata dal canonico del Duca Diacono, come Vicario delle monache (riluttando i canonici presbiteri) finita la messa $[\ldots]$ si cantò la libera dà Signori Canonici, attorno a detta Castellana [...] vi furono 17 sblendori, attorno con torcie [...] vi fü la musica del Duomo, e molti altri virtuosi, convitati dal Maestro di Cappella dell'Arcivescovato si cantò à Palestino, ${ }^{139}$ il tutto si fece da Sua Eminenza Arcivescovo. ${ }^{140}$

\section{Francescane}

\subsection{Santa Maria Donnaregina}

Le origini del complesso di Donnaregina risalgono all'VIII secolo, quando la zona era occupata da un complesso monastico denominato San Pietro del Monte di Donnaregina. Il monastero fu inizialmente impostato sulle regole di vita monastica basiliana, per poi passare alla regola benedettina dal XI fino al XIII secolo. Da quel momento in poi alle monache fu concesso di vivere secondo l'ordo Sanctae Clarae e la comunità venne affidata ai frati minori.

All'inizio del Seicento, le monache di Donnaregina, decisero di costruire una nuova chiesa barocca, più consona al gusto del tempo, annettendo l'antica chiesa gotica alla zona della clausura. I lavori per la costruzione del nuovo edificio sacro, detto perciò di Santa Maria Donnaregina 'nuova', iniziarono nel 1617, furono completati quasi del tutto nel 1626 , e la nuova chiesa divenne quella ufficiale. ${ }^{141}$

137 GDN, 10 aprile 1742, app. p. 164.

138 AsBn, BSS 1159 24/X/1726, app. p. 162.

139 L'espressione 'a Palestino' corrisponde allo stile 'alla Palestrina', denominazione ricorrente nel Seicento per indicare composizioni in stile antico a cappella.

140 AsDn, Cerimonieri, vol XVII, app. p. 162.

141 Situate nei pressi del palazzo arcivescovile, i due edifici di Donnaregina 'vecchia' e di Donnaregina 'nuova' comunicavano attraverso le due zone absidali. Nel 1646 vi fu un'opera di risanamento, attraverso la quale vennero abbattute diverse case popolari e venne creato il Largo Donnaregina. La costruzione di un nuovo campanile era stata avviata nel 1681, ma fu abbandonata in seguito all'opposizione delle suore del vicino convento di San Giuseppe dei Ruffi. La chiesa di 
Il monastero venne soppresso nel 1861 e le religiose si trasferirono nei monasteri di Santa Chiara e Donnalbina.

Già dalla sua fondazione il monastero accolse le figlie della più alta aristocrazia, beneficiò nel corso dei secoli di donazioni da parte dei sovrani e riuscì ad accumulare numerose proprietà terriere che si estendevano dalla provincia di Napoli fino a quella di Caserta.

L'ingente patrimonio archivistico del monastero, nei secoli disperso e smembrato, è oggi conservato in parte presso l'Archivio di Stato e in parte presso l'Archivio Diocesano.

I registri contabili riferiscono della festività di San Sebastiano per la quale erano previsiti «tre offizii, à tre chori, et nove istrumenti, et organo et portatura, incluso l'organista, inclusi li motetti, incluso anco il mastro di cappella, et la messa del octava à un choro»». ${ }^{142}$ Anche le festività di San Donato, l'Assunzione, San Bartolomeo e San Francesco prevedevano la presenza di musicisti, come conferma l'ordinazione ad un «mastro mannese» ${ }^{143}$ di due orchestri, ovvero i luoghi deputati ad ospitare le compagini musicali:

A Donna Vincenza de Silva duc. trenta e per Lei à Giovanni Grieco Mastro Mannese, sono in conto delli duc. Settantacinque convenuti doverseli da Lui pagare per L'intiero prezzo delli due Orchesti che si è obligato fare in conformità del disegno fattone dal Architetto Don Mutio Nacherio per Servitio delle Cinque Sollenni festività devono farsi nella Venerabile Chiesa di Santa Maria Donna Regina di questa Città durant'l'anno, che essa Vincenza è Sacristana di detta Chiesa, cioé quelle di San Francesco, Sant'Andrea, San Donato, Assunta, e San Bartolomeo, e detti Orchesti darli completi e perfetti ornati di tutto punto, e per tutta la fine di Settembre 1730 e ponere detti Orchesti in tempo di dette festività, e poi Levarli $[\ldots]]^{144}$

La festa di Sant'Andrea prevedeva la presenza del viceré che vi teneva Cappella Reale, attestata anche dalle cronache della «Gazzetta di Napoli»:

E, correndo mercordì trascorso [30 novembre] la solennità del glorioso apostolo S. Andrea, fu tra l'altre chiese singolarmente festeggiata in quella delle monache di Donna Regina, ove quella sacrestana D. Maria Gaetano d'Aragona, sorella del duca di Laurenzano, vi fé [...] spiccare la più ricca e sontuosa magnificenza di preziosi apparati e sceltissima musica che giammai siasi ivi goduta, conservandosi in detto tempio l'insigne reliquia della testa del santo $[\ldots]^{145}$.

Donnaregina è una delle più prestigiose di Napoli e ancora oggi conserva affreschi di Francesco Solimena e Luca Giordano. Attualmente è la sede del Museo Diocesano di Napoli. Si rimanda a: De Stefano 1560, pp. 184-85, D’Engenio 1624, pp. 169-170, 182; D’Aloe 1883, p. 543; De Lellis 1654, vol. II, ff. 79-90; Celano 2001, Giornata II, pp. 68-73; Galante 1985, Giornata II, pp. 66-68, 70, 73, 132, 169; Delfino 1983, pp. 81-121; Delfino 1990, pp. 101-114; Novi-Chavarria 2001, pp. 161-201; VAlerio 2006, pp. 287-294.

142 Asn, MS, f.3520, app. p. 166.

143 Questo termine si riferisce in genere agli artigiani che lavoravano il legno.

144 AsBn, BPI 1624 24/X/1731, app. pp. 173-174.

145 GDN, 6 dicembre 1712 (1), app. p. 174. 
Specifiche licenze venivano concesse per l'intervento musicale e processione delle reliquie, in occasione della festa di San Donato:

Si concede licenza alla Venerabile Santa Chiesa di Santa Maria Donna Regina farsi Festa nel giorno di San Donato V. e M. con musica de Poveri di Giesù Christo, come anco per la traslazione delle sacre reliquie dalla porta della clausura alla chiesa la processione di preti secolari e con messe di preti secolari $[\ldots] .{ }^{146}$

Per l'Assunta veniva concessa licenza per la partecipazione dei musicisti della Cappella della Cattedrale e l'aggiunta di cantanti:

Si concede licenza alla Venerabile Santa Chiesa di Santa Maria Donna Regina farsi Festa nel giorno dell'Assunta della Vergine Santissima con musica Arcivescovale et l'aggiunta di due voci non delle prohibite con soliti stromenti con messe di preti secolari $[\ldots]{ }^{147}$

Infine per San Bartolomeo apostolo, era concesso che le voci raggiungessero il numero di quattro:

Si concede licenza alla Venerabile Santa Chiesa di Santa Maria Donna Regina farsi Festa sollenne nel giorno di San Bartolomeo Apostolo à 29 Agosto con musica Arcivescovale con l'aggionta di quattro altre voci non delle prohibite con li soliti stromenti [...] e di più per la traslazione delle sante reliquie dalla Porta della Clausura alla Chiesa la processione di Padri Conventuali di San Lorenzo e Preti secolari con le solite flottole musicali [...]. ${ }^{148}$

Faceva seguito alle cerimonie liturgiche per San Bartolomeo anche un «trattenimento» organizzato la mattina dopo la festività, con la partecipazione dei musicisti della Cattedrale. ${ }^{149}$

Le festività di San Rocco, San Biagio e San Domenico erano probabilmente festività minori, di cui risultano soltanto pochi pagamenti generici per «musici»e assistenza dei «figlioli della pietà». ${ }^{150}$

Poche indicazioni riguardo i maestri dell'istituzione. Un solo riferimento a «Christofaro Caresano Mastro di Cappella» ${ }^{151}$ nel giugno del 1689 e agli organisti Marcello Patrizio fra 1645 e 1647 e Alfonso Cangi nel 1727.

Essendo uno dei più ricchi e prestigiosi cenobi non mancano cronache delle monacazioni in esso celebrate: "Assai decorosa [...] con famosissima musica di scelti virtuosi», fu la monacazione di «D. Carlotta Caracciolo, figlia degli eccellentissimi principe e principessa d'Avellino, ambasciator cattolico alla corte romana $[\ldots] \gg,{ }^{152}$ avvenuta il 2 dicembre 1710 , mentre per la monacazione di Donna Felice Carmignano vi fu «scelta musica delli più virtuosi professori, con

146 Asdn, Vicario, 258 D, app. pp. 170-171.

147 Asdn, Vicario, 258 D, app. p. 171.

148 Asdn, Vicario, 258 D, app. p. 171.

149 Asbn, BPV 1130 11/X/1731, app. p. 173.

150 Asn, MS, f. 3520, app. pp. 165-166.

151 Asn, MS, f. 3520 , app. p. 168.

152 GDN, 2 dicembre 1710 (2), app. p. 174. 
due mottetti cantati dal celebre musico conte [= marchese] Matteo Sassano» ${ }^{153}$ «sontuosa musica a quattro cori» ${ }^{154}$ fu predisposta per la professione della figlia del duca d'Atri.

Giovanni Battista Pinacci, Antonio Paci assieme al sopracitato Sassano, intervennero, nel 1721, alla monacazione di Caterina della Leonessa, figlia del duca di Ceppaloni. ${ }^{155}$ I tre artisti di passaggio a Napoli in quel periodo, erano infatti impegnati nell'opera Endimione in scena al San Bartolomeo.

\subsection{SS. Trinità}

«Tra le principali e belle chiese che sono in Napoli, questa è una situata, col suo nobile e magnifico monistero, su la falda del Monte di San Martino, cominciato ad habitar dalle monache francescane agli inizi di giugno del $1608 \gg{ }^{156}$

Così Pompeo Sarnelli descriveva il monastero della SS. Trinità eretto per volere di Donna Vittoria de Silva a inizio Seicento. Ottenuta un'autorizzazione da Papa Clemente VIII, Donna Vittoria, religiosa del monastero di San Girolamo, volle creare un proprio insediamento con regola francescana nella zona di Santa Maria di Costantinopoli. Successivamente fu scelta un'area più idonea e salubre, ai piedi della collina di San Martino. Grazie anche alla sua posizione panoramica, la struttura religiosa piacque molto alle famiglie aristocratiche dell'epoca che ne fecero una delle dimore privilegiate. ${ }^{157} \mathrm{Il}$ monastero divenne celebre anche per i vasti giardini, per le fontane e le peschiere che le monache avevano fatto costruire per divertirsi, cimentandosi nella cosiddetta 'pesca nelle fontane'. ${ }^{158}$

La documentazione di questo istituto oggi conservata è molto scarsa e lacunosa, tuttavia è sufficiente a far comprendere il grado di agiatezza nel quale le monache vivevano. Il monastero accoglieva solo fanciulle provenienti dalle famiglie aristocratiche Spinelli, Pignatelli, Medici, pertanto le carte d'archivio ci raccontano soprattutto delle fastosissime monacazioni. Le indicazioni spesso sono generiche e riferiscono semplicemente di spese «per la musica», in altri casi invece, qualche fortuito ritrovamento, ci permette di ricostruire l'intero organico intervenuto per questa tipologia di cerimonia:

153 GDN, 24 gennaio 1713 (2), app. pp. 174-175.

154 GDN, 9 aprile 1720 (3), app. p. 175.

155 GDN, 20 maggio 1721 (5), app. p. 175.

156 SARNELLI 1685 , p. 319.

157 La realizzazione architettonica del complesso fu affidata a importanti artisti partenopei come Cosimo Fanzago. Nel 1808 il complesso venne soppresso ed adibito ad ospedale militare fino al 1992. Sulle vicende storiche: D’Engenio 1624, p. 582; Celano 2001, Giornata VI, pp. 667-676. Galante 1985, Giornata X, p. 363; Sigismondo 1789, vol. II, pp. 255-257. Si veda anche: VALERIO 2006, pp. 463-469.

158 Si veda Novi-Chavarria 2009, pp. 95-96. 


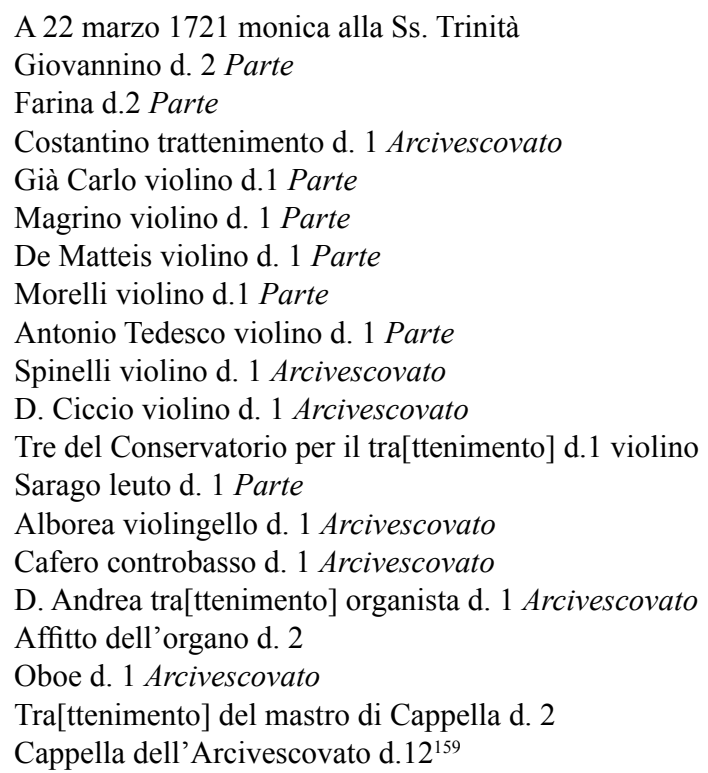

Il documento purtroppo non riporta il nominativo della novizia, né del compositore delle musiche né del maestro di cappella. Vengono indicati i musicisti intervenuti per la liturgia e per il «trattenimento». Accanto a ciascun musicista è presente la dicitura Parte o Arcivescovato che potrebbe forse indicare la provenienza del pagamento, se a carico del monastero o dell'Arcivescovado.

Più nutrito e maggiormente dettagliato l'organico della monacazione di Donna Maria Giuseppa Villapiana avvenuta nel 1744, che testimonia la ricchezza musicale dell'evento:

Coro dell'Arcivescovado:

Maestro di Cappella (D. Giacomo Sarcuni), organista, due soprani, contralto, tenore e basso, e tre violini per la messa cantata, ducati 12 .

Giovannini, trattenimento e messa, d. 4.50

Ciardini, soprano d. 2

Mariotta, contralto d. 2

Capuano, tenore d. 1.50

Pecoraro, basso d. 1.50 .

Trattenimenti alli Maestri dell'Arcivescovato:

Maestro di Cappella, d. 2

Organista d. 1

Palella, altro organista d. 1

D. Nicolino, soprano d.1

Signorile, soprano d.1

Sericci, contralto d.1

Tenore d. 0,50

Basso d. 0,50

159 Asn, MS, f. 5210, app. pp. 185-186. 


\author{
Istromenti: \\ Francesco Sopriano, violoncello d.1 \\ Ferdinando Pallino, 2o voloncello d.1 \\ Pugliese, Salterio et Arciliuto d. \\ De Vita, contrabasso d. 0.80 \\ Istrumenti di fiato: \\ Papa, oboè e flauto traversiero d. 0.80 \\ Bisuzzi, oboè d. 0.80 \\ Andreucci, corno da caccia d. 0.80 \\ De Angelis 2o corno da caccai d. 0.80 \\ Violini: \\ De Matteis, Fiorenza, Carcaiso, Gravina, Cladarano, Moro, Infantes, Cecere, Cammardella, \\ Carfonga, Fuscaldo d. 0.80 \\ Al soprano Palermitano d. $10^{160}$
}

Ancora una volta vengono differenziati gli artisti intervenuti nella liturgia e quelli per il «trattenimento» che seguiva la celebrazione, ed è interessante scorgere in questi documenti alcuni nomi di musicisti che saranno considerati dai posteri grandi esponenti della scuola strumentale napoletana, come il celebre violoncellista Francesco Supriani ${ }^{161}$ o il violinista Nicola Fiorenza. ${ }^{162}$

Non vi sono grandi indicazioni sulle festività celebrate presso l'istituto. Di certo in occasione della festività della SS. Trinità, il cerimoniale doveva essere particolarmente ricco e solenne, così come testimonia il Celano:

Per goder poi d'un paradiso in terra è di bisogno di vederla apparata et adornata ne' giorni festivi della Santissima Trinità, di san Francesco et altri. Vi si veggono famosissimi ricami, paleotti tutti ricamati di perle, quantità di vasi d'argento e candelieri per tutte le cappelle, e compartiti con polizie indicibili. La sacristia poi in detti giorni si rende così curiosa che si potrebbe venire da lontano a vederla, perché le suore vi espongono apparati per le messe che non han pari; vi si veggono un numero grande de càmisci con merletti grandi, e bianchi e d'oro e di ricami, così fini e nobilmente lavorati che sono di stupore. V'espongono ancora molte galanterie, come calici tutti d'oro, di cristallo di monte e d'argento, singularmente lavorati; anco un ostensorio per esponere la sacra eucharistia, con i suoi raggi tutti tempestati di rubini, il giro dove si colloca la sacra ostia, tutti di grossi diamanti e perle et altre gemme, che viene valutato 6.500 scudi; oltre de' pretiosi quadri che adornano le mura. ${ }^{163}$

160 Il documento appartenente al fascio Asn, MS, f. 5210 è citato in Strazzullo 1968, pp. 211-212. Oggi purtroppo, il documento non è più presente nella suddetta unità archivistica.

161 Francesco Supriani o Sopriano (1678-1753) si formò presso il conservatorio della Pietà de Turchini, violoncellista itinerante fra la Real Cappella di Napoli e quella di Barcellona, fu autore dei Principij da imparare a suonare il violoncello, da cui si evince l'altissimo grado di virtuosismo raggiunto nella città di Napoli a quel tempo. Si veda BACCiAgalupPI 2013, pp. 183-214; Olivieri 2009, pp. 109-136.

162 Nicola Fiorenza (1726-1764) fu docente di violino, violoncello e contrabbasso presso il conservatorio di Santa Maria del Loreto. Parallelamente all'insegnamento dovette far parte dell'orchestra del Teatro San Bartolomeo e della Real Cappella, in cui assunse il ruolo di primo violino dal 24 aprile 1758. Compose molta musica da camera e alcuni concerti per violino o violoncello ed archi.

163 Celano 2001, Giornata VI, pp. 667-676. 
I maestri di cappella presenti presso l'istituto risultano essere quelli attivi presso la Cattedrale: Angelo Durante dal 1719 al 1721, Giacomo Sarcuni, fra 1744 e 1745, Domenico de Floro nel 1730, Gennaro Manna nel 1776, ingaggiati per le festività di San Francesco d'Assisi, Sant'Orsola, la Santissima Trinità, Sepolcro, Candelora e Santo Natale.

Il monastero beneficiava inoltre dell'ausilio del conservatorio della Pietà de' Turchini, come lo stesso Rettore Matteo Lambiase attestava nel 1779, a riguardo dei servizi musicali prestati alle monache:

Attesto io qui sotto Rettore di questo real conservatorio di S. Maria dei Turchini come in tutto l'anno scorso 1778 si sono fatti nel venerabile monistero della SS. Trinità delle signore dame religiose i seguenti servizij di musiche, cioè: A 10 aprile venerdì di Passione, lo Stabat. A 16 detto Giovedì Santo. A 17 detto Venerdì Santo. A 18 detto Sabato Santo. A 19 domenica di Pasqua. A primo di giugno messa. Nel giorno del padrocinio di Maria, ss. messa. Nel giorno della Concezione messa. Nella notte del S. Natale messa. Nelli mesi di luglio, settembre, novembre messe tre di Requiem, quali alla ragione di carlini 30. Li detti servizij di Gloria [...] è di carlini 19. Quelli di Requiem giusta il convento, fanno la summa di doc. trentuno e gra. $50[\ldots] .{ }^{164}$

Le somme corrisposte ai musicisti sono abbastanza ingenti, ciascuna festa superava 100 ducati di spesa per la musica, tanto che nel «Sistema per il buon regolamento del Monastero» del 1763 veniva severamente ribadito alle monache di non superare «la somma di docati ottanta per la musica in ogn'una di esse festività [SS. Trinità, S. Francesco, sepolcro, candelora $[\ldots] \gg{ }^{165}$

\subsection{San Francesco delle Cappuccinelle}

Il monastero venne eretto nel XVI secolo, nella strada denominata «Pontecorvo», dalla vedova del Duca di Scarpato, Eleonora, per adempiere ad un ex voto, allo scopo di potervi ospitare ragazze madri. Inizialmente gestito da monache francescane, fu trasformato da conservatorio in monastero, essendo cresciuto il numero delle figlie ospiti. Venne così istituito ufficialmente nel 1671 sotto la regola di Santa Chiara, rivisitata secondo la regola cappuccina. Nel Settecento il numero delle religiose crebbe e grazie all'ingente patrimonio, costituito da donazioni e rendite, seguirono numerosi ed importanti restauri. ${ }^{166} \mathrm{Nel} 1866$ il monastero venne soppresso e convertito in riformatorio minorile.

Il monastero non sembra avere una propria attività musicale. Si affidava ai conservatori maschili per il supporto musicale e nello specifico al conservatorio della Pietà de' Turchini, come testimonia il seguente documento:

164 Asn, MS, f. 5201, app. p. 181.

165 Asdn, Vicario, 348 D, app. p. 190.

166 Il complesso, nel 1712, fu completamente ristrutturato da Giovan Battista Nauclerio. Sulla storia dell'istituzione si veda: Araldo 1998, p. 279; Galante 1985, Giornata XII, p. 408; VAlerio 2006, pp. 163-167. 


\begin{abstract}
Pagato al real conservatorio di Santa Maria della Pietà docati 30 con poliza per il banco della Pietà per l'annata maturanda alla fine del corrente mese di marzo 1769 , per tanti convenuti pagarli per tutte le funzioni in musica se fanno nella nostra chiesa, nelle quali devono intervenire i figlioli di detto Conservatorio col di loro maestro di cappella andando a peso di detto Conservatorio di portare quelle voci et istromenti acorrano nelle musiche suddette, senza che il nostro monastero sia ad altro tenuto, così appuntato e convenuto, e stante il presente pagamento resta detto Real Conservatorio soddisfatto. ${ }^{167}$
\end{abstract}

Si riscontrano solo generici pagamenti per «li Mostaccioli per li Musici», «libre di candele per li Musici», «dolci alli Musici», «affitti d'organo, tira mantici per l'organo, provviggioni all'organista», ${ }^{168}$ messe cantate.

\title{
4.4 San Francesco dell'Osservanza
}

Il complesso fu costruito nel 1325, per volontà del re Roberto d'Angiò e di sua moglie Sancia, quale residenza per le monache di Santa Chiara in via di costruzione. Una tradizione invece, ne fa risalire la nascita alla donazione di un ritratto dal vero del Santo, da parte di una monaca di Assisi alle consorelle napoletane. Le monache ivi residenti erano terziarie francescane e fino al 1568 il monastero non fu sottoposto alla clausura. ${ }^{169} \mathrm{Nel}$ monastero abitò per trent'anni Giulia Gonzaga, ricordata da Benedetto Croce come una delle protagoniste del fallito tentativo di riforma religiosa tentato nel XVI secolo. Scappata da Fondi, la Gonzaga a Napoli entrò in contatto con il circolo valdesiano e il suo fondatore, Juan de Valdés, facendo diventare questa chiesa centro del movimento francescano dell'Osservanza.

L'attività musicale dell'istituto è per lo più legata alle liturgie ufficiali come testimoniano gli esiti dei «trienni di badessato»:

Si sono spesi in tutto questo triennio d. secento settantasette t. 3.19 [...]. Consiste la sudetta spesa tanto nelle tre Musiche fatte nell'annuali feste di San Francesco, di dedicazione della nostra Chiesa, fattura di Sepolcro, Apparatura di detta Chiesa, Assistenti, Clerici estraordinari, ostie ed ogn'altro occorso per ogni funzione della Chiesa. Come più diffusamente si legge dal libro del esito f. 29. ${ }^{170}$

La festività più rilevante risulta essere quella di San Francesco, che prevedeva la partecipazione dei figlioli del conservatorio di Santa Maria di Loreto e dei Poveri di Gesù Cristo e musica «così di voce come d'istromenti» dell'Arcivescovado, con la presenza, tra 1725 e 1731, di Andrea Amendola e Angelo Durante.

167 Ass, MS, f. 4547, app. p. 192.

168 Asn, MS, f. 4577, app. pp. 193-195.

169 La chiesa è stata ripetutamente rimaneggiata. Nel 1629 venne eretto il muro di clausura e modificato l'interno. Nel 1646 venne restaurata e riconsacrata. Cfr. De Stefano 1560, p. 184, D'EnGenio 1624, p. 252; D’Aloe 1883, p. 294; Celano 2001, Giornata III, pp. 429-432; Galante 1985, Giornata IV, pp. 144-146; VALERIo 2006, pp. 169-173.

170 Ass, MS, f. 4492, app. p. 203. 
Oltre San Francesco le liturgie celebrate con musica risultano essere quella del Corpus Domini seguito dall'Ottava, San Silvano con processione, la festa della dedicazione della chiesa e il Santo Natale.

Nel decennio francese il monastero fu soppresso e destinato a quartiere militare, le monache furono trasferite a Santa Chiara. Nell'Ottocento divenne sede di un educandato femminile fondato da Rosalia Prota, dove si insegnava, assieme alla grammatica, aritmetica e arti donnesche, anche la musica. Probabilmente l'insegnamento musicale dovette avere un ruolo di primo piano, in quanto le esecuzioni delle fanciulle furono spesso segnalate dalle cronache dell'epoca. ${ }^{171}$

\subsection{Sant'Antonio a Port'Alba}

Il monastero denominato anche Sant'Antoniello delle Monache, venne fondato nel 1564 da suor Paola Cappellani. Col passare degli anni vennero annessi al monastero alcuni edifici adiacenti e venne anche realizzata una chiesa dedicata a Sant'Antonio da Padova. ${ }^{172}$ Era una comunità nota per la decadenza morale. Le festività solennizzate risultano essere quelle di Sant'Antonio e del Corpus Domini per le quali si riscontrano generici pagamenti per spese destinate alla musica della Cappella dell'Arcivescovado. Le «monache di Sant'Antoniello» venivano inoltre criticate per l'organizzazione di commedie in luoghi non consoni, ovvero, dove potevano essere viste dal vicinato:

Le Signore Monache di Sant'Antoniello si preparano à far la comedia, e han determinato farla in un luogo dà dove sono intese da alcuni secolari convicini. Che però si prega chi spetta, ò proibire in tutto, che non si faccia detta comedia, la quale caggionar suole non piccolo danno, ò veramente proibire, che non si faccia in quel luogo dà dove sono intese a tal luogo altre volte è stato proibito per il fine sudetto. ${ }^{173}$

\subsection{Santa Maria Maddalena Maggiore}

Sancia di Maiorca, impegnata verso il 1324 in un'opera di 'redenzione' delle prostitute napoletane, fondò, con tale scopo, un primo ritiro dedicato a Santa Maria

171 Rosalia Prota è una figura di notevole importanza nella storia dell'educazione musicale femminile. Agli inizi dell'Ottocento fondò e diresse a Napoli alcune case di educazione e collegi di musica. Sul ruolo svolto dalla Prota e la nascita degli educandati femminili ottocenteschi si veda ConTi 2003, pp. 43-46.

172 De Stefano 1560, p. 187, D’Engenio 1624, p. 222, 182; Celano 2001, Giornata VII, pp. 402-405; Galante 1985, Giornata III, p. 108; VAlerio 2006, pp. 103-109.

173 Il documento è citato nella corrispondenza fra arcivescovi e vicario delle monache, appartenente ad un fascio relativo al monastero della SS. Trinita. Cfr. AsDn, Vicario, 347 D, app. p. 188. 
Maddalena. ${ }^{174} \mathrm{Il}$ monastero fu ampliato nel 1343 e la comunità femminile fu sottoposta da Sancia alla regola agostiniana, ma la cura spirituale delle monache venne affidata ai frati minori francescani fin dal 1341. Intorno al 1478, le religiose vennero trasferite nel vicino monastero di Santa Caterina a Formello, per volere di Alfonso II d'Aragona. Qualche anno dopo le monache poterono far ritorno al loro monastero. Il complesso monastico fu restaurato integralmente nel 1721 e ampliato da Mario Gioffredo nel 1765, fu soppresso nel 1808 e demolito definitivamente nel 1955. ${ }^{175}$

Le testimonianze documentarie a noi giunte sono molto scarse. Trattandosi di monastero di regio patronato, non vi è traccia nei registri copiapolizze dei banchi napoletani. ${ }^{176} \mathrm{I}$ documenti interni dell'istituto indicano quasi esclusivamente l'attività degli organisti in servizio quali Ambrosio Molinaro nel 1682, Tommaso Tango fra 1697 e 1698, Giuseppe e Tommaso De Martino attivi nel Settecento. La «Gazzetta di Napoli» ci racconta invece della solennità di Santa Maria Maddalena per la quale, nel 1735, si era predisposto un «nobilissimo apparato e più cori di scelta musica, così ne' primi vesperi $[\ldots]$ come nel giorno della solennità [...]». Il cronista prosegue specificando che alla fine dei secondi vespri «vollero quelle dame monache far palese l'inesplicabile divozione che professono al nostro invittissimo, glorioso re [...], facendo cantare [...] da' primari virtuosi musici che sono in questa capitale solenne Te Deum [...] per lo felice arrivo della maestà sua nel Regno di Sicilia e resa di quella cittadella $[\ldots] \gg .{ }^{177}$ Le francescane di Santa Maria Maddalena usavano infatti dimostrare la loro benevolenza nei confronti della corte e dell'aristocrazia tutta, festeggiando vittorie, nascite e matrimoni reali. ${ }^{178}$

Qualche informazione sulle richieste che il monastero inviava alle autorità ecclesiastiche si trova nelle carte e nei permessi del monastero di Santa Chiara. Condividendo la stessa fondatrice ed essendo entrambe istituzioni regie, probabilmente l'ordinario diocesano che le tutelava doveva essere lo stesso. ${ }^{179}$ Pertanto troviamo domande di «puoter far Musica in occasione di dar l'Habito ad una Novitia», cui però facevano seguito ordini precisi circa le restrizioni da seguire in diverse situazioni liturgiche:

Molto Reverendo Padre. Altre volte hò ordinato, che nel Monastero della Madalena in occasione di vestizioni e Professioni non si permetta il cantare dialoghi volgari; che non si conceda l'istromento di cantare in musica la settimana santa; e che non si dia licenza alla Signora sagrestana di pigliare argenti in presto ò in affitto per occasione di qualsivoglia festa [...]. Roma 4 marzo $1690 .{ }^{180}$

174 Con la stessa finalità Sancia fondò anche S. M. Egiziaca a Forcella. Cfr. p. 138.

175 De Stefano 1560, p. 185; D’Engenio 1624, pp. 396-397; D’Aloe 1883, p. 693; Celano 2001, Giornata III, pp. 836-839; Galante 1985, Giornata VII, pp. 272-273.

176 Entrate e uscite delle istituzioni regie sembrano non passare per gli istituti di credito napoletani. Lo stesso accade per il monastero di Santa Chiara.

177 GDN,12 aprile 1735 (2), app. p. 211.

178 Cfr. cap. III, p. 74.

179 Sancia di Maiorca aveva fondato anche il monastero di Santa Chiara. Cfr. cap. VI, pp. 169-170. 180 AsN, MS, f. 2702, app. p. 264. 
Sembra che le autorità usassero divieti maggiori per la Maddalena, vista forse la finalità dell'istituto. Per entrambe le istituzioni era espressamente proibito «ogni sorte di canto figurato, ancorche senza suono», tuttavia alle monache di Santa Chiara era comunque concesso «cantar le lamentationi purche non vi intervengano secolari». Alle monache della Maddalena era invece indicato di usare «in detti giorni solamente il canto Gregoriano», affinché «il Popolo resti sempre dalle Religiose $[\ldots]$ in quei santi giorni di Passione, edificato»». ${ }^{181}$

\subsection{Santa Maria della Provvidenza ai Miracoli}

Nel 1616 i riformati conventuali di San Lorenzo ottennero un terreno dalla famiglia Vivaldi e vi edificarono una chiesa con annesso monastero dedicandolo a Santa Maria dei Miracoli. Il complesso fu ben presto abbandonato per essere acquistato, nel 1660, dai governatori del palazzo del Pio Monte della Misericordia, legali esecutori testamentari di Giovan Camillo Cacace, famoso uomo di legge, ${ }^{182}$ il quale, alla sua morte, aveva destinato tutta la sua sostanziosa eredità alla fondazione di un educandato femminile. Nel 1675 i governatori del Monte della Misericordia affidarono a monache del terz'ordine di San Francesco il nuovo complesso monastico che prese il nome di 'Santa Maria della Provvidenza', conosciuto però dal popolo fino al giorno d'oggi come 'I Miracoli'. Il monastero si popolò di monache provenienti da nobili famiglie. La prima badessa, suor Maria Agnese, proveniente dal monastero della Trinità fu la sorella del cardinale Caracciolo. Godendo di pieni poteri e rendite considerevoli, la badessa Caracciolo intraprese la decorazione di tutto il monastero servendosi dei maggiori artisti e non badando a spese. ${ }^{183} \mathrm{Fu}$ infatti la stessa badessa ad ingaggiare nel 1677 il famoso organaro Andrea Basso per la costruzione di un imponente organo, che divenne in breve tempo rinomato in tutta la città, essendo stato anche sottoposto al giudizio di due «esperti virtuosi»» ${ }^{14}$ prima di essere accettato dalla comunità. Purtroppo la vita di questo pregevole strumento fu breve. Nel 1723 la cantoria precipitò con musicisti e cantanti e l'organo andò di conseguenza distrutto:

Al Monastero della Provvidenza ducati 59.50, E per esso a Giacomo Pompilio per averli distribuiti alli musici et strumenti che han patito danno nella cascata che fecero con l'orchestra

181 Asn, MS, f. 2702, app. p. 264.

182 Giovan Camillo Cacace era presidente prima della Regia Camera della Sommaria e del Collaterale.

183 Sulla ricostruzione storica dell'istituto si veda: Celano 2001, Giornata VII, pp. 405-417; Galante 1985, Giornata XIV, p. 437; Sigismondo 1789, vol. II, pp. 34-36; Valerio 2007, pp. 285-293; NAPPI 1982.

184 NAPPI 1982, p. 206. 
dentro la loro chiesa il dì 21 prossimo passato mese di agosto per essernosi ritrovati li medesimi in quell'ora sopra di detto orchestro sonando e cantando. ${ }^{185}$

L'organo venne poi realizzato nuovamente solo nell'Ottocento. Inoltre presso il monastero vi erano altri due organi fittizi. Fra 1679 e 1680 si trovano infatti numerose provisioni ai mastri Nicola Schisano «per l'organo finto», ${ }^{186}$ a Francesco Antonio de Maria «per le canne di legname di ceraso tornite per l'organo finto», ${ }_{187}$ a Domenico Russomando per «l'indoratura delli choretti et organi»" ${ }^{188}$ e ancora «per lettorino de Balaustra dell'organo» e per «haver pittato li soffitti dell'organo». ${ }^{189}$ «Musici dell'Arcivescovado» e «musici et instrumentisti estraordinari» provvedevano al corredo musicale sotto la guida di maestri di cappella Andrea Amendola, Angelo Durante e Giacomo Sarcuni. Le principali feste celebrate con musica risultano essere legate ai culti mariani dell'Immacolata Concezione e dell'Assunta, alla Settimana Santa e alla SS. Trinità, alla devozione per San Giuseppe e San Francesco per cui era previsto anche l'intervento di flotte dei fanciulli del conservatorio dei Poveri di Gesù Cristo. La prassi dell'istituto, circa le feste in cui era concesso l'utilizzo della musica, avrebbe dovuto essere la seguente:

La Festa che devesi celebrare sollennemente è quella dell'Assunta con musica à un coro solo di poche voci e così ancora si celebri la Festa della SS. Trinità e del Glorioso Padre San Francesco nelli di loro proprj giorni; l'altre come quella della SS. Concezzione, di S. Giuseppe, de Santi Angeli, e San Gabriello, che si celebra a 14 di Marzo, di San Giovanni Battista e di San Giovanni Evangelista, di San Gennaro, e di Sant'Antonio di Padua, si celebrino sollennemente, però senza musica [...].Viene proibito dal Testatore il far Maschere in tempo di Carnevale, ma vuole, che s'abbiano da prendere divertimenti leciti $[\ldots] .{ }^{190}$

Le guide spirituali delle monache dei 'Miracoli' non si stancavano di tutelare l'onestà e la dignità monacale correggendo gli abusi allorché riscontrati. ${ }^{191}$ Concedevano infatti la dispensa per la festività dell'Immacolata Concezione affinché fosse celebrata adeguatamente e con musica, e nello stesso momento ribadivano la moderazione in tutte le altre festività:

A riserba di quelle Festività dichiarate dal Testatore, che si sollennizzino con pompa e Musica, e quella dell'Immacolata Concezione di Maria Santissima permessa da Nostri Predecessori celebrarsi parimenti con musica de Figliuoli di uno de Conservatori di questa nostra Capitale per segnalare grazie, che ne ha la comunità continuamente ottenute; tutte l'altre ordiniamo, che

185 NAPPI 1982, p. 198; p. 215. Il documento si trova in AsBN, BSS, 1118 1723, è riportato anche in AsN, MS, f. 3941, cfr. app. p. 215.

186 Asn, MS, f. 3940, app. p. 213.

187 Ass, MS, f. 3940, app. p. 212.

188 Asn, MS, f. 3940, app. p. 213.

189 Asn, MS, f. 3940, app. p. 213.

190 Asn, MS, f. 3959, app. pp. 215-216.

191 Si veda anche il decreto emesso dal cardinale Spinelli nel 1744 a seguito della Visita Pastorale presso la Provvidenza precedentemente citato. Cfr. cap. I, p. 40. 
siano celebrate con pompa giusto il volere espresso dal detto Testatore, ma senza Musica, acciò non resti aggravata la coscienza nostra, e quella della Madre Guardiana, e sue religiose [...]. ${ }^{192}$

Particolare moderazione era infine riservata ai riti di professione religiosa, non sembrava infatti opportuno che «per solennisare con magior pompa la vestizione e professione di qualche figliola», i parenti fossero «obbligati aggiungere altre voci a quelle che la comunità avea prese per cantare la Messa, e le Vesperi», per cui si ordinava che tali solennità venissero svolte senza «apparati superflui» e soprattutto senza che le famiglie delle novizie avessero «motivo di giustamente lagnarsi». ${ }^{193}$

\section{Canonichesse Lateranensi}

\subsection{Santa Maria Regina Coeli}

L'ordine dei canonici lateranensi possedeva tre monasteri a Napoli di cui Regina Coeli era quello femminile. La storia dell'istituzione ha inizio nel 1518, quando quattro suore del monastero benedettino di Santa Maria ad Agnone, decisero di convertirsi alla regola dei canonici di Sant'Agostino, ottenendo successivamente l'uso di un'antica chiesa in cui fondarono il loro monastero. ${ }^{194}$ Santa Maria Regina Coeli ospitò suore provenienti dalle famiglie nobili napoletane, indicate spesso con il nome di 'Rocchettine'. Le monache che sembra avessero raggiunto il numero di 100 nel XVII secolo, erano affidate alla direzione spirituale dei Canonici di San Pietro ad Aram.

Nonostante la sua importanza, la documentazione superstite è abbastanza lacunosa e le fonti archivistiche sembrano riferirsi esclusivamente ad un'attività musicale sostanzialmente ordinaria, legata alla preparazione delle principali feste del monastero. Il confronto con le cronache coeve e con altre fonti collaterali,

192 Asn, MS, f. 3959, app. p. 216.

193 Asn, MS, f. 3959, app. p. 216.

194 Caterina Mariconda, Margherita d'Aragona, Lucrezia e Aurelia Oliviero decisero di lasciare Santa Maria di Agnone e di fondare un nuovo monastero. Ad esse si aggiunse Francesca Gambacorta destinata poi a divenire la prima badessa di Regina Coeli. Due terremoti distrussero l'edificio che era stato scelto inizialmente come sede del monastero. Le monache furono dunque costrette a trasferirsi in una nuova dimora. L'ordine venne soppresso nel 1808 e le monache trasferite nel monastero di Gesù e Maria. Più tardi nell'antico edificio di Regina Coeli, ritornarono le suore della Carità di San Vincenzo de' Paoli fondate dalla francese Giovanna Antida Thouret che volle traferirsi nel monastero napoletano. Il monastero è tuttora attivo ed è considerato uno dei più belli della città conservando opere di Luca Giordano, Lorenzo Vaccaro, oltre ad un imponente chiostro cinquecentesco. Sulla storia dell'istituto si veda: De Stefano 1560, p. 171, D’Engenio 1624, p. 193; D’Aloe 1883, pp. 545-546; Celano 2001, Giornata II, pp. 68-73; Galante 1985, Giornata III, pp. 89-91; CeCi 1899, pp. 24-26; Miele 2001, pp. 91-138; VAleRIO 2006, pp. 263-268. 
ci porta invece comprendere che nonostante le esigue indicazioni, il monastero doveva avere un'importante vita musicale.

La solennità principale era quella del santo patrono dell'ordine Sant'Agostino, ${ }^{195}$ cui faceva seguito anche una solenne processione:

Si fa piena, et indubitata memoria [...] sotto li 27 del mese di agosto 1677 di della vigilia del glorioso Padre Sant'Agostino, si portò processionalmente la statua del detto glorioso santo con tutta pompa e musica dalla clausura del Venerabile monastero di Santa Maria Regina Coeli, e si portò per la strada del Venerabile Monastero di Sant'Andrea e si portò dentro la chiesa del detto Monastero di Regina Coeli per la porta grande, nella qual processione intervennero così li Reverendi Padri di Santa Maria delle Grazie, come quelli di San Pietro ad Aram et altri sacerdoti, et in fede del vero. Napoli primo di settembre $1685 .{ }^{196}$

Le principali feste celebrate risultano essere l'Assunta, il Natale e l'Epifania e la Settimana Santa. Alcune liturgie erano talmente sontuose da essere venire citate negli avvisi della «Gazzetta di Napoli»:

Nella settimana passata sono stati tutti gli ordini religiosi impiegati ne' divini uffici ed altre sacre funzioni eseguite con tutta la maggior decenza e proprietà, specialmente nel R. monistero di dame moniche di Reginacaeli [sic], dove è concorsa la più scelta nobiltà e la gente più culta di ogni ceto, per unire alla divozione il piacere della bene intesa musica, all'ultimo segno lodata e con universale sentimento creduta inarrivabile nel salmo Miserere. ${ }^{197}$

I maestri di cappella che si avvicendarono presso l'istituto furono Giuseppe e Nicola Conti, seguiti da Giuseppe de Magistris, Alessandro Speranza, Ferdinando de Donati. È da sottolineare inoltre l'esistenza di una messa per tre soprani composta da Nicola Conti che forse potrebbe essere destinata proprio a questo monastero. ${ }^{198}$ Nel 1739 la badessa Marianna Scalera consegnava 22 ducati a suor Maria Chiara D'Andrea da «spenderli in una composizione di Musica per la notte di Natale», singolare combinazione, in quanto, alcune monache D'Andrea risultano essere dedicatarie di alcune partiture di Johann Adolph Hasse, Leonardo Leo e Nicola Porpora, custodite presso la Biblioteca dei Girolamini. ${ }^{199}$

Essendo monastero della nobiltà napoletana, non mancavano celebrazioni per occasioni legate alle famiglie aristocratiche. Ancora una volta la «Gazzetta di Napoli» riporta che nel 1727, precisamente il 27 giugno, in occasione della nascita del figlio primogenito di Carlo di Borbone, il monastero aveva reso grazie festeggiando «con tre sere di magnifiche illuminazioni e con la solenne messa e $T e$

195 C'era una disputa fra i padri agostiniani del monastero di Sant'Agostino Maggiore e le monache di Regina Coeli sul privilegio di celebrare la festa di Sant'Agostino. La controversia venne risolta celebrando la festa del santo un anno ciascuno.

196 Asn, MS, f. 1976, app. p. 225.

197 Gdn, 30 aprile 1737, app. p. 232. Cfr. Magaudda/Costantini 2009, gli autori ipotizzano che autore di questo Miserere potrebbe essere lo stesso maestro di cappella Nicola Conti.

198 La messa è stata ritrovata da Claudio Bacciagaluppi. Si veda pertanto BaCciagaluppi 2010, pp. 69-72.

199 Cfr. cap. IV, pp. 115-116. 
Deum con scelta musica e sparo di mortaretti [...]». ${ }^{200}$ Il monastero si distingueva anche per le sontuose monacazioni. Ad esempio «primaria nobiltà ed altro numero indicibile di gente, che fu trattenuta con quattro cori di scelta musica, regolata dal maestro di cappella Niccolò Conte» ${ }^{201}$ era intervenuta per la professione religiosa della figlia del principe d'Ischitella. ${ }^{202}$ Inoltre, il maestro di cappella Alessandro Speranza compose nel 1781, cinque antifone per contralto per la monacazione della «Reverendissima Signora Mariantonia Pignatelli» avvenuta nel monastero. ${ }^{203}$ Allo stesso Speranza è attribuita la cantata sacra a tre voci La Gara tra la Fede e la Fortezza, composta nel 1776, in occasione «che veste l'abito religioso l'Eccellentissima Signora D. Maria Luisa de' Principi Pignatelli». ${ }^{204}$ Consuetudine di questa istituzione fu infatti quella di salutare l'ingresso delle fanciulle nel chiostro facendo eseguire cantate e oratori sacri a loro dedicati con relativa stampa dei libretti. Fra il 1747 e 1781 furono eseguiti il Trionfo dell'Amor divino, una cantata a due voci Nel vestire il sacro abito religioso e gli oratori Rut nel campo di Booz, con musica di Nicola Conti, Lo sposalizio di Rebecca, Il trionfo della croce e il fortunato incontro degli innocenti sposi Isacco e Rebecca su musica di Giuseppe de Magistris e Lo sposalizio di Abigaille con Davide su musica di Antonio Sacchini. ${ }^{205}$

\section{Carmelitane}

\subsection{La Croce di Lucca}

Nel 1534 venne costruita la chiesa con attiguo monastero voluto dai coniugi Andrea Sbarra e Cremona Spinelli, che dedicarono il complesso al crocifisso del volto Santo venerato nel Duomo di Lucca. Il monastero accolse già dai primi anni monache di regola carmelitana provenienti dalla piccola nobiltà di provincia. La fortuna del monastero la fece il Principe di Cellamare Nicola del Giudice, che volle collocare quattro delle sue figlie in questo istituto. «Il Principe», come ricorda il Celano, «oltre le doti et i larghi vitalitii che l'assignò per far conoscere al mondo che queste quattro figliuole solo per forza di spirito, e non per risparmio di dote, s'eran fatte religiose, fece tutto il monasterio di pianta, spendendovi più di cento venti mila scudi oltre le ricche cappellanie che vi fondò; e più havrebbe egli speso 
se più stato vi fusse di bisogno, in modo che questo monasterio è de' belli e de' magnifici che sia non solo nella nostra città, ma per l'Italia $[\ldots] \gg{ }^{206}$

Il legame con Lucca venne rafforzato anche dalla presenza, all'interno dell' $i$ stituzione, di una compagnia di Battenti «dell'istessa nazione Lucchese». Il monastero fu poi soppresso nel $1808 .^{207}$

Pur essendo ritenuto nei secoli un istituto esemplare per pietà e disciplina religiosa, le fonti esigue testimoniano al fianco di un'attività musicale ordinaria anche l'organizzazione di celebrazioni liturgiche e feste sontuose. Le maggiori informazioni sono relative agli organisti che prestarono servizio presso il monastero. Si avvicendano i nomi di Tommaso Pagano nel 1660, Vincenzo Pagano nel 1714, Nicola de Ruggiero attivo nel 1741.

Il servizio musicale era assicurato per le seguenti festività: Natale, la Santa Croce, Santa Maria del Carmine, la Candelora, la Settimana Santa, la Pentecoste indicata spesso come "Pasqua rosata", ${ }^{208}$ il Corpus Domini. Particolare attenzione veniva riservata alla festività della Santa Croce celebrata in due date: a settembre per 1' Esaltazione' e a maggio, in cui si celebrava il ritrovamento della Santa Croce. ${ }^{209}$ Il contributo musicale era assicurato dalla Cappella dell'Arcivescovo che per l'occasione si avvaleva di strumentisti e cantanti 'soprannumerari':

Per la musica per la festività di Santa Croce di Settembre dell'Arcivescovato d. sette t. 4.10

Per l'Istromenti aggionti d. tre t. 1.1.0

Per le voci aggionte d. 2.3.10

Per quello have havuto pensiero della Musica d. due [...]

Per li figlioli del Conservatorio de Poveri di Giesù Christo d.1.1 [...]

Per fare il coretto per li musici d.tre ${ }^{210}$

Il monastero inoltre era rinomato per essere sede di sontuose monacazioni, come riportano cronache e diari dei Cerimonieri. ${ }^{211}$ La professione della figlia del principe di San Vito, vide il concorso di «scelta musica, cantandovi il noto marchese

206 Celano 2001, Giornata II, pp. 278-281. Il principe pretese di conservare il patronato sulle cappelle della chiesa, di essere beneficiario di preghiere di suffragio, di godere dei privilegi di entrata per le visite in monastero.

207 Sulla storia del monastero: De Stefano 1560, p. 87; Caracciolo 1851; Celano 2001, Giornata II, pp. 278-281; D’Engenio 1624, pp. 72-73, Galante 1985, Giornata V, p. 158; VALERIO 2006, pp. 143-147; Novi-Chavarria 2001, pp. 131-149.

208 In alcune zone d'Italia esisteva l'uso, durante la messa di Pentecoste, di far piovere dall'alto sui fedeli dei petali di rose rosse, per evocare la discesa dello Spirito Santo. Per questo la festività prese il nome anche di "Pasqua rosata", tradizione che si conserva tuttora in alcune zone del centro e del sud dell'Italia.

209 La data di settembre celebrava il Trionfo della Croce, in cui si commemorava la conquista della Croce caduta in mano ai persiani e la data in maggio invece il ritrovamento della Santa Croce, ad opera di Sant'Elena. La festività in maggio è stata rimossa dal calendario della forma ordinaria del rito romano in seguito alla riforma liturgica del 1970.

210 Asn, MS, f. 3678, app. pp. 238-239.

211 Si veda a tal proposito la cronaca della monacazione della figlia del Principe di Cellamare, riportata dai cerimonieri dell'arcivescovo. Cfr. cap. III, p. 85. 
D. Matteo Sassano [...]», ${ }^{212}$ oppure la monacazione di «Irena Caracciola, figlia del marchese della Morosa» per la quale oltre alla «scelta musica a più cori» vi fu anche «un singolare apparato fatto dal celebre ingegnere Ignazio Cuomo, e detto apparato si è goduto anche il giorno appresso, per la sua magnificenza». ${ }^{213}$

\section{Clarisse}

\subsection{Santa Maria del Gesù}

Il monastero era probabilmente già esistente nel XV secolo e fu ampliato grazie alle cospicue donazioni della regina Giovanna III, moglie di re Ferrante I d'Aragona. In seguito divenne sede privata per le tombe della dinastia reale. Terminato il regno aragonese il complesso si ritrovò senza finanziamenti. Venne rifondato nel 1582 e intitolato a Santa Maria del Gesù da Lucrezia Dentice e della famiglia Montalto. ${ }^{214}$

L'attività musicale che si riscontra sembra essere del tutto ordinaria con pagamenti agli organari Francesco Cimino e Cesare Catarinozzi, cui era affidata la manutenzione degli organi presenti nella chiesa. Numerosi pagamenti riferiscono dell'organizzazione di solenni «esposizioni» ${ }^{215}$ in occasione di varie festività: per la Purificazione, per il Carnevale, San Giuseppe, la SS. Annunziata, la Beata Vergine delle Grazie, Sant'Anna, l'Immacolata. Inoltre, sempre in occasione delle festività suddette, si trovano pagamenti alle «sonatrici». ${ }^{216}$ Non vi sono però sufficienti indicazioni per comprendere se si trattasse di religiose musiciste che intervenivano musicalmente nelle celebrazioni o semplicemente di religiose addette al suono delle campane. I diari dei Cerimonieri e la «Gazzetta di Napoli» rimandano alle monacazioni avvenute nel monastero per le quali si riferisce sempre di «ricchissimi apparati e quattro cori di scelta musica», ${ }^{217}$ oppure di «sceltissima musica nella mattina e nel giorno, in cui vi cantarono li primari virtuosi di questa città [...]» come in occasione della professione religiosa di Grazia Capano, nipote della principessa di Bitetto. ${ }^{218}$

212 GDN, 14 gennaio 1721, app. p. 242.

213 GDN, 2 febbraio 1734 (2), app. p. 242.

214 Sulla storia del monastero di veda: De Stefano 1560, p. 173; D’Engenio 1624, pp. 499-501; D’Aloe 1883, pp. 512-513; Celano 2001, Giornata I, pp. 671-672; Valerio 2006, pp. 67-73.

$215 \mathrm{Si}$ allude con molta probabilità alle esposizioni del SS. Sacramento.

216 Ass, MS, f. 3126, app. p. 245, la stessa dicitura è presente anche nei documenti del monastero benedettino di Donnalbina, cfr. Asn, MS, f. 3316, app. p. 69.

217 GDN, 27 maggio 1710 (1), app. p. 246.

218 GDN, 30 luglio 1720, app. p. 246. 


\subsection{San Francesco degli Scarioni}

La chiesa venne fondata per volere di Leonardo Scarioni, un mercante di Prato trasferitosi a Napoli, il quale, morto nel 1701 senza lasciare eredi, stabilì con lascito testamentario, che le sue ricchezze venissero investite nella costruzione di un complesso monastico francescano destinato ad accogliere religiose provenienti dalla sua città natia. I lavori, sotto la direzione dell'architetto Giovan Battista Nauclerio, iniziarono nel $1704 \mathrm{e} \mathrm{si} \mathrm{conclusero} \mathrm{con} \mathrm{la} \mathrm{solenne} \mathrm{inaugurazione} \mathrm{del} \mathrm{1721..219}$

L'ingresso delle religiose fiorentine nel monastero partenopeo è segnalato anche dalla «Gazzetta di Napoli» che riporta la cronaca della solenne vestizione avvenuta il 7 ottobre del 1721:

Nel medesimo sabato [4 ottobre] nella chiesa del nuovo R. monastero di S. Francesco, detto delli Scarioni a Chiaia, da questo mons. Vicentini nunzio apostolico si fece la vestizione dell'abito religioso de' Minori Conventuali di S. Francesco delle 27 zitelle della città di Prato in Toscana, venute con le tre monache professe del medesimo ordine per la fondazione e direzione di detto R. monastero. La funzione riuscì decorosa per il concorso di molte dame e cavalieri, di nazionali fiorentini e di persone civili, e per la musica, e non meno di devozione [...] in veder tante verginelle passare processionalmente [...] dal monastero alla chiesa, di dove [...], dalla grata che in essa corrisponde, [mons. nunzio] [...] fece a tutte la fonzione de' capelli, terminata la quale si cantò il Te Deum [...]. E le novelle religiose, per gli auspici e protezione che godono del felicissimo governo del nostro augustissimo monarca, in adempimento del loro obbligo, nel suo fausto giorno natalizio [1 ottobre], con messa cantata e Te Deum, diedero grazie e suppliche all'Altissimo $[\ldots]^{220}$

Nel 1722 le clarisse commissionarono a Fabrizio Cimino la costruzione di un organo. Nelle «Spese de stromenti da suono» del monastero figura infatti la polizza di pagamento al celebre organaro per l'acquisto di «un organo che il medesimo ha venduto e consegnato per servizio di detto monastero». ${ }^{221}$ Cimino aveva inoltre l'incombenza di provvedere alla manutenzione degli strumenti:
Al Monasterio di San Francesco delli Scarioni d. Due, e con firma di Francesco della Posta am- ministratore a Fabrizio Cimino Mastro organaro disse esserno per un'annata maturata a ultimo decembre 1727 per causa di mantenere accomodato l'organo della Chiesa di detto Monastero com'ancora li cembali, ò siano Spinetti sistemati nel detto Monastero, per servitio di quelle religiose $[\ldots] .222$

Il documento dell'Archivio del Banco di Napoli suggerisce quindi un'attività musicale praticata dalle religiose e questa ipotesi è sostenuta anche dai pochi documenti dell'Archivio di Stato che mostrano i pagamenti dei maestri di cappella da cui apprendiamo delle lezioni di musica impartite alle religiose:

219 Galante 1985, Giornata IX, pp. 388-389; Sigismondo 1789, vol. I, pp. 141-143. Si veda anche: Valerio 2007, pp. 170-175; Strazzullo 1975, pp. 70-77.

220 GDN, 7 ottobre 1721 (3), app. p. 250.

221 Asn, MS, f. 4307, app. p. 249.

222 Asbn, BSA 830, 16/II/1728, app. p. 249. 
A Nicola Marinotti maestro di cappella docati 22, cioè: 20 per sua provvisione dal primo marzo 1723, per tutto dicembre a docati 12 il mese per aver dato lezione di canto fermo, e suono alle religiose di detto monastero, e carlini 10 per copiatura di carte $[\ldots] .{ }^{223}$

e ancora:

A Paolo Giugno maestro di cappella docati 18 per mesi dall'aprile 1726 per tutto il 1735, a carlini 20 il mese per dato lezione in detto tempo. ${ }^{224}$

Purtroppo mancano del tutto indicazioni sulle festività celebrate: piccoli accenni riferiscono della festività di San Francesco e Santa Chiara, con la sola indicazione delle messe cantate. 



\section{Il monastero delle clarisse di Santa Chiara}

\section{Il monastero e il suo archivio}

Il monastero delle clarisse di Santa Chiara sorse per volontà del Re Roberto d'Angiò e di sua moglie Sancia di Maiorca. I sovrani, entrambi devoti a San Francesco di Assisi e a Santa Chiara, vollero costruire una cittadella francescana in grado di accogliere nel monastero le clarisse e nel convento adiacente i frati minori. ${ }^{1}$ $\mathrm{Fu}$ la stessa regina Sancia a compilare la costituzione della comunità religiosa, ispirandosi alla regola di clausura data alle clarisse di San Damiano da Innocenzo IV. ${ }^{2}$ L'investitura del monastero di Santa Chiara a sede delle grandi cerimonie di Stato, delle incoronazioni e delle sepolture regali, fece sì che esso diventasse nel corso dei secoli segno tangibile del potere dei sovrani. ${ }^{3}$ La consacrazione del monastero come 'regio' avvenne nel 1317 con la dedica ufficiale al Sacro Corpo di Cristo o Ostia Santa, ma già nei documenti angioini del 1321 viene ricordato semplicemente con il nome di Santa Chiara. ${ }^{4}$

Le famiglie nobili della città erano solite riunirsi a Santa Chiara per assistere alle fastose cerimonie religiose, risiedendo in esso le figlie del patriziato napoletano. In questo monastero era infatti obbligatorio che la badessa appartenesse ad una delle più prestigiose famiglie aristocratiche napoletane quali i Caracciolo, $\mathrm{i}$ Carafa o i Pignatelli, ella era insignita del titolo di 'Regina di Pozzuoli', e, a riprova dell'autorevolezza della sua figura, era autorizzata a portare, in occasione di alcune festività, insegne reali quali manto, scettro e corona.

Il monastero era molto ricco, le doti delle monache, i proventi di arrendamenti e i numerosi censi che esso ricavava dalle case date in pigione, erano andati ad aggiungersi nel corso dei secoli, agli ingenti lasciti della fondatrice. A fine Settecento le monache arrivarono «quasi al numero di trecento, e delle prime famiglie napoletane», come riporta Giuseppe Sigismondo, aggiungendo inoltre che «il

1 Il monastero di Santa Chiara era in realtà un monastero doppio che accoglieva le clarisse e i frati minori francescani. Sull'argomento: GAGLIONE 2007, pp. 127-209.

2 Sancia stabilì l'età di ricezione delle monache, la formazione liturgica e spirituale, le regole interne e la disciplina da seguire all'interno della vita comunitaria.

3 I quattro re delle due Sicilie e gli altri membri della famiglia reale sono infatti sepolti nella chiesa del monastero.

4 Sulla storia di Santa Chiara si veda De Stefano 1560, pp. 180-181.184; D’Engenio 1624, pp. 234-236, 238-239; Celano 2001, Giornata III, pp. 393-426; Galante 1985, Giornata IV, p. 408; Pane 1954; Gallino 1963; Valerio 2006, pp. 127-139; D’Andrea 1987, pp. 39-78; Dell'Aja 1992; Gaglione 2003, pp. 399-431; Gaglione 2007, pp. 127-209. 
monistero è così grande che sembra una città. Vi è un chiostro di 18 archi in quadro, e vi sono dormitorj che da un capo all'altro appena si può ravvisare una persona $[\ldots] \gg{ }^{5}$ Le monache erano divise in due categorie: coriste e converse, le prime denominate signore, le seconde serve. Le coriste provenivano da famiglie nobili e avevano il compito di portare le doti più consistenti, ${ }^{6}$ per tal motivo, ad esse erano riservati gli uffici e gli incarichi maggiori, godevano di voce attiva e passiva, cantavano e recitavano l'ufficio in coro. Le converse disbrigavano invece gli uffici più umili, spesso a servizio delle stesse coriste. Vi erano inoltre, seppur in numero limitato, le educande, che vivevano nel monastero in educazione, sotto la responsabilità di una maestra ma comunque in clausura. Su tutte presiedeva la badessa che veniva eletta dal capitolo monasteriale ogni tre anni con conferma annuale, coadiuvata nel governo dalla vicaria e da sei discrete. ${ }^{7}$ Le monache di Santa Chiara erano inoltre soggette al padre guardiano dell'attiguo convento dei frati francescani, indicato anche come Commissario Apostolico o Vicario del Delegato Pontificio. ${ }^{8}$

La documentazione archivistica relativa al monastero purtroppo è abbastanza lacunosa, a causa anche di un grande bombardamento subito nel $1943^{9}$ che distrusse la chiesa e con essa archivio e biblioteca. ${ }^{10}$ Le informazioni a disposizione rinvenute nei diversi archivi napoletani, incrociate e confrontate con le cronache coeve, le numerose proibizioni e i provvedimenti disciplinari emanati dalla curia, permettono di colmare, anche in minima parte, queste perdite e ci donano informazioni sufficienti a tessere la storia musicale di questo celebre istituto che aveva

5 Sigismondo 1789, vol. II, p. 258.

6 La dote si versava in genere al momento della vestizione ed era conservata nella cassa comune del monastero fino alla professione solenne dei voti a seguito della quale la dote entrava nelle disponibilità del monastero stesso. Sembra infatti che a Santa Chiara fosse una consuetudine assai diffusa il posticipare la data della professione solenne dei voti per ritardare il più possibile la cessione della dote al monastero. Le monache che vivevano tra le professe senza aver fatto professione era chiamate 'indemanie'. Si veda D'ANDREA 1977.

7 La badessa e la camerlenga alla fine del loro triennio erano tenute a presentare i conti della loro triennale amministrazione al revisore dei conti. Si veda D'ANDREA 1977.

8 Le monache erano curate spiritualmente dai frati minori dell'annesso convento (frati minori riformati e frati minori alcantarini) che erano addetti anche all'ufficiatura della chiesa di fuori ovvero della basilica. I frati erano spesati dal monastero che provvedeva anche alla manutenzione ordinaria e straordinaria del loro convento. Cfr. D'ANDREA 1977.

9 I bombardamenti del 4 agosto 1943 non risparmiarono la cittadella di Santa Chiara, l'incendio che seguì divampò per giorni. Il restauro avvenuto nel 1953 restituì alla chiesa le primitive forme gotiche. Tuttavia i lavori di restauro proseguirono negli anni Sessanta e interessarono anche la demolizione delle case e delle botteghe edificate intorno al complesso. Cfr. GAGLIONE 2007, pp. 127-209.

10 L'archivio di Santa Chiara conservava documentazione relativa anche ad altri monasteri cittadini quali il Divino Amore, Santa Maria della Sapienza e Donnaregina. Per una ricostruzione di ciò che era custodito nella biblioteca precedentemente il bombardamento e per le sue vicende storiche si veda D'ANDrEA 1977, pp. 128-146; D'ANDrEA 1976, pp. 95-114. 
un'importante tradizione musicale, sia per quanto concerne la sfera pubblica che per quella privata. ${ }^{11}$

\section{La vita musicale dell'istituto}

A Santa Chiara più che negli altri monasteri femminili cittadini vi fu una certa resistenza alla clausura e alla riforma tridentina, accompagnata dalla rivendicazione e dalla difesa di privilegi e autonomie sul presupposto dell'esenzione dalla giurisdizione dell'ordinario diocesano trattandosi di monastero di regio patronato. Nel corso degli anni non mancarono infatti proteste e opposizioni ai vari tentativi di riforma o di visita degli arcivescovi napoletani. La documentazione del monastero riporta una numerosa serie di richiami e inviti a seguire le restrizioni regolamentari che avevano fatto seguito alla riforma. ${ }^{12}$ Eccessi musicali, divertimenti e ricreazioni non conformi ai regolamenti vengono segnalati a Santa Chiara già a fine Cinquecento e proseguono puntualmente per Sei e Settecento. ${ }^{13}$ Più di una volta il monastero di Santa Chiara aveva dato filo da torcere agli arcivescovi per il suo spirito d'intolleranza disciplinare che probabilmente derivava dalla consapevolezza di essere il più potente ed il più ricco monastero di Napoli. Nel 1724, ad esempio, giunse a Roma la notizia che a Santa Chiara si era fatta «una strepitosa musica [...] con essere stati ad essa chiamati per ostentazione maggiore di fasto ottanta musici», ${ }^{14}$ suscitando l'indignazione della Santa Sede. Scarsi risultati ebbe anche, nel 1724, l'editto del Cardinal Pignatelli in cui l'arcivescovo si pronunciava proprio riguardo al numero e alla tipologia di musicisti da impiegare nei monasteri in occasione delle festività in cui era 'diocesanamente' concessa la musica:

Per moderare gli eccessi introdotti dalle Monache nelle Musiche, concediamo loro che in quelle Feste, ed in quelle Chiese, nelle quali è loro permessa dalle Costituzioni Diocesane la Musica, e non altrimenti; e dove vi è il medesimo uso nella Monacazione, Professione, ò Velazione, e purché non siano solite di pigliare minor numero di voci, e d'istromenti, in tali casi solo possano

11 Il monastero di Santa Chiara è stato sempre considerato come una delle istituzioni più indisciplinate e prestigiose. L'apparente inesistenza di fonti documentarie relative alle diverse attività del monastero ha forse frenato gli studi in campo musicologico. Anche una fonte preziosa per numerose istituzioni religiose come l'Archivio Storico del Banco di Napoli trasmette pochi rimandi alle attività di Santa Chiara. Trattandosi di monastero di regio patronato è probabile che entrate e uscite dell'istituzione non passassero per gli istituti di credito napoletani.

12 Cfr. cap. I, pp. 33-36.

13 Si veda quanto detto in proposito al monastero di Santa Chiara nel cap. I, pp. 33-34.

14 Sono parole di rimprovero che il card. Segretario di Stato Paolucci rivolgeva, a nome del Papa, al cardinale Arcivescovo di Napoli Pignatelli, intimandogli, quale desiderio del Sommo Pontefice, di proibire tali manifestazioni. AsDn, Arcivescovi, Pignatelli, 24 novembre 1724. Il documento è citato anche in Strazzullo 1968, pp. 207-208. 
pigliare diece voci inclusevi anche quelle che dicono del Trattenimento, e quindeci istromenti; e niente più affatto: inclusovi però nel sudetto numero la musica della nostra Cattedrale, secondo il solito: sotto pena dell'Interdetto ipso facto alla Chiesa, e di scomunica à chi la fa fare, ò Monache, ò Secolari; ed anche a' Musici, e Sonatori [...]. 26 settembre 1724. Francesco Cardinale Arcivescovo. ${ }^{15}$

Il monastero inoltre non si assicurava soltanto un discreto numero di maestranze, ma ingaggiava costantemente i musicisti della Real Cappella di Palazzo. ${ }^{16}$ Giustificando la prassi, così rispondeva alle richieste del cardinale la badessa di Santa Chiara:

Si è degnata Vostra Eminenza partecipare con sua stimatissima lettera l'ordine dateli dà Nostro Signore per l'osservanza de' capi dell'editto ultimamente fatto pubblicare per li suoi monisteri, e chiese dà esse Religiose, però con tutta la venerazione dovuta à tali stimatissimi oracoli se li espongono con tutta rassegnatione le seguenti considerationi [...]: Passando al terzo dè divieti delle musiche con restrizioni à sole diece voci, e quindeci Istrumenti inclusa la musica dalla Catedrale. In questo deve riferire Vostra Eminenza la stravagante ampiezza di questa Regal Chiesa quale essendo diece volte più d'ogn'altra Chiesa di monache merita giusto permesso di maggior numero; né in questo vi è alcun disordine quando che esse Religiose non hanno alcuno aspetto in Chiesa, e così cessa ancora il ritrovato delle Gelosie, che già in tante Chiese non si sono adoprate né meno dopo l'editto, come in San Domenico Maggiore, San Sebastiano, ed altre, mentre cessando il motivo di zelo dell'editto, cessa ancora la necessità dell'effetto medesimo; Oltreché mai in detto Regio Monasterio si è chiamata, né si chiamerà la musica della Catedrale, non essendosi mai potuto comprendere nelle Costituzioni Diocesane dell'Arcivescovo; ma si avvale come Regio della musica della Regal Cappella; ed alle volte nelle funzioni Reali sono irreparabili di averne in maggior numero; onde su tali riflessi si rimettino alla prudente determinazione di Vostra Eminenza [...].17

Anche le liturgie di professione religiosa costituivano un'occasione per far musica e preparare ricevimenti. Le famiglie cercavano di conferire il maggior lustro possibile alle cerimonie e chiedevano pertanto dispense per poter far musica come nel caso seguente:

Magnifico Reverendo Padre La Signora Abbadessa di Santa Chiara, mi hà fatto instanza di permettere che in occasione di professare alcune Novizie possino fare la Musica al che sono condesceso per rimostrare nuovamente à coteste Signore il desiderio che hò di sodisfarle in tutte le loro convenienze. Incarico però a V. P. di avertire, che la funzione segua con tal' modestia, et esemplarità; onde non possa qua giungere alcun' richiamo $[\ldots] .^{18}$

La «Gazzetta di Napoli» riporta infatti alcune prestigiose monacazioni avvenute a Santa Chiara con la partecipazione di musici illustri. Nell'ottobre del 1719 per la monacazione di Donna Isabella Ravaschiero, principessa di Belvedere «vi fu

15 Asn, MS, f. 2702, app. p. 265.

16 Si veda il cap. I, p. 43 ove è possibile leggere cosa predisponeva l'arcivescovo Pignatelli nel sinodo del 1726.

17 Ass, MS, f. f. 2702, app. pp. 265-266.

18 Asn, MS, f. 2702, app. pp. 262-263. Si tratta della Copia delle licenze concesse dall'Eminentissimo Protettore circa la Musica da farsi in occasione di Vestire ò Professare, Novitie Chorali, datate Roma 18 ottobre 1683. 
una sceltissima musica, che forse simile non si è qui veduta, e per la quantità degl'istromenti, e per la qualità de' virtuosi, fra' quali vi cantorono il marchese D. Matteo Sassano, il cav. Niccolò Grimaldi e Francesco Vitale, tutti e tre nostri patrioti $[\ldots] \gg ;{ }^{19}$ qualche anno dopo nel 1722 in occasione della monacazione di Donna Vittoria della Leonessa «vi fu superbissimo apparato, con scelta musica a più cori che in questa città si trova, fra' quali anche il marchese Matteo Sassano, con vari concerti d'istromenti nuovi, fatta dal maestro di cappella Niccolò Veneziani $[\ldots]$; vi si portò ancora $\mathrm{S}$. Em. il sig. viceré, il quale volle assistere alla messa cantata, nella cui Gloria, nel Sanctus e nell'entrata della suddetta D. Vittoria nel monastero vi fu sparo d'innumerabili mortaletti, come anco all'atto che fece la detta monaca nel prendere gli abiti monastici, nel qual mentre si cantavano mottetti sacri dal detto marchese Sassano $[\ldots] \gg .{ }^{20}$ Alle monacazioni erano poi associate le rappresentazioni teatrali che spesso facevano seguito al termine della liturgia. A Santa Chiara si trovano numerosi riferimenti a vere e proprie rappresentazioni teatrali, operette spirituali, balletti mascherati e teatri di burattini. ${ }^{21}$

\section{Quotidianità liturgica e prassi musicale}

Ripetute furono, nel corso degli anni, le richieste per poter far ugualmente uso di musica. Per assecondare la volontà delle clarisse e per non scontentare le prestigiose famiglie di origine che sostenevano le monache stesse, si tentava di ovviare al problema 'musica' cercando di arrivare a dei compromessi:

\footnotetext{
Magnifico Reverendo Padre Illustrissimo Signor fece la proibizione della Musica per tutti li Monisteri di cotesta città prendendovi anche gli esenti, come à V. P. è noto. La Madre Abbadessa di Santa Chiara mi scrive pregandomi di levare ta[le] proibizione nel suo Monistero, ed io vorrei per quanto [possibile servirla] mà trattandosi d'un ordine di Sua Santità non dipende dal mio arbitrio il soddisfarla e stò però pensando, che potrebbero le Signore Monache introdurre l'uso del Canto Gregoriano nella maniera che si fa qui nella Cappella Pontificia, poiché con tal formalità avrebbero campo d'esercitare il canto che se bene non è della medesima specie della Musica è però egualmente virtuoso, e quello che importa, assai più divoto, e confacevole allo stato religioso. $[\ldots] .^{22}$
}

Come si evince dalla lettura del documento, datato 1682, il nunzio apostolico consigliava di introdurre anche a Santa Chiara la prassi del canto gregoriano maggiormente indicato al luogo ed espressione di devozione - «alla maniera che si fa [...] nella Cappella Pontificia». Intonare melodie gregoriane e adattare il

19 GDN, 10 ottobre 1719 (1), app. p. 274.

20 GDN, 22 dicembre 1722 (2), app. p. 274.

21 Si rimanda al cap. IV, pp. 102-105.

22 Asn, MS, f. 2554, app. p. 252. 
canto alla prassi dell'epoca era una consuetudine in diversi ordini monastici e in particolar modo in quelli francescani. Si tentava infatti di ottenere una musica liturgica che non sminuisse la sacralità del gregoriano e al contempo potesse dilettare il fedele, diventando orecchiabile attraverso l'utilizzo di stilemi propri della musica vocale e strumentale dell'epoca. La prassi musicale nella quotidianità liturgica era organizzata dai religiosi, dai membri del clero e, in questo caso, dalle monache che assicuravano il servizio musicale alle diverse celebrazioni giornaliere, seppur in maniera più contenuta e sobria. Il canto, assieme all'organo, svolgeva un ruolo decisivo all'interno della prassi liturgica e contribuiva a restituire alle differenti celebrazioni dignità e decoro. ${ }^{23}$

A questa prassi sembrano essere legati gli unici testimoni musicali rinvenuti presso l'istituzione. Si tratta di una messa a due voci femminili e tre antifone di compieta composte da suor Delia Bonito, maestra di coro delle clarisse del monastero. Dell'autrice si hanno solo poche informazioni biografiche. Non si conosce neanche la data della sua entrata a Santa Chiara. ${ }^{24}$ Probabilmente apparteneva alla famiglia dei Bonito, Marchesi di Petruro, nobili del Seggio di Nido. Sappiamo inoltre che nel 1738 firmò i conti presentati dalla badessa Antonia Capece alla fine del suo triennio di badessato. Dal 1735 al $1750 \mathrm{fu}$ discreta, camerlenga, vicaria e infine badessa dal 1751 al 1757, autrice inoltre del rinnovamento della chiesa trasformando l'antico tempio gotico in edificio barocco. ${ }^{25}$

23 Sulla questione musica e quotidianità liturgica si veda Torelli 2005, pp. 447-492; Torelli 2010, pp. 218-249.

24 Sulla figura di Delia Bonito si veda D'ANDREA 2005, tuttavia il breve saggio si sofferma soprattutto sulla storia del monastero, poche informazioni relative a Delia Bonito.

25 Cfr. Gaglione 2007. 


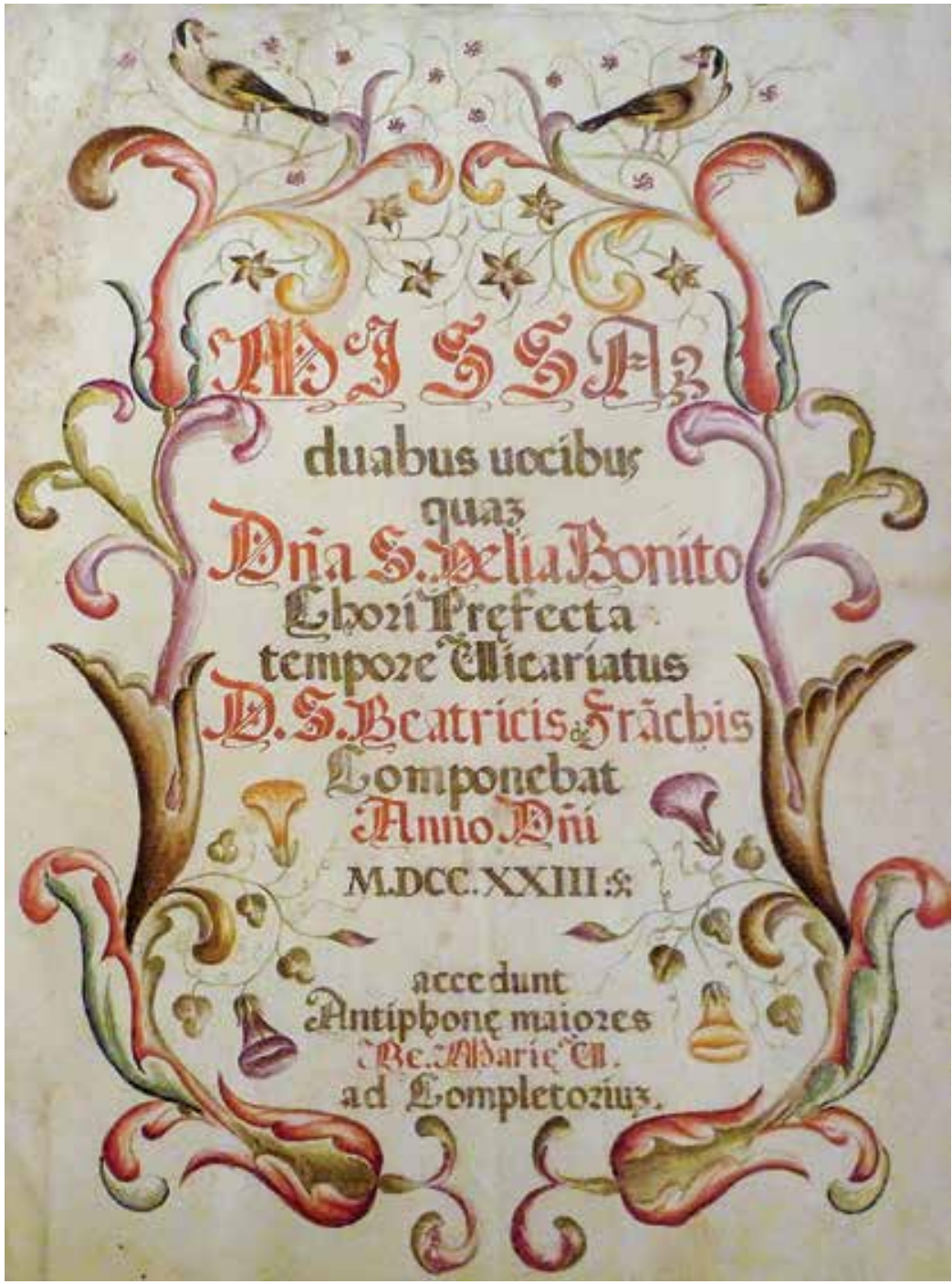

10. Messa a due voci composta da suor Delia Bonito. Frontespizio. Napoli, Biblioteca del monastero di Santa Chiara. ${ }^{26}$

La messa è custodita oggi presso la biblioteca del monastero. Il codice, di grandi dimensioni, si apre con un frontespizio ornato da motivi fitomorfi e zoomorfi in cui si legge: «MISSA/ duabus vocis/ quam/ Donna S. Delia Bonito/ Chori Praefecta/ Tempore Vicariatus/ D.S. Beatricis de Franchis/ Componebat/ Anno Domini/ MDCCXXIII/ accedunt/ Antiphonae maiores/ Beatae Mariae Virginis ad Completorium».

26 Si ringrazia la Biblioteca di Santa Chiara e il ministro provinciale dei Frati Minori di Napoli per aver permesso l'utilizzo delle riproduzioni. 


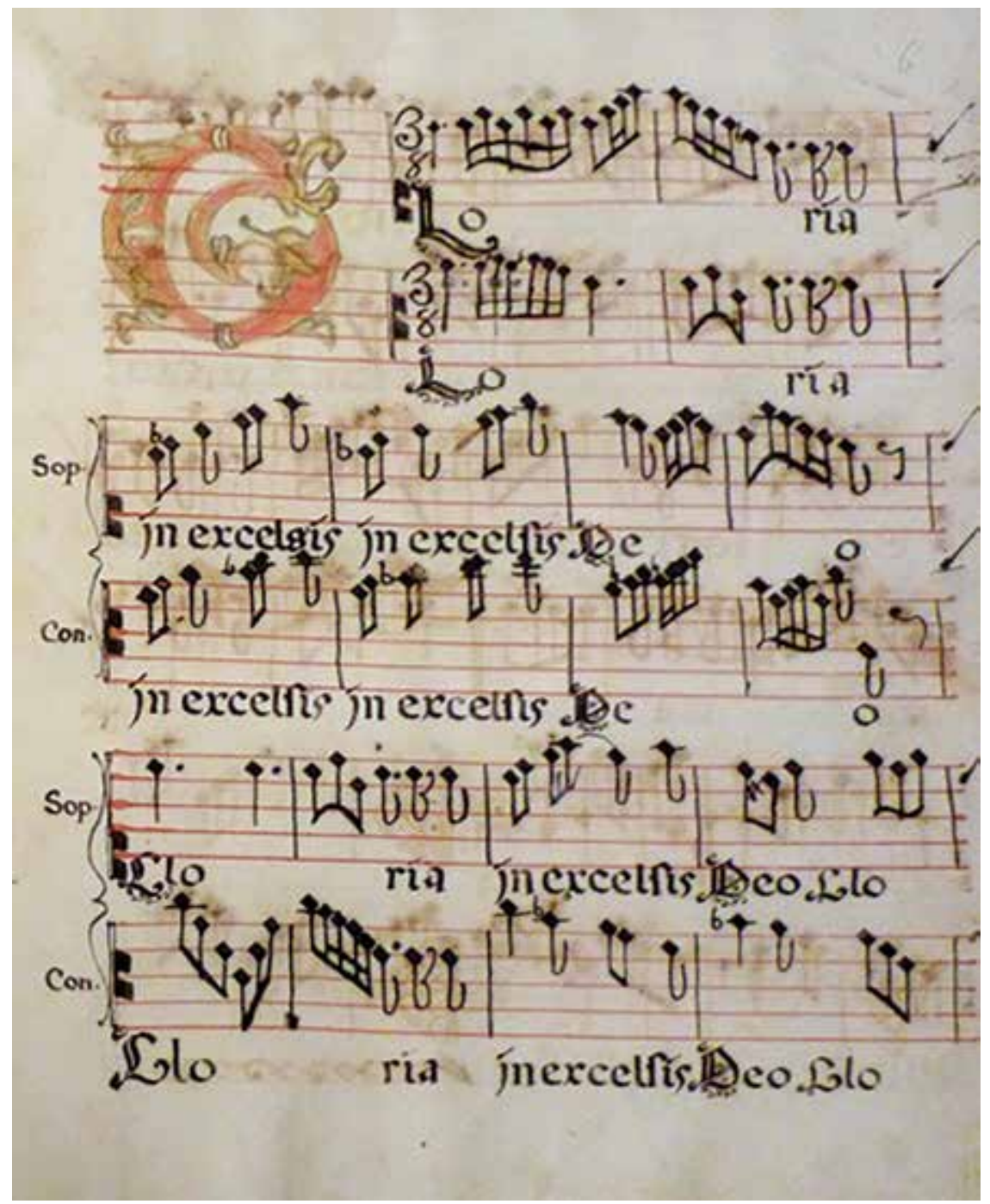

11. Messa a due voci. Gloria.

Napoli, Biblioteca del monastero di Santa Chiara.

Si tratta di una messa in canto figurato a due voci femminili comprendente le parti dell'ordinario (Kyrie, Gloria, Credo, Sanctus, Agnus Dei). Sebbene l'andamento delle voci, così come lo stile dell'intera composizione siano tipicamente settecentesche, l'utilizzo di una notazione romboidale, la presenza di un pentagramma rosso e del custos (che annuncia la posizione della nota successiva), rimanda ad una concezione del canto liturgico che tenta di far dialogare tratti distintivi della musica 'moderna' con la prassi gregoriana. 
Maggiormente ancorate al gregoriano sono le antifone presenti nello stesso manoscritto della Messa. Si tratta delle tre antifone mariane di compieta Salve Regina, Alma Redemptoris Mater, Regina Coeli. Le tre composizioni a due voci fanno utilizzo di una notazione mensurale nera cui si sovrappone una notazione bianca per la seconda voce, presente solo in alcune sezioni. Inoltre una di esse, la Salve Regina, fa utilizzo del tetragramma. Ciascuna antifona prevede la realizzazione della polifonia per terze parallele, con melodie fiorite e reminiscenze gregoriane su un impianto ormai tonale. Vi è inoltre la presenza di numerose progressioni, salti di ottava, alterazioni cromatiche e ornamentazioni virtuosistiche.

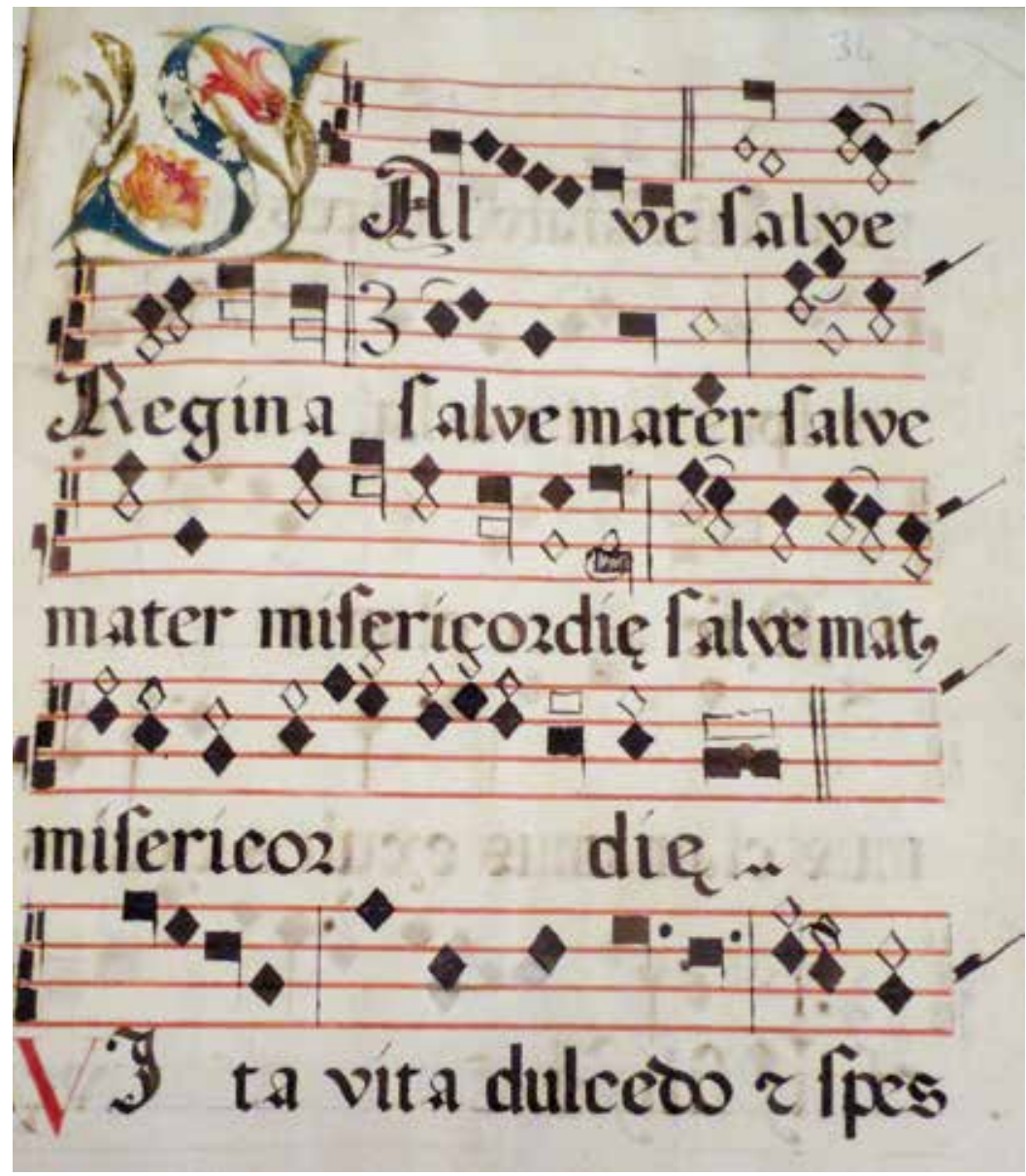

12. Antifone di compieta. Salve Regina.

Napoli, Biblioteca del monastero di Santa Chiara. 
Questi esempi sono assimilabili alla tradizione e all'utilizzo del canto fratto, che conobbe in alcune aree italiane una grande diffusione, soprattutto nella consuetudine liturgica di grandi o piccoli centri religiosi. L'ambiente francescano è stato di certo molto recettivo circa le forme più diversificate di questa tipologia di repertorio. ${ }^{27}$ È inoltre fondamentale sottolineare che siamo in presenza di testimonianze della prassi liturgica del monastero, espressione di usi e costumi di una comunità, ${ }^{28}$ tanto più interessanti in quanto composte da una mano femminile, una clarissa stessa del monastero. Questo ci informa anche sul livello di istruzione musicale delle religiose.

\section{Feste e cerimonie}

Nel corso dei secoli a Santa Chiara si assiste ad un costante e ricco impiego di musica nelle ricorrenze principali dell'anno. In particolare le celebrazioni del Corpus Domini avevano un grande significato per le clarisse così come racconta anche Giuseppe Sigismondo sottolineando la devozione del monastero al culto dell'Eucarestia: ${ }^{29}$

[...] Essendo dunque stata dedicata questa chiesa al Santissimo Corpo di Cristo, il re Roberto volle che la processione del Santissimo Sacramento, che usciva dalla Cattedrale nel giovedì dopo l'ottava della Pentecoste, passata fusse per questa chiesa, dentro della quale avesse l'arcivescovo data la benedizione alle suore ed al popolo, come anche oggi sta in uso; andando il re in questa chiesa in cui si attende nella mattina di detta giornata il Santissimo, indi ricevuta la benedizione dall'arcivescovo, accompagna il Sacramento sino all'Arcivescovato, col torchio acceso in mezzo al corpo della città che gli fa ala, preceduto da tutta la magistratura e tutti i cavalieri di corte per mezzo a due spalliere di soldati che da questa chiesa sino all'Arcivescovato sono dalla mattina situate sotto le loro respettive bandiere. Festa la quale fa onore alla nostra città, sì per la divozione con cui si esegue che per la pompa, degna di esser veduta da ogni forestiere. Dalla vigilia di questa festa per tutta l'ottava, dalle signore monache si fa una ben alta macchina colla esposizione del Santissimo sull'altare maggiore, tutta illuminata a cera, come parimenti sta illuminata d'intorno tutta la chiesa, e dura per sino alle due della notte con musica a più cori e immenso concorso di nobiltà e di popolo $[\ldots] .^{30}$

27 Sulla fioritura del canto fratto in ambito francescano si veda: GozZI 2012; GABRIELLI 2005.

28 Nelle comunità monastiche si tendeva alla creazione di repertori unici di canti liturgici, che sarebbero stati testimonianza nel corso dei secoli del 'suono' dell'istituzione nella quale venivano creati.

29 Sigismondo prosegue chiarendo il perché dell'intitolazione del monastero a Santa Chiara: «La chiesa però porta il nome di Santa Chiara, perché la regina Sancia v'introdusse le monache di santa Chiara d'Assisi della famiglia Lolli, sotto l'istituto del terz'ordine di san Francesco, e quindi venendo dette le suore monache di santa Chiara, così rimase questo nome alla chiesa». SigisMondo 1789, vol. II, p. 258.

30 Sigismondo 1789, vol.II, p. 258. 
Le celebrazioni del Corpus Domini a Napoli duravano una settimana e coinvolgevano la città tutta. La festa aveva inizio con una solenne e grandiosa processione capeggiata dall'arcivescovo, cui prendeva parte il viceré e tutte le autorità cittadine. ${ }^{31}$ Dopo aver fatto tappa nel seggio del Popolo nella piazza della Sellaria, detta anche del Pendino, nella quale era innalzato un sontuoso catafalco, veniva fatta eseguire musica dai «primi virtuosi della città» a cura dell'eletto del Popolo. Come anticipa Sigismondo, la processione terminava poi a Santa Chiara, dove avveniva la pubblica esposizione del Santissimo ${ }^{32}$ e veniva celebrata una messa solenne con «musica sceltissima» con la partecipazione del viceré, delle più importanti autorità cittadine, dell'esercito e della corte. La festa si concludeva con il canto del Pange lingua eseguito dai musici della Cappella Reale e una solenne benedizione finale. Nella settimana successiva al Corpus Domini aveva luogo l'ottavario. Le giornate erano articolate attraverso l'esposizione perpetua del Santissimo che prevedeva al termine dei vespri il canto del Pange lingua affidato ai fanciulli del conservatorio della Pietà de' Turchini. La parte curata dalle clarisse doveva essere alquanto ricca così come indicano anche cronache e avvisi riportando che le «Dame religiose di S. Chiara [...] si fanno sempre ammirare con general plauso, specialmente nel corrente Ottavario del SS. SAGRAMENTO con continua Musica a più cori, Panegirici, e con altre dimostranze fino all'ore notturne $[\ldots] \gg .^{33}$

La documentazione contabile dell'istituzione aggiunge dettagli relativamente alle spese che venivano sostenute in occasione del Corpus Domini. Nel 1736 la somma d'esito per la festività ammontava a 1863 ducati:

Per la Musica oltre l'estraordinario della sera, che si ponerà al suo luogo ed oltre quello si carica al Vicariato d. 528 [...].

Per convito di Messe, stampa d'Indulgenze, Figliuoli della Pietà, e serventi d.55.

E per la Processione dell'Ottava con Altar alla porta, Trombette, rinfresco, e parato, spese in tutto il triennio d. 85

Somma d'esito per detta Festività del Santissimo d. $1863^{34}$

Alcune preziose informazioni sui cerimoniali liturgici festivi a cui spesso prendeva parte il viceré con la Cappella Reale, sono presenti oltre che nei documenti contabili anche in un manuale di «istruzzioni di cerimonie». ${ }^{35} \mathrm{Il}$ padre francescano Ludovico di Bologna, che aveva presieduto all'organizzazione delle cerimonie

31 Sulla festa del Corpus Domini si veda anche Antonelli 2012, pp. 232-238; De Maio 1981; NAPPI 2001; MANCINI 1968.

32 L'esposizione del Sacramento avveniva grazie ad un'autorizzazione pontificia concessa su richiesta di re Roberto d'Angiò, particolarmente devoto al culto dell'Eucaristia, come conferma l'intitolazione del monastero al Corpo di Cristo.

33 Asn, MS, f. 2556, app. p. 256.

34 S. Chiara, Conto Capece, app. p. 269.

35 Le istruzioni di cerimonie scritte dal Padre Ludovico di Bologna si trovano in un fascio denominato Istrumenti, cause e censuazioni AsN, MS, f. 2555, app. p. 253. 
all'interno del monastero di Santa Chiara per una trentina di anni, prima di terminare il suo servizio aveva lasciato alle monache un memoriale delle cose necessarie per l'organizzazione di ciascuna festa. ${ }^{36}$

La festività su cui Padre Ludovico di Bologna si dilunga maggiormente è la ricorrenza della Beata Vergine delle Grazie, che cadeva il 5 di luglio e proseguiva per un mese intero. Per tale ricorrenza era prevista, dietro licenza della corte arcivescovile, l'esposizione del Santissimo da mattina a sera per ciascun giorno del mese:

[...] Il detto mese si comincia il giorno della festività, in cui dopo terminate le seconde vesperi si fa l'esposizione del Venerabile, vi si canta il Pange Lingua, dopo le litanie, e poi si depone con la benedizzione. La musica è stata solita nelle prime vesperi, e giorno della Festività essere a nove voci con quantità d'Istromenti, e però si fa il coro a due registri, poi si restringe ad uno registro, e siegue la musica dell'intiero mese a quattro voci, quattro violini, violoncello, liuto e controbasso. È solita farsi l'esposizione il giorno a circa l'ore ventidue, e mezza, si canta il Pange lingua, poi siegue sinfonia, poi un mottetto, in appresso altra sinfonia, ed in ultimo le litanie, e termina con la benedizzione verso l'Ave Maria. ${ }^{37}$

Al termine del mese festivo seguiva un'ulteriore processione del Sacramento. Nel documento che segue, si comprende come la pompa di tale festività e la musica protratta fino a tarda ora, avessero provocato l'indignazione dell'arcivescovo di Napoli e la conseguente chiusura forzata della porta del monastero. Anche in questo caso la risposta delle clarisse alla Curia di Napoli fece appello al regio patronato e all'impossibilità per un'altra autorità, che non fosse il nunzio apostolico, nel proclamare una qualche disposizione punitiva:

Eccellentissimo Signore

L'Abbadessa, Vicaria, e Discrete del Real Monasterio di Santa Chiara rappresentano à Vostra Eminenza, come hieri cinque del presente mese di luglio dal mastro d'atti della Corte Arcivescovale fù fatto ordine al Padre Guardiano, e Vicario di detto Convento, che al sono della Ave Maria havesero sevrato la detta Chiesa di Santa Chiara, quale al presente con l'occasione della Festività della Madonna della Gratia suole stare aperta per insino ad un'hora, e mezza di notte per causa della musica, litanie, et altre orationi, che si recitano in honore della Beata Vergine con gran devotione, edificatione di tutto il Populo non essendosi mai sentito ne tumulto, ne successo scandalo alcuno in detta Chiesa per tutto il tempo, che si celebra detto mese, nel quale benchè succedesse qualche disturbo, il che non è stato mai, per il che fusse necessario serrare la Chiesa prima del tempo non spettaria alla Corte Arcevescovale dare l'ordini necessari, mà à Monsignor Nuntio, al quale come Chiesa Regia viene affare immediatamente soggetta $[\ldots] .{ }^{38}$

36 Si può leggere in una sorta di prefazione al manuale di istruzioni: «Essendo stato in questo Regal Monastero di S. Chiara per lo spazio di anni 39 il Reverendo P. Lodovico da Bologna, ed ultimamente partito in Aprile 1733 per suo desiderio di voler ritornare alla sua Patria, si è stimato bene, come prattico delle funzioni di essa Regal Chiesa avesse lasciato memoria delle cose più necessarie a notarsi, onde si sono formati li seguenti fogli, con distinzione dell'occorso». AsN, MS, f. 2555, app. p. 253.

37 Asn, MS, f. 2555, app. p. 253.

38 Ass, MS, f. 2579, app. p. 257. Il documento è privo di data e non vi è alcun riferimento al nome dell'arcivescovo cui è indirizzata la lettera. Il documento è contenuto in un fascio del XVII sec. 
Il libello ci informa anche della festività di Santa Chiara, in occasione della quale, dal 10 al 12 agosto, era organizzato un imponente triduo. Le clarisse erano così potenti da ottenere nel 1729 che la solennità di Santa Chiara fosse dichiarata festa di precetto per Napoli e suoi borghi. Cronache e gazzette di Napoli ci descrivono per l'occasione la costruzione di una «maestosa invenzione della macchina», della «preziosità degl'argenti e gemme» e dell' «esquisitezza della musica a più cori $»^{39}$ cui spesso prendevano parte i virtuosi di Napoli come Sassano o Grimaldi.

La statua di Santa Chiara, custodita presso il Tesoro di San Gennaro, veniva portata processionalmente al monastero il 10 di agosto, e all'arrivo in Chiesa la Cappella di Palazzo intonava un solenne Te Deum. Il giorno successivo, $11 \mathrm{di}$ agosto, erano previste «le prime vesperi con musica a più cori».$^{40} \mathrm{Il}$ giorno della festa, 12 di agosto, aveva luogo nuovamente una messa cantata alla presenza del viceré e delle più alte autorità cittadine, infine il giorno 13 con un nuovo corteo composto da frati, ebdomadari, cantori, e figlioli della Pietà de' Turchini, si riportava la preziosa statua al Tesoro. Una relazione distinta della festa avvenuta proprio nel 1729 ci aiuta a comprendere le azioni principali dei tre giorni:

Dal Signor Vicerè oltre la solennità di Cappella Regale con le solite circostanze [...] si diede anche volontà di voler personalmente intervenire alla solenne Processione à quale effetto si fussero replicati li dispacci per altro sparo delle Regie Fortezze, ed intervento delle milizie, città, ministero, clero della Regal Cappella, e musica della medesima per cantarne all'arrivo in Chiesa solenne Te Deum. [...]. Dell'istesso modo si prevennero trà l'angustie del tempo da noi le diligenze più precise per un celebre parato, scelta musica, solenne illuminazione, e decorosi inviti per render plausibile detto triduo festivo [...]. Sporgeano più in su, due quattro grandi, altre tanto ben disposti Orchesti con lavor cesinato, parati dell'istesso nuovo drappo d'argento con francie d'oro per commodo della musica à quattro cori disposta dal maestro di Cappella Niccolò Fago volgarmente detto Tarantino [...]. Pervenuta detta statua all'Altar maggiore s'intuonò dal clero della Regal Cappella il Te Deum proseguito dà musici della medesima accompagnato dà altro gran sparo di 300 mortaletti e scarica del squadrone Alemanno [...]. La mattina seguente del giovedì 11 agosto oltre il gran convito di messe, e di gran numero di Cavalieri si vidde ingombra la Chiesa dà ogni ceto di persone e sul tardi ad ore quindeci vi si portò con le solite formalità l'Eccellentissima Città in corpo al di cui arrivo si cantò il solenne Te Deum intuonato dal maestro Padre Guardiano F. Vincenzo da Zagarolo, e proseguito come pure la messa cantata dà strepitosissima musica à quattro cori e da replicati spari di mortaretti [...]. Il giorno vi fù celebre Panegirico del grande Padre Angelo Maria Fabri de' Padri di S. Geronimo dopo di cui vi fu solennissimo Vespero con l'istessa musica, e gran convito di dame, terminandosi il tutto à circa un ora, e mezza di Notte. Nella mattina seguente del Venerdì [...] altri conviti per la Regal Cappella, serviti sempre con trattenimento della medesima musica [...]. Terminata la musica (seconde vesperi) dopo un ora di notte con l'istessa illuminazione s'istradò la processione conducendo la statua dalla porta della clausura dove fu presa dalle signore moniche, e si formò la solita processione dentro il monastero di cui vollero esserne spettatrici le medesime signore Dame dalla parte della Chiesa e fu nobil veduta l'osservare ancora tutto il cortile illuminato, e la processione istessa tutta con torcie à quattro lucigni [...]. Finalmente la mattina del sabbato 
seguente 13 agosto si ricondusse con solita Processione la statua al Tesoro dopo la messa cantata, ed in tal guisa è remasto adempiuto il detto triduo festivo [... $]^{41}$

\section{La venuta del nuovo Re}

Le clarisse non badavano a spese sia per le ricorrenze religiose che per avvenimenti politici, militari e civili del tempo. Così alle «dimostranze giulive» in occasione dell'elezione di un pontefice si alternavano feste sontuose per i parti delle viceregine, per le vittorie militari etc. ${ }^{42}$

Il mattino del 10 maggio 1734 Carlo di Borbone entrava trionfalmente a Napoli con l'intento di liberare il viceregno dall'oppressione e dal malgoverno austriaco. Il corteo attraversò il centro storico cittadino, accolto da una grande esultanza del popolo. Le clarisse annotano nei registri contabili che per l'occasione del passaggio del nuovo re «vi si mantennero, così nel giorno dell'ingresso come per tutte le trè sere de' lumi li suoni di oboè, corni di caccia, flauti e fagotti, con trombe, come pure altre 14 persone per il continuo suono delle campane». ${ }^{43}$

Dopo essersi insediato a Napoli Carlo di Borbone si recò in visita ai luoghi maggiormente significativi della città, fra cui il monastero di Santa Chiara. Al fine di ottenere il favore e l'attenzione del nuovo sovrano le monache avevano inviato numerosi regali a corte ricevendone in cambio il privilegio di una visita al monastero e ai giardini interni, avvenuta il 20 maggio del 1734. Data la straordinarietà dell'evento, avevano provveduto non solo ad adornare la Chiesa, ma anche i cortili e gli spazi esterni fino al largo del Gesù. ${ }^{44}$ Ecco come le clarisse riportano la cronaca di quei momenti: ${ }^{45}$

41 Asn, MS, f. 2554, app. pp. 251-252, si tratta della Relazione distinta del modo con cui siasi ottenuta la Festa di Precetto per la nostra gloriosa Santa Chiara e delle pompe festive praticate in quest'anno 1729.

42 Si veda anche il cap. III, pp. 72-75 in cui sono citate numerose liturgie per ricorrenze dei regnanti.

43 Asn, MS, f. 2555, app. pp. 254-255, si tratta della Relazione piena di tutto l'occorso in occasione del felice ingresso del nuovo Re Carlo di Borbone con le funzioni indi accadute, e spese occorsevi $[\ldots]$.

44 L'episodio è narrato anche in S. ChIARA, Conto Capece, app. p. 271; si riporta che «vi fu continuo suono di campana, ed oboè in tutto il giorno, ed entrato in chiesa s'intuonò solenne $\mathrm{Te}$ Deum, corrisposto da musica a quattro cori numerosa d'Istrumenti».

45 Testimonianze dell'evento si trovano anche in GDN, 25 maggio 1734 (1) «La M. del nostro re, avendo risoluto d'osservare i luoghi più cospicui di questa capitale, giovedì 20 del corrente mese dié principio dal rinomatissimo suo R. monistero di S. Chiara [...]; e in fatti verso le ore ventidue e mezza, portatasi sua maestà in detta chiesa [...], entrò in quella, sotto l'armonioso concerto di scelta musica a quattro cori e di altri suoni ivi preparati e, gionto sullo strato, s'intuonò il Te Deum da quel guardiano, padre Alessio da Roma, corrisposto da' musici [...]», cfr. Magaudda/Costantini 2009, appendice, p. 460. 
Dalla parte laterale vi si fece un ben disposto orchesto a due registri per la lunghezza de' palmi sessanta, finchè fosse stato capace per musica à quattro cori. [...] [all'esterno] si pose una corte di trombe di caccia, oboè, flauti, e fagotti, quale col suono festivo delle campane anticiparono i [...] segni di giubilo. Finalmente verso le ore ventidue e mezza giunse sua maestà [...]. S'incamminò verso l'Altar maggiore osservando ogni intorno [...] facendosi trambusto da musici una strepitossissima sinfonia con tutte sorti di istrumenti, con la direzione del maestro di Cappella Niccolò Tarantino. Appena giunto al suo posto, dal nostro M. Padre Guardiano Don Alessio di Roma, con l'assistenza delli due Padri Confessori [...] e con dodeci cantori vestiti di cotta e Chierico schierati con buon ordine, fatta prima riverenza a sua Maestà s'intuonò il Te Deum corrisposto dà scelta musica à quattro cori de' primi virtuosi qui si ritrovano, e dà grande sparo di mortaretti $[\ldots]^{46}$

Le monache auspicavano inoltre di poter ospitare presso il monastero la solenne incoronazione di Carlo di Borbone quale re di Napoli che era stata predisposta per l'estate del 1734. Nelle Notizie per la Coronazione del Re quando si avesse potuto destinare in nostra Chiesa apprendiamo che le monache si erano documentate «del modo e del luogo in cui fusser seguite le antiche precedenti coronazioni de' Serenissimi Rè di questo Regno» convincendosi inoltre che «per la Coronazione à Rè di questo Regno, non vi sia già mai stata certa legge di stabilimento di luogo ove debba farsi mà sia in totale arbitrio, non meno del Re istesso che deve coronarsi, che de Sommo Pontefice». Essendo dunque il monastero di Santa Chiara «Casa Regia» oltre che «luogo capacissimo» e «veneratissimo» dal Papa, le monache erano abbastanza certe di ottenere anche questa concessione da parte del futuro re. Dunque, per essere preparate a gestire un evento così prestigioso, avevano per tempo predisposto ogni cosa, annotando, nella relazione sopracitata, ogni dettaglio relativo all'eventuale organizzazione di tale cerimonia. Il cortile e la chiesa sarebbero stati «un Campidoglio festivo per le glorie Reali» e sulla porta d'ingresso della Chiesa avrebbero figurato «lettere illuminate» con su scritto «Viva il nostro Rè Carlo di Borbona». Ovviamente le monache avevano pensato anche all'apparato musicale, predisponendo di situare «due grandi orchestri laterali cominciando dall'atrio preliminare, e girando al di fuori, quali servirebbero per situarvi la musica delle sere, e nel giorno della solennità cupiosità di Istromenti, che facessero armonioso strepito di allegria. [...]». Infine sull'altare maggiore avrebbe dovuto trionfare una grande macchina costituita da «una gran Corona allusiva all'istessa Coronazione» sulla quale sarebbe stata posizionata anche «la Santissima Triade al di sopra e poi il corteggio di tutti li Trentatrè Santi Padroni Tutelari di questo pubblico, trà i quali la nostra Gloriosa Santa Chiara si assumesse il penziero di porger la corona, e scettro [...]. Tanto mi sembra sufficiente per abbozzo d'un opra così intricata $[\ldots] \gg{ }^{47}$ 
Malgrado ciò, la cerimonia di incoronazione di Carlo di Borbone quale re delle due Sicilie ebbe luogo soltanto il 3 giugno dell'anno successivo presso la Cattedrale di Palermo. ${ }^{48}$

48 L'incoronazione di Carlo di Borbone venne predisposta per l'estate del 1734, non molto dopo la sua entrata in Napoli, ma l'opposizione della corte romana, favorevole agli imperiali austriaci, consigliò, per evitare incidenti con il Papato, di rinviare la cerimonia. Questa venne celebrata il 3 giugno del 1735 nella Cattedrale di Palermo. Sull'argomento si veda Renda 1978, vol. VI, p. 187. 


\section{I conservatori e gli istituti assistenziali}

\section{Uno sguardo d'insieme}

Alla metà del Cinquecento i monasteri furono affiancati da istituti deputati all'educazione femminile. Sorsero così conservatori, collegi, ritiri, educandati, ospedali con l'intento di rispondere alle gravi situazioni di disagio sociale presenti a Napoli. Essendo istituzioni assistenziali si proponevano di accogliere orfane, donne ai margini da redimere e da proteggere, fanciulle da istruire e assistere in vista del matrimonio, garantendo loro un'adeguata formazione spirituale e culturale. ${ }^{1}$

Il numero dei conservatori sembra fosse già alto nel Cinquecento. Romeo De Maio annovera trentatré istituti a fine Seicento e quaranta nel 1779. ${ }^{2}$

Le ospiti dei conservatori, pur vestendo abiti pseudo-monacali e adeguandosi alle norme claustrali, emettevano spesso voti privati, non essendo obbligate alla proclamazione dei voti solenni. Gli istituti assistenziali avevano infatti una forte impronta monastica e preferivano adottare regole e schemi monastici per la tutela e l'educazione delle figlie che ospitavano. Il rigore claustrale sembrava infatti più idoneo alla vita interna di enti che svolgevano un ruolo di tutela per l'educazione di donne disagiate e prive di mezzi.

Una caratteristica assimilabile ai monasteri era la totale chiusura architettonica degli edifici verso l'esterno. Grate e panni scuri proteggevano le finestre garantendo l'isolamento totale. Inoltre le fanciulle non accedevano direttamente alla chiesa durante le celebrazioni, ma ascoltavano la messa da locali adiacenti accostandosi ai sacramenti attraverso un comunichino, detto anche 'comunicatorio'. ${ }^{3}$

Le fanciulle erano accolte verso i dieci anni e vi rimanevano fino al momento del matrimonio o della scelta monacale, intorno ai 20 anni. Le educande che decidevano di vivervi in perpetuo vennero poi nel tempo indicate come monache di conservatorio e chiamate più tardi con il termine di 'oblate' ${ }^{4}$ Esse diedero luogo al fenomeno dell'oblatismo, cioè di vestizioni religiose di comodo, che non richie-

1 Sulla storia della carità napoletana si rimanda a: FILANGIERI RAVASCHIERI 1879; ConTE 1884; Ceci 1896; Vecchione/Genovese 1908; Russo 1970; Illibato 1985; Valerio 2006; Valerio 2007; BocCadamo 1996; BocCadamo 1999, pp. 277-315; BocCadamo 2001, pp. 159-191; Facchiano 1992; Campanelli 2002; Guidi 1991; Vitale 1969.

2 De Maio, 1997, p. 26. Sul numero sproporzionato dei conservatori si veda anche Strazzullo 1968, pp. 170-172.

3 Boccadamo 1999, pp. 277-315.

4 Sulla distinzione fra monache di casa, di conservatorio, oblate, bizzoche e sull'interpretazione del nubilato e dei voti monacali nei conservatori si rimanda a BocCADAMO 2001, pp. 159-191. 
devano i voti perpetui del monastero, ma davano diritto ad alloggio e assistenza permanenti, e a ricoprire le cariche di maggior prestigio nelle comunità. Le oblate professavano pubblicamente il voler vivere in conservatorio attraverso la cerimonia definita "oblazione" corrispondente alla professione religiosa. ${ }^{5}$

Le ragazze invece, che erano accolte solo temporaneamente per 'educazione', partecipavano non solo alla vita religiosa e devozionale, ma potevano soprattutto beneficiare dell'opportunità di avvicinarsi al mondo della cultura.

Purtroppo vi è una grande difficoltà nello studio di queste istituzioni, dovuta principalmente al reperimento delle fonti. La documentazione interna degli istituti è praticamente inesistente, non vi sono tracce nel fondo Monasteri soppressi, pochi rimandi anche presso l'Archivio Diocesano. Le istituzioni assistenziali erano spesso gestite da congregazioni e confraternite di laici, e non ricadevano infatti sotto il controllo arcivescovile e pertanto potevano essere esentate da visite pastorali. I governatori delle varie istituzioni, sostenuti spesso dai viceré e dal Consiglio Collaterale, ${ }^{6}$ temevano inoltre qualsiasi intromissione o tentativo di usurpare le prerogative di regia giurisdizione. $^{7}$

Le principali fonti di riferimento per lo studio delle consuetudini musicali dei conservatori, rimangono quindi le polizze del Banco di Napoli, cui si aggiungono le regole interne degli istituti che ci danno qualche informazione sull'educazione e sugli incarichi delle monache. L'unica eccezione è costituita dal conservatorio della Solitaria che conserva, ancora oggi, il proprio archivio.

\section{L'educazione delle fanciulle}

La scansione del tempo, degli spazi, la distribuzione delle cariche interne e degli uffici all'interno dei conservatori, era organizzata sulla base di quella degli istituti con obbligo di clausura. ${ }^{8}$ Di norma le giovani venivano affidate alle cura di una maestra che non doveva superare i quarant' anni e doveva essere piena di attitudini spirituali e di moralità integerrima.

5 L'oblatismo verrà considerato una vera e propria piaga sociale ed avrà il suo culmine fra fine '700 e inizio '800. Si veda: Illibato 1985; Guidi 1991; VAlenzi 1995.

6 Il Consiglio Collaterale era uno dei più importanti organi politici e giurisdizionali del viceregno napoletano.

7 Sulla difficoltà delle visite pastorali nelle istituzioni assistenziali si veda BoccaDAmo 1999, pp. 279-283. In AsDn, Visite Pastorali, XIV [Card. Alfonso Gesualdo I], sono conservati i verbali delle visite di alcuni istituti che non si sottrassero alla visita pastorale dell'arcivescovo Gesualdo negli anni 1598-1599. Si tratta dei seguenti conservatori: Santa Maria della Concezione a Montecalvario, la Concezione di Orsola Benincasa, Ss. Filippo e Giacomo, Santa Maria del Rifugio, Ss. Crispino e Crispiano, Santa Maria della Carità, Spirito Santo, Santa Maria dello Splendore, Sant'Eligio, Tempio delle Paparelle, Conservatorio della Scorziata.

8 Sulle modalità di istruzione impartita nei conservatori si veda BocCADAMO 1996. 
Le educande venivano istruite nella grammatica, nella lettura e scrittura, oltreché avere tempo per la riflessione spirituale, l'apprendimento della dottrina cristiana, i momenti di preghiera personale, la lettura di libri spirituali. Come in un monastero, la preghiera era alla base di queste comunità, pur non essendoci un obbligo formale. Si partecipava dunque alla messa e si recitavano gli uffici divini. Le ragazze erano inoltre seguite da sacerdoti, cappellani e padri spirituali. Si occupavano anche del disbrigo della contabilità e delle pratiche amministrative, curavano il giardino e si dedicavano alle cosiddette arti donnesche, come il cucito e il ricamo. La disciplina musicale faceva parte del sistema formativo ed era inoltre elemento indispensabile per le funzioni liturgiche, pertanto era consuetudine impartire lezioni di canto o lo studio di uno strumento. ${ }^{9}$

I regolamenti interni ci danno numerose informazioni sull'educazione e sugli incarichi delle monache. Nelle regole del conservatorio dei Ss. Gennaro $e$ Clemente, ${ }^{10}$ vengono segnalati i requisiti per poter insegnare alle ospiti del conservatorio. La maestra delle novizie aveva il compito di formare, negli anni del noviziato, le educande alla «dottrina Cristiana», alle «regole dell'orazione, del Coro e del Conservatorio», e se le novizie non fossero ancora state istruite «nello scrivere, e nel leggere, o ne' lavori delle arti, [...] e non sapendo ancora il canto Gregoriano, le porteranno ogni dì nell'ora che s'insegna dalla maestra, e lo faranno apprendere $[\ldots] \gg .{ }^{11} \mathrm{Nel}$ capitolo «Delle Persone Particolari», accanto alla «Maestra delle Novizie e delle Educande», si trova anche l'indicazione della «Capo Corista, Organista, Maestra di Canto». A riguardo della «Capocorista» la regola prevedeva:

La Capocorista ha da essere una Religiosa ben pratica del Coro; mentre dovrà essa istruire, ed avvisare le sorelle di quale officio occorre la giornata secondo ' 1 calendario, che usano i Preti di questa nostra Città. Dovrà invigilare, che non si precipiti l'Offizio nel Coro, ma che dica con pausa, e divozione, fermandosi all'asterisco, come si è detto altrove [...]. Assegnerà di settimana in settimana quella che dovrà fare l'eddomanda, cioè intonare l'Offizio, e quelle che dovranno dire le lezioni, e quelle che dovranno dire le antifone, i versetti, ed intonare i salmi, e quella che dovrà leggere in Refettorio. ${ }^{12}$

L'organista invece doveva essere pratica nel «sonar gl'Inni, i Cantici, e tutte le Litanie secondo'l costume del Conservatorio, come anche delle messe, che si debbono solennemente cantare». Era inoltre necessario che ogni giorno venisse data «lezione del suono alle coriste giusta la nota Gregoriana», affinché ci fossero

9 Sull'educazione femminile e la nascita delle scuole di musica ed educandati a Napoli fra XVIII e XIX sec. si trovano informazioni in: Conti 2003; CAFIERo 2005.

10 Istituto sorto nel 1707 da una missione cattolica costituitasi per lo scampato pericolo da un'eruzione vesuviana nello stesso anno. Sedici giovani donne convertite dalla predicazione dei missionari si ritirarono in questo conservatorio sotto la direzione di alcuni sacerdoti. Cfr. ILLIBATO 1985, p. 6; VALERIO 2007, pp. 427-429.

11 Un estratto del documento è citato anche in Illibato 1985 e in Conti 2003, pp. 25-28.

12 AsDn, Vicario 427, app. pp. 307-308. 
molte educande in grado di saper suonare l'organo e in mancanza dell'organista titolare, non si ponesse mai il problema di chiamare musicisti esterni. A tal scopo si potevano «addestrare anche quelle Educande, che pajono abili», senza però «che l'organista sia soverchio gelosa, che altre riescano nell'ufizio suo, essendo ciò contro alle leggi della vera virtù».

Le regole dei Ss. Gennaro e Clemente prevedevano infine anche una maestra di canto gregoriano:

Deputiamo ancora una Maestra del canto Gregoriano, e vogliamo indispensabilmente ogni giorno nella medesima stanza del lavoro $\mathrm{o}$ in altra che sarà stimata più opportuna, che s'insegni a tutte quelle, che anno l'abilità, il detto canto Gregoriano giusta le note: nel quale impiego procuri la Maestra, che non si faccia confusione, e che si riporti quel profitto, che speriamo. ${ }^{13}$

Gli uffici di capocorista, organista e maestra di canto potevano essere svolti da tre persone differenti oppure, «se altra non si trovasse», l'onere sarebbe spettato alla sola capocorista.

La composizione musicale, il canto o lo studio di uno strumento avevano per lo più uno scopo e un uso liturgico, e a questo fine, l'istruzione delle figliole deputate all'ufficio del coro, doveva prevedere necessariamente lo studio del canto, così come si legge nelle regole del conservatorio di Santa Maria dello Splendore: ${ }^{14}$

La Chorista, ò à chi spetta il primario officio del Choro deve esser molto prattica del canto e delle rubriche, et anco prima dell'altre essere in choro, et anco anticipatamente deve far preparare il tutto, che fa bisogno per il choro, acciò non s'interrompa l'officio, che sarebbe difatto grande assignare le lettioni à chi le deve leggere, ò cantare, e far andare l'officio con le dovute pause [...]. Si deve ordinariamente andare quattro volte tra il giorno, e notte in choro cioè la mattina sonato il secondo segno si comincia l'orazione mentale [...] doppo si dichi Prima, Terza, e Sesta, e la litania dei Santi, e doppo si dicano le messe, e si facci la comunione, quale devesi fare tre volte la settimana, e tutte le feste di precetto, e vicino mezzo giorno si dichi nona: si dichino le vespri all'hora solita, doppo delle quali vi sia la lettione spirituale, e mezz'ora d'orazione mentale, e la litania della madonna ad hore 22 si dichi compieta l'estate, e alle 24 hore l'Inverno et appresso matutino e laudi. ${ }^{15}$

Le coriste non erano soltanto le monache o fanciulle deputate al coro, esse avevano il compito di accompagnare «col suono e col canto le liturgie». A tale scopo venivano affidate alle cure del maestro di cappella, secondo quanto affermano le regole del conservatorio di Santa Maria del Gran Trionfo: ${ }^{16}$

13 AsDn, Vicario 427, app. pp. 307-308.

14 Istituto fondato dalla nobildonna Lucia Caracciolo, con la volontà di radunare attorno a sé donne che volevano vivere traendo ispirazione dallo stile di vita delle cappuccine. Boccadamo 1999, pp. 277-315.

15 AsDn, Inventari di Chiese, II/15, app. p. 301.

16 Notizie su questo istituto si trovano in: D’Engenio 1624, p. 594; Galante 1985, Giornata XIII, pp. 429-430; VALERIO 2007, pp. 257-261. 
Ci saranno ancora un numero sufficiente di coriste, le quali accompagneranno col suono, e col canto le sacre funzioni. Esse esercitano l'ufficio degli Angeli i quali in Cielo cantano inni, e lodi al Signore per tutta l'eternità. Ravvivano perciò la loro fede nell'atto del loro esercizio, e si figurano di trovarsi in Cielo a cantare insieme cogli Angeli. Queste non sono esente da tutti gli altri esercizii della Comunità, se non nel solo tempo che debbono esercitare la loro carica, che se si debbono suonare e cantare litanie alla Vergine per li diversi bisogni, di quei fedeli, che ad esse ricorrono lasceranno (poiché la superiora vuole) solo per quel tempo, o la scuola, o altro, ed anderanno in coro a suonare, e cantare colla massima divozione per non defraudare la divozione dei fedeli che a tal fine lasciano le loro elemosine. Venendo il Maestro di Cappella ad istruirle stiano avanti a lui o con qualche accompagnatrice, o con qualche settimaniera ${ }^{17} \mathrm{a}$ disposizione della superiora, le quali ne risponderanno in caso d'inosservanza di regole; perciò stiano le figliuole con rispetto, decenza, e modestia, badando sempre ad essere a tutti di esempio. Imparino sempre la lezione, ed in mancanza saranno severamente punite. ${ }^{18}$

A testimonianza della reputazione di cui godevano questi enti vi sono le richieste inviate agli arcivescovi o ai governatori degli istituti da parte di personaggi di spicco della cultura per domandare di introdurre parenti, figlie o sorelle in educazione. Il conservatorio della Solitaria garantiva ad esempio l'educazione alle figlie del Giordano, così come curiosamente svela un documento del $1676,{ }^{19}$ e sempre alla Solitaria il maestro di cappella Cristofaro Caresana faceva richiesta perché sua sorella vi fosse accolta:

Illustrissimi Signori,

Christofaro Caresana Maestro di Musica delle Reverende Monache della Solitaria, Supplica Vostra Signoria Illustrissima à concederli licenza di poter introdurre in educazione nel detto Monastero Angiola sua sorella d'età d'anni 14 [...] pagando d. sei il mese conforme al solito [....$^{20}$

\section{Le diverse tipologie di istituti}

I conservatori si dividevano in due categorie: istituti 'chiusi' e 'misti'. Nei conservatori chiusi potevano ritirarsi solo ragazze o donne decise a viverci in perpetuo e in genere accoglievano ragazze che per mancanza di dote non potevano essere ricevute nei monasteri. ${ }^{21}$ Fra di essi i conservatori di Santa Maria della Carità, la Concezione a Montecalvario, Santa Maria dello Splendore. Erano invece conside-

17 Le "settimaniere" erano delle educande che a turno svolgevano servizi di pulizia o aiuto superiori, portinaie e accompagnatrici.

18 Asdn, Vicario delle Monache, 469, app. p. 302.

19 Il celebre pittore affida al Conservatorio l'«educazione de Anna, Angela et Agata Giordano sue figlie, de un'anno finito à 6 di detto decembre 1676 a ragione de d. 150 l'anno per causa di detta educatione, camera et alimenti che ricevono da detto monasterio [...]», Solitaria, Giornale di introito ed esito 1676-1680, 6/XII/1676, n.n.

20 Solitaria, Figlie della Casa, app. p. 310. Si tratta di una lettera inviata nel 1659 da Caresana ai governatori del conservatorio della Solitaria.

21 BocCADAmo 1999, pp. 277-315. 
rati misti quelli nati per accogliere ragazze in 'educazione', in grado di dare anche alloggio a vita a quante non riuscivano a contrarre un matrimonio conveniente al termine del periodo di educandato.

La destinazione dei vari istituti aveva scopi differenti: troviamo enti destinati a ragazze disagiate, alle orfane ragazze povere o semplici educande di particolari corporazioni di arti e mestieri.

Una serie di istituti deve la loro fondazione all'opera di benefattori e benefattrici che sostennero opere caritative verso fanciulle bisognose. Appartengono a questo gruppo il conservatorio della Scorziata, il Ritiro di Santa Maria delle Grazie di Mondragone, il Tempio delle Paparelle, il conservatorio di Santa Maria del Rifugio.

Il conservatorio della Scorziata, ${ }^{22}$ conosciuto anche sotto il nome di Presentazione di Maria al tempio, prese vita dal progetto educativo dalla nobildonna Giovanna Paparo e delle sorelle Luisa e Agata Paparo. L'istituto forniva un'istruzione cristiana a ragazze aristocratiche non accolte nei monasteri come educande. $\mathrm{Fu}$ fondato nel 1579 nei locali delle proprietà della famiglia Paparo e annoverava, nel 1728, la presenza di Domenico Sarro in qualità di maestro di cappella. ${ }^{23} \mathrm{Nel}$ 1585 Luisa Paparo, per incomprensioni sulla gestione del collegio, abbandonò l'istituto e fondò il cosiddetto Tempio delle Paparelle ${ }^{24}$ destinato a ragazze povere. Le uniche informazioni relative ad un'attività musicale dell'istituto, riguardano l'organizzazione della festa della Presentazione al tempio della Beata Vergine Maria che cadeva il 21 di novembre.

Il ritiro di Santa Maria delle Grazie di Mondragone fu istituito nel 1653 dalla Duchessa Elena Aldobrandini, con l'intento di ospitare gentildonne vedove ed educande. ${ }^{25}$ In esso Giuseppe De Bottis interveniva come maestro di cappella nella «musica fatta in loro Chiesa nella festa in essa celebrata di nostra Signora delle Grazie con prime vesperi, messa cantata, e seconde vesperi $[\ldots] \gg{ }^{26}$

Maggiori informazioni si hanno sul conservatorio di Santa Maria del Rifugio ${ }^{27}$ istituto situato in via Tribunali e fondato nel 1583 dalla principessa Costanza del Caretto Doria, una delle nobildonne più in vista di Napoli, per il suo impegno destinato alle opere di carità. L'istituto accoglieva ragazze non illibate, che avevano subito violenza o figlie di prostitute. Venivano ammesse anche donne in pericolo «dell'honore» e più tardi, nel Settecento verranno ammesse anche donne separate

22 Sulla storia dell'istituto: D’Engenio 1624, pp. 125-127; Celano 2001, Giornata II, pp. 208-209; Galante 1985, Giornata V, p. 180; Valerio 2006, pp. 327-331.

23 AsBn, BPV 1091 15/XII/1728, app. p. 304.

24 Sulla storia dell'istituto: D'Engenio 1624, pp. 126-127; VALERIo 2006, pp. 385-389.

25 Si veda anche: VALERIO 2007, pp. 331-335.

26 AsBn, BSE 938 5/VII/1728, app. p. 303.

27 Sulla storia dell'istituto: D'Engenio 1624, pp. 146-147; Celano 2001, Giornata I, p. 372; GALANTE 1985, Giornata I, p. 40. Si veda anche: Valerio 2006, pp. 215-219; Boccadamo 1999 pp. 277-315. 
dai mariti. ${ }^{28}$ Le regole dell'istituto prevedevano una rieducazione delle ragazze al fine matrimoniale o all'impiego lavorativo. La musica cooperava ai fini di una riabilitazione sociale e culturale, pertanto fra 1726 e 1736 si stipendiavano i maestri di cappella Giuseppe De Bottis e Andrea Barile per istruire «a cantare» le monache e le figlie ospiti. ${ }^{29} \mathrm{Il}$ «cembalaro» Francesco Andreasso aveva invece l'incombenza di «accomodare il Cembalo delle Monache Coriste del loro Conservatorio» ${ }^{30}$, mentre l'organaro Tommaso De Martino provvedeva ad «accordare l'organo della Chiesa del loro Conservatorio».$^{31}$ L'arte impartita alle figlie veniva poi impiegata in alcune liturgie, ad esempio in occasione dell'adorazione Eucaristica:

Alli Governatori del Real Conservatorio e Casa del Refugio duc. quindeci, e per essi ad Ottavio de Paolo razionale e segretario per doverli distribuire secondo il solito, tanto ad Andrea Barile Mastro di Cappella quanto a tre violini, ed un controbasso, [...] per aver accompagnato nella musica le moniche coriste di detto loro Conservatorio in tutti li quattro giorni dell'Espositione del Santissimo Sagramento per le quarantore circolari nella loro Venerabile Chiesa di Santa Maria del Rifugio $[\ldots] .^{32}$

Legati a specifiche corporazioni di arti e mestieri erano i conservatori dell'Arte della Lana e dell'Arte della Seta. In quest'ultimo, in particolare, doveva esserci una cospicua attività musicale. Il conservatorio della Nobil Arte della Seta, annesso alla chiesa dei Ss. Filippo e Giacomo, era stato istituito con l'intento di accogliere le ragazze degli affiliati alla corporazione dell'Arte della Seta, ${ }^{33}$ fra le più celebri corporazioni napoletane. ${ }^{34}$ Una delle principali occupazioni era per l'appunto quella di lavorare la seta. L'educazione delle figlie prevedeva anche l'insegnamento del violino e probabilmente di altri strumenti ad arco, secondo quanto riportato da diverse polizze dei banchi napoletani. Nel 1731 il «Virtuoso di Violino Nunzio Labruzzo», era tenuto ad «Insegniar alle Monache, e figliole della musica $[\ldots] »^{35}$, mentre nel 1736 si trovano pagamenti a Simone Giannini come maestro di cappella e al violinista Nicola Consolo, oppure ad anonimi musicisti la cui retribuzione avveniva a volte in maniera curiosa, ad esempio con galline:

A Fabiano Perrelli quondam Aniello d. Sette tarì 4.12 e per lui a Sor Barbara Piscopo moneca nel Real Conservatorio della Nobil arte della Seta e sono cioè d. 4 _ 12 per galline si regalano a coloro

BocCADAmo 1999, pp. 277-315.

29 AsBn, BSE 968 20/X/1730, app. p. 276.

30 AsBn, BPO 1086 12/V/1736, app. p. 278.

31 Asbn, BPO 1023 12/X/1731, app. p. 276.

32 AsBn, BPO, 1086 6/X/1735, app. p. 277.

33 Era infatti consuetudine che le diverse corporazioni di arti e mestieri provvedessero ai congiunti più bisognosi dei loro affiliati.

34 De Stefano 1564, p. 55; D’Engenio 1624, pp. 336-337; Celano 2001, Giornata III, pp. 698-702; Galante 1985, Giornata VI, pp. 205-207. Si veda anche: VALERIo 2006, pp. 352-355; BocCadAMO, 1999 pp. 277-315; Magaudda /Costantini 2001, pp. 79-201.

35 Asbn, BSE 982 6/X/1731, app. p. 280. 
della musica in detto Conservatorio per l'anno Corrente con essenci una pietanza di più del anno passato, d. 2 spesi per Corde e d. 1.20 spesi per accomodare delli Violini di detta musica [...]. ${ }^{36}$

Vi è inoltre la presenza di spese per la manutenzione degli strumenti così come per l'acquisto delle corde di ricambio

Ad Alfonzo Garofano d. sette t. 3 e per lui a Suor Barbara Piscosso Monaca del loro Real Conservatorio della nobil Arte della seta per averli spesi cioè d. 2 in Carte, e copiature al Mastro di Cappella d. 2. 1 per accomodatura di viola, violongello, e violini, carlini 9 per corde per 2 violongelli, d. 1. 2. 10 per il cambio del Mastro di violino in 3 servizij della festività di San Gennaro e d. 1 per accomodare l'organo per detta festa $[\ldots] .{ }^{37}$

La fondazione dei conservatori era spesso opera di confraternite o congregazioni preesistenti, come i conservatori della Solitaria, Ss. Bernardo e Margherita, lo Spirito Santo, Santa Maria della Carità, San Gennaro.

Il collegio di Santa Maria della Carità istituito nel 1548 e situato in via Toledo era destinato ad accogliere le "vergini povere onorate e di qualità", che per mancanza di dote non potevano essere ricevute negli altri monasteri. ${ }^{38}$ Dopo essere passato, nel XVII secolo, sotto la direzione di delegati del viceré e sotto la cura pastorale dei Pii Operai, l'istituto venne soppresso nel decennio francese e trasformato in albergo. La cura spirituale delle ragazze era garantita da un gruppo di sacerdoti sotto la guida di un cappellano. La vita del collegio era regolata come quella di un monastero: le fanciulle seguivano la liturgia delle ore, facevano orazione mentale, si confessavano e si comunicavano, e aggiungevano a questo la disciplina musicale. È documentata la presenza di anonimi maestri di musica e maestri di violino che avevano il compito di dare «lettione alle monache», ${ }^{39} \mathrm{ed} \mathrm{è} \mathrm{inoltre} \mathrm{interessante}$ notare la presenza di un pagamento destinato all'acquisto di «gumma armonica e ambra per servizio delle monache», ${ }^{40}$ che porterebbe a ipotizzare l'acquisto della pece da parte di monache che esercitavano un'attività violinistica.

A fine Cinquecento gli scopi assistenziali di alcuni di questi istituti si intrecciarono con la nascita dei banchi pubblici. ${ }^{41}$ La Casa Santa dell'Annunziata, celebre

36 AsBn, BSA 977 23/X/1736, app. p. 281.

37 AsBn, BSS 1188 23/XI/1728, app. p. 279.

38 Si vedano: Galante 1985, Giornata IX, pp. 351-352; VAlerio 2006, pp. 221-223; BocCadamo 1999, pp. 277-315.

39 AsBn, BSS $119622 / 1 / 1729$, app. p. 282.

40 AsBn, BSS 1189 13/VIII/1728, app. p. 282.

41 Per tentare di sollevare dall'usura le fasce più deboli di cittadini, sorsero a Napoli fra XVI e XVI sec. otto banchi pubblici: Banco della Pietà (1539-1808), Banco dei Poveri (1563-1808), Banco dell'Annunziata (1587-1702), Banco di Santa Maria del Popolo (1589-1808), Banco dello Spirito Santo (1590-1808), Banco di Sant'Eligio (1592-1808), Banco di San Giacomo (15971809), Banco del Salvatore (1640-1808). Successivamente essi si convertirono in veri istituti di credito. Le sedi dei banchi pubblici napoletani erano collocate nelle zone nevralgiche del centro storico di Napoli, da via Toledo a piazza Castelnuovo, da San Biagio dei Librai a piazza San Domenico Maggiore, da via Tribunali a piazza del Mercato. Sulla storia dei Banchi napoletani si veda: De Rosa 2002. 
istituto napoletano per l'infanzia abbandonata, per gestire più facilmente le diverse opere ad essa legate quali brefotrofio, conservatorio e ospedale, aveva chiesto che la "cassa di deposito" già aperta presso la sede dell'ente, venisse riconosciuta come banco pubblico avviando in tal modo il Banco dell' Ave Gratia Plena, in grado di gestire tutto il patrimonio dell'istituzione..$^{42}$

Tra gli scopi primari dell'ente, oltre all'accoglienza di bambini orfani vi era quello di dedicarsi alla tutela di giovani figlie. La formazione musicale ebbe un'importanza fondamentale nella storia dell'istituto. Gli studi più pertinenti relativi alle fonti musicali ${ }^{43}$ della Santa Casa testimoniano a fine Cinquecento - quindi prima della costituzione dei conservatori maschili - un'attività non professionale svolta dalle educande che apprendevano i primi rudimenti musicali dagli stessi sacerdoti della casa, chiamati, già nel 1584 , ad «imparare le figliuole di canto piano». ${ }^{44}$

Nel 1564 fu costruita la chiesa con annesso conservatorio dello Spirito Santo. ${ }^{45}$ L'istituzione era legata alla Confraternita degli Illuminati dello Spirito Santo. I confratelli infatti ebbero l'idea di erigere una piccola chiesa e di fondare due conservatori «uno per le figliuole vergini de' poveri confrati, l'altro per le figliuole, ed altre donzelle, che stavano in potere di donne prostitute, con pericolo di perdere la virginal pudicizia». ${ }^{46}$ Della raccolta di fondi per la costituzione del conservatorio si occuparono la compagnia dei Verdi e l'arciconfraternita dei Bianchi dello Spirito Santo che a sua volta diede vita, nel 1590, al Banco dello Spirito Santo. Nel primo ventennio del Seicento il conservatorio ospitava circa cinquecento religiose..$^{47}$ Nelle cronache riguardanti questo monastero, viene descritta l'attività musicale svolta in prima persona dalle monache durante le liturgie, a più riprese definite «virtuosissime», a proposito delle loro esibizioni musicali avvenute in occasione di eventi civili come il funerale di Carlo II e la gravidanza dell'imperatrice Elisabetta. È inoltre attestata la presenza di maestri come Gaetano Veneziano e Nicola Fago, conosciuto anche come «Signor Tarantino», ${ }^{48}$ indicato come «mastro di Cappella di detto loro Conservatorio», e ancora dei cembalari Antonio e Gaspare Sabatino. La festa maggiormente solennizzata era quella di Pentecoste alla quale prendevano parte i musicisti della Cappella Reale di Palazzo. Proprio in virtù

42 Sull'Annunziata e i suoi fini assistenziali si veda De Maio 1973, pp. 241-249; D'Ario 1994, pp. 515-568; CAMPANELli 2002, pp. 143-168; VALERIo 2006, pp. 57-65.

43 Sull'attività musicale del conservatorio dell'Annunziata si rimanda a: Columbro 2001, pp. $42-78$.

44 Cfr. Columbro 2001, pp. 42-78.

45 Spesso confuso con l'omonimo convento maschile, cenni storici si trovano in Illibato 1985, p. 55; BocCadamo 1999, pp. 277-315; Valerio 2006 pp. 333-339.

46 Celano 2001, Giornata II, pp. 21-22.

47 ILLibato 1985, p. 55.

48 Magaudda/Costantini 2009, p. 472. La presenza di Gaetano Veneziano in qualità di maestro di cappella e compositore di brani per le educande dello Spirito Santo è documentata intorno al 1713, cfr. Turano 1988, pp. 40-43. Sullattività di Antonio e Gaspare Sabatino si veda NocerINo 2001, pp. 212-213. Si rimanda inoltre all'appendice documentaria p. 293 e seg. 
di questa tradizione musicale, a inizio Ottocento, il conservatorio dello Spirito Santo veniva così descritto nella richiesta inviata al re dal Ministro dell'Interno per domandare che esso venisse riconosciuto ufficialmente come «stabilimento di musica per le donne»:

Vi è in Napoli un Conservatorio Laicale di Fanciulle, detto dello Spirito Santo: le medesime erano educate nelle Arti musicali, ma senza scopo, e senza regola: facevasi imparare alle ragazze diverse parti istrumentali, che punto non concordavano colla decenza femminile. ${ }^{49}$

Il re accolse la richiesta nel 1806 ed emanò un decreto con cui autorizzava la costituzione di questo collegio musicale femminile.

Nel 1546 per volontà del viceré Pedro de Toledo, venne edificato, presso la chiesa di Sant'Eligio, nel quartiere del Mercato, un istituto per accogliere le figlie del popolo rimaste orfane e in pericolo di cadere nella piaga della prostituzione: l'ospedale di Sant'Eligio. ${ }^{50} \mathrm{Si}$ conta che a metà Seicento le ragazze ospiti avessero raggiunto il numero di 150. Inoltre nel 1592 l'opera pia, convinta dell'utilità che la gestione di un banco pubblico avrebbe apportato agli istituti di beneficenza da essa governati (la chiesa, l'ospedale, e il conservatorio), diede vita al Banco di Sant'Eligio.

L'ospedale era posto sotto la direzione di un gruppo di oblate sotto la regola di Sant'Agostino, il cui compito consisteva nel prendersi cura delle ragazze ospiti e provvedere alla loro istruzione. Le ragazze venivano istruite anche nella musica. Si ha notizia nel 1729 di «letioni di musica» che venivano impartite dal maestro di cappella e organista Francesco Tringhera «alle monache e figliole destinate per il coro del nostro Conservatorio». ${ }^{51}$ Le fanciulle infatti si esibivano in occasione di liturgie e celebrazioni. La «Gazzetta di Napoli» documenta che, presso l'ospedale di Sant'Eligio, il viceré in persona aveva ascoltato «un famoso oratorio, cantato da quelle virtuose educande, in lode del nostro invittissimo monarca [...]». ${ }^{52}$ Si riscontra inoltre la presenza di Francesco Durante, che, in qualità di maestro di cappella, era chiamato a ripartire i compensi per sei violini, un contrabbasso, un violoncello e per «aver' lui sonato l'organo per tutti detti Cinque servitij, e similmente per aver' copiato la musica, per essere stata inferma la monica organista, il tutto in occasione delle quarantore Circolari fatte in nostra Chiesa nel corrente anno $1736[\ldots] \gg .{ }^{53}$

49 ConTi 2003, pp. 55-56.

50 Sulla storia dell'istituto si veda: De Stefano 1564, pp. 40-43; D'Engenio 1624, pp. 441-443; Celano 2001, Giornata IV, pp. 223-233; Galante 1985, Giornata VIII, pp. 294-296. Si veda anche: VALERIO 2006, pp. 155-161.

51 AsBn, BSE 950 5/IV/1729, app. p. 295.

52 GDN, 23 luglio 1715 (2), app. p. 296.

53 AsBn, BSE 1038 29/II/1736, app. p. 296. 
Vi sono ancora istituti la cui costituzione si deve a iniziative cittadine e alla devozione popolare, come i conservatori di Santa Maria Visita Poveri, Santa Maria di Costantinopoli, Santa Maria Antesaecula, Santa Monica.

Fra di esse, l'istituzione che risulta essere particolarmente attiva sotto il profilo della committenza e della formazione musicale, è il conservatorio e l'annessa chiesa di Santa Maria di Costantinopoli. La storia di questo conservatorio è legata alla diffusione a Napoli del culto della Madonna di Costantinopoli, durante gli anni in cui la città fu colpita gravemente dalla peste. ${ }^{54}$ Una leggenda racconta che, durante l'epidemia del 1527-1528, la Madonna di Costantinopoli apparve ad un'anziana donna, chiedendole di erigere un tempio lì dove avrebbe trovato una sua immagine dipinta su un muro. Rinvenuta per l'appunto un'effige lungo le mura di cinta sotto la rocca di Caponapoli, vi fu edificata una prima cappella dedicata a Santa Maria di Costantinopoli. In seguito, nel 1575, per allontanare il pericolo di una nuova epidemia, si decise di costruire una chiesa più grande lungo il tracciato di via Costantinopoli.

La «Gazzetta di Napoli» ci informa sulle attività pubbliche di questa istituzione riguardo le celebrazioni organizzate in occasione di ricorrenze particolari della famiglia reale. I sontuosi festeggiamenti per la «gloriosa memoria di Carlo II nostro monarca $[\ldots] »$, celebrati con «una musica degli migliori virtuosi che vi siano $[\ldots] »{ }^{55}$ si avvicendano ai funerali «per la defonta augustissima imperadrice $[\ldots]$ » che avevano previsto «un immenso numero di messe di requie» con l'ausilio di «esquisita musica [...]»;5 il 5 ottobre 1723 «fu intonato domenica 3 del corrente solennemente il Te Deum [...] per la gravidanza dell'augustissima imperadrice $[\ldots]\rangle^{57}$; mentre per il matrimonio di sua maestà avvenuto il 14 gennaio 1738 « ha voluto distinguersi la R. chiesa e collegio di Costantinopoli, in dove il rettore di quella, D. Girolamo Palomba, martedì la sera, 7 del corrente, cantò egli pontificalmente con tutto il suo clero in segno di giubilo un solenne Te Deum, con l'esposizione del Venerabile, replicato sparo di mortaretti ed illuminazioni $[\ldots] \gg{ }^{58}$

I dati più interessanti sono però quelli relativi alla musica praticata nel quotidiano di questa istituzione. Le carte d'archivio annotano la presenza di maestri di cappella salariati quali Giacomo Sarcuni, Michele de Falco, Simone Giannini, il maestro di coro Angelo Crisci. Negli stessi anni compaiono maestri di violino come Bonaventura Veneziano e Francesco Sabatini, e organisti come Carlo Codispoti e Matteo Vernucci, assieme alla presenza di alcune religiose che vengono denominate come «Monache Musiche», con molta probabilità violiniste ricompensate con dolci e «zuccari»: 
Alli Sudetti [scil. governatori del Monastero di Santa Maria di Costantinopoli] d. quattro [...] e sono per distribuirli alle Monache Musiche di detto Monastero alle quali spettano per li Zuccari soliti per le prossime feste del Santo Natale $[\ldots] .{ }^{59}$

Non mancano periodici pagamenti a monache «prefette della musica», il cui compito era quello di assolvere ad ogni esigenza per i diversi «servizi di musica» ${ }^{60} \mathrm{di}$ cui il conservatorio necessitava, dalla manutenzione e acquisto di strumenti, agli archetti e corde per strumenti ad arco, alla carta da musica, agli spartiti, come leggiamo da questo documento:

Alli Governatori della Casa Santa di Santa Maria Costantinopoli d. dieceotto 2.19 e per loro a Suor Anna Maria Sanges prefetta, che fu della musica del loro monastero, e sono per tanti da essa spesi in carta, corde, accomodatura d'istromenti, et altro bisognato per servitio della musica del detto monastero dal primo dicembre 1730 per tutta la fine di dicembre $1731[\ldots] .{ }^{61}$

Anche nelle realtà più piccole o di cui disponiamo di minori indicazioni si trova qualche informazione sulla pratica musicale: Filippo Prota era maestro di musica al conservatorio delle Figliole di San Gennaro $^{62}$ assieme a Nicola Di Napoli $^{63}$ che figura essere maestro di viola. Al conservatorio di Santa Maria Visita Poveri la festa del titolo si celebrava con «sceltissima musica» e con l'ausilio di prestigiosi maestri di cappella come Pietro Scarlatti, ${ }^{64}$ mentre al conservatorio della Concezione a Montecalvario, in occasione della festa dell'Immacolata nel 1718, «fu eseguita musica di scelte voci e ottimi istrumenti e specialmente del marchese Matteo Sassano, che si era esibito anche l'anno precedente in occasione di una monacazione». ${ }^{65}$

\section{Conservatori napoletani e ospedali veneziani: un possibile confronto?}

L'attività musicale dei conservatori femminili è un aspetto del tutto nuovo per la città di Napoli. Pur non essendoci ad oggi un concreto riscontro di un'attività professionale svolta da educande e religiose, i documenti d'archivio ci mostrano che vi era un'attività musicale formativa e performativa anche negli istituti caritativi napoletani.

AsBn, BSA 980 20/XII/1736, app. p. 289.

60 AsBn, BSG 752 1/XII/1728, app. p. 284.

61 Asвn, BSA 900 29/1/1732, app. p. 285.

62 Asbn, BPO 976 19/IV/1728, app. p. 297.

63 Cfr. app. pp. 297-298.

64 Si veda sul conservatorio di Santa Maria Visita Poveri anche il capitolo dedicato alle liturgie di monacazione, ove è descritta una cerimonia di professione religiosa in essa avvenuta, con la ricostruzione dell'organico musicale utilizzato. Cfr. cap. III, p. 89. 
Le realtà assistenziali femminili emerse consentono di allontanarci dallo stereotipo di una formazione musicale cittadina ad esclusivo privilegio dei quattro conservatori maschili, riconosciuti, per antonomasia, come uniche scuole di musica della capitale del viceregno. ${ }^{66}$ Anche le istituzioni maschili, erano in origine semplicemente enti assistenziali creati per accogliere ragazzi orfani e poveri, con finalità molto simili dunque alle realtà femminili.

In effetti l'origine storica dei conservatori vede queste istituzioni essenzialmente come luoghi di ricovero, destinati a ospitare ragazzi di entrambi i sessi provenienti da situazioni difficili. Gli ospiti di questi istituti venivano per l'appunto "conservati" per essere sottratti dai pericoli e dal degrado morale a cui la società poteva esporli, veniva impartita loro una educazione secondo i canoni della Dottrina cristiana, venivano istruiti e formati ad una attività lavorativa, nella visione di un futuro inserimento nella società.

Tuttavia, in alcuni contesi geografici, i conservatori assunsero nel tempo sempre più le caratteristiche di istituzioni finalizzate alla cultura. A Napoli, così come a Venezia, essi si trasformarono in vere e proprie scuole di musica universalmente riconosciute per i talenti che formarono.

Probabilmente proprio la presenza di queste celebri realtà maschili ha emarginato per troppo tempo le istituzioni femminili da indagini più approfondite $\mathrm{e}$ da ipotesi di confronto dirette con gli ospedali femminili veneziani. Nel tempo dunque la formazione professionalizzante dei conservatori maschili napoletani è stata sempre e solo tradizionalmente contrapposta a quella caritatevole degli ospedali femminili veneziani.

I quattro ospedali veneziani erano fra le istituzioni più importanti e propulsive della città lagunare. Nei diari dei viaggiatori in visita nella penisola italiana fra Sei e Settecento, ricorrono spesso i resoconti entusiastici degli intrattenimenti musicali che la città di Venezia offriva presso questi istituti: la celebre "Pietà", i Mendicanti, gli Incurabili e i Derelitti. ${ }^{67} \mathrm{Si}$ trattava infatti di enti assistenziali destinati all'accoglienza di fanciulle orfane o indigenti a cui veniva offerta un'educazione anche musicale. ${ }^{68}$

A ben guardare le istituzioni femminili veneziane e napoletane hanno numerose similitudini: i loro principi di costituzione e simili tipologie di attività formative possono essere messe a confronto. ${ }^{69}$

66 Sui quattro conservatori maschili napoletani si veda anche p. 61.

67 I quattro istituti ebbero nome di ospedali maggiori: i Derelitti sorse nel 1528 presso il complesso conventuale dei Santi Giovanni e Paolo; gli Incurabili fondato nel 1522; i Mendicanti istituito nel 1588; ed infine il complesso della Pietà, il più antico dato, creato nel 1336 dal frate francescano Pietro d'Assisi.

68 Sulla storia musicale degli ospedali veneziani si rimanda a: Gillio 2006; Geyer/Osthoff 2004; Over 1998 ; Selfridge-Field 1985; Giron Panel 2015.

69 I metodi utilizzati per educare le putte veneziane sembrano molto simili a quelli adottati nei conservatori maschili. Alcuni studiosi che si sono occupati delle istituzioni veneziane e dei 
Il primo elemento in comune riguarda le finalità istituzionali: conservatori e ospedali ponevano come istanza morale l'educazione della donna al fine di prepararla a svolgere il ruolo di moglie e madre o di religiosa. Come precedentemente citato, queste istituzioni nascono per accogliere ragazze in "educazione", per istruirle e riabilitarle anche attraverso la pratica musicale. La possibilità di essere accolte in un ospedale, così come un conservatorio, rappresentava una possibilità di salvaguardia temporanea dai pericoli della vita, e dunque la formazione impartita doveva mirare ad infondere nelle giovani figlie principi cristiani e abitudini comportamentali tali da favorire un reinserimento nella società. Oltre a ricevere un'educazione secondo i canoni della dottrina cristiana, le ospiti di questi istituti dovevano attendere ad una serie di obblighi devozionali secondo quanto prescritto nelle regole e costituzioni, garantendo anche la frequenza ai sacramenti.

Come per i conservatori napoletani, anche gli ospedali veneziani operarono sempre in totale autonomia dalla chiesa romana, e furono dunque enti indipendenti dal punto di vista amministrativo ed economico. Ad ogni modo, pur non essendo formalmente soggetti a clausura, ne sposavano comunque i principi. La giornata delle cosiddette "putte", era scandita da regole ben precise, vi erano restrizioni sulle uscite, sulla corrispondenza, sul comportamento. Le visite si svolgevano soltanto dietro autorizzazione e sotto vigilanza di una maestra o di una superiore. Raggiunta la maggiore età le figlie potevano decidere se rimanere in istituto, prendere i voti e monacarsi o altrimenti maritarsi.

Nel corso degli anni gli ospedali investirono buona parte delle loro risorse per assicurarsi una formazione musicale esclusiva: le performance musicali delle 'putte' costituivano difatti una grande forma di pubblicità per le istituzioni. Per provvedere all' 'istruzione musicale delle ragazze, i pii istituti impiegarono quindi nel corso dei secoli maestri rinomati come Vivaldi, Hasse, Galuppi, Traetta, Sacchini etc. Bisogna inoltre sottolineare la presenza di una numerosa serie di maestri napoletani che avevano in precedenza insegnato nei conservatori maschili.

Nel corso del XVIII secolo, i governatori degli ospedali scelsero di avvalersi molto spesso di compositori provenienti dal Sud Italia per rispondere al gusto del pubblico e dei teatri. Le frequenti interazioni fra Napoli e Venezia favorirono infatti, non solo una circolazione di compositori e di molta musica, ma con buona probabilità anche di metodi didattici. Nicola Porpora fu il primo napoletano ingaggiato da un ospedale veneziano, cui faranno seguito Jommelli, Latilla, Traetta, Sacchini, Anfossi.

Le educande dei conservatori napoletani usufruirono ugualmente dell'esperienza pedagogica dei grandi maestri della scuola napoletana. I documenti d'archivio

quattro conservatori napoletani hanno affrontato il tema del confronto fra Napoli e Venezia proponendo in alcuni casi anche un confronto fra i sistemi pedagogici utilizzati, si veda: GILlio 2006; Cafiero 2005; Giron Panel 2015. 
attestano la presenza di maestri di canto e strumento molto qualificati, gli stessi maestri che operarono nei conservatori maschili. Anche se le regole e le costituzioni ci danno ben poche informazioni sui contenuti dell'educazione, la sola presenza di questi maestri e il riscontro delle esibizioni delle educande che leggiamo nelle cronache, ci portano ad ipotizzare che la preparazione musicale delle fanciulle dovesse essere alquanto elevata. Fra le partiture superstiti custodite oggi presso la Biblioteca dei Girolamini troviamo ad esempio la presenza di alcune composizioni di Gaetano Veneziano maestro al conservatorio di Santa Maria di Loreto, dedicate alle fanciulle del Conservatorio dello Spirito Santo. Francesco Durante che sostituì Porpora sempre a Santa Maria di Loreto, figura essere maestro all'ospedale di Sant'Eligio. Anche un veneziano come Cristofaro Caresana, direttore al conservatorio di Sant'Onofrio, fu maestro delle figlie del Conservatorio della Solitaria.

Nei quattro ospedali l'insegnamento fondamentale impartito era quello degli strumenti ad arco. Ai Derelitti era presente un maestro di viola, violoncello, violino, violone. Al coro della Pietà l'insegnamento di violino fu istituzionalizzato nel 1703 con il conferimento dell'incarico ad Antonio Vivaldi. ${ }^{70}$ A Napoli la presenza del maestro di violino e di strumenti ad arco in generale è attestata in diverse istituzioni. Al Conservatorio della Solitaria, oltre a numerosi maestri di violino che si avvicendarono fra Sei e Settecento, vi era anche la presenza del maestro di ribeca. Presso le figliole di San Gennaro vi era un maestro di viola, mentre in altre cinque istituzioni figurano maestri di violino. Per Venezia così come per Napoli le carte d'archivio rivelano le spese sostenute dagli enti per le forniture periodiche e per la riparazione degli strumenti ad arco. La presenza di documenti che attestano il consumo e il relativo acquisto di corde costituisce un attendibile indicatore della pratica strumentale che veniva svolta in essi. ${ }^{71}$

Negli ospedali veneziani operava un maestro di solfeggio il cui insegnamento era indirizzato sia alle strumentiste sia alle cantanti. Spesso il maestro di solfeggio corrispondeva al maestro di canto. Anche a Napoli troviamo raramente la denominazione di maestro di solfeggio, incarico con buona probabilità espletato dai numerosi maestri di musica e canto presenti in quasi tutte le istituzioni prese in considerazione. A Venezia l'insegnamento delle tastiere era probabilmente impartito dagli stessi maestri di coro. Per Napoli troviamo qualche scarso riferimento in relazione all'acquisto di strumenti come clavicembali e spinette oppure a lezioni di strumenti da tasto date alle fanciulle.

A Venezia venivano impartite anche lezioni di strumenti a fiato. Inoltre, tra i maestri di strumento si trova talora una figura con incombenze assai diverse dalle altre: quella del maestro dei concerti, la cui funzione primaria era quella della com-

70 Probabilmente al maestro di violino competeva anche l'insegnamento della violetta e al maestro di violoncello quello del violone. Cfr. GiLLio 2006, pp. 175-184.

71 Si veda cap. IV, pp. 96-98. Si rimanda inoltre all'appendice documentaria, alle singole schede relative alle istituzioni sopracitate. 
posizione e della concertazione di musica strumentale. Negli ospedali veneziani il maestro di coro si occupava di comporre musica liturgica e paraliturgica, mentre la musica strumentale competeva a un maestro di strumenti o a un compositore esterno. ${ }^{72}$ Non esistono sufficienti informazioni che ci permettano di dedurre delle similitudini negli istituti napoletani. Le carte non forniscono alcuna indicazione relativa ad un 'maestro di concerti'. Molto raramente si trovano informazioni su compositori e composizioni di musica destinata alla liturgia e alle occasioni festive, stesso dicasi per la musica strumentale. Possiamo in qualche caso supporre che i numerosi maestri di cappella fossero incaricati di gestire le esecuzioni di musica con musicisti esterni alle istituzioni e probabilmente si occupassero anche delle composizioni, mentre i maestri di musica e di strumento erano dediti all'educazione musicale delle fanciulle e probabilmente componevano musica a scopo esclusivamente didattico. Se prendiamo in considerazione il conservatorio su cui abbiamo i maggiori riferimenti, quello della Solitaria, possiamo riscontrare che i maestri di strumento e di musica vengono retribuiti esclusivamente a scopi didattici e non in quanto maestri di cappella e viceversa. ${ }^{73}$

È probabile che le figlie di coro divenute maestre assumessero oltre ad incarichi didattici in ambito musicale più ampie responsabilità di ordine educativo $\mathrm{e}$ disciplinare, tuttavia mancano informazioni a riguardo. Anche a Napoli, come testimoniato anche da alcune regole citate nei paragrafi precedenti, ${ }^{74}$ troviamo la presenza di maestre che avevano incarichi didattici musicali nei confronti delle figlie più giovani. Quando una figlia dopo aver rinunciato al matrimonio e alla monacazione dimostrava di possedere requisiti adeguati diveniva maestra assumendo la responsabilità della formazione delle figlie più giovani.

Liturgie e festività costituivano poi il fiore all'occhiello dell'attività performativa e ricreativa degli ospedali veneziani: momenti nei quali le fanciulle potevano dare prova delle proprie abilità vocali o strumentali. Le cantanti e strumentiste impegnate nelle funzioni in musica erano parzialmente celate alla vista del pubblico da grate di legno o di metallo ancora oggi visibili nella loro originaria collocazione sulle balaustre dei cori.

Negli ospedali l'offerta musicale aveva luogo ogni sabato e domenica e in tutte le festività principali, con la celebrazione mattutina di messe solenni e dei vespri. Mottetti solistici e composizioni strumentali costituivano il repertorio principale. Il periodo in cui negli ospedali cadevano le celebrazioni più importanti, ad esempio quello quaresimale, era il tempo in cui l'opera non teneva rappresentazioni e quindi in chiesa era possibile ascoltare arie del tutto simili a quelle teatrali e perlopiù composte da maestri di coro che erano anche operisti di fama. Ovviamente non

73 Si veda il cap. VIII.

74 Si vedano le pp. 187-188. 
mancava un'offerta più tradizionale nelle chiese degli ospedali: nei giorni feriali la celebrazione ripristinava semplici esecuzioni in canto fermo.

Per Napoli, relativamente all'esiguità delle fonti a nostra disposizione, non possiamo desumere informazioni dettagliate circa $i$ tempi e le modalità delle performance musicali delle figlie dei conservatori. Tuttavia l'attività musicale di alcuni istituti assistenziali (Solitaria, Sant'Eligio, Spirito Santo, Santa Maria di Costantinopoli) riportate dalla documentazione d'archivio e in particolar modo dalla «Gazzetta di Napoli», lasciano presagire di essere in un ambiente non così dissimile da quello veneziano. La partecipazione delle educande è documentata durante celebrazioni straordinarie per qualche ricorrenza delle famiglie aristocratiche connesse ad un'istituzione, in occasione del tempo pasquale, nelle principali feste cittadine.

Le figlie di coro non si dedicavano soltanto ad un repertorio liturgico. Era infatti tradizione anche a Venezia che potessero recitare e cantare drammi in occasioni particolari o per loro diletto, sempre sotto la vigile attenzione della congregazione. A Napoli troviamo esattamente la stessa situazione:

\footnotetext{
Detto giorno ultimo dello scorso mese [31 maggio] [...] nella R. chiesa dello Spirito Santo cantossi il Te Deum per la nascita dell'augustissima prole al nostro imperadore, coll'esposizione del Venerabile. E fra l'altre cose vi fu da osservare un vaghissimo ornamento nella gran porta della chiesa [...]. Il disegno è stato del celebre letterato dipintore Francesco Solimena e l'invenzione del rinomato dott. Nicolò Amenta [...]. E la mattina seguente del lunedì, solennizzandosi la festa dello Spirito Santo con cappella reale, nella quale intervenne S. E. e sig. viceregina, co' ministri e numero grandioso di nobiltà, dentro il conservatorio da dieci figliuole di esso cantossi un bellissimo componimento del medesimo Amenta, per l'accennata nascita $[\ldots]^{75}$
}

Se molte figlie di coro ebbero carriere anonime, altre raggiunsero eccezionali traguardi di notorietà. Alcune di queste figliole riuscirono poi ad affrancarsi dai luoghi pii e fecero carriera. Per Napoli, non ci sono, ad oggi, testimonianze che facciano pensare a carriere svolte dalle educande al di fuori degli istituti. Da questo punto di vista si riscontra una grande reticenza delle fonti: la maggior parte dei documenti non fornisce quasi mai notizie precise riguardanti le monache 'musiciste', nomi e cognomi sono spesso sottaciuti. Tuttavia non si può escludere anche per Napoli la presenza di religiose compositrici o musiciste 'professioniste' così come riscontrato in altre aree italiane. Ad ogni modo tanta musica composta per occasioni liturgiche di chiese napoletane potrebbe tranquillamente essere ricondotta ai conservatori femminili napoletani. Molta musica per la liturgia del Settecento napoletano aspetta ancora di essere sondata e sufficientemente indagata.

Emerge, in conclusione, negli ospedali così come nei conservatori napoletani congiuntamente alla dimensione assistenziale, una forte dimensione pedagogica, volta al recupero e reinserimento sociale di donne cresciute in ambienti socialmente e moralmente inadatti. Questi enti permisero che la musica divenisse occasione 
di espressione artistica per donne che non avrebbero potuto, per estrazione sociale, percorrere la via della pratica musicale. La musica, a Napoli come a Venezia, diviene allora mezzo attraverso il quale rieducare giovani donne alla vita di società, donare loro una cultura che diversamente non avrebbero potuto avere. Essa coopera al riscatto dell'onore perduto e a restituire dignità al ruolo femminile e all'immagine stessa della donna nella società. 


\section{Il conservatorio di Nostra Signora della Solitaria}

\section{La fondazione}

Il conservatorio di Nostra Signora della Solitaria, conosciuto anche come Santa Maria della Solitudine o semplicemente Soledad, fu concepito per iniziativa del frate cappuccino spagnolo Pietro Trigoso e da Luis de Luxan y Enriquez, maestro di campo della fanteria spagnola, con l'idea di fondare a Napoli un istituto che avesse il compito di raccogliere ed educare le orfane dei militari spagnoli di stanza a Napoli.

Il primo fondo patrimoniale del conservatorio della Solitaria fu costituito dalle elemosine e dalle donazioni ottenute dai due fondatori, che lo aggregarono ad una confraternita di nobili spagnoli, istituita nel 1580 e dedicata al culto della Soledad, con sede in un palazzo alle pendici di Pizzofalcone, nella strada ancora oggi denominata via Solitaria. Per tutto il XVII secolo e fino alla fine del viceregno, la Casa Santa godeva del regio patronato ed era finanziata, per disposizione del viceré, con ritenute effettuate sul soldo dell'esercito regolare spagnolo, proporzionate all'entità della paga di ogni grado e di ogni arma.

Ritenuto erroneamente un monastero, il conservatorio fu soppresso durante il decennio francese, i suoi possedimenti venduti come beni ecclesiastici, e la sede della Solitaria a Pizzofalcone occupata dai militari e trasformata in paggeria.

Successivamente, verificato l'errore, l'istituzione veniva ripristinata e le era assegnata come sede il monastero soppresso delle monache di Santa Caterina da Siena, ${ }^{1}$ alla sommità dei gradoni di Chiaia dove, accanto alla chiesa sconsacrata, trova sede ancora oggi il suo archivio storico. ${ }^{2}$

1 Con la legge post-unitaria sulle istituzioni di beneficenza del 1862, il conservatorio, che era stato sempre considerato ente autonomo di regio patronato, fu sottoposto come tante altre istituzioni, al Ministero dell'Interno e considerato come opera di assistenza, avente lo scopo di educazione delle orfane spagnole. Dopo l'ultimo conflitto mondiale l'ente, perduta ogni forma di regio finanziamento, venne concesso a suore salesiane per tenervi asilo e scuole, conservando in tal modo l'ultimo collegamento all'originario scopo istitutivo, con l'impegno ad alloggiare, accudire e curare le monache domenicane spagnole superstiti. La sede originaria del conservatorio divenne nel 1882 Museo Artistico Industriale, prendendo il posto del Real Collegio della Marina Borbonica. Attualmente i locali dell'antico conservatorio sono occupati dall'istituto d'Arte "Filippo Palizzi" e dal Museo Artistico Industriale. Le uniche fonti relative alla storia della Solitaria sono riportate in: ARALDo 1998, p. 206; D'Aloe 1883, pp. 678-679; De Lellis 1654; Celano 2001, Giornata V, pp. 587-588; Galante 1985, Giornata XI, p. 379; in epoca moderna BocCADAmo 1999, pp. 277-315; VALERIO 2006, pp. 241-245.

2 La Fondazione Real Conservatorio della Solitaria costituitasi nel 1996, ha preservato i documenti dell'Archivio Storico nel corso degli anni, ed oggi prosegue alla valorizzazione del cospi- 
La ricchissima guida di Carlo Celano a proposito del conservatorio della Solitaria riporta:

In questo luogo vi sono molte monache che vivono sotto la Regola di S. Domenico; è molto dilettoso ed ampio avendo l'aspetto su la marina di S. Lucia. In questa chiesa vi sono molti belli quadri e particolarmente quello che sta nella prima cappella a destra quando s'entra, dove si vede la Vergine col suo morto Figliuolo in seno, è opera di Giuseppe Riviera; quello che sta nell'altare maggiore è del nostro Giordano. In questa chiesa vi sta una divota compagnia di nobili Spagnuoli sotto il titolo della Solidad $[\ldots] .^{3}$

Così come testimonia il canonico Celano, la chiesa doveva ospitare numerose opere di artisti di chiara fama. In un inventario degli oggetti utilizzati nella chiesa e nel conservatorio, risalente al luglio 1695 , sono infatti citati diversi dipinti di Luca Giordano come una Madonna del Rosario, San Ferdinando e la Deposizione dalla Croce, quest'ultima collocata, come indica il Celano, sull'altare maggiore. ${ }^{4}$

Il conservatorio della Solitaria si inserisce appieno nel circuito delle istituzioni assistenziali, divenendo a sua volta non solo un centro di esperienza religiosa, di tutela, di gestione economica, ma anche un centro di formazione culturale. Tuttavia, fino a qualche anno fa, sarebbe stato difficile tracciare una storia musicale del conservatorio: mancano testi o contributi specifici sia sulla sua fondazione che sull'argomento storico-musicale, pochi i riferimenti citati dalle cronache dell'epoca, purtroppo non si conservano nemmeno i documenti relativi alle visite pastorali. ${ }^{5}$ Accenni ad un'importante vita artistica e musicale alla Solitaria si riscontrano però nei giornali copiapolizze dell'Archivio Storico del Banco di Napoli ${ }^{6} \mathrm{e}$

cuo patrimonio storico e culturale del conservatorio. Un sincero ringraziamento va alla Fondazione del Real Conservatorio della Solitaria, e in particolare al presidente della Fondazione avv. Mario Mazza, nonché alla Soprintendenza archivistica campana per avermi concesso senza alcuna difficoltà di accedere all'archivio storico oggi chiuso al pubblico e studiarne i materiali.

3 Celano 2001, Giornata V, pp. 587-588. Il Chiarini a riguardo della Solitaria precisa che «La chiesa e il convento di Santa Maria della Solitudine, o della Solitaria ebbero per fondatori nel 1580 un cappuccino e un maestro di Campo entrambi spagnuoli, i quali avevano raccolto ampie elemosine. La destinarono a rinchiudere le orfane spagnuole, le quali uscendo per andare a marito ricevessero una somma di danaro in dote, o continuando a rimanervi, seguitassero la Regola di S. Domenico. Venne edificato il convento sul monte Echia, oggi Pizzofalcone, accessibile alle carrozze solamente per due strade che fiancheggiano la basilica di san francesco di Paola. La chiesa era ornata di pitture della scuola napolitana, del Giordano, del Ribera, del Vaccaro, e di Bernardino Siciliano. Demolita la chiesa ed abolito il monastero, nel 1824 venne l'edificio destinato ad altri usi».

4 La Solitaria ospitava le figlie del Giordano. Si veda quanto detto nel capitolo VII pp. Oltre al Giordano nella chiesa figuravano una Madonna con Cristo disceso dalla croce opera di Andrea Vaccaro posta nella cappella di Nostra Signora della Pietà, L'Agonia di San Giuseppe nella cappella dedicata al Santo di Francesco Caracciolo.

5 Le prime ricerche da me condotte, attraverso un'indagine a campionatura su alcuni materiali, hanno portato a chiarire e ad inquadrare il ruolo del conservatorio della Solitaria nel panorama musicale e spettacolare partenopeo fra Sei e Settecento. Cfr. Fiore 2010 e Fiore 2013. 
nelle cronache della «Gazzetta di Napoli». ${ }^{7}$ Essi fanno però riferimento, quasi esclusivamente, ad una tradizione musicale pubblica svolta dal conservatorio nel contesto cittadino, soprattutto in occasione dei riti della Settimana Santa. Pochi rimandi invece ad un uso della musica per così dire "privato", svolto fra le mura claustrali. La consultazione della documentazione interna dell'istituto custodita presso l'Archivio Storico della Solitaria, permette oggi di ricostruire l'attività del conservatorio e di determinarne l'importanza sul circuito cittadino. ${ }^{8}$

\section{La Soledad e la Cappella Reale}

Le prime testimonianze musicali rinvenute al conservatorio della Solitaria sono sorprendentemente legate alle attività e alla storia della più prestigiosa fra le istituzioni napoletane: la Real Cappella di Palazzo.

È necessario fare una breve precisazione sulla storia della Real Cappella per comprendere il legame stesso con la Solitaria. La compagine vicereale venne fondata nel 1555 da Pedro de Toledo, ma vi sono solo poche notizie relative ai primi periodi di attività. Numerose informazioni suffragate dagli studi di Prota-Giurleo, Di Giacomo, Maione, Fabris etc. ne osservano l'evoluzione dal 1650 in poi, quando essa in realtà ha già un secolo di vita. I registri di Mandatorum custoditi presso l'Archivio di Stato di Napoli da cui Ulisse Prota-Giurleo, prima dei disastri della seconda guerra mondiale, aveva tratto informazioni sulla Real Cappella, purtroppo sono andati distrutti. Si sa di certo che i membri della Real Cappella si raccolsero nel tempo in una propria confraternita sotto l'egida di Santa Cecilia che aveva sede nella chiesa di Santa Maria di Montesanto di Napoli. La confraternita era riservata ai musicisti della Cappella Reale che ogni anno celebravano, il 22 novembre, la festa della loro protettrice con apparati, musica e fastose celebrazioni liturgiche. Era dotata di un monte che tratteneva una percentuale sullo stipendio dei musicisti

7 Magaudda/Costantini 2009.

8 Il valore artistico dell'archivio storico della Solitaria è strettamente connesso a quello dell'intero complesso conventuale di Santa Caterina, attuale sede della Facoltà di lettere dell'Università degli Studi "Suor Orsola Benincasa". L'edificio custodisce opere di importanti artisti, che ne fanno un'antologia di grande portata storica della cultura del Settecento. I locali dell'Archivio Storico hanno il pregio di essere interamente affrescati da Vincenzo Diano e Fedele Fischietti. Attualmente la complessa integrità dell'archivio può essere riscontrata attraverso un inventario provvisorio che riporta la documentazione dal 1580 al 1890 circa. Oltre ai documenti relativi all'amministrazione dell'istituto, con la minuziosa descrizione di entrate e uscite, l'archivio raggruppa documenti sulla fondazione del conservatorio con le sue finalità e i vari passaggi di sede, la documentazione riguardante alcuni lasciti testamentari che i nobili spagnoli destinavano al conservatorio per costituire le doti delle fanciulle ospitate nel pio luogo, e soprattutto il fondo "Figlie della Casa", testimonianza storica di generazioni di fanciulle di origine spagnola che vissero nella Real Casa. 
impiegandola in investimenti sugli arrendamenti, compravendite di immobili e nell'organizzazione delle processioni cittadine della Solitaria e dei 'Battaglini'. I musicisti affiliati godevano del pagamento dei giorni di malattia, della sepoltura e di messe in suffragio della loro anima. ${ }^{9}$ Così come per la Real Cappella, anche sull'attività della confraternita dei musici di Palazzo esistono documenti certi dal 1655 in poi; difficile invece comprendere la fondazione e l'evoluzione di questo organismo nei primi anni del Seicento.

Il ritrovamento di alcuni documenti inediti risalenti ai primi quarant'anni del Seicento presso il conservatorio della Solitaria ha consentito di far luce sulla fondazione della confraternita dei musici di Palazzo e della cappella di Santa Cecilia, la cui sede originaria risulta essere per l'appunto la chiesa della Solitaria annessa all'omonimo conservatorio.

La documentazione del primo Seicento, presente all'interno dell'Archivio, raggruppa testimonianze che spiegano, seppur in maniera lacunosa, la storia della fondazione del conservatorio. Sono documenti redatti in spagnolo e di non facile lettura perché in cattivo stato di conservazione, ma è possibile riscontrare in essi numerose indicazioni circa il primo utilizzo della musica nel conservatorio; vi si fa riferimento infatti alla musica del viceré e ai musicisti della «Capilla di Santiago» una delle tante congregazioni napoletane, ${ }^{10}$ con sede nella chiesa di San Giacomo degli Spagnoli. ${ }^{11}$ I numerosi musicisti, provenienti da questa congregazione dedicata a San Giacomo, intervenivano nelle principali celebrazioni della Solitaria. I musici della «Capilla de Santiago» presero parte attivamente alla vita

9 I musicisti attivi nelle diverse istituzioni napoletane erano in genere affiliati a specifiche corporazioni e congregazioni. Si trattava di vere società assistenziali che svolgevano un ruolo di tutela nei confronti dei propri componenti e regolamentavano il diritto di esecuzione in pubblico e di insegnamento della musica. Nell'Italia meridionale, dalla fine del Cinquecento in poi, soprattutto nei territori soggetti al dominio spagnolo, vi era la consuetudine dell'associazione corporativa fra musicisti. Si veda: Magaudda/Costantini 2001, pp. 79-204; Columbro/Intini 1998, pp. 41-76; FABRIS 1994, pp. 779-800.

10 Si è sempre ipotizzato che la confraternita di Santa Cecilia dei Musici di Palazzo derivasse da quella di San Giorgio Maggiore, la più antica fra le congregazioni napoletane, cfr. FABRIs 1994, p. 783. Questi documenti invece aprono nuove ipotesi di indagine, e sembrerebbe più logico immaginare che il nucleo primitivo della Real Cappella insediatosi poi alla Solitaria, provenisse dalla congregazione di San Giacomo, non a caso una delle istituzioni spagnole presenti a Napoli. Ulteriori ipotesi su questo punto sono state presentate da Giulia Veneziano al convegno della International Musicological Society nel luglio 2012 con la relazione The soundscape of the "Nazione Spagnola": music activity at the church of San Giacomo degli Spagnoli and the "Hermandad de nobles espanoles" in Spanish Naples.

11 La costruzione della chiesa risale al 1540 e fu voluta dal viceré spagnolo Don Pedro de Toledo per associarla ad un ospedale destinato alla cura dei poveri già presente per volontà di alcuni nobili spagnoli e dedicato a San Giacomo; la chiesa di San Giacomo degli Spagnoli è riconosciuta come chiesa nazionale di Spagna, essendo oltretutto amministrata dalla Real Hermandad de Nobles Españoles de Santiago. 
del conservatorio e della Chiesa fino al 1608: dopo questa data non si riscontrano più riferimenti alla «musica de Santiago» bensì ai «Musici di Palazzo».

Dobbiamo sottolineare che già gli studi di Ulisse Prota-Giurleo collegavano le vicende della confraternita dei Musici di Palazzo alla Solitaria. ${ }^{12}$ Egli infatti aveva ipotizzato come anno di fondazione della confraternita il 1613 poiché si riteneva che in quello stesso anno Carlo Sellitto avesse dipinto una Santa Cecilia per l'omonima cappella sita nella chiesa della Solitaria, e quindi possibile prima sede di tale organismo. ${ }^{13}$

Tuttavia la stessa documentazione relativa alla chiesa e alla tela dedicata a Santa Cecilia è lacunosa e priva di validi riscontri scientifici. La tela raffigurante Santa Cecilia intenta a suonare l'organo, oggi custodita al Museo di Capodimonte di Napoli, non riporta né la firma dell'autore né la data. Nel corso dei secoli numerose furono le attribuzioni del quadro ad artisti quali Carlo Sellitto, Battistello Caracciolo, Andrea Vaccaro. ${ }^{14}$ L'attribuzione definitiva al Sellitto si ebbe nel corso degli studi per una mostra dedicata a Carlo Sellitto nel 1977, quando il gruppo di studiosi che si occupava del dipinto precisò la datazione della tela facendo riferimento alle memorie dello storico Carlo De Lellis. Questi, nelle sua Aggiunta alla Napoli sacra del d'Engenio del $1654,{ }^{15}$ ricordava che ai suoi tempi il quadro era collocato nella cappella di Santa Cecilia nella chiesa di Santa Maria della Solitaria non facendo però il nome dell'autore. L'epigrafe che il De Lellis sosteneva di ricordare sotto la tela, affermava che il Cappellano Maggiore e i confratelli della Real Cappella sotto il titolo di Santa Cecilia, dedicavano il dipinto alla santa nel 1613; tra le firme poste in calce all'epigrafe vi era anche il nome di Giovanni Maria Trabaci. La querelle ebbe quindi termine con l'assegnazione del quadro a Carlo Sellitto su di un'ipotetica commissione di Trabaci del 1613, avvenuta probabilmente poco prima

12 Prota-Giurleo 1952²; Prota-Giurleo 1984, vol. I, pp. 446-447; Fabris 1987, p. 60; Fabris 1994, p. 788.

13 Pittore di origini montemurresi attivo nei primi decenni del Seicento. Il padre lo introdusse come discepolo presso il pittore fiammingo Loise Croys, artista molto stimato a Napoli. Carlo Sellitto venne considerato dagli storici dell'arte del nostro tempo, il primo caravaggesco napoletano. Sellitto realizzò altre opere come il San Carlo Borromeo per la chiesa di Sant'Aniello a Caponapoli, l'Adorazione dei pastori per Santa Maria del Popolo agli Incurabili. Negli anni successivi, Sellitto realizzò molti altri dipinti come il Sant'Antonio con il Bambino del Museo di Capodimonte e la Visione di San Candida in Sant'Angelo a Nilo. Morì a Napoli nel 1614, a soli 33 anni, si veda Prota-Giurleo $1952^{2}$.

14 Domenico Antonio Parrino nel 1700 fu il primo ad attribuire il dipinto a Carlo Sellitto, successivamente contestato da Bernardo De Dominici che lo assegnava a Battistello Caracciolo. Ancora al Battistello la riferivano nel 1891 Riccardo Filangieri e Rinaldo De Rinaldis nel 1911. La tesi battistelliana fu ripresa e sostenuta nel 1915 da Roberto Longhi, mentre fu Hermann Voss a proporre il nome di Andrea Vaccaro. Ancora nel 1943 sempre Roberto Longhi riaprì il dibattito aderendo all'ipotesi del Voss e assegnando il quadro al Vaccaro. Cfr. Prota-GiURLeo 1984, pp. 446-447.

15 Si tratta dell'Aggiunta alla Napoli sacra del d'Engenio del sig. R. Carlo de Lellis, un manoscritto in 5 volumi conservato presso la Biblioteca Nazionale di Napoli con segnatura X B20-24. 
della morte dell'autore. Il quadro di Santa Cecilia nell'unico inventario dei beni del conservatorio della Solitaria risalente al 1695 è però attribuito al Caravaggio: ${ }^{16}$

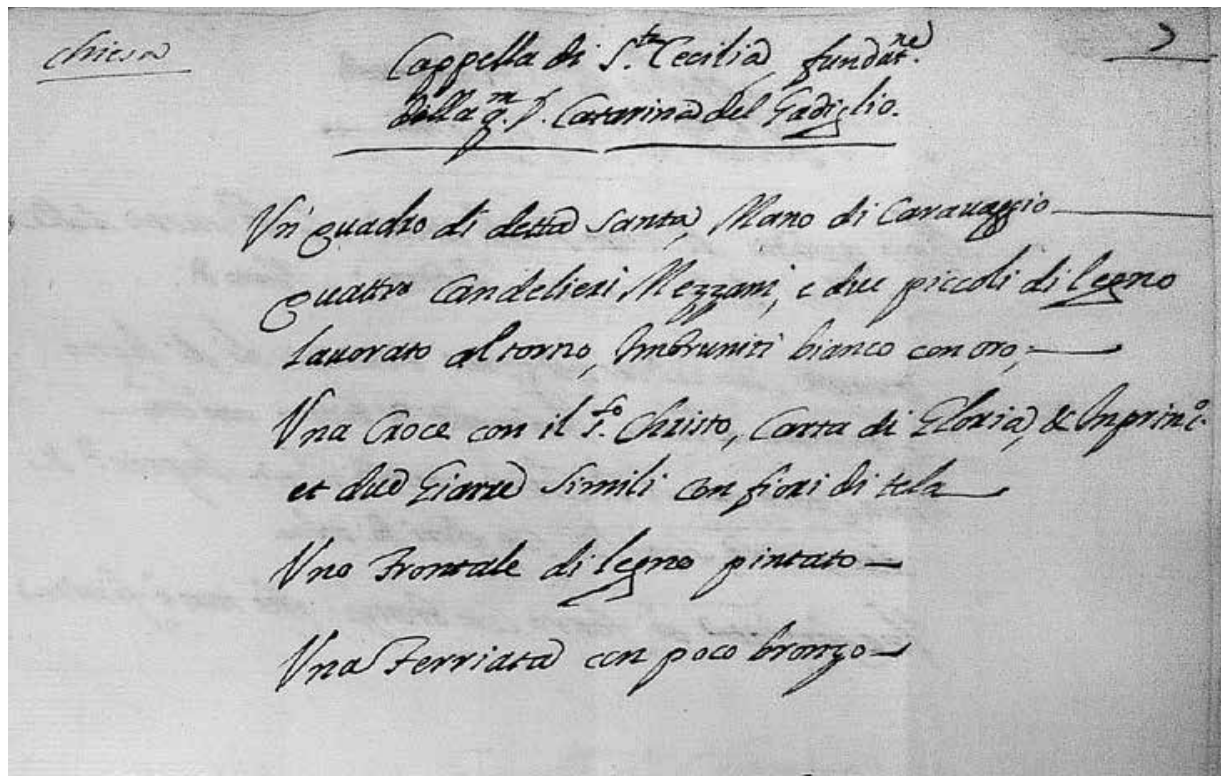

13. Inventario 4 luglio 1695.

Napoli, Archivio Storico della Solitaria.

L'anno di fondazione della cappella con l'insediamento ufficiale dei musici di Palazzo alla Solitaria risulta essere il 1609, come dimostra un documento presente nel Primero Libro de Rentas del 1607, in cui sono annotate le disposizioni e gli obblighi dei Musici di Palazzo nei confronti del conservatorio e chiesa della Soledad:

Capilla de Santa Cicilia de los musicos de Palacio

El primero dia de xbre 1608 ante notar Landolfo de Branche de Napoles los Señores Administratores desta Santa Casa Francesco Decañas don Diego Dequiñones Pedro de Hita y Garia de peña cedieron una capilla desta Santa yglesia que esta entrando por la puerta pequeña della amano derecha con titulo de la Confradia de Santa Cicilia a Jacobo Anelo Saso Gian Antonio Coradi y Joseph y Antonio Raymondo governadores y procuradores de la dicha confradia y capilla la qual es de los musicos y tañidores de la capilla real del palacio desta ciudad de Napoles de la qual tomaron el mismo dia la posesion y la començaron a aderecar para poder desir misa en ella y la primera Capilla que se dijo fu à 29 de março dell'año 1609 que fu domingo celebrola don Juan Domenico de Fura clerigo desta Santa Casa y el lunes siguente se canto misa con mucha veneracion y per toda la musica ansi de cantores como tañedores de palacio en la qual queden como propria hajer y dehajer por aumento de la dicha confradia de Santa Cecilia bienaventurada. ${ }^{17}$

16 Solitaria, Inventario, Coll. N.2.1.49.

17 Di seguito si riporta la traduzione del documento: «Cappella di Santa Cecilia dei musici di Palazzo. Il primo giorno del dicembre 1608 davanti notar Landolfo de Branche di Napoli i Signori Amministratori di questa Santa Casa Francesco Decañas, Don Diego Dequiñones, Pedro de Hita y Garia 
Il documento testimonia che nel dicembre del 1608 gli Amministratori della Soledad avevano ceduto una cappella a «Jacobo Anelo Saso Juan Antonio Coradi y Joseph y Antonio Raymondo», governatori e procuratori della confraternita di Santa Cecilia dei Musici della Real Cappella di Palazzo. Da esso si comprende che la celebrazione del possesso della cappella avvenne il 29 di marzo del 1609, cui seguì «una messa con molta venerazione e con tutta la musica di cantanti e suonatori di Palazzo». Da quel momento in poi i governatori della confraternita si impegnavano a far cantare e suonare gratuitamente presso la chiesa della Solitaria i musicisti della Cappella Reale di Palazzo in occasione dei primi e secondi vespri e la messa cantata nel giorno di Nostra Signora della Solitaria in settembre, oltre alla corresponsione di un censo di quindici ducati l'anno che erano tenuti a versare per il possesso della cappella. Nel caso avessero mancato questo obbligo gli amministratori della Solitaria si tutelavano mettendo in chiaro che avrebbero potuto chiamare altri musicisti per poter supplire a tale mancanza, il cui l'onorario sarebbe stato a carico della confraternita. ${ }^{18}$

Dal 1610 in poi si trovano una serie di pagamenti che attestano il censo dovuto dalla confraternita dei Musici alla Soledad, come il seguente:

Banco de Santojaco y Vittoria pagate per me a li governatori del monastero de la Solita di questa cita de napoli d. quindici e celi paga per nome e parte alli cantori e musici della real capella de palazzo per una anata che finira al primo de augusto prossimo venturo che deti musici e cantori

hanno ceduto una cappella di questa Santa Chiesa che sta entrando per la porta piccola di destra con il titolo della Confraternita di Santa Cecilia a Jacobo Anelo Saso Juan Antonio Coradi e Joseph e Antonio Raymondo governatori e procuratori de la detta confraternita e cappella la quale è dei musici e suonatori della Cappella Reale di Palazzo di questa città di Napoli di cui lo stesso giorno cominciarono a prendere possesso e a guarnirla per poter dire messa in quella e la prima cappella che è stato detto fu il 29 di marzo del 1609 che fu domenica la celebrò da don Juan Domenico de Dura clerico di questa Santa Casa e il lunedì seguente si cantò messa con molta venerazione e per tutta la musica di cantanti e suonatori di Palazzo [...]». Solitaria, 1 Libro de Rentas 1607, app. p. 315.

18 Se si considera che la bottega del Croys, ove Sellitto si era formato, era meta del compositore e musicista Jean de Macque, maestro della Real Cappella e dello stesso Trabaci, l'attribuzione al Sellitto sarebbe una logica conseguenza della commissione del Trabaci. Oggi però, con la nuova documentazione proveniente dall'Archivio della Solitaria, che sposta al 1609 la fondazione della cappella dedicata a Santa Cecilia, è forse lecito chiedersi se sia realmente possibile ipotizzare la commissione del quadro dedicato a Santa Cecilia nel 1613 ovvero cinque anni dopo l'inaugurazione della cappella. Bisogna dire inoltre che fino ad oggi la documentazione d'archivio indagata intorno all'anno 1613 non riporta alcun riferimento circa un arrivo tardivo dell'effige della santa, posteriore quindi all'inaugurazione della cappella. Inoltre, va anche sottolineato che l'Aggiunta del De Lellis, opera ambiziosa che si proponeva di emendare e aggiornare l'importantissima guida sacra all'antica capitale del Regno meridionale di Cesare d'Engenio Caracciolo, è di certo un'opera monumentale, ma non priva di errori, che non venne mai portata a termine e mai data alle stampe. I ricordi del De Lellis sulla chiesa della Solitaria, relativi allo spazio interno della chiesa e alla suddivisione delle cappelle sono infatti discordanti e la stessa epigrafe dedicata a Santa Cecilia è riportata in due differenti versioni, la prima delle quali non reca alcuna datazione della tela. L'ipotesi di Prota-Giurleo è probabilmente da retrodatare e con essa andrebbe forse ridiscussa l'attribuzione della tela al Sellitto e/o la datazione del dipinto, ricordando che proprio Caravaggio fu a Napoli dal 1606 al 1607 e una seconda volta dal 1609 al 1610. 
sono obligati pagare anui a detti governatori per la concesione che detti governatori hanno fato a deti musici e cantori de una capella de Santa Cicilia dentro detta eclesia della Soleda in virtu de cautela per mano de notar Landolfo de branca de Napoles a la qual me refero quali d. quindici mi sono pervenuti per mano del signor cappellano maggiore, con della ratione che detta ecclesia della Solita è stata sotisfata de tute le annate passate [...] 20 maggio 1615 Vincenzo Recco procuratore de la Regia Cappella. ${ }^{19}$

Questi documenti sono fondamentali anche perché ci consentono di meglio ricostruire le vicende della Cappella Reale. Ci indicano ad esempio i nominativi dei governatori e procuratori della confraternita: ${ }^{20}$ specificano il coinvolgimento dei cantori che assieme ai musici dal 1614 in poi furono presenti nelle differenti celebrazioni festive; ci danno informazioni sul differente impiego di strumentisti, suddivisi in musicisti e suonatori, a rimarcare la differenza fra gli strumentisti di livello che prendevano parte alle celebrazioni liturgiche e semplici musicisti di strada impiegati soprattutto nelle processioni, come «Juan Cola Picinino trompeta de palacio» cui venivano corrisposti sei ducati «por los trompetas y menestrilos que an servido en la fiesta de Nostra Señora [... 9 de settembre $1615 » .{ }^{21} \mathrm{Dal} 1623$ al 1632 le carte segnalano la presenza di Giovanni Maria Trabaci con la funzione di maestro di cappella, come si legge in questo documento:

Governadores dela Capilla de Santa Cecilia pagaron d.10 a Gioan Maria Trabachi Mastro dela Capilla real de Palacio por nostra quenta delo que senos deve del censo delos anos d. 15 dela dicha Capilla que seles paga por la musica que han hecho en notra Iglesia el dia de Nostra Señora a 8 de settembre 1630 de casa a 9 de settiembre $1630 .{ }^{22}$

Dal momento della costituzione della cappella di Santa Cecilia in poi, le vicende della Cappella Reale si legheranno, come vedremo nelle prossime pagine, a quelle del conservatorio della Solitaria. ${ }^{23}$

19 Solitaria, Registro 9, app. p. 314.

20 L'organizzazione interna di una congregazione era in genere articolata in alcuni governatori, un procuratore, tre deputati.

21 Solitaria, Registro 8, app. p. 314.

22 Solitaria, Registro 10, app. p. 317.

23 È da sottolineare l'importanza di questa istituzione relativamente al peso politico nella città di Napoli. Essa aveva per governatori personalità di spicco dell'ambiente militare e politico e rappresentava una sorta di sodalizio posto sotto la diretta protezione del viceré. La Soledad di Napoli si ricollega in modo straordinario alla cappella dei militari spagnoli di Nostra Signora della Soledad di Palermo. Le due istituzioni risultano simili non solo per tipologia di culto, per peso politico all'interno della città, e anche per il legame fra i musicisti presenti alla Soledad e quelli del Palazzo Reale. Cfr. Tedesco 2001. 


\section{Le festività}

La Solitaria vede la partecipazione dei musicisti della Real Cappella per ogni momento festivo dell'anno liturgico. «La musica del Señor Virrey» veniva impiegata per le Quarant'ore, per le ricorrenze mariane e per le monacazioni, in cui si cantavano «muchos motetes a dos coros». ${ }^{24}$

Ogni momento devozionale era occasione per fare musica presso l'istituzione spagnola. Nelle spese della chiesa del gennaio 1707, Gaetano Veneziano viene retribuito come maestro di cappella «per la musica hà fatto in questa chiesa nella notte di natale à 4 voci; due violini, viola, controbasso, et organo [...]». ${ }^{25}$

Negli stessi anni sempre per la notte di Natale e con organico simile vengono stipendiati come maestri di cappella Domenico De Majo e Antonio Raicola.

Numerose le celebrazioni commemorative dei santi titolari degli altari presenti nella chiesa come San Giuseppe, Sant'Antonio da Padova, San Giovanni Battista, San Raimondo, San Giacomo, San Pietro. Alla Solitaria inoltre era particolarmente diffuso il culto di San Michele Arcangelo che veniva celebrato in due distinte festività: l'8 maggio in occasione della rievocazione dell'apparizione del Santo sul monte Gargano, e il 29 settembre festa del Santo. Si predisponeva una novena con la celebrazione di una messa cantata al giorno con l'ausilio di cantanti e musicisti «per tutti li nove giorni di dette due feste $[\ldots] » .^{26}$

Il conservatorio era legato al culto di Nuestra Señora de la Soledad, corrispondente a quello della Madonna Addolorata. Il culto si diffuse infatti in Italia durante il periodo della dominazione spagnola,${ }^{27}$ in particolare nello stato di Milano e nei regni di Napoli e Sicilia. Sia a Milano che a Palermo esistevano infatti chiese e congregazioni con questa dedicazione. ${ }^{28}$ Rispetto ad altre celebrazioni, il ce-

24 Solitaria, Elenchi e Notamenti, app. p. 310.

25 Solitaria, Giornale di introito ed esito 1705-1716, app. p. 331.

26 Solitaria, Libro Maggiore 1695-1703, app. p. 328.

27 Le origini del culto della Soledad in Spagna risalgono al XVI sec. Con la fondazione a Madrid nel 1567 della "Confradia della Soledad y Angustias de Nuestra Señora". Presso questa confraternita è attestata già ad inizio del Cinquecento, e tuttora esistente, la processione del Santo Entierro y de los Siete Dolores, ossia della Santa Sepoltura e dei Sette dolori della Vergine, che commemorava la Passione di Cristo nella Settimana Santa. Sul culto della Soledad in Spagna si segnala: Hierro 1959, pp. 1-32; Martin/PÉrez 2000.

28 La festa dell'Addolorata si diffuse durante il XIII-XIV sec. in Occidente con tolleranza della Sede Apostolica con varie denominazioni: la Madonna della Pietà, la Madonna dello Spasimo il pianto della Beata Vergine, il martirio del cuore della Beata Vergine Maria, la compassione della Beata Vergine Maria, i Sette dolori di Maria etc. Era celebrata in varie date: il venerdì dopo la domenica in albis, il primo sabato dopo l'ottava di Pasqua, il venerdì antecedente la domenica di Passione. Il culto della Madonna dell'Addolorata era particolarmente diffuso nel viceregno di Napoli, basti pensare alle numerose congregazioni che ne prendevano il nome. L'istituzione più prestigiosa tra di esse era la Real Congregazione di Nostra Signora de' Sette Dolori eretta nella chiesa di San Luigi di Palazzo. Nota soprattutto per l'esecuzione dello Stabat Mater di Alessan- 
rimoniale liturgico e musicale per la festa «Dolori della madre Santissima» era particolarmente solenne. Ne troviamo conferma anche nella «Gazzetta di Napoli»:

\begin{abstract}
Venerdì trascorso di Passione [28 marzo], siccome ne' primi vesperi del giorno avanti [27 marzo] nella chiesa della Solitaria delle moniche spagnuole fu solennizzata la festa della Vergine Addolorata con singolarissima magnificenza, tanto pel numeroso concorso di nobiltà, regi ministri togati e militari, quanto per il ricchissimo apparato della chiesa, musica a raddoppiati cori di sceltissimi cantori ed istromenti, infiniti lumi e preziosissima argenteria. ${ }^{29}$
\end{abstract}

Nel 1712 e nel $1724^{30}$ vi aveva preso parte anche Matteo Sassano cantando «teneri e divoti mottetti». ${ }^{31}$ Per l'occasione «mastri indoratori» si cimentavano in arditi «lavori d'oro e argento», allestivano palii indorati, «coretti per li musici», macchine effimere di anno in anno modificate e altari ornati con baldacchini, fiori di seta o di carta, «fogliami traforati di argento», panni e parati di damasco, «puttini e angeloni», «christalli, festoni, buccali d'argento». Si trovano ancora spese per i maestri organari per «l'accomodatura» degli strumenti e per quelli che «hanno tirato l'organi il giorno della festa $[\ldots] »$, «per quello che hà sonato le campane», per i musicisti intervenuti e ancora spese «dar rinfresco a detti musici» e ai «40 figlioli della Pietà». ${ }^{32}$

La festa del Venerdì di Passione introduceva ai riti della Settimana Santa. Si iniziava la domenica delle Palme con la consueta processione che precedeva la liturgia eucaristica e si proseguiva con la fastosa preparazione dei sepolcri al Giovedì Santo. Ad Antonio Raicola spettavano «d. 25 per cinque musiche hà fatto in questa Chiesa a 4 voci, e 4 instrumenti dalla Domenica delle olive per tutto il giorno di Pasqua di Resurrettione», ${ }^{33}$ o Gennaro Ursino, veniva pagato «per due servitij della Musica fatta nella Settimana Santa», ${ }^{34} \mathrm{e}$ ancora Gaetano Veneziano stipendiato «per la Musica fatta in detta Chiesa nella Domenica dell'olivo; Giovedì, Venerdì, e Sabato Santo, e Domenica di Resurrettione [...]». ${ }^{35}$

Come citato in precedenza a proposito dei riti della Settimana Santa in uso presso le istituzioni partenopee, la Solitaria era rinomata soprattutto per la famosa e sug-

dro Scarlatti che avveniva nei Venerdì di Quaresima, e per aver commissionato a G. B. Pergolesi l'omonima opera. Sulle origini del culto della Addolorata e l'arciconfraternita napoletana dei Sette Dolori si veda: Magaudda/Costantini 2003, pp. 51-137.

29 GDN, 1 aprile 1692, app. p. 333.

30 GDN, 11 aprile 1724, app. p. 334. Magaudda-Costantini riportano inoltre che l'avvocato olandese Jan Alensoon nel suo diario di viaggio annotò che «in questa occasione furono eseguite composizioni di Francesco Mancini. Lo stesso personaggio riferisce che anche la sera precedente vi era stata eseguita musica «alquanto solenne e bella» dello stesso compositore, con la partecipazione di Sassano, che vi aveva cantato un brano solistico, probabilmente un mottetto. Nonostante al viaggiatore il famoso evirato fosse sembrato già abbastanza avanzato d'età, questi si era distinto per la bella voce e per l'improvvisazione di meravigliosi abbellimenti». Si veda Magaudda/Costantini 2009, appendice p. 409, nota 1143.

31 GDN, 22 marzo 1712 (1), app. p. 334.

32 Solitaria, Giornale di introito ed esito 1686-1691, app. p. 325.

33 Solitaria, Giornale di introito ed esito 1697-1704, app. p. 329.

34 Solitaria, Libro Maggiore 1711-1716, app. p. 331.

35 Solitaria, Libro Maggiore 1704-1710, app. p. 330. 
gestiva processione del Venerdì Santo detta 'della Solitaria' descritta da numerose fonti storiche come De Lellis, Celano etc. La processione a cui predeva parte la città tutta conduceva alla conclusione della lunga settimana, al termine della quale aveva luogo ancora una solenne messa e un'ulteriore processione il giorno di Pasqua. ${ }^{36}$

Inoltre, in quanto monastero di regio patronato non mancano riferimenti alle liturgie per la celebrazione di ricorrenze dell'aristocrazia partenopea. In alcuni casi la «Gazzetta di Napoli» sottolinea la partecipazione attiva delle stesse religiose durante queste ricorrenze festive:

Domenica mattina 4 del corrente [...], per [...] [la] gravidanza della M. dell'imperadrice [...] il segretario di Stato e Guerra D. Michele Orsi fé celebrare nella R. chiesa della Solitaria, monasterio di dame della nazione spagnuola di cui n'è primo governatore, con [...] messa cantata, e solennizzossi il Te Deum cantato dalle reverende madri $[\ldots] \cdot{ }^{37}$

\section{La formazione musicale}

Essendo un istituto di assistenza la Solitaria garantiva alle proprie ospiti un'educazione elevata grazie anche all'apprendimento della musica.

La gestione quotidiana dell'istituto era affidata a una governatrice, mediamente sui quarant'anni «di molte qualità et valore, et specialmente di molto spirito, oratione, mortificatione, prudentia, libera et disoccupata di figlioli». L'età per essere accolte andava dai 5 ai 18 anni e a fine '500 il conservatorio raggiunse tra le 25 e le 50 unità. Completata la loro formazione e raggiunta la maggiore età, alle giovani veniva assicurata una dote di 100 ducati per poter «trovare marito come si conviene», o in alternativa potevano divenire monache di conservatorio, seguendo la regola domenicana. Vestivano un abito bianco e nero: il bianco a simboleggiare la «nettezza», il nero «il dolore della Vergine per la morte del Figlio». La giornata era regolata dai rintocchi di un orologio e la preghiera era alla base della vita comunitaria, la frequenza della confessione era mensile. I confessori dovevano essere inoltre «santi spirituali e literati». ${ }^{38} \mathrm{La}$ struttura, simile a quella monastica, prevedeva doppia porta da superare per accedere all'interno, nonché una terza porta intermedia; c'erano anche un piccolo giardino e una cappellina per le devozioni.

La Solitaria garantiva una vera opera educativa sia alle ragazze in preparazione al matrimonio che alle religiose. Alla lettura di libri spirituali e vite dei santi, all'insegnamento della dottrina cristiana, alla cura del giardino, si affiancava anche

36 Sulla processione della Solitaria si rimanda al cap. III, pp. 70-71.

37 Il Te Deum venne celebrato in occasione della gravidanza di Elisabetta Cristina di BrunswickWolfenbüttel, moglie di Carlo III, re di Napoli. Cfr. GDN, 11 dicembre 1715 (10).

38 La gestione quotidiana del conservatorio si apprende dalle regole che si conservano in AsDN, Visite Pastorali, XVI. 
l'insegnamento della musica. La pratica musicale non era un evento occasionale, ma consuetudine giornaliera e centrale nella vita del conservatorio.

Erano proprio i musicisti della Real Cappella ad essere tenuti a provvedere alla formazione musicale delle monache e delle figlie ospiti del conservatorio. I primi documenti che accennano a questa pratica risalgono al 1601. Essi rivelano i ruoli ricoperti dai diversi musicisti con l'indicazione delle paghe corrispettive. Il «mastro de canto de las virgines» della Solitaria, don Domenico Manso, veniva stipendiato con «çinco ducados [...] por su salario de cinco meses de primero Agosto 1601 por todo dicembre siguente [...]», ${ }^{39}$ e qualche anno dopo nel 1607 è don Aurelio Sassano che insegna «de cantar [...] a las Virgines». ${ }^{40} \mathrm{Nel} 1652$ troviamo Andrea Ansalone, esponente di una delle famiglie di musicisti più note nella città di Napoli ${ }^{41}$ con il compito di «enseñar a las monjas de esta Santa Casa de cantar». ${ }^{42}$

«Maestro di musica delle monache» della Solitaria dal 1659 al 1670 fu il veneziano Cristoforo Caresana, allievo del celebre Pietro Andrea Ziani:

don Cristofaro Caresana Maestro di Musica delle monache di questo Monastero deve a 11 Agosto 1659 d. 30 pagarli lo banco del popolo per conto delle fatighe che ha fatto dalli 22 maggio 1659 che ha cominciato ad insegnare dette monache et che sta facendo per l'advenire $[\ldots]{ }^{43}$

Trasferitosi a Napoli nel 1658, Caresana entrò a far parte della Real Cappella di Palazzo come tenore e organista, fu inoltre maestro di cappella presso il conservatorio di Sant'Onofrio dal 1688 al 1690, e nel 1699 maestro presso la Cappella del Tesoro di San Gennaro. A Caresana subentrò Pietro Bartilotti, ${ }^{44}$ per breve tempo direttore del conservatorio di Santa Maria di Loreto, attivo alla Solitaria dal 1671 al 1698 col titolo di maestro di musica e di cappella, stipendiato per «la musica che insegna alle Reverende Monache». ${ }^{45}$ Dal 1708 al 1724 fu Gennaro Ursino ${ }^{46}$

39 Solitaria, Registro Copiapolizze 1601-1603, app. p. 310.

40 Solitaria, Registro Copiapolizze 1603-1616, app. p. 312.

41 Ben quattordici membri di questa famiglia napoletana nel corso di quattro generazioni furono attivi fra XVI e XVII sec, soprattutto in veste di strumentisti a fiato. Alcuni di essi hanno fatto parte della Cappella Reale altri si dedicarono maggiormente alla didattica. Cfr. Grove 2001.

42 Solitaria, Manual 2 1644-1654, app. pp. 319-320.

43 Solitaria, Libro Maggiore 1656-1662, app. p. 322.

44 Brevi notizie su Pietro Barilotti si ritrovano in: Di Giacomo 1928.

45 Solitaria, Libro Maggiore 1679-1685, app. p. 325.

46 Gennaro Ursino fu allievo di Giovanni Salvatore presso il conservatorio della Pietà de' Turchini di Napoli, dal 1675 fu assistente di Francesco Provenzale al conservatorio della Pietà de' Turchini, e assistente dello stesso Salvatore al conservatorio dei Poveri di Gesù Cristo, oltre alla Solitaria prestò servizio dal 1701 al 1715 come maestro di cappella presso la Santa Casa dell'Annunziata, la chiesa di Santa Maria in Portico e la Casa Professa e Collegio dei Gesuiti. Di Ursino non si conosce la data di morte, la voce a lui dedicata presente all'interno del Grove Dictionary of Music and Musician e curata da K.A. Larson, ne attesta l'attività fino al 1715. È quindi da sottolineare che invece Ursino fu attivo alla Solitaria fino al 1724, prolungando in tal modo la sua presenza sulla scena musicale partenopea. 
a ricoprire la carica di maestro di musica e ad insegnare a cantare alle religiose. Giuseppe De Bottis, proveniente sempre dalla prestigiosa Real Cappella, invece arrivò alla Solitaria nel 1725 e vi rimase fino al 1736.

I maestri di musica erano affiancati da quelli di strumenti ad arco. Nel 1652 Onofrio Mirabello insegnava la ribeca ${ }^{47}$ seguito dal maestro di violino Giovanni Angelo D'Urso che avea il compito «de enseñar las monjas de esta Santa Casa de tocar violin $[\ldots] \gg{ }^{48}$ Francesco Antonio D'Angelo, musicista del tutto obliato, probabilmente attivo presso la Cappella Reale intorno al 1650, figura essere «maestro de violini $\gg^{49}$ delle monache dal 1656 al 1670. Infine, nel 1695, è Pietro Marchitelli ad essere investito della stessa carica.

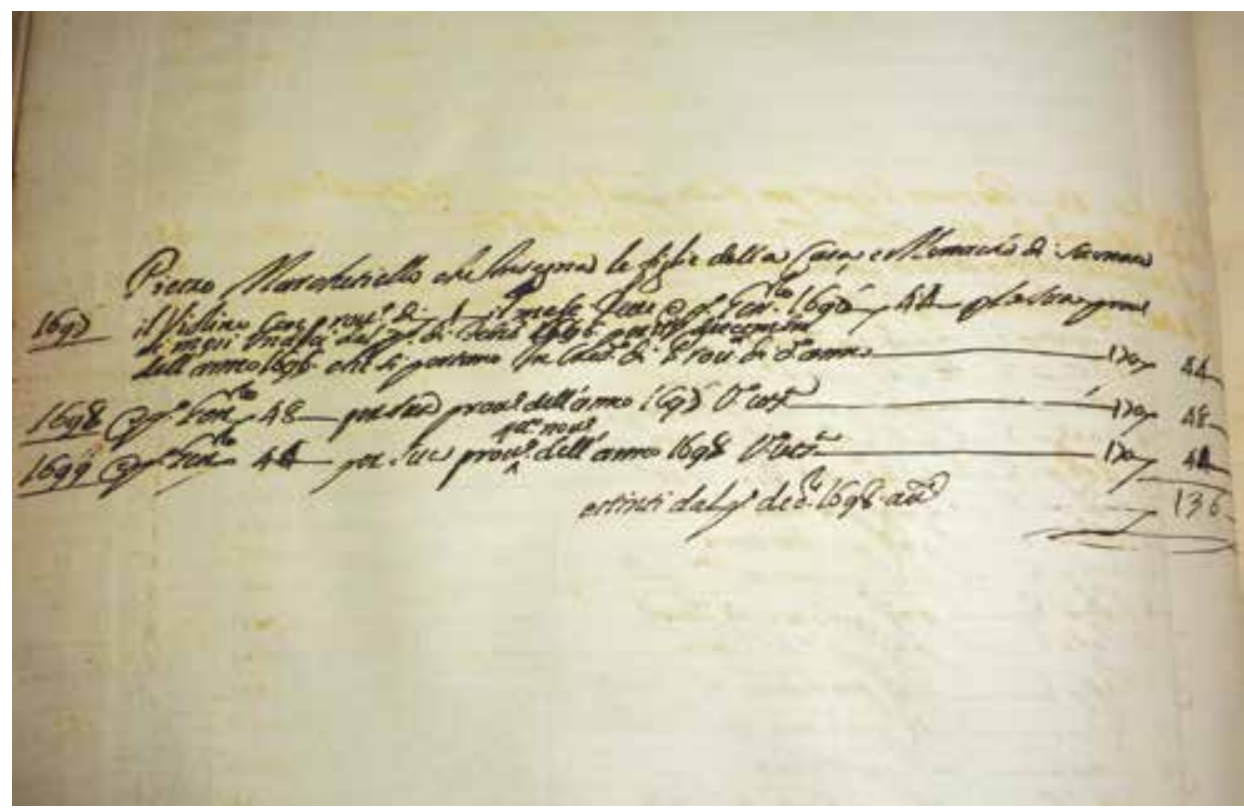

14. Libro Maggiore $1695-1703,1 / \mathrm{I} / 1697$, c. $144 \mathrm{v} .{ }^{50}$ Napoli, Archivio Storico della Solitaria.

Relativamente alla presenza di una scuola violinistica alla Solitaria non mancano pagamenti per tutto ciò che necessitava l'esercizio della disciplina musicale, dall'acquisto di strumenti ad arco e a tastiera, alle corde, alla carta da musica e a spartiti: Pietro Bartilotti si occupava dell'acquisto di «libri e carta rigata per la mu-

47 Solitaria, Manual 2 1644-1654, app. pp. 318-321. Sull'introduzione del violino alla Solitaria e negli altri istituti si rimanda al cap. IV, pp. 96-97.

48 Solitaria, Manual 2 1644-1654, app. p. 319.

49 Solitaria, Libro Maggiore 1656-1662, app. p. 322.

50 Cfr. app. p. 327. 
sica $\rangle^{51}$ assieme a «corde di violone, et violini copie de sonate di organo, violone et violini» e ad «accomodare l'archi di detti Instrumenti [... $] \gg .{ }^{52}$ In un libro maggiore del 1696 vengono infatti corrisposti sei ducati alla «Rettora» del conservatorio «per comprare 2 violini; uno boffettino, uno lettorino, corde et accomodatura di una viola $[\ldots] \gg .^{53}$

Non mancano anche i pagamenti per i maestri organari e cembalari stipendiati per garantire l'ordinaria accordatura degli strumenti in uso sia presso la chiesa che presso il conservatorio. La manutenzione di questi strumenti avveniva con una certa periodicità, gli organari così come i cembalari, venivano pagati mensilmente con regolarità. Fra i maestri di «cimbalo» troviamo uno sconosciuto Andrea De Ragona e Bartolomeo Amoruso attivo anche presso la congregazione dell'Oratorio di San Filippo Neri, chiamato anche a costruire nel 1661 «uno cimbalo nuovo da lui fatto et venduto per uso di detto monastero a due registri $[\ldots] \gg .{ }^{54}$ Mentre fra gli organari attivi figurano alcuni fra i più grandi esponenti della scuola organaria napoletana come Giovanni Gualberto Ferreri organaro della prestigiosa Cappella del Tesoro di San Gennaro e della Cappella Reale, il rinomato organaro e organista Andrea Basso attivo dal 1671 e Felice Cimino. ${ }^{55}$

Purtroppo manca presso l'Archivio della Solitaria un fondo musicale, ma la fitta trama di contenuti racchiusa nei documenti perfettamente conservati, possiede, al di là di uno straordinario valore storico, una grandissima capacità evocativa, grazie all'energia e alla suggestione che trasmettono queste carte, che cessano così di essere dei semplici oggetti per acquistare significati, divenire simboli e custodi della memoria storica, trasmettendo un qualche "sonoro" riflesso di un'istituzione un tempo gloriosa.

51 Solitaria, Libro Maggiore 1679-1685, app. p. 325.

52 Solitaria, Libro Maggiore 1679-1685, app. p. 325.

53 Solitaria, Libro Maggiore 1695-1703, app. p. 327.

54 Solitaria, Libro Maggiore 1656-1662, app. p. 322.

55 Sull'arte organaria e cembalaria napoletana: si rimanda al cap. IV, pp. 98-99. 


\section{Conclusioni}

Da quanto detto nelle pagine precedenti risulta evidente che le istituzioni religiose femminili non furono soltanto una parte significativa del tessuto musicale della città, ma la loro funzione fu decisamente cruciale. Lo studio delle fonti archivistiche chiarisce il peso e la funzione delle comunità monastiche e testimonia che esse, anche per Napoli, divennero nel tempo centri indiscutibili di cultura, fucine di mecenatismo, in alcuni casi laboratori privilegiati di attività artistiche e di produzioni musicali, e diedero un contributo incisivo alla vita culturale e artistica della città.

Il numero dei monasteri nei quali vi era un'attività musicale potrebbe anche essere più alto di quelli presi in esame in questa sede. Bisogna infatti ricordare e precisare che per alcune istituzioni il materiale documentario pervenuto è lacunoso, in alcuni casi praticamente inesistente. Questo però non esclude la possibilità che la pratica musicale fosse consuetudine di tutte le realtà monastiche partenopee.

Le fonti archivistiche non si limitano a donarci informazioni sulla ricostruzione di usi e costumi delle sole comunità monastiche, al contrario sono utili a cogliere i multiformi rapporti tra monasteri, gerarchie ecclesiastiche e la realtà esterna; offrono numerosi dettagli sull'intero entourage che orbitava attorno ai chiostri; fanno luce su pratiche e tradizioni locali. La ricostruzione della storia musicale dei grandi e prestigiosi monasteri così come delle comunità 'minori' e del loro impegno nell'organizzazione festiva cittadina, amplia la prospettiva della storiografia musicale e aggiunge informazioni non secondarie sulle carriere di musicisti, su usi e costumi delle singole comunità e della città.

L'attività e il ruolo dei monasteri femminili rientra così di diritto nel circuito delle grandi istituzioni napoletane - la Cattedrale, la Cappella reale, i teatri cittadini, il Tesoro di San Gennaro - su cui si sono incentrati la maggior parte dei contributi sulla storia della musica napoletana. I monasteri infatti risultano essere promotori di pratiche devote che coinvolgevano la città tutta, e le stesse monache non si limitavano ad essere spettatrici passive, ma si preoccupavano di provvedere all'organizzazione spettacolare di questi grandi eventi. Pensiamo alla celebrazione del Corpus Domini, festività che coinvolgeva città, diocesi e viceré e che era fortemente patrocinata dalle clarisse di Santa Chiara. Stesso dicasi per la processione del Venerdì Santo alla Solitaria o le processioni di San Giovanni Battista e San Benedetto organizzate dal monastero di San Gregorio Armeno.

L'indagine sulle consuetudini musicali degli ordini monastici femminili apre nuovi filoni di ricerca e pone al contempo nuovi interrogativi e nuove ipotesi.

Le monache non si affidavano solo a compagini esterne per le esecuzioni musicali, ma ne commissionavano anche le composizioni da eseguire. Questo denota 
una collaborazione fra religiose e musicisti. Nella documentazione e nella ricostruzione storica degli istituti emergono riferimenti che permettono di ipotizzare questo tipo di sinergia. Il sostegno di musicisti professionisti per le liturgie però non ha ostacolato la pratica musicale da parte delle stesse religiose. Pensiamo ancora una volta alla realtà di Santa Chiara, che faceva costantemente ricorso a musicisti accreditati per le proprie celebrazioni liturgiche. Proprio a Santa Chiara è presente l'unica figura di monaca compositrice di cui si ha notizia per il periodo indagato. Inoltre, la documentazione interna degli istituti, porta ad ipotizzare la presenza di musicisti esterni che cantavano e suonavano insieme alle monache. Si veda la testimonianza delle Lamentazioni per il triduo delle Tenebre nella Settimana Santa di Gaetano Veneziano di cui si conservano le partiture presso il celebre Archivio dei Girolamini. Si tratta di composizioni per voce solista e piccolo ensemble strumentale che recano esplicite dediche a religiose. Chi avrebbe potuto accompagnare le religiose durante il rito delle Tenebre? È probabile dunque che fossero gli stessi musicisti esterni provenienti dalle compagini cittadine. D'altra parte abbiamo un chiaro riferimento a questa pratica nella testimonianza di alcune polizze del Banco di Napoli, in cui alcuni musicisti venivano stipendiati al conservatorio di Santa Maria del Rifugio per «aver accompagnato nella musica le moniche coriste di detto loro Conservatorio». ${ }^{1}$

Alcune fonti archivistiche e musicali inoltre documentano, oltre all'utilizzo della musica in occasione delle feste principali, una più o meno costante attività interna alle istituzioni, 'quotidiana', nel senso letterale del termine. Di tale attività sembra trovarsi riscontro nelle composizioni musicali legate alla pratica della polifonia semplice, rinvenute presso la biblioteca di Santa Chiara. Il concetto di decoro proprio dell'atto liturgico rivolto al Divino, imponeva di fare ricorso ad ogni mezzo in grado di realizzare al meglio ogni celebrazione e alle religiose spettava la scelta di soluzioni esecutive e di repertorio che meglio corrispondessero alle esigenze della liturgia. La pratica della polifonia semplice e del canto fratto permetteva di adattare il canto gregoriano all'estetica corrente, ottenendo una musica liturgica che non intaccava il rito e che allo stesso tempo era in grado di allietare il fedele, diventando orecchiabile grazie all'uso di caratteristiche proprie della musica vocale e strumentale dell'epoca. Questo porta a ipotizzare quanto diverse e quanto numerose fossero nella musica per la liturgia, anche in epoca barocca, le alternative alla grande polifonia dominio dei musicisti professionisti e delle compagini organizzate e soprattutto, quanto le diverse forme di canto liturgico rappresentassero, ognuna, una soluzione di differente efficacia per rispondere in maniera adeguata ai vari gradi di festività che le riforme liturgiche post-tridentine avevano istituito.

Uno dei dati maggiormente interessanti è l'aver riscoperto la funzione dei conservatori femminili in qualità di scuole di musica, da cui scaturisce obbliga- 
toriamente una revisione di alcuni clichés storiografici di grande peso. Nel tempo si è infatti consolidato il mito dei quattro antichi conservatori maschili come vere e proprie scuole di musica, lasciando passare l'idea che l'insegnamento della musica fosse esclusivamente loro appannaggio. I conservatori femminili nascono per accogliere ragazze in "educazione", per istruirle e riabilitarle anche attraverso la pratica dell'insegnamento del canto, della teoria musicale, di strumenti a corda e a tastiera. Le educande venivano affidate alla tutela del maestro di cappella o di una maestra e, in alcuni casi, le fanciulle potevano essere esentate da alcuni compiti ed esercizi giornalieri per poter prendere lezioni di musica e di canto. L'aspetto fondamentale è dunque la funzione che questi istituti svolsero a livello locale ed educativo, la cultura e la formazione che essi diedero a queste giovani sorelle, allieve spesso di grandi nomi della scuola napoletana. I conservatori permisero che la musica divenisse occasione di espressione artistica per donne che non avrebbero potuto, per estrazione sociale, percorrere la via della pratica musicale.

Dall'analisi proposta si comprende quanto la vita monastica donasse alla donna la possibilità di appropriarsi di una cultura di prim'ordine. Il chiostro non è più luogo di reclusione ma diviene risorsa e ricchezza, cenacolo di donne che gestiscono tempi, spazi, lavori, economie in autonomia, microcosmo in cui si trovano a convivere donne di differenti ceti sociali. Monache di alto rango e nobildonne in grado di amministrare ingenti patrimoni e gestire spazi di potere e cultura affiancano converse di umili origini o fanciulle da redimere. Emerge una presenza femminile viva e significativa che trae dagli atteggiamenti e dalle funzioni dell'attività musicale diversi benefici, a seconda se essa venga intesa come mezzo di espressione artistica, passatempo o strumento di riabilitazione sociale. Le vicende e le attività dei monasteri partenopei non solo fanno luce su quanto fosse estremamente varia l'offerta culturale da essi proposta, ma permettono soprattutto di recuperare una dimensione attiva e costruttiva dell'identità femminile nel contesto napoletano. 


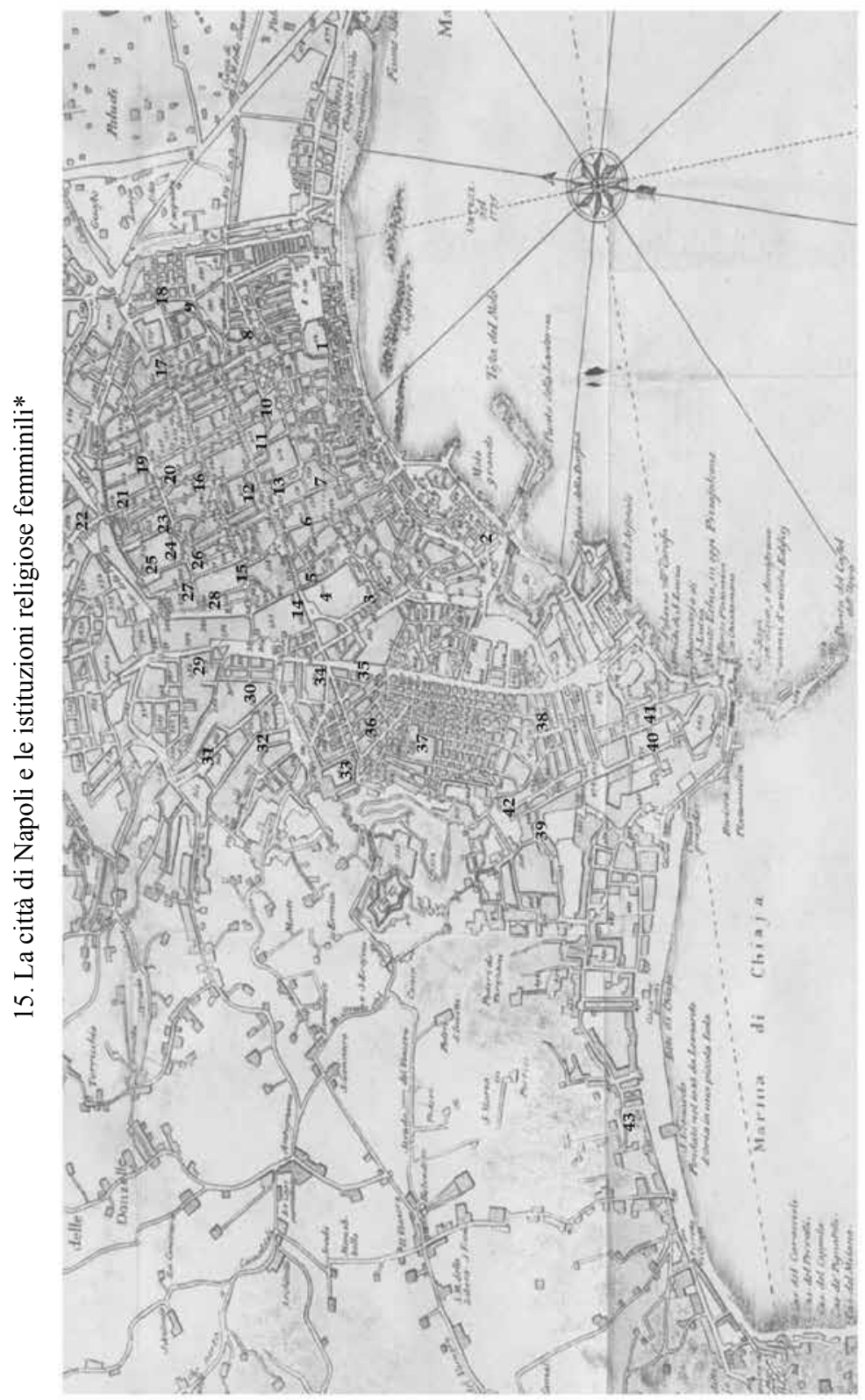

ํํㄹ के

萢的要

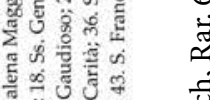

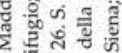

$\sum \frac{2}{3} \bar{z}$ व

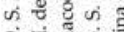

¿ $\Sigma$ 대

的㣢

政步的

造

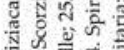

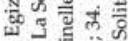

的昆

i

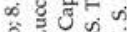

은

० 8 \&

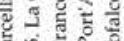

$\sum \frac{10}{4} \div$

कं हैं

部苟弯

के है

政

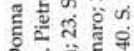

员

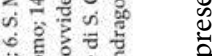

अँ.

ए人)

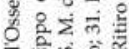

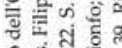

ठक्ष

造运

ह हैं एँ

in है

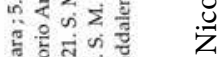

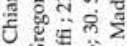

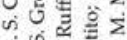

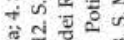

हैं के

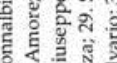

ถิ

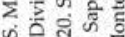

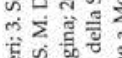

二

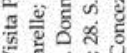

흘

w

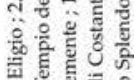

w.

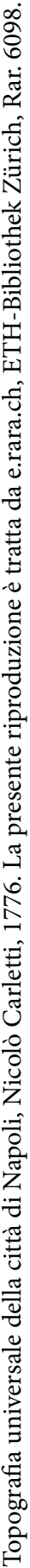




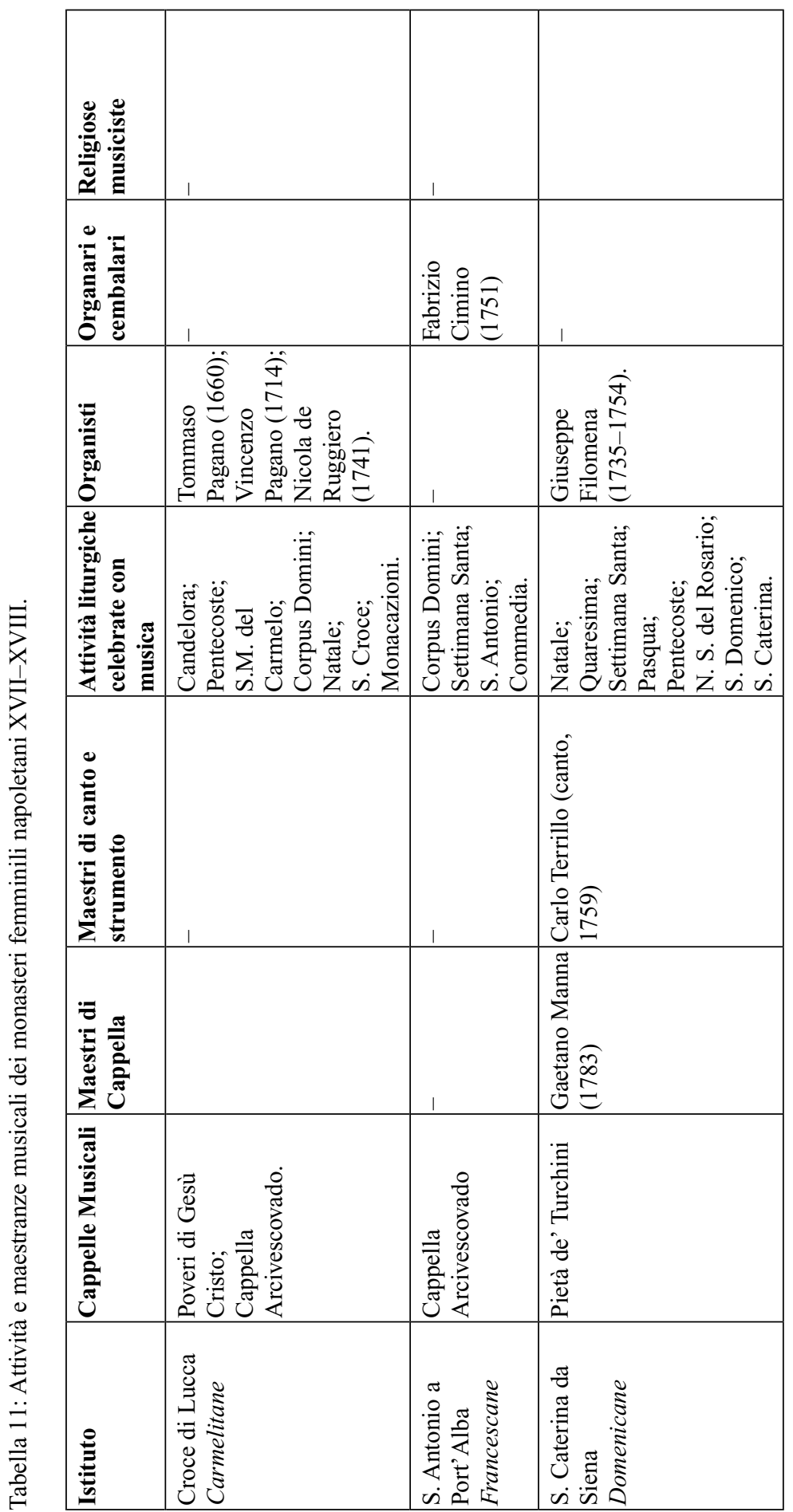




\begin{tabular}{|c|c|c|c|}
\hline 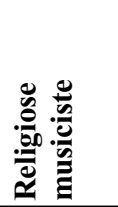 & 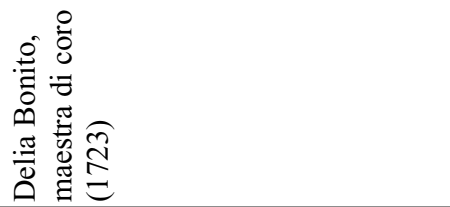 & 1 & 1 \\
\hline 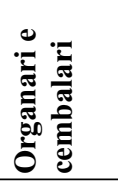 & & 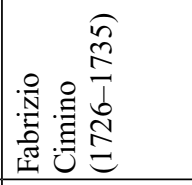 & \\
\hline 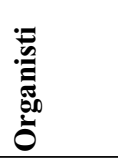 & & 1 & \\
\hline 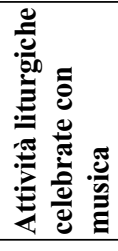 & 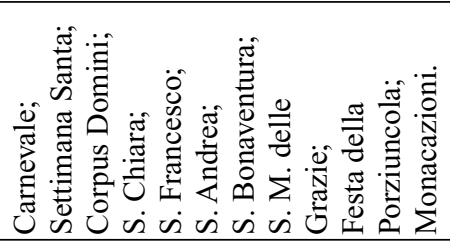 & 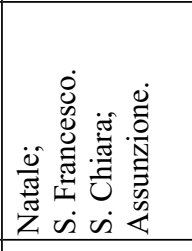 & 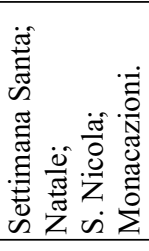 \\
\hline 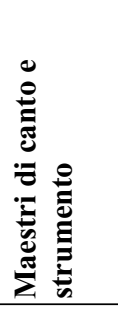 & & 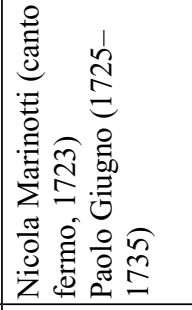 & 1 \\
\hline 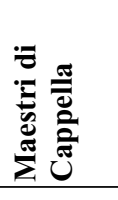 & 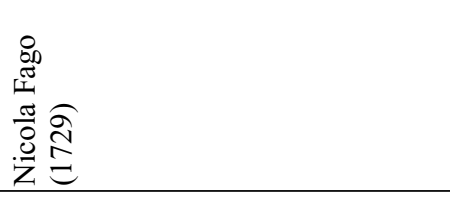 & 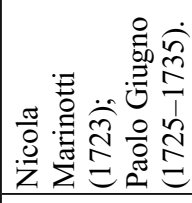 & 1 \\
\hline 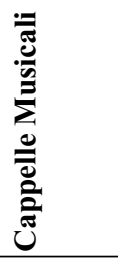 & 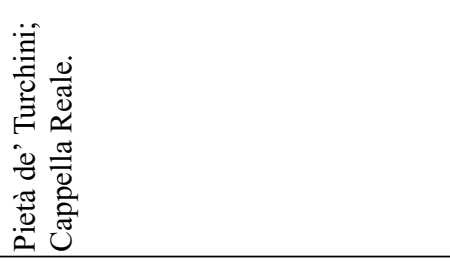 & 1 & $\begin{array}{l}. \\
0 \\
0 \\
0 \\
0 \\
0 \\
0 \\
0 \\
0 \\
0 \\
0\end{array}$ \\
\hline 兑 & 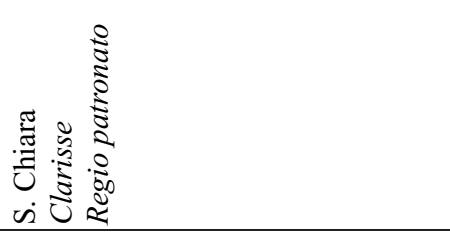 & 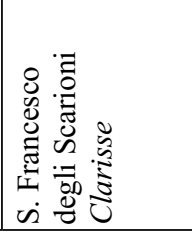 & 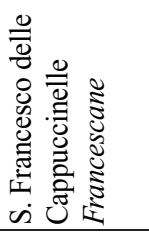 \\
\hline
\end{tabular}




\begin{tabular}{|c|c|c|c|}
\hline 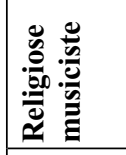 & 1 & 1 & 1 \\
\hline 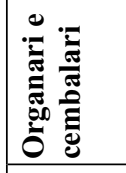 & 1 & & 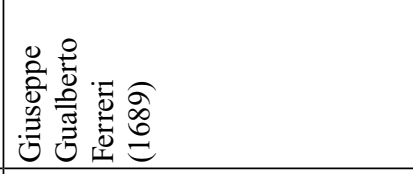 \\
\hline : & 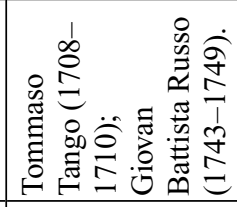 & 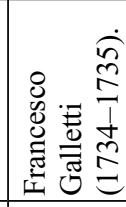 & 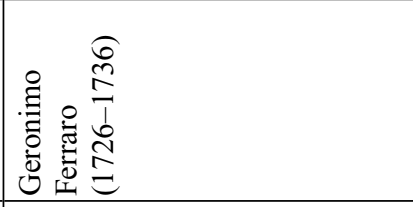 \\
\hline 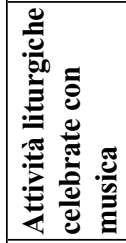 & 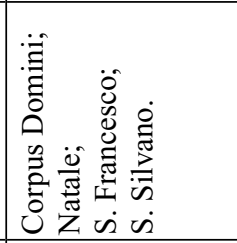 & 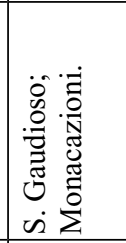 & 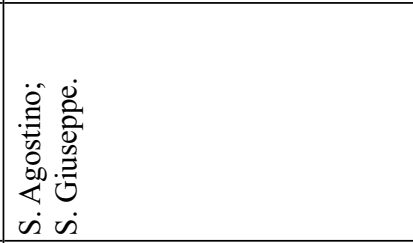 \\
\hline 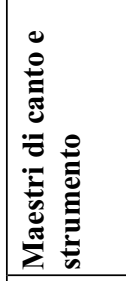 & 1 & 1 & 1 \\
\hline 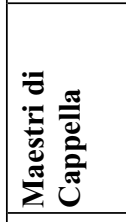 & 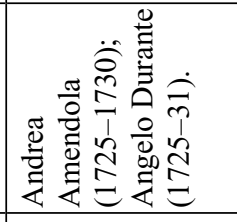 & 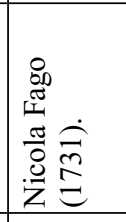 & 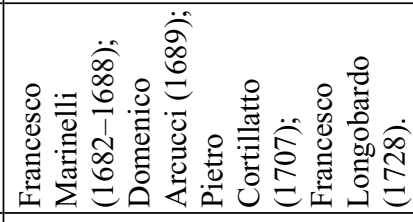 \\
\hline 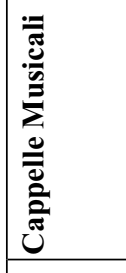 & 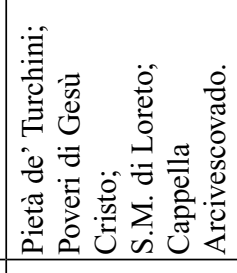 & 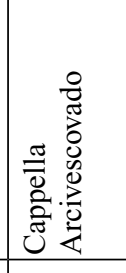 & 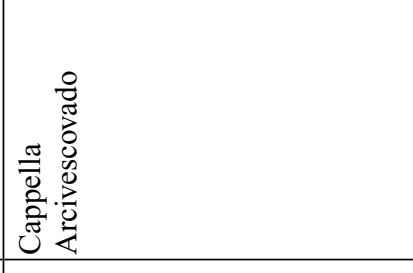 \\
\hline 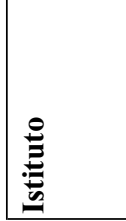 & 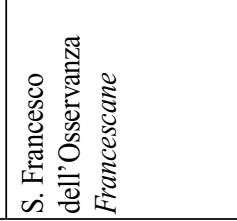 & 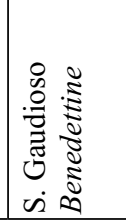 & 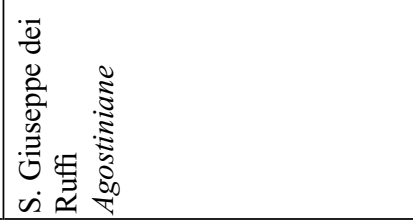 \\
\hline
\end{tabular}




\begin{tabular}{|c|c|}
\hline 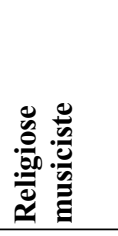 & 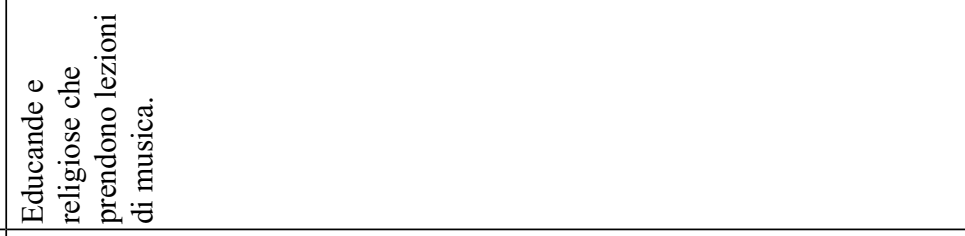 \\
\hline 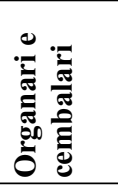 & 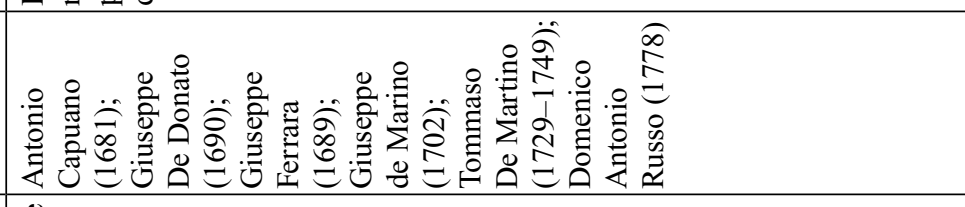 \\
\hline : & 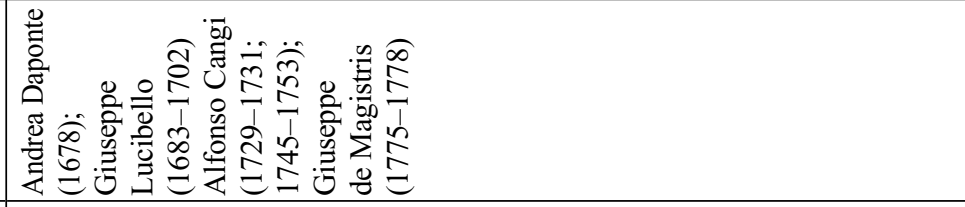 \\
\hline 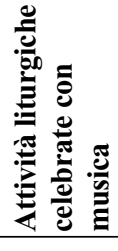 & 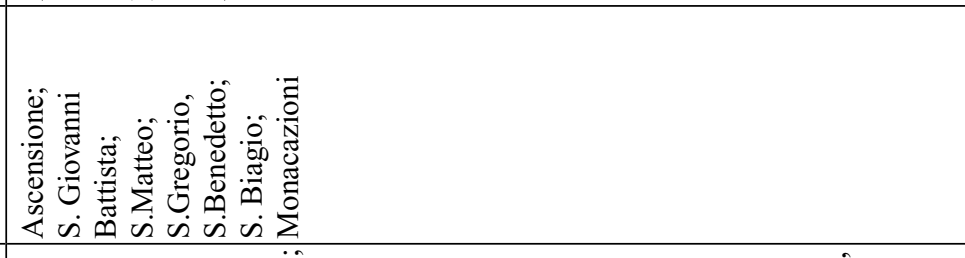 \\
\hline 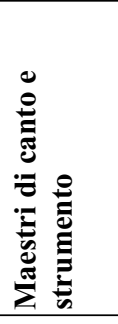 & 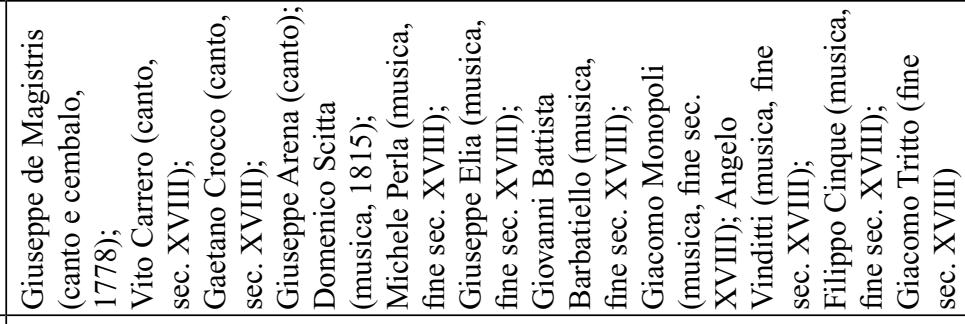 \\
\hline 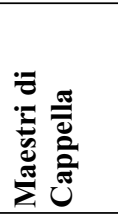 & 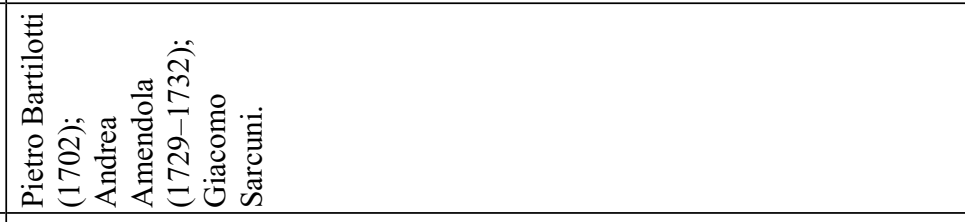 \\
\hline 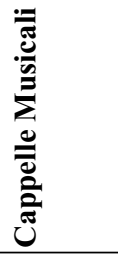 & 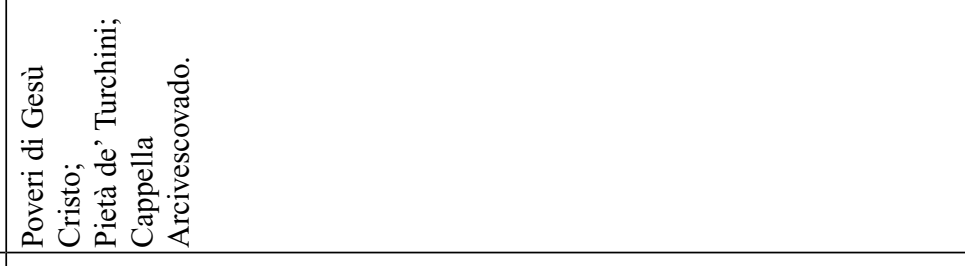 \\
\hline 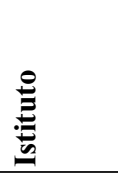 & 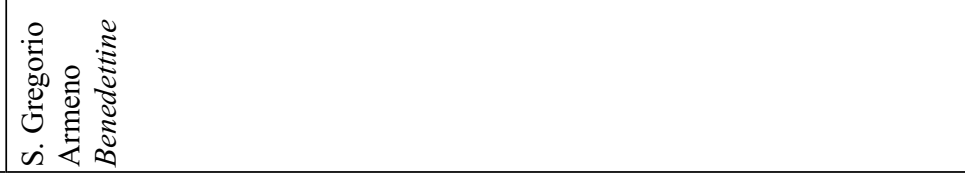 \\
\hline
\end{tabular}




\begin{tabular}{|c|c|c|c|}
\hline 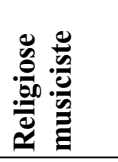 & 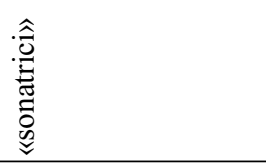 & 1 & 1 \\
\hline 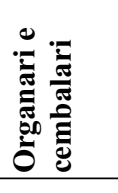 & 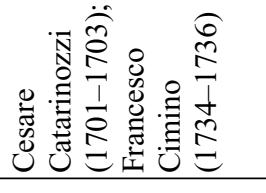 & 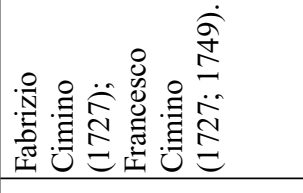 & 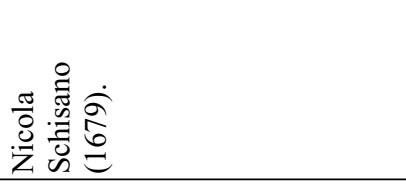 \\
\hline 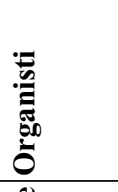 & 1 & 1 & 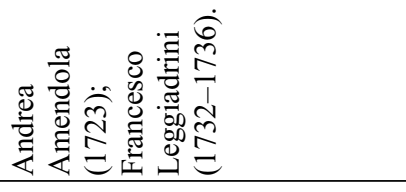 \\
\hline 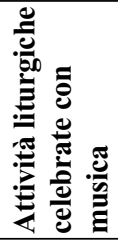 & 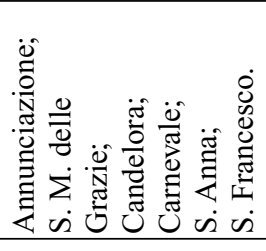 & 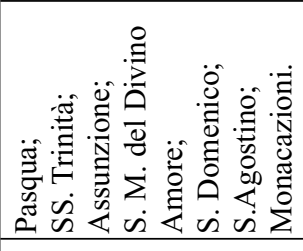 & 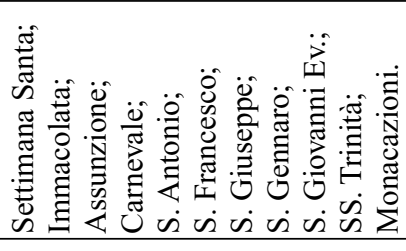 \\
\hline 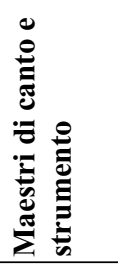 & 1 & & \\
\hline 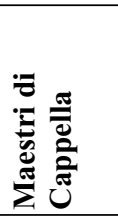 & 1 & 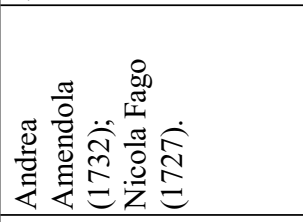 & 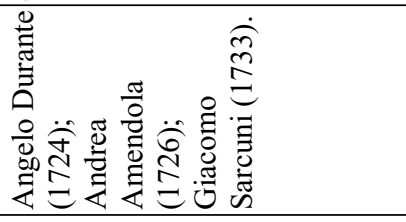 \\
\hline 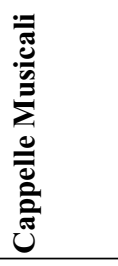 & 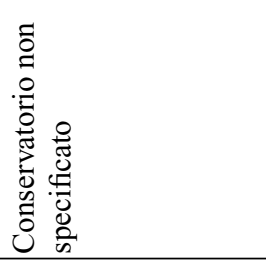 & 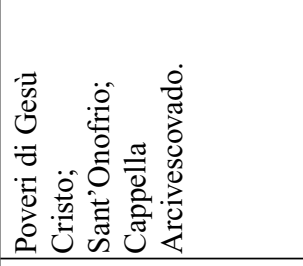 & 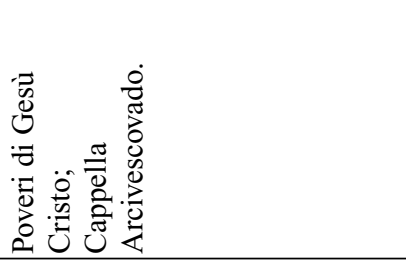 \\
\hline 竧 & 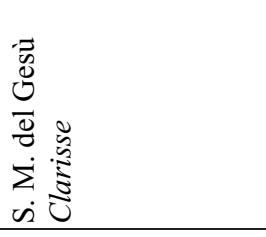 & 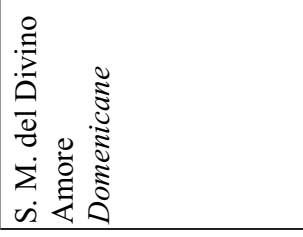 & 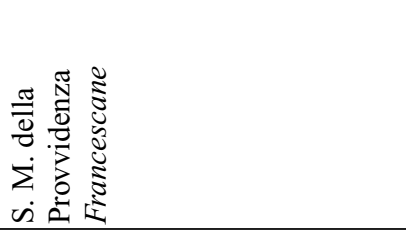 \\
\hline
\end{tabular}




\begin{tabular}{|c|c|c|c|}
\hline 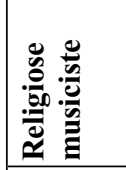 & 1 & & 1 \\
\hline 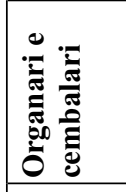 & 1 & 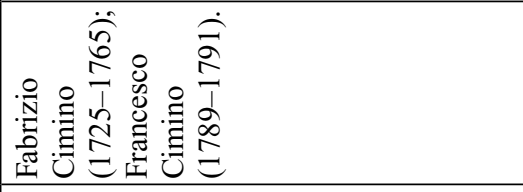 & \\
\hline 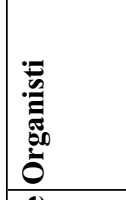 & 1 & 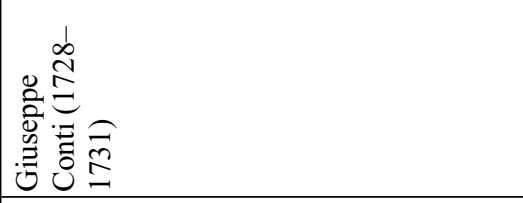 & 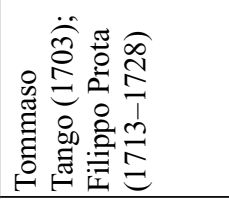 \\
\hline 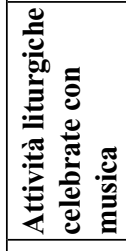 & 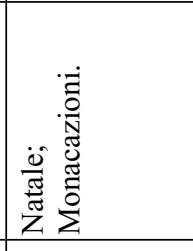 & 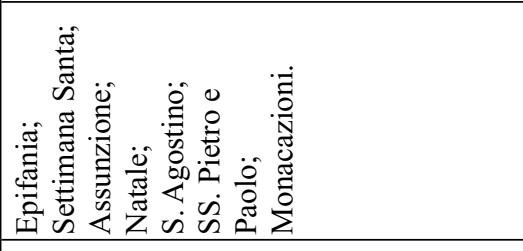 & 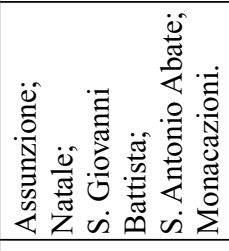 \\
\hline 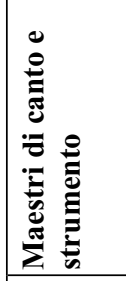 & 1 & & 1 \\
\hline 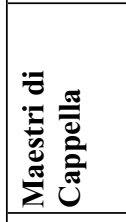 & 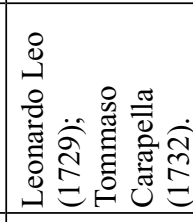 & 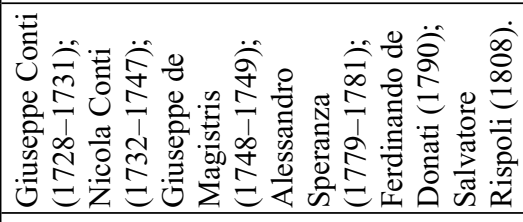 & \\
\hline 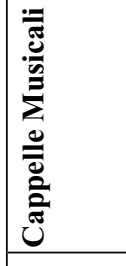 & 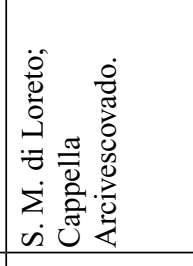 & 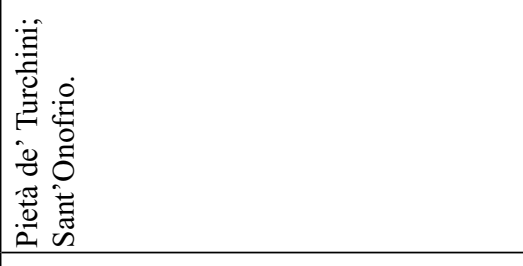 & 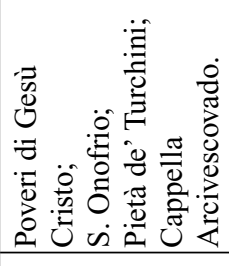 \\
\hline 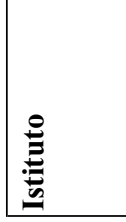 & 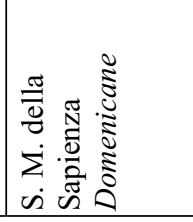 & 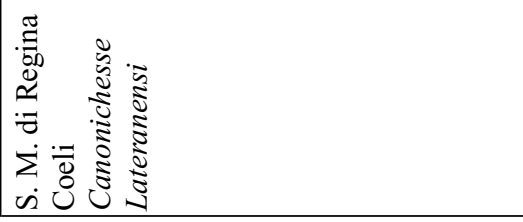 & 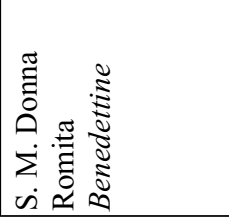 \\
\hline
\end{tabular}




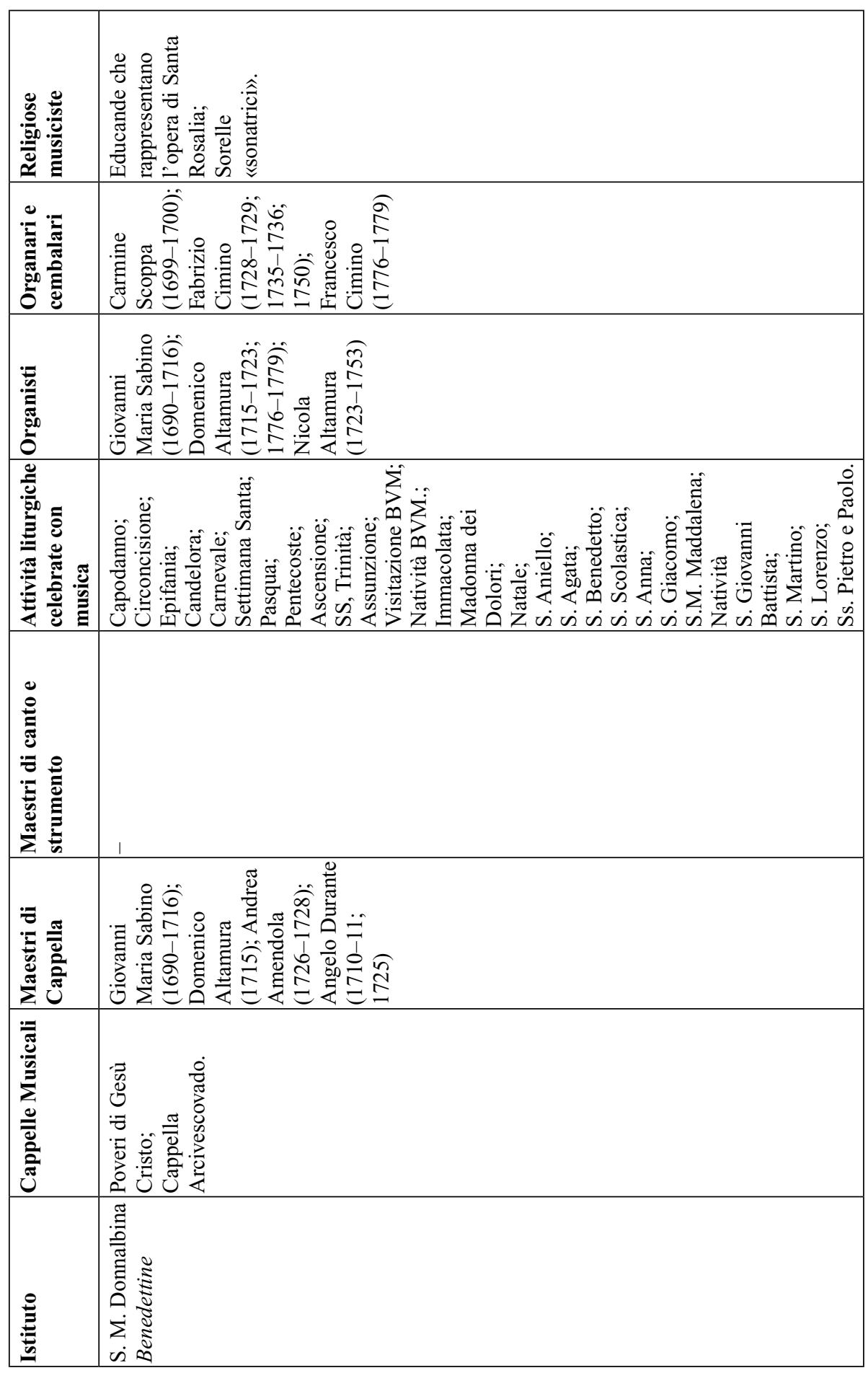




\begin{tabular}{|c|c|c|c|}
\hline 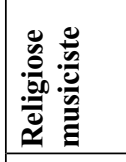 & 1 & 1 & 1 \\
\hline 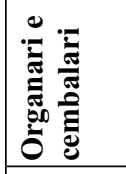 & 1 & 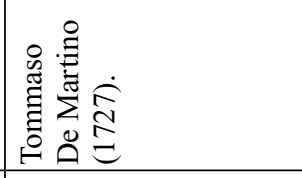 & 1 \\
\hline 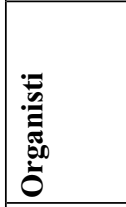 & 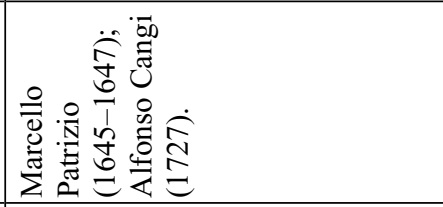 & & 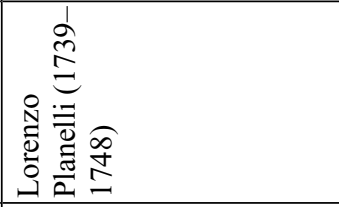 \\
\hline 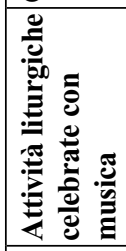 & 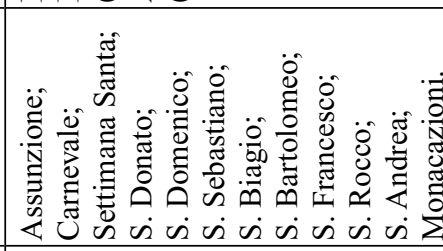 & 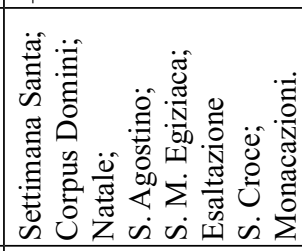 & 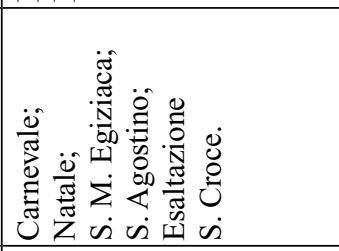 \\
\hline 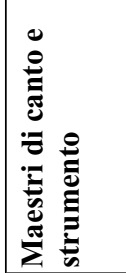 & 1 & & \\
\hline 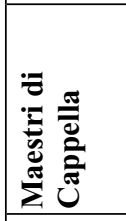 & 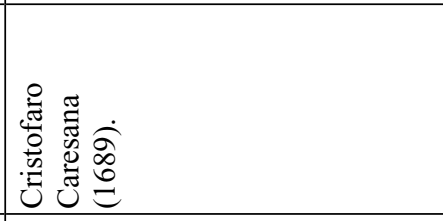 & 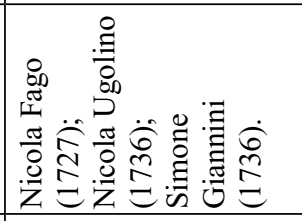 & 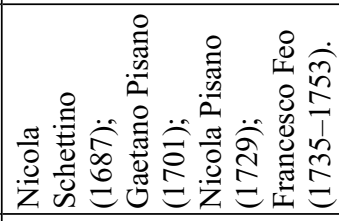 \\
\hline 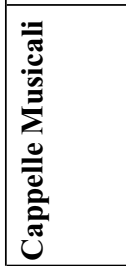 & 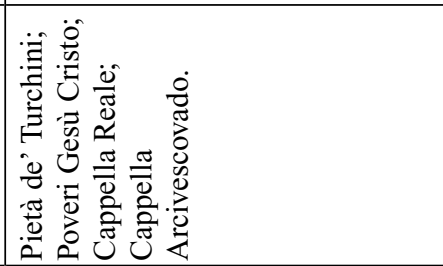 & 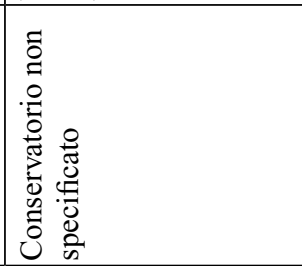 & 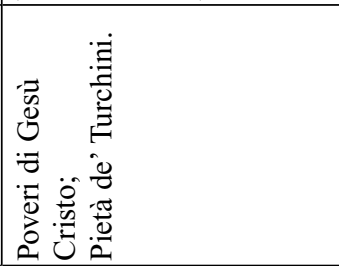 \\
\hline 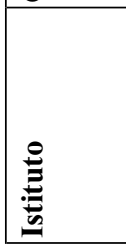 & 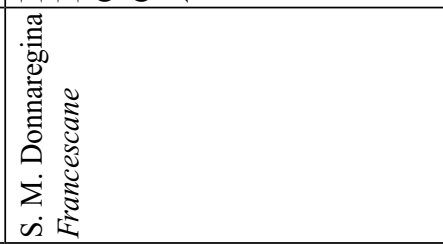 & 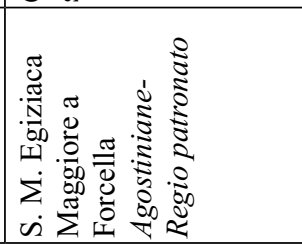 & 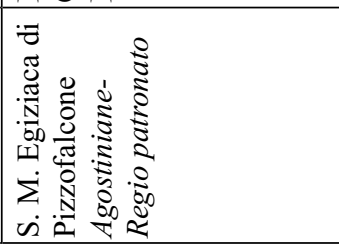 \\
\hline
\end{tabular}




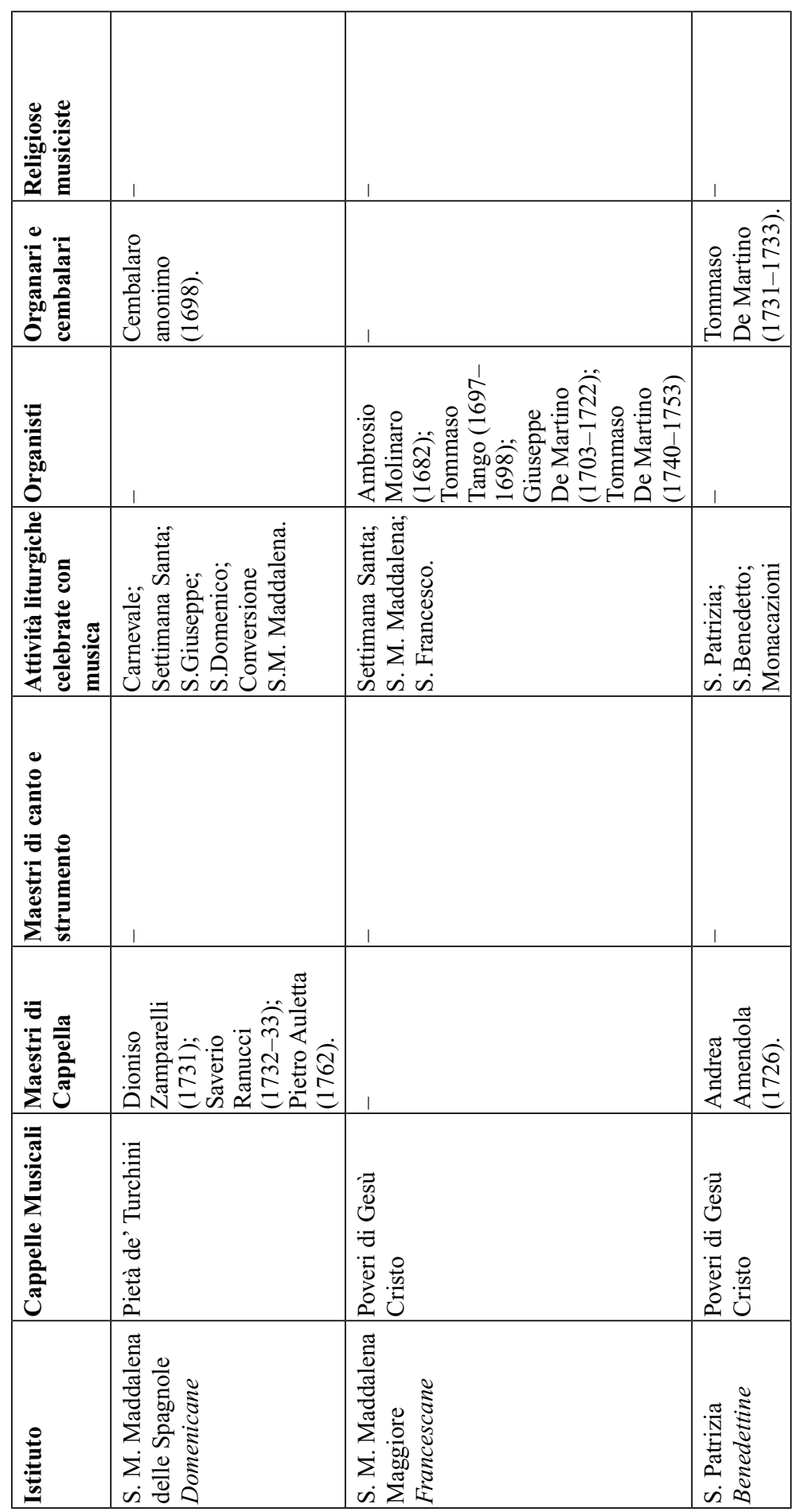




\begin{tabular}{|c|c|c|c|}
\hline 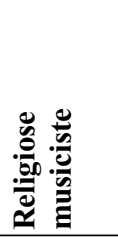 & 1 & 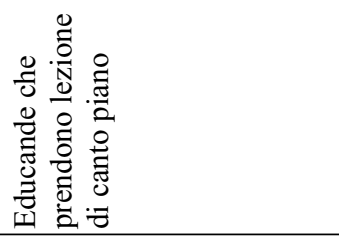 & 1 \\
\hline 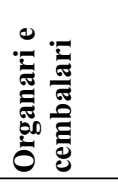 & 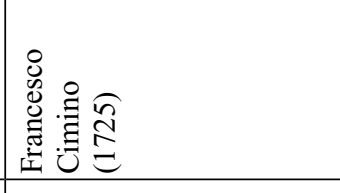 & 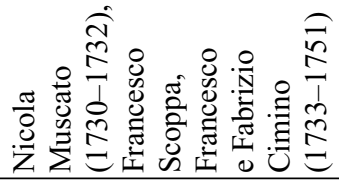 & 1 \\
\hline : & 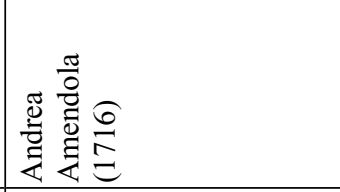 & 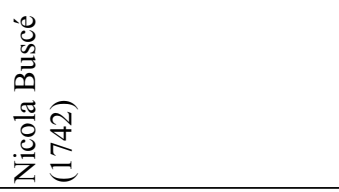 & 1 \\
\hline 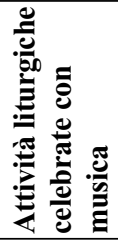 & 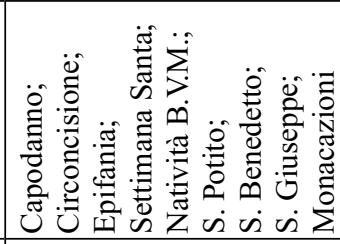 & 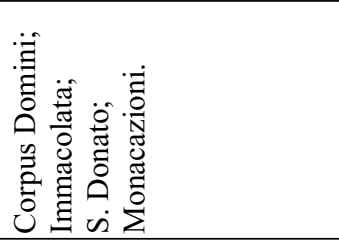 & 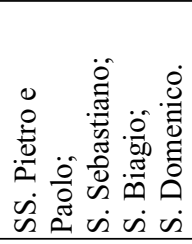 \\
\hline 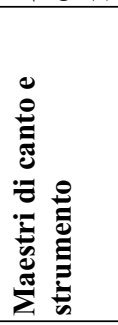 & & 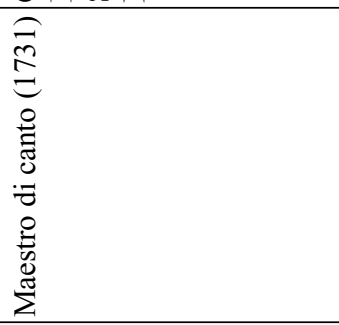 & \\
\hline 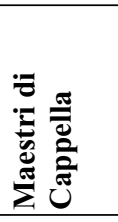 & 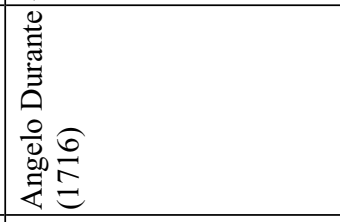 & 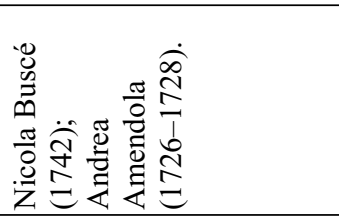 & 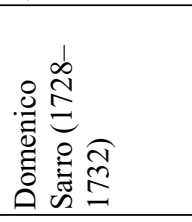 \\
\hline 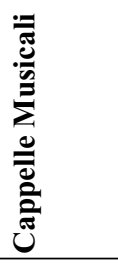 & 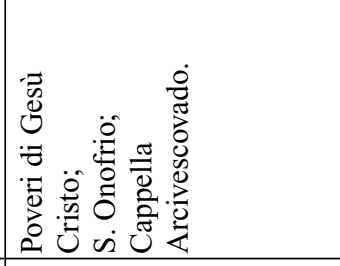 & 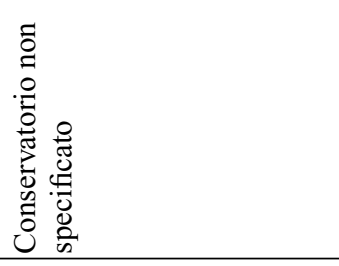 & 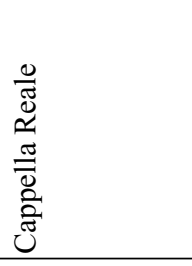 \\
\hline 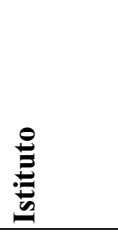 & 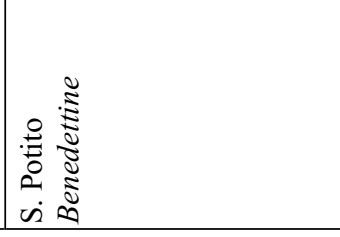 & 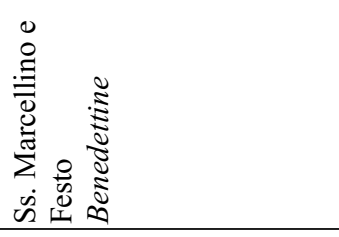 & 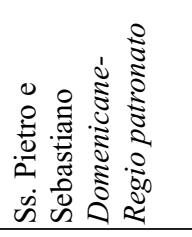 \\
\hline
\end{tabular}




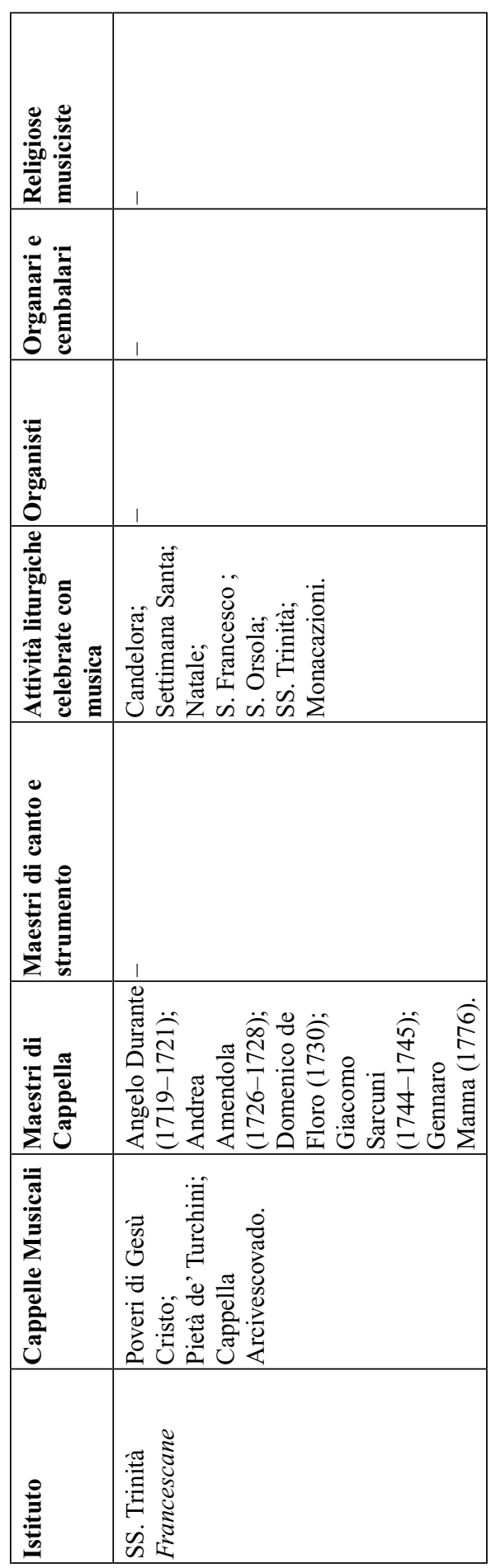




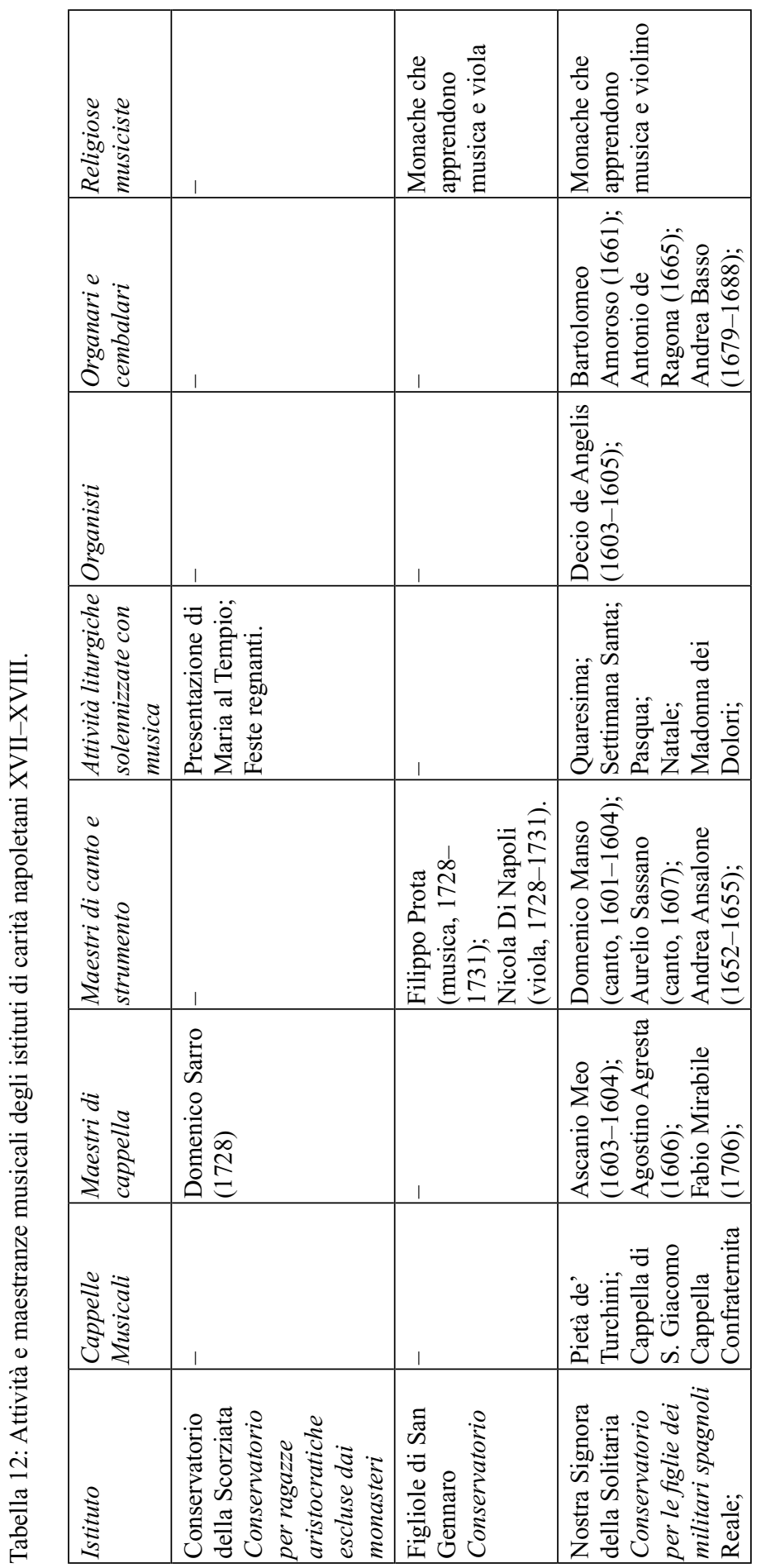




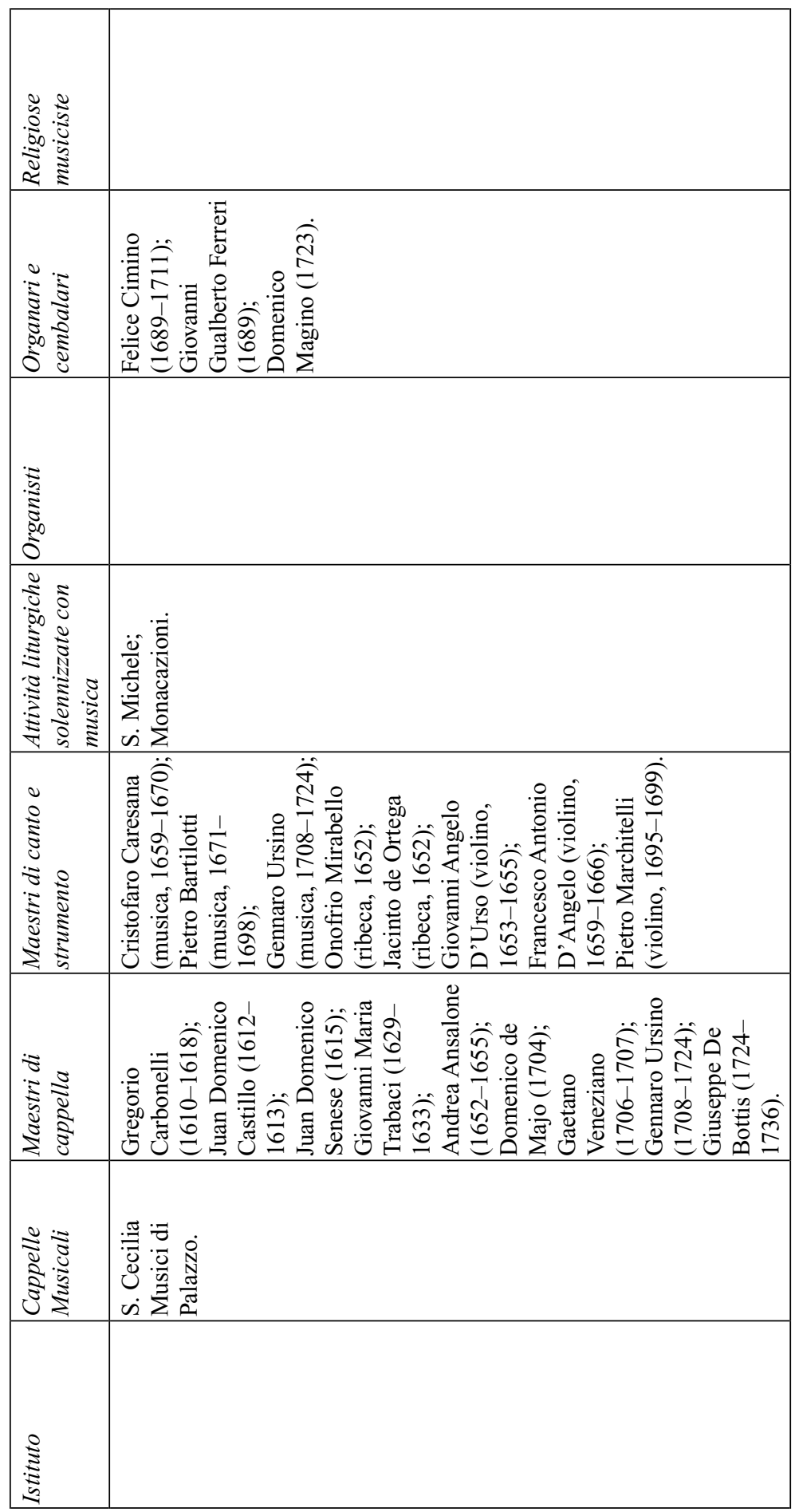




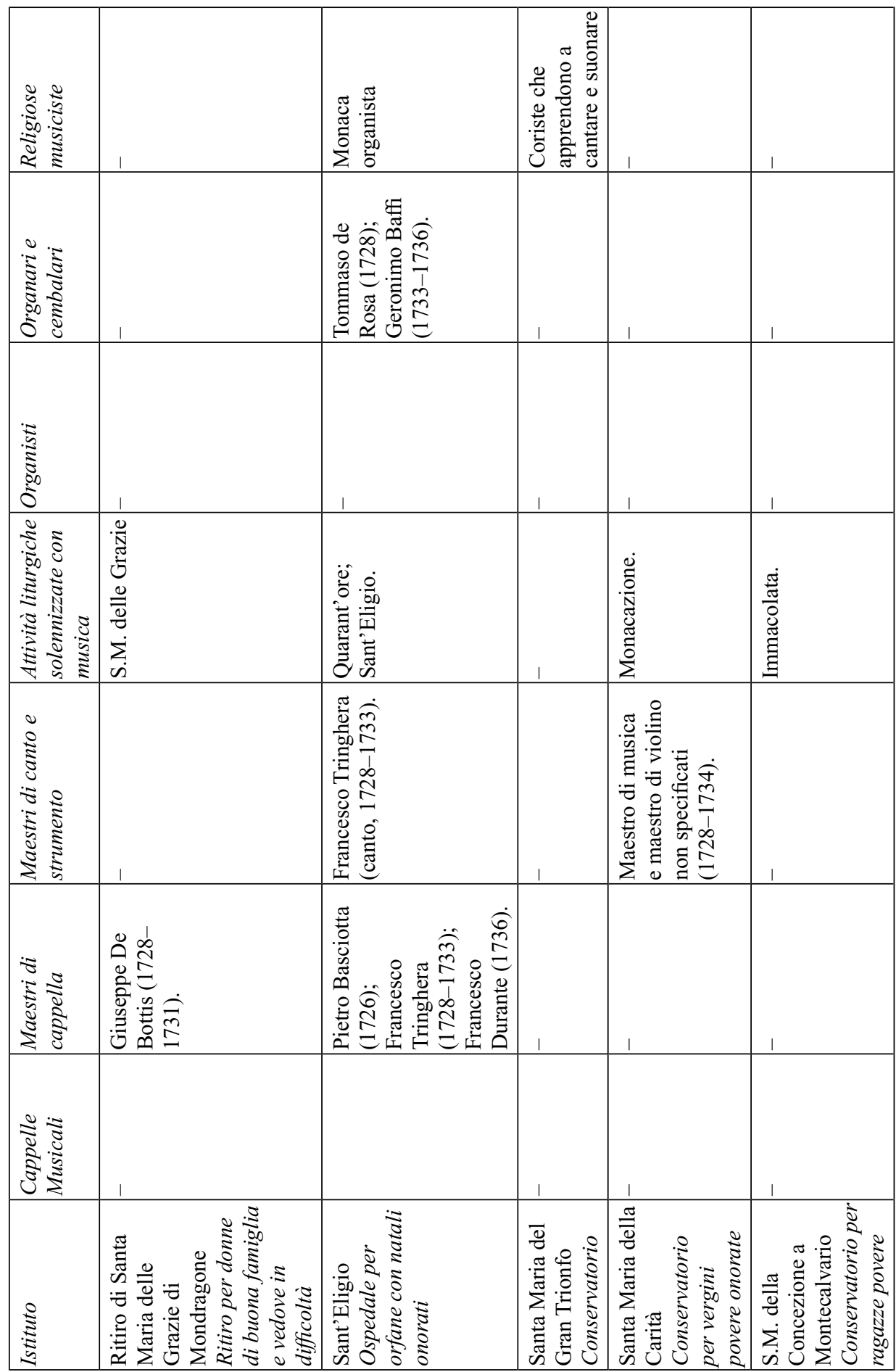




\begin{tabular}{|c|c|c|c|c|}
\hline 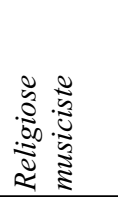 & 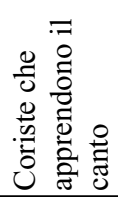 & 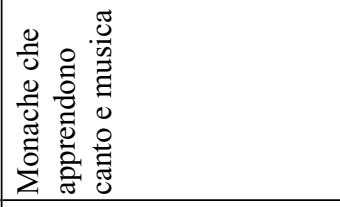 & 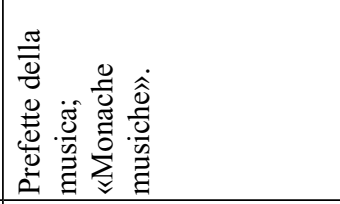 & 1 \\
\hline 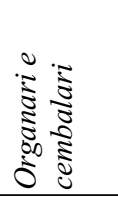 & 1 & 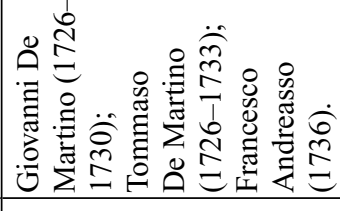 & 1 & 1 \\
\hline 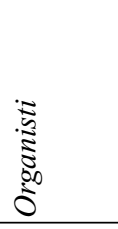 & 1 & & 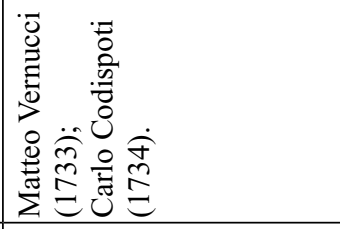 & \\
\hline 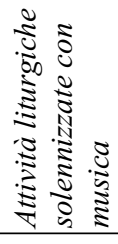 & $\perp$ & 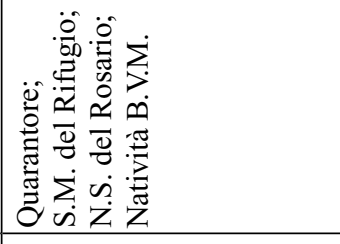 & 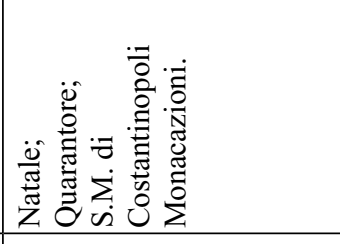 & 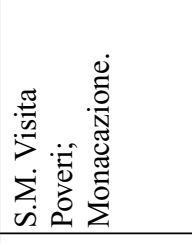 \\
\hline 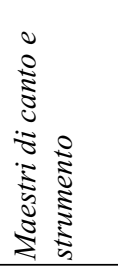 & & 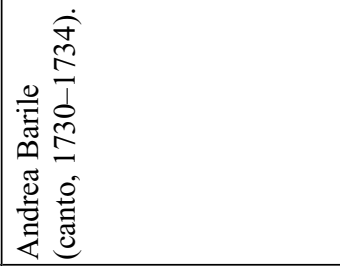 & 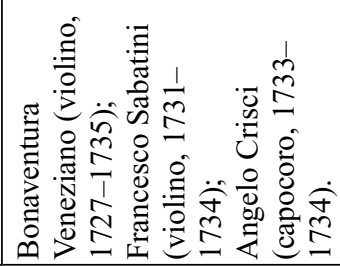 & \\
\hline 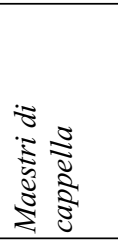 & & 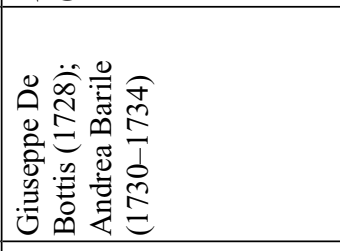 & 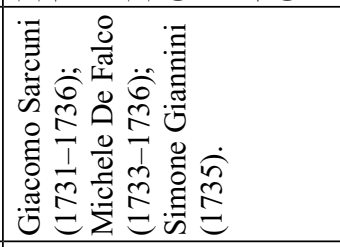 & 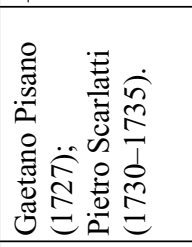 \\
\hline 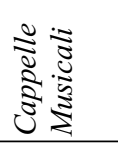 & & 1 & । & \\
\hline 咅 & 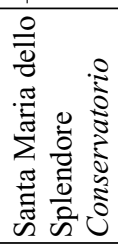 & 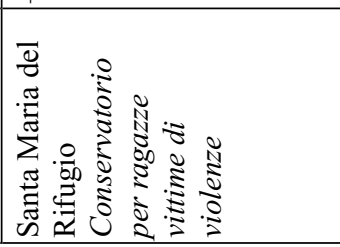 & 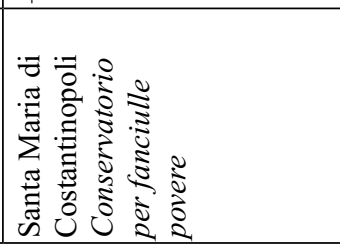 & 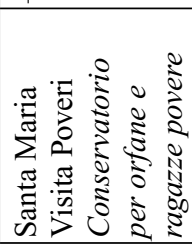 \\
\hline
\end{tabular}




\begin{tabular}{|c|c|c|c|c|}
\hline 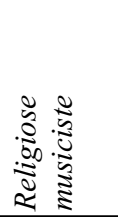 & 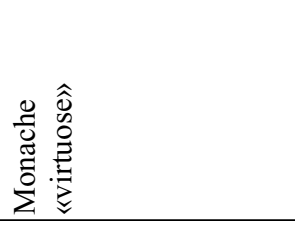 & 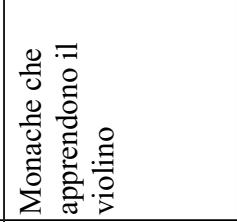 & 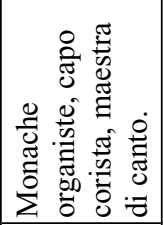 & \\
\hline 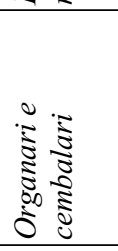 & 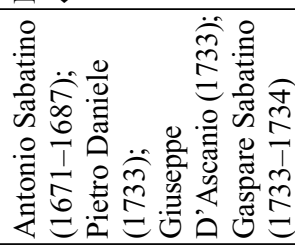 & 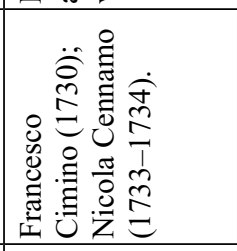 & 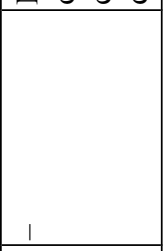 & 1 \\
\hline $\begin{array}{c}0 \\
0 \\
0 \\
0 \\
0 \\
0 \\
0 \\
0\end{array}$ & 1 & 1 & 1 & 1 \\
\hline 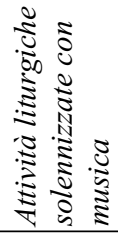 & 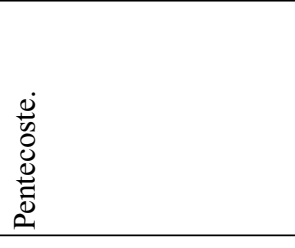 & 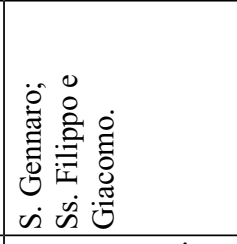 & 1 & 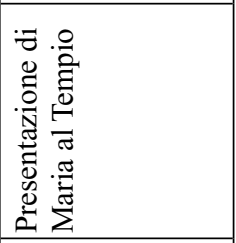 \\
\hline 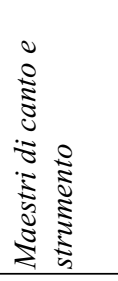 & & 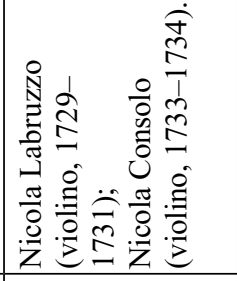 & 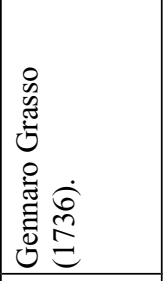 & \\
\hline 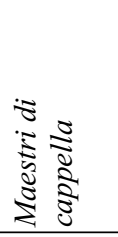 & 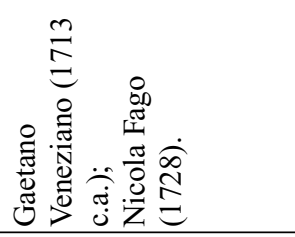 & 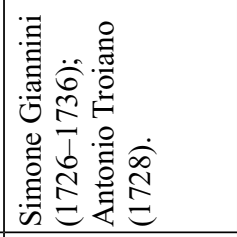 & 1 & 1 \\
\hline 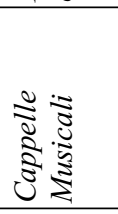 & $-1,0-1$ & 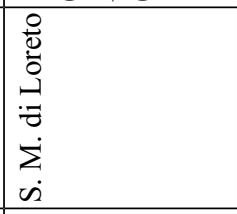 & & \\
\hline 怘 & 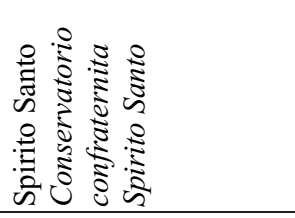 & 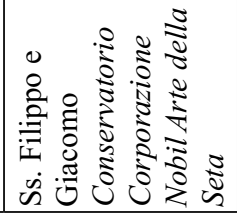 & 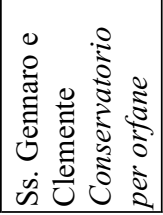 & 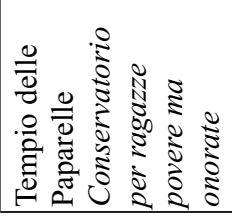 \\
\hline
\end{tabular}




\section{Fonti storiche a stampa}

ARALDO 1998

Giovan Francesco Araldo, Cronica della Compagnia di Giesù di Napoli [1595], edito in Francesco Divenuto, Napoli, l'Europa e la Compagnia di Gesù nella "Cronica” di Giovan Francesco Araldo, Napoli, Edizioni scientifiche italiane, 1998.

BOUCHARD 1977

JEAN-JACQUES BouchaRD, Journal: Voyage dans le royaume de Naples, in Oeuvres de Jean-Jacques Bouchard, a cura di Emanuele Kanceff, II vol., Torino, Giappichelli, 1977.

BULIFON 1932

Antonio Bulifon, Giornali di Napoli dal 1547 al 1706, Napoli, Società di Storia Patria, 1932.

BUOMMATTEI 1622

Benedetto Buommattei, Modo di consacrare le vergini secondo l'uso del pontifical romano con la dichiarazione de' Misteri delle cerimonie, che in quell'azion si fanno, Venezia, Antonio Pinelli, 1622.

BURNEY 1957

Charles Burney, A General History of Music from Earliest Ages to the Present Period (1776-1789), 2 voll., New York, F. Mercer, 1957.

CARACCIOLO 1864

Enrichetta Caracciolo, Misteri del chiostro napoletano. Memorie di Enrichetta Caracciolo de' Principi di Forino, Firenze, Barbera Editore, 1864.

CARACCIOLO 1851

Fulvia Caracciolo, Breve compendio della fondazione del Ministero di San Gregorio Armeno detto San Ligorio di Napoli messo a stampa con annotazioni di Raffaele Maria Zito, Napoli, Manfredi, 1851.

CAPASSO 1882

Bartolomeo Capasso, Sulla circoscrizione civile ed ecclesiastica e sulla popolazione della città di Napoli dalla fine del secolo XIII fino al 1809. Ricerche e documenti, Napoli, Tipografia della Regia Università, 1882.

CAPASSO 1895

Bartolomeo Capasso, Topografia della città di Napoli nell' XI secolo, Napoli, Forni, 1895. 
CeCI 1896

Giuseppe CeCI, I Reali Educandati Femminili a Napoli, Trani, Vecchi, 1896.

CECI 1899

Giuseppe Ceci, Un convento di canonichesse. Regina Coeli, «Napoli Nobilissima», 8, 1899, pp. 24-26.

Celano 2001

Carlo Celano, Notizie del bello dell'antico e del curioso della città di Napoli (1692) con aggiunzioni di Giovanni Battista Chiarini (1856-60), fedele ristampa anastatica, Napoli, Edizioni dell'Anticaglia, 2001.

CONFUORTo 1930

Domenico Confuorto, Giornali di Napoli, Napoli, Luigi Lubrano, 1930.

CONTE 1884

Carlo Conte, Gli stabilimenti di beneficenza di Napoli, Napoli, Stabilimento tipografico dell'Ancora, 1884.

CROCE 1966

Benedetto Croce, I teatri di Napoli, (1891), Roma-Bari, Laterza, 1966.

D’Aloe 1883

Stanislao D'Aloe, Catalogo di tutti gli edifici sacri della città di Napoli e dei suoi sobborghi [metà '600], «Archivio Storico per le Province Napoletane», VIII, 1883, pp. 111-152, 287-315, 499-566, 670-737.

De LeLLIS 1654

Carlo De Lellis, Supplimento a Napoli Sacra di D. Cesare D'Engenio Caracciolo, Napoli, Roberto Mollo, 1654.

De Magistris 1678

Francesco De Magistris, Status Rerum Memorabilium tam Eclesiasticarum, quam Politicarum, ac etiam aedificiorum Fedelissima Civitatis Neapolitanae, Napoli, Fusco, 1678.

D'ENGENIO 1624

Cesare D’Engenio Caracciolo, Napoli Sacra, Napoli, Beltrano, 1624.

De Rosa 1840

Carlo Antonio De Rosa, Memorie de' Compositori di Musica del Regno di Napoli, Napoli, Stamperia Reale, 1840.

De Stefano 1560

Pietro De Stefano, Descrittione dei luoghi sacri della città di Napoli, Napoli, Amato, 1560. 
ECKENSTEIN 1896

Lina ECKenstein, Woman under Monasticism, Chapters on Saint- Lore and Convent Life between a. D. 500 and a. D. 150, New York, Cambridge University Press, 1896.

FILANGIERI 1888

Gaetano Filangieri, Chiesa e Monastero di S. Gaudioso in Napoli, Napoli, Tipografia dell'Accademia Reale delle Scienze, 1888.

FilANGIERI RAVASCHIERI 1879

Teresa Filangieri Ravaschieri Fieschi, Storia della carità napoletana. Conservatorii, Ritiri, Collegi, Convitti, Napoli, Morano, 1879.

FLORIMO 1869

Francesco Florimo, Cenno storico sulla scuola musicale di Napoli, 2 voll., Napoli, Tipografia di Lorenzo Rocco, 1869-1871.

FLORIMO 1882

Francesco Florimo, La scuola musicale napoletana e i suoi 4 conservatori, 4 voll., Napoli, Stabilimento tipografico di Vicenzo Morano, 1882.

FoRTUNATO 1861

Gennaro Fortunato, Statistica dei monasteri d'ambo i sessi esistenti nelle Province Napoletane all'epoca del decreto di soppressione del 17 febbraio 1861, Napoli, Stabilimento tipografico del cav. Gaetano Nobile, 1861.

FUIDORO 1943

InNOCEnZo Fuidoro, Giornali di Napoli dal 1660 al 1680, Napoli, Società Storia Patria, 1943.

GaLANTE 1985

Gennaro Aspreno Galante, Guida Sacra della città di Napoli, edizione a cura di Nicola Spinosa, Napoli, Società editrice napoletana, 1985.

GALANTI 1792

Giuseppe Maria Galanti, Breve descrizione della città di Napoli e del suo contorno, Napoli, Gabinetto Letterario, 1792.

MANCINI 1777

Giambattista Mancini, Riflessioni pratiche sul canto figurato [...] rivedute e corrette ed aumentate, Milano, Giuseppe Galeazzi, 1777.

PARRINO 1700

Domenico Antonio Parrino, Napoli città nobilissima, antica e fedelissima..., Napoli, Nuova stampa del Parrino a Toledo, 1700. 
PARRINO 1725

Domenico Antonio Parrino, Nuova guida de' forastieri. Pero osservare e godere le curiosità più vaghe e più rare della fedelissima gran Napoli [...], Napoli, Parrino, 1725.

SAINT-Non 1786

Jean-Claude Richard de Saint-Non, Voyage pittoresque ou Description des Royaumes de Naples et de Sicilie, 4 voll., Paris, Clousier, 1781-1786.

SARNELLI 1685

Pompeo SARNelli, Guida de' forestieri curiosi di vedere e d'intendere le cose più notabili della regal città di Napoli e del suo amenissimo distretto, Napoli, Roselli, 1685.

SERAO 1970

Matilde Serao, Leggende napoletane, Napoli, Edizioni Bideri, 1970.

SERIO 1865

CARlo SerIo, La vita del chiostro e le religiose in Napoli per Carlo Serio, Napoli, Manfredi, 1865.

SigISMONDO 1789

Giuseppe Sigismondo, Descrizione della città di Napoli e suoi borghi, 2 voll., Napoli, Terres, 1788-89.

VARGAS 1748

Francesco Vargas Macciucca, Dissertazione intorno la Riforma degli abusi introdotti ne' Monisteri delle Monache per le Doti e per le spese che vogliono dalle Donzelle che ne veston l'abito, Napoli-Lucca, Marescandoli, 1748.

VeCCHIONE/GENOVESE 1908

ERnesto VeCChiONE - EtTORe Genovese, Le istituzioni di beneficenza nella città di Napoli, Napoli, Premiata Scuola Tipografica dei Sordomuti, 1908. 


\section{Fonti liturgiche}

CAerimoniale EPISCOPORUM 1744

Caerimoniale Episcoporum in duos libros distributum. Clementis VIII. Et Innocentii X. Auctoritate recognitum. A Benedicto XIII. In multis correctum. Nunc vero primum Commentariis illustratum. Ad Sanctissimum Patrem Benedictum XIV. Pontificem Maximum. Cura et Studio Josephi Catalani Presbyteri. Romae. MDCCXLIV.

Missale 1994

Missale romanum: anno 1962 promulgatum, Subsidia Instrumenta Liturgica quarreriensia, Roma, C.L.V. Ed. liturgiche, 1994.

Missale 1998

Missale romanum: editio princeps (1570) / ed. anastatica, Città del Vaticano, Libreria Editrice Vaticana, 1998.

Pontificale 1663

Pontificale Romanum Clementis VIII. Primum, nunc denuo Urbani Papae Octavi Auctoritate Recognitum. Antuerpiae, Ex Officina Plantiniana Balthasaris Moreti. M. DC. LXIII. 



\section{Bibliografia}

ANTONELLi 2012

Attilio Antonelli, Cerimoniale del viceregno spagnolo e austriaco di Napoli. 1650-1717, Crotone, Rubbettino, 2012.

ANTONELLI 2014

Attilio Antonelli, Cerimoniale del viceregno austriaco di Napoli. 1707-1734, Napoli, Arte'm, 2014.

BACCIAGALUPPI 2009

Claudio Bacciagaluppi, Dignas laudes resonemus di Pergolesi e il 'mottettone' napoletano nel primo Settecento, «Studi Musicali», XXXVIII, 2, 2009, pp. 329-360.

BACCIAGALUPPI 2010

Claudio Bacciagaluppi, Rom, Prag, Dresden Pergolesi und die Neapolitanische Messe in Europa, Kassel, Bärenreiter, 2010.

BACCIAGALUPPI 2013

Claudio Bacciagaluppi, Doubles Ensembles and Cello Continuo in EighteenthCentury Naples, in Barockmusik: diskurs zu einem Interpretationsprofil, vol. 10, a cura di Thomas Hochradner, Freiburg, Romback Verlag, 2013, pp. $183-214$.

BACCIAGALUPPI/FiORE/ZiTELLINI 2015

Claudio Bacciagalupi - Angela Fiore - Rodolfo Zitellini, 'Musico Napolitano'-An Online Biographical Index of Music Professions in Naples, «Early Music», Oxford University Press, 2015, pp. 673-676.

BALDAUF-BERDES 1996

Jane L. Baldauf-Berdes, Woman Musician of Venice: Musical Foundations 1525-1700, Oxford, Clarendon Press, 1996.

BERNARDI 1991

Claudio Bernardi, La drammaturgia della Settimana Santa in Italia, Milano, Vita e Pensiero, 1991.

BOCCADAMO 1990

GIUliana BocCaDAmo, Una riforma impossibile? I papi e primi tentativi di riforma dei monasteri femminili di Napoli nel '500, Roma, Dehoniana, 1990. 
BOCCADAMO 1996

GiUlana BocCadamo, Istruzione ed educazione a Napoli tra il Concilio di Trento e l'espulsione dei Gesuiti, «Annali di storia dell'educazione e delle istituzioni scolastiche», 3, 1996, pp. 48-52.

BOCCADAMO 1999

Giuliana Boccadamo, Un 'palombaro di palombe sante'. Squarci di vita quotidiana nei conservatori femminili napoletani sul finire del Cinquecento, in Munera Parva. Studi in onore di Boris Ulianich, a cura di Gennaro Luongo, vol. II, Napoli, Fridericiana Editrice Universitaria, 1999, pp. 277-315.

BOCCADAMO 2001

GiUlana Boccadamo, Dinamiche di potere e vita comunitaria nella gestione dei monasteri di clausura, in Oltre le grate. Comunità regolari femminili nel Mezzogiorno moderno tra vissuto religioso, gestione economica e potere urbano, a cura di Mario Spedicato e Angelo D’Ambrosio, Bari, 2001, pp. 77-106.

Boesch/Scaraffia 1990

Sofia Boesch Gajano - Lucetta Scaraffia (a cura di), Luoghi sacri e spazi della santità, Rosenberg \& Sellier, Torino 1990.

BOLCHI 2002

Elena LuCia Bolchi, La consacrazione nell'ordo virginum: forma di vita e disciplina canonica, Roma, Editrice Pontificia Università Gregoriana, 2002.

Bonsante/PasquandRea 2010

Annamaria Bonsante - Roberto Pasquandrea (a cura di), Celesti Sirene. Musica e Monachesimo dal Medioevo all'Ottocento, Foggia, Claudio Grenzi Editore, 2010.

BONSANTE 2013

ANNAMARIa Bonsante, Settecento napoletano a san Gregorio Armeno: ricreazioni musicali, in San Gregorio Armeno. Storia, architettura, arte e tradizioni, a cura di Nicola Spinosa, Aldo Pinto e Adriano Valerio, Napoli, Fridericiana Editrice Universitaria, 2013, pp. 267-281.

BRYANT/QUARANTA 2005

David Bryant-Elena Quaranta (a cura di), Produzione, circolazione e consumo. Consuetudine e quotidianità della polifonia sacra nelle chiese monastiche e parrocchiali dal tardo Medioevo alla fine degli Antichi Regimi, Bologna, Il Mulino, 2005.

CAFIERO/MARINo 1987

Rosa CAFIERo-Marina Marino, Materiali per una definizione di «oratorio» a Napoli nel Seicento: primi accertamenti, in La musica a Napoli durante il 
Seicento, a cura di Domenico Antonio D'Alessandro e Agostino Ziino, Roma, Torre d'Orfeo, 1987, pp. 465-510.

CAFiERo/Marino 1987

Rosa Cafiero-Marina Marino (a cura di), Francesco Florimo e l'Ottocento musicale, Reggio Calabria, Jason, 1999.

CAFIERo 1993

Rosa CAFIERo, Una biblioteca per la biblioteca: la collezione musicale di Giuseppe Sigismondo, in Napoli e il teatro musicale in Europa tra Sette e Ottocento: studi in onore di Friedrich Lippmann, a cura di Bianca Maria Antolini e Wolfgang Witzenmann, Firenze, Olschki, 1993, pp. 293-367.

CAFIERO 2005

Rosa CAFIERo, Conservatories and the Napolitan School: a European Model at the End of the Eighteenth Century?, in Music Education in Europe (1770-1914). Compositional, Institutional and Political Challenges, a cura di Michael FeldMichel Noiray, Berlin, Berlin Wissenschafts-Verlag, 2005.

Campanelli 2001

Marcella Campanelli, "Una virtù soda, maschia, robusta". Il monachesimo femminile nel Settecento napoletano, in Donne e religione a Napoli. Secoli XVI-XVIII, a cura di Giuseppe Galasso e Adriana Valerio, Milano, Franco Angeli, 2001, pp. 139-158.

CAMPANELli 2002

MarCella Campanelli, Chiesa e assistenza pubblica a Napoli nel Cinquecento, in Gli inizi della circolazione della cartamoneta e i banchi pubblici napoletani, a cura di Luigi de Rosa, Napoli 2002, pp. 143-168.

CAmpanelli 2009

Marcella Campanelli, Spazio sacro e spazio urbano nelle cerimonie religiose della Napoli barocca, «Archivio Storico per le Province Napoletane», 129, 2009, pp. 241-256.

CANOSA 1991

Romano Canosa, Il velo e il cappuccio, Monacazioni forzate e sessualità nei conventi femminili in Italia tra Quattrocento e Settecento, Roma, Sapere 2000, 1991.

\section{CAPUTI 1994}

Maria CAPUTI, Napoli rivelata. Gli spazi sacri del centro antico, Napoli, D'Auria, 1994.

CARPinello 2002

Mariella Carpinello, Il monachesimo femminile, Mondadori, Milano, 2002. 
CARRINO 2013

CANDIDA CARRINo (a cura di), Le monache ribelli raccontate da suor Fulvia Caracciolo, Napoli, Intra Moenia, 2013.

CASERTA 1983

Aldo Caserta, Sinodi della Chiesa di Napoli (sec. XVI-XX), Napoli, Editoriale comunicazioni sociali, 1983.

CiCALESE 1972

Angela Cicalese, Note ed appunti sui monasteri di Napoli tra il Cinque e l'Ottocento (dal fondo Monasteri dell'Archivio Storico Diocesano), «Campania Sacra», 3, 1972, pp. 208-240.

COLUMBro/INTINI 1998

Marta Columbro - Eloisa Intini, Congregazioni e Corporazioni di musici a Napoli tra Sei e Settecento, «Rivista Italiana di Musicologia», 33, 1998, pp. 41-76.

Columbro/INTINi 2000

Marta Columbro-Eloisa Intini, Considerazioni sulla condizione sociale e lavorativa del musicista napoletano nel Sei Settecento, in Pietro Metastasio. Il testo e il contesto, a cura di Marta Columbro e Paologiovanni Maione, Napoli, Altrastampa, 2000.

Columbro/Maione 2008

Marta Columbro-Paologiovanni Maione, La Cappella musicale del Tesoro di San Gennaro di Napoli tra Sei e Settecento, Napoli, Turchini edizioni, 2008.

Columbro 2005

Marta Columbro, Organi e Organisti: sguardo su alcune fonti napoletane del XVI e del XVII secolo, in Napoli e L'Europa: gli strumenti, $i$ costruttori e la musica per organo dal XV al XX secolo, a cura di Luigi Sisto e Emanuele Cardi, Battipaglia (Sa), Accademia Organistica Campana, 2005.

Colusso/D'Alessandro 2003

Flavio Colusso-Domenico Antonio D'Alessandro (a cura di), Cataldo Amodei-Composizioni Liturgiche, Musica Theatina vol. I, Lucca, LIM, 2003.

CONCILIORUM DECRETA 1973

Conciliorum Oecumenicorum Decreta, Bologna, Istituto per le scienze religiose, 1973.

Coniglio 1959

GIUSEPPE CONIGLIO, Gli archivi dei monasteri soppressi napoletani nell'Archivio di Stato di Napoli, «Rassegna degli Archivi di Stato», 19, 1959, pp. 103-147. 
CONTI 2003

Carla Conti, Nobilissime allieve: della musica a Napoli fra Sette e Ottocento, Napoli, Guida, 2003.

Cotticelli/Maione 1993

Francesco Cotticelli - Paologiovanni Maione, Le istituzioni musicali a Napoli durante il Viceregno Austriaco (1707-1734). Materiali inediti sulla Real Cappella ed il Teatro di San Bartolomeo, Napoli, Luciano Editore, 1993.

Cotticelli/Maione 1996

Francesco Cotticelli - Paologiovanni Maione, Onesto divertimento, ed allegria de' popoli: materiali per una storia dello spettacolo a Napoli nel primo Settecento, Milano, Ricordi, 1996.

Cotticelli/Maione 2006

Francesco Cotticelli - PaOlogiovanni Maione, Le carte degli antichi banchi e il panorama musicale e teatrale della Napoli di primo Settecento: 1732-1733, «Studi pergolesiani / Pergolesi studies», 5, 2006, pp. 21-54.

Cotticelli/Maione 2009

Francesco Cotticelli - Paologiovanni Maione (a cura di), Storia della musica e dello spettacolo a Napoli. Il Settecento, Napoli, Turchini, 2009.

Cotticelli/Maione 2015

Francesco Cotticelli - Paologiovanni Maione, Le carte degli antichi banchie il panorama musicale e teatrale della Napoli di primo Settecento: 1726-1736, «Studi pergolesiani / Pergolesi studies», 9, 2015.

Creytens 1965

RAYMond CReytens, La riforma dei monasteri femminili dopo i Decreti Tridentini, in Il Concilio di Trento e la riforma tridentina, Roma, Herder, 1965, I, pp. $45-84$.

D'AlESSANDRO/ZIINO 1987

Domenico Antonio D'Alessandro - Agostino ZiIno (a cura di), La musica a Napoli durante il Seicento, Roma, Torre d'Orfeo, 1987.

D'AlessANDRo 1983

Domenico Antonio D'Alessandro, La musica nel secolo XVII attraverso gli «avvisi» e i giornali, in Musica e cultura a Napoli dal XV al XIX sec., a cura di Lorenzo Bianconi e Renato Bossa, Firenze, Olschki, 1983 (Quaderni della Rivista Italiana di Musicologia, 9), pp. 145-164.

D’Alessio 2007

Silvana D'Alessio, Masaniello. La sua vita e il mito in Europa, Roma, Salerno, 2007. 
D'ANDREa 1976

Gioncchino Francesco D'Andrea, Un libro di conti dell'ex monastero delle clarisse di S. Chiara di Napoli, «Rivista storica dei Cappuccini di Napoli», I/ III, 1976, pp. 95-114.

D'ANDREA 1977

Gioacchino Francesco D'Andrea, Ciò che resta dell 'antico archivio di S. Chiara de Napoli, «Archivum franciscanum historicum», 70, 1977, pp. 128-146.

D'ANDREA 1987

Gioacchino Francesco D'Andrea, Il monastero napoletano di S. Chiara secondo i registri dell'Archivio di Stato di Napoli, «Archivum franciscanum historicum», 80, 1987, pp. 39-78.

D'ANDREA 2005

Gioacchino D'Andrea, Delia Bonito nella storia del complesso monumentale S. Chiara di Napoli, in All'ombra della chiara luce, a cura di Aleksander Horowski, Roma, Istituto storico dei Cappuccini, 2005 pp. 201-232.

DA NADRO 1960

Silvino da Nadro (a cura di), Sinodi diocesani italiani, Catalogo bibliografico degli atti a stampa 1534-1878, Città del Vaticano, Biblioteca Apostolica Vaticana, 1960.

D'ARIO 1994

CARMEla D'Ario, Gli esposti a Napoli nel XVIII sec., in Chiesa, assistenza e società nel Mezzogiorno moderno, a cura di Carla Russo, Galatina, Congedo, 1994, pp. 515-568.

DELFINO 1983

Antonio Delfino, La chiesa di Donnaregina Nuova, «Ricerche sul Seicento Napoletano», 1983, pp. 81-121.

DELFINO 1990

Antonio Delfino, La chiesa nuova ed il monastero di Donnaregina nel '600, «Ricerche sul Seicento Napoletano», 1990, pp. 101-114.

Dell'Aja 1992

Gaudenzio Dell'Aja, Per la storia del monastero di S.Chiara in Napoli, Napoli, Giannini, 1992.

Dell'Olio 2013

Antonio Dell'Olio, Drammi sacri e oratori musicali in Puglia nei secoli XVII e XVIII, Galatina, Congedo Editore, 2013. 
Del Prete 1999

Rossella Del Prete, La trasformazione di un istituto benefico-assistenziale in scuola di musica: una lettura dei libri contabili del conservatorio di S. Maria di Loreto di Napoli (1583-1703), in Francesco Florimo e l'Ottocento musicale, a cura di Rosa Cafiero e Marina Marino, Reggio Calabria, Jason, 1999, vol. II, pp. 671-715.

Del PRete $1999^{2}$

Rossella Del Prete, Un'azienda musicale a Napoli tra Cinque e Settecento: il Conservatorio della Pietà de’ Turchini, «Storia economica», II, 1999, pp. 413-464.

De Maio 1973

Romeo De Maio, Riforme e miti nella Chiesa del '500, Napoli, Guida, 1973.

De Maio 1981

Romeo De MaIo, Società e vita religiosa a Napoli nell'età moderna (1658-1799), Napoli, Edizioni scientifiche italiane, 1981.

De Maio 1997

Romeo De MaIo, Religiosità a Napoli 1656-1799, Napoli, Edizioni scientifiche italiane, 1997.

DE Rosa 2002

LUIGI DE Rosa (a cura di), Gli inizi della circolazione della cartamoneta e i banchi pubblici napoletani nella società del loro tempo, 1540-1650, Napoli, Istituto Banco di Napoli, 2002.

DiETZ 1972

Hanns-Bertold Dietz, 'Zur Frage der musikalischen Leitung des Conservatorio di Santa Maria di Loreto in Neapel im 18. Jahrhundert', «Die Musikforschung», XXV, 1972, pp. 419-429.

DIETZ $1972^{2}$

Hanns-Bertold Dietz, A Chronology of Maestri and Organisti at the Cappella Reale in Naples, 1745-1800, «Journal of the American Musicological Society», 35, 1972, pp. 379-406.

Di Giacomo 1918

Salvatore Di Giacomo (a cura di), Catalogo delle opere musicali teoriche e pratiche di autori italiani vissuti sino ai primi decenni del secolo XIX, esistenti nelle biblioteche e negli archivi pubblici e privati d'Italia. Città di Napoli, Archivio dell'Oratorio dei Filippini, Parma, Fresching, 1918.

Di Giacomo 1920

Salvatore Di Giacomo, Maestri di cappella, musici \& istromenti al Tesoro di San Gennaro nei secoli XVII \& XVIII, Napoli, a spese dell'autore, 1920. 
Di Giacomo 1928

Salvatore Di Giacomo, I quattro antichi Conservatori di musica a Napoli, 2 voll., Palermo, Sandron, 1924-28.

DOMPNIER 2009

BERNARD DOMPNIER (a cura di), Les ceremonies extraordinaires du catholicisme baroque, Clermont Ferrand, Presses universitaires Blaise-Pascal, 2009.

DOMINGUEZ 2015

José Maria Dominguez, Oltre il viceré: mecenatismo musicale della nobiltà di corte a Napoli alla fine del Seicento, «Studi Pergolesiani/Pergolesi Studies», 10, 2015, pp. 63-98.

DONADI 2003

PaOla Donadi, La regola e lo spirito: arte, cultura, quotidianità nei monasteri femminili, Milano, Franco Angeli, 2003.

DONELla 1991

Valentino Donella, Musica e liturgia, Bergamo, Carrara, 1991.

EVANGELISTI 2000

Silvia Evangelisti, Wives, Widows, and Brides of Christ: Marriage and the Convent in the Historiography of Early Modern Italy, «The Historical Journal», 43, I, 2000, Cambridge University Press, pp. 233-247.

FABRIS 1983

DinKo FABRIs, Strumenti di corde, musici e congregazioni a Napoli alla metà del Seicento, «Note d'Archivio», 1, 1983, pp. 63-110.

FABRIS 1987

Dinko Fabris, Andrea Falconieri Napoletano. Un liutista-compositore del Seicento, Roma, Torre D’Orfeo, 1987.

FABRIS $1987^{2}$

Dinko FABRIS, Generi e fonti della musica sacra a Napoli nei Seicento, in La musica a Napoli durante il Seicento, a cura di Domenico Antonio D'Alessandro e Agostino Ziino, Roma, Torre d'Orfeo, 1987, pp. 415-454.

FABRIS 1994

DINKo FABRIS, Istituzioni assistenziali e congregazioni di musici a Napoli e nell'Italia meridionale durante il viceregno spagnolo, in Confraternite, Chiesa e società. Aspetti e problemi dell'associazionismo laicale europeo in età moderna e contemporanea, a cura di Liana Bertoldi Lenoci, Fasano, Schena, 1994, pp. 779-800. 
FABRIS 2001

Dinko Fabris, La Capilla Real en las etiquetas de la corte virreinal de Nápoles durante el siglo XVII, in La Capilla Real de los Austrias. Música y ritual de corte en la Europa moderna, a cura di Juan José Carreras e Bernardo José García, Madrid, Fundación Carlos de Amberes, 2001, pp. 235-250.

FABRIS 2005

Dinko Fabris e Gruppo di LaVoro “Napoli” dell'Università Ca' Foscari, Dal Medioevo al decennio napoleonico e oltre: metamorfosi e continuità nella tradizione napoletana, in Produzione, circolazione e consumo. Consuetudine e quotidianità della polifonia sacra nelle chiese monastiche e parrocchiali dal tardo Medioevo alla fine degli Antichi Regimi, a cura di David Bryant e Elena Quaranta, Bologna, Il Mulino, 2005, pp. 227-281.

FABRIS 2007

Dinko Fabris, Music in Seventeenth-Century Naples. Francesco Provenzale (1624-1704), Aldershot, Ashgate, 2007.

FACCHIANO 1992

Annamaria Facchiano, Monasteri femminili e nobiltà a Napoli tra medioevo ed età moderna, Altavilla Silentina, Edizioni Studi Storici Meridionali, 1992.

FACCHIANO 1997

Annamaria Facchiano, Monachesimo femminile nel Mezzogiorno medievale e moderno, in Il monachesimo femminile in Italia dall'Alto Medioevo al secolo XVII a confronto con l'oggi, a cura di Gabriella Zarri, S. Pietro in Cariano, Il segno dei Gabrielli Editore, 1997, pp. 169-191.

FERNANDES 1998

Cristina Fernandes, A música no contexto de cerimónia de Profissão nos mosteiros femininos portugueses (1768-1828), «Revista Portuguesa de Musicologia», 7/8, Lisboa, 1997/98, pp. 59-94.

FERNANDES 2015

Cristina Fernandes, Patronage monarchico e pratica musicale monastica: rapporti tra la Cappella Reale e Patriarcale, gli strumentisti della Reale Camera e la rete di monasteri di Lisbona durante il Settecento, in Celesti Sirene: Musica e Monachesimo dal Medioevo all'Ottocento, Barletta, Cafagna editore, 2015, pp. 341-372.

FIORE 2012

Angela Fiore, La Cappella di Santa Cecilia dei Musici di Palazzo di Napoli. Nuove acquisizioni dall'Archivio del Conservatorio della Solitaria, «Fonti Musicali Italiane», 17, 2012, pp. 25-44. 
FIORE 2013

Angela Fiore, Cerimoniali musicali presso il Conservatorio di Nuestra Señora della Soledad di Napoli, in Fiesta y Cerimonia en la corte virreinal de Napoles (siglos XVI y XVII), Madrid, Centro de Estudios Europa Ispanica, 2013, pp. 491-512.

FIORE 2015

ANGELA FIORE, Et nunc sequimur in toto corde: riti e cerimonie di monacazione nei chiostri napoletani fra XVII e XVIII sec., in Celesti Sirene: Musica e Monachesimo dal Medioevo all'Ottocento, Barletta, Cafagna editore, 2015, pp. 399-428.

FIORE $2015^{2}$

Angela Fione, La tradizione musicale del monastero delle Clarisse di Santa Chiara di Napoli, «Rivista Italiana di Musicologia», 50, 2015, pp. 33-60.

FIORE forth. ${ }^{1}$

ANgela Fiore, Divertissements secrets : traditions et interdictions dans les pratiques musicales des institutions féminines napolitaines sous l'Ancien Régime, in Réalités et fictions de la musique religieuse à l'époque moderne. Essais d'analyse des discours, Musèfrem, Presses Universitaires de Rennes, forthcoming.

FIORE forth. ${ }^{2}$

Angela Fiore, La Chapelle du Palais Royal, la cour et les couvents dans le viceroyaume de Naples, in Musique de la foi, musique du pouvoir - Musiques religieuses d'apparat dans les cours régnantes d'Europe au temps de Louis XIV, Muséfrem, Centre de Musique Baroque de Versailles, forthcoming.

FioRe/IACONO 2016

Angela Fiore-Sarah Iacono, L'Amor divino di Alessandro Speranza. Antifone e Cantate nel monastero di Regina Coeli, in Alessandro Speranza e la musica sacra a Napoli nel Settecento, Avellino, Il Cimarosa, 2016, pp. 55-74.

FIORELLI 2001

VitTORIA Fiorelli, Una santa della città. Suor Orsola Benincasa e la devozione napoletana tra Cinquecento e Seicento, Napoli, Editoriale Scientifica, 2001.

FIORELLI 2003

VITTORIA FIORELli, Una esperienza religiosa periferica. I monasteri di madre Serafina di Dio da Capri alla terraferma, Napoli, Guida, 2003.

GABRIELLI 2005

GiUlia Gabrielli (a cura di), Il canto fratto nei manoscritti della Fondazione Biblioteca S. Bernardino di Trento, Trento, Soprintendenza ai Beni librari e archivistici della Provincia autonoma di Trento, 2005. 
GaGLiONE 2003

Mario Gaglione, Quattro documenti per la storia di S.Chiara in Napoli, «Archivio Storico per le Province Napoletane», 121, 2003, pp. 399-431.

GAGLiONE 2007

Mario Gaglione, La basilica e il Monastero doppio di S.Chiara a Napoli in studi recenti, «Archivio per la Storia delle Donne», IV, 2007, pp. 127-209.

GALASSO/VALERIO 2001

Giuseppe Galasso-Adriana Valerio, Donne e religione a Napoli. Secoli XVIXVIII, Milano, Franco Angeli, 2001.

GaLASSO 1982

GiUSEPPE Galasso, Napoli spagnola dopo Masaniello: politica, cultura, società, Sansoni Editore, Firenze, 1982.

GALlino 1951

Tommaso Maria Gallino, Il chiostro maiolicato del monastero di Santa Chiara in Napoli, Napoli, Giannini, 1951.

GaLLINO 1963

Tommaso Maria Gallino, Il complesso monumentale di S. Chiara in Napoli, Napoli, Pontificio Istituto Superiore di Scienze e Lettere S. Chiara dei Frati Minori, 1963.

GeYER/OsthofF 2004

Helen Geyer- Wolfgang Osthoff (a cura di), La Musica negli Ospedali. Conservatori veneziani tra Sette e Ottocento/Musik an den venezianischen Ospedali. Konservatorien vom 17. bis zum frühen 19. Jahrhundert, Roma, Edizioni di Storia e Letteratura, 2004.

GILLIO 2006

Pier GiusepPe Gillio, L'attività musicale negli ospedali di Venezia nel Settecento, Firenze, Olschki, 2006.

Giron-Panel 2015

Caroline Giron-Panel, Musique et musiciennes à Venise. Histoire sociale des ospedali (XVI $-X V I I I^{e}$ siècles), Roma, École française de Rome, 2015.

Gozzi 2012

Marco Gozzi (a cura di), Cantus fractus italiano: un 'antologia, in Musica Mensurabilis 4, Hidelscheim-Zürich-New York, Olms, 2012.

GRECO 1998

Franco Carmelo Greco, Drammaturgia della santità a Napoli in età barocca, in Scrivere di Santi, a cura di Gennaro Luongo, Roma, Viella, 1998, pp. 227-245. 
GRIECO/ZARRI 2000

Sara Matthews-Grieco-Gabriella Zarri (a cura di), Committenza artistica femminile, «Quaderni storici», 35, 2000, pp. 283-412.

GRIFFIN 1993

Thomas E. GRIFFIn, Musical references in the Gazzetta di Napoli, 1681-1725, Berkeley, CA: Fallen Leaf Press, 1993.

Grove 2001

The New Grove Dictionary of Music and Musicians, ed. by Stanley Sadie, 20 voll., Macmillian, London.

GUIDI 1991

LAURA GUIDI, L'onore in pericolo, Napoli, Liguori, 1991.

HAMELINE 2009

JeAn-Yves Hameline, La distinction ordinaire/extraordinaire dans les textes rubricaux, les cérémoniaux, et chez leurs commentateurs autorisés, in Les ceremonies extraordinaires du catholicisme baroque, a cura di Bernard Dompnier, Clermont Ferrand, Presses universitaires Blaise-Pascal, 2009, pp. 19-31.

HIERRo 1959

Diego Díaz Hierro, Compendio histórico de la devocion española a la Soledad de Maria, «El Lábaro», 1959, pp. 1-32.

HiLLS 2004

Helen Hills, Invisible City. The Architecture of Devotion in Seventeenth-Century Neapolitan Convents, New York, Oxford University Press, 2004.

ILLIBATO 1985

Antonio Illibato, La donna a Napoli nel Settecento: aspetti dell'educazione e dell'istruzione, Napoli, D'Auria Editore, 1985.

KENDRICK 1996

Robert Kendrick, Celestial Sirens. Nuns and their Music in Early Modern Milan, Oxford, Clarendon Press, 1996.

KENDRICK 2002

Robert Kendrick, The Sounds of Milan 1585-1650, Oxford-New York, Oxford University Press, 2002.

KRAUSE 1987

RalPh Krause, Die Kirchenmusik von Leonardo Leo (1694-1744): ein Beitrag zur Musikgeschichte Neapels im 18. Jahrhundert, Regensburg, Bosse, 1987. 
KraUSE 1993

RalPh Krause, Documenti per la storia della Real Cappella di Napoli nella prima metà del Settecento, «Annali dell'Istituto Italiano per gli studi Storici», 11, 1993, pp. 235-257.

KraUSE 1998

RalPH Krause, Das musikalische Panorama am neapolitanischen Hofe: zur Real Cappella di Palazzo im frühen 18. Jahrhundert, «Studien zur italienischen Musikgeschichte», 15, Laaber: Laaber, 1998 (Analecta Musicologica, 30), pp. 271-295.

\section{LARSON 1983}

Keith A. LaRson, Condizione sociale dei musicisti e dei loro committenti nella Napoli del Cinque e Seicento, in Musica e cultura a Napoli dal XV al XIX secolo, a cura di Lorenzo Bianconi e Renato Bossa, Firenze, Olschki, 1983, (Quaderni della Rivista Italiana di Musicologia, 9), pp. 63-77.

LOWE 2002

Kate J. P. Lowe, Secular brides and convent brides: wedding ceremonies in Italy during the Renaissance and Counter Reformation, in Marriage in Italy (1300-1650), ed. by Trevor Dean and Kate J. P. Lowe, Cambridge and New York, Cambridge University Press, 2002, pp. 41-65.

LOWE 2004

Kate J.P. Lowe. Nuns' Chronicles and Convent Culture in Renaissance and Counter-Reformation Italy, Cambridge and New York, Cambridge University Press, 2004.

MAGaudda/COSTANTINI 2001

Ausilia Magaudda - Danilo Costantini, Attività musicali promosse dalle confraternite laiche nel Regno di Napoli (1677-1763), in Fonti d'archivio per la storia della musica e dello spettacolo a Napoli tra XVI e XVIII secolo, a cura di Paologiovanni Maione, Napoli, Editoriale Scientifica, 2001, pp. 79-204.

Magaudda/Costantini 20012

Ausilia Magaudda e Danilo Costantini, Aurora Sanseverino (1669-1726) e la sua attività di committente musicale nel Regno di Napoli. Con notizie inedite sulla napoletana congregazione dei sette Dolori, in Giacomo Francesco Milano ed il ruolo dell'aristocrazia nel patrocinio delle attività musicali nel secolo XVIII, a cura di Gaetano Pitarresi, Reggio Calabria, Laruffa editore, 2001, pp. 297-416.

Magaudda/Costantini 2003

Ausilia Magaudda - Danilo Costantini, L'arciconfraternita napoletana dei Sette Dolori (1602-1778). Notizie musicali inedite da un archivio inesplorato, «Musica e Storia», XI, 1, 2003, pp. 51-137. 
Magaudda/Costantini 2007

Ausilia Magaudda - Danilo Costantini, Serenate e componimenti celebrativi nel Regno di Napoli (1677-1754), in La Serenata tra Seicento e Settecento: musica, poesia, scenotecnica, a cura di Nicolò Maccavino, Reggio Calabria, Laruffa Editore, 2007.

Magaudda/Costantini 2009

Ausilia Magaudda - Danilo Costantini, Musica e spettacolo nel Regno di Napoli attraverso lo spoglio della «Gazzetta» (1675-1768), Roma, Ismez, 2009.

Magaudda/COSTANTINI 2015

Ausilia Magaudda - Danilo Costantini, Antecedenti pergolesiani nei drammi sacri di Giovanni Fischetti, «Studi pergolesiani / Pergolesi studies», 9, 2015, pp. 243-278.

MAIONE 2000

Paologiovanni Maione, Le carte degli antichi banchi e il panorama musicale e teatrale della Napoli di primo Settecento, «Studi pergolesiani / Pergolesi studies», 4, 2000, pp. 1-129.

MAIONE 2001

Paologiovanni Maione (a cura di), Fonti d'archivio per la storia della musica e dello spettacolo a Napoli tra XVI e XVIII secolo, Napoli, Editoriale Scientifica, 2001, pp. 79-204.

MAIONE 2005

Paologiovanni Maione, Il mondo musicale seicentesco e le sue istituzioni: la Cappella Reale di Napoli (1650-1700), in La circolazione dell'opera veneziana nei Seicento, a cura di Dinko Fabris, Napoli, Turchini Edizioni, 2005, pp. 309-341.

MAMY 1994

SiLVIE Mamy, Les grands castrats napolitains à Venise au XVIIIe siècle, Liège, Mardaga 1994.

MANCINI 1968

Franco Mancini, Feste ed apparati civili e religiosi in Napoli, Napoli, Edizioni scientifiche italiane, 1968.

MANCINI 1998

FranCo MANCINI, «L'immaginario di regime». Apparati e scenografie alla corte del viceré, in Civiltà del Seicento a Napoli, Napoli, Electa, 1998, pp. 27-35.

MAFRICI 2010

Mirella MAFrici (a cura di), Donne e potere nella Napoli borbonica (1734-1860), Napoli, Fridericiana Editrice Universitaria, 2010. 
MAFRICI 2012

Mirella Mafrici (a cura di), Alla corte napoletana: Donne e potere dall'età aragonese al viceregno austriaco, Napoli, Fridericiana Editrice Universitaria, 2012.

MARINO 2009

Marina Marino, La musica sacra nel Settecento a Napoli in Storia della musica e dello spettacolo a Napoli. Il Settecento, a cura di Francesco Cotticelli e Paologiovanni Maione, Napoli, Turchini Edizioni, 2009, pp. 823-923.

MARTIN/PÉREZ 2000

Pedro Calleja Martín - Enrique Guevara Pérez, Semana Santa en Madrid, Madrid, La Librería, 2000.

MasetTi ZanNini 1980

Gian Lodovico Masetti Zannini, Motivi storici della educazione femminile (1500-1650), I, Morale, religione, lettere, arte, musica, Bari, Editoriale Bari, 1980 .

MaSeTti ZanNini 1993

Gian Lodovico Masetti Zannini, «Suavità di canto» e purità di cuore. Aspetti della musica nei monasteri femminili romani, in La cappella musicale nell'Italia della Controriforma, a cura di Oscar Mischiati e Paolo Russo, Firenze, Olschki, 1993, pp. 123-141.

Megale 2013

Teresa Megale, Teatro e spettacolo nella Napoli vicereale: modelli e ritualità, in Fiesta y Cerimonia en la corte virreinal de Napoles (siglos XVI y XVII), Madrid, Centro de Estudios Europa Ispanica, 2013, pp. 387-414.

MelucCi/Morgese 1993

Maria Grazia Melucci-Angela Morgese (a cura di), Il fondo musicale del monastero delle benedettine di San Severo, San Severo, Gerni, 1993.

Metz 2001

RenÉ Metz, La consécration des vierges. Hier, aujourd'hui, demain, Paris, Cerf, 2001.

MIELE 1979

Michele Miele, Il primo Sinodo di Napoli dopo il concilio di Trento (1595), in Sinodi della Chiesa di Napoli (sec. XVI-XX), a cura di Aldo Caserta, Napoli, Editoriale comunicazioni sociali, 1983.

MIELE 1990

Michele Miele, Sisto V e la riforma dei monasteri femminili di Napoli, «Campania Sacra», 21, 1990, pp. 123-204. 
MIELE 1991

Michele Miele, Religiosi e monache nei concili post-tridentini del Regno di Napoli (1565-1729), «Annuarium Historiae Conciliorum», 23, 1991, pp. 360-372.

MIELE 2001

Michele Miele, Monache e monasteri del Cinque-Seicento tra riforme imposte e nuove esperienze, in Donne e religione a Napoli, a cura di Giuseppe Galasso e Adriana Valerio, Milano 2001, pp. 91-138.

Miller LAWRENCE 1996

Cynthia Miller LaWrence, Women and art in early modern Europe: patrons, collectors, and connoisseurs, Pennsylvania, Pennsylvania State University Press, 1996.

Mischiati 2004

Oscar Mischiati, Un'indagine statistica sulla professione di musicista negli ordini religiosi tra XVI e XVIII secolo, in Laeta dies. Musiche per San Benedetto e attività musicale nei centri benedettini di età moderna, a cura di Saverio Franchi e Biancamaria Brumana, Roma, IBIMUS, 2004, pp. 1-20.

Monson 1992

Craig A. Monson (a cura di), The Crannied Wall: Women, Religion, and the Arts in Early Modern Europe, Ann Arbor, Michigan, 1992.

Monson 1993

Craig A. Monson, La pratica della musica nei monasteri femminili bolognesi, in La cappella musicale nell'Italia della Controriforma, atti del convegno internazionale di studi nel IV Centenario di fondazione della Cappella Musicale di S. Biagio di Cento, a cura di Oscar Mischiati e Paolo Russo, Firenze, Olschki, 1993, pp. 143-160.

Monson 1995

Craig A. Monson, Disembodied Voices: Music and Culture in an Early Modern Italian Convent, Berkeley, University of California Press, 1995.

Monson 1996

Craig A. Monson, Organi e organiste nei monasteri femminili di Bologna, «L'Organo. Rivista di cultura organaria e organistica», XXX, 1996, pp. 37-118.

MORELli 1997

Arnaldo Morelli, La circolazione dell'oratorio italiano nel Seicento, «Studi musicali», XXVI, 1997, pp. 105-189.

MORELli 2004

Arnaldo Morelli, «Musica nobile e copiosa di voci et istromenti». Spazio architettonico, cantorie e palchi in relazione ai mutamenti di stile e prassi nella musica da chiesa fra Sei e Settecento, in Musik in Rom im 17. und 18. Jahr- 
hundert: Kirche und Fest, a cura di Markus Engelhardt e Christoph Flamm, Laaber, Laaber-Verlag, 2004, pp. 293-334 (Analecta musicologica 33).

NAPPI 1982

Eduardo NAPPI, La Chiesa di Santa Maria dei Miracoli, «Napoli Nobilissima», XXI, 1982, pp. 196-218.

NAPPI 2001

EDUARDO NAPPI, Antiche feste napoletane, «Ricerche sul ' 600 napoletano, Saggi e documenti 2001», 2001, Napoli, Electa, pp. 100-120.

NiCOLELLA 1986

DARIo Nicolella, I cento chiostri di Napoli, Napoli, Edizioni scientifiche italiane, 1986.

NOCERINO 1998

FranCeSCo Nocerino, Arte cembalaria a Napoli. Documenti e notizie su costruttori e strumenti napoletani, «Ricerche sul ' 600 napoletano. Saggi e documenti 1996-1997», 1998, Napoli, Electa, pp. 85-109.

NOCERINO 2001

FRANCESCO Nocerino, Napoli centro di produzione cembalaria alla luce delle recenti ricerche archivistiche, in Fonti d'archivio per la storia della musica e dello spettacolo a Napoli tra XVI e XVIII secolo, Napoli, a cura di Paologiovanni Maione, Napoli, Editoriale Scientifica, 2001, pp. 205-225.

NOCERINO 2005

FRANCESCO NOCERINO, L'attività cembalaria dell'organaro a Napoli nei secoli XVI-XVIII. Contributi documentari, in Napoli e L'Europa: gli strumenti, i costruttori e la musica per organo dal XV al XX secolo, a cura di Luigi Sisto e Emanuele Cardi, Battipaglia (Sa), Accademia Organistica Campana, 2005, pp. $105-115$.

NOCERINO 2009

FranCESCO Nocerino, Gli strumenti musicali a Napoli nel secolo XVIII, in Storia della musica e dello spettacolo a Napoli. Il Settecento, a cura di Francesco Cotticelli e Paologiovanni Maione, Napoli, Turchini, 2009, pp. 773-804.

NOVI-CHAVARRIA 1993

Elisa Novi ChaVARria, Nobiltà di seggio, nobiltà nuova e monasteri femminili in Napoli in età moderna, «Dimensioni e problemi della ricerca storica», 2, 1993, pp. 84-111.

NOVI-CHAVARRIA 1997

Elisa Novi ChaVARRIA, Monachesimo femminile nel Mezzogiorno nei secoli XVI$X V I I$, in Il monachesimo femminile in Italia dall'alto medioevo al secolo XVII, 
a confronto con l'oggi, a cura di Gabriella Zarri, San Pietro in Cariano, Il segno dei Gabrielli, 1997, pp. 339-367.

Novi-ChaVARria 2001

Elisa Novi Chavarria, Monache e gentildonne. Un labile confine. Poteri politici e identità religiose nei monasteri napoletani, sec. XVI-XVII, Milano, Franco Angeli, 2001.

NOVI-ChAVARRIA 2005

ELISA Novi CHAVARRIA (a cura di), La città e il monastero: comunità femminili cittadine nel Mezzogiorno moderno, Napoli, Edizioni scientifiche italiane, 2005.

NOVI-CHAVARria 2009

Elisa Novi-Chavarria, Sacro, pubblico e privato. Donne nei secoli $X V-X V I I I$, Napoli, Guida, 2009.

NOVI-CHAVARRIA 2009²

Elisa Novi-Chavarria, Les rituel de vêture à Naples à l'époque baroque, in Les ceremonies extraordinaires du catholicisme baroque, a cura di Bernard Dompnier, Clermont Ferrand, Presses universitaires Blaise-Pascal, 2009, pp. 349-364.

Nubola/TurChini 1999

Cecilia Nubola- Angelo Turchini (a cura di), Fonti ecclesiastiche per la storia sociale e religiosa d'Europa: XVXVIII secolo, Bologna, Il Mulino, 1999.

OLIVIERI 1999

GuIDo Olivieri, Aggiunte a 'La scuola musicale di Napoli' di F. Florimo: i contratti dei figlioli della Pietà dei Turchini nei protocolli notarili (1677-1713), in Francesco Florimo e l'Ottocento musicale, a cura di Rosa Cafiero e Marina Marino, Reggio Calabria, Jason, 1999, pp. 728-752.

OLIVIERI 2000

GUIDo OlIVIERI, Musica strumentale a Napoli nell'età di Pergolesi: le sonate per tre violini e basso, «Studi pergolesiani / Pergolesi studies», 4, 2000, pp. 193208.

OLIVIERI 2002

GUIDo Olivieri, Tra Napoli e Vienna: musicisti e organici strumentali nel Viceregno austriaco (1701-1736), in Italienische Instrumentalmusik des 18. Jahrhunderts: alte und neue Protagonisten, a cura di Enrico Careri e Markus Engelhardt, Laaber, Laaber Verlag, 2002, pp. 161-182 (Analecta musicologica, $32)$.

OLIVIERI 2009

Guido Olivieri, Cello teaching and playing in Naples in the Early Eigtheenth Century: Francesco Paolo Supriani's «Principij da imparare a suonare il 
violoncello», in Performance Practice: issues and approaches, ed. by Timothy D. Watkins, AnnArbor, Steglein Publishing 2009, pp. 109-136.

OVER 1998

Berthold Over, Per la gloria di Dio: solistische Kirchenmusik an den venezianischen Ospedali im 18.Jahrhundert, Bonn, Orpheus-Verlag, 1998.

PANe 1954

Roberto PANe, Il chiostro di Santa Chiara in Napoli, Napoli, L'arte tipografica, 1954.

PAOLIN 1996

Giovanna Paolin, Lo spazio del silenzio. Monacazioni forzate, clausura e proposte di vita religiosa femminile nell'eta moderna, Pordenone, Biblioteca dell'Immagine, 1996.

Paschini 1960

Pio Paschini, I monasteri femminili in Italia nel '500, in Problemi di vita religiosa in Italia nel Cinquecento, Padova, Antenore, 1960, pp. 31-59.

PASTORE 2007

GiUsepPe PAStore, Due composizioni sacre di Alessandro Speranza: raccolta di documenti, in Archivi e Storia di Terra d'Otranto, a cura di Mario Spedicato, Galatina, EdiPan, 2007, pp. 55-64.

Pellegrino/Gaudioso 1987

Bruno Pellegrino-Francesco Gaudioso, Ordini religiosi e Società nel Mezzogiorno moderno, Galatina, Congedo, 1987.

PENCO 1961

Gregorio Penco, Storia del monachesimo in Italia dalle origini alla fine del medioevo, Roma, Paoline, 1961.

PEVERADA 1991

Enrico PeVerada, Vita musicale nella chiesa ferrarese del Quattrocento, Ferrara, Archivio Capitolare, 1991.

PEVERADA 1997

Enrico Peverada, Documenti per la storia organaria dei monasteri femminili ferraresi (sec. 16.-17.), Bologna, Patron, 1997.

Prota-Giurleo 1952

Ulisse Prota-Giurleo, Breve storia del Teatro di Corte e della musica a Napoli nei secoli XVII-XVIII, in Il Teatro di Corte del Palazzo Reale di Napoli, a cura di Felice De Filippis e Ulisse Prota-Giurleo, Napoli, L'Arte Tipografica, 1952, pp. 19-77. 
Prota-Giurleo $1952^{2}$

Ulisse Prota-Giurleo, Pittori montemurresi del '600, Montemurro, Comune di Montemurro, 1952.

PROTA-GIURLEO 1961

Ulisse Prota-Giurleo, Organari napoletani del XVII e XVIII secolo, «L'Organo», II, 1961, pp. 109-126.

Prota-Giurleo 1966

Ulisse Prota-Giurleo, Matteo Sassano detto «Matteuccio» (Documenti napoletani), «Rivista italiana di musicologia», I, 1966, pp. 97-119.

Prota-GiURLeo 1984

Ulisse Prota-Giurleo, Civiltà del Seicento a Napoli, 2 voll., Napoli, Electa, 1984.

REARDON 2000

COLlEEN REARDON, "Ho innalzato un muro attorno a me»: Lamentazioni per monache senesi, in I monasteri femminili come centri di cultura fra Rinascimento e barocco, a cura di Gabriella Zarri e Gianna Pomata, Roma, Edizioni di storia e letteratura, 2000, pp. 27-44.

REARDON 2002

Colleen Reardon, Holy Concord within Sacred Walls: Nuns and Music in Siena, 1575-1700, New York, Oxford University Press, 2002.

REARDON 2005

COLLEEN ReARdon, I monasteri femminili e la vita musicale a Siena, 1550-1700 circa, in Produzione, circolazione e consumo. Consuetudine e quotidianità della polifonia sacra nelle chiese monastiche e parrocchiali dal tardo Medioevo alla fine degli Antichi Regimi, a cura di David Bryant e Elena Quaranta, Bologna, Il Mulino, 2005, pp. 167-192.

RENDA 1978

FRANCESCO ReNDA, Dalle riforme al periodo costituzionale 1734-1816, in Storia della Sicilia, vol. VI, a cura di Rosario Romeo, Napoli, Società editrice Storia di Napoli e della Sicilia, 1978.

RigHETTI 1955

Mario Righetti, Manuale di storia liturgica, Milano, Ancora, 1955.

Rosa 1990

MARIo Rosa, L'onda che ritorna: interno ed esterno sacro nella Napoli del '600, in Luoghi sacri e spazi della santità, a cura di Sofia Boesch Gajano e Lucetta Scarrafia, Torino, Rosenberg, 1990, pp. 397-417. 
ROMANO 1980

Stefano Romano, L'arte organaria a Napoli: dalle origini al secolo XX, Napoli, Società Editrice Napoletana, 1980.

Rossi 2000

Pasquale Rossi (a cura di), La Croce di Lucca in Napoli. Vicende storiche e artistiche di un monastero carmelitano, Roma, Elio de Rosa, 2000.

Russo 1970

CARLa Russo, I monasteri femminili di clausura a Napoli nel secolo XVII, Napoli, Istituto di Storia Medievale e Moderna, 1970.

SARTORI 1994

Claudio SARTORI, I libretti italiani a stampa dalle origini al 1800: catalogo analitico con 16 indici, Cuneo, Bertola \& Locatelli, 1994.

SCARAFFIA/ZARRI 1994

Lucetta Scaraffia-Gabriella Zarri (a cura di), Donne e fede. Santità e vita religiosa in Italia, Bari, Laterza, 1994.

SELFRIDGE-FIELD 1985

Eleanor Selfridge-Field, Pallade veneta: Writings on Music in Venetian Society 1650-1750, Venezia, Fondazione Levi, 1985.

SHEARON 1993

StePHEN MARK SHEARon, Latin sacred music and Nicola Fago: the career and sources of an early eighteenth-century Neapolitan maestro di cappella, Ann Arbor, Michigan, 1993.

Sisto/CARDI 2005

Luigi Sisto-Emanuele CARDi (a cura di), Napoli e L'Europa: gli strumenti, i costruttori e la musica per organo dal XV al XX secolo, Battipaglia, Accademia Organistica Campana, 2005.

SPINOSA/Pinto/VAlerio 2013

Nicola Spinosa-Aldo Pinto-Adriana Valerio (a cura di), San Gregorio Armeno. Storia, architettura, arte e tradizioni, Napoli, Fridericiana Editrice Universitaria, 2013.

STEFANI 1975

Gino Stefani, Musica e religione nell'Italia barocca, Milano, Flaccovio, 1975.

STRAZZULLO 1955

Franco Strazzullo, Il monastero e la chiesa dei Ss. Marcellino e Festo (Documenti inediti), «Archivio Storico per le Province Napoletane», 35, 1955, p. 438. 
STRAZZULLO 1961

Franco Strazzullo, I diari dei cerimonieri della Cattedrale di Napoli: una fonte per la storia napoletana, Napoli, Agar, 1961.

STRAZZULlo 1968

Franco Strazzullo, Edilizia e urbanistica a Napoli dal '500 al '700, Napoli, Berisio 1968.

STRAZZULLO 1975

Franco Strazzullo, Documenti per la chiesa e il monastero di San Francesco degli Scarioni, «Napoli Nobilissima», 14, 1975, pp. 70-77.

STRAZZULlo 1978

Franco Strazzullo, Dietro le grate del divino amore: il Settecento religioso a Napoli, Napoli, Edizioni di Napoli nobilissima, 1978.

TeDEsco 2001

Anna Tedesco, La cappella de' militari spagnoli di Nostra Signora della Soledad di Palermo, in Giacomo Francesco Milano ed il ruolo dell'aristocrazia nel patrocinio delle attività musicali nel secolo XVIII, a cura di Gaetano Pitarresi, Reggio Calabria, Laruffa, 2001, pp. 169-254.

\section{TORELLI 2005}

DANIELE TORELl, Notazioni ritmiche e canto fratto nelle edizioni non liturgiche tra Cinquecento e Seicento, in Il canto fratto. L'altro gregoriano, a cura di Marco Gozzi e Francesco Luisi, Roma, Torre d'Orfeo, 2005 («Miscellanea musicologica», 7), pp. 447-492.

TORELLI 2010

Daniele Torelli, «Cantores inchoent sequentem antiphonam». Canto piano e canto figurato nella liturgia quotidiana tra Cinque e Seicento, «Barocco Padano», 6, a cura di Alberto Colzani, Andrea Luppi, Maurizio Padoan, Como, A.M.I.S., 2010, pp. 218-249.

Tufano 2009

Lucio Tufano, Il mestiere del musicista: formazione, mercato, consapevolezza, immagine, in Storia della musica e dello spettacolo a Napoli. Il Settecento, a cura di Francesco Cotticelli e Paologiovanni Maione, Napoli, Turchini Edizioni, 2009, pp. 773-804.

TURANO 1988

Francesca TURANo, La musica sacra di Gaetano Veneziano: qualche verifica e ipotesi di ricerca, in Musicisti nati in Puglia ed emigrazione musicale tra Seicento e Settecento, a cura di Detty Bozzi e Luisa Cosi, Roma, Torre d'Orfeo, 1988, pp. 37-48. 
VALENZI 1995

Lucia ValenZI, Poveri, ospizi e potere a Napoli (XVIII-XIX sec.), Milano, Franco Angeli, 1995.

VALERIO 2006

Adriana VALERIO, Istituti religiosi femminili a Napoli dal IV al XVI sec., Napoli, Voyage pittoresque, 2006.

VALERIO 2007

AdRIANA VALERIO, Istituti religiosi femminili a Napoli dal 1600 al 1861, Napoli, Voyage pittoresque, 2007.

VINCENZI 1984

Marcella Vincenzi, La musica a Napoli, Napoli, Arturo Berisio Editore, 1984.

VitALE 1969

GiUliana Vitale, Ricerche sulla vita religiosa e caritativa a Napoli tra medioevo ed età moderna, "Archivio storico per le province napoletane», 85/86, 1968-69, pp. 265-292.

WEAVER 2002

Elissa Weaver, Convent Theatre in Early Modern Italy: Spiritual Fun and Learning for Woman, Cambridge, Cambridge Univesity Press, 2002.

ZARRI/POMATA 2000

GABRIELla ZARRI - GIANNA POMATA, I monasteri femminili come centri di cultura fra Rinascimento e barocco, Roma, Edizioni di storia e letteratura, 2000.

ZARRI 1986

GabrIella ZARRI, Monasteri femminili e città (secoli XV-XVIII), in Storia d'Italia. Annali IX. La Chiesa e il potere politico dal medioevo all'età contemporanea, a cura di Giorgio Chittolini e Giovanni Miccoli, Torino, 1986, pp. 357-429.

ZARRI 1990

GABRIELLA ZARRI, Le sante vive. Cultura e religiosità femminile nella prima età moderna, Torino, Rosenberg \& Sellier, 1990.

ZARRI 1996

GABrIELla ZARri (a cura di), Donna, disciplina e creanza cristiana dal XV al XVII secolo, Roma, Studi e testi a stampa, 1996.

ZARRI 1997

GABRIELLA ZARRI (a cura di), Il monachesimo femminile in Italia dall'alto medioevo al secolo XVII, a confronto con l'oggi, San Pietro in Cariano, Il segno dei Gabrielli, 1997. 


\section{ZARRI 1999}

GABRIELla ZARRI, Ordini religiosi e autorità episcopale: le visite pastorali a chiese esenti e monasteri, in Fonti ecclesiastiche per la storia sociale e religiosa d'Europa: XVXVIII secolo, a cura di Cecilia Nubola e Angelo Turchini, Bologna, Il Mulino, 1999, pp. 347-368.

ZARRI 2000

GABRIELla ZARRI, Recinti. Donne, clausura e matrimonio nella prima età moderna, Bologna, Il Mulino, 2000. 


\section{Indice dei Nomi}

Il presente indice comprende i nomi di persone citati nel testo. Non sono stati indicizzati i nomi contenuti in citazioni di documenti d'archivio. In corsivo sono riportati i riferimenti a tavole e appendici.

\section{A}

Abos, Girolamo 112, 118, 135, 135n Acquaviva, Foresta 90

Acquaviva, Ottavio d'Aragona (arcivescovo) 36, 36n, 95n

Agresta, Agustin (Agostino) 71, 232

Alarcon y Mendoza, Isabella 145

Aldobrandini, Elena 190

Alensoon, Jan 212n

Alfieri, Giuseppe $117 \mathrm{n}$

Alfonso d'Aragona 146

Alfonso II d'Aragona 158

Altamura, Domenico 127, 227

Altamura, Nicola 227

Amendola, Andrea 63, 88, 93, 123,

$124,132,136,156,160,223$,

$224,225,227,229,230,231$

Amenta, Nicolò 73, 73n

Amorevoli, Angelo Maria 86, 87, 87n

Amoruso (Amoroso), Bartolomeo

216, 232

Andreasso, Francesco 191, 235

Andrisani, Pietro 101n

Anfossi, Pasquale 198

Ansalone, Andrea 97, 214, 232, 233

Antonelli, Attilio 88n, 179n

Apice (violino) 89

Aprile, Giuseppe 135

Aragona (famiglia) 148

Araldo, Giovan Francesco 155n, 203n

Arcucci, Domenico 143, 223
Arena, Giuseppe 98, 224

Auletta, Pietro 145, 145n, 229

Avitrano, Giuseppe 125, 125n, 135

\section{B}

Bacciagaluppi, Claudio 43n, 86n, 94n, 122n, 130n, 135n, 154n, $162 \mathrm{n}$

Bach, Johann Christian 135

Baffi, Geronimo 99, 234

Baldi, Giuseppe 106

Bandiera, Giovan Nicola 43

Barbatiello, Giovanni Battista 98, 224

Barile, Andrea 98, 191, 235

Barone di Toraldo 126

Bartilotti, Pietro 70, 97, 132, 214, 215, 224, 233

Basciotta, Pietro 234

Basso, Andrea 99, 159, 216, 232

Benedetto XIII (pontefice) 149

Benedetto XIV (pontefice) 80n

Bernardi, Claudio 68n, 69n

Bilanzoni, Francesco 106

Boccadamo, Giuliana 22, 22n, 34n, 36n, 37n, 38n, 124n, 126n, 129n, 185n, 186n, 188n, 189n, 190n, 191n, 192n, 193n, 203n

Bolchi, Elena Lucia 78n, 80n

Boncompagni, Francesco (arcivesco-

vo) $36,36 n, 39 n$

Bonito (famiglia) 174 
Bonito, Delia 112, 117, 174, 174n, 175,222

Bonno, Giuseppe 106

Bonsante, Annamaria 22, 22n, 135n

Bouchard, Jean-Jacques 68, 68n, 69, 69n, 70, 70n

Broggia, Giovan Battista 124n

Broschi, Carlo 87

Bruno, Gioacchino 89

Bryant, David 23, 23n, 59n

Bucci, Angiolo 107

Bulifon, Antonio 146n

Buommattei, Benedetto 78n

Buscé, Nicola 122, 230

\section{C}

Cacace, Giovan Camillo 159, 159n

Cafaro, Pasquale 106, 114, 116, 135n

Cafiero, Rosa 61n, 99n, 187n, 198n

Cailò, Giancarlo 125n

Campanelli, Marcella 61n, 185n, $193 n$

Cangi, Alfonso 151, 224, 228

Canonico (violino) 89

Canosa, Romano 33n

Cantalon (violino) 89

Cantelmo Stuart, Giacomo (arcivescovo) $39,42,42 n, 45 n, 130$

Capano, Grazia 165

Capasso, Bartolomeo 129, 129n

Capece Minutolo (famiglia) 23, 56

Capece Zurlo, Giuseppe Maria (arcivescovo) $47,47 \mathrm{n}$

Capece, Antonia 103, 174

Cappellani, Paola 157

Caprile, Agata 127n

Capuano, Antonio 224

Caracciolo (famiglia) 56, 129, 169

Caracciolo, Aurelia 88

Caracciolo, Battistello 137n, 207, 207n

Caracciolo, Enrichetta 131n
Caracciolo, Francesco 204n

Caracciolo, Fulvia 34n, 131, 132n, $133,164 \mathrm{n}$

Caracciolo, Innico (arcivescovo) 41, 41n, 42, 42n, 159

Caracciolo, Isabella 101

Caracciolo, Lucia 188n

Caracciolo, Maria Agnese 159

Carafa (famiglia) 85, 148, 169

Carafa, Agnese Maria 136n

Carafa, Alfonso (arcivescovo) 34, 34n, 121n, 126, 126n, 131, 148

Carafa, Decio (arcivescovo) 36, 36n, $39 n$

Carafa, Emilia 87

Carafa, Isabella 85

Carafa, Sancia 148

Carapella, Tommaso 226

Caravaggio, Michelangelo Merisi 208, 209n

Carbonelli, Gregorio 233

Carcais, Saverio 89

Cardi, Emanuele 98n

Caresana, Cristofaro 28, 29, 69, 94, 97, 109, 117n, 189, 189n, 199, 214, 228, 233

Caricato, Giuseppe 107

Carletti, Nicolò 220

Carlo di Borbone (re di Napoli) poi Carlo III (re di Spagna) 42, 44n, 73, 74, 75, 86, 137, 162, 182, $183,184,184 n, 213 n$

Carlo II (re di Spagna) 72, 193

Carlo IV di Borbone (re di Spagna) 75

Carlo VI d'Asburgo (re d'Ungheria) 73

Carmignano, Felice 151

Carrero, Vito 98, 224

Carrino, Candida 34n, 132n, 133n

Caserta, Aldo 40n

Castaldo, Giuseppe 101, 105 
Castillo, Juan Domenico 233

Cataneo (violino) 89

Catarinozzi, Cesare 99, 165, 225

Ceci, Giuseppe 161n, 185n

Celano, Carlo 24, 24n, 54, 54n, 70, 70n, 99n, 121n, 124n, 126n, 129n, 131n, 136n, 137n, 139n, 140n, 141n, 144n, 146n, 147n, $148 \mathrm{n}, 150 \mathrm{n}, 152 \mathrm{n}, 154,154 \mathrm{n}$, 156n, 157n, 158n, 159n, 161n, $163,164 n, 165 n, 169 n, 190 n$, 191n, 193n, 194n, 195n, 203n, 204, 204n, 213

Celio Gaudioso, Settimio (vescovo) 137

Cennamo, Nicola 99, 236

Chiarini, Giovanni Battista 204n

Chirico, Petronilla 89

Cicalese, Angela 41n

Cigala, Scipione 143

Cimino, Fabrizio 99, 122, 128, 144, $166,221,222,225,226,227,230$

Cimino, Felice 216, 233

Cimino, Francesco 99, 122, 133, 144, $165,225,226,227,230,236$

Cinque, Filippo 98, 224

Clemente VIII (pontefice) 79n, 152

Clemente XI (pontefice) 42n, 121

Cloche, Antonino 147

Codispoti, Carlo 195, 235

Columbro, Marta 60n, 93n, 98n, 193n, 206n

Confuorto, Domenico 24, 24n, 87n

Consolo, Nicola 96, 98, 191, 236

Conte di Potenza 87

Conte, Carlo 185n

Conti, Carla 23, 23n, 51n, 157n, $187 \mathrm{n}, 194 \mathrm{n}$

Conti, Giuseppe 93, 162, 226

Conti, Nicola (Nicolò) 93, 107, 162, 162n, 163, 116, 226

Coppola, Filippo 60
Corelli, Arcangelo 96

Cortillatto, Pietro 143, 223

Costantini, Danilo 27, 27n, 72n, 73n, 74n, 75n, 99n, 138n, 143n, 162n, 182n, 191n, 193n, 205n, 206n, $212 n$

Cotticelli, Francesco 24n, 25n, 60n, 61n, 71n, 88n, 90n, 93n, 100n, 130 n, 204n

Cozzolani, Chiara Margherita 21

Crescentini, Gerolamo 135

Creytens, Raymond 33n, 34n

Crisci, Angelo 195, 235

Crocco, Gaetano 98, 224

Croce, Benedetto 100n, 156

Croys, Loise 207n, 209n

\section{D}

D'Alessandro, Domenico Antonio $101 \mathrm{n}$

D'Alessio, Silvana 41n

D'Aloe, Stanislao 124n, 126n, 146n, 147n, 148n, 150n, 156n, 158n, $161 n, 165 n, 203 n$

D'Amalfi, Tommaso Aniello 40, 40n, $41 n$

d'Ancona, Giacomo 33

D'Andrea, Gioacchino 169n, 170n, $174 n$

D'Andrea, Maria Chiara 162

D'Angelo, Francesco Antonio 97, 215, 233

D'Ario, Carmela 193n

d'Ascanio, Giuseppe 236

d'Assisi, Pietro 197n

D'Engenio Caracciolo, Cesare 24n, 121n, 124n, 126n, 131n, 136n, 137n, 139n, 141n, 146n, 147n, 148n, 150n, 152n, 156n, 157n, $158 \mathrm{n}, 161 \mathrm{n}, 164 \mathrm{n}, 165 \mathrm{n}, 169 \mathrm{n}$, 188n, 190n, 191n, 194n, 209n 
D’Urso, Gian Angelo (Giovanni Angelo) 97, 215, 233

Da Nadro, Silvino 40n

Daniele, Pietro 236

Daponte, Andrea 224

Daun, Wirich Philipp von (viceré) 73 de Angelis, Decio 232

De Bottis, Giuseppe 93, 98, 190, 191, 215, 233, 234, 235

De Dominici, Bernardo 207n

de Donati, Ferdinando 162, 226

De Donato, Giuseppe 224

de Falco, Michele 98, 195, 235

de Floro, Domenico 155, 231

De Lellis, Carlo 24, 146n, 150n, 203n, 207, 209n, 213

de Luxan y Enriquez, Luis 203

de Macque, Jean 60, 209n

de Magistris, Gennaro 106

de Magistris, Giuseppe 98, 108, 133, $162,163,224,226$

De Maio, Romeo 34n, 36n, 38n, 39n, 40n, 41n, 42n, 44n, 100n, 179n, $185,185 n, 193 n$

De Majo, Domenico 97, 211, 233

de Majo, Francesco 116

de Marco, Pasquale 107

de Maria, Francesco Antonio 160

de Marino, Giuseppe 224

de Marino, Pietro 124, 124n

De Martino, Giovanni 235

De Martino, Giuseppe 158, 229

De Martino, Tommaso 99, 133, 158, 191, 224, 228, 229, 235

De Matteis, Paolo 146n

De Mura, Francesco 55n, 144n, 146n

de Ortega, Jacinto 97, 233

De Ragona, Andrea 216, 232

De Rinaldis, Rinaldo 207n

De Rosa, Luigi 192n

de Rosa, Tommaso 234 de Ruggiero, Nicola 164, 221

De Silva, Vittoria 152

de Simone, Nicolo' 106

De Stefano, Pietro 121n, 124n, 126n, 129n, 136n, 137n, 139n, 147n, $148 \mathrm{n}, 150 \mathrm{n}, 156 \mathrm{n}, 157 \mathrm{n}, 158 \mathrm{n}$, $161 \mathrm{n}, 164 \mathrm{n}, 165 \mathrm{n}, 169 \mathrm{n}, 191 \mathrm{n}$, $194 \mathrm{n}$

de Toledo, Pedro 60n, 194, 205, 206n

de Valdés, Juan 156

De Vita, Francesco 89

Degrada, Francesco 24n

del Giudice, Nicola (principe di Cellamare) $85,85 n, 163,164 n$

Del Prete, Rossella 61n

Delfino, Antonio 150n

dell'Aja, Gaudenzio 169n

Dell'Olio, Antonio 99n

della Leonessa, Caterina 152

della Leonessa, Vittoria 173

Della Marra, Chiara 133

Della Torre, Clemente Filomarino 108

Delmonti, Nicola 107

Dentice, Lucrezia 148, 165

di (da) Bologna, Ludovico 179, 179n, 180

di Gennaro, Agnese 107

Di Giacomo, Salvatore 28, 61n, 96n, 109, 109n, 115, 117n, 130n, 205, $214 n$

Di Napoli, Nicola 97, 97, 196, 232

di Sangro, Beatrice 133

Diano, Giacinto 146n

Diano, Vincenzo 205n

Dietz, Hanns-Bertold 60n, 61n, 146n

Dominguez, José Maria 125n

Dompnier, Bernard 72n

Donadi, Paola 34n

Doria, Costanza del Caretto 190

Duca d'Atri 90, 152

Duca di Bagnara 149 
Duca di Ceppaloni 152

Duca di Gravina 148

Duca di Noja 85

Duca di Parete 145n

Duca di Scarpato 155

Durante, Angelo 63, 124, 127, 155, $156,160,223,225,227,230$, 231

Durante, Francesco 29, 93, 194, 199, 234

\section{$\mathbf{E}$}

Elia, Giuseppe 98, 224

Elisabetta Cristina di Brunswick-

Wolfenbüttel (imperatrice) 193, $213 n$

Elisabetta d'Ungheria 147

Evangelisti, Silvia 75n

\section{$\mathbf{F}$}

Fabris, Dinko 23, 60n, 61n, 62n, 68n, 69n, 85n, 93n, 96n, 127n, 205, 206n, 207n

Facchiano, Annamaria 22n, 77n, 136n, $185 \mathrm{n}$

Fago, Nicola 88, 93, 114, 135n, 138, $144,193,222,223,225,228$, 236

Falconieri, Andrea 60, 85n

Fanzago, Cosimo 55n, 137n, 148n, $152 \mathrm{n}$

Farinelli $\rightarrow$ Carlo Broschi

Federico Cristiano di Sassonia 74n

Federico, Gennarantonio 106

Fenaroli, Fedele 135n, 146n

Feo, Francesco 29, 87n, 93, 112, 117, $117 \mathrm{n}, 140,228$

Fernandes, Cristina 82n

Ferrante I d'Aragona 165

Ferrara, Giuseppe 224

Ferrari, Bartolomeo 115
Ferraro, Geronimo 99, 223

Ferreri, Giovanni Gualberto 216, 233

Ferreri, Giuseppe Gualberto 143, 223

Filangieri, Gaetano 137n

Filangieri Ravaschieri Fieschi, Teresa $185 n$

Filangieri, Maria Anna 135

Filangieri, Marianna 135

Filangieri, Riccardo 207n

Filangieri, Serafino (arcivescovo) 47n

Filippo V di Borbone (re di Spagna) 96

Filippo di Borbone (principe) 75

Filomarino, Ascanio 22, 36, 38n, 39, 39n, 40, 40n, 41, 41n, 45, 85

Filomena, Giuseppe 146, 221

Fiore, Angela 56n, 60n, 80n, 84n, $94 n, 113 n, 204 n$

Fiorelli, Vittoria 22, 22n

Fiorenza, Nicola 154, 154n

Fiorillo (basso) 89

Fisanio Teutronio 107, 108

Fischietti, Fedele 205n

Florimo, Francesco 61n

Fuidoro, Innocenzo 24, 24n

G

Gabrielli, Giulia 178n

Gaglione, Mario 169n, 170n, 174n

Galante, Gennaro Aspreno 121n, 124n, 126n, 129n, 131n, 136n, 137n, 139n, 140n, 141n, 144n, 145n, 146n, 147n, 148n, 150n, $152 \mathrm{n}, 155 \mathrm{n}, 156 \mathrm{n}, 157 \mathrm{n}, 158 \mathrm{n}$, 159n, 161n, 164n, 166n, 169n, 188n, 190n, 191n, 192n, 194n, 195n, 203n

Galasso, Giuseppe 22n, 34n, 36n, 41n

Galletti, Francesco 137, 223

Gallino, Tommaso Maria 52n,169n

Gallo, Antonio Maria (cardinale) 95 
Gallo, Pietro Antonio 146n

Galuppi, Baldassarre 198

Gambacorta, Francesca 161n

Genovese, Ettore 185n

Gesualdo, Alfonso 38n, 186n

Geyer, Helen 21, 21n, 197n

Giacchi, Francesco 106

Giannini, Simone 191, 195, 228, 235, 236

Giannoni, Cesare 135

Gillio, Pier Giuseppe 21, 21n, 22, 94n, 197n, 198n, 199n, 200n

Gioffredo, Mario 146n, 158

Giordano, Luca 55n, 131, 137n, 146n, 150n, 161n, 189, 204, 204n

Giovanna III d'Aragona 165

Giovanni d'Austria 146

Giron-Panel, Caroline 197n, 198n

Giugno, Paolo 97, 222

Giuliano, Luigi 106

Giuseppa d'Aragona 140

Gizzi, Domenico 89, 130, 130n

Gluck, Christoph Willibald 87n

Gonzaga, Giulia 156

Gozzi, Marco 178n

Grasso, Gennaro 98, 236

Grillo, Nicola 115

Grimaldi, Livia 140

Grimaldi, Nicola (Nicolino) 181

Grimaldi, Placida 140

Grippaudo, Ilaria 60n

Guéranger, Prosper 80n

Guidi, Laura 185n, 186n

Gurriero (violino) 89

\section{H}

Hameline, Jean-Yves 72n

Handel, Georg Friedrich 135

Hasse, Johann Adolph 87n, 109, 115, 116, 162, 198

Hills, Helen 22, 22n, 53n
I

Iacono, Sarah M. 80n, 113n

Illibato, Antonio 22n, 51n, 53n, 54n, 185n, 186n, 187n, 193n

Innocenzo IV (pontefice) 169

Innocenzo XII (pontefice) 42n, 46, 148

Insanguine, Giacomo 135

Intini, Eloisa 93n, 206n

\section{J}

Jommelli, Niccolò 198

K

Kendrick, Robert 21, 21n

Krause, Ralph 60n

\section{$\mathbf{L}$}

Labruzzo, Nicola 97, 236

Lambiase, Matteo 155

Larson, Keith A. 93n, 214n

Latilla, Gaetano 198

Latro, Giovanni 148

Lazzari, Dioniso 148n

Leclerc, Simone 107

Leo, Leonardo 88, 94, 109, 114, 116, 130n, 162, 226

Leone XIII 80n

Leopoldo Giovanni d'Asburgo (arciduca d'Austria) 73

Longhi, Roberto 207n

Lowe, Kate 21, 21n, 75n

Lucibello, Giuseppe 224

Luigi III d'Angiò (re di Napoli) 146

\section{M}

Magaudda, Ausilia 27, 27n, 72n, 73n, 74n, 75n, 99n, 138n, 143n, 162n, 182n, 191n, 193n, 205n, 206n, $212 \mathrm{n}$ 
Magino, Domenico 233

Maione, Paologiovanni 24n, 25n, 60n, 61n, 71n, 88n, 90n, 93n, 100n, 130n, 204n, 205

Majella (canonico) $87 \mathrm{n}$

Majone, Ascanio 60

Malizia, Domenico Geronimo 106

Mallozzi, Taddeo 89

Mamy, Silvie 87n

Mancinelli, Giuseppe 107

Mancini, Francesco 212n

Mancini, Franco 71n, 179n

Manna, Andrea 89

Manna, Gaetano 111, 117, 140, 141, $141 \mathrm{n}, 146,146 \mathrm{n}, 221$

Manna, Gennaro 109, 112, 116, 117, $135,155,231$

Manso, Domenico 97, 214, 232

Manzuoli, Giovanni 106

Marchitelli, Pietro 29, 96, 97, 125n, 215, 233

Margherita d'Aragona 161n

Maria Amalia di Sassonia (regina di Napoli) 73, 74, 74n, 75, 86, 148

Maria Isabella Antonia di Borbone (principessa) 75

Maria Josefa Antoinette di Borbone (principessa) 75

Maria Teresa Anna di Borbone (principessa) 75

Mariconda, Caterina 161n

Marinelli, Francesco 143, 223

Marino, Marina 61n, 99n, 109n

Marinotti, Nicola 97, 222

Martin, Pedro Calleja 211n

Martino V (pontefice) 146

Masaniello $\rightarrow$ Tommaso Aniello

D'Amalfi

Mazza, Mario 204n

Medici (famiglia) 152

Megale, Teresa 62n
Melchiorre, Domenico 125

Melospirito, Pompeo 106

Melucci, Maria Grazia 22, 22n

Meo, Ascanio 71, 232

Mero (violino) 89

Metastasio, Pietro 106

Metz, René 76n, 78n

Miele, Michele 33n, 34n, 35n, 40n, $41 \mathrm{n}, 161 \mathrm{n}$

Milano, Cornelia 133

Miller Lawrence, Cyntia 21, 21n

Mirabello (Mirabella), Onofrio 96, 97, 215, 233

Mirabile, Fabio 232

Molinaro, Ambrosio 158, 229

Monopoli, Giacomo 98, 224

Monson, Craig 21, 21n, 33n

Montalto (famiglia) 165

Monticelli, Angelo Maria 138, 138n

Morgese, Angela 22, 22n

Muscato, Nicola 99, 122, 230

Mysliveček, Josef 135

\section{$\mathbf{N}$}

Nappi, Eduardo 71n, 159n, 160n, 179 n

Nauclerio, Giovan Battista 155n, 166

Nelvi, Pantalone 107

Nicoletti, Giuliano 106

Nocerino, Francesco 98n, 193n

Nola, Antonio 117

Novi-Chavarria, Elisa 22, 22n, 33n, $34 \mathrm{n}, 36 \mathrm{n}, 55 \mathrm{n}, 56 \mathrm{n}, 77 \mathrm{n}, 85 \mathrm{n}$, $150 \mathrm{n}, 152 \mathrm{n}, 164 \mathrm{n}$

\section{O}

Olivieri, Guido 61n, 125n, 154n

Oliviero, Aurelia 161n

Oliviero, Lucrezia 161n

Orsini (famiglia) 148

Orsini Vizzani, Lucrezia 21 
Ortiz, Diego 60n

Osthoff, Wolfgang 21n, 197n

Over, Berthold 21n, 197n

\section{$\mathbf{P}$}

Paci, Antonio 152

Pagano, Tommaso 164, 221

Pagano, Vincenzo 164, 221

Paisiello, Giovanni 61, 135, 135n

Pallino, Ferdinando 89

Pane, Roberto 52n, 169n

Paolin, Giovanna 33n, 53n

Paolo IV (pontefice) 121n

Paolucci, Fabrizio (cardinale) 171n

Paparo (famiglia) 190

Paparo, Agata 190

Paparo, Giovanna 190

Paparo, Luisa 190

Papebrochio Fungoni 106

Parrino, Domenico Antonio 207n

Paschini, Pio 34n

Pasquale, Francesco 106

Pasquandrea, Roberto 22n

Pastore, Giuseppe 80n, 81n, 113n

Patrizio, Marcello 151, 228

Pellegrino, Nicola 106

Pérez, Enrique Guevara 211n

Pergolesi, Giovanni Battista 135, 212n

Perla, Michele 98, 224

Pertici (tenore) 89

Peverada, Enrico 21, 21n

Picchiatti, Francesco Antonio 143

Piccinni, Niccolò 61

Piccolomini (famiglia) 148

Pignatelli (famiglia) 56, 152, 169

Pignatelli, Antonio 42n

Pignatelli, Caterina 135

Pignatelli, Eleonora 133

Pignatelli, Francesco (arcivescovo)

$42,42 n, 43,44 n, 46,68,90,121$, $149,171,171 \mathrm{n}, 172 \mathrm{n}$
Pignatelli, Maria Antonia 108n

Pinacci, Giovanni Battista 152

Pinto, Aldo 22n, 34n, 51n, 54n, 131n, $132 n$

Pinto, Francesco Emanuele (principe di Ischitella) 163, 163n

Pio V (pontefice) 34

Pio, Antonio 107

Pisano, Gaetano 89, 140, 228, 235

Pisano, Nicola 89, 140, 228

Planelli, Lorenzo 140, 228

Pomata, Gianna 21n

Porpora, Nicola Antonio 87n, 109, 116, 130n, 162, 198, 199

Preti, Mattia 144n

Principe di Belvedere 145n

Principe di Ischitella $\rightarrow$ Francesco Emanuele Pinto

Principe di Marano 138

Principe di Marzano 149

Principessa della Riccia 148

Prota-Giurleo, Ulisse 60n, 87n, 98n, 130n, 205, 207, 207n, 209n

Prota, Filippo 97, 130, 196, 226, 232

Prota, Rosalia 157, 157n

Provenzale, Francesco 61, 101, 105, $109,109 n, 214 n$

\section{Q}

Quaranta, Elena 23n, 59n

$\mathbf{R}$

Raicola, Antonio 211, 212

Ranucci, Saverio 145, 229

Ravaschiero, Isabella 172

Reardon, Colleen 21, 21n, 70n

Recco, Nicolò 107

Renda, Francesco 184n

Ricchezza, Donato 116

Righini, Vincenzo 135

Rispoli, Salvatore 226 
Roberto d'Angiò (re di Napoli) 138, $156,169,179 \mathrm{n}$

Romano, Stefano 98n

Rossi, Domenico Antonio 133

Ruffo, Caterina 141

Ruffo, Ippolita 141

Russo, Carla 22, 22n, 37n, 38n, $56 n, 185 n$

Russo, Domenico Antonio 224

Russo, Giovan Battista 223

Russomando, Domenico 160

\section{$\mathbf{S}$}

Sabatini, Francesco 96, 98, 195, 235

Sabatino, Antonio 193, 193n, 236

Sabatino, Gaspare 193, 193n, 236

Sabatino, Nicola 135

Sabino, Giovanni Maria 126, 126n, $127,127 \mathrm{n}, 227$

Sacchini, Antonio 108, 163, 198

Salvatore, Giovanni 214n

Sancia di Maiorca (regina) 138, 156, $157,158,158 \mathrm{n}, 169,169 \mathrm{n}, 178 \mathrm{n}$

Sarcuni, Giacomo 98, 112, 118, 118n, $155,160,195,224,225,231$, 235

Sarnelli, Pompeo 152, 152n

Sarnicchiaro, Gennaro 106

Sarraca (arciliuto) 89

Sarro, Domenico 87, 88, 94, 190, 230,232

Sartori, Claudio 103, 103n, 105, 105, 106, 107, 108

Sassano, Aurelio 97, 214, 232

Sassano, Matteo 87, 87n, 125, 140, $144,152,181,196,212,212 \mathrm{n}$

Sbarra, Andrea 163

Scalera, Marianna 162

Scarioni, Leonardo 166

Scarlatti, Alessandro 60, 61, 111n, 130n, 212
Scarlatti, Pietro 196, 235

Schisano, Nicola 160, 225

Schuster, Joseph 135

Scitta, Domenico 98, 224

Scoppa, Carmine 99, 128, 227

Scoppa, Francesco 99, 122, 230

Selfridge-Field, Eleanor 87n, 197n

Sellitto, Carlo 207, 207n, 209n

Senese, Juan Domenico 233

Serao, Matilde 126n

Sersale, Caterina Maria 106

Sersale, Maddalena 135

Sigismondo, Giuseppe 141n, 145n, 146n, 152n, 159n, 166n, 169, 170n, 178, 178n, 179

Sisto, Luigi $98 \mathrm{n}$

Solimena, Francesco 55n, 73, 137n, $150 \mathrm{n}$

Speranza, Alessandro 80, 81, 82, 108, 108n, 113, 113n, 114, 116, 162, 163,226

Spinelli (famiglia) 152

Spinelli, Cremona 163

Spinelli, Giuseppe (arcivescovo) 40, $44 \mathrm{n}, 86,87 \mathrm{n}, 122,152,160 \mathrm{n}$

Spinosa, Nicola 22n, 34n, 51n, 54n, $131 \mathrm{n}, 132 \mathrm{n}$

Stendardo, Marino 148

Stendardo, Pietro 148

Strazzullo, Franco 36n, 37n, 38n, 40n, 44n, 54n, 121n, 154n, 166n, $171 \mathrm{n}, 185 \mathrm{n}$

Stuck, Jean-Baptiste 125

Supriani (Sopriano), Francesco 154, $154 n$

\section{$\mathbf{T}$}

Tango, Antonio 127n

Tango, Tommaso 130, 158, 223, 226, 229

Tedesco, Anna 210n 
Terillo (Terrillo), Carlo 98, 146, 221

Thouret, Giovanna Antida 161n

Tissoate, Prineo 108

Tomacelli, Caterina 141

Torelli, Daniele 174n

Trabaci, Giovanni Maria 60, 85n, 207, 209n, 210, 233

Traetta, Tommaso 198

Trigoso, Pietro 203

Tringhera (Trinchera), Francesco 97, 194, 234

Tritto, Giacomo 98, 107, 117, 135, $135 \mathrm{n}, 224$

Troiano, Antonio 236

Trojano, Ferdinando 106

Tufano, Lucio 60n, 93n

Turano, Francesca 109n, 193n

\section{$\mathbf{U}$}

Urbano VIII (pontefice) 40n, 79n

Ursini, Maria Scolastica 149

Ursino, Gennaro 28, 70, 97, 212, 214, 214n, 233

\section{V}

Vaccaro, Andrea 204n, 207, 207n

Vaccaro, Domenico Antonio 52n, 55n

Vaccaro, Lorenzo 161n

Valenzi, Lucia 186n

Valerio, Adriana 22, 22n, 34n, 36n, $37 \mathrm{n}, 38 \mathrm{n}, 51 \mathrm{n}, 54 \mathrm{n}, 55 \mathrm{n}, 121 \mathrm{n}$, 124n, 126n, 129n, 131n, 132n, 136n, 137n, 139n, 140n, 141n, $144 \mathrm{n}, 145 \mathrm{n}, 146 \mathrm{n}, 147 \mathrm{n}, 148 \mathrm{n}$, $150 \mathrm{n}, 152 \mathrm{n}, 155 \mathrm{n}, 156 \mathrm{n}, 157 \mathrm{n}$, 159n, 161n, 164n, 165n, 166n, $169 \mathrm{n}, 185 \mathrm{n}, 187 \mathrm{n}, 188 \mathrm{n}, 190 \mathrm{n}$, 191n, 192n, 193n, 194n, 195n, $203 n$
Vanvitelli, Luigi 55n

Vecchione, Ernesto 185n

Veneziano, Bonaventura 96, 97, 195, 235

Veneziano, Gaetano 28, 29, 69, 70, 94, 97, 109, 109n, 110, 111, 115, 117, 117n, 193, 193n, 199, 211, 212, 218, 233, 236

Veneziano, Nicolò 118

Veneziano, Giulia 206n

Vernucci, Matteo 195, 235

Villani, Beatrice 143

Villani, Eugenia 126

Villapiana, Maria Giuseppa 153

Vinci, Leonardo 130n

Vinditti, Angelo 98, 224

Vitale, Francesco 140, 173

Vitale, Giuliana $185 \mathrm{n}$

Vivaldi, Antonio 87n, 159, 198, 199

Voss, Hermann 207n

\section{W}

Weaver, Elissa 100n, 102n

\section{$\mathbf{Z}$}

Zamparelli, Dioniso 145, 229

Zarri, Gabriella 21, 21n, 33n, 34n, 36n, 53n, 75n, 76n, 78n

Ziani, Pietro Andrea 60, 214

Zingone, Battista 106

Zitellini, Rodolfo 94n

Zuppardi, Feliciano 146 